\title{
Constructive methods of invariant manifolds for kinetic problems
}

\author{
Alexander N. Gorban ${ }^{\mathrm{a}, \mathrm{b}, \mathrm{c}, *}$, Iliya V. Karlina ${ }^{\mathrm{a}, \mathrm{b}}$, Andrei Yu. Zinovyev ${ }^{\mathrm{b}, \mathrm{c}}$ \\ ${ }^{a}$ ETH-Zentrum, Department of Materials, Institute of Polymers, Sonneggstr. 3, ML J19, CH-8092 Zürich, \\ Switzerland \\ ${ }^{\mathrm{b}}$ Institute of Computational Modeling SB RAS, Akademgorodok, Krasnoyarsk 660036, Russia \\ ${ }^{\mathrm{c}}$ Institut des Hautes Etudes Scientifiques, Le Bois-Marie, 35, route de Chartres, F-91440, Bures-sur-Yvette, France
}

Accepted 13 March 2004

editor: I. Procaccia

\begin{abstract}
The concept of the slow invariant manifold is recognized as the central idea underpinning a transition from micro to macro and model reduction in kinetic theories. We present the Constructive Methods of Invariant Manifolds for model reduction in physical and chemical kinetics, developed during last two decades. The physical problem of reduced description is studied in the most general form as a problem of constructing the slow invariant manifold. The invariance conditions are formulated as the differential equation for a manifold immersed in the phase space (the invariance equation). The equation of motion for immersed manifolds is obtained (the film extension of the dynamics). Invariant manifolds are fixed points for this equation, and slow invariant manifolds are Lyapunov stable fixed points, thus slowness is presented as stability.

A collection of methods to derive analytically and to compute numerically the slow invariant manifolds is presented. Among them, iteration methods based on incomplete linearization, relaxation method and the method of invariant grids are developed. The systematic use of thermodynamics structures and of the quasi-chemical representation allow to construct approximations which are in concordance with physical restrictions.

The following examples of applications are presented: nonperturbative derivation of physically consistent hydrodynamics from the Boltzmann equation and from the reversible dynamics, for Knudsen numbers $K n \sim 1$; construction of the moment equations for nonequilibrium media and their dynamical correction (instead of extension of list of variables) to gain more accuracy in description of highly nonequilibrium flows; determination of molecules dimension (as diameters of equivalent hard spheres) from experimental viscosity data;
\end{abstract}

\footnotetext{
* Corresponding author. ETH-Zentrum, Department of Materials, Institute of Polymers, Sonneggstr. 3, ML J19, CH-8092 Zürich, Switzerland.

E-mail addresses: agorban@mat.ethz.ch (A.N. Gorban), ikarlin@mat.ethz.ch (I.V. Karlin), zinovyev@ihes.fr (A.Y. Zinovyev).
} 
model reduction in chemical kinetics; derivation and numerical implementation of constitutive equations for polymeric fluids; the limits of macroscopic description for polymer molecules, etc.

(c) 2004 Elsevier B.V. All rights reserved.

PACS: 05.20.Dd; 02.30.Mv; 02.70.Dh; 05.70.Ln

Keywords: Model reduction; Invariant manifold; Entropy; Kinetics; Boltzmann equation; Fokker-Planck equation; Navier-Stokes equation; Burnett equation; Quasi-chemical approximation; Oldroyd equation; Polymer dynamics; Molecular individualism; Accuracy estimation; Post-processing

\section{Contents}

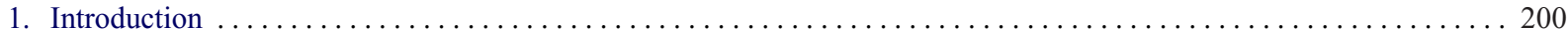

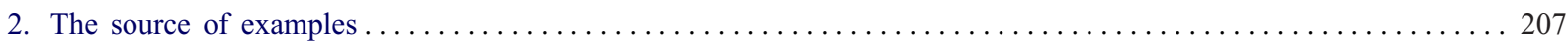

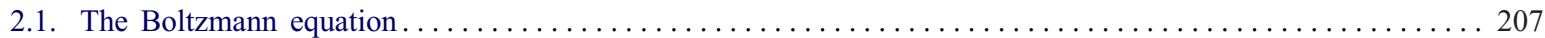

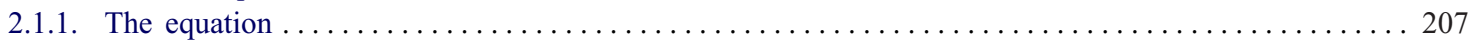

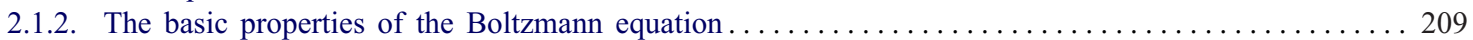

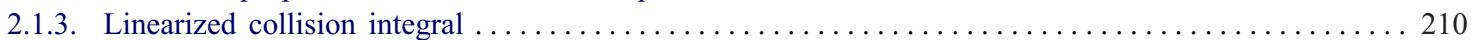

2.2. Phenomenology and Quasi-chemical representation of the Boltzmann equation $\ldots \ldots \ldots \ldots \ldots \ldots \ldots \ldots \ldots$

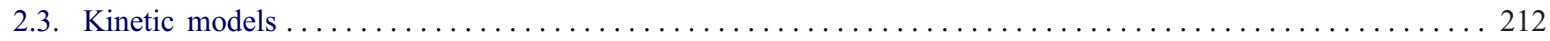

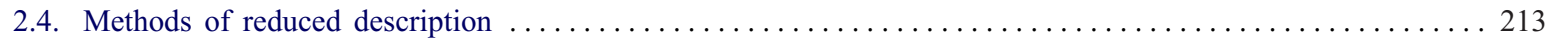

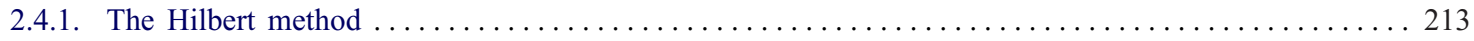

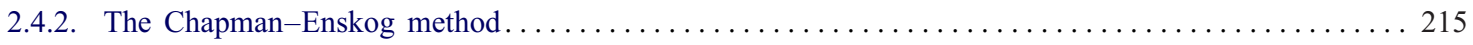

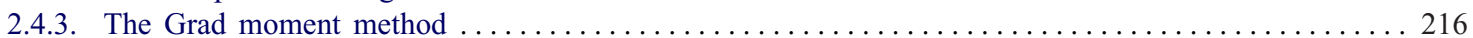

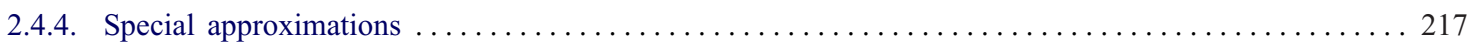

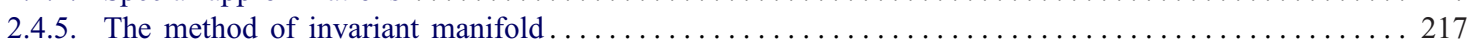

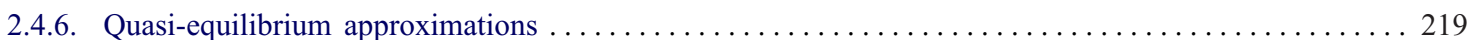

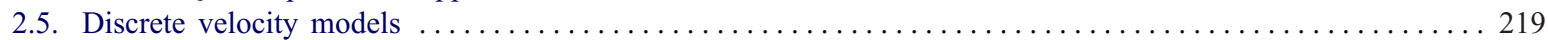

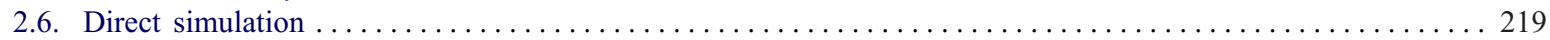

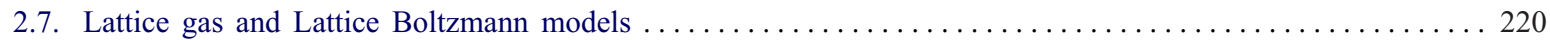

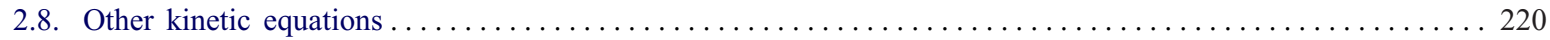

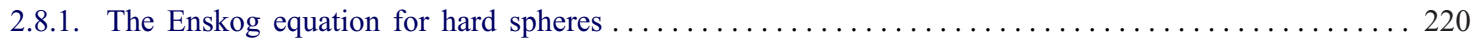

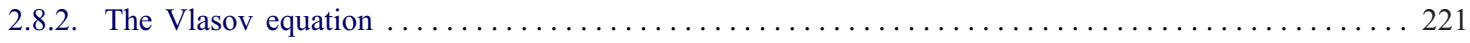

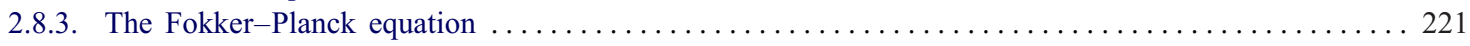

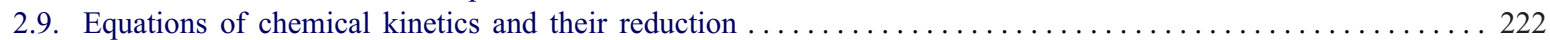

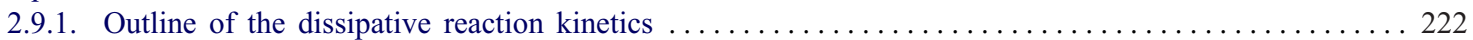

2.9.2. The problem of reduced description in chemical kinetics $\ldots \ldots \ldots \ldots \ldots \ldots \ldots \ldots \ldots$

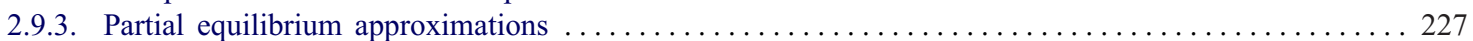

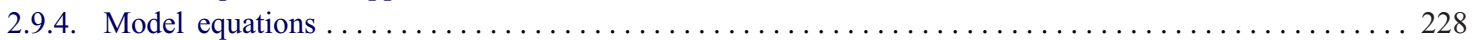

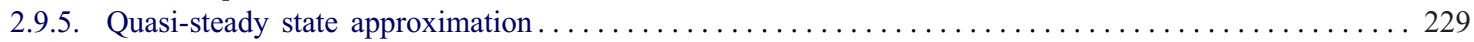

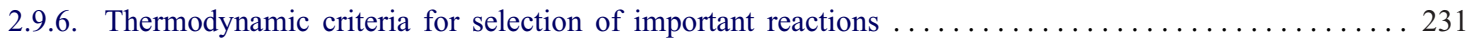

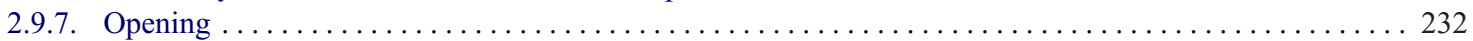

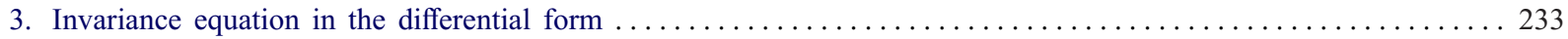

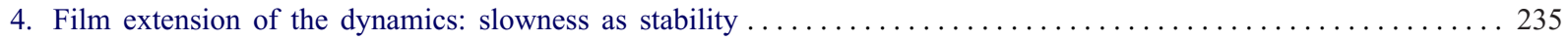

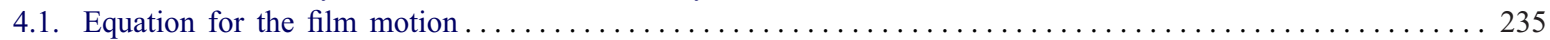

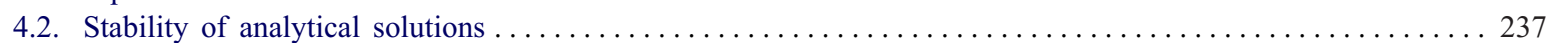

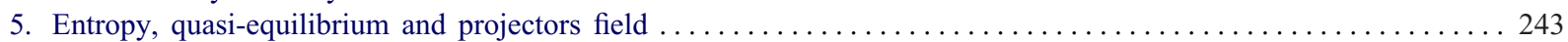

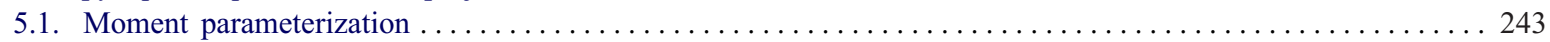




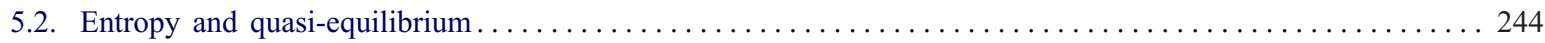

5.3. Thermodynamic projector without a priori parameterization $\ldots \ldots \ldots \ldots \ldots \ldots \ldots \ldots \ldots \ldots \ldots \ldots \ldots \ldots$

Example 1: Quasi-equilibrium projector and defect of invariance for the local Maxwellians manifold of the

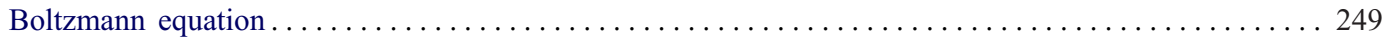

Example 2: Scattering rates versus moments: alternative Grad equations $\ldots \ldots \ldots \ldots \ldots \ldots \ldots \ldots \ldots \ldots \ldots 253$

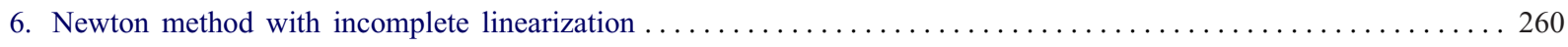

Example 3: Nonperturbative correction of Local Maxwellian manifold and derivation of nonlinear hydrodynamics

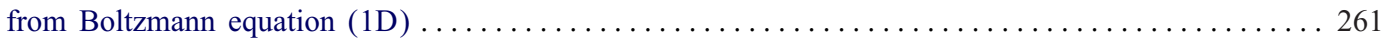

Example 4: Nonperturbative derivation of linear hydrodynamics from Boltzmann equation (3D) $\ldots \ldots \ldots \ldots 278$

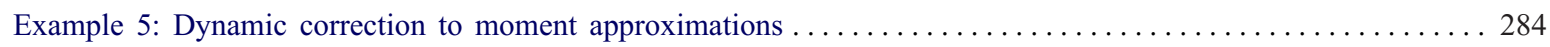

7. Decomposition of motions, nonuniqueness of selection of fast motions, self-adjoint linearization, Onsager filter

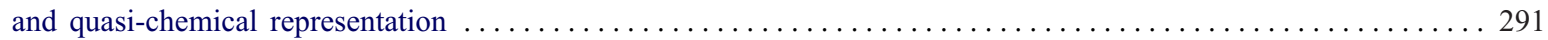

Example 6: Quasi-chemical representation and self-adjoint linearization of the Boltzmann collision

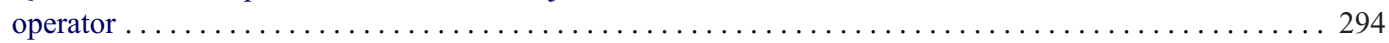

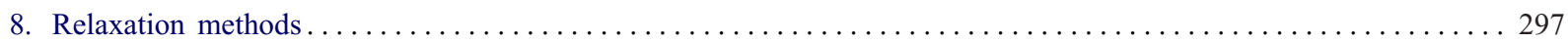

Example 7: Relaxation method for the Fokker-Planck equation $\ldots \ldots \ldots \ldots \ldots \ldots \ldots \ldots \ldots \ldots \ldots \ldots \ldots$

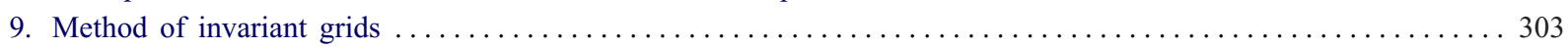

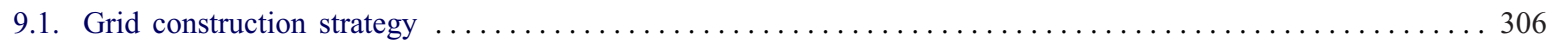

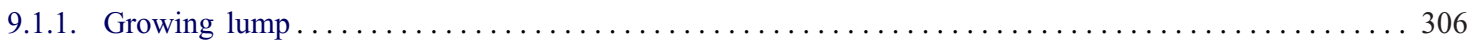

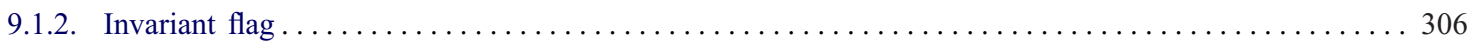

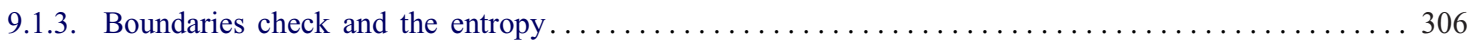

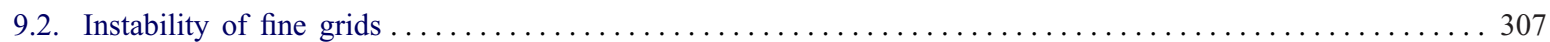

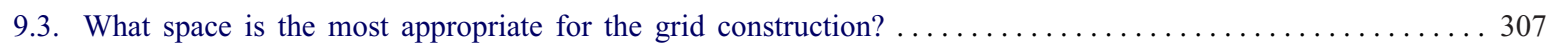

9.4. Carleman's formulae in the analytical invariant manifolds approximations. First benefit of analyticity:

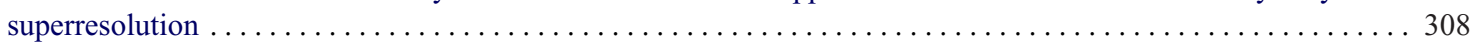

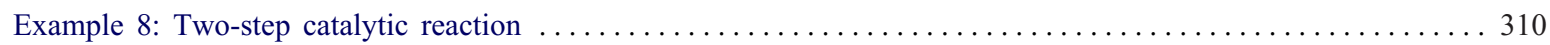

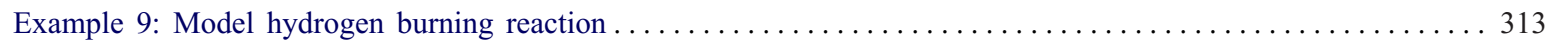

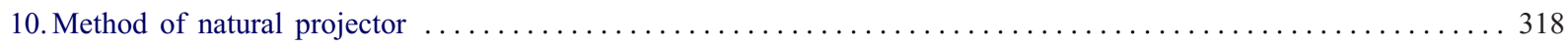

Example 10: From reversible dynamics to Navier-Stokes and post-Navier-Stokes hydrodynamics by natural

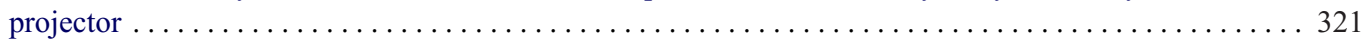

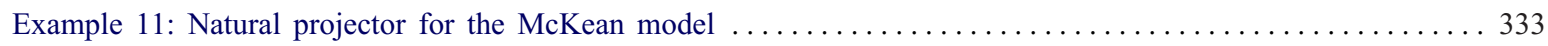

11. Slow invariant manifold for a closed system has been found. What next? ..................... 339

11.1. Slow dynamics in open systems. Zero-order approximation and the thermodynamic projector . . . . . . . 340

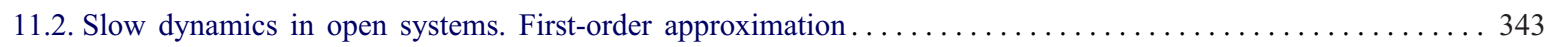

11.3. Beyond the first-order approximation: higher-order dynamical corrections, stability loss and invariant

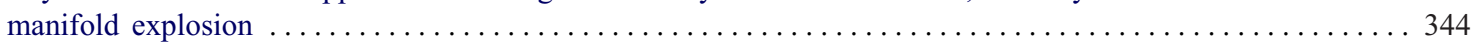

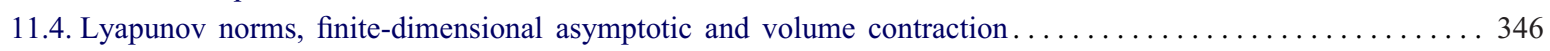

Example 12: The universal limit in dynamics of dilute polymeric solutions $\ldots \ldots \ldots \ldots \ldots \ldots \ldots \ldots \ldots \ldots \ldots$

Example 13: Explosion of invariant manifold, limits of macroscopic description for polymer molecules,

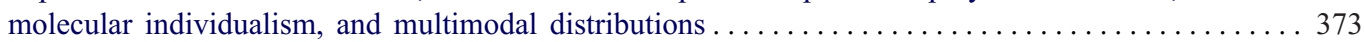

12. Accuracy estimation and postprocessing in invariant manifolds construction $\ldots \ldots \ldots \ldots \ldots \ldots \ldots \ldots \ldots \ldots$

Example 14: Defect of invariance estimation and switching from the microscopic simulations to

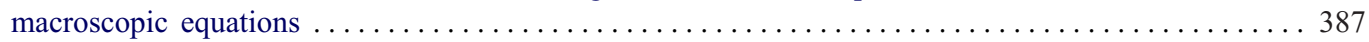

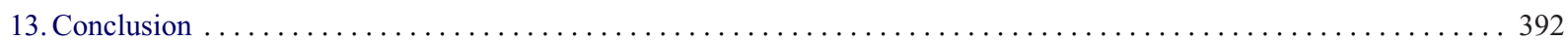

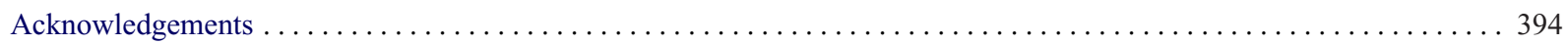

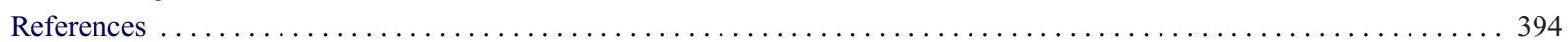




\section{Introduction}

In this review, we present a collection of constructive methods to study slow (stable) positively invariant manifolds of dynamic systems. The main objects of our study are dissipative dynamic systems (finite or infinite) which arise in various problems of kinetics. Some of the results and methods presented herein may have a more general applicability, and can be useful not only for dissipative systems but also, for example, for conservative systems.

Nonequilibrium statistical physics is a collection of ideas and methods to extract slow invariant manifolds. Reduction of description for dissipative systems assumes (explicitly or implicitly) the following picture: There exists a manifold of slow motions in the phase space of the system. From the initial conditions the system goes quickly in a small neighborhood of the manifold, and after that moves slowly along this manifold (see, for example, [1]). The manifold of slow motion must be positively invariant: if the motion starts on the manifold at $t_{0}$, then it stays on the manifold at $t>t_{0}$. Frequently used wording "invariant manifold" is not really exact: for the dissipative systems, the possibility of extending the solutions (in a meaningful way) backwards in time is limited. So, in nonequilibrium statistical physics we study positively invariant slow manifolds. The necessary invariance condition can be written explicitly as the differential equation for the manifold immersed into the phase space. ${ }^{1}$

A dissipative system may have many closed positively invariant sets. For example, for every set of initial conditions $K$, unification of all the trajectories $\{x(t), t \geqslant 0\}$ with initial conditions $x(0) \in K$ is positively invariant. Thus, selection of the slow (stable) positively invariant manifolds becomes an important problem. ${ }^{2}$

One of the difficulties in the problem of reducing the description is pertinent to the fact that there exists no commonly accepted formal definition of slow (and stable) positively invariant manifold. This difficulty is resolved in Section 4 of our review in the following way: First, we consider manifolds immersed into a phase space and study their motion along trajectories. Second, we subtract from this motion the motion of immersed manifolds along themselves, and obtain a new equation for dynamics of manifolds in phase space: the film extension of the dynamics. Invariant manifolds are fixed points for this extended dynamics, and slow invariant manifolds are Lyapunov stable fixed points.

\footnotetext{
${ }^{1}$ This picture is directly applicable to dissipative systems. Time separation for conservative systems and the way from the reversible mechanics (for example, from the Liouville equation) to dissipative systems (for example, to the Boltzmann equation) requires some additional ideas and steps. For any conservative system, a restriction of its dynamics onto any invariant manifold is conservative again. We should represent a dynamics of a large conservative system as a result of dynamics in its small subsystems, and it is necessary to take into account that a macroscopically small interval of time can be considered as an infinitely large interval for a small subsystem, i.e. microscopically. It allows us to represent a relaxation of such a large systems as an ensemble of indivisible events (for example, collision) which happen to its small subsystems. The Bogolyubov-Born-Green-Kirkwood-Yvon (BBGKY) hierarchy and Bogolyubov method for derivation of the Boltzmann equation give us the unexcelled realization of this approach [2].

${ }^{2}$ Nevertheless, there exists a different point of view: "nonuniqueness, when it arises, is irrelevant for modeling" [3], because the differences between the possible manifolds are of the same order as the differences we set out to ignore in establishing the low-dimensional model.
} 
The main body of this review is about how to actually compute the slow invariant manifold. Here we present three approaches to constructing slow (stable) positively invariant manifolds.

- Iteration method (the Newton method subject to incomplete linearization);

- Relaxation methods based on a film extension of the original dynamic system;

- The method of natural projector

The Newton method (with incomplete linearization) is convenient for obtaining the explicit formulas - even one iteration can give a good approximation.

Relaxation methods are oriented more at the numerical implementation. Nevertheless, several first steps also can give appropriate analytical approximations, competitive with other methods.

Finally, the method of natural projector constructs not the manifold itself but a projection of slow dynamics from the slow manifold onto some set of variables.

The Newton method subject to incomplete linearization was developed for the construction of slow (stable) positively invariant manifolds for the following problems:

- Derivation of the post-Navier-Stokes hydrodynamics from the Boltzmann equation [4,6,7].

- Description of the dynamics of polymers solutions [8].

- Correction of the moment equations [9].

- Reduced description for the chemical kinetics $[10,11,81]$.

Relaxation methods based on a film extension of the original dynamic system were applied for the analysis of the Fokker-Planck equation [12]. Applications of these methods in the theory of the Boltzmann equation can benefit from the estimations, obtained in the papers [14,15].

The method of natural projector was initially applied to derivation of the dissipative equations of macroscopic dynamics from the conservative equations of microscopic dynamics [16-21]. Using this method, new equations were obtained for the post-Navier-Stokes hydrodynamics, equations of plasma hydrodynamics and others [17,21]. This short-memory approximation is applied to the Wigner formulation of quantum mechanics [22]. The dissipative dynamics of a single quantum particle in a confining external potential is shown to take the form of a damped oscillator whose effective frequency and damping coefficients depend on the shape of the quantum-mechanical potential [22]. The method of natural projector can also be applied effectively for the dissipative systems: instead of the Chapman-Enskog method in theory of the Boltzmann equation, etc.

A natural initial approximation for the methods under consideration is a quasi-equilibrium manifold. It is the manifold of conditional maxima of the entropy. Most of the works on nonequilibrium thermodynamics deal with corrections to quasi-equilibrium approximations, or with applications of these approximations (with or without corrections). The construction of the quasi-equilibrium allows for the following generalization: Almost every manifold can be represented as a set of minimizers of the entropy under linear constrains. However, in contrast to the standard quasi-equilibrium, these linear constrains will depend on the point on the manifold. We describe the quasi-equilibrium manifold and a quasi-equilibrium projector on the tangent space of this manifold. This projector is orthogonal with respect to entropic scalar product (the bilinear form defined by the negative second differential of the entropy). We construct the thermodynamical projector, which transforms the arbitrary vector field equipped with the given Lyapunov function (the entropy) into a vector field with 
the same Lyapunov function for an arbitrary ansatz manifold which is not tangent to the level of the Lyapunov function. The uniqueness of this construction is demonstrated.

Here, a comment on the status of the most of the statements in this text is in order. Just like the absolute majority of all claims concerning such things as general solutions of the NavierStokes and Boltzmann equations, etc., they have the status of being plausible. They can become theorems only if one restricts essentially the set of the objects under consideration. Among such restrictions we should mention cases of exact reduction, i.e. exact derivation of the hydrodynamics from the kinetics $[23,24]$. In these (still infinite-dimensional) examples one can compare different methods, for example, the Newton method with the methods of series summation in the perturbation theory [24,25].

Also, it is necessary to stress here, that even if in the limit all the methods lead to the same results, they can give rather different approximations "on the way".

The rigorous grounds of the constructive methods of invariant manifolds should, in particular, include the theorems about persistence of invariant manifolds under perturbations. The most known result of this type is the Kolmogorov-Arnold-Moser theory about persistence of almost all invariant tori of completely integrable system under small perturbation [28-30]. Such theorems exist for some classes of infinite dimensional dissipative systems too [31]. Unfortunately, it is not proven until now that many important systems (the Boltzmann equation, the 3D Navier-Stokes equations, the Grad equations, etc.) belong to these classes. So, it is necessary to act with these systems without a rigorous basis.

Two approaches are widely known to the construction of the invariant manifolds: the Taylor series expansion [32,33] and the method of renormalization group [34-40]. The advantages and disadvantages of the Taylor expansion are well-known: constructivity against the absence of physical meaning for the high-order terms (often) and divergence in the most interesting cases (often).

In the paper [37] a geometrical formulation of the renormalization group method for global analysis was given. It was shown that the renormalization group equation can be interpreted as an envelope equation. Recently [38] the renormalization group method was formulated in terms of the notion of invariant manifolds. This method was applied to derive kinetic and transport equations from the respective microscopic equations [39]. The derived equations include the Boltzmann equation in classical mechanics (see also the paper [36], where it was shown for the first time that kinetic equations such as the Boltzmann equation can be understood naturally as renormalization group equations), Fokker-Planck equation, a rate equation in a quantum field theoretical model, etc.

The renormalization group approach was applied to the stochastic Navier-Stokes equation that describes fully developed fluid turbulence [41-43]. For the evaluation of the relevant degrees of freedom the renormalization group technique was revised for discrete systems in the recent paper Ref. [40].

The kinetic approach to subgrid modeling of fluid turbulence became more popular during the last decade [44-47]. A mean-field approach (filtering out subgrid scales) is applied to the Boltzmann equation in order to derive a subgrid turbulence model based on kinetic theory. It is demonstrated [47] that the only Smagorinsky type model which survives in the hydrodynamic limit on the viscosity time scale is the so-called tensor-diffusivity model [48].

The new quantum field theory formulation of the problem of persistence of invariant tori in perturbed completely integrable systems was obtained, and the new proof of the KAM theorem for analytic Hamiltonians based on the renormalization group method was given [49]. 
From the authors of the paper Ref. [36] point of view, the relation of renormalization group theory and reductive perturbation theory has simultaneously been recognized: renormalization group equations are actually the slow-motion equations which are usually obtained by reductive perturbation methods.

The first systematic and (at least partially) successful method of constructing invariant manifolds for dissipative systems was the celebrated Chapman-Enskog method [51] for the Boltzmann kinetic equation. The Chapman-Enskog method results in a series development of the so-called normal solution (the notion introduced by Hilbert [52]) where the one-body distribution function depends on time and space through its locally conserved moments. To the first approximation, the Chapman-Enskog series leads to hydrodynamic equations with transport coefficients expressed in terms of molecular scattering cross-sections. However, next terms of the Chapman-Enskog bring in the "ultra-violet catastrophe" (noticed first by Bobylev [53]) and negative viscosity. These drawbacks pertinent to the Taylor-series expansion disappear as soon as the Newton method is used to construct the invariant manifold [6].

The Chapman-Enskog method was generalized many times [54] and gave rise to a host of subsequent works and methods, such as the famous method of the quasi-steady state in chemical kinetics, pioneered by Bodenstein and Semenov and explored in considerable detail by many authors (see, for example, [55-59,10]), and the theory of singularly perturbed differential equations [55,60-65].

There exist a group of methods to construct an ansatz for the invariant manifold based on the spectral decomposition of the Jacobian. The idea to use the spectral decomposition of Jacobian fields in the problem of separating the motions into fast and slow originates from methods of analysis of stiff systems [66], and from methods of sensitivity analysis in control theory [67,68]. One of the currently most popular methods based on the spectral decomposition of Jacobian fields is the construction of the so-called intrinsic low-dimensional manifold (ILDM) [69].

These methods were thoroughly analyzed in two papers [70,71]. It was shown that the successive applications of the computational singular perturbation algorithm (developed in [68]) generate, order by order, the asymptotic expansion of a slow manifold, and the manifold identified by the ILDM technique (developed in [69]) agrees with the invariant manifold to some order.

The theory of inertial manifold is based on the special linear dominance in higher dimensions. Let an infinite-dimensional system have a form: $\dot{u}+A u=R(u)$, where $A$ is self-adjoint, and has discrete spectrum $\lambda_{i} \rightarrow \infty$ with sufficiently big gaps between $\lambda_{i}$, and $R(u)$ is continuous. One can build the slow manifold as the graph over a root space of $A$ [72]. The textbook [76] provides an exhaustive introduction to the scope of main ideas and methods of this theory. Systems with linear dominance have limited utility in kinetics. Often neither a big spectral gaps between $\lambda_{i}$ exists, no $\lambda_{i} \rightarrow \infty$ (for example, for the simplest model BGK equations, or for the Grad equations). Nevertheless, the concept of inertial attracting manifold has more wide field of applications than the theory, based on the linear dominance assumption.

The Newton method with incomplete linearization as well as the relaxation method allow us to find an approximate slow invariant manifolds without the preliminary stage of Jacobian field spectral decomposition. Moreover, a necessary slow invariant subspace of Jacobian in equilibrium point appears as a by-product of the Newton iterations (with incomplete linearization), or of the relaxation method.

It is of importance to search for minimal (or subminimal) sets of natural parameters that uniquely determine the long-time behavior of a system. This problem was first discussed by Foias and Prodi 
[73] and by Ladyzhenskaya [74] for the 2D Navier-Stokes equations. They have proved that the long-time behavior of solutions is completely determined by the dynamics of sufficiently large amount of the first Fourier modes. A general approach to the problem on the existence of a finite number of determining parameters has been discussed [75,76].

Past decade witnessed a rapid development of the so-called set oriented numerical methods [77]. The purpose of these methods is to compute attractors, invariant manifolds (often, computation of stable and unstable manifolds in hyperbolic systems [78-80]). Also, one of the central tasks of these methods is to gain statistical information, i.e. computations of physically observable invariant measures. The distinguished feature of the modern set-oriented methods of numerical dynamics is the use of ensembles of trajectories within a relatively short propagation time instead of a long time single trajectory.

In this paper we systematically consider a discrete analogue of the slow (stable) positively invariant manifolds for dissipative systems, invariant grids. These invariant grids were introduced in [10]. Here we will describe the Newton method subject to incomplete linearization and the relaxation methods for the invariant grids [81].

It is worth to mention, that the problem of the grid correction is fully decomposed into the tasks of the grid's nodes correction. The edges between the nodes appear only in the calculation of the tangent spaces at the nodes. This fact determines high computational efficiency of the invariant grids method.

Let the (approximate) slow invariant manifold for a dissipative system be found. What for have we constructed it? One important part of the answer to this question is: We have constructed it to create models of open system dynamics in the neighborhood of this manifold. Different approaches for this modeling are described. We apply these methods to the problem of reduced description in polymer dynamics and derive the universal limit in dynamics of dilute polymeric solutions. It is represented by the revised Oldroyd 8 constants constitutive equation for the polymeric stress tensor. Coefficients of this constitutive equation are expressed in terms of the microscopic parameters. This limit of dynamics of dilute polymeric solutions is universal in the same sense, as Korteweg-De-Vries equation is universal in the description of the dispersive dissipative nonlinear waves: any physically consistent equation should contain the obtained equation as a limit.

The phenomenon of invariant manifold explosion in driven open systems is demonstrated on the example of dumbbell models of dilute polymeric solutions [84]. This explosion gives us a possible mechanism of drag reduction in dilute polymeric solutions [85].

Suppose that for the kinetic system the approximate invariant manifold has been constructed and the slow motion equations have been derived. Suppose that we have solved the slow motion system and obtained $x_{\mathrm{sl}}(t)$. We consider the following two questions:

- How well this solution approximates the true solution $x(t)$ given the same initial conditions?

- How is it possible to use the solution $x_{\mathrm{sl}}(t)$ for it's refinement without solving the slow motion system (or it's modifications) again?

These two questions are interconnected. The first question states the problem of the accuracy estimation. The second one states the problem of postprocessing [244-246,277]. We propose various algorithms for postprocessing and accuracy estimation, and give an example of application. 


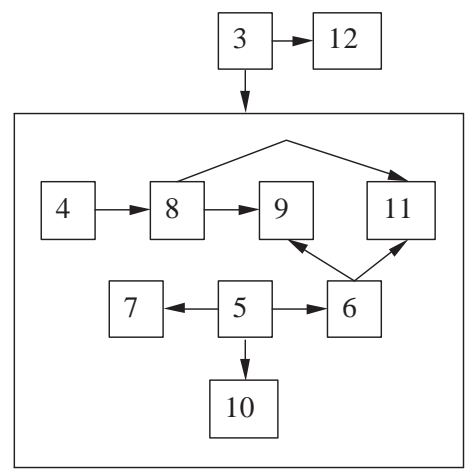

Fig. 1. Logical connections between sections. All the sections depend on Section 3. There are many possible routes for reading, for example the following route gives the invariance equation and one of the main methods for its solution with applications to the Boltzmann equation: Sections 3-5 (with Example 1), Section 6 (with Example 3). Another possibility gives the shortest way to rheology applications: Sections 3, 5, 6 and 11 (with Example 12). The formalization of the classical Ehrenfests idea of coarse-graining and its application for derivation of the correct high-order hydrodynamic equations can be reached in such a way: Sections 3, 5, 10 (with Examples 10, 11). The shortest road to numerical representation of invariant manifolds and to the method of invariant grids is as follows: Sections 3-6, 8, 9 (with Examples 8, 9).

Our collection of methods and algorithms can be incorporated into recently developed technologies of computer-aided multiscale analysis which enable the "level jumping" between microscopic and macroscopic (system) levels. It is possible both for traditional technique based on transition from microscopic equations to macroscopic equations and for the "equation-free" approach [82]. This approach developed in recent series of work [83], when successful, can bypass the derivation of the macroscopic evolution equations when these equations conceptually exist but are not available in closed form. The mathematics-assisted development of a computational superstructure may enable alternative descriptions of the problem physics (e.g. Lattice Boltzmann (LB), kinetic Monte-Carlo (KMC) or Molecular Dynamics (MD) microscopic simulators, executed over relatively short time and space scales) to perform systems level tasks (integration over relatively large time and space scales, coarse bifurcation analysis, optimization, and control) directly. In effect, the procedure constitutes a system identification based, closure-on-demand computational toolkit. It is possible to use macroscopic invariant manifolds in this environment without explicit equations.

The present paper comprises sections of the two kinds. Numbered sections contain basic notions, methods and algorithmic realizations. Sections entitled "Examples" contain various case studies where the methods are applied to specific equations. Exposition in the "Examples" sections is not as consequent as in the numbered sections. Most of the examples can be read more or less independently. Logical connections between sections are presented in Figs. 1, 2.

The list of cited literature is by no means complete although we spent effort in order to reflect at least the main directions of studies related to computations of the invariant manifolds. We think that this list is more or less exhaustive in the second-order approximation. 


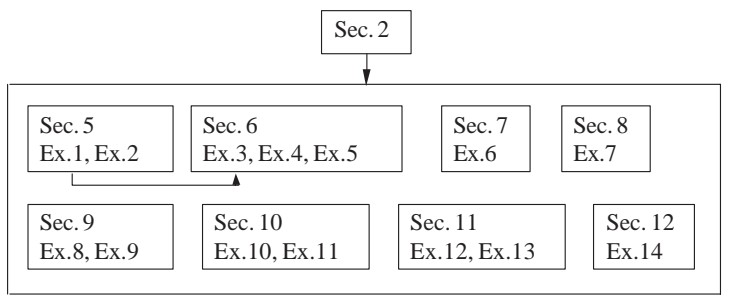

Fig. 2. Logical connections between sections and examples. Only one connection between examples is significant: Example 3 depends on Example 1. All the examples depend on corresponding subsections of Section 2.

\section{Mathematical notation and some terminology}

- The operator $L$ from space $W$ to space $E: L: W \rightarrow E$.

- The kernel of a linear operator $L: W \rightarrow E$ is a subspace $\operatorname{ker} L \subset W$ that transforms by $L$ into 0 : $\operatorname{ker} L=\{x \in W \mid L x=0\}$.

- The image of a linear operator $L: W \rightarrow E$ is a subspace $\operatorname{im} L=L(W) \subset E$.

- Projector is a linear operator $P: E \rightarrow E$ with the property $P^{2}=P$. Projector $P$ is orthogonal one, if $\operatorname{ker} P \perp \operatorname{im} P$ (the kernel of $P$ is orthogonal to the image of $P$ ).

- If $F: U \rightarrow V$ is a map of domains in normed spaces ( $U \subset W, V \subset E$ ) then the differential of $F$ at a point $x$ is a linear operator $D_{x} F: W \rightarrow E$ with the property: $\| F(x+\delta x)-F(x)-$ $\left(D_{x} F\right)(\delta x) \|=\mathrm{o}(\|\delta x\|)$. This operator (if it exists) is the best linear approximation of the map $F(x+\delta x)-F(x)$.

- The differential of the function $f(x)$ is the linear functional $D_{x} f$. The gradient of the function $f(x)$ can be defined, if there is a given scalar product $\langle\mid\rangle$, and if there exists a Riesz representation for functional $D_{x} f:\left(D_{x} f\right)(a)=\left\langle\operatorname{grad}_{x} f \mid a\right\rangle$. The gradient $\operatorname{grad}_{x} f$ is a vector.

- The second differential of a map $F: U \rightarrow V$ is a bilinear operator $D_{x}^{2} F: W \times W \rightarrow E$ which can be defined by Taylor formula: $F(x+\delta x)=F(x)+\left(D_{x} F\right)(\delta x)+\frac{1}{2}\left(D_{x}^{2} F\right)(\delta x, \delta x)+\mathrm{o}\left(\|\delta x\|^{2}\right)$.

- The differentiable map of domains in normed spaces $F: U \rightarrow V$ is an immersion, if for any $x \in U$ the operator $D_{x} F$ is injective: $\operatorname{ker} D_{x} F=\{0\}$. In this case the image of $F$, i.e. $(F(U))$ is called the immersed manifold, and the image of $D_{x} F$ is called the tangent space to the immersed manifold $F(U)$. We shall use the notation $T_{x}$ for this tangent space: $\operatorname{im} D_{x} F=T_{x}$.

- The subset $U$ of the vector space $E$ is convex, if for every two points $x_{1}, x_{2} \in U$ it contains the segment between $x_{1}$ and $x_{2}: \lambda x_{1}+(1-\lambda) x_{2} \in U$ for every $\lambda \in[0,1]$.

- The function $f$, defined on the convex set $U \subset E$, is convex, if its epigraph, i.e. the set of pairs Epi $f=\{(x, g) \mid x \in U, g \geqslant f(x)\}$, is the convex set in $E \times R$. The twice differentiable function $f$ is convex if and only if the quadratic form $\left(D_{x}^{2} f\right)(\delta x, \delta x)$ is nonnegative.

- The convex function $f$ is called strictly convex if in the domain of definition there is no line segment on which it is constant and finite $(f(x)=$ const $\neq \infty)$. The sufficient condition for the twice differentiable function $f$ to be strictly convex is that the quadratic form $\left(D_{x}^{2} f\right)(\delta x, \delta x)$ is positive defined (i.e. it is positive for all $\delta x \neq 0$ ).

- We use summation convention for vectors and tensors, $c_{i} g_{i}=\sum_{i} c_{i} g_{i}$, when it cannot cause a confusion, in more complicated cases we use the sign $\sum$. 


\section{The source of examples}

In this section we follow, partially, the paper [50], where nonlinear kinetic equations and methods of reduced description are reviewed for a wide audience of specialists and postgraduate students in physics, mathematical physics, material science, chemical engineering and interdisciplinary research.

\subsection{The Boltzmann equation}

\subsubsection{The equation}

The Boltzmann equation is the first and the most celebrated nonlinear kinetic equation introduced by the great Austrian scientist Ludwig Boltzmann in 1872 [86]. This equation describes the dynamics of a moderately rarefied gas, taking into account the two processes: the free flight of the particles, and their collisions. In its original version, the Boltzmann equation has been formulated for particles represented by hard spheres. The physical condition of rarefaction means that only pair collisions are taken into account, a mathematical specification of which is given by the Grad-Boltzmann limit: If $N$ is the number of particles, and $\sigma$ is the diameter of the hard sphere, then the Boltzmann equation is expected to hold when $N$ tends to infinity, $\sigma$ tends to zero, $N \sigma^{3}$ (the volume occupied by the particles) tends to zero, while $N \sigma^{2}$ (the total collision cross section) remains constant. The microscopic state of the gas at time $t$ is described by the one-body distribution function $P(\boldsymbol{x}, \boldsymbol{v}, t)$, where $\boldsymbol{x}$ is the position of the center of the particle, and $v$ is the velocity of the particle. The distribution function is the probability density of finding the particle at time $t$ within the infinitesimal phase space volume centered at the phase point $(\boldsymbol{x}, \boldsymbol{v})$. The collision mechanism of two hard spheres is presented by a relation between the velocities of the particles before $[\boldsymbol{v}$ and $\boldsymbol{w}]$ and after $\left[\boldsymbol{v}^{\prime}\right.$ and $\left.\boldsymbol{w}^{\prime}\right]$ their impact:

$$
\begin{gathered}
\boldsymbol{v}^{\prime}=v-n(n, v-w) \\
\boldsymbol{w}^{\prime}=w+n(n, v-w),
\end{gathered}
$$

where $\boldsymbol{n}$ is the unit vector along $\boldsymbol{v}-\boldsymbol{v}^{\prime}$. Transformation of the velocities conserves the total momentum of the pair of colliding particles $\left(\boldsymbol{v}^{\prime}+\boldsymbol{w}^{\prime}=\boldsymbol{v}+\boldsymbol{w}\right)$, and the total kinetic energy $\left(\boldsymbol{v}^{\prime 2}+\boldsymbol{w}^{\prime 2}=\boldsymbol{v}^{2}+\boldsymbol{w}^{2}\right)$ The Boltzmann equation reads:

$$
\begin{aligned}
\frac{\partial P}{\partial t} & +\left(\boldsymbol{v}, \frac{\partial P}{\partial \boldsymbol{x}}\right) \\
& =N \sigma^{2} \int_{R^{3}} \int_{B^{-}}\left(P\left(\boldsymbol{x}, \boldsymbol{v}^{\prime}, t\right) P\left(\boldsymbol{x}, \boldsymbol{w}^{\prime}, t\right)-P(\boldsymbol{x}, \boldsymbol{v}, t) P(\boldsymbol{x}, \boldsymbol{w}, t)\right)|(\boldsymbol{w}-\boldsymbol{v}, \boldsymbol{n})| \mathrm{d} \boldsymbol{w} \mathrm{d} \boldsymbol{n}
\end{aligned}
$$

where integration in $\boldsymbol{w}$ is carried over the whole space $R^{3}$, while integration in $\boldsymbol{n}$ goes over a hemisphere $B^{-}=\{\boldsymbol{n} \mid(\boldsymbol{w}-\boldsymbol{v}, \boldsymbol{n})<0\}$. This hemisphere corresponds to the particles entering the collision. The nonlinear integral operator on the right hand side of Eq. (1) is nonlocal in the velocity variable, and local in space. The Boltzmann equation for arbitrary hard-core interaction is a generalization of the Boltzmann equation for hard spheres under the proviso that the true infinite-range interaction potential between the particles is cut-off at some distance. This generalization amounts to 
a replacement,

$$
\sigma^{2}|(\boldsymbol{w}-\boldsymbol{v}, \boldsymbol{n})| \mathrm{d} \boldsymbol{n} \rightarrow B(\theta,|\boldsymbol{w}-\boldsymbol{v}|) \mathrm{d} \theta \mathrm{d} \varepsilon,
$$

where function $B$ is determined by the interaction potential, and vector $\boldsymbol{n}$ is identified with two angles, $\theta$ and $\varepsilon$. In particular, for potentials proportional to the $n$th inverse power of the distance, the function $B$ reads,

$$
B(\theta,|\boldsymbol{v}-\boldsymbol{w}|)=\beta(\theta)|\boldsymbol{v}-\boldsymbol{w}|^{(n-5) /(n-1)} .
$$

In the special case $n=5$, function $B$ is independent of the magnitude of the relative velocity (Maxwell molecules). Maxwell molecules occupy a distinct place in the theory of the Boltzmann equation, they provide exact results. Three most important findings for the Maxwell molecules are mentioned here: (1) The exact spectrum of the linearized Boltzmann collision integral, found by Truesdell and Muncaster, (2) Exact transport coefficients found by Maxwell even before the Boltzmann equation was formulated, (3) Exact solutions to the space-free model version of the nonlinear Boltzmann equation. Pivotal results in this domain belong to Galkin who has found the general solution to the system of moment equations in a form of a series expansion, to Bobylev, Krook and Wu who have found an exact solution of a particular elegant closed form, and to Bobylev who has demonstrated the complete integrability of this dynamic system.

A broad review of the Boltzmann equation and analysis of analytical solutions to kinetic models is presented in the book of Cercignani [87]. A modern account of rigorous results on the Boltzmann equation is given in the book [88]. Proof of the existence theorem for the Boltzmann equation was done by DiPerna and Lions [92].

It is customary to write the Boltzmann equation using another normalization of the distribution function, $f(\boldsymbol{x}, \boldsymbol{v}, t) \mathrm{d} \boldsymbol{x} \mathrm{d} \boldsymbol{v}$, taken in such a way that the function $f$ is compliant with the definition of the hydrodynamic fields: the mass density $\rho$, the momentum density $\rho \boldsymbol{u}$, and the energy density $\varepsilon$ :

$$
\begin{aligned}
& \int f(\boldsymbol{x}, \boldsymbol{v}, t) m \mathrm{~d} \boldsymbol{v}=\rho(\boldsymbol{x}, t), \\
& \int f(\boldsymbol{x}, \boldsymbol{v}, t) m \boldsymbol{v} \mathrm{d} \boldsymbol{v}=\rho \boldsymbol{u}(\boldsymbol{x}, t), \\
& \int f(\boldsymbol{x}, \boldsymbol{v}, t) m \frac{v^{2}}{2} \mathrm{~d} \boldsymbol{v}=\varepsilon(\boldsymbol{x}, t) .
\end{aligned}
$$

Here $m$ is the particle's mass.

The Boltzmann equation for the distribution function $f$ reads,

$$
\frac{\partial f}{\partial t}+\left(\boldsymbol{v}, \frac{\partial}{\partial \boldsymbol{x}} f\right)=Q(f, f),
$$

where the nonlinear integral operator on the right hand side is the Boltzmann collision integral,

$$
Q=\int_{R^{3}} \int_{B^{-}}\left(f\left(\boldsymbol{v}^{\prime}\right) f\left(\boldsymbol{w}^{\prime}\right)-f(\boldsymbol{v}) f(\boldsymbol{w})\right) B(\theta, \boldsymbol{v}) \mathrm{d} \boldsymbol{w} \mathrm{d} \theta \mathrm{d} \varepsilon .
$$


Finally, we mention the following form of the Boltzmann collision integral (sometimes referred to as the scattering or the quasi-chemical representation),

$$
Q=\int W\left(\boldsymbol{v}, \boldsymbol{w} \mid \boldsymbol{v}^{\prime}, \boldsymbol{w}^{\prime}\right)\left[\left(f\left(\boldsymbol{v}^{\prime}\right) f\left(\boldsymbol{w}^{\prime}\right)-f(\boldsymbol{v}) f(\boldsymbol{w})\right)\right] \mathrm{d} \boldsymbol{w} \mathrm{d} \boldsymbol{w}^{\prime} \mathrm{d} \boldsymbol{v}^{\prime},
$$

where $W$ is a generalized function which is called the probability density of the elementary event,

$$
W=w\left(\boldsymbol{v}, \boldsymbol{w} \mid \boldsymbol{v}^{\prime}, \boldsymbol{w}^{\prime}\right) \delta\left(\boldsymbol{v}+\boldsymbol{w}-\boldsymbol{v}^{\prime}-\boldsymbol{w}^{\prime}\right) \delta\left(v^{2}+w^{2}-v^{\prime 2}-{w^{\prime 2}}^{2} .\right.
$$

\subsubsection{The basic properties of the Boltzmann equation}

Generalized function $W$ has the following symmetries:

$$
W\left(\boldsymbol{v}^{\prime}, \boldsymbol{w}^{\prime} \mid \boldsymbol{v}, \boldsymbol{w}\right) \equiv W\left(\boldsymbol{w}^{\prime}, \boldsymbol{v}^{\prime} \mid \boldsymbol{v}, \boldsymbol{w}\right) \equiv W\left(\boldsymbol{v}^{\prime}, \boldsymbol{w}^{\prime} \mid \boldsymbol{w}, \boldsymbol{v}\right) \equiv W\left(\boldsymbol{v}, \boldsymbol{w} \mid \boldsymbol{v}^{\prime}, \boldsymbol{w}^{\prime}\right) .
$$

The first two identities reflect the symmetry of the collision process with respect to labeling the particles, whereas the last identity is the celebrated detail balance condition which is underpinned by the time-reversal symmetry of the microscopic (Newton's) equations of motion. The basic properties of the Boltzmann equation are:

1. Additive invariants of collision operator:

$$
\int Q(f, f)\left\{1, \boldsymbol{v}, v^{2}\right\} \mathrm{d} \boldsymbol{v}=0
$$

for any function $f$, assuming integrals exist. Equality (10) reflects the fact that the number of particles, the three components of particle's momentum, and the particle's energy are conserved by the collision. Conservation laws (10) imply that the local hydrodynamic fields (4) can change in time only due to redistribution in the space.

2. Zero point of the integral $(Q=0)$ satisfy the equation (which is also called the detail balance): for almost all velocities,

$$
f\left(\boldsymbol{v}^{\prime}, \boldsymbol{x}, t\right) f\left(\boldsymbol{w}^{\prime}, \boldsymbol{x}, t\right)=f(\boldsymbol{v}, \boldsymbol{x}, t) f(\boldsymbol{w}, \boldsymbol{x}, t) .
$$

3. Boltzmann's local entropy production inequality:

$$
\sigma(x, t)=-k_{\mathrm{B}} \int Q(f, f) \ln f \mathrm{~d} \boldsymbol{v} \geqslant 0
$$

for any function $f$, assuming integrals exist. Dimensional Boltzmann's constant $\left(k_{\mathrm{B}} \approx 1.38 \cdot 10^{-23} \mathrm{~J} / \mathrm{K}\right)$ in this expression serves for a recalculation of the energy units into the absolute temperature units. Moreover, equality sign takes place if $\ln f$ is a linear combination of the additive invariants of collision.

Distribution functions $f$ whose logarithm is a linear combination of additive collision invariants, with coefficients dependent on $x$, are called local Maxwell distribution functions $f_{\mathrm{LM}}$,

$$
f_{\mathrm{LM}}=\frac{\rho}{m}\left(\frac{2 \pi k_{\mathrm{B}} T}{m}\right)^{-3 / 2} \exp \left(\frac{-m(\boldsymbol{v}-\boldsymbol{u})^{2}}{2 k_{\mathrm{B}} T}\right) \text {. }
$$


Local Maxwellians are parametrized by values of five hydrodynamic variables, $\rho, \boldsymbol{u}$ and $T$. This parametrization is consistent with the definitions of the hydrodynamic fields $(4), \int f_{\mathrm{LM}}\left(m, m \boldsymbol{v}, m v^{2} / 2\right)$ $=(\rho, \rho \boldsymbol{u}, \varepsilon)$ provided the relation between the energy and the kinetic temperature $T$, holds, $\varepsilon=3 \rho / 2 m k_{\mathrm{B}} T$.

4. Boltzmann's $H$ theorem: The function

$$
S[f]=-k_{\mathrm{B}} \int f \ln f \mathrm{~d} \boldsymbol{v},
$$

is called the entropy density. The local $H$ theorem for distribution functions independent of space states that the rate of the entropy density increase is equal to the nonnegative entropy production,

$$
\frac{\mathrm{d} S}{\mathrm{~d} t}=\sigma \geqslant 0
$$

Thus, if no space dependence is concerned, the Boltzmann equation describes relaxation to the unique global Maxwellian (whose parameters are fixed by initial conditions), and the entropy density grows monotonically along the solutions. Mathematical specifications of this property has been initialized by Carleman, and many estimations of the entropy growth were obtained over the past two decades. In the case of space-dependent distribution functions, the local entropy density obeys the entropy balance equation:

$$
\frac{\partial S(\boldsymbol{x}, t)}{\partial t}+\left(\frac{\partial}{\partial \boldsymbol{x}}, \mathbf{J}_{s}(\boldsymbol{x}, t)\right)=\sigma(\boldsymbol{x}, t) \geqslant 0,
$$

where $\mathbf{J}_{s}$ is the entropy flux, $\mathbf{J}_{s}(\boldsymbol{x}, t)=-k_{\mathrm{B}} \int \ln f(\boldsymbol{x}, t) \boldsymbol{v} f(\boldsymbol{x}, t) \mathrm{d} \boldsymbol{v}$. For suitable boundary conditions, such as, specularly reflecting or at the infinity, the entropy flux gives no contribution to the equation for the total entropy, $S_{\text {tot }}=\int S(\boldsymbol{x}, t) \mathrm{d} \boldsymbol{x}$ and its rate of changes is then equal to the nonnegative total entropy production $\sigma_{\text {tot }}=\int \sigma(\boldsymbol{x}, t) \mathrm{d} \boldsymbol{x}$ (the global $H$ theorem). For more general boundary conditions which maintain the entropy influx the global $H$ theorem needs to be modified. A detailed discussion of this question is given by Cercignani. The local Maxwellian is also specified as the maximizer of the Boltzmann entropy function (13), subject to fixed hydrodynamic constraints (4). For this reason, the local Maxwellian is also termed as the local equilibrium distribution function.

\subsubsection{Linearized collision integral}

Linearization of the Boltzmann integral around the local equilibrium results in the linear integral operator,

$$
\begin{aligned}
& \operatorname{Lh}(\boldsymbol{v}) \\
& \qquad=\int W\left(\boldsymbol{v}, \boldsymbol{w} \mid \boldsymbol{v}^{\prime}, \boldsymbol{w}^{\prime}\right) f_{\mathrm{LM}}(\boldsymbol{v}) f_{\mathrm{LM}}(\boldsymbol{w})\left[\frac{h\left(\boldsymbol{v}^{\prime}\right)}{f_{\mathrm{LM}}\left(\boldsymbol{v}^{\prime}\right)}+\frac{h\left(\boldsymbol{w}^{\prime}\right)}{f_{\mathrm{LM}}\left(\boldsymbol{w}^{\prime}\right)}-\frac{h(\boldsymbol{v})}{f_{\mathrm{LM}}(\boldsymbol{v})}-\frac{h(\boldsymbol{w})}{f_{\mathrm{LM}}(\boldsymbol{w})}\right] \mathrm{d} \boldsymbol{w}^{\prime} \mathrm{d} \boldsymbol{v}^{\prime} \mathrm{d} \boldsymbol{w} .
\end{aligned}
$$

Linearized collision integral is symmetric with respect to scalar product defined by the second derivative of the entropy functional,

$$
\int f_{\mathrm{LM}}^{-1}(\boldsymbol{v}) g(\boldsymbol{v}) L h(\boldsymbol{v}) \mathrm{d} \boldsymbol{v}=\int f_{\mathrm{LM}}^{-1}(\boldsymbol{v}) h(\boldsymbol{v}) L g(\boldsymbol{v}) \mathrm{d} \boldsymbol{v},
$$


it is nonpositively definite,

$$
\int f_{\mathrm{LM}}^{-1}(\boldsymbol{v}) h(\boldsymbol{v}) \operatorname{Lh}(\boldsymbol{v}) \mathrm{d} \boldsymbol{v} \leqslant 0
$$

where equality sign takes place if the function $h f_{\mathrm{LM}}^{-1}$ is a linear combination of collision invariants, which characterize the null-space of the operator $L$. Spectrum of the linearized collision integral is well studied in the case of the small angle cut-off.

\subsection{Phenomenology and Quasi-chemical representation of the Boltzmann equation}

Boltzmann's original derivation of his collision integral was based on a phenomenological "bookkeeping" of the gain and of the loss of probability density in the collision process. This derivation postulates that the rate of gain $G$ equals

$$
G=\int W^{+}\left(\boldsymbol{v}, \boldsymbol{w} \mid \boldsymbol{v}^{\prime}, \boldsymbol{w}^{\prime}\right) f\left(\boldsymbol{v}^{\prime}\right) f\left(\boldsymbol{w}^{\prime}\right) \mathrm{d} \boldsymbol{v}^{\prime} \mathrm{d} \boldsymbol{w}^{\prime} \mathrm{d} \boldsymbol{w},
$$

while the rate of loss is

$$
L=\int W^{-}\left(\boldsymbol{v}, \boldsymbol{w} \mid \boldsymbol{v}^{\prime}, \boldsymbol{w}^{\prime}\right) f(\boldsymbol{v}) f(\boldsymbol{w}) \mathrm{d} \boldsymbol{v}^{\prime} \mathrm{d} \boldsymbol{w}^{\prime} \mathrm{d} \boldsymbol{w} .
$$

The form of the gain and of the loss, containing products of one-body distribution functions in place of the two-body distribution, constitutes the famous Stosszahlansatz. The Boltzmann collision integral follows now as $(G-L)$, subject to the detail balance for the rates of individual collisions,

$$
W^{+}\left(\boldsymbol{v}, \boldsymbol{w} \mid \boldsymbol{v}^{\prime}, \boldsymbol{w}^{\prime}\right)=W^{-}\left(\boldsymbol{v}, \boldsymbol{w} \mid \boldsymbol{v}^{\prime}, \boldsymbol{w}^{\prime}\right)
$$

This representation for interactions different from hard spheres requires also the cut-off of functions $\beta$ (3) at small angles. The gain-loss form of the collision integral makes it evident that the detail balance for the rates of individual collisions is sufficient to prove the local $H$ theorem. A weaker condition which is also sufficient to establish the $H$ theorem was first derived by Stueckelberg (so-called semi-detailed balance), and later generalized to inequalities of concordance:

$$
\begin{gathered}
\int \mathrm{d} \boldsymbol{v}^{\prime} \int \mathrm{d} \boldsymbol{w}^{\prime}\left(W^{+}\left(\boldsymbol{v}, \boldsymbol{w} \mid \boldsymbol{v}^{\prime}, \boldsymbol{w}^{\prime}\right)-W^{-}\left(\boldsymbol{v}, \boldsymbol{w} \mid \boldsymbol{v}^{\prime}, \boldsymbol{w}^{\prime}\right)\right) \geqslant 0, \\
\int \mathrm{d} \boldsymbol{v} \int \mathrm{d} \boldsymbol{w}\left(W^{+}\left(\boldsymbol{v}, \boldsymbol{w} \mid \boldsymbol{v}^{\prime}, \boldsymbol{w}^{\prime}\right)-W^{-}\left(\boldsymbol{v}, \boldsymbol{w} \mid \boldsymbol{v}^{\prime}, \boldsymbol{w}^{\prime}\right)\right) \leqslant 0 .
\end{gathered}
$$

The semi-detailed balance follows from these expressions if the inequality signs are replaced by equalities. 
The pattern of Boltzmann's phenomenological approach is often used in order to construct nonlinear kinetic models. In particular, nonlinear equations of chemical kinetics are based on this idea: If $n$ chemical species $A_{i}$ participate in a complex chemical reaction,

$$
\sum_{i} \alpha_{s i} A_{i} \leftrightarrow \sum_{i} \beta_{s i} A_{i}
$$

where $\alpha_{s i}$ and $\beta_{s i}$ are nonnegative integers (stoichiometric coefficients) then equations of chemical kinetics for the concentrations of species $c_{j}$ are written

$$
\frac{\mathrm{d} c_{i}}{\mathrm{~d} t}=\sum_{s=1}^{n}\left(\beta_{s i}-\alpha_{s i}\right)\left[\varphi_{s}^{+} \exp \left(\sum_{j=1}^{n} \frac{\partial G}{\partial c_{j}} \alpha_{s j}\right)-\varphi_{s}^{-} \exp \left(\sum_{j=1}^{n} \frac{\partial G}{\partial c_{j}} \beta_{s j}\right)\right] .
$$

Functions $\varphi_{s}^{+}$and $\varphi_{s}^{-}$are interpreted as constants of the direct and of the inverse reactions, while the function $G$ is an analog of the Boltzmann's $H$-function. Modern derivation of the Boltzmann equation, initialized by the seminal work of N.N. Bogoliubov, seeks a replacement condition, and which would be more closely related to many-particle dynamics. Such conditions are applied to the $N$-particle Liouville equation should factorize in the remote enough past, as well as in the remote infinity (the hypothesis of weakening of correlations). Different conditions has been formulated by D.N. Zubarev, J. Lewis and others. The advantage of these formulations is the possibility to systematically find corrections not included in the Stosszahlansatz.

\subsection{Kinetic models}

Mathematical complications caused by the nonlinearly Boltzmann collision integral are traced back to the Stosszahlansatz. Several approaches were developed in order to simplify the Boltzmann equation. Such simplifications are termed kinetic models. Various kinetic models preserve certain features of the Boltzmann equation, while sacrificing the rest of them. The most well known kinetic model which preserves the $H$ theorem is the nonlinear Bhatnagar-Gross-Krook model (BGK) [89]. The BGK collision integral reads:

$$
Q_{\mathrm{BGK}}=-\frac{1}{\tau}\left(f-f_{\mathrm{LM}}(f)\right) \text {. }
$$

The time parameter $\tau>0$ is interpreted as a characteristic relaxation time to the local Maxwellian. The BGK is a nonlinear operator: parameters of the local Maxwellian are identified with the values of the corresponding moments of the distribution function $f$. This nonlinearly is of "lower dimension" than in the Boltzmann collision integral because $f_{\mathrm{LM}}(f)$ is a nonlinear function of only the moments of $f$ whereas the Boltzmann collision integral is nonlinear in the distribution function $f$ itself. This type of simplification introduced by the BGK approach is closely related to the family of so-called mean-field approximations in statistical mechanics. By its construction, the BGK collision integral preserves the following three properties of the Boltzmann equation: additive invariants of collision, uniqueness of the equilibrium, and the $H$ theorem. A class of kinetic models which generalized the BGK model to quasi-equilibrium approximations of a general form is described as follows: The quasi-equilibrium $f^{*}$ for the set of linear functionals $M(f)$ is a distribution function $f^{*}(M)(\boldsymbol{x}, \boldsymbol{v})$ 
which maximizes the entropy under fixed values of functions $M$. The quasi-equilibrium (QE) models are characterized by the collision integral [90],

$$
Q_{\mathrm{QE}}(f)=-\frac{1}{\tau}\left[f-f^{*}(M(f))\right]+Q_{\mathrm{B}}\left(f^{*}(M(f)), f^{*}(M(f))\right) .
$$

Same as in the case of the BGK collision integral, operator $Q_{\mathrm{QE}}$ is nonlinear in the moments $M$ only. The QE models preserve the following properties of the Boltzmann collision operator: additive invariants, uniqueness of the equilibrium, and the $H$ theorem, provided the relaxation time $\tau$ to the quasi-equilibrium is sufficiently small. A different nonlinear model was proposed by Lebowitz et al. [91]:

$$
Q_{D}=D\left(\frac{\partial}{\partial \boldsymbol{v}} \frac{\partial}{\partial \boldsymbol{v}} f+\frac{m}{k_{\mathrm{B}} T} \frac{\partial}{\partial \boldsymbol{v}}(\boldsymbol{v}-\boldsymbol{u}(f)) f\right) .
$$

The collision integral has the form of the self-consistent Fokker-Planck operator, describing diffusion (in the velocity space) in the self-consistent potential. Diffusion coefficient $D>0$ may depend on the distribution function $f$. Operator $Q_{D}$ preserves the same properties of the Boltzmann collision operator as the BGK model. The kinetic BGK model has been used for obtaining exact solutions of gasdynamic problems, especially its linearized form for stationary problems. Linearized BGK collision model has been extended to model more precisely the linearized Boltzmann collision integral.

\subsection{Methods of reduced description}

One of the major issues raised by the Boltzmann equation is the problem of the reduced description. Equations of hydrodynamics constitute a closed set of equations for the hydrodynamic field (local density, local momentum, and local temperature). From the standpoint of the Boltzmann equation, these quantities are low-order moments of the one-body distribution function, or, in other words, the macroscopic variables. The problem of the reduced description consists in giving an answer to the following two questions:

1. What are the conditions under which the macroscopic description sets in?

2. How to derive equations for the macroscopic variables from kinetic equations?

The classical methods of reduced description for the Boltzmann equation are: the Hilbert method, the Chapman-Enskog method, and the Grad moment method.

\subsubsection{The Hilbert method}

In 1911, David Hilbert introduced the notion of normal solutions,

$$
f_{\mathrm{H}}(\boldsymbol{v}, n(\boldsymbol{x}, t), \boldsymbol{u}(\boldsymbol{x}, t), T(\boldsymbol{x}, t)),
$$

that is, solution to the Boltzmann equation which depends on space and time only through five hydrodynamic fields [52].

The normal solutions are found from a singularly perturbed Boltzmann equation,

$$
D_{t} f=\frac{1}{\varepsilon} Q(f, f),
$$


where $\varepsilon$ is a small parameter, and

$$
D_{t} f \equiv \frac{\partial}{\partial t} f+\left(\boldsymbol{v}, \frac{\partial}{\partial \boldsymbol{x}}\right) f .
$$

Physically, parameter $\varepsilon$ corresponds to the Knudsen number, the ratio between the mean free path of the molecules between collisions, and the characteristic scale of variation of the hydrodynamic fields. In the Hilbert method, one seeks functions $n(\boldsymbol{x}, t), \boldsymbol{u}(\boldsymbol{x}, t), T(\boldsymbol{x}, t)$, such that the normal solution in the form of the Hilbert expansion,

$$
f_{\mathrm{H}}=\sum_{i=0}^{\infty} \varepsilon^{i} f_{\mathrm{H}}^{(i)}
$$

satisfies the Eq. (16) order by order. Hilbert was able to demonstrate that this is formally possible. Substituting (17) into (16), and matching various order in $\varepsilon$, we have the sequence of integral equations

$$
\begin{aligned}
& Q\left(f_{\mathrm{H}}^{(0)}, f_{\mathrm{H}}^{(0)}\right)=0, \\
& L f_{\mathrm{H}}^{(1)}=D_{t} f_{\mathrm{H}}^{(0)}, \\
& L f_{\mathrm{H}}^{(2)}=D_{t} f_{\mathrm{H}}^{(1)}-Q\left(f_{\mathrm{H}}^{(0)}, f_{\mathrm{H}}^{(1)}\right),
\end{aligned}
$$

and so on for higher orders. Here $L$ is the linearized collision integral. From Eq. (18), it follows that $f_{\mathrm{H}}^{(0)}$ is the local Maxwellian with parameters not yet determined. The Fredholm alternative, as applied to the second Eq. (19) results in

(a) Solvability condition,

$$
\int D_{t} f_{\mathrm{H}}^{(0)}\left\{1, \boldsymbol{v}, v^{2}\right\} \mathrm{d} \boldsymbol{v}=0
$$

which is the set of compressible Euler equations of the nonviscous hydrodynamics. Solution to the Euler equation determine the parameters of the Maxwellian $f_{\mathrm{H}}^{0}$.

(b) General solution $f_{\mathrm{H}}^{(1)}=f_{\mathrm{H}}^{(1) 1}+f_{\mathrm{H}}^{(1) 2}$, where $f_{\mathrm{H}}^{(1) 1}$ is the special solution to the linear integral equation (19), and $f_{\mathrm{H}}^{(1) 2}$ is yet undetermined linear combination of the additive invariants of collision.

(c) Solvability condition to the next equation (19) determines coefficients of the function $f_{\mathrm{H}}^{(1) 2}$ in terms of solutions to the linear hyperbolic differential equations,

$$
\int D_{t}\left(f_{\mathrm{H}}^{(1) 1}+f_{\mathrm{H}}^{(1) 2}\right)\left\{1, \boldsymbol{v}, v^{2}\right\} \mathrm{d} \boldsymbol{v}=0 .
$$

Hilbert was able to demonstrate that this procedure of constructing the normal solution can be carried out to arbitrary order $n$, where the function $f_{\mathrm{H}}^{(n)}$ is determined from the solvability condition at the next, $(n+1)$ th order. In order to summarize, implementation of the Hilbert method requires solutions for the function $n(\boldsymbol{x}, t), \boldsymbol{u}(\boldsymbol{x}, t)$, and $T(\boldsymbol{x}, t)$ obtained from a sequence of partial differential equations. 


\subsubsection{The Chapman-Enskog method}

A completely different approach to the reduced description was invented in 1917 by David Enskog [93], and independently by Sidney Chapman [51]. The key innovation was to seek an expansion of the time derivatives of the hydrodynamic variables rather than seeking the time-space dependencies of these functions as in the Hilbert method.

The Chapman-Enskog method starts also with the singularly perturbed Boltzmann equation, and with the expansion

$$
f_{\mathrm{CE}}=\sum_{n=0}^{\infty} \varepsilon^{n} f_{\mathrm{CE}}^{(n)} \text {. }
$$

However, the procedure of evaluation of the functions $f_{\mathrm{CE}}^{(n)}$ differs from the Hilbert method:

$$
\begin{aligned}
& Q\left(f_{\mathrm{CE}}^{(0)}, f_{\mathrm{CE}}^{(0)}\right)=0, \\
& L f_{\mathrm{CE}}^{(1)}=-Q\left(f_{\mathrm{CE}}^{(0)}, f_{\mathrm{CE}}^{(0)}\right)+\frac{\partial^{(0)}}{\partial t} f_{\mathrm{CE}}^{(0)}+\left(\boldsymbol{v}, \frac{\partial}{\partial \boldsymbol{x}}\right) f_{\mathrm{CE}}^{(0)} .
\end{aligned}
$$

Operator $\partial^{(0)} / \partial t$ is defined from the expansion of the right hand side of hydrodynamic equation,

$$
\frac{\partial^{(0)}}{\partial t}\{\rho, \rho \boldsymbol{u}, e\} \equiv-\int\left\{m, m \boldsymbol{v}, \frac{m v^{2}}{2}\right\}\left(\boldsymbol{v}, \frac{\partial}{\partial \boldsymbol{x}}\right) f_{\mathrm{CE}}^{(0)} \mathrm{d} \boldsymbol{v} .
$$

From Eq. (21), function $f_{\mathrm{CE}}^{(0)}$ is again the local Maxwellian, whereas (23) is the Euler equations, and $\partial^{(0)} / \partial t$ acts on various functions $g(\rho, \rho \boldsymbol{u}, e)$ according to the chain rule,

$$
\frac{\partial^{(0)}}{\partial t} g=\frac{\partial g}{\partial \rho} \frac{\partial^{(0)}}{\partial t} \rho+\frac{\partial g}{\partial(\rho \boldsymbol{u})} \frac{\partial^{(0)}}{\partial t} \rho \boldsymbol{u}+\frac{\partial g}{\partial e} \frac{\partial^{(0)} e}{\partial t},
$$

while the time derivatives $\partial^{(0)} / \partial t$ of the hydrodynamic fields are expressed using the right hand side of Eq. (23).

The result of the Chapman-Enskog definition of the time derivative $\partial^{(0)} / \partial t$, is that the Fredholm alternative is satisfied by the right hand side of Eq. (22). Finally, the solution to the homogeneous equation is set to be zero by the requirement that the hydrodynamic variables as defined by the function $f^{(0)}+\varepsilon f^{(1)}$ coincide with the parameters of the local Maxwellian $f^{(0)}$ :

$$
\int\left\{1, \boldsymbol{v}, v^{2}\right\} f_{\mathrm{CE}}^{(1)} \mathrm{d} \boldsymbol{v}=0
$$

The first correction $f_{\mathrm{CE}}^{(1)}$ of the Chapman-Enskog method adds the terms

$$
\frac{\partial^{(1)}}{\partial t}\{\rho, \rho \boldsymbol{u}, e\}=-\int\left\{m, m \boldsymbol{v}, \frac{m v^{2}}{2}\right\}\left(\boldsymbol{v}, \frac{\partial}{\partial \boldsymbol{x}}\right) f_{\mathrm{CE}}^{(1)} \mathrm{d} \boldsymbol{v}
$$

to the time derivatives of the hydrodynamic fields. These terms correspond to the dissipative hydrodynamics where viscous momentum transfer and heat transfer are in the Navier-Stokes and Fourier form. The Chapman-Enskog method was the first true success of the Boltzmann equation since it had made it possible to derive macroscopic equation without a priori guessing (the generalization of the Boltzmann equation onto mixtures predicted existence of the thermodiffusion before it has been found experimentally), and to express the kinetic coefficient in terms of microscopic particle's interaction. 
However, higher-order corrections of the Chapman-Enskog method, resulting in hydrodynamic equations with derivatives (Burnett hydrodynamic equations) face serve difficulties both from the theoretical, as well as from the practical sides. In particular, they result in unphysical instabilities of the equilibrium.

\subsubsection{The Grad moment method}

In 1949, Harold Grad extended the basic assumption behind the Hilbert and the Chapman-Enskog methods (the space and time dependence of the normal solutions is mediated by the five hydrodynamic moments) [158]. A physical rationale behind the Grad moment method is an assumption of the decomposition of motions:

(i) During the time of order $\tau$, a set of distinguished moments $M^{\prime}$ (which include the hydrodynamic moments and a subset of higher-order moments) does not change significantly as compared to the rest of the moments $M^{\prime \prime}$ (the fast evolution).

(ii) Towards the end of the fast evolution, the values of the moments $M^{\prime \prime}$ become unambiguously determined by the values of the distinguished moments $M^{\prime}$.

(iii) On the time of order $\theta \gg \tau$, dynamics of the distribution function is determined by the dynamics of the distinguished moments while the rest of the moments remain to be determined by the distinguished moments (the slow evolution period).

Implementation of this picture requires an ansatz for the distribution function in order to represent the set of states visited in the course of the slow evolution. In Grad's method, these representative sets are finite-order truncations of an expansion of the distribution functions in terms of Hermite velocity tensors:

$$
f_{\mathrm{G}}\left(M^{\prime}, \boldsymbol{v}\right)=f_{\mathrm{LM}}(\rho, \boldsymbol{u}, E, \boldsymbol{v})\left[1+\sum_{(\alpha)}^{N} a_{(\alpha)}\left(M^{\prime}\right) H_{(\alpha)}(\boldsymbol{v}-\boldsymbol{u})\right],
$$

where $H_{(\alpha)}(\boldsymbol{v}-\boldsymbol{u})$ are various Hermite tensor polynomials, orthogonal with the weight $f_{\mathrm{LM}}$, while coefficient $a_{(\alpha)}\left(M^{\prime}\right)$ are known functions of the distinguished moments $M^{\prime}$, and $N$ is the highest order of $M^{\prime}$. Other moments are functions of $M^{\prime}: M^{\prime \prime}=M^{\prime \prime}\left(f_{\mathrm{G}}\left(M^{\prime}\right)\right)$.

Slow evolution of distinguished moments is found upon substitution of Eq. (24) into the Boltzmann equation and finding the moments of the resulting expression (Grad's moment equations). Following Grad, this extremely simple approximation can be improved by extending the list of distinguished moments. The most well known is Grad's 13-moment approximation where the set of distinguished moments consists of five hydrodynamic moments, five components of the traceless stress tensor $\sigma_{i j}=\int m\left[\left(v_{i}-u_{i}\right)\left(v_{j}-u_{j}\right)-\delta_{i j}(\boldsymbol{v}-\boldsymbol{u})^{2} / 3\right] f \mathrm{~d} \boldsymbol{v}$, and of the three components of the heat flux vector $q_{i}=\int\left(v_{i}-u_{i}\right) m(\boldsymbol{v}-\boldsymbol{u})^{2} / 2 f \mathrm{~d} \boldsymbol{v}$.

The time evolution hypothesis cannot be evaluated for its validity within the framework of Grad's approach. It is not surprising therefore that Grad's methods failed to work in situations where it was (unmotivatedly) supposed to, primarily, in the phenomena with sharp time-space dependence such as the strong shock wave. On the other hand, Grad's method was quite successful for describing transition between parabolic and hyperbolic propagation, in particular, the second sound effect in massive solids at low temperatures, and, in general, situations slightly deviating from the classical Navier-Stokes-Fourier domain. Finally, the Grad method has been important background for 
development of phenomenological nonequilibrium thermodynamics based on hyperbolic first-order equation, the so-called EIT (extended irreversible thermodynamics).

\subsubsection{Special approximations}

Special approximation of the solutions to the Boltzmann equation has been found for several problems, and which perform better than results of "regular" procedures. The most well known is the ansatz introduced independently by Mott-Smith and Tamm for the strong shock wave problem: The (stationary) distribution function is thought as

$$
f_{\mathrm{TMS}}(a(x))=(1-a(x)) f_{+}+a(x) f_{-},
$$

where $f_{ \pm}$are upstream and downstream Maxwell distribution functions, whereas $a(x)$ is an undetermined scalar function of the coordinate along the shock tube.

Equation for function $a(x)$ has to be found upon substitution of Eq. (25) into the Bolltzmann equation, and upon integration with some velocity-dependent function $\varphi(v)$. Two general problems arise with the special approximation thus constructed: Which function $\varphi(v)$ should be taken, and how to find correction to the ansatz like Eq. (25).

\subsubsection{The method of invariant manifold}

The general approach to the problem of reduced description for dissipative system was recognized as the problem of finding stable invariant manifolds in the space of distribution functions [4-6]. The notion of invariant manifold generalizes the normal solution in the Hilbert and in the Chapman -Enskog method, and the finite-moment sets of distribution function in the Grad method: If $\Omega$ is a smooth manifold in the space of distribution function, and if $f_{\Omega}$ is an element of $\Omega$, then $\Omega$ is invariant with respect to the dynamic system,

$$
\begin{aligned}
& \frac{\partial f}{\partial t}=J(f), \\
& \text { if } J\left(f_{\Omega}\right) \in T \Omega \text { for all } f_{\Omega} \in \Omega,
\end{aligned}
$$

where $T \Omega$ is the tangent bundle of the manifold $\Omega$. Application of the invariant manifold idea to dissipative systems is based on iterations, progressively improving the initial approximation, involves the following steps:

Thermodynamic projector. Given a manifold $\Omega$ (not obligatory invariant), the macroscopic dynamics on this manifold is defined by the macroscopic vector field, which is the result of a projection of vectors $J\left(f_{\Omega}\right)$ onto the tangent bundle $T \Omega$. The thermodynamic projector $P_{f_{\Omega}}^{*}$ takes advantage of dissipativity:

$$
\left.\operatorname{ker} P_{f_{\Omega}}^{*} \subseteq \operatorname{ker} D_{f} S\right|_{f_{\Omega}},
$$

where $\left.D_{f} S\right|_{f_{\Omega}}$ is the differential of the entropy evaluated in $f_{\Omega}$.

This condition of thermodynamicity means that each state of the manifold $\Omega$ is regarded as the result of decomposition of motions occurring near $\Omega$ : The state $f_{\Omega}$ is the maximum entropy state on the set of states $f_{\Omega}+\operatorname{ker} P_{f_{\Omega}}^{*}$. Condition of thermodynamicity does not define projector completely; rather, it is the condition that should be satisfied by any projector used to define the macroscopic vector field, $J_{\Omega}^{\prime}=P_{f_{\Omega}}^{*} J\left(f_{\Omega}\right)$. For, once the condition (28) is met, the macroscopic vector field 
preserves dissipativity of the original microscopic vector field $J(f)$ :

$$
\left.D_{f} S\right|_{f_{\Omega}} \cdot P_{f_{\Omega}}^{*}\left(J\left(f_{\Omega}\right)\right) \geqslant 0 \text { for all } f_{\Omega} \in \Omega .
$$

The thermodynamic projector is the formalization of the assumption that $\Omega$ is the manifold of slow motion: If a fast relaxation takes place at least in a neighborhood of $\Omega$, then the states visited in this process before arriving at $f_{\Omega}$ belong to $\operatorname{ker} P_{f_{\Omega}}^{*}$. In general, $P_{f_{\Omega}}^{*}$ depends in a nontrivial way on $f_{\Omega}$.

Iterations for the invariance condition. The invariance condition for the manifold $\Omega$ reads

$$
P_{\Omega}\left(J\left(f_{\Omega}\right)\right)-J\left(f_{\Omega}\right)=0,
$$

here $P_{\Omega}$ is arbitrary (not obligatory thermodynamic) projector onto the tangent bundle of $\Omega$. The invariance condition is considered as an equation which is solved iteratively, starting with initial approximation $\Omega_{0}$. On the $(n+1)$ th iteration, the correction $f^{(n+1)}=f^{(n)}+\delta f^{(n+1)}$ is found from linear equations,

$$
\begin{aligned}
& D_{f} J_{n}^{*} \delta f^{(n+1)}=P_{n}^{*} J\left(f^{(n)}\right)-J\left(f^{(n)}\right), \\
& P_{n}^{*} \delta f^{(n+1)}=0,
\end{aligned}
$$

here $D_{f} J_{n}^{*}$ is the linear selfajoint operator with respect to the scalar product by the second differential of the entropy $\left.D_{f}^{2} S\right|_{f^{(n)}}$.

Together with the above-mentioned principle of thermodynamic projecting, the selfadjoint linearization implements the assumption about the decomposition of motions around the $n$th approximation. The selfadjoint linearization of the Boltzmann collision integral $Q$ (7) around a distribution function $f$ is given by the formula,

$$
\begin{aligned}
D_{f} Q^{*} \delta f= & \int W\left(\boldsymbol{v}, \boldsymbol{w}, \mid \boldsymbol{v}^{\prime}, \boldsymbol{w}^{\prime}\right) \frac{f(\boldsymbol{v}) f(\boldsymbol{w})+f\left(\boldsymbol{v}^{\prime}\right) f\left(\boldsymbol{w}^{\prime}\right)}{2} \\
& \times\left[\frac{\delta f\left(\boldsymbol{v}^{\prime}\right)}{f\left(\boldsymbol{v}^{\prime}\right)}+\frac{\delta f\left(\boldsymbol{w}^{\prime}\right)}{f\left(\boldsymbol{w}^{\prime}\right)}-\frac{\delta f(\boldsymbol{v})}{f(\boldsymbol{v})}-\frac{\delta f(\boldsymbol{w})}{f(\boldsymbol{w})}\right] \mathrm{d} \boldsymbol{w}^{\prime} \mathrm{d} \boldsymbol{v}^{\prime} \mathrm{d} \boldsymbol{w} .
\end{aligned}
$$

If $f=f_{\mathrm{LM}}$, the self-adjoint operator (30) becomes the linearized collision integral.

The method of invariant manifold is the iterative process:

$$
\left(f^{(n)}, P_{n}^{*}\right) \rightarrow\left(f^{(n+1)}, P_{n}^{*}\right) \rightarrow\left(f^{(n+1)}, P_{n+1}^{*}\right) .
$$

On the each 1st part of the iteration, the linear equation (29) is solved with the projector known from the previous iteration. On the each 2 nd part, the projector is updated, following the thermodynamic construction.

The method of invariant manifold can be further simplified if smallness parameters are known.

The proliferation of the procedure in comparison to the Chapman-Enskog method is essentially twofold:

First, the projector is made dependent on the manifold. This enlarges the set of admissible approximations.

Second, the method is based on iteration rather than a series expansion in a smallness parameter. Importance of iteration procedures is well understood in physics, in particular, in the renormalization group approach to reducing the description in equilibrium statistical mechanics, and in the Kolmogorov-Arnold-Moser theory of finite-dimensional Hamiltonian systems. 


\subsubsection{Quasi-equilibrium approximations}

Important generalization of the Grad moment method is the concept of the quasi-equilibrium approximations already mentioned above (we will discuss this approximation in detail in a separate section). The quasi-equilibrium distribution function for a set of distinguished moment $M=m(f)$ maximizes the entropy density $S$ for fixed $M$. The quasi-equilibrium manifold $\Omega^{*}(M)$ is the collection of the quasi-equilibrium distribution functions for all admissible values of $M$. The quasi-equilibrium approximation is the simplest and extremely useful (not only in the kinetic theory itself) implementation of the hypothesis about a decomposition of motions: if $M$ are considered as slow variables, then states which could be visited in the course of rapid motion in the vicinity of $\Omega^{*}(M)$ belong to the planes $\Gamma_{M}=\left\{f \mid m\left(f-f^{*}(M)\right)=0\right\}$. In this respect, the thermodynamic construction in the method of invariant manifold is a generalization of the quasi-equilibrium approximation where the given manifold is equipped with a quasi-equilibrium structure by choosing appropriately the macroscopic variables of the slow motion. In contrast to the quasi-equilibrium, the macroscopic variables thus constructed are not obligatory moments. A text book example of the quasi-equilibrium approximation is the generalized Gaussian function for $M=\{\rho, \rho \boldsymbol{u}, P\}$ where $P_{i j}=\int v_{i} v_{j} f \mathrm{~d} \boldsymbol{v}$ is the pressure tensor.

The thermodynamic projector $P^{*}$ for a quasi-equilibrium approximation was first introduced by Robertson [95] (in a different context of conservative dynamics and for a special case of the Gibbs-Shannon entropy). It acts on a function $\Psi$ as follows:

$$
P_{M}^{*} \Psi=\sum_{i} \frac{\partial f^{*}}{\partial M_{i}} \int m_{i} \Psi \mathrm{d} \boldsymbol{v},
$$

where $M=\int m_{i} f \mathrm{~d} \boldsymbol{v}$. The quasi-equilibrium approximation does not exist if the highest order moment is an odd polynomial of velocity (therefore, there exists no quasi-equilibrium for thirteen Grad's moments). Otherwise, the Grad moment approximation is the first-order expansion of the quasi-equilibrium around the local Maxwellian.

\subsection{Discrete velocity models}

If the number of microscopic velocities is reduced drastically to only a finite set, the resulting discrete velocity, continuous time and continuous space models can still mimic the gas-dynamic flows. This idea was introduced in Broadwell's paper in 1963 to mimic the strong shock wave [94].

Further important development of this idea was due to Cabannes and Gatignol in the seventies who introduced a systematic class of discrete velocity models [98]. The structure of the collision operators in the discrete velocity models mimics the polynomial character of the Boltzmann collision integral. Discrete velocity models are implemented numerically by using the natural operator splitting in which each update due to free flight is followed by the collision update, the idea which dates back to Grad. One of the most important recent results is the proof of convergence of the discrete velocity models with pair collisions to the Boltzmann collision integral.

\subsection{Direct simulation}

Besides the analytical approach, direct numerical simulation of Boltzmann-type nonlinear kinetic equations have been developed since mid of 1960s [96,97]. The basis of the approach is a representation of the Boltzmann gas by a set of particles whose dynamics is modeled as a sequence of free 
propagation and collisions. The modeling of collisions uses a random choice of pairs of particles inside the cells of the space, and changing the velocities of these pairs in such a way as to comply with the conservation laws, and in accordance with the kernel of the Boltzmann collision integral. At present, there exists a variety of this scheme known under the common title of the direct simulation Monte-Carlo method [96,97]. The DSMC, in particular, provides data to test various analytical theories.

\subsection{Lattice gas and Lattice Boltzmann models}

Since mid of $1980 \mathrm{~s}$, the kinetic theory based approach to simulation of complex macroscopic phenomena such as hydrodynamics has been developed. The main idea of the approach is construction of minimal kinetic system in such a way that their long-time and large-scale limit matches the desired macroscopic equations. For this purpose, the fully discrete (in time-space-velocity) nonlinear kinetic equations are considered on sufficiently isotropic lattices, where the links represent the discrete velocities of fictitious particles. In the earlier version of the lattice methods, the particle-based picture has been exploited, subject to the exclusion rule (one or zero particle per lattice link) [the lattice gas model [99]]. Most of the present versions use the distribution function picture, where populations of the links are noninteger [the Lattice Boltzmann model [100-104]]. Discrete-time dynamics consists of a propagation step where populations are transmitted to adjacent links and collision step where populations of the links at each node of the lattice are equilibrated by a certain rule. Most of the present versions use the BGK-type equilibration, where the local equilibrium is constructed in such a way as to match desired macroscopic equations. The Lattice Boltzmann method is a useful approach for computational fluid dynamics, effectively compliant with parallel architectures. The proof of the $H$ theorem for the Lattice gas models is based on the semi-detailed (or Stueckelberg's) balance principle. The proof of the $H$ theorem in the framework of the Lattice Boltzmann method has been only very recently achieved [105-110].

\subsection{Other kinetic equations}

\subsubsection{The Enskog equation for hard spheres}

The Enskog equation for hard spheres is an extension of the Boltzmann equation to moderately dense gases. The Enskog equation explicitly takes into account the nonlocality of collisions through a two-fold modification of the Boltzmann collision integral: First, the one-particle distribution functions are evaluated at the locations of the centers of spheres, separated by the nonzero distance at the impact. This makes the collision integral nonlocal in space. Second, the equilibrium pair distribution function at the contact of the spheres enhances the scattering probability. The proof of the $H$ theorem for the Enskog equation has posed certain difficulties, and has led to a modification of the collision integral.

Methods of solution of the Enskog equation are immediate generalizations of those developed for the Boltzmann equation, but there is one additional difficulty. The Enskog collision integral is nonlocal in space. The Chapman-Enskog method, when applied to the Enskog equation, is supplemented with a gradient expansion around the homogeneous equilibrium state. 


\subsubsection{The Vlasov equation}

The Vlasov equation (or kinetic equation for a self-consistent force) is the nonlinear equation for the one-body distribution function, which takes into account a long-range interaction between particles:

$$
\frac{\partial}{\partial t} f+\left(\boldsymbol{v}, \frac{\partial}{\partial \boldsymbol{x}} f\right)+\left(\mathbf{F}, \frac{\partial}{\partial \boldsymbol{v}} f\right)=0,
$$

where $\mathbf{F}=\int \Phi\left(\left|\boldsymbol{x}-\boldsymbol{x}^{\prime}\right|\right)\left(\boldsymbol{x}-\boldsymbol{x}^{\prime}\right) /\left(\left|\boldsymbol{x}-\boldsymbol{x}^{\prime}\right|\right) n\left(\boldsymbol{x}^{\prime}\right) \mathrm{d} \boldsymbol{x}^{\prime}$ is the self-consistent force. In this expression $\Phi\left(\left|\boldsymbol{x}-\boldsymbol{x}^{\prime}\right|\right)\left(\boldsymbol{x}-\boldsymbol{x}^{\prime}\right) /\left(\left|\boldsymbol{x}-\boldsymbol{x}^{\prime}\right|\right)$ is the microscopic force between the two particles, and $n\left(\boldsymbol{x}^{\prime}\right)$ is the density of particles, defined self-consistently, $n\left(\boldsymbol{x}^{\prime}\right)=\int f\left(\boldsymbol{x}^{\prime}, \boldsymbol{v}\right) \mathrm{d} \boldsymbol{v}$.

The Vlasov equation is used for a description of collisionless plasmas in which case it is completed by a set of Maxwell equation for the electromagnetic field [135]. It is also used for a description of the gravitating gas.

The Vlasov equation is an infinite-dimensional Hamiltonian system. Many special and approximate (wave-like) solutions to the Vlasov equation are known and they describe important physical effects. One of the most well known effects is the Landau damping: The energy of a volume element dissipates with the rate

$$
Q \approx-\left.|E|^{2} \frac{\omega(k)}{k^{2}} \frac{\mathrm{d} f_{0}}{\mathrm{~d} v}\right|_{v=\omega / k},
$$

where $f_{0}$ is the Maxwell distribution function, $|E|$ is the amplitude of the applied monochromatic electric field with the frequency $\omega(k), k$ is the wave vector. The Landau damping is thermodynamically reversible effect, and it is not accompanied with an entropy increase. Thermodynamically reversed to the Landau damping is the plasma echo effect.

\subsubsection{The Fokker-Planck equation}

The Fokker-Planck equation (FPE) is a familiar model in various problems of nonequilibrium statistical physics [111,112]. We consider the FPE of the form

$$
\frac{\partial W(\boldsymbol{x}, t)}{\partial t}=\frac{\partial}{\partial \boldsymbol{x}}\left\{D\left[W \frac{\partial}{\partial \boldsymbol{x}} U+\frac{\partial}{\partial \boldsymbol{x}} W\right]\right\} .
$$

Here $W(\boldsymbol{x}, t)$ is the probability density over the configuration space $x$, at the time $t$, while $U(\boldsymbol{x})$ and $D(\boldsymbol{x})$ are the potential and the positively semi-definite $((\boldsymbol{y}, D \boldsymbol{y}) \geqslant 0)$ diffusion matrix.

The FPE (31) is particularly important in studies of polymer solutions [113-115]. Let us recall the two properties of the FPE (31), important to what will follow: (i). Conservation of the total probability: $\int W(\boldsymbol{x}, t) \mathrm{d} x=1$. (ii). Dissipation: The equilibrium distribution, $W_{\mathrm{eq}} \propto \exp (-U)$, is the unique stationary solution to the FPE (31). The entropy,

$$
S[W]=-\int W(\boldsymbol{x}, t) \ln \left[\frac{W(\boldsymbol{x}, t)}{W_{\mathrm{eq}}(\boldsymbol{x})}\right] \mathrm{d} x,
$$


is a monotonically growing function due to the FPE (31), and it arrives at the global maximum in the equilibrium. These properties are most apparent when the FPE (31) is rewritten as follows:

$$
\partial_{t} W(\boldsymbol{x}, t)=\hat{M}_{W} \frac{\delta S[W]}{\delta W(\boldsymbol{x}, t)},
$$

where

$$
\hat{M}_{W}=-\frac{\partial}{\partial \boldsymbol{x}}\left[W(\boldsymbol{x}, t) D(\boldsymbol{x}) \frac{\partial}{\partial \boldsymbol{x}}\right]
$$

is a positive semi-definite symmetric operator with kernel 1. Form (33) is the dissipative part of a structure termed GENERIC (the dissipative vector field is a metric transform of the entropy gradient) [116,117].

The entropy does not depend on kinetic constants. It is the same for different details of kinetics and depends only on equilibrium data. Let us call this property "universality". It is known that for the Boltzmann equation there exists only one universal Lyapunov functional. It is the entropy (we do not distinguish functionals which are connected by multiplication on a constant or adding a constant). But for the FPE there exists a big family of universal Lyapunov functionals. Let $h(a)$ be a convex function of one variable $a \geqslant 0, h^{\prime \prime}(a)>0$,

$$
S_{h}[W]=-\int W_{\mathrm{eq}}(\boldsymbol{x}) h\left[\frac{W(\boldsymbol{x}, t)}{W_{\mathrm{eq}}(\boldsymbol{x})}\right] \mathrm{d} x .
$$

The production of the generalized entropy $S_{h}, \sigma_{h}$ is nonnegative:

$$
\sigma_{h}(\boldsymbol{x})=W_{\mathrm{eq}}(\boldsymbol{x}) h^{\prime \prime}\left[\frac{W(\boldsymbol{x}, t)}{W_{\mathrm{eq}}(\boldsymbol{x})}\right]\left(\frac{\partial}{\partial \boldsymbol{x}} \frac{W(\boldsymbol{x}, t)}{W_{\mathrm{eq}}(\boldsymbol{x})}, D \frac{\partial}{\partial \boldsymbol{x}} \frac{W(\boldsymbol{x}, t)}{W_{\mathrm{eq}}(\boldsymbol{x})}\right) \geqslant 0 .
$$

The most important variants for choice of $h$ :

$h(a)=a \ln a, S_{h}$ is the Boltzmann-Gibbs-Shannon entropy (in the Kullback form [118,119]),

$h(a)=a \ln a-\epsilon \ln a, S_{h}^{\epsilon}$ is the maximal family of additive entropies [120-122] (these entropies are additive for composition of independent subsystems).

$h(a)=\left(1-a^{q}\right) /(1-q), S_{h}^{q}$ is the family of Tsallis entropies [123,124]. These entropies are not additive, but become additive after nonlinear monotonous transformation. This property can serve as definition of the Tsallis entropies in the class of generalized entropies (34) [122].

\subsection{Equations of chemical kinetics and their reduction}

\subsubsection{Outline of the dissipative reaction kinetics}

We begin with an outline of the reaction kinetics (for details see e.g. the book [59]). Let us consider a closed system with $n$ chemical species $\mathrm{A}_{1}, \ldots, \mathrm{A}_{n}$, participating in a complex reaction. The complex reaction is represented by the following stoichiometric mechanism:

$$
\alpha_{s 1} \mathrm{~A}_{1}+\cdots+\alpha_{s n} \mathrm{~A}_{n} \rightleftharpoons \beta_{s 1} \mathrm{~A}_{1}+\cdots+\beta_{s n} \mathrm{~A}_{n},
$$

where the index $s=1, \ldots, r$ enumerates the reaction steps, and where integers, $\alpha_{s i}$ and $\beta_{s i}$, are stoichiometric coefficients. For each reaction step $s$, we introduce $n$-component vectors $\boldsymbol{\alpha}_{s}$ and $\boldsymbol{\beta}_{s}$ with components $\alpha_{s i}$ and $\beta_{s i}$. Notation $\gamma_{s}$ stands for the vector with integer components $\gamma_{s i}=\beta_{s i}-\alpha_{s i}$ (the stoichiometric vector). 
For every $A_{i}$ an extensive variable $N_{i}$, "the number of particles of that species", is defined. The concentration of $A_{i}$ is $c_{i}=N_{i} / V$, where $V$ is the volume.

Given the stoichiometric mechanism (36), the reaction kinetic equations read:

$$
\dot{\boldsymbol{N}}=V \boldsymbol{J}(\boldsymbol{c}), \quad \boldsymbol{J}(\boldsymbol{c})=\sum_{s=1}^{r} \gamma_{s} W_{s}(\boldsymbol{c}),
$$

where dot denotes the time derivative, and $W_{s}$ is the reaction rate function of the step $s$. In particular, the mass action law suggests the polynomial form of the reaction rates:

$$
W_{s}(\boldsymbol{c})=W_{s}^{+}(\boldsymbol{c})-W_{s}^{-}(\boldsymbol{c})=k_{s}^{+}(T) \prod_{i=1}^{n} c_{i}^{\alpha_{i}}-k_{s}^{-}(T) \prod_{i=1}^{n} c_{i}^{\beta_{i}},
$$

where $k_{s}^{+}(T)$ and $k_{s}^{-}(T)$ are the constants of the direct and of the inverse reactions rates of the $s$ th reaction step, $T$ is the temperature. The (generalized) Arrhenius equation gives the most popular form of dependence $k_{s}^{+}(T)$ :

$$
k_{s}^{ \pm}(T)=a_{s}^{ \pm} T^{b_{s}^{ \pm}} \exp \left(S_{s}^{ \pm} / k_{\mathrm{B}}\right) \exp \left(-H_{s}^{ \pm} / k_{\mathrm{B}} T\right),
$$

where $a_{s}^{ \pm}, b_{s}^{ \pm}$are constants, $H_{s}^{ \pm}$are activation enthalpies, $S_{s}^{ \pm}$are activation entropies.

The rate constants are not independent. The principle of detail balance gives the following connection between these constants: There exists such a positive vector $c^{\mathrm{eq}}(T)$ that

$$
W_{s}^{+}\left(c^{\mathrm{eq}}\right)=W_{s}^{-}\left(c^{\mathrm{eq}}\right) \quad \text { for all } s=1, \ldots, r .
$$

The necessary and sufficient conditions for existence of such $c^{\text {eq }}$ can be formulate as the system of polynomial equalities for $\left\{k_{s}^{ \pm}\right\}$, if the stoichiometric vectors $\left\{\gamma_{s}\right\}$ are linearly dependent (see, for example, [59]).

The reaction kinetic equations (37) do not give us a closed system of equations, because dynamics of the volume $V$ is not defined still. Four classical conditions for closure of this system are well studied: $U, V=$ const (isolated system, $U$ is the internal energy); $H, P=$ const (thermal isolated isobaric system, $P$ is the pressure, $H=U+P V$ is the enthalpy), $V, T=$ const (isochoric isothermal conditions); $P, T=$ const (isobaric isothermal conditions). For $V, T=$ const we do not need additional equations and data. It is possible just to divide Eq. (37) on the constant volume and write

$$
\dot{\boldsymbol{c}}=\sum_{s=1}^{r} \gamma_{s} W_{s}(\boldsymbol{c}) \text {. }
$$

For nonisothermal and nonisochoric conditions we do need addition formulae to derive $T$ and $V$. For all four classical conditions the thermodynamic Lyapunov functions $G$ for kinetic equations are known:

$$
\begin{aligned}
& U, V=\text { const }, G_{U, V}=-S / k_{\mathrm{B}} ; \quad V, T=\mathrm{const}, G_{V, T}=F / k_{\mathrm{B}} T=U / k_{\mathrm{B}} T-S / k_{\mathrm{B}} ; \\
& H, P=\mathrm{const}, G_{H, P}=-S / k_{\mathrm{B}} ; \quad P, T=\mathrm{const}, G_{P, T}=G / T=H / k_{\mathrm{B}} T-S / k_{\mathrm{B}},
\end{aligned}
$$

where $F=U-T S$ is the free energy (Helmholtz free energy), $G=H-T S$ is the free enthalpy (Gibbs free energy). All the thermodynamic Lyapunov functions are normalized to dimensionless scale (if one measures the number of particles in moles, then it is necessary to change $k_{\mathrm{B}}$ to $R$ ). All these functions decrease in time. For classical conditions the correspondent thermodynamic Lyapunov 
functions can be written in the form: $G_{\bullet}($ const, $\boldsymbol{N})$. The derivatives $\partial G_{\bullet}($ const, $\boldsymbol{N}) / \partial N_{i}$ are the same functions of $c$ and $T$ for all classical conditions:

$$
\mu_{i}(\boldsymbol{c}, T)=\frac{\partial G_{\bullet}(\text { const }, \boldsymbol{N})}{\partial N_{i}}=\frac{\mu_{\text {chem } i}(\boldsymbol{c}, T)}{k_{\mathrm{B}} T},
$$

where $\mu_{\mathrm{chem} i}(\boldsymbol{c}, T)$ is the chemical potential of $A_{i}$.

Usual $G_{\bullet}($ const, $\boldsymbol{N})$ are strictly convex functions of $\boldsymbol{N}$, and the matrix $\partial \mu_{i} / \partial c_{j}$ is positively defined. The dissipation inequality (44) holds

$$
\frac{\mathrm{d} G_{\bullet}}{\mathrm{d} t}=V(\boldsymbol{\mu}, \boldsymbol{J}) \leqslant 0 .
$$

This inequality is the restriction on possible kinetic low and on possible values of kinetic constants.

The most important generalization of the mass action law (38) is the Marcelin-De Donder kinetic function. This generalization [187,188] is based on ideas of the thermodynamic theory of affinity [189]. We use the kinetic function suggested in its final form in [188]. Within this approach, the functions $W_{s}$ are constructed as follows: For a given $\boldsymbol{\mu}(\boldsymbol{c}, T)(43)$, and for a given stoichiometric mechanism (36), we define the gain $(+)$ and the loss $(-)$ rates of the sth step,

$$
W_{s}^{+}=\varphi_{s}^{+} \exp \left(\boldsymbol{\mu}, \boldsymbol{\alpha}_{s}\right), \quad W_{s}^{-}=\varphi_{s}^{-} \exp \left(\boldsymbol{\mu}, \boldsymbol{\beta}_{s}\right)
$$

where $\varphi_{s}^{ \pm}>0$ are kinetic factors. The Marcelin-De Donder kinetic function reads: $W_{s}=W_{s}^{+}-W_{s}^{-}$, and the right hand side of the kinetic equation (37) becomes,

$$
\boldsymbol{J}=\sum_{s=1}^{r} \gamma_{s}\left\{\varphi_{s}^{+} \exp \left(\boldsymbol{\mu}, \boldsymbol{\alpha}_{s}\right)-\varphi_{s}^{-} \exp \left(\boldsymbol{\mu}, \boldsymbol{\beta}_{s}\right)\right\} .
$$

For the Marcelin-De Donder reaction rate (45), the dissipation inequality (44) reads:

$$
\dot{G}=\sum_{s=1}^{r}\left[\left(\boldsymbol{\mu}, \boldsymbol{\beta}_{s}\right)-\left(\boldsymbol{\mu}, \boldsymbol{\alpha}_{s}\right)\right]\left\{\varphi_{s}^{+} \mathrm{e}^{\left(\boldsymbol{\mu}, \boldsymbol{\alpha}_{s}\right)}-\varphi_{s}^{-} \mathrm{e}^{\left(\boldsymbol{\mu}, \boldsymbol{\beta}_{s}\right)}\right\} \leqslant 0 .
$$

The kinetic factors $\varphi_{s}^{ \pm}$should satisfy certain conditions in order to make valid the dissipation inequality (47). A well known sufficient condition is the detail balance:

$$
\varphi_{s}^{+}=\varphi_{s}^{-},
$$

other sufficient conditions are discussed in detail elsewhere [125,59,126].

For ideal systems, function $G_{\bullet}$ is constructed from the thermodynamic data of individual species. It is convenient to start from the isochoric isothermal conditions. The Helmholtz free energy for ideal system is

$$
F=k_{\mathrm{B}} T \sum_{i} N_{i}\left[\ln c_{i}-1+\mu_{0 i}\right]+\operatorname{const}_{T, V},
$$

where the internal energy is assumed to be a linear function:

$$
U=\sum_{i} N_{i} u_{i}(T)=\sum_{i} N_{i}\left(u_{0 i}+C_{V i} T\right)
$$


in given interval of $\boldsymbol{c}, T, u_{i}(T)$ is the internal energy of $A_{i}$ per particle. It is well known that $S=-(\partial F / \partial T)_{V, N=\text { const }}, U=F+T S=F-T(\partial F / \partial T)_{V, N=\text { const }}$, hence, $u_{i}(T)=-k_{\mathrm{B}} T^{2} \mathrm{~d} \mu_{0 i} / \mathrm{d} T$ and

$$
\mu_{0 i}=\delta_{i}+u_{0 i} / k_{\mathrm{B}} T-\left(C_{V i} / k_{\mathrm{B}}\right) \ln T,
$$

where $\delta_{i}=$ const, $C_{V i}$ is the $A_{i}$ heat capacity at constant volume (per particle).

In concordance with the form of ideal free energy (49) the expression for $\boldsymbol{\mu}$ is:

$$
\mu_{i}=\ln c_{i}+\delta_{i}+u_{0 i} / k_{\mathrm{B}} T-\left(C_{V i} / k_{\mathrm{B}}\right) \ln T .
$$

For the function $\boldsymbol{\mu}$ of form (51), the Marcelin-De Donder equation casts into the more familiar mass action law form (38). Taking into account the principle of detail balance (48) we get the ideal rate functions:

$$
\begin{aligned}
& W_{s}(\boldsymbol{c})=W_{s}^{+}(\boldsymbol{c})-W_{s}^{-}(\boldsymbol{c}), \\
& W_{s}^{+}(\boldsymbol{c})=\varphi(\boldsymbol{c}, T) T^{-\sum_{i} \alpha_{s i} C_{V i} / k_{\mathrm{B}}} \mathrm{e}^{\sum_{i} \alpha_{s i}\left(\delta_{i}+u_{0 i} / k_{\mathrm{B}} T\right)} \prod_{i=1}^{n} c_{i}^{\alpha_{i}}, \\
& W_{s}^{-}(\boldsymbol{c})=\varphi(\boldsymbol{c}, T) T^{-\sum_{i} \beta_{s i} C_{V i} / k_{\mathrm{B}}} \mathrm{e}^{\sum_{i} \beta_{s i}\left(\delta_{i}+u_{0 i} / k_{\mathrm{B}} T\right)} \prod_{i=1}^{n} c_{i}^{\beta_{i}} .
\end{aligned}
$$

where $\varphi(c, T)$ is an arbitrary positive function (from thermodynamic point of view).

Let us discuss further the vector field $\boldsymbol{J}(\boldsymbol{c})$ in the concentration space (41). Conservation laws (balances) impose linear constrains on admissible vectors $\mathrm{d} c / \mathrm{d} t$ :

$$
\left(\boldsymbol{b}_{i}, \boldsymbol{c}\right)=B_{i}=\text { const }, \quad i=1, \ldots, l,
$$

where $\boldsymbol{b}_{i}$ are fixed and linearly independent vectors. Let us denote as $\boldsymbol{B}$ the set of vectors which satisfy the conservation laws (53) with given $B_{i}$ :

$$
\boldsymbol{B}=\left\{\boldsymbol{c} \mid\left(\boldsymbol{b}_{1}, \boldsymbol{c}\right)=B_{1}, \ldots,\left(\boldsymbol{b}_{l}, \boldsymbol{c}\right)=B_{l}\right\} .
$$

The natural phase space $\boldsymbol{X}$ of system (41) is the intersection of the cone of $n$-dimensional vectors with nonnegative components, with the set $\boldsymbol{B}$, and $\operatorname{dim} \boldsymbol{X}=d=n-l$. In the sequel, we term a vector $\boldsymbol{c} \in \boldsymbol{X}$ the state of the system. In addition, we assume that each of the conservation laws is supported by each elementary reaction step, that is

$$
\left(\gamma_{s}, \boldsymbol{b}_{i}\right)=0 \text {, }
$$

for each pair of vectors $\gamma_{s}$ and $\boldsymbol{b}_{i}$.

Reaction kinetic equations describe variations of the states in time. The phase space $\boldsymbol{X}$ is positiveinvariant of system (41): If $\boldsymbol{c}(0) \in \boldsymbol{X}$, then $\boldsymbol{c}(t) \in \boldsymbol{X}$ for all the times $t>0$.

In the sequel, we assume that the kinetic equation (41) describes evolution towards the unique equilibrium state, $\boldsymbol{c}^{\mathrm{eq}}$, in the interior of the phase space $\boldsymbol{X}$. Furthermore, we assume that there exists a strictly convex function $G(c)$ which decreases monotonically in time due to Eq. (41):

Here $\nabla G$ is the vector of partial derivatives $\partial G / \partial c_{i}$, and the convexity assumes that the $n \times n$ matrices

$$
\boldsymbol{H}_{\boldsymbol{c}}=\left\|\partial^{2} G(\boldsymbol{c}) / \partial c_{i} \partial c_{j}\right\|
$$


are positive definite for all $\boldsymbol{c} \in \boldsymbol{X}$. In addition, we assume that the matrices (55) are invertible if $\boldsymbol{c}$ is taken in the interior of the phase space.

The function $G$ is the Lyapunov function of system (37), and $c^{\mathrm{eq}}$ is the point of global minimum of the function $G$ in the phase space $X$. Otherwise stated, the manifold of equilibrium states $c^{\mathrm{eq}}\left(B_{1}, \ldots, B_{l}\right)$ is the solution to the variational problem,

$$
G \rightarrow \min \text { for }\left(\boldsymbol{b}_{i}, \boldsymbol{c}\right)=B_{i}, \quad i=1, \ldots, l .
$$

For each fixed value of the conserved quantities $B_{i}$, the solution is unique. In many cases, however, it is convenient to consider the whole equilibrium manifold, keeping the conserved quantities as parameters.

For example, for perfect systems in a constant volume under a constant temperature, the Lyapunov function $G$ reads

$$
G=\sum_{i=1}^{n} c_{i}\left[\ln \left(c_{i} / c_{i}^{\mathrm{eq}}\right)-1\right] .
$$

It is important to stress that $c^{\mathrm{eq}}$ in Eq. (57) is an arbitrary equilibrium of the system, under arbitrary values of the balances. In order to compute $G(\boldsymbol{c})$, it is unnecessary to calculate the specific equilibrium $c^{\text {eq }}$ which corresponds to the initial state $c$. Let us compare the Lyapunov function $G$ (57) with the classical formula for the free energy (49). This comparison gives a possible choice for $c^{\mathrm{eq}}$ :

$$
\ln c_{i}^{\mathrm{eq}}=-\delta_{i}-u_{0 i} / k_{\mathrm{B}} T+\left(C_{V i} / k_{\mathrm{B}}\right) \ln T .
$$

\subsubsection{The problem of reduced description in chemical kinetics}

What does it mean, "to reduce the description of a chemical system"? This means the following:

1. To shorten the list of species. This, in turn, can be achieved in two ways:

(i) To eliminate inessential components from the list;

(ii) To lump some of the species into integrated components.

2. To shorten the list of reactions. This also can be done in several ways:

(i) To eliminate inessential reactions, those which do not significantly influence the reaction process;

(ii) To assume that some of the reactions "have been already completed", and that the equilibrium has been reached along their paths (this leads to dimensional reduction because the rate constants of the "completed" reactions are not used thereafter, what one needs are equilibrium constants only).

3. To decompose the motions into fast and slow, into independent (almost-independent) and slaved, etc. As the result of such a decomposition, the system admits a study "in parts". After that, results of this study are combined into a joint picture. There are several approaches which fall into this category. The famous method of the quasi-steady state (QSS), pioneered by Bodenstein and Semenov, follows the Chapman-Enskog method. The partial equilibrium approximations are predecessors of the Grad method and quasi-equilibrium approximations in physical kinetics. These two family of methods have different physical backgrounds and mathematical forms. 


\subsubsection{Partial equilibrium approximations}

Quasi-equilibrium with respect to reactions is constructed as follows: from the list of reactions (36), one selects those which are assumed to equilibrate first. Let they be indexed with the numbers $s_{1}, \ldots, s_{k}$. The quasi-equilibrium manifold is defined by the system of equations,

$$
W_{s_{i}}^{+}=W_{s_{i}}^{-}, \quad i=1, \ldots, k .
$$

This system of equations looks particularly elegant when written in terms of conjugated (dual) variables, $\boldsymbol{\mu}=\nabla G$ :

$$
\left(\gamma_{s_{i}}, \boldsymbol{\mu}\right)=0, \quad i=1, \ldots, k \text {. }
$$

In terms of conjugated variables, the quasi-equilibrium manifold forms a linear subspace. This subspace, $L^{\perp}$, is the orthogonal completement to the linear envelope of vectors, $L=\operatorname{lin}\left\{\gamma_{s_{1}}, \ldots, \gamma_{s_{k}}\right\}$.

Quasi-equilibrium with respect to species is constructed practically in the same way but without selecting the subset of reactions. For a given set of species, $A_{i_{1}}, \ldots, A_{i_{k}}$, one assumes that they evolve fast to equilibrium, and remain there. Formally, this means that in the $k$-dimensional subspace of the space of concentrations with the coordinates $c_{i_{1}}, \ldots, c_{i_{k}}$, one constructs the subspace $L$ which is defined by the balance equations, $\left(\boldsymbol{b}_{i}, \boldsymbol{c}\right)=0$. In terms of the conjugated variables, the quasi-equilibrium manifold, $L^{\perp}$, is defined by equations,

$$
\boldsymbol{\mu} \in L^{\perp}, \quad\left(\boldsymbol{\mu}=\left(\mu_{1}, \ldots, \mu_{n}\right)\right) .
$$

The same quasi-equilibrium manifold can be also defined with the help of fictitious reactions: Let $\boldsymbol{g}_{1}, \ldots, \boldsymbol{g}_{q}$ be a basis in $L$. Then Eq. (61) may be rewritten as follows:

$$
\left(\boldsymbol{g}_{i}, \boldsymbol{\mu}\right)=0, \quad i=1, \ldots, q .
$$

Illustration: Quasi-equilibrium with respect to reactions in hydrogen oxidation: Let us assume equilibrium with respect to dissociation reactions, $\mathrm{H}_{2} \rightleftharpoons 2 \mathrm{H}$, and, $\mathrm{O}_{2} \rightleftharpoons 2 \mathrm{O}$, in some subdomain of reaction conditions. This gives

$$
k_{1}^{+} c_{\mathrm{H}_{2}}=k_{1}^{-} c_{\mathrm{H}}^{2}, \quad k_{2}^{+} c_{\mathrm{O}_{2}}=k_{2}^{-} c_{\mathrm{O}}^{2} .
$$

Quasi-equilibrium with respect to species: for the same reaction, let us assume equilibrium over $\mathrm{H}, \mathrm{O}, \mathrm{OH}$, and $\mathrm{H}_{2} \mathrm{O}_{2}$, in a subdomain of reaction conditions. Subspace $L$ is defined by balance constraints:

$$
c_{\mathrm{H}}+c_{\mathrm{OH}}+2 c_{\mathrm{H}_{2} \mathrm{O}_{2}}=0, \quad c_{\mathrm{O}}+c_{\mathrm{OH}}+2 c_{\mathrm{H}_{2} \mathrm{O}_{2}}=0 .
$$

Subspace $L$ is two-dimensional. Its basis, $\left\{\boldsymbol{g}_{1}, \boldsymbol{g}_{2}\right\}$ in the coordinates $c_{\mathrm{H}}, c_{\mathrm{O}}, c_{\mathrm{OH}}$, and $c_{\mathrm{H}_{2} \mathrm{O}_{2}}$ reads:

$$
\boldsymbol{g}_{1}=(1,1,-1,0), \quad \boldsymbol{g}_{2}=(2,2,0,-1) .
$$

Corresponding Eq. (62) is

$$
\mu_{\mathrm{H}}+\mu_{\mathrm{O}}=\mu_{\mathrm{OH}}, \quad 2 \mu_{\mathrm{H}}+2 \mu_{\mathrm{O}}=\mu_{\mathrm{H}_{2} \mathrm{O}_{2}} .
$$

General construction of the quasi-equilibrium manifold: In the space of concentration, one defines a subspace $L$ which satisfies the balance constraints:

$$
\left(\boldsymbol{b}_{i}, L\right) \equiv 0 \text {. }
$$


The orthogonal complement of $L$ in the space with coordinates $\boldsymbol{\mu}=\nabla G$ defines then the quasiequilibrium manifold $\boldsymbol{\Omega}_{L}$. For the actual computations, one requires the inversion from $\boldsymbol{\mu}$ to $\boldsymbol{c}$. Duality structure $\boldsymbol{\mu} \leftrightarrow \boldsymbol{c}$ is well studied by many authors $[127,126]$.

Quasi-equilibrium projector. It is not sufficient to just derive the manifold, it is also required to define a projector which would transform the vector field defined on the space of concentrations to a vector field on the manifold. Quasi-equilibrium manifold consists of points which minimize $G$ on the affine spaces of the form $c+L$. These affine planes are hypothetic planes of fast motions ( $G$ is decreasing in the course of the fast motions). Therefore, the quasi-equilibrium projector maps the whole space of concentrations on $\boldsymbol{\Omega}_{L}$ parallel to $L$. The vector field is also projected onto the tangent space of $\boldsymbol{\Omega}_{L}$ parallel to $L$.

Thus, the quasi-equilibrium approximation implies the decomposition of motions into the fast-parallel to $L$, and the slow-along the quasi-equilibrium manifold. In order to construct the quasi-equilibrium approximation, knowledge of reaction rate constants of "fast" reactions is not required (stoichiometric vectors of all these fast reaction are in $L, \gamma_{\text {fast }} \in L$, thus, knowledge of $L$ suffices), one only needs some confidence in that they all are sufficiently fast [128]. The quasiequilibrium manifold itself is constructed based on the knowledge of $L$ and of $G$. Dynamics on the quasi-equilibrium manifold is defined as the quasi-equilibrium projection of the "slow component" of kinetic equations (37).

\subsubsection{Model equations}

The assumption behind the quasi-equilibrium is the hypothesis of the decomposition of motions into fast and slow. The quasi-equilibrium approximation itself describes slow motions. However, sometimes it becomes necessary to restore to the whole system, and to take into account the fast motions as well. With this, it is desirable to keep intact one of the important advantages of the quasi-equilibrium approximation-its independence of the rate constants of fast reactions. For this purpose, the detailed fast kinetics is replaced by a model equation (single relaxation time approximation).

Quasi-equilibrium models (QEM) are constructed as follows: For each concentration vector $\boldsymbol{c}$, consider the affine manifold, $\boldsymbol{c}+L$. Its intersection with the quasi-equilibrium manifold $\boldsymbol{\Omega}_{L}$ consists of one point. This point delivers the minimum to $G$ on $\boldsymbol{c}+L$. Let us denote this point as $\boldsymbol{c}_{L}^{*}(\boldsymbol{c})$. The equation of the quasi-equilibrium model reads:

$$
\dot{\boldsymbol{c}}=-\frac{1}{\tau}\left[\boldsymbol{c}-\boldsymbol{c}_{L}^{*}(\boldsymbol{c})\right]+\sum_{\text {slow }} \gamma_{s} W_{s}\left(\boldsymbol{c}_{L}^{*}(\boldsymbol{c})\right),
$$

where $\tau>0$ is the relaxation time of the fast subsystem. Rates of slow reactions are computed at the points $c_{L}^{*}(\boldsymbol{c})$ (the second term on the right hand side of Eq. (63)), whereas the rapid motion is taken into account by a simple relaxational term (the first term on the right hand side of Eq. (63)). The most famous model kinetic equation is the BGK equation in the theory of the Boltzmann equation [89]. The general theory of the quasi-equilibrium models, including proofs of their thermodynamic consistency, was constructed in the paper [90].

Single relaxation time gradient models (SRTGM) were considered in the context of the lattice Boltzmann method for hydrodynamics [109,129]. These models are aimed at improving the obvious drawback of quasi-equilibrium models (63): In order to construct the QEM, one needs to compute 
the function,

$$
\boldsymbol{c}_{L}^{*}(\boldsymbol{c})=\arg \min _{\boldsymbol{x} \in \boldsymbol{c}+L, \boldsymbol{x}>0} G(\boldsymbol{x}) .
$$

This is a convex programming problem. It does not always has a closed-form solution.

Let $\boldsymbol{g}_{1}, \ldots, \boldsymbol{g}_{k}$ is the orthonormal basis of $L$. We denote as $\boldsymbol{D}(\boldsymbol{c})$ the $k \times k$ matrix with the elements $\left(\boldsymbol{g}_{i}, \boldsymbol{H}_{\boldsymbol{c}} \boldsymbol{g}_{j}\right)$, where $\boldsymbol{H}_{\boldsymbol{c}}$ is the matrix of second derivatives of $G(55)$. Let $\boldsymbol{C}(\boldsymbol{c})$ be the inverse of $\boldsymbol{D}(\boldsymbol{c})$. The single relaxation time gradient model has the form:

$$
\dot{\boldsymbol{c}}=-\frac{1}{\tau} \sum_{i, j} \boldsymbol{g}_{i} \boldsymbol{C}(\boldsymbol{c})_{i j}\left(\boldsymbol{g}_{j}, \nabla G\right)+\sum_{\text {slow }} \gamma_{s} W_{s}(\boldsymbol{c}) .
$$

The first term drives the system to the minimum of $G$ on $c+L$, it does not require solving the problem (64), and its spectrum in the quasi-equilibrium is the same as in the quasi-equilibrium model (63). Note that the slow component is evaluated in the "current" state $c$.

The first term in Eq. (65) has a simple form

$$
\dot{\boldsymbol{c}}=-\frac{1}{\tau} \operatorname{grad} G,
$$

if one calculates $\operatorname{grad} G$ with the entropic scalar $\operatorname{product}^{3}\langle\boldsymbol{x}, \boldsymbol{y}\rangle=\left(\boldsymbol{x}, \boldsymbol{H}_{\boldsymbol{c}} \boldsymbol{y}\right)$.

Models (63) and (65) lift the quasi-equilibrium approximation to a kinetic equation by approximating the fast dynamics with a single "reaction rate constant"-relaxation time $\tau$.

\subsubsection{Quasi-steady state approximation}

The quasi-steady state approximation (QSS) is a tool used in a major number of works. Let us split the list of species in two groups: The basic and the intermediate (radicals etc). Concentration vectors are denoted accordingly, $\boldsymbol{c}^{\mathrm{s}}$ (slow, basic species), and $\boldsymbol{c}^{\mathrm{f}}$ (fast, intermediate species). The concentration vector $\boldsymbol{c}$ is the direct sum, $\boldsymbol{c}=\boldsymbol{c}^{\mathrm{s}} \oplus \boldsymbol{c}^{\mathrm{f}}$. The fast subsystem is Eq. (37) for the component $c^{\mathrm{f}}$ at fixed values of $\boldsymbol{c}^{\mathrm{s}}$. If it happens that this way defined fast subsystem relaxes to a stationary state, $\boldsymbol{c}^{\mathrm{f}} \rightarrow \boldsymbol{c}_{\mathrm{qsS}}^{\mathrm{f}}\left(\boldsymbol{c}^{\mathrm{s}}\right)$, then the assumption that $\boldsymbol{c}^{\mathrm{f}}=\boldsymbol{c}_{\mathrm{qSS}}^{\mathrm{f}}(\boldsymbol{c})$ is precisely the QSS assumption. The slow subsystem is the part of system (37) for $c^{\mathrm{s}}$, on the right hand side of which the component $\boldsymbol{c}^{\mathrm{f}}$ is replaced with $\boldsymbol{c}_{\mathrm{qss}}^{\mathrm{f}}(\boldsymbol{c})$. Thus, $\boldsymbol{J}=\boldsymbol{J}_{\mathrm{s}} \oplus \boldsymbol{J}_{\mathrm{f}}$, where

$$
\begin{aligned}
& \dot{c}^{\mathrm{f}}=J_{\mathrm{f}}\left(c^{\mathrm{s}} \oplus \boldsymbol{c}^{\mathrm{f}}\right), \quad c^{\mathrm{s}}=\mathrm{const} ; \quad \boldsymbol{c}^{\mathrm{f}} \rightarrow c_{\mathrm{qss}}^{\mathrm{f}}\left(c^{\mathrm{s}}\right) ; \\
& \dot{c}^{\mathrm{s}}=J_{\mathrm{s}}\left(c^{\mathrm{s}} \oplus \boldsymbol{c}_{\mathrm{qss}}^{\mathrm{f}}\left(c^{\mathrm{s}}\right)\right) .
\end{aligned}
$$

Bifurcations in system (67) under variation of $\boldsymbol{c}^{\mathrm{s}}$ as a parameter are confronted to kinetic critical phenomena. Studies of more complicated dynamic phenomena in the fast subsystem (67) require various techniques of averaging, stability analysis of the averaged quantities, etc.

Various versions of the QSS method are well possible, and are actually used widely, for example, the hierarchical QSS method. There, one defines not a single fast subsystem but a hierarchy of them, $c^{f_{1}}, \ldots, c^{f_{k}}$. Each subsystem $c^{f_{i}}$ is regarded as a slow system for all the foregoing subsystems, and it

\footnotetext{
${ }^{3}$ Let us remind that $\operatorname{grad} G$ is the Riesz representation of the differential of $G$ in the phase space $\boldsymbol{X}: G(\boldsymbol{c}+\Delta \boldsymbol{c})=$ $G(\boldsymbol{c})+\langle\operatorname{grad} G(\boldsymbol{c}), \Delta \boldsymbol{c}\rangle+\mathrm{o}(\Delta \boldsymbol{c})$. It depends on the scalar product, and from thermodynamic point of view there is only one distinguished scalar product in concentration space. Usual definition of $\operatorname{grad} G$ as the vector of partial derivatives corresponds to the standard scalar product $(\bullet, \bullet)$.
} 
is regarded as a fast subsystem for the following members of the hierarchy. Instead of one system of Eqs. (67), a hierarchy of systems of lower-dimensional equations is considered, each of these subsystem is easier to study analytically.

Theory of singularly perturbed systems of ordinary differential equations is used to provide a mathematical background and further development of the QSS approximation. In spite of a broad literature on this subject, it remains, in general, unclear, what is the smallness parameter that separates the intermediate (fast) species from the basic (slow). Reaction rate constants cannot be such a parameter (unlike in the case of the quasi-equilibrium). Indeed, intermediate species participate in the same reactions, as the basic species (for example, $\mathrm{H}_{2} \rightleftharpoons 2 \mathrm{H}, \mathrm{H}+\mathrm{O}_{2} \rightleftharpoons \mathrm{OH}+\mathrm{O}$ ). It is therefore incorrect to state that $\boldsymbol{c}^{\mathrm{f}}$ evolve faster than $\boldsymbol{c}^{\mathrm{s}}$. In the sense of reaction rate constants, $\boldsymbol{c}^{\mathrm{f}}$ is not faster.

For catalytic reactions, it is not difficult to figure out what is the smallness parameter that separates the intermediate species from the basic, and which allows to upgrade the QSS assumption to a singular perturbation theory rigorously [59]. This smallness parameter is the ratio of balances: Intermediate species include the catalyst, and their total amount is simply significantly less than the amount of all the $\boldsymbol{c}_{i}$ 's. After renormalizing to the variables of one order of magnitude, the small parameter appears explicitly. The simplest example gives the catalytic reaction $A+Z \rightleftharpoons A Z \rightleftharpoons P+Z$ (here $Z$ is a catalyst, $A$ and $P$ are an initial substrate and a product). The kinetic equations are (in obvious notations):

$$
\begin{aligned}
& \dot{c}_{A}=-k_{1}^{+} c_{A} c_{Z}+k_{1}^{-} c_{A Z}, \\
& \dot{c}_{Z}=-k_{1}^{+} c_{A} c_{Z}+k_{1}^{-} c_{A Z}+k_{2}^{+} c_{A Z}-k_{2}^{-} c_{Z} c_{P}, \\
& \dot{c}_{A Z}=k_{1}^{+} c_{A} c_{Z}-k_{1}^{-} c_{A Z}-k_{2}^{+} c_{A Z}+k_{2}^{-} c_{Z} c_{P}, \\
& \dot{c}_{P}=k_{2}^{+} c_{A Z}-k_{2}^{-} c_{Z} c_{P} .
\end{aligned}
$$

The constants and the reactions rates are the same for concentrations $c_{A}, c_{P}$, and for $c_{Z}, c_{A Z}$, and cannot give a reason for relative slowness of $c_{A}, c_{P}$ in comparison with $c_{Z}, c_{A Z}$, but there may be another source of slowness. There are two balances for this kinetics: $c_{A}+c_{P}+c_{A Z}=B_{A}, c_{Z}+c_{A Z}=B_{Z}$. Let us go to dimensionless variables:

$$
\begin{aligned}
& \varsigma_{A}=c_{A} / B_{A}, \quad \varsigma_{P}=c_{P} / B_{A}, \quad \varsigma_{Z}=c_{Z} / B_{Z}, \quad \varsigma_{A Z}=c_{A Z} / B_{Z} ; \\
& \dot{\varsigma_{A}}=B_{Z}\left[-k_{1}^{+} \varsigma_{A} \varsigma_{Z}+\frac{k_{1}^{-}}{B_{A}} \varsigma_{A Z}\right], \\
& \dot{\varsigma_{Z}}=B_{A}\left[-k_{1}^{+} \varsigma_{A} \varsigma_{Z}+\frac{k_{1}^{-}}{B_{A}} \varsigma_{A Z}+\frac{k_{2}^{+}}{B_{A}} \varsigma_{A Z}-k_{2}^{-} \varsigma_{Z} \varsigma_{P}\right] \\
& \varsigma_{A}+\varsigma_{P}+\frac{B_{Z}}{B_{A}} \varsigma_{A Z}=1, \quad \varsigma_{Z}+\varsigma_{A Z}=1 ; \quad \varsigma_{\bullet} \geqslant 0 .
\end{aligned}
$$

For $B_{Z} \ll B_{A}$ the slowness of $\varsigma_{A}, \varsigma_{P}$ is evident from these Eqs. (70).

For usual radicals, the origin of the smallness parameter is quite similar. There are much less radicals than the basic species (otherwise, the QSS assumption is inapplicable). In the case of radicals, however, the smallness parameter cannot be extracted directly from balances $B_{i}(53)$. Instead, one can come up with a thermodynamic estimate: Function $G$ decreases in the course of reactions, 
whereupon we obtain the limiting estimate of concentrations of any species:

$$
c_{i} \leqslant \max _{G(c) \leqslant G(c(0))} c_{i},
$$

where $c(0)$ is the initial composition. If the concentration $c_{\mathrm{R}}$ of the radical $\mathrm{R}$ is small both initially and in the equilibrium, then it should remain small also along the path to the equilibrium. For example, in the case of ideal $G(57)$ under relevant conditions, for any $t>0$, the following inequality is valid:

$$
c_{\mathrm{R}}\left[\ln \left(c_{\mathrm{R}}(t) / c_{\mathrm{R}}^{\mathrm{eq}}\right)-1\right] \leqslant G(\boldsymbol{c}(0)) .
$$

Inequality (72) provides the simplest (but rather coarse) thermodynamic estimate of $c_{\mathrm{R}}(t)$ in terms of $G(c(0))$ and $c_{\mathrm{R}}^{\text {eq }}$ uniformly for $t>0$. Complete theory of thermodynamic estimates of dynamics has been developed in the book [125].

One can also do computations without a priori estimations, if one accepts the QSS assumption until the values $\boldsymbol{c}^{\mathrm{f}}$ stay sufficiently small. It is the simplest way to operate with QSS: Just use it until $\boldsymbol{c}^{\mathrm{f}}$ are small.

Let us assume that an a priori estimate has been found, $c_{i}(t) \leqslant c_{i} \max$, for each $c_{i}$. These estimate may depend on the initial conditions, thermodynamic data etc. With these estimates, we are able to renormalize the variables in the kinetic equations (37) in such a way that the renormalized variables take their values from the unit segment $[0,1]: \tilde{c}_{i}=c_{i} / c_{i}$ max. Then system (37) can be written as follows:

$$
\frac{\mathrm{d} \tilde{c}_{i}}{\mathrm{~d} t}=\frac{1}{c_{i \max }} J_{i}(\boldsymbol{c}) .
$$

The system of dimensionless parameters, $\epsilon_{i}=c_{i \max } / \max _{i} c_{i \max }$ defines a hierarchy of relaxation times, and with its help one can establish various realizations of the QSS approximation. The simplest version is the standard QSS assumption: Parameters $\epsilon_{i}$ are separated in two groups, the smaller ones, and of the order 1. Accordingly, the concentration vector is split into $\boldsymbol{c}^{\mathrm{s}} \oplus \boldsymbol{c}^{\mathrm{f}}$. Various hierarchical QSS are possible, with this, the problem becomes more tractable analytically.

There exist a variety of ways to introduce the smallness parameter into kinetic equations, and one can find applications to each of the realizations. However, the two particular realizations remain basic for chemical kinetics: (i) fast reactions (under a given thermodynamic data); (ii) small concentrations. In the first case, one is led to the quasi-equilibrium approximation, in the second case-to the classical QSS assumption. Both of these approximations allow for hierarchical realizations, those which include not just two but many relaxation time scales. Such a multi-scale approach essentially simplifies analytical studies of the problem.

\subsubsection{Thermodynamic criteria for selection of important reactions}

One of the problems addressed by the sensitivity analysis is the selection of the important and discarding the unimportant reactions. A simple principle was suggested in the paper [130] to compare importance of different reactions according to their contribution to the entropy production (or, which is the same, according to their contribution to $\dot{G}$ ). Based on this principle, Dimitrov [133] described domains of parameters in which the reaction of hydrogen oxidation, $\mathrm{H}_{2}+\mathrm{O}_{2}+\mathrm{M}$, proceeds due to different mechanisms. For each elementary reaction, he has derived the domain inside 
which the contribution of this reaction is essential (nonnegligible). Due to its simplicity, this entropy production principle is especially well suited for analysis of complex problems. In particular, recently, a version of the entropy production principle was used in the problem of selection of boundary conditions for Grad's moment equations [131,132]. For ideal systems (57), as well, as for the Marcelin-De Donder kinetics (47) the contribution of the sth reaction to $\dot{G}$ has a particularly simple form:

$$
\dot{G}_{s}=-W_{s} \ln \left(\frac{W_{s}^{+}}{W_{s}^{-}}\right), \quad \dot{G}=\sum_{s=1}^{r} \dot{G}_{s} .
$$

\subsubsection{Opening}

One of the problems to be focused on when studying closed systems is to prepare extensions of the result for open or driven by flows systems. External flows are usually taken into account by additional terms in the kinetic equations (37):

$$
\dot{N}=V \boldsymbol{J}(\boldsymbol{c})+\Pi(\boldsymbol{c}, t) .
$$

It is important to stress here that the vector field $\boldsymbol{J}(\boldsymbol{c})$ in Eqs. (75) is the same, as for the closed system, with thermodynamic restrictions, Lyapunov functions, etc. The thermodynamic structures are important for analysis of open systems (75), if the external flow $\boldsymbol{\Pi}$ is small in some sense, is linear function of $c$, has small time derivatives, etc. There are some general results for such "weakly open" systems, for example the Prigogine minimum entropy production theorem [134] and the estimations of possible of steady states and limit sets for open systems, based on thermodynamic functions and stoichiometric equations [125].

There are general results for another limit case: for very intensive flow the dynamics is very simple again [59]. Let the flow have a natural structure: $\boldsymbol{\Pi}(\boldsymbol{c}, t)=v_{\text {in }}(t) \boldsymbol{c}_{\text {in }}(t)-v_{\text {out }}(t) \boldsymbol{c}(t)$, where $v_{\text {in }}$ and $v_{\text {out }}$ are the rates of inflow and outflow, $\boldsymbol{c}_{\text {in }}(t)$ is the concentration vector for inflow. If $v_{\text {out }}$ is sufficiently big, $v_{\text {out }}(t)>v_{0}$ for some critical value $v_{0}$ and all $t>0$, then for the open system (75) the Lyapunov norm exists: for any two solutions $\boldsymbol{c}^{1}(t)$ and $\boldsymbol{c}^{2}(t)$ the function $\left\|\boldsymbol{c}^{1}(t)-\boldsymbol{c}^{2}(t)\right\|$ monotonically decreases in time. Such a critical value $v_{0}$ exists for any norm, for example, for usual Euclidian norm $\|\bullet\|^{2}=(\bullet, \bullet)$.

For arbitrary form of $\boldsymbol{\Pi}$, system (75) can lose all signs of being thermodynamic one. Nevertheless, thermodynamic structures often can help in the study of open systems.

The seminal questions are: What happens with slow/fast motion separation after opening? Which slow invariant manifold for the closed system can be deformed to the slow invariant manifold for the open system? Which slow invariant manifold for the closed system can be used as approximate slow invariant manifold for the open system? There is more or less useful technique to seek the answers for specific systems under consideration.

The way to study an open system as the result of opening a closed system may be fruitful. In any case, out of this way we have just a general dynamical system (75) and no hints what to do with.

Basic introductory textbook on physical kinetics of the Landau L.D. and Lifshitz E.M. Course of Theoretical Physics [135] contains many further examples and their applications.

Modern development of kinetics follows the route of specific numerical methods, such as direct simulations. An opposite tendency is also clearly observed, and the kinetic theory based schemes are 
increasingly used for the development of numerical methods and models in mechanics of continuous media.

\section{Invariance equation in the differential form}

The notions and notations of this section will apply elsewhere below.

Definition of the invariance in terms of motions and trajectories assumes, at least, existence and uniqueness theorems for solutions of the original dynamic system. This prerequisite causes difficulties when one studies equations relevant to physical and chemical kinetics, such as, for example, equations of hydrodynamics. Nevertheless, there exists a necessary differential condition of invariance: The vector field of the original dynamic system touches the manifold in every point. Let us write down this condition in order to set up notation.

Let $E$ be a linear space, let $U$ (the phase space) be a domain in $E$, and let a vector field $J: U \rightarrow E$ be defined in $U$. This vector field defines the original dynamic system,

$$
\frac{\mathrm{d} x}{\mathrm{~d} t}=J(x), \quad x \in U
$$

In the sequel, we consider submanifolds in $U$ which are parameterized with a given set of parameters. Let a linear space of parameters $L$ be defined, and let $W$ be a domain in $L$. We consider differentiable maps, $F: W \rightarrow U$, such that, for every $y \in W$, the differential of $F, D_{y} F: L \rightarrow E$, is an isomorphism of $L$ on a subspace of $E$. That is, $F$ are the manifolds, immersed in the phase space of the dynamic system (76), and parametrized by parameter set $W$.

Remark. One never discusses the choice of norms and topologies are such a general setting. It is assumed that the corresponding choice is made appropriately in each specific case.

We denote $T_{y}$ the tangent space at the point $y, T_{y}=\left(D_{y} F\right)(L)$. The differential condition of invariance has the following form: For every $y \in W$,

$$
J(F(y)) \in T_{y} .
$$

Let us rewrite the differential condition of invariance (77) in a form of a differential equation. In order to achieve this, one needs to define a projector $P_{y}: E \rightarrow T_{y}$ for every $y \in W$. Once a projector $P_{y}$ is defined, then condition (77) takes the form:

$$
\Delta_{y}=\left(1-P_{y}\right) J(F(y))=0 .
$$

Obviously, by $P_{y}^{2}=P_{y}$ we have, $P_{y} \Delta_{y}=0$. We refer to the function $\Delta_{y}$ as the defect of invariance at the point $y$. The defect of invariance will be encountered oft in what will follow.

Eq. (78) is the first-order differential equation for the function $F(y)$. Projectors $P_{y}$ should be tailored to the specific physical features of the problem at hand. A separate section below is devoted to the construction of projectors. There we shall demonstrate how to construct a projector, 
$P(x, T): E \rightarrow T$, given a point $x \in U$ and a specified subspace $T$. We then set $P_{y}=P\left(F(y), T_{y}\right)$ in Eq. (78). ${ }^{4}$

There are two possible senses of the notion "approximate solution of invariance equations" (78):

1. The approximation of the solution;

2. The map $F$ with small defect of invariance (the right hand side approximation).

If someone is looking for the approximation of the first kind, then he needs theorems about existence of solutions, he should find the estimations of deviations from the exact solution, because the right hand side not always gives the good estimation, etc. The second kind of approximations does not require hypothesis of existence of solutions. Moreover, the manifold with sufficiently small defect of invariance can serve as a slow manifold by itself. So, we shall accept the concept of approximate invariant manifold (the manifold with small defect of invariance) instead of the approximation of the invariant manifold (see also [13,245] and other works about approximate inertial manifolds). Sometimes these approximate invariant manifolds will give approximations of the invariant manifolds, sometimes not, but it is additional and often difficult problem to make a distinction between these situations. In addition to defect of invariance, the key role in analysis of motion separation into the fast and the slow components play Jacobians, the differentials of $J(x)$. Some estimations of errors of this separation will be presented below in the subsection devoted to post-processing.

Our paper is focused on nonperturbative methods for computing invariant manifolds, but it should be mentioned that in the huge amount of applications the Taylor expansion is in use, and sometimes it works rather well. The main idea is the continuation of slow manifold with respect to a small parameter: Let our system depends on the parameter $\varepsilon$, and let a manifold of steady states exist for $\varepsilon=0$, as well, as fibers of motions towards these steady states, for example

$$
\dot{x}=\varepsilon f(x, y) ; \quad \dot{y}=g(x, y) .
$$

For $\varepsilon=0$ a value of (vector) variable $x$ is a vector of conserved quantities. Let for every $x$ the equation of fast motion, $\dot{y}=g(x, y)$, be globally stable: Its solution $y(t)$ tends to the unique (for given $x$ ) stable fixed point $y_{x}$. If the function $g(x, y)$ meets the conditions of the implicit function theorem, then the graph of the map $x \mapsto y_{x}$ forms a manifold $\Omega_{0}=\left\{\left(x, y_{x}\right)\right\}$ of steady states. For small $\varepsilon>0$ we can look for the slow manifold in a form of a series in powers of $\varepsilon: \Omega_{\varepsilon}=\{x, y(x, \varepsilon)\}$, $y(x, \varepsilon)=y_{x}+\varepsilon y^{1}(x)+\varepsilon^{2} y^{2}(x)+\cdots$. The fibers of fast motions can be constructed in a form of a power series too (the zero term is the fast motion $\dot{y}=g(x, y)$ in the affine planes $x=$ const). This analytic continuation with respect to the parameter $\varepsilon$ for small $\varepsilon>0$ is studied in the "Geometric singular perturbation theory" [247,248]. As it was mentioned above, the first successful application

\footnotetext{
${ }^{4}$ One of the main routes to define the field of projectors $P(x, T)$ would be to make use of a Riemannian structure. To this end, one defines a scalar product in $E$ for every point $x \in U$, that is, a bilinear form $\langle p \mid q\rangle_{x}$ with a positive definite quadratic form, $\langle p \mid p\rangle_{x}>0$, if $p \neq 0$. A good candidate for such a scalar product is the bilinear form defined by the negative second differential of the entropy at the point $x,-D^{2} S(x)$. As we demonstrate it later in this review, this choice is essentially the only correct one close to the equilibrium. However, far from the equilibrium, an improvement is required in order to guarantee the thermodymamicity condition, $\operatorname{ker} P_{y} \subset \operatorname{ker}\left(D_{x} S\right)_{x=F(y)}$, for the field of projectors, $P(x, T)$, defined for any $x$ and $T$, if $T \not \subset \operatorname{ker} D_{x} S$. The thermodymamicity condition provides the preservation of the type of dynamics: if $\mathrm{d} S / \mathrm{d} t>0$ for initial vector field (76) in point $x=F(y)$, then $\mathrm{d} S / \mathrm{d} t>0$ in this point $x$ for projected vector field $P_{y}(J(F(y)))$ too.
} 
of such an approach to construction of a slow invariant manifold in a form of Taylor expansion in powers of small parameter of singular perturbation $\varepsilon$ was the Chapman-Enskog expansion [51].

It is well-known in various applications that there are many different ways to introduce a small parameter into a system, there are many ways to present a given system as a member of a one-parametric family with a manifold of fixed points for zero value of a parameter. And different ways of specification of such a parameter result in different definitions of slowness of positively invariant manifold. Therefore it is desirable to study the notion of separation of motions without such an artificial specification. The notion of slow positively invariant manifold should be intrinsic. At least we should try to invent such a notion.

\section{Film extension of the dynamics: slowness as stability}

\subsection{Equation for the film motion}

One of the difficulties in the problem of reducing the description is caused by the fact that there exists no commonly accepted formal definition of slow (and stable) positively invariant manifolds. Classical definitions of stability and of the asymptotic stability of the invariant sets sound as follows: Let a dynamic system be defined in some metric space, (so that we can measure distances between points), and let $x\left(t, x_{0}\right)$ be a motion of this system at time $t$ with the initial condition $x(0)=x_{0}$ at time $t=0$. The subset $S$ of the phase space is called invariant if it is made of whole trajectories, that is, if $x_{0} \in S$ then $x\left(t, x_{0}\right) \in S$ for all $t \in(-\infty, \infty)$.

Let us denote as $\rho(x, y)$ the distance between the points $x$ and $y$. The distance from $x$ to a closed set $S$ is defined as usual: $\rho(x, S)=\inf \{\rho(x, y) \mid y \in S\}$. The closed invariant subset $S$ is called stable, if for every $\epsilon>0$ there exists $\delta>0$ such that if $\rho\left(x_{0}, S\right)<\delta$, then for every $t>0$ it holds $\rho\left(x\left(t, x_{0}\right), S\right)<\epsilon$. A closed invariant subset $S$ is called asymptotically stable if it is stable and attractive, that is, there exists $\epsilon>0$ such that if $\rho\left(x_{0}, S\right)<\epsilon$, then $\rho\left(x\left(t, x_{0}\right), S\right) \rightarrow 0$ as $t \rightarrow \infty$.

Formally, one can reiterate the definitions of stability and of the asymptotic stability for positively invariant subsets. Moreover, since in the definitions mentioned above it goes only about $t \geqslant 0$ or $t \rightarrow \infty$, it might seem that positively invariant subsets can be a natural object of study for stability issues. Such conclusion is misleading, however. The study of the classical stability of the positively invariant subsets reduces essentially to the notion of stability of invariant sets - maximal attractors.

Let $Y$ be a closed positively invariant subset of the phase space. The maximal attractor for $Y$ is the set $M_{Y}$,

$$
M_{Y}=\bigcap_{t \geqslant 0} T_{t}(Y)
$$

where $T_{t}$ is the shift operator for the time $t$ :

$$
T_{t}\left(x_{0}\right)=x\left(t, x_{0}\right) \text {. }
$$

The maximal attractor $M_{Y}$ is invariant, and the stability of $Y$ defined classically is equivalent to the stability of $M_{Y}$ under any sensible assumption about homogeneous continuity (for example, it is so for a compact phase space).

For systems which relax to a stable equilibrium, the maximal attractor is simply one and the same for any bounded positively invariant subset, and it consists of a single stable point. 
It is important to note that in definition (80) one considers motions of a positively invariant subset to equilibrium along itself: $T_{t} Y \subset Y$ for $t \geqslant 0$. It is precisely this motion which is uninteresting from the perspective of the comparison of stability of positively invariant subsets. If one subtracts this motion along itself out of the vector field $J(x)$ (76), one obtains a less trivial picture.

We again assume submanifolds in $U$ parameterized with a single parameter set $F: W \rightarrow U$. Note that there exists a wide class of transformations which do not alter the geometric picture of motion: For a smooth diffeomorphism $\varphi: W \rightarrow W$ (a smooth coordinate transform), maps $F$ and $F \circ \varphi$ define the same geometric pattern in the phase space.

Let us consider motions of the manifold $F(W)$ along solutions of Eq. (76). Denote as $F_{t}$ the time-dependent map, and write equation of motion for this map:

$$
\frac{\mathrm{d} F_{t}(y)}{\mathrm{d} t}=J\left(F_{t}(y)\right) \text {. }
$$

Let us now subtract the component of the vector field responsible for the motion of the map $F_{t}(y)$ along itself from the right hand side of Eq. (81). In order to do this, we decompose the vector field $J(x)$ in each point $x=F_{t}(y)$ as

$$
J(x)=J_{\|}(x)+J_{\perp}(x),
$$

where $J_{\|}(x) \in T_{t, y} T_{t, y}=\left(D_{y} F_{t}(y)(L)\right)$. If projectors are well defined, $P_{t, y}=P\left(F_{t}(y), T_{t, y}\right)$, then decomposition (82) has the form

$$
J(x)=P_{t, y} J(x)+\left(1-P_{t, y}\right) J(x) .
$$

Subtracting the component $J_{\|}$from the right hand side of Eq. (81), we obtain

$$
\frac{\mathrm{d} F_{t}(y)}{\mathrm{d} t}=\left(1-P_{t, y}\right) J\left(F_{t}(y)\right) \text {. }
$$

Note that the geometric pictures of motion corresponding to Eqs. (81) and (84) are identical locally in $y$ and $t$. Indeed, the infinitesimal shift of the manifold $W$ along the vector field is easily computed:

$$
\left(D_{y} F_{t}(y)\right)^{-1} J_{\|}\left(F_{t}(y)\right)=\left(D_{y} F_{t}(y)\right)^{-1}\left(P_{t, y} J\left(F_{t}(y)\right)\right) .
$$

This defines a smooth change of the coordinate system (assuming all solutions exist). In other words, the component $J_{\perp}$ defines the motion of the manifold in $U$, while we can consider (locally) the component $J_{\|}$as a component which locally defines motions in $W$ (a coordinate transform).

The positive semi-trajectory of motion (for $t>0$ ) of any submanifold in the phase space along the solutions of initial differential equation (76) (without subtraction of $J_{\|}(x)$ ) is the positively invariant manifold. The closure of such semi-trajectory is an invariant subset. The construction of the invariant manifold as a trajectory of an appropriate initial edge may be useful for producing invariant exponentially attracting set $[136,137]$. Very recently, the notion of exponential stability of invariants manifold for ODEs was revised by splitting motions into tangent and transversal (orthogonal) components in the work [138].

We further refer to Eq. (84) as the film extension of the dynamic system (76). The phase space of the dynamic system (84) is the set of maps $F$ (films). Fixed points of Eq. (84) are solutions to the invariance equation in the differential form (78). These include, in particular, all positively invariant manifolds. Stable or asymptotically stable fixed points of Eq. (84) are slow manifolds we 
are interested in. It is the notion of stability associated with the film extension of the dynamics which is relevant to our study. Below in Section 8, we consider relaxation methods for constructing slow positively invariant manifolds on the basis of the film extension (84).

\subsection{Stability of analytical solutions}

When studying the Cauchy problem for Eq. (84), one should ask a question of how to choose the boundary conditions: which conditions the function $F$ must satisfy at the boundary of $W$ ? Without fixing the boundary conditions, the general solution of the Cauchy problem for the film extension equations (84) in the class of smooth functions on $W$ is essentially ambiguous.

The boundary of $W, \partial W$, splits in two pieces: $\partial W=\partial W_{+} \cup \partial W_{-}$. For a smooth boundary these parts can be defined as

$$
\begin{gathered}
\partial W_{+}=\left\{y \in \partial W \mid\left(v(y),(D F(y))^{-1}\left(P_{y} J(F(y))\right)\right)<0\right\}, \\
\partial W_{-}=\left\{y \in \partial W \mid\left(v(y),(D F(y))^{-1}\left(P_{y} J(F(y))\right)\right) \geqslant 0\right\} .
\end{gathered}
$$

where $v(y)$ denotes the unit outer normal vector in the boundary point $y,(D F(y))^{-1}$ is the isomorphism of the tangent space $T_{y}$ on the linear space of parameters $L$.

One can understand the boundary splitting (86) in such a way: The projected vector field $P_{y} J(F(y))$ defines dynamics on the manifold $F(W)$, this dynamics is the image of some dynamics on $W$. The corresponding vector field on $W$ is $v(y)=(D F(y))^{-1}\left(P_{y} J(F(y))\right)$. The boundary part $\partial W_{+}$consists of points $y$, where the velocity vector $v(y)$ is pointed inside $W$, and for $y \in \partial W_{-}$this vector $v(y)$ is directed outside of $W$ (or is tangent to $\partial W$ ). The splitting $\partial W=\partial W_{+} \cup \partial W_{-}$depends on $t$ with the vector field $v(y)$ :

$$
v_{t}(y)=\left(D F_{t}(y)\right)^{-1}\left(P_{y} J\left(F_{t}(y)\right)\right),
$$

and dynamics of $F_{t}(y)$ is determined by Eq. (84).

If we would like to derive a solution of the film extension, (84) $F(y, t)$ for $(y, t) \in W \times[0, \tau]$, for some time $\tau>0$, then it is necessary to fix some boundary conditions on $\partial W_{+}$(for the "incoming from abroad" part of the function $F(y))$.

Nevertheless, there is a way to study Eq. (84) without introducing any boundary conditions. It is in the spirit of the classical Cauchy-Kovalevskaya theorem [139-141] about analytical Cauchy problem solutions with analytical data, as well as in the spirit of the classical Lyapunov auxiliary theorem about analytical invariant manifolds in the neighborhood of a fixed point [144,33] and Poincaré [145] theorem about analytical linearization of analytical nonresonant contractions (see [146]).

We note in passing that recently, the interest to the classical analytical Cauchy problem revived in the mathematical physics literature $[142,143]$. In particular, analogs of the Cauchy-Kovalevskaya theorem were obtained for generalized Euler equations [142]. A technique to estimate the convergence radii of the series emerging therein was also developed.

Analytical solutions to Eq. (84) do not require boundary conditions on the boundary of $W$. The analycity condition itself allows finding unique analytical solutions of the Eq. (84) with the analytical right hand side $(1-P) J$ for analytical initial conditions $F_{0}$ in $W$ (assuming that such solutions exist). Of course, the analytical continuation without additional regularity conditions is an 
ill-posed problem. However, it may be useful to go from functions to germs: ${ }^{5}$ we can solve chains of ordinary differential equations for Taylor coefficients instead of partial differential equations for functions (84), and after that it may be possible to prove the convergence of the Taylor series thus obtained. This is the way to prove the Lyapunov auxiliary theorem [144], and one of the known ways to prove the Cauchy-Kovalevskaya theorem.

Let us consider system (1) with stable equilibrium point $x^{*}$, real analytical right hand side $J$, and real analytical projector field $P(x, T): E \rightarrow T$. We shall study real analytical sub-manifolds, which include the equilibrium point $x^{*}\left(0 \in W, F(0)=x^{*}\right)$. Let us expand $F$ in a Taylor series in the neighborhood of zero:

$$
F(y)=x^{*}+A_{1}(y)+A_{2}(y, y)+\cdots+A_{k}(y, y, \ldots, y)+\cdots,
$$

where $A_{k}(y, y, \ldots, y)$ is a symmetric $k$-linear operator $(k=1,2, \ldots)$.

Let us expand also the right hand side of the film equation (84). Matching operators of the same order, we obtain a chain of equations for $A_{1}, \ldots, A_{k}, \ldots$ :

$$
\frac{\mathrm{d} A_{k}}{\mathrm{~d} t}=\Psi_{k}\left(A_{1}, \ldots, A_{k}\right) .
$$

It is crucially important, that the dynamics of $A_{k}$ does not depend on $A_{k+1}, \ldots$, and Eqs. (88) can be studied in the following order: we first study the dynamics of $A_{1}$, then the dynamics of $A_{2}$ with the $A_{1}$ motion already given, then $A_{3}$ and so on.

Let the projector $P_{y}$ in Eq. (84) be analytical function of the derivative $D_{y} F(y)$ and of the deviation $x-x^{*}$. Let the correspondent Taylor expansion at the point $\left(A_{1}^{0}(\bullet), x^{*}\right)$ have the form

$$
\begin{aligned}
& D_{y} F(y)(\bullet)=A_{1}(\bullet)+\sum_{k=2}^{\infty} k A_{k}(y, \ldots, \bullet), \\
& P_{y}=\sum_{k, m=0}^{\infty} P_{k, m}(\underbrace{D_{y} F(y)(\bullet)-A_{1}^{0}(\bullet), \ldots, D_{y} F(y)(\bullet)-A_{1}^{0}(\bullet)}_{k} ; \underbrace{F(y)-x^{*}, \ldots, F(y)-x^{*}}_{m}),
\end{aligned}
$$

where $A_{1}^{0}(\bullet), A_{1}(\bullet), A_{k}(y, \ldots, \bullet)$ are linear operators. $P_{k, m}$ is a $k+m$-linear operator $(k, m=0,1,2, \ldots)$ with values in the space of linear operators $E \rightarrow E$. The operators $P_{k, m}$ depend on the operator $A_{1}^{0}(\bullet)$ as on a parameter. Let the point of expansion $A_{1}^{0}(\bullet)$ be the linear part of $F: A_{1}^{0}(\bullet)=A_{1}(\bullet)$.

Let us represent the analytical vector field $J(x)$ as a power series:

$$
J(x)=\sum_{k=1}^{\infty} J_{k}\left(x-x^{*}, \ldots, x-x^{*}\right),
$$

where $J_{k}$ is a symmetric $k$-linear operator $(k=1,2, \ldots)$.

Let us write, for example, the first two equations of the equation chain (88):

$$
\begin{aligned}
\frac{\mathrm{d} A_{1}(y)}{\mathrm{d} t}=\left(1-P_{0,0}\right) J_{1}\left(A_{1}(y)\right) & \\
\frac{\mathrm{d} A_{2}(y, y)}{\mathrm{d} t}= & \left(1-P_{0,0}\right)\left[J_{1}\left(A_{2}(y, y)\right)+J_{2}\left(A_{1}(y), A_{1}(y)\right)\right] \\
& -\left[2 P_{1,0}\left(A_{2}(y, \bullet)\right)+P_{0,1}\left(A_{1}(y)\right)\right] J_{1}\left(A_{1}(y)\right) .
\end{aligned}
$$

\footnotetext{
${ }^{5}$ The germ is the sequences of Taylor coefficients that represent an analytical function near a given point.
} 
Here operators $P_{0,0}, P_{1,0}\left(A_{2}(y, \bullet)\right), P_{0,1}\left(A_{1}(y)\right)$ parametrically depend on the operator $A_{1}(\bullet)$, hence, the first equation is nonlinear, and the second is linear with respect to $A_{2}(y, y)$. The leading term on the right hand side has the same form for all equations of the sequence (88):

$$
\frac{\mathrm{d} A_{n}(y, \ldots, y)}{\mathrm{d} t}=\left(1-P_{0,0}\right) J_{1}\left(A_{n}(y, \ldots, y)\right)-n P_{1,0}\left(A_{n}(\underbrace{y, \ldots, y}_{n-1}, \bullet)\right) J_{1}\left(A_{1}(y)\right)+\cdots .
$$

There are two important conditions on $P_{y}$ and $D_{y} F(y): P_{y}^{2}=P_{y}$, because $P_{y}$ is a projector, and $\operatorname{im} P_{y}=\operatorname{im} D_{y} F(y)$, because $P_{y}$ projects on the image of $D_{y} F(y)$. If we expand these conditions in the power series, then we get the conditions on the coefficients. For example, from the first condition we get

$$
\begin{aligned}
& P_{0,0}^{2}=P_{0,0} \\
& P_{0,0}\left[2 P_{1,0}\left(A_{2}(y, \bullet)\right)+P_{0,1}\left(A_{1}(y)\right)\right]+\left[2 P_{1,0}\left(A_{2}(y, \bullet)\right)+P_{0,1}\left(A_{1}(y)\right)\right] P_{0,0} \\
& \quad=2 P_{1,0}\left(A_{2}(y, \bullet)\right)+P_{0,1}\left(A_{1}(y)\right), \ldots .
\end{aligned}
$$

After multiplication the second equation in (93) with $P_{0,0}$ we get

$$
P_{0,0}\left[2 P_{1,0}\left(A_{2}(y, \bullet)\right)+P_{0,1}\left(A_{1}(y)\right)\right] P_{0,0}=0 .
$$

Similar identities can be obtained for any oder of the expansion. These equalities allow us to simplify the stationary equation for sequence (88). For example, for the first two equations of this sequence (91) we obtain the following stationary equations:

$$
\begin{aligned}
& \left(1-P_{0,0}\right) J_{1}\left(A_{1}(y)\right)=0, \\
& \left(1-P_{0,0}\right)\left[J_{1}\left(A_{2}(y, y)\right)+J_{2}\left(A_{1}(y), A_{1}(y)\right)\right] \\
& \quad-\left[2 P_{1,0}\left(A_{2}(y, \bullet)\right)+P_{0,1}\left(A_{1}(y)\right)\right] J_{1}\left(A_{1}(y)\right)=0 .
\end{aligned}
$$

The operator $P_{0,0}$ is the projector on the space $\operatorname{im} A_{1}$ (the image of $A_{1}$ ), hence, from the first equation in (95) it follows: $J_{1}\left(\operatorname{im} A_{1}\right) \subseteq \operatorname{im} A_{1}$. So, im $A_{1}$ is a $J_{1}$-invariant subspace in $E\left(J_{1}=\right.$ $\left.\left.D_{x} J(x)\right|_{x^{*}}\right)$ and $P_{0,0}\left(J_{1}\left(A_{1}(y)\right)\right) \equiv J_{1}\left(A_{1}(y)\right)$. It is equivalent to the first equation of (95). Let us multiply the second equation of (95) with $P_{0,0}$ on the left. As a result we obtain the condition

$$
P_{0,0}\left[2 P_{1,0}\left(A_{2}(y, \bullet)\right)+P_{0,1}\left(A_{1}(y)\right)\right] J_{1}\left(A_{1}(y)\right)=0,
$$

for solution of Eqs. (95), because $P_{0,0}\left(1-P_{0,0}\right) \equiv 0$. If $A_{1}(y)$ is a solution of the first equation of (95), then this condition becomes an identity, and we can write the second equation of (95) in the form

$$
\begin{aligned}
(1- & \left.P_{0,0}\right) \\
& \times\left[J_{1}\left(A_{2}(y, y)\right)+J_{2}\left(A_{1}(y), A_{1}(y)\right)-\left(2 P_{1,0}\left(A_{2}(y, \bullet)\right)+P_{0,1}\left(A_{1}(y)\right)\right) J_{1}\left(A_{1}(y)\right)\right]=0 .
\end{aligned}
$$

It should be stressed, that the choice of projector field $P_{y}$ (89) has impact only on the $F(y)$ parametrization, whereas the invariant geometrical properties of solutions of (84) do not depend on projector field if some transversality and analycity conditions hold. The conditions of thermodynamic structures preservation significantly reduce ambiguousness of the projector choice. One of the most important condition is $\operatorname{ker} P_{y} \subset \operatorname{ker} D_{x} S$, where $x=F(y)$ and $S$ is the entropy (see the section 
about the entropy below). The thermodynamic projector is the unique operator which transforms the arbitrary vector field equipped with the given Lyapunov function into a vector field with the same Lyapunov function on the arbitrary submanifold which is not tangent to the level of the Lyapunov function. For the thermodynamic projectors $P_{y}$ the entropy $S(F(y))$ conserves on solutions $F(y, t)$ of Eq. (84) for any $y \in W$.

If projectors $P_{y}$ in Eqs. (89)-(96) are thermodynamic, then $P_{0,0}$ is the orthogonal projector with respect to the entropic scalar product. ${ }^{6}$ For orthogonal projectors the operator $P_{1,0}$ has a simple explicit form. Let $A: L \rightarrow E$ be an isomorphic injection (an isomorphism on the image), and $P: E \rightarrow E$ be the orthogonal projector on the image of $A$. The orthogonal projector on the image of perturbed operator $A+\delta A$ is $P+\delta P$,

$$
\begin{aligned}
& \delta P=(1-P) \delta A A^{-1} P+\left(\delta A A^{-1} P\right)^{+}(1-P)+\mathrm{o}(\delta A) \\
& P_{1,0}(\delta A(\bullet))=(1-P) \delta A(\bullet) A^{-1} P+\left(\delta A(\bullet) A^{-1} P\right)^{+}(1-P) .
\end{aligned}
$$

Here, in (97), the operator $A^{-1}$ is defined on $\operatorname{im} A, \operatorname{im} A=\operatorname{im} P$, the operator $A^{-1} P$ acts on $E$.

Formula for $\delta P$ (97) follows from the three conditions:

$$
(P+\delta P)(A+\delta A)=A+\delta A, \quad(P+\delta P)^{2}=P+\delta P, \quad(P+\delta P)^{+}=P+\delta P .
$$

Every $A_{k}$ is driven by $A_{1}, \ldots, A_{k-1}$. Stability of the germ of the positively invariant analytical manifold $F(W)$ at the point $0\left(F(0)=x^{*}\right)$ is defined as stability of the solution of the corresponding equations sequence (88). Moreover, the notion of the $k$-jet stability can be useful: let's call $k$-jet stable such a germ of positively invariant manifold $F(M)$ at the point $0\left(F(0)=x^{*}\right)$, if the corresponding solution of the equations sequence $(88)$ is stable for $k=1, \ldots, n$. The simple "triangle" structure of the equation sequence (88) with form (92) of principal linear part makes the problem of jets stability very similar for all orders $n>1$.

Let us demonstrate the stability conditions for the 1 -jets in a $n$-dimensional space $E$. Let the Jacobian matrix $J_{1}=\left.D_{x} J(x)\right|_{x^{*}}$ be self-adjoint with a simple spectrum $\lambda_{1}, \ldots, \lambda_{n}$, and the projector $P_{0,0}$ be orthogonal (this is a typical "thermodynamic" situation). Eigenvectors of $J_{1}$ form a basis in $E:\left\{e_{i}\right\}_{i=1}^{n}$. Let a linear space of parameters $L$ be a $k$-dimensional real space, $k<n$. We shall study stability of a operator $A_{1}^{0}$ which is a fixed point for the first equation of sequence (88). The operator $A_{1}^{0}$ is a fixed point of this equation, if $\operatorname{im} A_{1}^{0}$ is a $J_{1}$-invariant subspace in $E$. We discuss full-rank operators, so, for some order of $\left\{e_{i}\right\}_{i=1}^{n}$ numbering, the matrix of $A_{1}^{0}$ should have a form: $a_{1 i j}^{0}=0$, if $i>k$. Let us choose the basis in $L: l_{j}=\left(A_{1}^{0}\right)^{-1} e_{j},(j=1, \ldots, k)$. For this basis $a_{1 i j}^{0}=\delta_{i j}, \quad\left(i=1, \ldots, n, j=1, \ldots, k, \delta_{i j}\right.$ is the Kronecker symbol). The corresponding projectors $P$ and $1-P$ have the matrices:

$$
P=\operatorname{diag}(\underbrace{1, \ldots, 1}_{k}, \underbrace{0, \ldots, 0}_{n-k}), \quad 1-P=\operatorname{diag}(\underbrace{0, \ldots, 0}_{k}, \underbrace{1, \ldots, 1}_{n-k}),
$$

where $\operatorname{diag}\left(\alpha_{1}, \ldots, \alpha_{n}\right)$ is the $n \times n$ diagonal matrix with numbers $\alpha_{1}, \ldots, \alpha_{n}$ on the diagonal.

\footnotetext{
${ }^{6}$ This scalar product is the bilinear form defined by the negative second differential of the entropy at the point $x^{*}$, $-D^{2} S(x)$.
} 
Equations of the linear approximation for the dynamics of the deviations $\delta A$ read:

$$
\frac{\mathrm{d} \delta A}{\mathrm{~d} t}=\operatorname{diag}(\underbrace{0, \ldots, 0}_{k}, \underbrace{1, \ldots, 1}_{n-k})\left[\operatorname{diag}\left(\lambda_{1}, \ldots, \lambda_{n}\right) \delta A-\delta A \operatorname{diag}(\underbrace{\lambda_{1}, \ldots, \lambda_{k}}_{k})\right] .
$$

The time derivative of $A$ is orthogonal to $A$ : for any $y, z \in L$ the equality $(\dot{A}(y), A(x))=0$ holds, hence, for the stability analysis it is necessary and sufficient to study $\delta A$ with $\operatorname{im} \delta A_{1}^{0} \perp \operatorname{im} A$. The matrix for such a $\delta A$ has a form

$$
\delta a_{i j}=0 \quad \text { if } i \leqslant k .
$$

For $i=k+1, \ldots, n, j=1, \ldots, k$ Eq. (100) gives

$$
\frac{\mathrm{d} \delta a_{i j}}{\mathrm{~d} t}=\left(\lambda_{i}-\lambda_{j}\right) \delta a_{i j}
$$

From Eq. (101), the stability condition follows:

$$
\lambda_{i}-\lambda_{j}<0 \text { for all } i>k, j \leqslant k .
$$

This means that the relaxation towards $\operatorname{im} A$ (with the spectrum of relaxation times $\left|\lambda_{i}\right|^{-1}(i=$ $k+1, \ldots, n)$ ) is faster, then the relaxation along $\operatorname{im} A$ (with the spectrum of relaxation times $\left.\left|\lambda_{j}\right|^{-1}(j=1, \ldots, k)\right)$.

Let condition (102) holds. The relaxation time for the film (in the first approximation) is

$$
\tau=1 /\left(\min _{i>k}\left|\lambda_{i}\right|-\max _{j \leqslant k}\left|\lambda_{j}\right|\right),
$$

thus it depends on the spectral gap in the spectrum of the operator $J_{1}=\left.D_{x} J(x)\right|_{x^{*}}$.

It is the gap between spectra of two restrictions of the operator $J_{1}, J_{1}^{\|}$and $J_{1}^{\perp}$, respectively. The operator $J_{1}^{\|}$is the restriction of $J_{1}$ on the $J_{1}$-invariant subspace $\operatorname{im} A_{1}^{0}$ (it is the tangent space to the slow invariant manifold at the point $x^{*}$ ). The operator $J_{1}^{\perp}$ is the restriction of $J_{1}$ on the orthogonal complement to $\operatorname{im} A_{1}^{0}$. This subspace is also $J_{1}$-invariant, because $J_{1}$ is selfadjoint. The spectral gap between spectra of these two operators is the spectral gap between relaxation towards the slow manifold and relaxation along this manifold.

The stability condition (102) demonstrates that our formalization of the slowness of manifolds as the stability of fixed points for the film extension (84) of initial dynamics met the intuitive expectations.

For the analysis of system (88) in the neighborhood of some manifold $F_{0}\left(F_{0}(0)=x^{*}\right)$, the following parametrization can be convenient. Let's consider $F_{0}(y)=A_{1}(y)+\cdots, T_{0}=A_{1}(L)$ is a tangent space to $F_{0}(W)$ at the point $x^{*}, E=T_{0} \oplus H$ is the direct sum decomposition.

We shall consider analytical sub-manifolds in the form

$$
x=x^{*}+(y, \Phi(y)),
$$

where $y \in W_{0} \subset T_{0}, W_{0}$ is neighborhood of zero in $T_{0}, \Phi(y)$ is an analytical map of $W_{0}$ in $H$, $\Phi(0)=0$.

Any analytical manifold close to $F_{0}$ can be represented in this form. 
Let us define the projector $P_{y}$ that corresponds to decomposition (103), as the projector on $T_{y}$ parallel to $H$. Furthermore, let us introduce the corresponding decomposition of the vector field $J=J_{y} \oplus J_{z}, J_{y} \in T_{0}, J_{z} \in H$. Then

$$
P_{y}(J)=\left(J_{y},\left(D_{y} \Phi(y)\right) J_{y}\right) .
$$

The corresponding equation of motion of film (84) has the following form:

$$
\frac{\mathrm{d} \Phi(y)}{\mathrm{d} t}=J_{z}(y, \Phi(y))-\left(D_{y} \Phi(y)\right) J_{y}(y, \Phi(y)) .
$$

If $J_{y}$ and $J_{z}$ depend analytically on their arguments, then from (105) one can easily obtain a hierarchy of equations of form (88) (of course, $J_{y}\left(x^{*}\right)=0, J_{z}\left(x^{*}\right)=0$ ).

Using these notions, it is convenient to formulate the Lyapunov Auxiliary Theorem [144]. Let $T_{0}=$ $R^{m}, H=R^{p}$, and in $U$ an analytical vector field is defined $J(y, z)=J_{y}(y, z) \oplus J_{z}(y, z),\left(y \in T_{0}, z \in H\right)$, and the following conditions are satisfied:

(1) $J(0,0)=0$;

(2) $\left.D_{z} J_{y}(y, z)\right|_{(0,0)}=0$;

(3) $0 \notin \operatorname{conv}\left\{k_{1}, \ldots, k_{m}\right\}$, where $k_{1}, \ldots, k_{m}$ are the eigenvalues of $\left.D_{y} J_{y}(y, z)\right|_{(0.0)}$, and $\operatorname{conv}\left\{k_{1}, \ldots, k_{m}\right\}$ is the convex envelope of $\left\{k_{1}, \ldots, k_{m}\right\}$;

(4) the numbers $k_{i}$ and $\lambda_{j}$ are not related by any equation of the form

$$
\sum_{i=1}^{m} m_{i} k_{i}=\lambda_{j}
$$

where $\lambda_{j}(j=1, \ldots, p)$ are eigenvalues of $\left.D_{z} J_{z}(y, z)\right|_{(0,0)}$, and $m_{i} \geqslant 0$ are integers, $\sum_{i=1}^{m} m_{i}>0$.

Let us consider analytical manifold $(y, \Phi(y))$ in $U$ in the neighborhood of zero $(\Phi(0)=0)$ and write for it the differential invariance equation with projector (104):

$$
\left(D_{y} \Phi(y)\right) J_{y}(y, \Phi(y))=J_{z}(y, \Phi(y)) .
$$

Lyapunov auxiliary theorem. Given conditions 1-4, Eq. (103) has the unique analytical in the neighborhood of zero solution, satisfying condition $\Phi(0)=0$.

Recently various new applications of this theorem were developed [33,147-149].

Studying germs of invariant manifolds using Taylor expansion in a neighborhood of a fixed point is definitely useful from the theoretical as well as from the practical perspective. But the well known difficulties pertinent to this approach, of convergence, of small denominators (connected with proximity to resonances (106)) and others call for development of different methods. A hint can be found in the famous KAM theory: one should use iterative methods instead of the Taylor expansion [28-30]. Below we present two such methods:

- The Newton method subject to incomplete linearization;

- The relaxation method which is the Galerkin-type approximation to Newton's method with projection on defect of invariance (78), i.e. on the right hand side of Eq. (84). 


\section{Entropy, quasi-equilibrium and projectors field}

Projector operators $P_{y}$ contribute both to the invariance equation (77), and to the film extension of dynamics (84). Limiting results, exact solutions, etc. only weakly depend on the particular choice of projectors, or do not depend at all on it. However, the validity of approximations obtained in each iteration step towards the limit does strongly depend on the choice of the projector. Moreover, if we want each approximate solution to be consistent with such physically crucial conditions as the second law of thermodynamics (the entropy of the isolated systems increases), then the choice of the projector becomes practically unique.

In this section we consider the main ingredients for constructing the projector, based on the two additional structures: (a) the moment parameterization, and (b) the entropy and the entropic scalar product.

\subsection{Moment parameterization}

Same as in the previous section, let a regular map (projection) is defined, $\Pi: U \rightarrow W$. We consider only maps $F: W \rightarrow U$ which satisfy $\Pi \circ F=1$. We seek slow invariant manifolds among such maps. (A natural remark is in order here: sometimes one has to consider $F$ which are defined not on the whole $W$ but only on some subset of it.) In this case, the unique projector consistent with the given structure is the superposition of the differentials:

$$
P_{y} J=\left(D_{y} F\right)_{y} \circ\left(D_{x} \Pi\right)_{F(y)} J .
$$

In the language of differential equations, formula (108) has the following significance: First, Eq. (76) is projected,

$$
\frac{\mathrm{d} y}{\mathrm{~d} t}=\left(D_{x} \Pi\right)_{F(y)} J(F(y)) .
$$

Second, the latter equation is lifted back to $U$ with the help of $F$ and its differential,

$$
x(t)=F(y(t)),\left.\frac{\mathrm{d} x}{\mathrm{~d} t}\right|_{x=F(y)}=\left(D_{y} F\right)_{y}\left(\frac{\mathrm{d} y}{\mathrm{~d} t}\right)=\left(D_{y} F\right)_{y}\left(\left(D_{x} \Pi\right)_{F(y)} J(F(y))\right)=P_{y} J .
$$

The most standard example of the construction just described is as follows: $x$ is the distribution density, $y=\Pi(x)$ is the set of selected moments of this density, $F: y \rightarrow x$ is a "closure assumption", which constructs a distribution density parameterized by the values of the moments $y$. Another standard example is relevant to problems of chemical kinetics: $x$ is a detailed description of the reacting species (including all the intermediates and radicals), $y$ are concentrations of stable reactants and products of the reaction.

The moment parameterization and moment projectors (108) are often encountered in the applications. However, they have some shortcomings. In particular, it is by far not always happens that the moment projection transforms a dissipative system into another dissipative system. Of course, for invariant $F(y)$ any projector transforms the dissipative system into a dissipative system. However, for various approximations to invariant manifolds (closure assumptions) this is not readily the case. ${ }^{7}$ The property of projectors to preserve the type of the dynamics will be imposed below as one of the requirements.

\footnotetext{
${ }^{7}$ See, e.g. a discussion of this problem for the Tamm-Mott-Smith approximation for the strong shock wave in [5].
} 


\subsection{Entropy and quasi-equilibrium}

The dissipation properties of system (76) are described by specifying the entropy $S$, the distinguished Lyapunov function which monotonically increases along solutions of Eq. (76). In a certain sense, this Lyapunov function is more fundamental than system (76) itself. That is, usually, the entropy is known much better than the right hand side of Eq. (76). For example, in chemical kinetics, the entropy is obtained from the equilibrium data. The same holds for other Lyapunov functions, which are defined by the entropy and by specification of the reaction conditions (the free energy, $U-T S$, for the isothermal isochoric processes, the free enthalpy, $U-T H$, for the isothermal isobaric processes, etc.). On physical grounds, all these entropic Lyapunov functions are proportional (up to additive constants) to the entropy of the minimal isolated system which includes the system under study [125]. In general, with some abuse of language, we term the Lyapunov functional $S$ the entropy elsewhere below, although it is a different functional for nonisolated systems.

Thus, we assume that a concave functional $S$ is defined in $U$, such that it takes maximum in an inner point $x^{*} \in U$. This point is termed the equilibrium.

For any dissipative system (76) under consideration in $U$, the derivative of $S$ due to Eq. (76) must be nonnegative,

$$
\left.\frac{\mathrm{d} S}{\mathrm{~d} t}\right|_{x}=\left(D_{x} S\right)(J(x)) \geqslant 0,
$$

where $D_{x} S$ is the linear functional, the differential of the entropy, while the equality in (111) is achieved only in the equilibrium $x=x^{*}$.

Most of the works on nonequilibrium thermodynamics deal with corrections to quasi-equilibrium approximations, or with applications of these approximations (with or without corrections). This viewpoint is not the only possible but it proves very efficient for the construction of a variety of useful models, approximations and equations, as well as methods to solve them. ${ }^{8} \mathrm{We}$ shall now introduce the quasi-equilibrium approximation in the most general setting.

\footnotetext{
${ }^{8}$ From time to time it is discussed in the literature, who was the first to introduce the quasi-equilibrium approximations, and how to interpret them. At least a part of the discussion is due to a different rôle the quasi-equilibrium plays in the entropy-conserving and the dissipative dynamics. The very first use of the entropy maximization dates back to the classical work of Gibbs [166], but it was first claimed for a principle of informational statistical thermodynamics by Jaynes [153]. Probably the first explicit and systematic use of quasi-equilibria to derive dissipation from entropy-conserving systems is due to the works of Zubarev. Recent detailed exposition is given in [155]. The method of nonequilibrium ensemble was developed also by Eu [173]. For dissipative systems, the use of the quasi-equilibrium to reduce description can be traced to the works of Grad on the Boltzmann equation [158]. A review of the ideas of the underlying method behind informational statistical thermodynamics was presented in Ref. [172]. The connection between entropy maximization and (nonlinear) Onsager formalism was also studied [171,127]. The viewpoint of two of the present authors (ANG and IVK) was influenced by the papers by L.I. Rozonoer and co-workers, in particular, [167-169]. A detailed exposition of the quasi-equilibrium approximation for Markov chains is given in the book [125] (Chapter 3, Quasi-equilibrium and entropy maximum, pp. 92-122), and for the BBGKY hierarchy in the paper Ref. [170]. We have applied maximum entropy principle to the description the universal dependence the 3-particle distribution function $F_{3}$ on the 2-particle distribution function $F_{2}$ in classical systems with binary interactions [174]. For a discussion the quasi-equilibrium moment closure hierarchies for the Boltzmann equation [168] see the papers [178,179,175]. A very general discussion of the maximum entropy principle with applications to dissipative kinetics is given in the review [176]. Recently the quasi-equilibrium approximation with some further correction was applied to description of rheology of polymer solutions [198,199] and of ferrofluids $[200,201]$.
} 
A linear moment parameterization is a linear operator, $\Pi: E \rightarrow L$, where $L=\mathrm{im} \Pi=E / \operatorname{ker} \Pi$, ker $\Pi$ is a closed linear subspace of space $E$, and $\Pi$ is the projection of $E$ onto factor-space $L$. Let us denote $W=\Pi(U)$. quasi-equilibrium (or restricted equilibrium, or conditional equilibrium) is the embedding, $F^{*}: W \rightarrow U$, which puts into correspondence to each $y \in W$ the solution to the entropy maximization problem:

$$
S(x) \rightarrow \max , \quad \Pi(x)=y .
$$

We assume that, for each $y \in \operatorname{int} W$, there exists the unique solution $F^{*}(y) \in \operatorname{int} U$ to problem (112). This solution, $F^{*}(y)$, is called the quasi-equilibrium, corresponding to the value $y$ of the macroscopic variables. The set of quasi-equilibria $F^{*}(y), y \in W$, forms a manifold in int $U$, parameterized by the values of the macroscopic variables $y \in W$.

Let us specify some notations: $E^{T}$ is the adjoint to the $E$ space. Adjoint spaces and operators will be indicated by ${ }^{T}$, whereas notation ${ }^{*}$ is earmarked for equilibria and quasi-equilibria.

Furthermore, $[l, x]$ is the result of application of the functional $l \in E^{T}$ to the vector $x \in E$. We recall that, for an operator $A: E_{1} \rightarrow E_{2}$, the adjoint operator, $A^{T}: E_{1}^{T} \rightarrow E_{2}^{T}$ is defined by the following relation: For any $l \in E_{2}^{T}$ and $x \in E_{1}$,

$$
[l, A x]=\left[A^{T} l, x\right] .
$$

Next, $D_{x} S(x) \in E^{T}$ is the differential of the entropy functional $S(x), D_{x}^{2} S(x)$ is the second differential of the entropy functional $S(x)$. The corresponding quadratic functional $D_{x}^{2} S(x)(z, z)$ on $E$ is defined by the Taylor formula,

$$
S(x+z)=S(x)+\left[D_{x} S(x), z\right]+\frac{1}{2} D_{x}^{2} S(x)(z, z)+\mathrm{o}\left(\|z\|^{2}\right) .
$$

We keep the same notation for the corresponding symmetric bilinear form, $D_{x}^{2} S(x)(z, p)$, and also for the linear operator, $D_{x}^{2} S(x): E \rightarrow E^{T}$, defined by the formula

$$
\left[D_{x}^{2} S(x) z, p\right]=D_{x}^{2} S(x)(z, p) .
$$

In the latter formula, on the left hand side, there is an operator, on the right hand side there is a bilinear form. Operator $D_{x}^{2} S(x)$ is symmetric on $E, D_{x}^{2} S(x)^{T}=D_{x}^{2} S(x)$.

Concavity of the entropy $S$ means that for any $z \in E$, the inequality holds,

$$
D_{x}^{2} S(x)(z, z) \leqslant 0 ;
$$

in the restriction onto the affine subspace parallel to $\operatorname{ker} \Pi$ we assume the strict concavity,

$$
D_{x}^{2} S(x)(z, z)<0 \quad \text { if } z \in \operatorname{ker} \Pi \text { and if } z \neq 0 .
$$

In the remainder of this subsection we are going to construct the important object, the projector onto the tangent space of the quasi-equilibrium manifold.

Let us compute the derivative $D_{y} F^{*}(y)$. For this purpose, let us apply the method of Lagrange multipliers: There exists such a linear functional $\Lambda(y) \in(L)^{T}$, that

$$
\left.D_{x} S(x)\right|_{F^{*}(y)}=\Lambda(y) \cdot \Pi, \quad \Pi\left(F^{*}(y)\right)=y
$$

or

$$
\left.D_{x} S(x)\right|_{F^{*}(y)}=\Pi^{T} \cdot \Lambda(y), \quad \Pi\left(F^{*}(y)\right)=y .
$$


From Eq. (115) we get

$$
\Pi\left(D_{y} F^{*}(y)\right)=1_{L},
$$

where we have indicated the space in which the unit operator acts. Next, using the latter expression, we transform the differential of Eq. (114),

$$
D_{y} \Lambda=\left(\Pi\left(D_{x}^{2} S\right)_{F^{*}(y)}^{-1} \Pi^{T}\right)^{-1},
$$

and, consequently,

$$
D_{y} F^{*}(y)=\left(D_{x}^{2} S\right)_{F^{*}(y)}^{-1} \Pi^{T}\left(\Pi\left(D_{x}^{2} S\right)_{F^{*}(y)}^{-1} \Pi^{T}\right)^{-1} .
$$

Notice that, elsewhere in Eq. (118), operator $\left(D_{x}^{2} S\right)^{-1}$ acts on the linear functionals from $L^{T}$. These functionals are precisely those which become zero on $\operatorname{ker} \Pi$ or, that is the same, those which can be represented as linear functionals of macroscopic variables.

The tangent space to the quasi-equilibrium manifold at the point $F^{*}(y)$ is the image of the operator $D_{y} F^{*}(y)$ :

$$
\operatorname{im}\left(D_{y} F^{*}(y)\right)=\left(D_{x}^{2} S\right)_{F^{*}(y)}^{-1} L^{T}=\left(D_{x}^{2} S\right)_{F^{*}(y)}^{-1} \operatorname{Ann}(\operatorname{ker} \Pi)
$$

where $\operatorname{Ann}(\operatorname{ker} \Pi)$ is the set of linear functionals which become zero on $\operatorname{ker} \Pi$. Another way to write Eq. (119) is the following:

$$
x \in \operatorname{im}\left(D_{y} F^{*}(y)\right) \Leftrightarrow\left(D_{x}^{2} S\right)_{F^{*}(y)}(z, p)=0, \quad p \in \operatorname{ker} \Pi .
$$

This means that $\operatorname{im}\left(D_{y} F^{*}(y)\right)$ is the orthogonal completement of $\operatorname{ker} \Pi$ in $E$ with respect to the scalar product,

$$
\langle z \mid p\rangle_{F^{*}(y)}=-\left(D_{x}^{2} S\right)_{F^{*}(y)}(z, p) .
$$

The entropic scalar product (121) appears often in the constructions below. (Usually, it becomes the scalar product indeed after the conservation laws are excluded). Let us denote as $T_{y}=$ $\operatorname{im}\left(D_{y} F^{*}(y)\right)$ the tangent space to the quasi-equilibrium manifold at the point $F^{*}(y)$. Important role in the construction of quasi-equilibrium dynamics and its generalizations is played by the quasi-equilibrium projector, an operator which projects $E$ on $T_{y}$ parallel to ker $\Pi$. This is the orthogonal projector with respect to the entropic scalar product, $P_{y}^{*}: E \rightarrow T_{y}$ :

$$
P_{y}^{*}=D_{y} F^{*}(y) \cdot \Pi=\left(\left.D_{x}^{2} S\right|_{F^{*}(y)}\right)^{-1} \Pi^{T}\left(\Pi\left(\left.D_{x}^{2} S\right|_{F^{*}(y)}\right)^{-1} \Pi^{T}\right)^{-1} \Pi .
$$

It is straightforward to check the equality $P_{y}^{* 2}=P_{y}^{*}$, and the self-adjointness of $P_{y}^{*}$ with respect to the entropic scalar product (121). Thus, we have introduced the basic constructions: the quasi-equilibrium manifold, the entropic scalar product, and the quasi-equilibrium projector.

The construction of the quasi-equilibrium allows for the following generalization: Almost every manifold can be represented as a set of minimizers of the entropy under linear constrains. However, in contrast to the standard quasi-equilibrium, these linear constrains will depend, generally speaking, on the point on the manifold.

So, let the manifold $\Omega=F(W) \subset U$ be given. This is a parametric set of distribution functions. However, now macroscopic variables $y$ are not functionals on $R$ or $U$ but just parameters defining points on the manifold. The problem is how to extend the definitions of $y$ onto a neighborhood of 
$F(W)$ in such a way that $F(W)$ will appear as the solution to the variational problem:

$$
S(x) \rightarrow \max , \quad \Pi(x)=y .
$$

For each point $F(y)$, we identify $T_{y} \in E$, the tangent space to the manifold $\Omega$ in $F_{y}$, and the subspace $Y_{y} \subset E$, which depends smoothly on $y$, and which has the property, $Y_{y} \oplus T_{y}=E$. Let us define $\Pi(x)$ in the neighborhood of $F(W)$ in such a way, that

$$
\Pi(x)=y \quad \text { if } x-F(y) \in Y_{y} .
$$

The point $F(y)$ is the solution of the quasi-equilibrium problem (123) if and only if

$$
\left.D_{x} S(x)\right|_{F(y)} \in \text { Ann } Y_{y} \text {. }
$$

That is, if and only if $\left.Y_{y} \subset \operatorname{ker} D_{x} S(x)\right|_{F(y)}$. It is always possible to construct subspaces $Y_{y}$ with the properties just specified, at least locally, if the functional $\left.D_{x} S\right|_{F(y)}$ is not identically equal to zero on $T_{y}$.

The construction just described allows to consider practically any manifold as a quasi-equilibrium. This construction is required when one seeks the induced dynamics on a given manifold. Then the vector fields are projected on $T_{y}$ parallel to $Y_{y}$, and this preserves intact the basic properties of the quasi-equilibrium approximations.

Let us return to the usual linear moment parametrization. quasi-equilibrium entropy $S(y)$ is a functional on $W$. It is defined as the value of the entropy on the corresponding quasi-equilibrium $x=F^{*}(y)$ :

$$
S(y)=S\left(F^{*}(y)\right)
$$

Quasi-equilibrium dynamics is a dynamics on $W$, defined by Eq. (109) for the quasi-equilibrium $F^{*}(y)$ :

$$
\frac{\mathrm{d} y}{\mathrm{~d} t}=\Pi J\left(F^{*}(y)\right) .
$$

Here $\Pi$ is constant linear operator (in the general case (109), it may become nonlinear). The corresponding quasi-equilibrium dynamics on the quasi-equilibrium manifold $F^{*}(W)$ is defined using the projector (108):

$$
\frac{\mathrm{d} x}{\mathrm{~d} t}=\left.P_{y}^{*}\right|_{x=F^{*}(y)} J(x)=\left(D_{y} F^{*}\right)_{x=F^{*}(y)} \Pi J(x), \quad x \in F^{*}(W) .
$$

The orthogonal projector $P_{v}^{*}$ on the right hand side of Eq. (128) can be explicitly written using the second derivative of $S$ and the operator $\Pi$ (122). Let us remind that the only distinguished scalar product in $E$ is the entropic scalar product (121):

$$
\langle z, p\rangle_{x}=-\left(D_{x}^{2} S\right)_{x}(z, p)
$$

It depends on the point $x \in U$. This dependence $\langle\mid\rangle_{x}$ endows $U$ with the structure of a Riemann space.

The most important property of the quasi-equilibrium system (127), (128) is highlighted by the conservation of the dynamics type theorem: if for the original dynamic system (76) $\mathrm{d} S / \mathrm{d} t \geqslant 0$, then for the quasi-equilibrium dynamics $\mathrm{d} S / \mathrm{d} t \geqslant 0$. If for the original dynamic system (76) $\mathrm{d} S / \mathrm{d} t=0$ (conservative system), then for the quasi-equilibrium dynamics $\mathrm{d} S / \mathrm{d} t=0$ as well. 


\subsection{Thermodynamic projector without a priori parameterization}

Quasi-equilibrium manifolds is a place where the entropy and the moment parameterization meet each other. The projectors $P_{y}$ for a quasi-equilibrium manifold is nothing but the orthogonal with respect to the entropic scalar product $\langle\mid\rangle_{x}$ projector (122). The quasi-equilibrium projector preserves the type of dynamics. Note that in order to preserve the type of dynamics we needed only one condition to be satisfied,

$$
\operatorname{ker} P_{y} \subset \operatorname{ker}\left(D_{x} S\right)_{x=F(y)}
$$

Let us require that the field of projectors, $P(x, T)$, is defined for any $x$ and $T$, if

$$
T \not \subset \operatorname{ker} D_{x} S \text {. }
$$

It follows immediately from these conditions that in the equilibrium, $P\left(x^{*}, T\right)$ is the orthogonal projector onto $T$ (ortogonality is with respect to the entropic scalar product $\langle\mid\rangle_{x^{*}}$ ).

The field of projectors is constructed in the neighborhood of the equilibrium based on the requirement of the maximal smoothness of $P$ as a function of $g_{x}=D_{x} S$ and $x$. It turns out that to the first order in the deviations $x-x^{*}$ and $g_{x}-g_{x^{*}}$, the projector is defined uniquely. Let us first describe the construction of the projector, and next discuss its uniqueness.

Let the subspace $T \subset E$, the point $x$, and the differential of the entropy at this point, $g=D_{x} S$, be defined in such a way that the transversality condition (131) is satisfied. Let us define $T_{0}=T \cap \operatorname{ker} g_{x}$. By condition (131), $T_{0} \neq T$. Let us denote, $e_{g}=e_{g}(T) \in T$ the vector in $T$, such that $e_{g}$ is orthogonal to $T_{0}$, and is normalized by the condition $g\left(e_{g}\right)=1$. The vector $e_{g}$ is defined unambiguously. The projector $P_{S, x}=P(x, T)$ is defined as follows: For any $z \in E$,

$$
P_{S, x}(z)=P_{0}(z)+e_{g} g_{x}(z)
$$

where $P_{0}$ is the orthogonal projector on $T_{0}$ (orthogonality is with respect to the entropic scalar product $\left.\langle\mid\rangle_{x}\right)$. The entropic projector (132) depends on the point $x$ through the $x$-dependence of the scalar product $\langle\mid\rangle_{x}$, and also through the differential of $S$ in $x$, the functional $g_{x}$.

Obviously, $P(z)=0$ implies $g(z)=0$, that is, the thermodynamicity requirement (130) is satisfied. Uniqueness of the thermodynamic projector (132) is supported by the requirement of the maximal smoothness (analyticity) [10] of the projector as a function of $g_{x}$ and $\langle\mid\rangle_{x}$, and is done in two steps which we sketch here (detailed proof is given in Ref. [177]):

1. Considering the expansion of the entropy in the equilibrium up to the quadratic terms, one demonstrates that in the equilibrium the thermodynamic projector is the orthogonal projector with respect to the scalar product $\langle\mid\rangle_{x^{*}}$.

2. For a given $g$, one considers auxiliary dissipative dynamic systems (76), which satisfy the condition: For every $x^{\prime} \in U$, it holds, $g_{x}\left(J\left(x^{\prime}\right)\right)=0$, that is, $g_{x}$ defines an additional linear conservation law for the auxiliary systems. For the auxiliary systems, the point $x$ is the equilibrium. Eliminating the linear conservation law $g_{x}$, and using the result of the previous point, we end up with formula (132). 
Thus, the entropic structure defines unambiguously the field of projectors (132), for which the dynamics of any dissipative system (76) projected on any closure assumption remains dissipative.

\section{Example 1: Quasi-equilibrium projector and defect of invariance for the local Maxwellians} manifold of the Boltzmann equation

The Boltzmann equation is one of the everlasting equations. It remains the most inspiring source for the model reduction problems. With this subsection we start a series of examples for the Boltzmann equation.

Difficulties of classical methods of the Boltzmann equation theory. As was mentioned above, the first systematic and (at least partially) successful method of constructing invariant manifolds for dissipative systems was the celebrated Chapman-Enskog method [51] for the Boltzmann kinetic equation. The main difficulties of the Chapman-Enskog method [51] are "nonphysical" properties of high-order approximations. This was stated by a number of authors and was discussed in detail in [87]. In particular, as it was noted in [53], the Burnett approximation results in a short-wave instability of the acoustic spectra. This fact contradicts the $H$-theorem (cf. in [53]). The Hilbert expansion contains secular terms [87]. The latter contradicts the $H$-theorem.

The other difficulties of both of these methods are: the restriction upon the choice of initial approximation (the local equilibrium approximation), the demand for a small parameter, and the usage of slowly converging Taylor expansion. These difficulties never allow a direct transfer of these methods on essentially nonequilibrium situations.

The main difficulty of the Grad method [158] is the uncontrollability of the chosen approximation. An extension of the list of moments can result in a certain success, but it cannot also give anything. Difficulties of moment expansion in the problems of shock waves and sound propagation can be seen in [87].

Many attempts were made to refine these methods. For the Chapman-Enskog and Hilbert methods these attempts are based in general on some "good" rearrangement of expansions (e.g. neglecting high-order derivatives [87], reexpanding [87], Pade approximations and partial summing [25,178,165], etc.). This type of work with formal series is wide spread in physics. Sometimes the results are surprisingly good - from the renormalization theory in quantum fields to the Percus-Yevick equation and the ring-operator in statistical mechanics. However, one should realize that success could not be guaranteed. Moreover, rearrangements never remove the restriction upon the choice of the initial local equilibrium approximation.

Attempts to improve the Grad method are based on quasi-equilibrium approximations [167,168]. It was found in [168] that the Grad distributions are linearized versions of appropriate quasi-equilibrium approximations (see also the late papers $[178,179,175]$ ). A method which treats fluxes (e.g. moments with respect to collision integrals) as independent variables in a quasi-equilibrium description was introduced in [190,178,192,179].

An important feature of quasi-equilibrium approximations is that they are always thermodynamic, i.e. they are concordant with the $H$-theorem due to their construction. However, quasi-equilibrium approximations do not remove the uncontrollability of the Grad method.

Boltzmann Equation (BE). The phase space $E$ consists of distribution functions $f(\boldsymbol{v}, \boldsymbol{x})$ which depend on the spatial variable $\boldsymbol{x}$ and on velocity variable $\boldsymbol{v}$. The variable $\boldsymbol{x}$ spans an open domain $\Omega_{\boldsymbol{x}}^{3} \subseteq \mathbf{R}_{\boldsymbol{x}}$, and the variable $\boldsymbol{v}$ spans the space $\mathbf{R}_{\boldsymbol{v}}^{3}$. We require that $f(\boldsymbol{v}, \boldsymbol{x}) \in F$ are nonnegative 
functions, and also that the following integrals are finite for every $\boldsymbol{x} \in \Omega_{\boldsymbol{x}}$ (the existence of moments and of the entropy):

$$
\begin{aligned}
& I_{\boldsymbol{x}}^{\left(i_{1} i_{2} i_{3}\right)}(f)=\int v_{1}^{i_{1}} v_{2}^{i_{2}} v_{3}^{i_{3}} f(\boldsymbol{v}, \boldsymbol{x}) \mathrm{d}^{3} \boldsymbol{v}, \quad i_{1} \geqslant 0, i_{2} \geqslant 0, i_{3} \geqslant 0 ; \\
& H_{\boldsymbol{x}}(f)=\int f(\boldsymbol{v}, \boldsymbol{x})(\ln f(\boldsymbol{v}, \boldsymbol{x})-1) \mathrm{d}^{3} \boldsymbol{v}, \quad H(f)=\int H_{\boldsymbol{x}}(f) \mathrm{d}^{3} \boldsymbol{x} .
\end{aligned}
$$

Here and below integration in $\boldsymbol{v}$ is made over $\mathbf{R}_{\boldsymbol{v}}^{3}$, and it is made over $\Omega_{\boldsymbol{x}}$ in $\boldsymbol{x}$. For every fixed $x \in \Omega_{x}, I_{x}^{(\cdots)}$ and $H_{x}$ might be treated as functionals defined in $F$.

We write $\mathrm{BE}$ in the form of (76) using standard notations [87]:

$$
\frac{\partial f}{\partial t}=J(f), \quad J(f)=-v_{s} \frac{\partial f}{\partial x_{s}}+Q(f, f) .
$$

Here and further a summation in two repeated indices is assumed, and $Q(f, f)$ stands for the Boltzmann collision integral [1]. The latter represents the dissipative part of the vector field $J(f)(135)$.

In this paper we consider the case when boundary conditions for Eq. (135) are relevant to the local with respect to $\boldsymbol{x}$ form of the $H$-theorem.

For every fixed $\boldsymbol{x}$, we denote as $H_{\boldsymbol{x}}^{0}(f)$ the space of linear functionals $\sum_{i=0}^{4} a_{i}(\boldsymbol{x}) \int \psi_{i}(\boldsymbol{v}) f(\boldsymbol{v}, \boldsymbol{x}) \mathrm{d}^{3} \boldsymbol{v}$, where $\psi_{i}(\boldsymbol{v})$ represent summational invariants of a collision [1,2] $\left(\psi_{0}=1, \psi_{i}=v_{i}, i=1,2,3, \psi_{4}=v^{2}\right)$. We write $\left(\bmod H_{x}^{0}(f)\right)$ if an expression is valid within the accuracy of adding a functional from $H_{x}^{0}(f)$. The local $H$-theorem states: for any functional

$$
H_{x}(f)=\int f(\boldsymbol{v}, \boldsymbol{x})(\ln f(\boldsymbol{v}, \boldsymbol{x})-1) \mathrm{d}^{3} \boldsymbol{v}\left(\bmod H_{x}^{0}(f)\right)
$$

the following inequality is valid:

$$
\mathrm{d} H_{x}(f) /\left.\mathrm{d} t \equiv \int Q(f, f)\right|_{f=f(\boldsymbol{v}, \boldsymbol{x})} \ln f(\boldsymbol{v}, \boldsymbol{x}) \mathrm{d}^{3} \boldsymbol{v} \leqslant 0 .
$$

Expression (137) is equal to zero if and only if $\ln f=\sum_{i=0}^{4} a_{i}(\boldsymbol{x}) \psi_{i}(\boldsymbol{v})$.

Although all functionals (136) are equivalent in the sense of the $H$-theorem, it is convenient to deal with the functional

$$
H_{x}(f)=\int f(\boldsymbol{v}, \boldsymbol{x})(\ln f(\boldsymbol{v}, \boldsymbol{x})-1) \mathrm{d}^{3} \boldsymbol{v} .
$$

All what was said in the previous sections can be applied to BE (135). Now we will discuss some specific points.

Local manifolds. Although the general description of manifolds $\Omega \subset F$ (Section 2.1) holds as well for BE, a specific class of manifolds might be defined due to the different character of spatial and of velocity dependencies in BE vector field (135). These manifolds will be called local manifolds, and they are constructed as follows. Denote as $F_{\text {loc }}$ the set of functions $f(\boldsymbol{v})$ with finite integrals

(a)

$$
I^{\left(i_{1} i_{2} i_{3}\right)}(f)=\int v_{1}^{i_{1}} v_{2}^{i_{2}} v_{3}^{i_{3}} f(\boldsymbol{v}) \mathrm{d}^{3} \boldsymbol{v}, \quad i_{1} \geqslant 0, \quad i_{2} \geqslant 0, \quad i_{3} \geqslant 0
$$


(b)

$$
H(f)=\int f(\boldsymbol{v}) \ln f(\boldsymbol{v}) \mathrm{d}^{3} \boldsymbol{v} .
$$

In order to construct a local manifold in $F$, we, firstly, consider a manifold in $F_{\text {loc }}$. Namely, we define a domain $A \subset B$, where $B$ is a linear space, and consider a smooth immersion $A \rightarrow F_{\text {loc }}$ : $a \rightarrow f(a, v)$. The set of functions $f(a, v) \in F_{\text {loc }}$, where $a$ spans the domain $A$, is a manifold in $F_{\text {loc }}$. Secondly, we consider all bounded and sufficiently smooth functions $a(\boldsymbol{x}): \Omega_{\boldsymbol{x}} \rightarrow A$, and we define the local manifold in $F$ as the set of functions $f(a(\boldsymbol{x}), \boldsymbol{v})$. Roughly speaking, the local manifold is a set of functions which are parameterized with $\boldsymbol{x}$-dependent functions $a(\boldsymbol{x})$. A local manifold will be called a locally finite-dimensional manifold if $B$ is a finite-dimensional linear space.

Locally finite-dimensional manifolds are a natural source of initial approximations for constructing dynamic invariant manifolds in BE theory. For example, the Tamm-Mott-Smith (TMS) approximation gives us locally two-dimensional manifold $\left\{f\left(a_{-}, a_{+}\right)\right\}$which consists of distributions

$$
f\left(a_{-}, a_{+}\right)=a_{-} f_{-}+a_{+} f_{+} .
$$

Here $a_{-}$and $a_{+}$(the coordinates on the manifold $\Omega_{\mathrm{TMS}}=\left\{f\left(a_{-}, a_{+}\right)\right\}$) are nonnegative real functions of the position vector $\boldsymbol{x}$, and $f_{-}$and $f_{+}$are fixed Maxwellians.

Next example is locally five-dimensional manifold $\{f(n, \boldsymbol{u}, T)\}$ which consists of local Maxwellians (LM). The LM manifold consists of distributions $f_{0}$ which are labeled with parameters $n, \boldsymbol{u}$, and $T$ :

$$
f_{0}(n, \boldsymbol{u}, T)=n\left(\frac{2 \pi k_{\mathrm{B}} T}{m}\right)^{-3 / 2} \exp \left(-\frac{m(\boldsymbol{v}-\boldsymbol{u})^{2}}{2 k_{\mathrm{B}} T}\right) .
$$

Parameters $n, \boldsymbol{u}$, and $T$ in (140) are functions depending on $\boldsymbol{x}$. In this section we will not indicate this dependency explicitly.

Distribution $f_{0}(n, \boldsymbol{u}, T)$ is the unique solution of the variational problem:

$$
H(f)=\int f \ln f \mathrm{~d}^{3} \boldsymbol{v} \rightarrow \min
$$

for

$$
\begin{aligned}
& M_{0}(f)=\int 1 \cdot f \mathrm{~d}^{3} \boldsymbol{v} \\
& M_{i}(f)=\int v_{i} f \mathrm{~d}^{3} \boldsymbol{v}=n u_{i}, \quad i=1,2,3 ; \\
& M_{4}(f)=\int v^{2} f \mathrm{~d}^{3} \boldsymbol{v}=\frac{3 n k_{\mathrm{B}} T}{m}+n u^{2} .
\end{aligned}
$$

Hence, the LM manifold is a quasi-equilibrium manifold. Considering $n, \boldsymbol{u}$, and $T$ as five scalar parameters (see the remark on locality in Section 3), we see that LM manifold is parameterized with the values of $M_{s}(f), s=0, \ldots, 4$, which are defined in the neighborhood of LM manifold. It is sometimes convenient to consider the variables $M_{s}\left(f_{0}\right), s=0, \ldots, 4$, as new coordinates on LM manifold. The relationship between the sets $\left\{M_{s}\left(f_{0}\right)\right\}$ and $\{n, \boldsymbol{u}, T\}$ is

$$
n=M_{0} ; \quad u_{i}=M_{0}^{-1} M_{i}, \quad i=1,2,3 ; \quad T=\frac{m}{3 k_{\mathrm{B}}} M_{0}^{-1}\left(M_{4}-M_{0}^{-1} M_{i} M_{i}\right) .
$$

This is the standard moment parametrization of the quasi-equilibrium manifold. 
Thermodynamic quasi-equilibrium projector. Thermodynamic quasi-equilibrium projector $P_{f_{0}(n, \boldsymbol{u}, T)}(J)$ onto the tangent space $T_{f_{0}(n, u, T)}$ is defined as

$$
P_{f_{0}(n, \boldsymbol{u}, T)}(J)=\sum_{s=0}^{4} \frac{\partial f_{0}(n, \boldsymbol{u}, T)}{\partial M_{s}} \int \psi_{s} J \mathrm{~d}^{3} \boldsymbol{v} .
$$

Here we have assumed that $n, \boldsymbol{u}$, and $T$ are functions of $M_{0}, \ldots, M_{4}$ (see relationship (142)), and

$$
\psi_{0}=1, \quad \psi_{i}=v_{i}, \quad i=1,2,3, \quad \psi_{4}=v^{2} .
$$

Calculating derivatives in (143), and next returning to variables $n, \boldsymbol{u}$, and $T$, we obtain

$$
\begin{aligned}
P_{f_{0}(n, \boldsymbol{u}, T)}(J)= & f_{0}(n, \boldsymbol{u}, T)\left\{\left[\frac{1}{n}-\frac{m u_{i}}{n k_{\mathrm{B}} T}\left(v_{i}-u_{i}\right)+\left(\frac{m u^{2}}{3 n k_{\mathrm{B}}}-\frac{T}{n}\right)\left(\frac{m(\boldsymbol{v}-\boldsymbol{u})^{2}}{2 k_{\mathrm{B}} T^{2}}-\frac{3}{2 T}\right)\right]\right. \\
& \times \int 1 \cdot J \mathrm{~d}^{3} \boldsymbol{v}+\left[\frac{m}{n k_{\mathrm{B}} T}\left(v_{i}-u_{i}\right)-\frac{2 m u_{i}}{3 n k_{\mathrm{B}}}\left(\frac{m(\boldsymbol{v}-\boldsymbol{u})^{2}}{2 k_{\mathrm{B}} T^{2}}-\frac{3}{2 T}\right)\right] \\
& \left.\times \int v_{i} J \mathrm{~d}^{3} \boldsymbol{v}+\frac{m}{3 n k_{\mathrm{B}}}\left(\frac{m(\boldsymbol{v}-\boldsymbol{u})^{2}}{2 k_{\mathrm{B}} T^{2}}-\frac{3}{2 T}\right) \int \boldsymbol{v}^{2} J \mathrm{~d}^{3} \boldsymbol{v}\right\} .
\end{aligned}
$$

It is sometimes convenient to rewrite $(145)$ as

$$
P_{f_{0}(n, \boldsymbol{u}, T)}(J)=f_{0}(n, \boldsymbol{u}, T) \sum_{s=0}^{4} \psi_{f_{0}(n, \boldsymbol{u}, T)}^{(s)} \int \psi_{f_{0}(n, \boldsymbol{u}, T)}^{(s)} J \mathrm{~d}^{3} \boldsymbol{v}
$$

Here

$$
\begin{aligned}
& \psi_{f_{0}(n, \boldsymbol{u}, T)}^{(0)}=n^{-1 / 2}, \quad \psi_{f_{0}(n, \boldsymbol{u}, T)}^{(i)}=(2 / n)^{1 / 2} c_{i}, \quad i=1,2,3 \\
& \psi_{f_{0}(n, \boldsymbol{u}, T)}^{(4)}=(2 / 3 n)^{1 / 2}\left(c^{2}-(3 / 2)\right) ; \quad c_{i}=\left(m / 2 k_{\mathrm{B}} T\right)^{1 / 2}\left(v_{i}-u_{i}\right) .
\end{aligned}
$$

It is easy to check that

$$
\int f_{0}(n, \boldsymbol{u}, T) \psi_{f_{0}(n, \boldsymbol{u}, T)}^{(k)} \psi_{f_{0}(n, \boldsymbol{u}, T)}^{(l)} \mathrm{d}^{3} \boldsymbol{v}=\delta_{k l} .
$$

Here $\delta_{k l}$ is the Kronecker delta.

Defect of invariance for the LM manifold. The defect of invariance for the LM manifold at the point $f_{0}(n, \boldsymbol{u}, T)$ for the $B E$ is

$$
\begin{aligned}
\Delta\left(f_{0}(n, \boldsymbol{u}, T)\right)= & P_{f_{0}(n, \boldsymbol{u}, T)}\left(-\left(v_{s}-u_{s}\right) \frac{\partial f_{0}(n, \boldsymbol{u}, T)}{\partial x_{s}}+Q\left(f_{0}(n, \boldsymbol{u}, T)\right)\right) \\
& -\left(-\left(v_{s}-u_{s}\right) \frac{\partial f_{0}(n, \boldsymbol{u}, T)}{\partial x_{s}}+Q\left(f_{0}(n, \boldsymbol{u}, T)\right)\right) \\
= & P_{f_{0}(n, \boldsymbol{u}, T)}\left(-\left(v_{s}-u_{s}\right) \frac{\partial f_{0}(n, \boldsymbol{u}, T)}{\partial x_{s}}\right)+\left(v_{s}-u_{s}\right) \frac{\partial f_{0}(n, \boldsymbol{u}, T)}{\partial x_{s}}
\end{aligned}
$$


Substituting (145) into (149), we obtain

$$
\begin{aligned}
\Delta\left(f_{0}(n, \boldsymbol{u}, T)\right)= & f_{0}(n, \boldsymbol{u}, T)\left\{\left(\frac{m(\boldsymbol{v}-\boldsymbol{u})^{2}}{2 k_{\mathrm{B}} T}-\frac{5}{2}\right)\left(v_{i}-u_{i}\right) \frac{\partial \ln T}{\partial x_{i}}\right. \\
& \left.+\frac{m}{k_{\mathrm{B}} T}\left(\left(v_{i}-u_{i}\right)\left(v_{s}-u_{s}\right)-\frac{1}{3} \delta_{i s}(\boldsymbol{v}-\boldsymbol{u})^{2}\right) \frac{\partial u_{s}}{\partial x_{i}}\right\} .
\end{aligned}
$$

The LM manifold is not a dynamic invariant manifold of the Boltzmann equation and defect (150) is not identical to zero.

\section{Example 2: Scattering rates versus moments: alternative Grad equations}

In this subsection scattering rates (moments of collision integral) are treated as new independent variables, and as an alternative to moments of the distribution function, to describe the rarefied gas near local equilibrium. A version of entropy maximum principle is used to derive the Grad-like description in terms of a finite number of scattering rates. New equations are compared to the Grad moment system in the heat nonconductive case. Estimations for hard spheres demonstrate, in particular, some $10 \%$ excess of the viscosity coefficient resulting from the scattering rate description, as compared to the Grad moment estimation.

In 1949, Harold Grad [158] has extended the basic assumption behind the Hilbert and ChapmanEnskog methods (the space and time dependence of the normal solutions is mediated by the five hydrodynamic moments [51]). A physical rationale behind the Grad moment method is an assumption of the decomposition of motion (i). During the time of order $\tau$, a set of distinguished moments $M^{\prime}$ (which include the hydrodynamic moments and a subset of higher-order moment) does not change significantly as compared to the rest of the moments $M^{\prime \prime}$ (the fast evolution) (ii). Towards the end of the fast evolution, the values of the moments $M^{\prime \prime}$ become unambiguously determined by the values of the distinguished moments $M^{\prime}$, and (iii). On the time of order $\theta \gg \tau$, dynamics of the distribution function is determined by the dynamics of the distinguished moments while the rest of the moments remains to be determined by the distinguished moments (the slow evolution period).

Implementation of this picture requires an ansatz for the distribution function in order to represent the set of states visited in the course of the slow evolution. In Grad's method, these representative sets are finite-order truncations of an expansion of the distribution functions in terms of Hermit velocity tensors:

$$
f_{\mathrm{C}}\left(M^{\prime}, \boldsymbol{v}\right)=f_{\mathrm{LM}}(\rho, \boldsymbol{u}, E, \boldsymbol{v})\left[1+\sum_{(\alpha)}^{N} a_{\alpha}\left(M^{\prime}\right) H_{(\alpha)}(\boldsymbol{v}-\boldsymbol{u})\right],
$$

where $H_{(\alpha)}(\boldsymbol{v}-\boldsymbol{u})$ are various Hermit tensor polynomials, orthogonal with the weight $f_{\mathrm{LM}}$, while coefficient $a_{(\alpha)}\left(M^{\prime}\right)$ are known functions of the distinguished moments $M^{\prime}$, and $N$ is the highest order of $M^{\prime}$. Other moments are functions of $M^{\prime}: M^{\prime \prime}=M^{\prime \prime}\left(f_{\mathrm{C}}\left(M^{\prime}\right)\right)$.

Slow evolution of distinguished moments is found upon substitution of Eq. (151) into the Boltzmann equation and finding the moments of the resulting expression (Grad's moment equations). 
Following Grad, this extremely simple approximation can be improved by extending the list of distinguished moments. The most well known is Grad's thirteen-moment approximation where the set of distinguished moments consists of five hydrodynamic moments, five components of the traceless stress tensor $\sigma_{i j}=\int m\left[\left(v_{i}-u_{i}\right)\left(v_{j}-u_{j}\right)-\delta_{i j}(\boldsymbol{v}-\boldsymbol{u})^{2} / 3\right] f \mathrm{~d} \boldsymbol{v}$, and of the three components of the heat flux vector $q_{i}=\int\left(v_{i}-u_{i}\right) m(\boldsymbol{v}-\boldsymbol{u})^{2} / 2 f \mathrm{~d} \boldsymbol{v}$.

The time evolution hypothesis cannot be evaluated for its validity within the framework of Grad's approach. It is not surprising therefore that Grad's methods failed to work in situations where it was (unmotivatedly) supposed to, primarily, in the phenomena with sharp time-space dependence such as the strong shock wave. On the other hand, Grad's method was quite successful for describing transition between parabolic and hyperbolic propagation, in particular the second sound effect in massive solids at low temperatures, and, in general, situations slightly deviating from the classical Navier-Stokes-Fourier domain. Finally, the Grad method has been important background for development of phenomenological nonequilibrium thermodynamics based on hyperbolic first-order equation, the so-called EIT (extended irreversible thermodynamics [180]).

Important generalization of the Grad moment method is the concept of quasi-equilibrium approximations already mentioned above. The quasi-equilibrium distribution function for a set of distinguished moment $M^{\prime}$ maximizes the entropy density $S$ for fixed $M^{\prime}$. The quasi-equilibrium manifold $\Omega^{3}(M)$ is the collection of the quasi-equilibrium distribution functions for all admissible values of $M$. The quasi-equilibrium approximation is the simplest and extremely useful (not only in the kinetic theory itself) implementation of the hypothesis about the decomposition: If $M^{\prime}$ are considered as slow variables, then states which could be visited in the course of rapid motion in the neighbored of $\Omega^{*}\left(M^{\prime}\right)$ belong to the planes $\Gamma_{M^{\prime}}=\left\{f \mid m^{\prime}\left(f-f^{*}\left(M^{\prime}\right)\right)=0\right\}$. In this respect, the thermodynamic construction in the method of invariant manifold is a generalization of the quasi-equilibrium approximation where the given manifold is equipped with a quasi-equilibrium structure by choosing appropriately the macroscopic variables of the slow motion. In contrast to the quasi-equilibrium, the macroscopic variables thus constructed are not obligatory moments. A text book example of the quasi-equilibrium approximation is the generalized Gaussian function for $M^{\prime}=\{\rho, \rho \boldsymbol{u}, P\}$ where $P_{i j}=\int v_{i} v_{j} f \mathrm{~d} \boldsymbol{v}$ is the pressure tensor. The quasi-equilibrium approximation does not exist if the highest order moment is an odd polynomial of velocity (therefore, there exists no quasi-equilibrium for thirteen Grad's moments). Otherwise, the Grad moment approximation is the first-order expansion of the quasi-equilibrium around the local Maxwellian.

The classical Grad moment method [158] provides an approximate solution to the Boltzmann equation, and leads to a closed system of equations where hydrodynamic variables $\rho, \boldsymbol{u}$, and $P$ (density, mean flux, and pressure) are coupled to a finite set of nonhydrodynamic variables. The latter are usually the stress tensor $\sigma$ and the heat flux $\mathbf{q}$ constituting 10 and 13 moment Grad systems. The Grad method was originally introduced for diluted gases to describe regimes beyond the normal solutions [51], but later it was used, in particular, as a prototype of certain phenomenological schemes in nonequilibrium thermodynamics [180].

However, the moments do not constitute the unique system of nonhydrodynamic variables, and the exact dynamics might be equally expressed in terms of other infinite sets of variables (possibly, of a nonmoment nature). Moreover, as long as one shortens the description to only a finite subset of variables, the advantage of the moment description above other systems is not obvious. 
Nonlinear functionals instead of moments in the closure problem. Here we consider a new system of nonhydrodynamic variables, scattering rates $M^{w}(f)$ :

$$
\begin{aligned}
& M_{i_{1} i_{2} i_{3}}^{w}(f)=\int \mu_{i_{1} i_{2} i_{3}} Q^{w}(f) \mathrm{d} \boldsymbol{v} ; \\
& \mu_{i_{1} i_{2} i_{3}}=m v_{1}^{i_{1}} v_{2}^{i_{2}} v_{3}^{i_{3}},
\end{aligned}
$$

which, by definition, are the moments of the Boltzmann collision integral $Q^{w}(f)$ :

$$
Q^{w}(f)=\int w\left(\boldsymbol{v}^{\prime}, \boldsymbol{v}_{1}^{\prime}, \boldsymbol{v}, \boldsymbol{v}_{1}\right)\left\{f\left(\boldsymbol{v}^{\prime}\right) f\left(\boldsymbol{v}_{1}^{\prime}\right)-f(\boldsymbol{v}) f\left(\boldsymbol{v}_{1}\right)\right\} \mathrm{d} \boldsymbol{v}^{\prime} \mathrm{d} \boldsymbol{v}_{1}^{\prime} \mathrm{d} \boldsymbol{v}_{1} .
$$

Here $w$ is the probability density of a change of the velocities, $\left(\boldsymbol{v}, \boldsymbol{v}_{1}\right) \rightarrow\left(\boldsymbol{v}^{\prime}, \boldsymbol{v}_{1}^{\prime}\right)$, of the two particles after their encounter, and $w$ is defined by a model of pair interactions. The description in terms of the scattering rates $M^{w}$ (152) is alternative to the usually treated description in terms of the moments $M: M_{i_{1} i_{2} i_{3}}(f)=\int \mu_{i_{1} i_{2} i_{3}} f \mathrm{~d} \boldsymbol{v}$.

A reason to consider scattering rates instead of the moments is that $M^{w}$ (152) reflect features of the interactions because of the $w$ incorporated in their definition, while the moments do not. For this reason we can expect that, in general, a description with a finite number of scattering rates will be more informative than a description provided by the same number of their moment counterparts.

To come to the Grad-like equations in terms of the scattering rates, we have to complete the following two steps:

(i) To derive a hierarchy of transport equations for $\rho, \boldsymbol{u}, P$, and $M_{i_{1} i_{2} i_{3}}^{w}$ in a neighborhood of the local Maxwell states $f_{0}(\rho, \boldsymbol{u}, P)$.

(ii) To truncate this hierarchy, and to come to a closed set of equations with respect to $\rho, \boldsymbol{u}, P$, and a finite number of scattering rates.

In step (i), we derive a description with infinite number of variables, which is formally equivalent both to the Boltzmann equation near the local equilibrium, and to the description with an infinite number of moments. The approximation comes into play in step (ii) where we reduce the description to a finite number of variables. The difference between the moment and the alternative description occurs at this point.

Program (i) and (ii) is similar to what is done in the Grad method [158], with the only exception (and this is important) that we should always use scattering rates as independent variables and not to expand them into series in moments. Consequently, we will use a method of a closure in the step (ii) that does not refer to the moment expansions. Major steps of the computation will be presented below.

Linearization. To complete step (i), we represent $f$ as $f_{0}(1+\varphi)$, where $f_{0}$ is the local Maxwellian, and we linearize the scattering rates (152) with respect to $\varphi$ :

$$
\begin{aligned}
& \Delta M_{i_{1} i_{2} i_{3}}^{w}(\varphi)=\int \Delta \mu_{i_{1} i_{2} i_{3}}^{w} f_{0} \varphi \mathrm{d} v ; \\
& \Delta \mu_{i_{1} i_{2} i_{3}}^{w}=L^{w}\left(\mu_{i_{1} i_{2} i_{3}}\right) .
\end{aligned}
$$

Here $L^{w}$ is the usual linearized collision integral, divided by $f_{0}$. Though $\Delta M^{w}$ are linear in $\varphi$, they are not moments because their microscopic densities, $\Delta \mu^{w}$, are not velocity polynomials for a general case of $w$. 
It is not difficult to derive the corresponding hierarchy of transport equations for variables $\Delta M_{i_{1} i_{2} i_{3}}^{w}$, $\rho, \boldsymbol{u}$, and $P$ (we will further refer to this hierarchy as to the alternative chain): one has to calculate the time derivative of the scattering rates (152) due to the Boltzmann equation, in the linear approximation (153), and to complete the system with the five known balance equations for the hydrodynamic moments (scattering rates of the hydrodynamic moments are equal to zero due to conservation laws). The structure of the alternative chain is quite similar to that of the usual moment transport chain, and for this reason we do not reproduce it here (details of calculations can be found in [181]). One should only keep in mind that the stress tensor and the heat flux vector in the balance equations for $\boldsymbol{u}$ and $P$ are no more independent variables, and they are expressed in terms of $\Delta M_{i_{1} i_{2} i_{3}}^{w}, \rho, \boldsymbol{u}$, and $P$.

Truncating the chain. To truncate the alternative chain (step (ii)), we have, first, to choose a finite set of "essential" scattering rates (153), and, second, to obtain the distribution functions which depend parametrically only on $\rho, \boldsymbol{u}, P$, and on the chosen set of scattering rates. We will restrict our consideration to a single nonhydrodynamic variable, $\sigma_{i j}^{w}$, which is the counterpart of the stress tensor $\sigma_{i j}$. This choice corresponds to the polynomial $m v_{i} v_{j}$ in expressions (152) and (153), and the resulting equations will be alternative to the 10 moment Grad system. ${ }^{9}$ For a spherically symmetric interaction, the expression for $\sigma_{i j}^{w}$ may be written as

$$
\begin{aligned}
\sigma_{i j}^{w}(\varphi) & =\int \Delta \mu_{i j}^{w} f_{0} \varphi \mathrm{d} \boldsymbol{v} \\
\Delta \mu_{i j}^{w} & =L^{w}\left(m v_{i} v_{j}\right)=\frac{P}{\eta_{0}^{w}(T)} S^{w}\left(c^{2}\right)\left\{c_{i} c_{j}-\frac{1}{3} \delta_{i j} c^{2}\right\} .
\end{aligned}
$$

Here $\eta_{0}^{w}(T)$ is the first Sonine polynomial approximation of the Chapman-Enskog viscosity coefficient (VC) [51], and, as usual, $\mathbf{c}=\sqrt{m / 2 k T}(\boldsymbol{v}-\boldsymbol{u})$. The scalar dimensionless function $S^{w}$ depends only on $c^{2}$, and its form depends on the choice of interaction $w$.

Entropy maximization. Next, we find the functions $f^{*}\left(\rho, \boldsymbol{u}, P, \sigma_{i j}^{w}\right)=f_{0}(\rho, \boldsymbol{u}, P)\left(1+\varphi^{*}\left(\rho, \boldsymbol{u}, P, \sigma_{i j}^{w}\right)\right)$ which maximize the Boltzmann entropy $S(f)$ in a neighborhood of $f_{0}$ (the quadratic approximation to the entropy is valid within the accuracy of our consideration), for fixed values of $\sigma_{i j}^{w}$. That is, $\varphi^{*}$ is a solution to the following conditional variational problem:

$$
\begin{aligned}
& \Delta S(\varphi)=-\frac{k_{\mathrm{B}}}{2} \int f_{0} \varphi^{2} \mathrm{~d} \boldsymbol{v} \rightarrow \max , \\
& \text { (i) } \int \Delta \mu_{i j}^{w} f_{0} \varphi \mathrm{d} \boldsymbol{v}=\sigma_{i j}^{w} ; \quad \text { (ii) } \int\left\{1, \boldsymbol{v}, v^{2}\right\} f_{0} \varphi \mathrm{d} \boldsymbol{v}=0 .
\end{aligned}
$$

The second (homogeneous) condition in (155) reflects that a deviation $\varphi$ from the state $f_{0}$ is due only to nonhydrodynamic degrees of freedom, and it is straightforwardly satisfied for $\Delta \mu_{i j}^{w}(154)$.

Notice, that if we turn to the usual moment description, then condition (i) in (155) would fix the stress tensor $\sigma_{i j}$ instead of its scattering counterpart $\sigma_{i j}^{w}$. Then the resulting function $f^{*}\left(\rho, \boldsymbol{u}, P, \sigma_{i j}\right)$ will be exactly the 10 moment Grad approximation. It can be shown that a choice of any finite set of higher moments as the constraint (i) in (155) results in the corresponding Grad approximation.

\footnotetext{
${ }^{9}$ To get the alternative to the 13 moment Grad equations, one should take into account the scattering counterpart of the heat flux, $q_{i}^{w}=m \int v_{i}\left(v^{2} / 2\right) Q^{w}(f) \mathrm{d} \boldsymbol{v}$.
} 
In that sense our method of constructing $f^{*}$ is a direct generalization of the Grad method onto the alternative description.

The Lagrange multipliers method gives straightforwardly the solution to the problem (155). After the alternative chain is closed with the functions $f^{*}\left(\rho, \boldsymbol{u}, P, \sigma_{i j}^{w}\right)$, the step (ii) is completed, and we arrive at a set of equations with respect to the variables $\rho, \boldsymbol{u}, P$, and $\sigma_{i j}^{w}$. Switching to the variable $\zeta_{i j}=n^{-1} \sigma_{i j}^{w}$, we have

$$
\begin{aligned}
& \partial_{t} n+\partial_{i}\left(n u_{i}\right)=0 ; \\
& \rho\left(\partial_{t} u_{k}+u_{i} \partial_{i} u_{k}\right)+\partial_{k} P+\partial_{i}\left\{\frac{\eta_{0}^{w}(T) n}{2 r^{w} P} \zeta_{i k}\right\}=0 ; \\
& \frac{3}{2}\left(\partial_{t} P+u_{i} \partial_{i} P\right)+\frac{5}{2} P \partial_{i} u_{i}+\left\{\frac{\eta_{0}^{w}(T) n}{2 r^{w} P} \zeta_{i k}\right\} \partial_{i} u_{k}=0 ; \\
& \partial_{t} \zeta_{i k}+\partial_{s}\left(u_{s} \zeta_{i k}\right)+\left\{\zeta_{k s} \partial_{s} u_{i}+\zeta_{i s} \partial_{s} u_{k}-\frac{2}{3} \delta_{i k} \zeta_{r s} \partial_{s} u_{r}\right\} \\
& \quad+\left\{\gamma^{w}-\frac{2 \beta^{w}}{r^{w}}\right\} \zeta_{i k} \partial_{s} u_{s}-\frac{P^{2}}{\eta_{0}^{w}(T) n}\left(\partial_{i} u_{k}+\partial_{k} u_{i}-\frac{2}{3} \delta_{i k} \partial_{s} u_{s}\right) \\
& \quad-\frac{\alpha^{w} P}{r^{w} \eta_{0}^{w}(T)} \zeta_{i k}=0 .
\end{aligned}
$$

Here $\partial_{t}=\partial / \partial t, \partial_{i}=\partial / \partial x_{i}$, summation in two repeated indices is assumed, and the coefficients $r^{w}, \beta^{w}$, and $\alpha^{w}$ are defined with the help of the function $S^{w}(154)$ as follows:

$$
\begin{aligned}
r^{w} & =\frac{8}{15 \sqrt{\pi}} \int_{0}^{\infty} \mathrm{e}^{-c^{2}} c^{6}\left(S^{w}\left(c^{2}\right)\right)^{2} \mathrm{~d} c \\
\beta^{w} & =\frac{8}{15 \sqrt{\pi}} \int_{0}^{\infty} \mathrm{e}^{-c^{2}} c^{6} S^{w}\left(c^{2}\right) \frac{\mathrm{d} S^{w}\left(c^{2}\right)}{\mathrm{d}\left(c^{2}\right)} \mathrm{d} c \\
\alpha^{w} & =\frac{8}{15 \sqrt{\pi}} \int_{0}^{\infty} \mathrm{e}^{-c^{2}} c^{6} S^{w}\left(c^{2}\right) R^{w}\left(c^{2}\right) \mathrm{d} c .
\end{aligned}
$$

The function $R^{w}\left(c^{2}\right)$ in the last expression is defined due to the action of the operator $L^{w}$ on the function $S^{w}\left(c^{2}\right)\left(c_{i} c_{j}-\frac{1}{3} \delta_{i j} c^{2}\right)$ :

$$
\frac{P}{\eta_{0}^{w}} R^{w}\left(c^{2}\right)\left(c_{i} c_{j}-\frac{1}{3} \delta_{i j} c^{2}\right)=L^{w}\left(S^{w}\left(c^{2}\right)\left(c_{i} c_{j}-\frac{1}{3} \delta_{i j} c^{2}\right)\right) .
$$

Finally, the parameter $\gamma^{w}$ in (156)-(160) reflects the temperature dependence of the VC:

$$
\gamma^{w}=\frac{2}{3}\left(1-\frac{T}{\eta_{0}^{w}(T)}\left(\frac{\mathrm{d} \eta_{0}^{w}(T)}{\mathrm{d} T}\right)\right) .
$$

The set of 10 equations (156)-(160) is alternative to the 10-moment Grad equations. 
A new determination of molecular dimensions (revisited). The first observation to be made is that for Maxwellian molecules we have: $S^{\mathrm{MM}} \equiv 1$, and $\eta_{0}^{\mathrm{MM}} \propto T$; thus $\gamma^{\mathrm{MM}}=\beta^{\mathrm{MM}}=0, r^{\mathrm{MM}}=\alpha^{\mathrm{MM}}=\frac{1}{2}$, and (156)-(160) becomes the 10-moment Grad system under a simple change of variables $\lambda \zeta_{i j}=\sigma_{i j}$, where $\lambda$ is the proportionality coefficient in the temperature dependence of $\eta_{0}^{\mathrm{MM}}$.

These properties (the function $S^{w}$ is a constant, and the $\mathrm{VC}$ is proportional to $T$ ) are true only for Maxwellian molecules. For all other interactions, the function $S^{w}$ is not identical to one, and the $\mathrm{VC} \eta_{0}^{w}(T)$ is not proportional to $T$. Thus, the shortened alternative description is not equivalent indeed to the Grad moment description. In particular, for hard spheres, the exact expression for the function $S^{\mathrm{HS}}(154)$ reads

$$
S^{\mathrm{HS}}=\frac{5 \sqrt{2}}{16} \int_{0}^{1} \exp \left(-c^{2} t^{2}\right)\left(1-t^{4}\right)\left(c^{2}\left(1-t^{2}\right)+2\right) \mathrm{d} t, \quad \eta_{0}^{\mathrm{HS}} \propto \sqrt{T} .
$$

Thus, $\gamma^{\mathrm{HS}}=\frac{1}{3}$, and $\beta^{\mathrm{HS}} / r^{\mathrm{HS}} \approx 0.07$, and the equation for the function $\zeta_{i k}(160)$ contains a nonlinear term

$$
\theta^{\mathrm{HS}} \zeta_{i k} \partial_{s} u_{s}
$$

where $\theta^{\mathrm{HS}} \approx 0.19$. This term is missing in the Grad 10 moment equation.

Finally, let us evaluate the VC which results from the alternative description (156)-(160). Following Grad's arguments [158], we see that, if the relaxation of $\zeta_{i k}$ is fast compared to the hydrodynamic variables, then the two last terms in the equation for $\zeta_{i k}(156)-(160)$ become dominant, and the equation for $\boldsymbol{u}$ casts into the standard Navier-Stokes form with an effective VC $\eta_{\mathrm{eff}}^{w}$ :

$$
\eta_{\mathrm{eff}}^{w}=\frac{1}{2 \alpha^{w}} \eta_{0}^{w}
$$

For Maxwellian molecules, we easily derive that the coefficient $\alpha^{w}$ in Eq. (164) is equal to $\frac{1}{2}$. Thus, as one expects, the effective VC (164) is equal to the Grad value, which, in turn, is equal to the exact value in the frames of the Chapman-Enskog method for this model.

For all interactions different from the Maxwellian molecules, the VC $\eta_{\mathrm{eff}}^{w}(164)$ is not equal to $\eta_{0}^{w}$. For hard spheres, in particular, a computation of the VC (164) requires information about the function $R^{\mathrm{HS}}$ (161). This is achieved upon a substitution of the function $S^{\mathrm{HS}}$ (162) into Eq. (161). Further, we have to compute the action of the operator $L^{\mathrm{HS}}$ on the function $S^{\mathrm{HS}}\left(c_{i} c_{j}-\frac{1}{3} \delta_{i j} c^{2}\right)$, which is rather complicated. However, the $\mathrm{VC} \eta_{\mathrm{eff}}^{\mathrm{HS}}$ can be relatively easily estimated by using a function $S_{a}^{\mathrm{HS}}=\frac{1}{\sqrt{2}}\left(1+\frac{1}{7} c^{2}\right)$, instead of the function $S^{\mathrm{HS}}$, in Eq. (161). Indeed, the function $S_{a}^{\mathrm{HS}}$ is tangent to the function $S^{\mathrm{HS}}$ at $c^{2}=0$, and is its majorant (see Fig. 3). Substituting $S_{a}^{\mathrm{HS}}$ into Eq. (161), and computing the action of the collision integral, we find the approximation $R_{a}^{\mathrm{HS}}$; thereafter we evaluate the integral $\alpha^{\mathrm{HS}}(160)$, and finally come to the following expression:

$$
\eta_{\mathrm{eff}}^{\mathrm{HS}} \geqslant \frac{75264}{67237} \eta_{0}^{\mathrm{HS}} \approx 1.12 \eta_{0}^{\mathrm{HS}}
$$

Thus, for hard spheres, the description in terms of scattering rates results in the $\mathrm{VC}$ of more than $10 \%$ higher than in the Grad moment description.

A discussion of the results concerns the following two items.

1. Having two not equivalent descriptions which were obtained within one method, we may ask: which is more relevant? A simple test is to compare characteristic times of an approach to hydrodynamic regime. We have $\tau_{\mathrm{G}} \sim \eta_{0}^{\mathrm{HS}} / P$ for 10 -moment description, and $\tau_{a} \sim \eta_{\mathrm{eff}}^{\mathrm{HS}} / P$ for alternative 


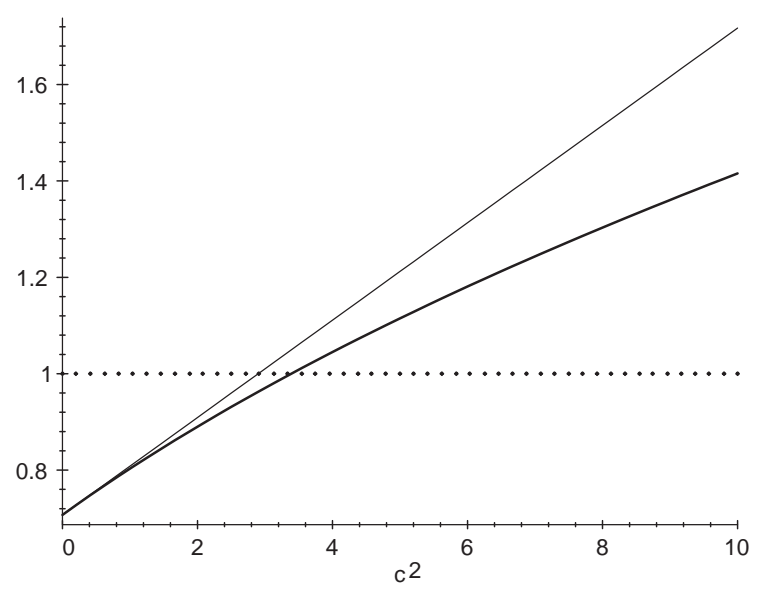

Fig. 3. Approximations for hard spheres: bold line-function $S^{\mathrm{HS}}$, solid line-approximation $S_{a}^{\mathrm{HS}}$, dotted line- Grad moment approximation.

Table 1

Three virial coefficients: experimental $B_{\text {exp }}$, classical $B_{0}$ [182], and reduced $B_{\text {eff }}$ for three gases at $T=500 \mathrm{~K}$

\begin{tabular}{lccc}
\hline & $B_{\exp }$ & $B_{0}$ & $B_{\text {eff }}$ \\
\hline Argon & 8.4 & 60.9 & 50.5 \\
Helium & 10.8 & 21.9 & 18.2 \\
Nitrogen & 168 & 66.5 & 55.2 \\
\hline
\end{tabular}

description. As $\tau_{a}>\tau_{\mathrm{G}}$, we see that scattering rate decay slower than corresponding moment, hence, at least for rigid spheres the alternative description is more relevant. For Maxwellian molecules both the descriptions are, of course, equivalent.

2. The $\mathrm{VC} \eta_{\mathrm{eff}}^{\mathrm{HS}}(165)$ has the same temperature dependence as $\eta_{0}^{\mathrm{HS}}$, and also the same dependence on a scaling parameter (a diameter of the sphere). In the classical book [51] (pp. 228-229), "sizes" of molecules are presented, assuming that a molecule is represented with an equivalent sphere and $\mathrm{VC}$ is estimated as $\eta_{0}^{\mathrm{HS}}$. Since our estimation of $\mathrm{VC}$ differs only by a dimensionless factor from $\eta_{0}^{\mathrm{HS}}$, it is straightforward to conclude that effective sizes of molecules will be reduced by the factor $b$, where

$$
b=\sqrt{\eta_{0}^{\mathrm{HS}} / \eta_{\mathrm{eff}}^{\mathrm{HS}}} \approx 0.94
$$

Further, it is well known that sizes of molecules estimated via viscosity in [51] disagree with the estimation via the virial expansion of the equation of state. In particular, in the book [182, p. 5] the measured second virial coefficient $B_{\text {exp }}$ was compared with the calculated $B_{0}$, in which the diameter of the sphere was taken from the viscosity data. The reduction of the diameter by factor $b$ gives $B_{\text {eff }}=b^{3} B_{0}$. The values $B_{\text {exp }}$ and $B_{0}$ [182] are compared with $B_{\text {eff }}$ in Table 1 for three gases at $T=500 \mathrm{~K}$. The results for argon and helium are better for $B_{\text {eff }}$, while for nitrogen $B_{\text {eff }}$ is worth than $B_{0}$. However, both $B_{0}$ and $B_{\text {eff }}$ are far from the experimental values. 
Hard spheres is, of course, an oversimplified model of interaction, and the comparison presented does not allow for a decision between $\eta_{0}^{\mathrm{HS}}$ and $\eta_{\mathrm{eff}}^{\mathrm{HS}}$. However, this simple example illustrates to what extend the correction to the $\mathrm{VC}$ can affect a comparison with experiment. Indeed, as it is well known, the first-order Sonine polynomial computation for the Lennard-Jones (LJ) potential gives a very good fit of the temperature dependence of the VC for all noble gases [183], subject to a proper choice of the two unknown scaling parameters of the LJ potential. ${ }^{10} \mathrm{We}$ may expect that a dimensionless correction of the VC for the LJ potential might be of the same order as above for rigid spheres. However, the functional character of the temperature dependence will not be affected, and a fit will be obtained subject to a different choice of the molecular parameters of the LJ potential.

There remains, however, a general question how the estimation of the VC (164) responds to the exact value [51,184]. Since the analysis performed above does not immediately appeal to the exact Chapman-Enskog expressions just mentioned, this question remains open for a further work.

\section{Newton method with incomplete linearization}

Let us come back to the invariance equation (78),

$$
\Delta_{y}=\left(1-P_{y}\right) J(F(y))=0 \text {. }
$$

One of the most efficient methods to solve this equation is the Newton method with incomplete linearization. Let us linearize the vector field $J$ around $F(y)$ :

$$
J(F(y)+\delta F(y))=J(F(y))+(D J)_{F(y)} \delta F(y)+\mathrm{o}(\delta F(y)) .
$$

Equation of the Newton method with incomplete linearization makes it possible to determine $\delta F(y)$ :

$$
\begin{aligned}
& P_{y} \delta F(y)=0, \\
& \left(1-P_{y}\right)(D J)_{F(y)} \delta F(y)=\left(1-P_{y}\right) J(F(y)) .
\end{aligned}
$$

The crucial point here is that the same projector $P_{y}$ is used as in Eq. (78), that is, without computing the variation of the projector $\delta P$ (hence, the linearization of Eq. (78) is incomplete). We recall that projector $P_{y}$ depends on the tangent space $T_{y}=\operatorname{Im}(D F)_{y}$. If the thermodynamic projector (132) is used here, then $P_{y}$ depends also on $\langle\mid\rangle_{F(y)}$ and on $g=(D S)_{F(y)}$.

Equations of the Newton method with incomplete linearization (167) are not differential equations in $y$ anymore, they do not contain derivatives of the unknown $\delta F(y)$ with respect to $y$ (which would be the case if the variation of the projector $\delta P$ has been taken into account). The absence of the derivatives in Eq. (167) significantly simplifies its solving. However, even this is not the main advantage of the incomplete linearization. More essential is the fact that iterations of the Newton method with incomplete linearization are expected to converge to slow invariant manifolds, unlike the usual Newton method. This has been demonstrated in [6] in the linear approximation.

In order to illustrate the nature of Eq. (167), let us consider the case of linear manifolds for linear systems. Let a linear evolution equation be given in the finite-dimensional real space: $\dot{\boldsymbol{x}}=\mathbf{A} \boldsymbol{x}$, where

\footnotetext{
${ }^{10}$ A comparison of molecular parameters of the LJ potential, as derived from the viscosity data, to those obtained from independent sources, can be found elsewhere, e.g. in Ref. [51, p. 237].
} 
A is negatively definite symmetric matrix with a simple spectrum. Let us further assume quadratic Lyapunov function, $S(\boldsymbol{x})=\langle\boldsymbol{x}, \boldsymbol{x}\rangle$. The manifolds we consider are lines, $\boldsymbol{l}(y)=y \boldsymbol{e}$, where $\boldsymbol{e}$ is the unit vector, and $y$ is a scalar. The invariance equation for such manifolds reads: $\boldsymbol{e}\langle\boldsymbol{e}, \mathbf{A} \boldsymbol{e}\rangle-\mathbf{A} \boldsymbol{e}=0$, and is simply the eigenvalue problem for the operator A. Solutions to the latter equation are eigenvectors $\boldsymbol{e}_{i}$, corresponding to eigenvalues $\lambda_{i}$.

Assume that we have chosen a line, $\boldsymbol{l}_{0}=y \boldsymbol{e}_{0}$, defined by the unit vector $\boldsymbol{e}_{0}$, and that $\boldsymbol{e}_{0}$ is not an eigenvector of $\mathbf{A}$. We seek another line, $\boldsymbol{l}_{1}=a \boldsymbol{e}_{1}$, where $\boldsymbol{e}_{1}$ is another unit vector, $\boldsymbol{e}_{1}=\boldsymbol{x}_{1} /\left\|\boldsymbol{x}_{1}\right\|$, $\boldsymbol{x}_{1}=\boldsymbol{e}_{0}+\delta \boldsymbol{x}$. The additional condition in (167) reads: $P_{y} \delta F(y)=0$, i.e. $\left\langle\boldsymbol{e}_{0}, \delta \boldsymbol{x}\right\rangle=0$. Then Eq. (167) becomes $\left[1-\boldsymbol{e}_{0}\left\langle\boldsymbol{e}_{0}, \cdot\right\rangle\right] \mathbf{A}\left[\boldsymbol{e}_{0}+\delta \boldsymbol{x}\right]=0$. Subject to the additional condition, the unique solution is as follows: $\boldsymbol{e}_{0}+\delta \boldsymbol{x}=\left\langle\boldsymbol{e}_{0}, \mathbf{A}^{-1} \boldsymbol{e}_{0}\right\rangle^{-1} \mathbf{A}^{-1} \boldsymbol{e}_{0}$. Rewriting the latter expression in the eigenbasis of $\mathbf{A}$, we have: $\boldsymbol{e}_{0}+\delta \boldsymbol{y} \propto \sum_{i} \lambda_{i}^{-1} \boldsymbol{e}_{i}\left\langle\boldsymbol{e}_{i}, \boldsymbol{e}_{0}\right\rangle$. The leading term in this sum corresponds to the eigenvalue with the minimal absolute value. The example indicates that the method (167) seeks the direction of the slowest relaxation. For this reason, the Newton method with incomplete linearization (167) can be recognized as the basis of iterative construction of the manifolds of slow motions.

In an attempt to simplify computations, the question which always can be asked is as follows: to what extend is the choice of the projector essential in Eq. (167)? This question is a valid one, because, if we accept that iterations converge to a relevant slow manifold, and also that the projection on the true invariant manifold is insensible to the choice of the projector, then should one care of the projector on each iteration? In particular, for the moment parameterizations, can one use in Eq. (167) the projector (108)? Experience gained from some of the problems studied by this method indicates that this is possible. However, in order to derive physically meaningful equations of motion along the approximate slow manifolds, one has to use the thermodynamic projector (132). Otherwise we are not guaranteed from violating the dissipation properties of these equations of motion.

\section{Example 3: Nonperturbative correction of Local Maxwellian manifold and derivation of nonlinear hydrodynamics from Boltzmann equation (1D)}

This section is a continuation of Example 1. Here we apply the method of invariant manifold to a particular situation when the initial manifold consists of local Maxwellians (140) (the LM manifold). This manifold and its corrections play the central role in the problem of derivation of hydrodynamics from BE. Hence, any method of approximate investigation of BE should be tested with the LM manifold. Classical methods (the Chapman-Enskog and Hilbert methods) use Taylor-type expansions into powers of a small parameter (the Knudsen number expansion). However, as we have mentioned above, the method of invariant manifold, generally speaking, assumes no small parameters, at least in its formal part where convergency properties are not discussed. We will develop an appropriate technique to consider the invariance equation of the first iteration. This involves ideas of parametrix expansions of the theory of pseudodifferential and Fourier integral operators [193,194]. This approach will make it possible to reject the restriction of using small parameters.

We search for a correction to the LM manifold as

$$
f_{1}(n, \boldsymbol{u}, T)=f_{0}(n, \boldsymbol{u}, T)+\delta f_{1}(n, \boldsymbol{u}, T) .
$$

We will use the Newton method with incomplete linearization for obtaining the correction $\delta f_{1}(n, \boldsymbol{u}, T)$, because we search for a manifold of slow (hydrodynamic) motions. We introduce the 
representation:

$$
\delta f_{1}(n, \boldsymbol{u}, T)=f_{0}(n, \boldsymbol{u}, T) \varphi(n, \boldsymbol{u}, T) .
$$

Positivity and normalization. When searching for a correction, we should be ready to face two problems that are typical for any method of successive approximations in BE theory. Namely, the first of this problems is that the correction

$$
f_{\Omega_{k+1}}=f_{\Omega_{k}}+\delta f_{\Omega_{k+1}}
$$

obtained from the linearized invariance equation of the $(k+1)$ th iteration may be not a nonnegatively defined function and thus it cannot be used directly to define the thermodynamic projector for the $(k+1)$ th approximation. In order to overcome this difficulty, we can treat the procedure as a process of correcting the dual variable $\mu_{f}=D_{f} H(f)$ rather than the process of immediate correcting the distribution functions.

The dual variable $\mu_{f}$ is

$$
\left.\mu_{f}\right|_{f=f(\boldsymbol{x}, \boldsymbol{v})}=\left.D_{f} H(f)\right|_{f=f(\boldsymbol{x}, \boldsymbol{v})}=\left.D_{f} H_{x}(f)\right|_{f=f(\boldsymbol{x}, \boldsymbol{v})}=\ln f(\boldsymbol{v}, \boldsymbol{x}) .
$$

Then, at the $(k+1)$ th iteration, we search for new dual variables $\left.\mu_{f}\right|_{\Omega_{k+1}}$ :

$$
\left.\mu_{f}\right|_{\Omega_{k+1}}=\left.\mu_{f}\right|_{\Omega_{k}}+\left.\delta \mu_{f}\right|_{\Omega_{k+1}} .
$$

Due to the relationship $\mu_{f} \leftrightarrow f$, we have

$$
\left.\delta \mu_{f}\right|_{\Omega_{k+1}}=\varphi_{\Omega_{k+1}}+\mathrm{O}\left(\delta f_{\Omega_{k+1}}^{2}\right), \quad \varphi_{\Omega_{k+1}}=f_{\Omega_{k}}^{-1} \delta f_{\Omega_{k+1}} .
$$

Thus, solving the linear invariance equation of the $k$ th iteration with respect to the unknown function $\delta f_{\Omega_{k+1}}$, we find a correction to the dual variable $\varphi_{\Omega_{k+1}}$ (172), and we derive the corrected distributions $f_{\Omega_{k+1}}$ as

$$
f_{\Omega_{k+1}}=\exp \left(\left.\mu_{f}\right|_{\Omega_{k}}+\varphi_{\Omega_{k+1}}\right)=f_{\Omega_{k}} \exp \left(\varphi_{\Omega_{k+1}}\right) .
$$

Functions (173) are positive, and they satisfy the invariance equation and the additional conditions within the accuracy of $\varphi_{\Omega_{k+1}}$.

However, the second difficulty which might occur is that functions (173) might have no finite integrals (134). In particular, this difficulty can be a result of some approximations used in solving equations. Hence, we have to "regularize" functions (173). A sketch of an approach to make this regularization might be as follows: instead of $f_{\Omega_{k+1}}(173)$, we consider functions

$$
f_{\Omega_{k+1}}^{(\beta)}=f_{\Omega_{k}} \exp \left(\varphi_{\Omega_{k+1}}+\varphi^{\mathrm{reg}}(\beta)\right) .
$$

Here $\varphi^{\text {reg }}(\beta)$ is a function labeled with $\beta \in B$, and $B$ is a linear space. Then we derive $\beta_{*}$ from the condition of coincidence of macroscopic parameters. Further consideration of this procedure [6] is out of frames of this paper.

The two difficulties mentioned here are not specific for the approximate method developed. For example, corrections to the LM distribution in the Chapman-Enskog method [51] and the 13-moment Grad approximation [158] are not nonnegatively defined functions, while the 13-moment quasi-equilibrium approximation [168] has no finite integrals (133) and (134). 
Galilean invariance of invariance equation. In some cases, it is convenient to consider BE vector field in a reference system which moves with the flow velocity. In this reference system, we define the $\mathrm{BE}$ vector field as

$$
\frac{\mathrm{d} f}{\mathrm{~d} t}=J_{u}(f), \quad \frac{\mathrm{d} f}{\mathrm{~d} t}=\frac{\partial f}{\partial t}+u_{\boldsymbol{x}, s}(f) \frac{\partial f}{\partial x_{s}} ; \quad J_{u}(f)=-\left(v_{s}-u_{\boldsymbol{x}, s}(f)\right) \frac{\partial f}{\partial x_{s}}+Q(f, f) .
$$

Here $u_{x, s}(f)$ stands for the sth component of the flow velocity:

$$
u_{x, s}(f)=n_{\boldsymbol{x}}^{-1}(f) \int v_{s} f(\boldsymbol{v}, \boldsymbol{x}) \mathrm{d}^{3} \boldsymbol{v} ; \quad n_{\boldsymbol{x}}(f)=\int f(\boldsymbol{v}, \boldsymbol{x}) \mathrm{d}^{3} \boldsymbol{v} .
$$

In particular, this form of BE vector field is convenient when the initial manifold $\Omega_{0}$ consists of functions $f_{\Omega_{0}}$ which depend explicitly on $\left(\boldsymbol{v}-u_{x}(f)\right)$ (i.e., if functions $f_{\Omega_{0}} \in \Omega_{0}$ do not change under velocity shifts: $\boldsymbol{v} \rightarrow \boldsymbol{v}+c$, where $c$ is a constant vector).

Substituting $J_{u}(f)(175)$ instead of $J(f)$ (135) into all expressions which depend on the BE vector field, we transfer all procedures developed above into the moving reference system. In particular, we obtain the following analog of the invariance equation of the first iteration:

$$
\begin{aligned}
\left(P_{a(\boldsymbol{x})}^{0 *}(\cdot)-1\right) & J_{u, \operatorname{lin}, a(\boldsymbol{x})}^{0}\left(\delta f_{1}(a(\boldsymbol{x}), \boldsymbol{v})\right)+\Delta\left(f_{0}(a(\boldsymbol{x}), \boldsymbol{v})\right)=0 \\
J_{u, \operatorname{lin}, a(\boldsymbol{x})}^{0}(g)= & \left\{n_{\boldsymbol{x}}^{-1}\left(f_{0}(a(\boldsymbol{x}))\right) \int v_{s} g \mathrm{~d}^{3} \boldsymbol{v}\right. \\
& \left.+u_{\boldsymbol{x}, s}\left(f_{0}(a(\boldsymbol{x}))\right) n_{\boldsymbol{x}}^{-1}\left(f_{0}(a(\boldsymbol{x}))\right) \int g \mathrm{~d}^{3} \boldsymbol{v}\right\} \frac{\partial f_{0}(a(\boldsymbol{x}), \boldsymbol{v})}{\partial x_{s}} \\
& -\left(v_{s}-u_{\boldsymbol{x}, s}\left(f_{0}(a(\boldsymbol{x}))\right)\right) \frac{\partial g}{\partial x_{s}}+L_{f_{0}(a(\boldsymbol{x}), \boldsymbol{v})}(g) ; \\
\Delta\left(f_{0}(a(\boldsymbol{x}), \boldsymbol{v})\right) & =\left(P_{a(\boldsymbol{x})}^{*}(\cdot)-1\right) J_{u}\left(f_{0}(a(\boldsymbol{x}), \boldsymbol{v})\right) .
\end{aligned}
$$

Additional conditions do not depend on the vector field, and thus they remain valid for Eq. (177).

The equation of the first iteration. The equation of the first iteration in the form of (172) for the correction $\varphi(n, \boldsymbol{u}, T)$ is

$$
\begin{aligned}
& \left\{P_{f_{0}(n, \boldsymbol{u}, T)}(\cdot)-1\right\}\left\{-\left(v_{s}-u_{s}\right) \frac{\partial f_{0}(n, \boldsymbol{u}, T)}{\partial x_{s}}+f_{0}(n, \boldsymbol{u}, T) L_{f_{0}(n, \boldsymbol{u}, T)}(\varphi)\right. \\
& -\left(v_{s}-u_{s}\right) \frac{\partial\left(f_{0}(n, \boldsymbol{u}, T) \varphi\right)}{\partial x_{s}}-n^{-1}\left(f_{0}(n, \boldsymbol{u}, T)\right)\left(\int v_{s} f_{0}(n, \boldsymbol{u}, T) \varphi \mathrm{d}^{3} \boldsymbol{v}\right. \\
& \left.\left.\quad+u_{s}\left(f_{0}(n, \boldsymbol{u}, T)\right) \int f_{0}(n, \boldsymbol{u}, T) \varphi \mathrm{d}^{3} \boldsymbol{v}\right) \frac{\partial f_{0}(n, \boldsymbol{u}, T)}{\partial x_{s}}\right\}=0 .
\end{aligned}
$$

Here $f_{0}(n, \boldsymbol{u}, T) L_{f_{0}(n, \boldsymbol{u}, T)}(\varphi)$ is the linearized Boltzmann collision integral:

$$
\begin{aligned}
f_{0}(n, \boldsymbol{u}, T) L_{f_{0}(n, \boldsymbol{u}, T)}(\varphi)= & \int w\left(\mathbf{v}^{\prime}, \boldsymbol{v} \mid \boldsymbol{v}, \boldsymbol{v}_{1}\right) f_{0}(n, \boldsymbol{u}, T) \\
& \times\left\{\varphi^{\prime}+\varphi_{1}^{\prime}-\varphi_{1}-\varphi\right\} \mathrm{d}^{3} \mathbf{v}^{\prime} \mathrm{d}^{3} \mathbf{v}_{1}^{\prime} \mathrm{d}^{3} \boldsymbol{v}_{1} .
\end{aligned}
$$

and $w\left(\mathbf{v}^{\prime}, \boldsymbol{v}_{1}^{\prime} \mid \boldsymbol{v}, \boldsymbol{v}_{1}\right)$ is the kernel of the Boltzmann collision integral, standard notations label the velocities before and after collision. 
Additional condition for Eq. (178) has the form

$$
P_{f_{0}(n, \boldsymbol{u}, T)}\left(f_{0}(n, \boldsymbol{u}, T) \varphi\right)=0 \text {. }
$$

In detail notation:

$$
\begin{aligned}
& \int 1 \cdot f_{0}(n, \boldsymbol{u}, T) \varphi \mathrm{d}^{3} \boldsymbol{v}=0, \quad \int v_{i} f_{0}(n, \boldsymbol{u}, T) \varphi \mathrm{d}^{3} \boldsymbol{v}=0, \quad i=1,2,3, \\
& \int \boldsymbol{v}^{2} f_{0}(n, \boldsymbol{u}, T) \varphi \mathrm{d}^{3} \boldsymbol{v}=0 .
\end{aligned}
$$

Eliminating in (178) the terms containing $\int v_{s} f_{0}(n, \boldsymbol{u}, T) \varphi \mathrm{d}^{3} \boldsymbol{v}$ and $\int f_{0}(n, \boldsymbol{u}, T) \varphi \mathrm{d}^{3} \boldsymbol{v}$ with the aid of (181), we obtain the following form of Eq. (178):

$$
\begin{aligned}
& \left\{P_{f_{0}(n, \boldsymbol{u}, T)}(\cdot)-1\right\} \\
& \quad \times\left(-\left(v_{s}-u_{s}\right) \frac{\partial f_{0}(n, \boldsymbol{u}, T)}{\partial x_{s}}+f_{0}(n, \boldsymbol{u}, T) L_{f_{0}(n, \boldsymbol{u}, T)}(\varphi)-\left(v_{s}-u_{s}\right) \frac{\partial\left(f_{0}(n, \boldsymbol{u}, T) \varphi\right)}{\partial x_{s}}\right)=0 .
\end{aligned}
$$

In order to consider the properties of Eq. (182), it is useful to introduce real Hilbert spaces $G_{f_{0}(n, \boldsymbol{u}, T)}$ with scalar products:

$$
(\varphi, \psi)_{f_{0}(n, \boldsymbol{u}, T)}=\int f_{0}(n, \boldsymbol{u}, T) \varphi \psi \mathrm{d}^{3} \boldsymbol{v} .
$$

Each Hilbert space is associated with the corresponding LM distribution $f_{0}(n, \boldsymbol{u}, T)$.

The projector $P_{f_{0}(n, \boldsymbol{u}, T)}(146)$ is associated with a projector $\Pi_{f_{0}(n, \boldsymbol{u}, T)}$ which acts in the space $G_{f_{0}(n, \boldsymbol{u}, T)}$ :

$$
\Pi_{f_{0}(n, \boldsymbol{u}, T)}(\varphi)=f_{0}^{-1}(n, \boldsymbol{u}, T) P_{f_{0}(n, \boldsymbol{u}, T)}\left(f_{0}(n, \boldsymbol{u}, T) \varphi\right) .
$$

It is an orthogonal projector, because

$$
\Pi_{f_{0}(n, \boldsymbol{u}, T)}(\varphi)=\sum_{s=0}^{4} \psi_{f_{0}(n, \boldsymbol{u}, T)}^{(s)}\left(\psi_{f_{0}(n, \boldsymbol{u}, T)}^{(s)}, \varphi\right)_{f_{0}(n, \boldsymbol{u}, T)} .
$$

Here $\psi_{f_{0}(n, \boldsymbol{u}, T)}^{(s)}$ are given by expression (147).

We can rewrite the equation of the first iteration (182) in the form

$$
L_{f_{0}(n, \boldsymbol{u}, T)}(\varphi)+K_{f_{0}(n, \boldsymbol{u}, T)}(\varphi)=D_{f_{0}(n, \boldsymbol{u}, T)} .
$$

Notations used here are:

$$
\begin{aligned}
& D_{f_{0}(n, \boldsymbol{u}, T)}=f_{0}^{-1}(n, \boldsymbol{u}, T) \Delta\left(f_{0}(n, \boldsymbol{u}, T)\right) \\
& K_{f_{0}(n, \boldsymbol{u}, T)}(\varphi)=\left\{\Pi_{f_{0}(n, \boldsymbol{u}, T)}(\cdot)-1\right\} f_{0}^{-1}(n, \boldsymbol{u}, T)\left(v_{s}-u_{s}\right) \frac{\partial\left(f_{0}(n, \boldsymbol{u}, T) \varphi\right)}{\partial x_{s}} .
\end{aligned}
$$

The additional condition for Eq. (186) is

$$
\left(\psi_{f_{0}(n, \boldsymbol{u}, T)}^{(s)}, \varphi\right)_{f_{0}(n, \boldsymbol{u}, T)}=0, \quad s=0, \ldots, 4 .
$$


Now we will list the properties of Eq. (186) for usual collision models [51]:

(a) The linear integral operator $L_{f_{0}(n, \boldsymbol{u}, T)}$ is selfadjoint with respect to the scalar product $(\cdot, \cdot)_{f_{0}(n, \boldsymbol{u}, T)}$, and the quadratic form $\left(\varphi, L_{f_{0}(n, \boldsymbol{u}, T)}(\varphi)\right)$ is negatively defined in $\operatorname{Im}_{f_{0}(n, \boldsymbol{u}, T)}$.

(b) The kernel of $L_{f_{0}(n, \boldsymbol{u}, T)}$ does not depend on $f_{0}(n, \boldsymbol{u}, T)$, and it is the linear envelope of the polynomials $\psi_{0}=1, \psi_{i}=v_{i}, i=1,2,3$, and $\psi_{4}=v^{2}$.

(c) The RHS $D_{f_{0}(n, \boldsymbol{u}, T)}$ is orthogonal to $\operatorname{ker} L_{f_{0}(n, \boldsymbol{u}, T)}$ in the sense of the scalar product $(\cdot, \cdot)_{f_{0}(n, \boldsymbol{u}, T)}$.

(d) The projecting operator $\Pi_{f_{0}(n, \boldsymbol{u}, T)}$ is the selfadjoint projector onto $\operatorname{ker} L_{f_{0}(n, \boldsymbol{u}, T)}$ :

$$
\Pi_{f_{0}(n, \boldsymbol{u}, T)}(\varphi) \in \operatorname{ker} L_{f_{0}(n, \boldsymbol{u}, T)} .
$$

Projector $\Pi_{f_{0}(n, \boldsymbol{u}, T)}$ projects orthogonally.

(e) The image of the operator $K_{f_{0}(n, \boldsymbol{u}, T)}$ is orthogonal to $\operatorname{ker} L_{f_{0}(n, \boldsymbol{u}, T)}$.

(f) Additional condition (188) requires the solution of Eq. (186) to be orthogonal to $\operatorname{ker} L_{f_{0}(n, \boldsymbol{u}, T)}$.

These properties result in the necessity condition for solving Eq. (186) with the additional constraint (188). This means the following: Eq. (186), provided with constraint (188), satisfies the necessary condition for to have an unique solution in $\operatorname{Im} L_{f_{0}(n, \boldsymbol{u}, T)}$.

Remark. Because of the differential part of the operator $K_{f_{0}(n, \boldsymbol{u}, T)}$, we are not able to apply the Fredholm alternative to obtain the necessary and sufficient conditions for solvability of Eq. (188). Thus, the condition mentioned here is, rigorously speaking, only the necessity condition. Nevertheless, we will still develop a formal procedure for solving Eq. (186).

To this end, we paid no attention to the dependency of all functions, spaces, operators, etc, on $\mathbf{x}$. It is useful to rewrite once again Eq. (186) in order to separate the local in $x$ operators from those differential. Furthermore, we shall replace the subscript $f_{0}(n, \boldsymbol{u}, T)$ with the subscript $\boldsymbol{x}$ in all expressions. We represent (186) as

$$
\begin{aligned}
& A_{\mathrm{loc}}(\boldsymbol{x}, \boldsymbol{v}) \varphi-A_{\mathrm{diff}}\left(\boldsymbol{x}, \frac{\partial}{\partial \boldsymbol{x}}, \boldsymbol{v}\right) \varphi=-D(\boldsymbol{x}, \boldsymbol{v}) ; \\
& A_{\mathrm{loc}}(\boldsymbol{x}, \boldsymbol{v}) \varphi=-\left\{L_{\boldsymbol{x}}(\boldsymbol{v}) \varphi+\left(\Pi_{\boldsymbol{x}}(\boldsymbol{v})-1\right) r_{\boldsymbol{x}} \varphi\right\} ; \\
& A_{\mathrm{diff}}\left(\boldsymbol{x}, \frac{\partial}{\partial \boldsymbol{x}}, \boldsymbol{v}\right) \varphi=\left(\Pi_{\boldsymbol{x}}(\cdot)-1\right)\left(\left(v_{s}-u_{s}\right) \frac{\partial}{\partial x_{s}} \varphi\right) ; \\
& \Pi_{\boldsymbol{x}}(\boldsymbol{v}) g=\sum_{s=0}^{4} \psi_{\boldsymbol{x}}^{(s)}\left(\psi_{\boldsymbol{x}}^{(s)}, g\right) ; \\
& \psi_{\boldsymbol{x}}^{(0)}=n^{-1 / 2}, \quad \psi_{\boldsymbol{x}}^{(s)}=(2 / n)^{1 / 2} c_{s}(\boldsymbol{x}, \boldsymbol{v}), \quad s=1,2,3, \\
& \psi_{\boldsymbol{x}}^{(4)}=(2 / 3 n)^{1 / 2}\left(c^{2}(\boldsymbol{x}, \boldsymbol{v})-3 / 2\right) ; \quad c_{i}(\boldsymbol{x}, \boldsymbol{v})=\left(m / 2 k_{\mathrm{B}} T(\boldsymbol{x})\right)^{1 / 2}\left(v_{i}-u_{i}(\boldsymbol{x})\right) \\
& r_{\boldsymbol{x}}=\left(v_{s}-u_{s}\right)\left(\frac{\partial \ln n}{\partial x_{s}}+\frac{m}{k_{\mathrm{B}} T}\left(v_{i}-u_{i}\right) \frac{\partial u_{i}}{\partial x_{s}}+\left(\frac{m(\boldsymbol{v}-\boldsymbol{u})^{2}}{2 k_{\mathrm{B}} T}-\frac{3}{2}\right) \frac{\partial \ln T}{\partial x_{s}}\right) ;
\end{aligned}
$$




$$
\begin{aligned}
D(\boldsymbol{x}, \boldsymbol{v})= & \left\{\left(\frac{m(\boldsymbol{v}-\boldsymbol{u})^{2}}{2 k_{\mathrm{B}} T}-\frac{5}{2}\right)\left(v_{i}-u_{i}\right) \frac{\partial \ln T}{\partial x_{i}}\right. \\
& \left.+\frac{m}{k_{\mathrm{B}} T}\left(\left(v_{i}-u_{i}\right)\left(v_{s}-u_{s}\right)-\frac{1}{3} \delta_{i s}(\boldsymbol{v}-\boldsymbol{u})^{2}\right) \frac{\partial u_{s}}{\partial x_{i}}\right\} .
\end{aligned}
$$

Here we have omitted the dependence on $\boldsymbol{x}$ in the functions $n(\boldsymbol{x}), u_{i}(\boldsymbol{x})$, and $T(\boldsymbol{x})$. Further, if no discrepancy might occur, we will always assume this dependence, and we will not indicate it explicitly.

The additional condition for this equation is

$$
\Pi_{x}(\varphi)=0 .
$$

Eq. (190) is linear in $\varphi$. However, the main difficulty in solving this equation is caused by the differential in $\boldsymbol{x}$ operator $A_{\text {diff }}$ which does not commutate with the local in $\boldsymbol{x}$ operator $A_{\text {loc }}$.

Parametrix expansion. In this subsection we introduce a procedure to construct approximate solutions of Eq. (189). This procedure involves an expansion similar to the parametrix expansion in the theory of pseudo-differential (PDO) and Fourier integral operators (FIO).

Considering $\varphi \in \operatorname{Im} L_{x}$, we write a formal solution of Eq. (190) as

$$
\varphi(\boldsymbol{x}, \boldsymbol{v})=\left(A_{\mathrm{loc}}(\boldsymbol{x}, \boldsymbol{v})-A_{\mathrm{diff}}\left(\boldsymbol{x}, \frac{\partial}{\partial \boldsymbol{x}}, \boldsymbol{v}\right)\right)^{-1}(-D(\boldsymbol{x}, \boldsymbol{v})) .
$$

It is useful to extract the differential operator $\partial / \partial \boldsymbol{x}$ from the operator $A_{\text {diff }}(\boldsymbol{x}, \partial / \partial \boldsymbol{x}, \boldsymbol{v})$ :

$$
\varphi(\boldsymbol{x}, \boldsymbol{v})=\left(1-B_{s}(\boldsymbol{x}, \boldsymbol{v}) \frac{\partial}{\partial x_{s}}\right)^{-1} \varphi_{\mathrm{loc}}(\boldsymbol{x}, \boldsymbol{v}) .
$$

Notations used here are:

$$
\begin{aligned}
\varphi_{\mathrm{loc}}(\boldsymbol{x}, \boldsymbol{v}) & =A_{\mathrm{loc}}^{-1}(\boldsymbol{x}, \boldsymbol{v})(-D(\boldsymbol{x}, \boldsymbol{v})) \\
& =\left[-L_{\boldsymbol{x}}(\boldsymbol{v})-\left(\Pi_{x}(\boldsymbol{v})-1\right) r_{x}\right]^{-1}(-D(\boldsymbol{x}, \boldsymbol{v})) \\
B_{s}(\boldsymbol{x}, \boldsymbol{v}) & =A_{\mathrm{loc}}^{-1}(\boldsymbol{x}, \boldsymbol{v})\left(\Pi_{x}(\boldsymbol{v})-1\right)\left(v_{s}-u_{s}\right) \\
& =\left[-L_{x}(\boldsymbol{v})-\left(\Pi_{x}(\boldsymbol{v})-1\right) r_{x}\right]^{-1}\left(\Pi_{x}(\boldsymbol{v})-1\right)\left(v_{s}-u_{s}\right) .
\end{aligned}
$$

We will now discuss in more details the character of expressions in (194).

For every $\boldsymbol{x}$, the function $\varphi_{\text {loc }}(\boldsymbol{x}, \boldsymbol{v})$, considered as a function of $\boldsymbol{v}$, is an element of the Hilbert space $G_{\boldsymbol{x}}$. It gives a solution to the integral equation:

$$
-L_{x}(\boldsymbol{v}) \varphi_{\text {loc }}-\left(\Pi_{x}(\boldsymbol{v})-1\right)\left(r_{x} \varphi_{\text {loc }}\right)=(-D(\boldsymbol{x}, \boldsymbol{v}))
$$

This latter linear integral equation has an unique solution in $\operatorname{Im} L_{\boldsymbol{x}}(\boldsymbol{v})$. Indeed,

$$
\begin{aligned}
\operatorname{ker} A_{\mathrm{loc}}^{+}(\boldsymbol{x}, \boldsymbol{v}) & =\operatorname{ker}\left(L_{x}(\boldsymbol{v})+\left(\Pi_{x}(\boldsymbol{v})-1\right) r_{x}\right)^{+} \\
& =\operatorname{ker}\left(L_{x}(\boldsymbol{v})\right)^{+} \cap \operatorname{ker}\left(\left(\Pi_{x}(\boldsymbol{v})-1\right) r_{x}\right)^{+} \\
& =\operatorname{ker}\left(L_{x}(\boldsymbol{v})\right)^{+} \cap \operatorname{ker}\left(r_{x}\left(\Pi_{x}(\boldsymbol{v})-1\right)\right) \quad \text { and } \quad G_{x} \cap \Pi_{x}(\boldsymbol{v}) G_{x}=\{0\} .
\end{aligned}
$$

Thus, the existence of the unique solution of Eq. (195) follows from the Fredholm alternative. 
Let us consider the operator $R(\boldsymbol{x}, \partial / \partial \boldsymbol{x}, \boldsymbol{v})$ :

$$
R\left(\boldsymbol{x}, \frac{\partial}{\partial \boldsymbol{x}}, \boldsymbol{v}\right)=\left(1-B_{S}(\boldsymbol{x}, \boldsymbol{v}) \frac{\partial}{\partial x_{s}}\right)^{-1} .
$$

One can represent it as a formal series:

$$
R\left(\boldsymbol{x}, \frac{\partial}{\partial \boldsymbol{x}}, \boldsymbol{v}\right)=\sum_{m=0}^{\infty}\left[B_{s}(\boldsymbol{x}, \boldsymbol{v}) \frac{\partial}{\partial x_{s}}\right]^{m} .
$$

Here

$$
\left[B_{s}(\boldsymbol{x}, \boldsymbol{v}) \frac{\partial}{\partial x_{s}}\right]^{m}=B_{s_{1}}(\boldsymbol{x}, \boldsymbol{v}) \frac{\partial}{\partial x_{s_{1}}} \cdots B_{s_{m}}(\boldsymbol{x}, \boldsymbol{v}) \frac{\partial}{\partial x_{s_{m}}} .
$$

Every term of type (199) can be represented as a finite sum of operators which are superpositions of the following two operations: of the integral in $\boldsymbol{v}$ operations with kernels depending on $\boldsymbol{x}$, and of differential in $\boldsymbol{x}$ operations.

Our goal is to obtain an explicit representation of the operator $R(\boldsymbol{x}, \partial / \partial \boldsymbol{x}, \boldsymbol{v})(197)$ as an integral operator. If the operator $B_{s}(\boldsymbol{x}, \boldsymbol{v})$ would not depend on $\boldsymbol{x}$ (i.e., if no dependence on spatial variables would occur in kernels of integral operators, in $\left.B_{s}(\boldsymbol{x}, \boldsymbol{v})\right)$, then we could reach our goal via usual Fourier transformation. However, operators $B_{s}(\boldsymbol{x}, \boldsymbol{v})$ and $\partial / \partial x_{k}$ do not commutate, and thus this elementary approach does not work. We will develop a method to obtain the required explicit representation using the ideas of PDO and IOF technique.

We start with representation (198). Our strategy is to transform every summand (199) in order to place integral in $\boldsymbol{v}$ operators $B_{s}(\boldsymbol{x}, \boldsymbol{v})$ left to differential operators $\partial / \partial x_{k}$. The transposition of every pair $\partial / \partial x_{k} B_{s}(\boldsymbol{x}, \boldsymbol{v})$ yields an elementary transform:

$$
\frac{\partial}{\partial x_{k}} B_{s}(\boldsymbol{x}, \boldsymbol{v}) \rightarrow B_{s}(\boldsymbol{x}, \boldsymbol{v}) \frac{\partial}{\partial x_{k}}-\left[B_{s}(\boldsymbol{x}, \boldsymbol{v}), \frac{\partial}{\partial x_{k}}\right] \text {. }
$$

Here $[M, N]=M N-N M$ denotes the commutator of operators $M$ and $N$. We can represent (199) as

$$
\left[B_{s}(\boldsymbol{x}, \boldsymbol{v}) \frac{\partial}{\partial x_{s}}\right]^{m}=B_{s_{1}}(\boldsymbol{x}, \boldsymbol{v}) \cdots B_{s_{m}}(\boldsymbol{x}, \boldsymbol{v}) \frac{\partial}{\partial x_{s_{1}}} \cdots \frac{\partial}{\partial x_{s_{m}}}+\mathrm{O}\left(\left[B_{s_{i}}(\boldsymbol{x}, \boldsymbol{v}), \frac{\partial}{\partial x_{s_{k}}}\right]\right) \text {. }
$$

Here $\mathrm{O}\left(\left[B_{s_{i}}(\boldsymbol{x}, \boldsymbol{v}), \partial / \partial x_{s_{k}}\right]\right)$ denotes the terms which contain one or more pairs of brackets $[\cdot, \cdot]$. The first term in (201) contains none of these brackets. We can continue this process of selection and extract the first-order in the number of pairs of brackets terms, the second-order terms, etc. Thus, we arrive at the expansion into powers of commutator of expressions (199).

In this paper we will consider explicitly the zeroth-order term of this commutator expansion. Neglecting all terms with brackets in (201), we write

$$
\left[B_{s}(\boldsymbol{x}, \boldsymbol{v}) \frac{\partial}{\partial x_{s}}\right]_{0}^{m}=B_{s_{1}}(\boldsymbol{x}, \boldsymbol{v}) \cdots B_{s_{m}}(\boldsymbol{x}, \boldsymbol{v}) \frac{\partial}{\partial x_{s_{1}}} \cdots \frac{\partial}{\partial x_{s_{m}}} .
$$

Here the subscript zero indicates the zeroth order with respect to the number of brackets. 
We now substitute expressions $\left[B_{s}(\boldsymbol{x}, \boldsymbol{v}) \partial / \partial x_{s}\right]_{0}^{m}(202)$ instead of expressions $\left[B_{s}(\boldsymbol{x}, \boldsymbol{v}) \partial / \partial x_{s}\right]^{m}(199)$ into the series (198):

$$
R_{0}\left(\boldsymbol{x}, \frac{\partial}{\partial x}, \boldsymbol{v}\right)=\sum_{m=0}^{\infty}\left[B_{s}(\boldsymbol{x}, \boldsymbol{v}) \frac{\partial}{\partial x_{s}}\right]_{0}^{m} .
$$

The action of every summand (202) might be defined via the Fourier transform with respect to spatial variables.

Denote as $F$ the direct Fourier transform of a function $g(\boldsymbol{x}, \boldsymbol{v})$ :

$$
F g(\boldsymbol{x}, \boldsymbol{v}) \equiv \hat{g}(\boldsymbol{k}, \boldsymbol{v})=\int g(\boldsymbol{x}, \boldsymbol{v}) \exp \left(-\mathrm{i} k_{s} x_{s}\right) \mathrm{d}^{p} \boldsymbol{x} .
$$

Here $p$ is the spatial dimension. Then the inverse Fourier transform is

$$
g(x, \boldsymbol{v}) \equiv F^{-1} \hat{g}(\boldsymbol{k}, \boldsymbol{v})=(2 \pi)^{-p} \int \hat{g}(\boldsymbol{k}, \boldsymbol{v}) \exp \left(\mathrm{i} k_{s} x_{s}\right) \mathrm{d}^{p} \boldsymbol{k} .
$$

The action of operator (202) on a function $g(\boldsymbol{x}, \boldsymbol{v})$ is defined as

$$
\begin{aligned}
& {\left[B_{s}(\boldsymbol{x}, \boldsymbol{v}) \frac{\partial}{\partial x_{s}}\right]_{0}^{m} g(\boldsymbol{x}, \boldsymbol{v})} \\
& \quad=\left(B_{s_{1}}(\boldsymbol{x}, \boldsymbol{v}) \cdots B_{s_{m}}(\boldsymbol{x}, \boldsymbol{v}) \frac{\partial}{\partial x_{s_{1}}} \cdots \frac{\partial}{\partial x_{s_{m}}}\right)(2 \pi)^{-p} \int \hat{g}(\boldsymbol{k}, \boldsymbol{v}) \mathrm{e}^{\mathrm{i} k_{s} x_{s}} \mathrm{~d}^{p} \boldsymbol{k} \\
& \quad=(2 \pi)^{-p} \int \exp \left(\mathrm{i} k_{s} x_{s}\right)\left[\mathrm{i} k_{l} B_{l}(\boldsymbol{x}, \boldsymbol{v})\right]^{m} \hat{g}(\boldsymbol{k}, \boldsymbol{v}) \mathrm{d}^{p} \boldsymbol{k} .
\end{aligned}
$$

The account of (206) in formula (203) yields the following definition of the operator $R_{0}$ :

$$
R_{0} g(\boldsymbol{x}, \boldsymbol{v})=(2 \pi)^{-p} \int \mathrm{e}^{\mathrm{i} k_{s} x_{s}}\left(1-\mathrm{i} k_{l} B_{l}(\boldsymbol{x}, \boldsymbol{v})\right)^{-1} \hat{g}(\boldsymbol{k}, \boldsymbol{v}) \mathrm{d}^{p} \boldsymbol{k} .
$$

This is the Fourier integral operator (note that the kernel of this integral operator depends on $\boldsymbol{k}$ and on $\boldsymbol{x}$ ). The commutator expansion introduced above is a version of the parametrix expansion $[193,194]$, while expression (207) is the leading term of this expansion. The kernel $\left(1-\mathrm{i} k_{l} B_{l}(\boldsymbol{x}, \boldsymbol{v})\right)^{-1}$ is called the main symbol of the parametrix.

The account of (207) in formula (193) yields the zeroth-order term of parametrix expansion $\varphi_{0}(\boldsymbol{x}, \boldsymbol{v})$ :

$$
\varphi_{0}(\boldsymbol{x}, \boldsymbol{v})=F^{-1}\left(1-\mathrm{i} k_{l} B_{l}(\boldsymbol{x}, \boldsymbol{v})\right)^{-1} F \varphi_{\mathrm{loc}} .
$$

In detail notation:

$$
\begin{aligned}
\varphi_{0}(\boldsymbol{x}, \boldsymbol{v})= & (2 \pi)^{-p} \iint \exp \left(\mathrm{i} k_{s}\left(x_{s}-y_{s}\right)\right) \\
& \times\left(1-\mathrm{i} k_{s}\left[-L_{\boldsymbol{x}}(\boldsymbol{v})-\left(\Pi_{\boldsymbol{x}}(\boldsymbol{v})-1\right) r_{\boldsymbol{x}}\right]^{-1}\left(\Pi_{\boldsymbol{x}}(\boldsymbol{v})-1\right)\left(v_{s}-u_{s}(\boldsymbol{x})\right)\right)^{-1} \\
& \times\left[-L_{\boldsymbol{y}}(\boldsymbol{v})-\left(\Pi_{\boldsymbol{y}}(\boldsymbol{v})-1\right) r_{\boldsymbol{y}}\right]^{-1}(-D(\boldsymbol{y}, \boldsymbol{v})) \mathrm{d}^{p} \boldsymbol{y} \mathrm{d}^{p} \boldsymbol{k} .
\end{aligned}
$$

We now will list the steps to calculate the function $\varphi_{0}(\boldsymbol{x}, \boldsymbol{v})(209)$. 
Step 1: Solve the linear integral equation

$$
\left[-L_{x}(\boldsymbol{v})-\left(\Pi_{x}(\boldsymbol{v})-1\right) r_{x}\right] \varphi_{\mathrm{loc}}(\boldsymbol{x}, \boldsymbol{v})=-D(\boldsymbol{x}, \boldsymbol{v}) .
$$

and obtain the function $\varphi_{\text {loc }}(\boldsymbol{x}, \boldsymbol{v})$.

Step 2: Calculate the Fourier transform $\hat{\varphi}_{\text {loc }}(\boldsymbol{k}, \boldsymbol{v})$ :

$$
\hat{\varphi}_{\text {loc }}(\boldsymbol{k}, \boldsymbol{v})=\int \varphi_{\text {loc }}(\boldsymbol{y}, \boldsymbol{v}) \exp \left(-\mathrm{i} k_{s} y_{s}\right) \mathrm{d}^{p} \boldsymbol{y} .
$$

Step 3: Solve the linear integral equation

$$
\begin{aligned}
& {\left[-L_{x}(\boldsymbol{v})-\left(\Pi_{x}(\boldsymbol{v})-1\right)\left(r_{x}+\mathrm{i} k_{s}\left(v_{s}-u_{s}(\boldsymbol{x})\right)\right)\right] \hat{\varphi}_{0}(\boldsymbol{x}, \boldsymbol{k}, \boldsymbol{v})=-\hat{D}(\boldsymbol{x}, \boldsymbol{k}, \boldsymbol{v}) ;} \\
& -\hat{D}(\boldsymbol{x}, \boldsymbol{k}, \boldsymbol{v})=\left[-L_{\boldsymbol{x}}(\boldsymbol{v})-\left(\Pi_{\boldsymbol{x}}(\boldsymbol{v})-1\right) r_{x}\right] \hat{\varphi}_{\mathrm{loc}}(\boldsymbol{k}, \boldsymbol{v}) .
\end{aligned}
$$

and obtain the function $\hat{\varphi}_{0}(\boldsymbol{x}, \boldsymbol{k}, \boldsymbol{v})$.

Step 4: Calculate the inverse Fourier transform $\varphi_{0}(\boldsymbol{x}, \boldsymbol{v})$ :

$$
\varphi_{0}(\boldsymbol{x}, \boldsymbol{v})=(2 \pi)^{-p} \int \hat{\varphi}_{0}(\boldsymbol{x}, \boldsymbol{k}, \boldsymbol{v}) \exp \left(\mathrm{i} k_{s} x_{s}\right) \mathrm{d}^{p} \boldsymbol{k} .
$$

Completing these four steps, we obtain an explicit expression for the zeroth-order term of parametrix expansion $\varphi_{0}(\boldsymbol{x}, \boldsymbol{v})(208)$.

As we have already mentioned above, Eq. (210) of Step 1 has a unique solution in $\operatorname{Im} L_{x}(\boldsymbol{v})$. Eq. (212) of Step 3 has the same property. Indeed, for every $\boldsymbol{k}$, the right hand side $-\hat{D}(\boldsymbol{x}, \boldsymbol{k}, \boldsymbol{v})$ is orthogonal to $\operatorname{Im} \Pi_{x}(\boldsymbol{v})$, and thus the existence and the uniqueness of formal solution $\hat{\varphi}_{0}(\boldsymbol{x}, \boldsymbol{k}, \boldsymbol{v})$ follows again from the Fredholm alternative.

Thus, in Step 3, we obtain the unique solution $\hat{\varphi}_{0}(\boldsymbol{x}, \boldsymbol{k}, \boldsymbol{v})$. For every $\boldsymbol{k}$, this is a function which belongs to $\operatorname{Im} L_{x}(\boldsymbol{v})$. Accounting that $f_{0}(\boldsymbol{x}, \boldsymbol{v})=f_{0}(n(\boldsymbol{x}), \boldsymbol{u}(\boldsymbol{x}), T(\boldsymbol{x}), \boldsymbol{v})$ exposes no explicit dependency on $\boldsymbol{x}$, we see that the inverse Fourier transform of Step 4 gives $\varphi_{0}(\boldsymbol{x}, \boldsymbol{v}) \in \operatorname{Im} L_{\boldsymbol{x}}(\boldsymbol{v})$.

Eqs. (210)-(213) provide us with the scheme of constructing the zeroth-order term of parametrix expansion. Finishing this section, we will outline briefly the way to calculate the first-order term of this expansion.

Consider a formal operator $R=(1-A B)^{-1}$. Operator $R$ is defined by a formal series:

$$
R=\sum_{m=0}^{\infty}(A B)^{m} .
$$

In every term of this series, we want to place operators $A$ left to operators $B$. In order to do this, we have to commutate $B$ with $A$ from left to right. The commutation of every pair $B A$ yields the elementary transform $B A \rightarrow A B-[A, B]$ where $[A, B]=A B-B A$. Extracting the terms with no commutators $[A, B]$ and with a single commutator $[A, B]$, we arrive at the following representation:

$$
R=R_{0}+R_{1}+\text { (terms with more than two brackets) . }
$$

Here

$$
\begin{aligned}
& R_{0}=\sum_{m=0}^{\infty} A^{m} B^{m} \\
& R_{1}=-\sum_{m=2}^{\infty} \sum_{i=2}^{\infty} \mathrm{i} A^{m-i}[A, B] A^{i-1} B^{i-1} B^{m-i} .
\end{aligned}
$$


Operator $R_{0}$ (216) is the zeroth-order term of parametrix expansion derived above. Operator $R_{1}$ (the first-order term of parametrix expansion) can be represented as follows:

$$
R_{1}=-\sum_{m=1}^{\infty} m A^{m}[A, B]\left(\sum_{i=0}^{\infty} A^{i} B^{i}\right) B^{m}=-\sum_{m=1}^{\infty} m A^{m} C B^{m}, \quad C=[A, B] R_{0} .
$$

This expression can be considered as an ansatz for the formal series (214), and it gives the most convenient way to calculate $R_{1}$. Its structure is similar to that of $R_{0}$. Continuing in this manner, we can derive the second-order term $R_{2}$, etc. We will not discuss these questions in this paper.

In the next subsection we will consider in more detail the first-order term of parametrix expansion.

Finite-dimensional approximations to integral equations. Dealing further only with the zeroth-order term of parametrix expansion (209), we have to solve two linear integral equations, (210) and (212). These equations satisfy the Fredholm alternative, and thus they have unique solutions. The problem we face here is exactly of the same level of complexity as that of the Chapman-Enskog method [51]. The usual approach is to replace integral operators with some appropriate finite-dimensional operators.

First we will recall standard objectives of finite-dimensional approximations, considering Eq. (210). Let $p_{i}(\boldsymbol{x}, \boldsymbol{v})$, where $i=1,2, \ldots$, be a basis in $\operatorname{Im} L_{x}(\boldsymbol{v})$. Every function $\varphi(\boldsymbol{x}, \boldsymbol{v}) \in \operatorname{Im} L_{x}(\boldsymbol{v})$ might be represented in this basis as

$$
\varphi(\boldsymbol{x}, \boldsymbol{v})=\sum_{i=1}^{\infty} a_{i}(\boldsymbol{x}) p_{i}(\boldsymbol{x}, \boldsymbol{v}) ; \quad a_{i}(\boldsymbol{x})=\left(\varphi(\boldsymbol{x}, \boldsymbol{v}), p_{i}(\boldsymbol{x}, \boldsymbol{v})\right)_{\boldsymbol{x}} .
$$

Eq. (210) is equivalent to an infinite set of linear algebraic equations with respect to unknowns $a_{i}(\boldsymbol{x})$ :

$$
\sum_{i=1}^{\infty} m_{k i}(\boldsymbol{x}) a_{i}(\boldsymbol{x})=d_{k}(\boldsymbol{x}), \quad k=1,2, \ldots .
$$

Here

$$
\begin{aligned}
& m_{k i}(\boldsymbol{x})=\left(p_{k}(\boldsymbol{x}, \boldsymbol{v}), A_{\mathrm{loc}}(\boldsymbol{x}, \boldsymbol{v}) p_{i}(\boldsymbol{x}, \boldsymbol{v})\right)_{\boldsymbol{x}} ; \\
& d_{k}(\boldsymbol{x})=-\left(p_{k}(\boldsymbol{x}, \boldsymbol{v}), D(\boldsymbol{x}, \boldsymbol{v})\right)_{\boldsymbol{x}} .
\end{aligned}
$$

For a finite-dimensional approximation of Eq. (220) we use a projection onto a finite number of basis elements $p_{i}(\boldsymbol{x}, \boldsymbol{v}), i=i_{1}, \ldots, i_{n}$. Then, instead of (219), we search for the function $\varphi_{\text {fin }}$ :

$$
\varphi_{\text {fin }}(\boldsymbol{x}, \boldsymbol{v})=\sum_{s=1}^{n} a_{i_{s}}(\boldsymbol{x}) p_{i_{s}}(\boldsymbol{x}, \boldsymbol{v}) .
$$

Infinite set of Eqs. (220) is replaced with a finite set of linear algebraic equations with respect to $a_{i_{s}}(\boldsymbol{x})$, where $s=1, \ldots, n$ :

$$
\sum_{l=1}^{n} m_{i_{s i} l_{l}}(\boldsymbol{x}) a_{i_{l}}(\boldsymbol{x})=d_{i_{s}}(\boldsymbol{x}), \quad s=1, \ldots, n .
$$

There are no a priori restrictions upon the choice of the basis, as well as upon the choice of its finite-dimensional approximations. In this paper we use the standard basis of unreducible Hermite 
tensors (see, for example, [87,158]). The simplest appropriate version of a finite-dimensional approximation occurs if the finite set of Hermite tensors is chosen as:

$$
\begin{aligned}
& p_{k}(\boldsymbol{x}, \boldsymbol{v})=c_{k}(\boldsymbol{x}, \boldsymbol{v})\left(c^{2}(\boldsymbol{x}, \boldsymbol{v})-(5 / 2)\right), \quad k=1,2,3 \\
& p_{i j}(\boldsymbol{x}, \boldsymbol{v})=c_{i}(\boldsymbol{x}, \boldsymbol{v}) c_{j}(\boldsymbol{x}, \boldsymbol{v})-\frac{1}{3} \delta_{i j} c^{2}(\boldsymbol{x}, \boldsymbol{v}), \quad i, j=1,2,3 \\
& c_{i}(\boldsymbol{x}, \boldsymbol{v})=\boldsymbol{v}_{T}^{-1}(\boldsymbol{x})\left(v_{i}-u_{i}(\boldsymbol{x})\right), \quad v_{T}(\boldsymbol{x})=\left(2 k_{\mathrm{B}} T(\boldsymbol{x}) / m\right)^{1 / 2} .
\end{aligned}
$$

It is important to stress here that "good" properties of orthogonality of Hermite tensors, as well as of other similar polynomial systems in BE theory, have the local in $\boldsymbol{x}$ character, i.e. when these functions are treated as polynomials in $c(\boldsymbol{x}, \boldsymbol{v})$ rather than polynomials in $\boldsymbol{v}$. For example, functions $p_{k}(\boldsymbol{x}, \boldsymbol{v})$ and $p_{i j}(\boldsymbol{x}, \boldsymbol{v})(224)$ are orthogonal in the sense of the scalar product $(\cdot, \cdot)_{\boldsymbol{x}}$ :

$$
\left(p_{k}(\boldsymbol{x}, \boldsymbol{v}), p_{i j}(\boldsymbol{x}, \boldsymbol{v})\right)_{\boldsymbol{x}} \propto \int \mathrm{e}^{-c^{2}(\boldsymbol{x}, \boldsymbol{v})} p_{k}(\boldsymbol{x}, \boldsymbol{v}) p_{i j}(\boldsymbol{x}, \boldsymbol{v}) \mathrm{d}^{3} c(\boldsymbol{x}, \boldsymbol{v})=0 .
$$

On contrary, functions $p_{k}(\boldsymbol{y}, \boldsymbol{v})$ and $p_{i j}(\boldsymbol{x}, \boldsymbol{v})$ are not orthogonal neither in the sense of the scalar product $(\cdot, \cdot)_{\boldsymbol{y}}$, nor in the sense of the scalar product $(\cdot, \cdot)_{\boldsymbol{x}}$, if $\boldsymbol{y} \neq \boldsymbol{x}$. This distinction is important for constructing the parametrix expansion. Further, we will omit the dependencies on $\boldsymbol{x}$ and $\boldsymbol{v}$ in the dimensionless velocity $c_{i}(\boldsymbol{x}, \boldsymbol{v})(224)$ if no misunderstanding might occur.

In this section we will consider the case of one-dimensional in $\boldsymbol{x}$ equations. We assume that:

$$
u_{1}(\boldsymbol{x})=u\left(x_{1}\right), \quad u_{2}=u_{3}=0, \quad T(\boldsymbol{x})=T\left(x_{1}\right), \quad n(\boldsymbol{x})=n\left(x_{1}\right) .
$$

We write $x$ instead of $x_{1}$ below. Finite-dimensional approximation (224) requires only two functions:

$$
\begin{aligned}
& p_{3}(x, \boldsymbol{v})=c_{1}^{2}(x, \boldsymbol{v})-\frac{1}{3} c^{2}(x, \boldsymbol{v}), \quad p_{4}(x, \boldsymbol{v})=c_{1}(x, \boldsymbol{v})\left(c^{2}(x, \boldsymbol{v})-(5 / 2)\right), \\
& c_{1}(x, \boldsymbol{v})=v_{T}^{-1}(x)\left(v_{1}-u(x)\right), \quad c_{2,3}(x, \boldsymbol{v})=v_{T}^{-1}(x) v_{2,3} .
\end{aligned}
$$

Now we will make a step-by-step calculation of the zeroth-order term of parametrix expansion, in the one-dimensional case, for the finite-dimensional approximation (227).

Step 1. Calculation of $\varphi_{\mathrm{loc}}(x, \boldsymbol{v})$ from $E q$. (210).

We search for the function $\varphi_{\text {loc }}(x, v)$ in approximation $(227)$ as

$$
\varphi_{\mathrm{loc}}(x, \boldsymbol{v})=a_{\mathrm{loc}}(x)\left(c_{1}^{2}-(1 / 3) c^{2}\right)+b_{\mathrm{loc}}(x) c_{1}\left(c^{2}-(5 / 2)\right) .
$$

Finite-dimensional approximation (223) of integral equation (210) in basis (227) yields

$$
\begin{aligned}
& m_{33}(x) a_{\mathrm{loc}}(x)+m_{34}(x) b_{\mathrm{loc}}(x)=\alpha_{\mathrm{loc}}(x) ; \\
& m_{43}(x) a_{\mathrm{loc}}(x)+m_{44}(x) b_{\mathrm{loc}}(x)=\beta_{\mathrm{loc}}(x) .
\end{aligned}
$$

Notations used are:

$$
\begin{aligned}
& m_{33}(x)=n(x) \lambda_{3}(x)+\frac{11}{9} \frac{\partial u}{\partial x} ; \quad m_{44}(x)=n(x) \lambda_{4}(x)+\frac{27}{4} \frac{\partial u}{\partial x} ; \\
& m_{34}(x)=m_{43}(x)=\frac{v_{T}(x)}{3}\left(\frac{\partial \ln n}{\partial x}+\frac{11}{2} \frac{\partial \ln T}{\partial x}\right) ;
\end{aligned}
$$




$$
\begin{aligned}
& \lambda_{3,4}(x)=-\frac{1}{\pi^{3 / 2}} \int \mathrm{e}^{-c^{2}(\boldsymbol{x}, \boldsymbol{v})} p_{3,4}(x, \boldsymbol{v}) L_{x}(\boldsymbol{v}) p_{3,4}(\boldsymbol{x}, \boldsymbol{v}) \mathrm{d}^{3} c(\boldsymbol{x}, \boldsymbol{v})>0 \\
& \alpha_{\mathrm{loc}}(x)=-\frac{2}{3} \frac{\partial u}{\partial x} ; \quad \beta_{\mathrm{loc}}(x)=-\frac{5}{4} v_{T}(x) \frac{\partial \ln T}{\partial x} .
\end{aligned}
$$

Parameters $\lambda_{3}(x)$ and $\lambda_{4}(x)$ are easily expressed via Enskog integral brackets, and they are calculated in [51] for a wide class of molecular models.

Solving Eq. (229), we obtain coefficients $a_{\text {loc }}(x)$ and $b_{\text {loc }}(x)$ in expression (228):

$$
\begin{aligned}
& a_{\mathrm{loc}}=\frac{A_{\mathrm{loc}}(x)}{Z(x, 0)} ; \quad b_{\mathrm{loc}}=\frac{B_{\mathrm{loc}}(x)}{Z(x, 0)} ; \quad Z(x, 0)=m_{33}(x) m_{44}(x)-m_{34}^{2}(x) ; \\
& A_{\mathrm{loc}}(x)=\alpha_{\mathrm{loc}}(x) m_{44}(x)-\beta_{\mathrm{loc}}(x) m_{34}(x) ; \\
& B_{\mathrm{loc}}(x)=\beta_{\mathrm{loc}}(x) m_{33}(x)-\alpha_{\mathrm{loc}}(x) m_{34}(x) ; \\
& a_{\mathrm{loc}}=\frac{-\frac{2}{3} \partial u / \partial x\left(n \lambda_{4}+\frac{27}{4} \partial u / \partial x\right)+\frac{5}{12} v_{T}^{2} \partial \ln T / \partial x\left(\partial \ln n / \partial x+\frac{11}{2} \partial \ln T / \partial x\right)}{\left(n \lambda_{3}+\frac{11}{9} \partial u / \partial x\right)\left(n \lambda_{4}+\frac{27}{4} \partial u / \partial x\right)-\frac{v_{T}^{2}}{9}\left(\partial \ln n / \partial x+\frac{11}{2} \partial \ln T / \partial x\right)^{2}} \\
& b_{\mathrm{loc}}=\frac{-\frac{5}{4} v_{T} \partial \ln T / \partial x\left(n \lambda_{3}+\frac{11}{9} \partial u / \partial x\right)+\frac{2}{9} v_{T} \partial u / \partial x\left(\partial \ln n / \partial x+\frac{11}{2} \partial \ln T / \partial x\right)}{\left(n \lambda_{3}+\frac{11}{9} \partial u / x\right)\left(n \lambda_{4}+\frac{27}{4} \partial u / \partial x\right)-\frac{v_{T}^{2}}{9}\left(\partial \ln n / x+\frac{11}{2} \partial \ln T / x\right)^{2}}
\end{aligned}
$$

These expressions complete Step 1.

Step 2. Calculation of Fourier transform of $\varphi_{\mathrm{loc}}(x, \boldsymbol{v})$ and its expression in the local basis.

In this step we make two operations:

(i) The Fourier transformation of the function $\varphi_{\text {loc }}(x, v)$ :

$$
\hat{\varphi}_{\text {loc }}(k, v)=\int_{-\infty}^{+\infty} \exp (-\mathrm{i} k y) \varphi_{\mathrm{loc}}(y, \boldsymbol{v}) \mathrm{d} y .
$$

(ii) The representation of $\hat{\varphi}_{\text {loc }}(k, v)$ in the local basis $\left\{p_{0}(x, \boldsymbol{v}), \ldots, p_{4}(x, \boldsymbol{v})\right\}$ :

$$
\begin{array}{ll}
p_{0}(x, \boldsymbol{v})=1, \quad p_{1}(x, \boldsymbol{v})=c_{1}(x, \boldsymbol{v}), & p_{2}(x, \boldsymbol{v})=c^{2}(x, \boldsymbol{v})-(3 / 2), \\
p_{3}(x, \boldsymbol{v})=c_{1}^{2}(x, \boldsymbol{v})-(1 / 3) c^{2}(x, \boldsymbol{v}), & p_{4}(x, \boldsymbol{v})=c_{1}(x, \boldsymbol{v})\left(c^{2}(x, \boldsymbol{v})-(5 / 2)\right) .
\end{array}
$$

Operation (ii) is necessary for completing Step 3 because there we deal with $x$-dependent operators. Obviously, the function $\hat{\varphi}_{\text {loc }}(k, v)(232)$ is a finite-order polynomial in $\boldsymbol{v}$, and thus the operation (ii) is exact.

We obtain in (ii):

$$
\hat{\varphi}_{\mathrm{loc}}(x, k, \boldsymbol{v}) \equiv \hat{\varphi}_{\mathrm{loc}}(x, k, c(x, \boldsymbol{v}))=\sum_{i=0}^{4} \hat{h}_{i}(x, k) p_{i}(x, \boldsymbol{v}) .
$$


Here

$$
\hat{h}_{i}(x, k)=\left(p_{i}(x, \boldsymbol{v}), p_{i}(x, \boldsymbol{v})\right)_{x}^{-2}\left(\hat{\varphi}_{\mathrm{loc}}(k, \boldsymbol{v}), p_{i}(x, \boldsymbol{v})\right)_{x} .
$$

Let us introduce notations:

$$
\vartheta \equiv \vartheta(x, y)=(T(x) / T(y))^{1 / 2}, \quad \gamma \equiv \gamma(x, y)=\frac{u(x)-u(y)}{v_{T}(y)} .
$$

Coefficients $\hat{h}_{i}(x, k)(235)$ have the following explicit form:

$$
\begin{aligned}
& \hat{h}_{i}(x, k)=\int_{-\infty}^{+\infty} \exp (-\mathrm{i} k y) h_{i}(x, y) \mathrm{d} y ; \quad h_{i}(x, y)=Z^{-1}(y, 0) g_{i}(x, y) \\
& g_{0}(x, y)=B_{\mathrm{loc}}(y)\left(\gamma^{3}+\frac{5}{2} \gamma\left(\vartheta^{2}-1\right)\right)+\frac{2}{3} A_{\mathrm{loc}}(y) \gamma^{2} \\
& g_{1}(x, y)=B_{\mathrm{loc}}(y)\left(3 \vartheta \gamma^{2}+\frac{5}{2} \vartheta\left(\vartheta^{2}-1\right)\right)+\frac{4}{3} A_{\mathrm{loc}}(y) \vartheta \gamma \\
& g_{2}(x, y)=\frac{5}{3} B_{\mathrm{loc}}(y) \vartheta^{2} \gamma \\
& g_{3}(x, y)=B_{\mathrm{loc}}(y) 2 \vartheta \gamma+A_{\mathrm{loc}}(y) \vartheta^{2} \\
& g_{4}(x, y)=B_{\mathrm{loc}}(y) \vartheta^{3}
\end{aligned}
$$

Here $Z(y, 0), B_{\text {loc }}(y)$ and $A_{\text {loc }}(y)$ are the functions defined in (231).

Step 3: Calculation of the function $\hat{\varphi}_{0}(x, k, v)$ from Eq. (212).

Linear integral equation (212) has a form similar to that of Eq. (210). We search for the function $\hat{\varphi}_{0}(x, k, \boldsymbol{v})$ in basis $(227)$ as

$$
\hat{\varphi}_{0}(x, k, \boldsymbol{v})=\hat{a}_{0}(x, k) p_{3}(x, \boldsymbol{v})+\hat{b}_{0}(x, k) p_{4}(x, \boldsymbol{v}) .
$$

Finite-dimensional approximation of the integral Eq. (212) in basis (227) yields the following equations for unknowns $\hat{a}_{0}(x, k)$ and $\hat{b}_{0}(x, k)$ :

$$
\begin{aligned}
& m_{33}(x) \hat{a}_{0}(x, k)+\left[m_{34}(x)+\frac{1}{3} \mathrm{i} k v_{T}(x)\right] \hat{b}_{0}(x, k)=\hat{\alpha}_{0}(x, k) ; \\
& {\left[m_{43}(x)+\frac{1}{3} \mathrm{i} k v_{T}(x)\right] \hat{a}_{0}(x, k)+m_{44}(x) \hat{b}_{0}(x, k)=\hat{\beta}_{0}(x, k) .}
\end{aligned}
$$

Notations used here are

$$
\begin{aligned}
& \hat{\alpha}_{0}(x, k)=m_{33}(x) \hat{h}_{3}(x, k)+m_{34}(x) \hat{h}_{4}(x, k)+\hat{s}_{\alpha}(x, k) ; \\
& \hat{\beta}_{0}(x, k)=m_{43}(x) \hat{h}_{3}(x, k)+m_{44}(x) \hat{h}_{4}(x, k)+\hat{s}_{\beta}(x, k) ;
\end{aligned}
$$




$$
\begin{aligned}
& \hat{s}_{\alpha, \beta}(x, k)=\int_{-\infty}^{+\infty} \exp (-\mathrm{i} k y) s_{\alpha, \beta}(x, y) \mathrm{d} y \\
& s_{\alpha}(x, y)=\frac{1}{3} v_{T}(x)\left(\frac{\partial \ln n}{\partial x}+2 \frac{\partial \ln T}{\partial x}\right) h_{1}(x, y)+\frac{2}{3} \frac{\partial u}{\partial x}\left(h_{0}(x, y)+2 h_{2}(x, y)\right) \\
& s_{\beta}(x, y)=\frac{5}{4} v_{T}(x)\left(\frac{\partial \ln n}{\partial x} h_{2}(x, y)+\frac{\partial \ln T}{\partial x}\left(3 h_{2}(x, y)+h_{0}(x, y)\right)\right)+\frac{2 \partial u}{3 \partial x} h_{1}(x, y)
\end{aligned}
$$

Solving Eqs. (239), we obtain functions $\hat{a}_{0}(x, k)$ and $\hat{b}_{0}(x, k)$ in $(238)$ :

$$
\begin{aligned}
& \hat{a}_{0}(x, k)=\frac{\hat{\alpha}_{0}(x, k) m_{44}(x)-\hat{\beta}_{0}(x, k)\left(m_{34}(x)+\frac{1}{3} \mathrm{i} k v_{T}(x)\right)}{Z\left(x, \frac{1}{3} \mathrm{i} k v_{T}(x)\right)} ; \\
& \hat{b}_{0}(x, k)=\frac{\hat{\beta}_{0}(x, k) m_{33}(x)-\hat{\alpha}_{0}(x, k)\left(m_{34}(x)+\frac{1}{3} \mathrm{i} k v_{T}(x)\right)}{Z\left(x, \frac{1}{3} \mathrm{i} k v_{T}(x)\right)} .
\end{aligned}
$$

Here

$$
\begin{aligned}
Z\left(x, \frac{1}{3} \mathrm{i} k v_{T}(x)\right)= & Z(x, 0)+\frac{k^{2} v_{T}^{2}(x)}{9}+\frac{2}{3} \mathrm{i} k v_{T}(x) m_{34}(x) \\
= & \left(n \lambda_{3}+\frac{11 \partial u}{9 \partial x}\right)\left(n \lambda_{4}+\frac{27 \partial u}{4 \partial x}\right)-\frac{v_{T}^{2}(x)}{9}\left(\frac{\partial \ln n}{\partial x}+\frac{11 \partial \ln T}{2 \partial x}\right)^{2} \\
& +\frac{k^{2} v_{T}^{2}(x)}{9}+\frac{2}{9} \mathrm{i} k v_{T}^{2}(x)\left(\frac{\partial \ln n}{\partial x}+\frac{11 \partial \ln T}{2 \partial x}\right) .
\end{aligned}
$$

Step 4: Calculation of the inverse Fourier transform of the function $\hat{\varphi}_{0}(x, k, v)$.

The inverse Fourier transform of the function $\hat{\varphi}_{0}(x, k, v)(238)$ yields:

$$
\varphi_{0}(x, \boldsymbol{v})=a_{0}(x) p_{3}(x, \boldsymbol{v})+b_{0}(x) p_{4}(x, \boldsymbol{v}) .
$$

Here

$$
\begin{aligned}
& a_{0}(x)=\frac{1}{2 \pi} \int_{-\infty}^{+\infty} \exp (\mathrm{i} k x) \hat{a}_{0}(x, k) \mathrm{d} k \\
& b_{0}(x)=\frac{1}{2 \pi} \int_{-\infty}^{+\infty} \exp (\mathrm{i} k x) \hat{b}_{0}(x, k) \mathrm{d} k
\end{aligned}
$$


Taking into account expressions (231), (240)-(243), and (237), we obtain the explicit expression for the finite-dimensional approximation of the zeroth-order term of parametrix expansion (244):

$$
\begin{aligned}
a_{0}(x)= & \frac{1}{2 \pi} \int_{-\infty}^{+\infty} \mathrm{d} y \int_{-\infty}^{+\infty} \mathrm{d} k \exp (\mathrm{i} k(x-y)) Z^{-1}\left(x, \frac{1}{3} \mathrm{i} k v_{T}(x)\right) \\
& \times\left\{Z(x, 0) h_{3}(x, y)+\left[s_{\alpha}(x, y) m_{44}(x)-s_{\beta}(x, y) m_{34}(x)\right]\right. \\
& \left.-\frac{1}{3} \mathrm{i} k v_{T}(x)\left[m_{34}(x) h_{3}(x, y)+m_{44}(x) h_{4}(x, y)+s_{\beta}(x, y)\right]\right\} ; \\
b_{0}(x)= & \frac{1}{2 \pi} \int_{-\infty}^{+\infty} \mathrm{d} y \int_{-\infty}^{+\infty} \mathrm{d} k \exp (\mathrm{i} k(x-y)) Z^{-1}\left(x, \frac{1}{3} \mathrm{i} k v_{T}(x)\right) \\
& \times\left\{Z(x, 0) h_{4}(x, y)+\left[s_{\beta}(x, y) m_{33}(x)-s_{\alpha}(x, y) m_{34}(x)\right]\right. \\
& \left.-\frac{1}{3} \mathrm{i} k v_{T}(x)\left[m_{34}(x) h_{4}(x, y)+m_{33}(x) h_{3}(x, y)+s_{\alpha}(x, y)\right]\right\} .
\end{aligned}
$$

Hydrodynamic equations. Now we will discuss briefly the utility of obtained results for hydrodynamics.

The correction to LM functions $f_{0}(n, \boldsymbol{u}, T)(140)$ obtained has the form

$$
f_{1}(n, \boldsymbol{u}, T)=f_{0}(n, \boldsymbol{u}, T)\left(1+\varphi_{0}(n, \boldsymbol{u}, T)\right) .
$$

Here the function $\varphi_{0}(n, \boldsymbol{u}, T)$ is given explicitly with expressions (244)-(246).

The usual form of closed hydrodynamic equations for $n, \boldsymbol{u}$, and $T$, where the traceless stress tensor $\sigma_{i k}$ and the heat flux vector $q_{i}$ are expressed via hydrodynamic variables, will be obtained if we substitute function (247) into balance equations of the density of the momentum, and of the energy. For LM approximation, these balance equations result in Euler equation of the nonviscid liquid (i.e. $\sigma_{i k}\left(f_{0}\right) \equiv 0$, and $\left.q_{i}\left(f_{0}\right) \equiv 0\right)$. For the correction $f_{1}(247)$, we obtain the following expressions of $\sigma=\sigma_{x x}\left(f_{1}\right)$ and $q=q_{x}\left(f_{1}\right)$ (all other components are equal to zero in the one-dimensional situation under consideration):

$$
\sigma=\frac{1}{3} n a_{0}, \quad q=\frac{5}{4} n b_{0} .
$$

Here $a_{0}$ and $b_{0}$ are given by expression (246).

From the geometrical viewpoint, hydrodynamic equations with the stress tensor and the heat flux vector (248) have the following interpretation: we take the corrected manifold $\Omega_{1}$ which consists of functions $f_{1}$ (247), and we project the BE vectors $J_{u}\left(f_{1}\right)$ onto the tangent spaces $T_{f_{1}}$ using the LM projector $P_{f_{0}}(145)$.

Although a detailed investigation of these hydrodynamic equations is a subject of a special study and it is not the goal of this paper, some points should be mentioned.

Nonlocality. Expressions (246) expose a nonlocal spatial dependency, and, hence, the corresponding hydrodynamic equations are nonlocal. This nonlocality appears through two contributions. The 
first of these contributions might be called a frequency-response contribution, and it comes through explicit nonpolynomial $k$-dependency of integrands in (246). This latter dependency has the form

$$
\int_{-\infty}^{+\infty} \frac{A(x, y)+\mathrm{i} k B(x, y)}{C(x, y)+\mathrm{i} k D(x, y)+k^{2} E(x, y)} \exp (\mathrm{i} k(x-y)) \mathrm{d} k .
$$

Integration over $k$ in (249) can be completed via auxiliary functions.

The second nonlocal contribution might be called correlative, and it is due to relationships via $(u(x)-u(y)$ ) (the difference of flow velocities in points $x$ and $y$ ) and via $T(x) / T(y)$ (the ratio of temperatures in points $x$ and $y$ ).

Acoustic spectra. The purely frequency-response contribution to hydrodynamic equations is relevant to small perturbations of equilibria. The stress tensor $\sigma$ and the heat flux $q$ (248) are:

$$
\begin{aligned}
& \sigma=-\left(\frac{2}{3}\right) n_{0} T_{0} R\left(2 \varepsilon \frac{\partial u^{\prime}}{\partial \xi}-3 \varepsilon^{2} \frac{\partial^{2} T}{\partial \xi^{2}}\right) ; \\
& q=-\left(\frac{5}{4}\right) T_{0}^{3 / 2} n_{0} R\left(3 \varepsilon \frac{\partial T^{\prime}}{\partial \xi}-\left(\frac{8}{5}\right) \varepsilon^{2} \frac{\partial^{2} u}{\partial \xi^{2}}\right) .
\end{aligned}
$$

Here

$$
R=\left(1-\left(\frac{2}{5}\right) \varepsilon^{2} \frac{\partial^{2}}{\partial \xi^{2}}\right)^{-1} .
$$

In (250), we have expressed parameters $\lambda_{3}$ and $\lambda_{4}$ via the viscosity coefficient $\mu$ of the ChapmanEnskog method [51] (it is easy to see from (230) that $\lambda_{3}=\lambda_{4} \propto \mu^{-1}$ for spherically symmetric models of a collision), and we have used the following notations: $T_{0}$ and $n_{0}$ are the equilibrium temperature and density, $\xi=\left(\eta T_{0}^{1 / 2}\right)^{-1} n_{0} x$ is the dimensionless coordinate, $\eta=\mu\left(T_{0}\right) / T_{0}, u^{\prime}=T_{0}^{-1 / 2} \delta u$, $T^{\prime}=\delta T / T_{0}, n^{\prime}=\delta n / n_{0}$, and $\delta u, \delta T, \delta n$ are the deviations of the flux velocity, of the temperature and of the density from their equilibrium values $u=0, T=T_{0}$ and $n=n_{0}$. We also use the system of units with $k_{\mathrm{B}}=m=1$.

In the linear case, the parametrix expansion degenerates, and its zeroth-order term (213) gives the solution of Eq. (190).

The dispersion relationship for approximation (250) is

$$
\begin{aligned}
& \omega^{3}+\left(23 k^{2} / 6 D\right) \omega^{2}+\left\{k^{2}+\left(2 k^{4} / D^{2}\right)+\left(8 k^{6} / 5 D^{2}\right)\right\} \omega+\left(5 k^{4} / 2 D\right)=0 ; \\
& D=1+\left(\frac{4}{5}\right) k^{2} .
\end{aligned}
$$

Here $k$ is the wave vector.

Acoustic spectra given by dispersion relationship (252) contains no nonphysical short-wave instability characteristic to the Burnett approximation (Fig. 4). The regularization of the Burnett approximation $[25,26]$ has the same feature. Both of these approximations predict a limit of the decrement $\operatorname{Re} \omega$ for short waves.

Nonlinearity. Nonlinear dependency on $\partial u / \partial x$, on $\partial \ln T / \partial x$, and on $\partial \ln n / \partial x$ appears already in the local approximation $\varphi_{\text {loc }}(231)$. In order to outline some peculiarities of this nonlinearity, we represent the zeroth-order term of the expansion of $a_{\text {loc }}$ (231) into powers of $\partial \ln T / \partial x$ and $\partial \ln n / \partial x$ :

$$
a_{\mathrm{loc}}=-\frac{2}{3} \frac{\partial u}{\partial x}\left(n \lambda_{3}+\frac{11}{9} \frac{\partial u}{\partial x}\right)^{-1}+\mathrm{O}\left(\frac{\partial \ln T}{\partial x}, \frac{\partial \ln n}{\partial x}\right) \text {. }
$$




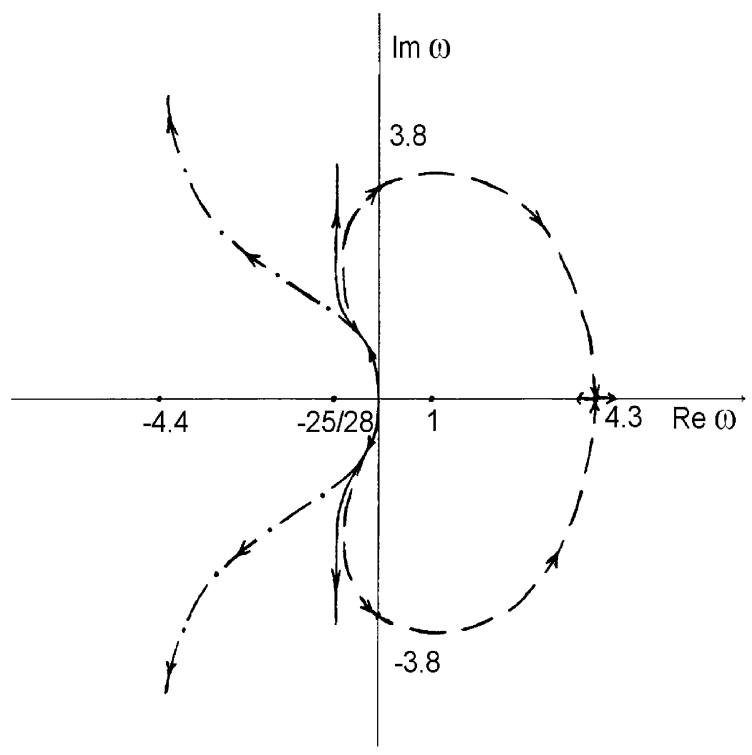

Fig. 4. Acoustic dispersion curves for approximation 250 (solid line), for second (the Burnett) approximation of the Chapman-Enskog expansion [53] (dashed line) and for the regularization of the Burnett approximation via partial summing of the Chapman-Enskog expansion $[25,26]$ (punctuated dashed line). Arrows indicate an increase of $k^{2}$.

This expression describes the asymptotic of the "purely nonlinear" contribution to the stress tensor $\sigma$ (248) for a strong divergency of a flow. The account of nonlocality yields instead of (250):

$$
\begin{aligned}
a_{0}(x)= & -\frac{1}{2 \pi} \int_{-\infty}^{+\infty} \mathrm{d} y \int_{-\infty}^{+\infty} \mathrm{d} k \exp (\mathrm{i} k(x-y)) \frac{2}{3} \frac{\partial u}{\partial y}\left(n \lambda_{3}+\frac{11}{9} \frac{\partial u}{\partial y}\right)^{-1} \\
& \times\left[\left(n \lambda_{3}+\frac{11}{9} \frac{\partial u}{\partial x}\right)\left(n \lambda_{4}+\frac{27}{4} \frac{\partial u}{\partial x}\right)+\frac{k^{2} v_{T}^{2}}{9}\right]^{-1}\left[\left(n \lambda_{3}+\frac{11}{9} \frac{\partial u}{\partial x}\right)\left(n \lambda_{4}+\frac{27}{4} \frac{\partial u}{\partial x}\right)\right. \\
& \left.+\frac{4}{9}\left(n \lambda_{4}+\frac{27}{4} \frac{\partial u}{d y}\right) \frac{\partial u}{\partial x} v_{T}^{-2}(u(x)-u(y))^{2}-\frac{2}{3} \mathrm{i} k \frac{\partial u}{\partial x}(u(x)-u(y))\right] \\
& +\mathrm{O}\left(\frac{\partial \ln T}{\partial x}, \frac{\partial \ln n}{\partial x}\right) .
\end{aligned}
$$

Both expressions, (253) and (254) become singular when

$$
\frac{\partial u}{\partial y} \rightarrow\left(\frac{\partial u}{\partial y}\right)^{*}=-\frac{9 n \lambda_{3}}{11}
$$

Hence, the stress tensor (249) becomes infinite if $\partial u / \partial y$ tends to $\partial u^{*} / \partial y$ in any point $y$. In other words, the flow becomes infinitely viscid when $\partial u / \partial y$ approaches the negative value $-9 n \lambda_{3} / 11$. This infinite viscosity threshold prevents a transfer of the flow into nonphysical region of negative viscosity if $\partial u / \partial y>\partial u^{*} / \partial y$ because of the infinitely strong dumping at $\partial u^{*} / \partial y$. This peculiarity was detected in $[25,26]$ as a result of partial summing of the Chapman-Enskog expansion. In particular, 
partial summing for the simplest nonlinear situation [27,178] yields the following expression for the stress tensor $\sigma$ :

$$
\begin{aligned}
& \sigma=\sigma_{\mathrm{I} R}+\sigma_{\mathrm{II} R} ; \quad \sigma_{\mathrm{I} R}=-\frac{4}{3}\left(1-\frac{5}{3} \varepsilon^{2} \frac{\partial^{2}}{\partial \xi^{2}}\right)^{-1}\left(\varepsilon \frac{\partial u^{\prime}}{\partial \xi}+\varepsilon^{2} \frac{\partial^{2} \theta^{\prime}}{\partial \xi^{2}}\right) ; \quad \theta^{\prime}=T^{\prime}+n^{\prime} ; \\
& \sigma_{\mathrm{II} R}=\frac{28}{9}\left(1+\frac{7}{3} \varepsilon \frac{\partial u^{\prime}}{\partial \xi}\right)^{-1} \frac{\partial^{2} u^{\prime}}{\partial \xi^{2}} .
\end{aligned}
$$

Notations here follow (250) and (251). Expression (256) might be considered as a "rough draft" of the "full" stress tensor defined by $a_{0}$ (246). It accounts both the frequency-response and the nonlinear contributions $\left(\sigma_{\mathrm{I} R}\right.$ and $\sigma_{\mathrm{II} R}$, respectively) in a simple form of a sum. However, the superposition of these contributions in (246) is more complicated. Moreover, the explicit correlative nonlocality of expression (246) was never detected neither in [27], nor in numerous examples of partial summing [178].

Nevertheless, approximation (256) contains the peculiarity of viscosity similar to that in (253) and (254). In dimensionless variables and $\varepsilon=1$, expression (256) predicts the infinite threshold at velocity divergency equal to $-\left(\frac{3}{7}\right)$, rather than $-\left(\frac{9}{11}\right)$ in (253) and (254). Viscosity tends to zero as the divergency tends to positive infinity in both approximations. Physical interpretation of these phenomena was given in [27]: large positive values of $\partial u / \partial x$ means that the gas diverges rapidly, and the flow becomes nonviscid because the particles retard to exchange their momentum. On contrary, its negative values (such as $-\left(\frac{3}{7}\right)$ for $(256)$ and $-\left(\frac{9}{11}\right)$ ) for (253) and (254)) describe a strong compression of the flow. Strong deceleration results in "solid fluid" limit with an infinite viscosity (Fig. 5).

Thus, hydrodynamic equations for approximation (247) are both nonlinear and nonlocal. This result is not surprising, accounting the integro-differential character of Eq. (190).

It is important that no small parameters were used neither when we were deriving Eq. (190) nor when we were obtaining correction (247).

\section{Example 4: Nonperturbative derivation of linear hydrodynamics from Boltzmann equation (3D)}

Using the Newton method instead of power series, a model of linear hydrodynamics is derived from the Boltzmann equation for regimes where the Knudsen number is of order unity. The model demonstrates no violation of stability of acoustic spectra in contrast to Burnett hydrodynamics.

The Knudsen number $\varepsilon$ (a ratio between the mean free path, $l_{c}$, and the scale of hydrodynamic flows, $\left.l_{h}\right)$ is a recognized order parameter when hydrodynamics is derived from the Boltzmann equation [183]. The Chapman-Enskog method [51] establishes the Navier-Stokes hydrodynamic equations as the first-order correction to Euler hydrodynamics at $\varepsilon \rightarrow 0$, and it also derives formal corrections of order $\varepsilon^{2}, \varepsilon^{3}, \ldots$ (known as Burnett and super-Burnett corrections). These corrections are important outside the strictly hydrodynamic domain $\varepsilon \ll 1$, and has to be considered for an extension of hydrodynamic description into a highly nonequilibrium domain $\varepsilon \leqslant 1$. Not much is known about high-order in $\varepsilon$ hydrodynamics, especially in a nonlinear case. Nonetheless, in a linear case, some definite information can be obtained. On the one hand, experiments on sound propagation in noble gases are considerably better explained with the Burnett and the super-Burnett hydrodynamics rather than with the Navier-Stokes approximation alone [185]. On the other hand, a direct calculation shows 


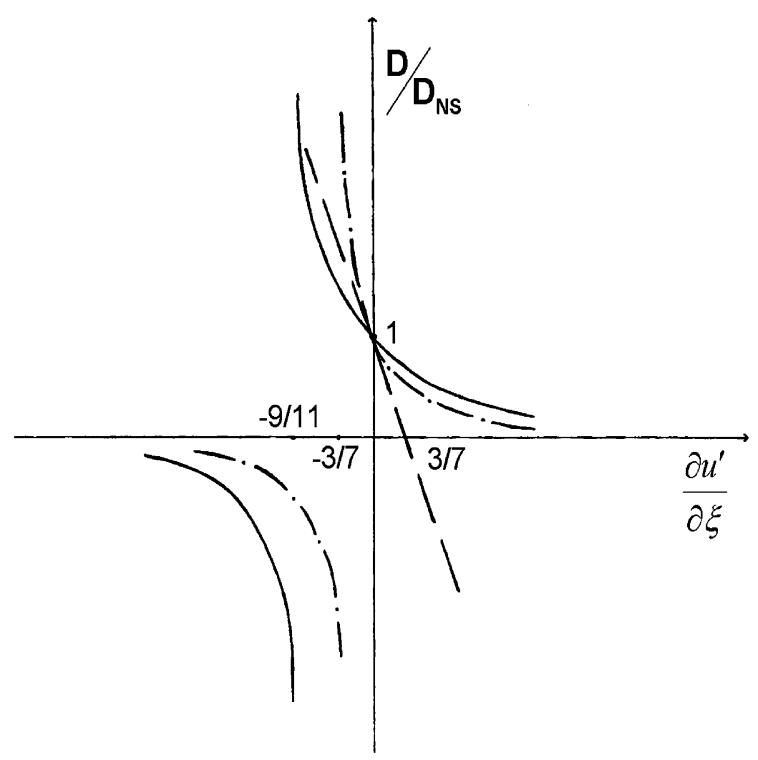

Fig. 5. Dependency of viscosity on compression for approximation (253) (solid line), for partial summing (256) (punctuated dashed line), and for the Burnett approximation [27,178] (dashed line). The latter changes the sign at a regular point and, hence, nothing prevents the flow to transfer into the nonphysical region.

nonphysical behavior of the Burnett hydrodynamics for ultra-short waves: acoustic waves increase instead of decay [53]. The latter failure of the Burnett approximation cannot be rejected on a basis that for such regimes they might be not applicable because for the Navier-Stokes approximation, which is formally still less valid, no such violation is observed.

These two results indicate that, at least in a linear regime, it makes sense to consider hydrodynamics at $\varepsilon \leqslant 1$, but Enskog way of deriving such hydrodynamics is problematic. The problem of constructing solutions to the Boltzmann equation valid when $\varepsilon$ is of order unity is one of the main open problems of classical kinetic theory [183].

In this example we suggest a new approach to derive hydrodynamics at $\varepsilon \leqslant 1$. The main idea is to pose a problem of a finding a correction to the Euler hydrodynamics in such a fashion that expansions in $\varepsilon$ do not appear as a necessary element of analysis. This will be possible by using the Newton method instead of Taylor expansions to get such correction. We restrict our consideration to a linear case. Resulting hydrodynamic equations do not exhibit the mentioned violation.

The starting point is the set of local Maxwell distribution functions (LM) $f_{0}(n, \boldsymbol{u}, T ; \boldsymbol{v})$, where $\boldsymbol{v}$ is the particle's velocity, and $n, \boldsymbol{u}$, and $T$ are local number density, average velocity, and temperature. We write the Boltzmann equation as

$$
\frac{\mathrm{d} f}{\mathrm{~d} t}=J(f), \quad J(f)=-(v-u)_{i} \cdot \partial_{i} f+Q(f),
$$

where $\mathrm{d} / \mathrm{d} t=\partial / \partial t+u_{i} \cdot \partial_{i}$ is the material derivative, $\partial_{i}=\partial / \partial x_{i}$, while $Q$ is the Boltzmann collision integral [183]. 
On the one hand, calculating r.h.s. of Eq. (257) in LM-states, we obtain $J\left(f_{0}\right)$, a time derivative of LM-states due to Boltzmann equation. On the other hand, calculating a time derivative of LM-states due to Euler dynamics, we obtain $P_{0} J\left(f_{0}\right)$, where $P_{0}$ is a projector operator onto the LM manifold (see [6]):

$$
P_{0} J=\frac{f_{0}}{n}\left\{\int J \mathrm{~d} \boldsymbol{c}+2 c_{i} \cdot \int c_{i} J \mathrm{~d} \boldsymbol{c}+\frac{2}{3}\left(c^{2}-\frac{3}{2}\right) \int\left(c^{2}-\frac{3}{2}\right) J \mathrm{~d} \boldsymbol{c}\right\},
$$

Since the LM functions are not solutions to the Boltzmann equation (257) (except for constant $n$, $\boldsymbol{u}$, and $T)$, difference between $J\left(f_{0}\right)$ and $P_{0} J\left(f_{0}\right)$ is not equal to zero:

$$
\Delta\left(f_{0}\right)=J\left(f_{0}\right)-P_{0} J\left(f_{0}\right)=-f_{0}\left\{2\left(\partial_{i} u_{k}\right)\left(c_{i} c_{k}-\frac{1}{3} \delta_{i k} c^{2}\right)+v_{T} \frac{\partial_{i} T}{T} c_{i}\left(c^{2}-\frac{5}{2}\right)\right\} .
$$

here $\boldsymbol{c}=v_{T}^{-1}(\boldsymbol{v}-\boldsymbol{u})$, and $v_{T}=\sqrt{2 k_{\mathrm{B}} T / m}$ is the thermal velocity. Note that the latter expression gives a complete discrepancy of the linearized local Maxwell approximation, and it is neither big nor small in itself. An unknown hydrodynamic solution of Eq. (257), $f_{\infty}(n, \boldsymbol{u}, T ; \boldsymbol{v})$, satisfies the following equation:

$$
\Delta\left(f_{\infty}\right)=J\left(f_{\infty}\right)-P_{\infty} J\left(f_{\infty}\right)=0
$$

where $P_{\infty}$ is an unknown projecting operator. Both $P_{\infty}$ and $f_{\infty}$ are unknown in Eq. (260), but, nontheless, one is able to consider a sequence of corrections $\left\{f_{1}, f_{2}, \ldots\right\},\left\{P_{1}, P_{2}, \ldots\right\}$ to the initial approximation $f_{0}$ and $P_{0}$. A method to deal with equations of a form (260) was developed in [6] for a general case of dissipative systems. In particular, it was shown, how to ensure the $H$-theorem on every step of approximations by choosing appropriate projecting operators $P_{n}$. In the present illustrative example we will not consider projectors other than $P_{0}$, rather, we will use an iterative procedure to find $f_{1}$.

Let us apply the Newton method with incomplete linearization to Eq. (260) with $f_{0}$ as initial approximation for $f_{\infty}$ and with $P_{0}$ as an initial approximation for $P_{\infty}$. Writing $f_{1}=f_{0}+\delta f$, we get the first Newton iterate:

$$
L\left(\delta f / f_{0}\right)+\left(P_{0}-1\right)(v-u)_{i} \partial_{i} \delta f+\Delta\left(f_{0}\right)=0,
$$

where $L$ is a linearized collision integral.

$$
L(g)=f_{0}(\boldsymbol{v}) \int w\left(\boldsymbol{v}_{1}^{\prime}, \boldsymbol{v}^{\prime} ; \boldsymbol{v}_{1}, \boldsymbol{v}\right) f_{0}\left(\boldsymbol{v}_{1}\right)\left\{g\left(\boldsymbol{v}_{1}^{\prime}\right)+g\left(\boldsymbol{v}^{\prime}\right)-g\left(\boldsymbol{v}_{1}\right)-g(\boldsymbol{v})\right\} \mathrm{d} \boldsymbol{v}_{1}^{\prime} \mathrm{d} \boldsymbol{v}^{\prime} \mathrm{d} \boldsymbol{v}_{1} .
$$

Here $w$ is a probability density of velocities change, $\left(\boldsymbol{v}, \boldsymbol{v}_{1}\right) \leftrightarrow\left(\boldsymbol{v}^{\prime}, \boldsymbol{v}_{1}^{\prime}\right)$, of a pair of molecules after their encounter. When deriving (261), we have accounted $P_{0} L=0$, and an additional condition which fixes the same values of $n, \boldsymbol{u}$, and $T$ in states $f_{1}$ as in LM states $f_{0}$ :

$$
P_{0} \delta f=0 .
$$

Eq. (261) is basic in what follows. Note that it contains no Knudsen number explicitly. Our strategy will be to treat Eq. (261) in such a way that the Knudsen number will appear explicitly only at the latest stage of computations. 
The two further approximations will be adopted. The first concerns a linearization of Eq. (261) about a global equilibria $F_{0}$. The second concerns a finite-dimensional approximation of integral operator in (261) in velocity space. It is worthwhile noting here that none of these approximations concerns an assumption about the Knudsen number.

Following the first of the approximations mentioned, denote as $\delta n, \delta \boldsymbol{u}$, and $\delta T$ deviations of hydrodynamic variables from their equilibrium values $n_{0}, \boldsymbol{u}_{0}=0$, and $T_{0}$. Introduce also nondimensional variables $\Delta n=\delta n / n_{0}, \Delta \boldsymbol{u}=\delta \boldsymbol{u} / v_{T}^{0}$, and $\Delta T=\delta T / T_{0}$, where $v_{T}^{0}$ is a heat velocity in equilibria, and a nondimensional relative velocity $\boldsymbol{\xi}=\boldsymbol{v} / v_{T}^{0}$. Correction $f_{1}$ in the approximation, linear in deviations from $F_{0}$ reads:

$$
f_{1}=F_{0}\left(1+\varphi_{0}+\varphi_{1}\right),
$$

where

$$
\varphi_{0}=\Delta n+2 \Delta u_{i} \xi_{i}+\Delta T\left(\xi^{2}-3 / 2\right)
$$

is a linearized deviation of LM from $F_{0}$, and $\varphi_{1}$ is an unknown function. The latter is to be obtained from a linearized version of Eq. (261).

Following the second approximation, we search for $\varphi_{1}$ in a form

$$
\varphi_{1}=A_{i}(\boldsymbol{x}) \xi_{i}\left(\xi^{2}-\frac{5}{2}\right)+B_{i k}(\boldsymbol{x})\left(\xi_{i} \xi_{k}-\frac{1}{3} \delta_{i k} \xi^{2}\right)+\cdots
$$

where dots denote terms of an expansion of $\varphi_{1}$ in velocity polynomials, orthogonal to $\xi_{i}\left(\xi^{2}-5 / 2\right)$ and $\xi_{i} \xi_{k}-1 / 3 \delta_{i k} \xi^{2}$, as well as to 1 , to $\xi$, and to $\xi^{2}$. These terms do not contribute to shear stress tensor and heat flux vector in hydrodynamic equations. Independency of functions $A$ and $B$ from $\xi^{2}$ amounts to the first Sonine polynomial approximation of viscosity and heat transfer coefficients. Put another way, we consider a projection onto a finite-dimensional subspace spanned by $\xi_{i}\left(\xi^{2}-5 / 2\right)$ and $\xi_{i} \xi_{k}-1 / 3 \delta_{i k} \xi^{2}$. Our goal is to derive functions $A$ and $B$ from a linearized version of Eq. (261). Knowing $A$ and $B$, we get the following expressions for shear stress tensor $\boldsymbol{\sigma}$ and heat flux vector $\boldsymbol{q}$ :

$$
\sigma=p_{0} B, \quad \boldsymbol{q}=\frac{5}{4} p_{0} v_{T}^{0} A
$$

where $p_{0}$ is equilibrium pressure of ideal gas.

Linearizing Eq. (261) near $F_{0}$, using an ansatz for $\varphi_{1}$ cited above, and turning to Fourier transform in space, we derive

$$
\begin{aligned}
& \frac{5 p_{0}}{3 \eta_{0}} a_{i}(\boldsymbol{k})+\mathrm{i} v_{T}^{0} b_{i j}(\boldsymbol{k}) k_{j}=-\frac{5}{2} \mathrm{i} v_{T}^{0} k_{i} \tau(\boldsymbol{k}) ; \\
& \frac{p_{0}}{\eta_{0}} b_{i j}(\boldsymbol{k})+\mathrm{i} v_{T}^{0} \overline{k_{i} a_{j}(\boldsymbol{k})}=-2 \mathrm{i} v_{T}^{0} \overline{k_{i} \gamma_{j}(\boldsymbol{k})},
\end{aligned}
$$

where $\mathrm{i}=\sqrt{-1}, \boldsymbol{k}$ is the wave vector, $\eta_{0}$ is the first Sonine polynomial approximation of shear viscosity coefficient, $\boldsymbol{a}(\boldsymbol{k}), \boldsymbol{b}(\boldsymbol{k}), \tau(\boldsymbol{k})$ and $\boldsymbol{\gamma}(\boldsymbol{k})$ are Fourier transforms of $\boldsymbol{A}(\boldsymbol{x}), \boldsymbol{B}(\boldsymbol{x}), \Delta T(\boldsymbol{x})$, and $\Delta \boldsymbol{u}(\boldsymbol{x})$, respectively, and the over-bar denotes a symmetric traceless dyad:

$$
\overline{a_{i} b_{j}}=2 a_{i} b_{j}-\frac{2}{3} \delta_{i j} a_{s} b_{s} .
$$


Introducing a dimensionless wave vector $\boldsymbol{f}=\left[\left(v_{T}^{0} \eta_{0}\right) /\left(p_{0}\right)\right] \boldsymbol{k}$, solution to Eq. (266) may be written as

$$
\begin{aligned}
b_{l j}(\boldsymbol{k})= & -\frac{10}{3} \mathrm{i} \overline{\gamma_{l}(\boldsymbol{k}) f_{j}}\left[(5 / 3)+(1 / 2) f^{2}\right]^{-1} \\
& +\frac{5}{3} \mathrm{i}\left(\gamma_{s}(\boldsymbol{k}) f_{s}\right) \overline{f_{l} f_{j}}\left[(5 / 3)+(1 / 2) f^{2}\right]^{-1}\left[5+2 f^{2}\right]^{-1}-\frac{15}{2} \tau(\boldsymbol{k}) \overline{f_{l} f_{j}}\left[5+2 f^{2}\right]^{-1} \\
a_{l}(\boldsymbol{k})= & -\frac{15}{2} \mathrm{i} f_{l} \tau(\boldsymbol{k})\left[5+2 f^{2}\right]^{-1} \\
& -\left[5+2 f^{2}\right]^{-1}\left[(5 / 3)+(1 / 2) f^{2}\right]^{-1}\left[(5 / 3) f_{l}\left(\gamma_{s}(\boldsymbol{k}) f_{s}\right)+\gamma_{l}(\boldsymbol{k}) f^{2}\left(5+2 f^{2}\right)\right] .
\end{aligned}
$$

Considering $z$-axis as a direction of propagation and denoting $k_{z}$ as $k, \gamma$ as $\gamma_{z}$, we obtain from (266) the $k$-dependence of $a=a_{z}$ and $b=b_{z z}$ :

$$
\begin{gathered}
a(k)=-\frac{\frac{3}{2} p_{0}^{-1} \eta_{0} v_{T}^{0} \mathrm{i} k \tau(k)+\frac{4}{5} p_{0}^{-2} \eta_{0}^{2}\left(v_{T}^{0}\right)^{2} k^{2} \gamma(k)}{1+\frac{2}{5} p_{0}^{-2} \eta_{0}^{2}\left(v_{T}^{0}\right)^{2} k^{2}}, \\
b(k)=-\frac{\frac{4}{3} p_{0}^{-1} \eta_{0} v_{T}^{0} \mathrm{i} k \gamma(k)+p_{0}^{-2} \eta_{0}^{2}\left(v_{T}^{0}\right)^{2} k^{2} \tau(k)}{1+\frac{2}{5} p_{0}^{-2} \eta_{0}^{2}\left(v_{T}^{0}\right)^{2} k^{2}} .
\end{gathered}
$$

Using expressions for $\sigma$ and $\mathbf{q}$ cited above, and also using (268), it is an easy matter to close the linearized balance equations (given in Fourier terms):

$$
\begin{aligned}
& \frac{1}{v_{T}^{0}} \partial_{t} v(k)+\mathrm{i} k \gamma_{k}=0, \\
& \frac{2}{v_{T}^{0}} \partial_{t} \gamma(k)+\mathrm{i} k(\tau(k)+v(k))+\mathrm{i} k b(k)=0, \\
& \frac{3}{2 v_{T}^{0}} \partial \tau+\mathrm{i} k \gamma(k)+\frac{5}{4} \mathrm{i} k a(k)=0 .
\end{aligned}
$$

Eqs. (269), together with expressions (268), complete our derivation of hydrodynamic equations.

To this end, the Knudsen number was not penetrating our derivations. Now it is worthwhile to introduce it. The Knudsen number will appear most naturally if we turn to dimensionless form of Eq. (268). Taking $l_{c}=v_{T}^{0} \eta_{0} / p_{0}\left(l_{c}\right.$ is of order of a mean free path), and introducing a hydrodynamic scale $l_{h}$, so that $k=\kappa / l_{h}$, where $\kappa$ is a nondimensional wave vector, we obtain in (268):

$$
\begin{gathered}
a(\kappa)=-\frac{\frac{3}{2} \mathrm{i} \varepsilon \kappa \tau(\kappa)+\frac{4}{5} \varepsilon^{2} \kappa^{2} \gamma_{\kappa},}{1+\frac{2}{5} \varepsilon^{2} \kappa^{2}}, \\
b(\kappa)=-\frac{\frac{4}{3} \mathrm{i} \varepsilon \kappa \gamma(\kappa)+\varepsilon^{2} \kappa^{2} \tau(\kappa)}{1+\frac{2}{5} \varepsilon^{2} \kappa^{2}},
\end{gathered}
$$

where $\varepsilon=l_{c} / l_{h}$. Considering the limit $\varepsilon \rightarrow 0$ in (270), we come back to the familiar Navier-Stokes expressions: $\sigma_{z z}^{\mathrm{NS}}=-\frac{4}{3} \eta_{0} \partial_{z} \delta u_{z}, q_{z}^{\mathrm{NS}}=-\lambda_{0} \partial_{z} \delta T$, where $\lambda_{0}=15 k_{\mathrm{B}} \eta_{0} / 4 m$ is the first Sonine polynomial approximation of heat conductivity coefficient. 


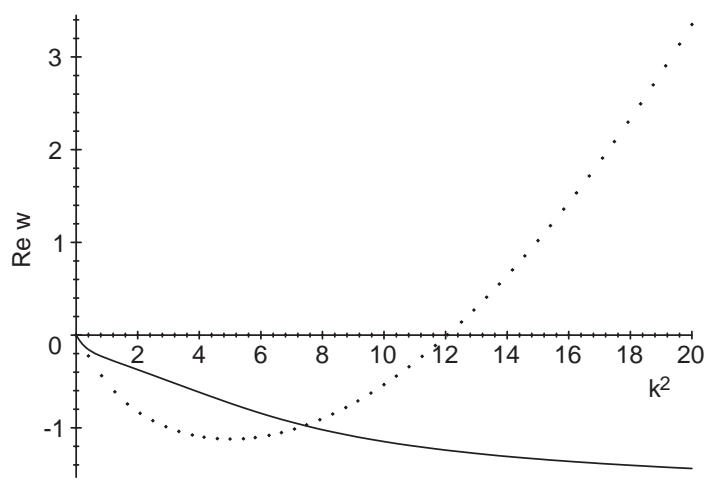

Fig. 6. Attenuation rate of sound waves. Dotts: Burnett approximation. Bobylev's instability occurs when the curve intersects the horizontal axis. Solid: First iteration of the Newton method on the invariance equation.

Since we were not assuming smallness of the Knudsen number $\varepsilon$ while deriving (270), we are completely legal to put $\varepsilon=1$. With all the approximations mentioned above, Eqs. (269) and (268) (or, equivalently, (269) and (270)) may be considered as a model of a linear hydrodynamics at $\varepsilon$ of order unity. The most interesting feature of this model is a nonpolynomial dependence on $\kappa$. This amounts to that share stress tensor and heat flux vector depend on spatial derivatives of $\delta \boldsymbol{u}$ and of $\delta T$ of an arbitrary high order.

To find out a result of nonpolynomial behavior (270), it is most informative to calculate a dispersion relation for planar waves. It is worthwhile introducing dimensionless frequency $\lambda=\omega l_{h} / v_{T}^{0}$, where $\omega$ is a complex frequency of a wave $\sim \exp (\omega t+\mathrm{i} k z)$ ( $\operatorname{Re} \omega$ is a damping rate, and $\operatorname{Im} \omega$ is a circular frequency). Making use of Eqs. (269) and (270), writing $\varepsilon=1$, we obtain the following dispersion relation $\lambda(\kappa)$ :

$$
12\left(1+\frac{2}{5} \kappa^{2}\right)^{2} \lambda^{3}+23 \kappa^{2}\left(1+\frac{2}{5} \kappa^{2}\right) \lambda^{2}+2 \kappa^{2}\left(5+5 \kappa^{2}+\frac{6}{5} \kappa^{4}\right) \lambda+\frac{15}{2} \kappa^{4}\left(1+\frac{2}{5} \kappa^{2}\right)=0 .
$$

Fig. 6 presents a dependence $\operatorname{Re} \lambda\left(\kappa^{2}\right)$ for acoustic waves obtained from (271) and for the Burnett approximation [53]. The violation in the latter occurs when the curve overcomes the horizontal axis. In contrast to the Burnett approximation [53], the acoustic spectrum (271) is stable for all $\kappa$. Moreover, $\operatorname{Re} \lambda\left(\kappa^{2}\right)$ demonstrates a finite limit, as $\kappa^{2} \rightarrow \infty$.

A discussion of results concerns the following two items:

1. The approach used avoids expansion into powers of the Knudsen number, and thus we obtain a hydrodynamics valid (at least formally) for moderate Knudsen numbers as an immediate correction to Euler hydrodynamics. This is in contrast to usual treatment of high-order hydrodynamics as "(the well established) Navier-Stokes approximation + high-order terms". The Navier-Stokes hydrodynamics is recovered a posteriori, as a limiting case, but not as a necessary intermediate step of computations.

2. Linear hydrodynamics derived is stable for all $k$, same as the Navier-Stokes hydrodynamics alone. The $\left(1+\alpha k^{2}\right)^{-1}$ "cut-off", as in (268) and (270), was earlier found in a "partial summing" of Enskog series [25,24]. 
Thus, we come to the following two conclusions:

1. A preliminary positive answer is given to the question of whether is it possible to construct solutions of the Boltzmann equation valid for the Knudsen number of order unity.

2. Linear hydrodynamics derived can be used as a model for $\varepsilon=1$ with no danger to get a violation of acoustic spectra at large $k$.

\section{Example 5: Dynamic correction to moment approximations}

Dynamic correction or extension of the list of variables? Considering the Grad moment ansatz as a suitable first approximation to a closed finite-moment dynamics, the correction is derived from the Boltzmann equation. The correction consists of two parts, local and nonlocal. Locally corrected 13-moment equations are demonstrated to contain exact transport coefficients. Equations resulting from the nonlocal correction give a microscopic justification to some phenomenological theories of extended hydrodynamics.

A considerable part of the modern development of nonequilibrium thermodynamics is based on the idea of extension of the list of relevant variables. Various phenomenological and semi-phenomenological theories in this domain are known under the common title of the extended irreversible thermodynamics (EIT) [180]. With this, the question of a microscopic justification of the EIT becomes important. Recall that a justification for some of the versions of the EIT was found within the well known Grad moment method [158].

Originally, the Grad moment approximation was introduced for the purpose of solving the Boltzmann-like equations of the classical kinetic theory. The Grad method is used in various kinetic problems, e.g., in plasma and in phonon transport. We mention also that Grad equations assist in understanding asymptotic features of gradient expansions, both in linear and nonlinear domains $[178,163,162,23,24]$.

The essence of the Grad method is to introduce an approximation to the one-particle distribution function $f$ which would depend only on a finite number $N$ of moments, and, subsequently, to use this approximation to derive a closed system of $N$ moment equations from the kinetic equation. The number $N$ (the level at which the moment transport hierarchy is truncated) is not specified in the Grad method. One particular way to choose $N$ is to obtain an estimation of the transport coefficients (viscosity and heat conductivity) sufficiently close to their exact values provided by the ChapmanEnskog method (CE) [51]. In particular, for the 13-moment (13M) Grad approximation it is well known that transport coefficients are equal to the first Sonine polynomial approximation to the exact CE values. Accounting for higher moments with $N>13$ can improve this approximation (good for neutral gases but poor for plasmas [176]). However, what should be done, starting with the $13 \mathrm{M}$ approximation, to come to the exact CE transport coefficients is an open question. It is also well known [161] that the Grad method provides a poorly converging approximation when applied to strongly nonequilibrium problems (such as shock and kinetic layers).

Another question comes from the approximate character of the Grad equations, and is discussed in frames of the EIT: while the Grad equations are strictly hyperbolic at any level $N$ (i.e., predicting a finite speed of propagation), whether this feature will be preserved in the further corrections.

These two questions are special cases of a more general one, namely, how to derive a closed description with a given number of moments? Such a description is sometimes called mesoscopic 
[195] since it occupies an intermediate level between the hydrodynamic (macroscopic) and the kinetic (microscopic) levels of description.

Here we aim at deriving the mesoscopic dynamics of 13 moments [9] in the simplest case when the kinetic description satisfies the linearized Boltzmann equation. Our approach will be based on the two assumptions: (i). The mesoscopic dynamics of thirteen moments exists, and is invariant with respect to the microscopic dynamics, and (ii). The $13 \mathrm{M} \mathrm{Grad} \mathrm{approximation} \mathrm{is} \mathrm{a} \mathrm{suitable} \mathrm{first}$ approximation to this mesoscopic dynamics. The assumption (i) is realized as the invariance equation for the (unknown) mesoscopic distribution function. Following the assumption (ii), we solve the invariance equation iteratively, taking the $13 \mathrm{M}$ Grad approximation for the input approximation, and consider the first iteration (further we refer to this as to the dynamic correction, to distinguish from constructing another ansatz). We demonstrate that the correction results in the exact CE transport coefficients. We also demonstrate how the dynamic correction modifies the hyperbolicity of the Grad equations. A similar viewpoint on derivation of hydrodynamics was earlier developed in [6] (see previous examples). We will return to a comparison below.

Invariance equation for $13 \mathrm{M}$ parameterization. We denote as $n_{0}, \boldsymbol{u}_{0}=0$, and $p_{0}$ the equilibrium values of the hydrodynamic parameters ( $n$ is the number density, $\boldsymbol{u}$ is the average velocity, and $p=n k_{\mathrm{B}} T$ is the pressure). The global Maxwell distribution function $F$ is

$$
F=n_{0}\left(v_{T}\right)^{-3} \pi^{-3 / 2} \exp \left(-c^{2}\right)
$$

where $v_{T}=\sqrt{2 k_{\mathrm{B}} T_{0} m^{-1}}$ is the equilibrium thermal velocity, and $\boldsymbol{c}=\boldsymbol{v} / v_{T}$ is the peculiar velocity of a particle. The near-equilibrium dynamics of the distribution function, $f=F(1+\varphi)$, is due to the linearized Boltzmann equation:

$$
\begin{aligned}
\partial_{t} \varphi & =\hat{J} \varphi \equiv-v_{T} c_{i} \partial_{i} \varphi+\hat{L} \varphi, \\
\hat{L} \varphi & =\int w F\left(\boldsymbol{v}_{1}\right)\left[\varphi\left(\boldsymbol{v}_{1}^{\prime}\right)+\varphi\left(\boldsymbol{v}^{\prime}\right)-\varphi\left(\boldsymbol{v}_{1}\right)-\varphi(\boldsymbol{v})\right] \mathrm{d} \boldsymbol{v}_{1}^{\prime} \mathrm{d} \boldsymbol{v}^{\prime} \mathrm{d} \boldsymbol{v}_{1},
\end{aligned}
$$

where $\hat{L}$ is the linearized collision operator, and $w$ is the probability density of pair encounters. Furthermore, $\partial_{i}=\partial / \partial x_{i}$, and summation convention in two repeated indices is assumed.

Let $n=\delta n / n_{0}, \boldsymbol{u}=\delta \boldsymbol{u} / v_{T}, p=\delta p / p_{0}\left(p=n+T, T=\delta T / T_{0}\right)$, be dimensionless deviations of the hydrodynamic variables, while $\boldsymbol{\sigma}=\delta \boldsymbol{\sigma} / p_{0}$ and $\boldsymbol{q}=\delta \boldsymbol{q} /\left(p_{0} v_{T}\right)$ are dimensionless deviations of the stress tensor $\boldsymbol{\sigma}$, and of the heat flux $\boldsymbol{q}$. The linearized $13 \mathrm{M}$ Grad distribution function is $f_{0}=F(\boldsymbol{c})\left[1+\varphi_{0}\right]$, where

$$
\begin{aligned}
\varphi_{0} & =\varphi_{1}+\varphi_{2}, \\
\varphi_{1} & =n+2 u_{i} c_{i}+T\left[c^{2}-(3 / 2)\right], \\
\varphi_{2} & =\sigma_{i k} \overline{c_{i} c_{k}}+(4 / 5) q_{i} c_{i}\left[c^{2}-(5 / 2)\right] .
\end{aligned}
$$

The overline denotes a symmetric traceless dyad. We use the following convention:

$$
\begin{aligned}
& \overline{a_{i} b_{k}}=a_{i} b_{k}+a_{k} b_{i}-\frac{2}{3} \delta_{i k} a_{l} b_{l}, \\
& \overline{\partial_{i} f_{k}}=\partial_{i} f_{k}+\partial_{k} f_{i}-\frac{2}{3} \delta_{i k} \partial_{l} f_{l} .
\end{aligned}
$$

The 13M Grad's equations are derived in two steps: first, the 13M Grad's distribution function (272) is inserted into the linearized Boltzmann equation to give a formal expression, $\partial_{t} \varphi_{0}=\hat{J} \varphi_{0}$, 
second, projector $P_{0}$ is applied to this expression, where $P_{0}=P_{1}+P_{2}$, and operators $P_{1}$ and $P_{2}$ act as follows:

$$
\begin{aligned}
& P_{1} J=\frac{F}{n_{0}}\left\{X_{0} \int X_{0} J \mathrm{~d} \boldsymbol{v}+X_{i} \int X_{i} J \mathrm{~d} \boldsymbol{v}+X_{4} \int X_{4} J \mathrm{~d} \boldsymbol{v}\right\}, \\
& P_{2} J=\frac{F}{n_{0}}\left\{Y_{i k} \int Y_{i k} J \mathrm{~d} \boldsymbol{v}+Z_{i} \int Z_{i} J \mathrm{~d} \boldsymbol{v}\right\} .
\end{aligned}
$$

Here $X_{0}=1, X_{i}=\sqrt{2} c_{i}$, where $i=1,2,3, X_{4}=\sqrt{2 / 3}\left(c^{2}-\frac{3}{2}\right), Y_{i k}=\sqrt{2} \overline{c_{i} c_{k}}$, and $Z_{i}=\frac{2}{\sqrt{5}} c_{i}\left(c^{2}-\frac{5}{2}\right)$. The resulting equation,

$$
P_{0}\left[F \partial_{t} \varphi_{0}\right]=P_{0}\left[F \hat{J} \varphi_{0}\right],
$$

is a compressed representation for the $13 \mathrm{M}$ Grad equations for the macroscopic variables $M_{13}=\{n, \boldsymbol{u}, T, \boldsymbol{\sigma}, \boldsymbol{q}\}$.

Now we turn to the main purpose of this paper, and derive the dynamic correction to the $13 \mathrm{M}$ distribution function (272). Assumption (i) [existence of closed dynamics of thirteen moments] implies the invariance equation for the true mesoscopic distribution function, $\tilde{f}\left(M_{13}, \boldsymbol{c}\right)=F\left[1+\tilde{\varphi}\left(M_{13}, \boldsymbol{c}\right)\right]$, where we have stressed that this function depends parametrically on the same 13 macroscopic parameters, as the original Grad approximation. The invariance condition for $\tilde{f}\left(M_{13}, \boldsymbol{c}\right) \operatorname{reads}[6]$

$$
(1-\tilde{P})[F \hat{J} \tilde{\varphi}]=0,
$$

where $\tilde{P}$ is the projector associated with $\tilde{f}$. Generally speaking, the projector $\tilde{P}$ depends on the distribution function $\tilde{f}[6,176]$. In the following, we use the projector $P_{0}$ (273) which will be consistent with our approximate treatment of Eq. (274).

Following assumption (ii) [13M Grad's distribution function (272) is a good initial approximation], the Grad's function $f_{0}$, and the projector $P_{0}$, are chosen as the input data for solving Eq. (274) iteratively. The dynamic correction amounts to the first iterate. Let us consider these steps in a more detail.

Substituting $\varphi_{0}(272)$ and $P_{0}(273)$ instead of $\varphi$ and $P$ in the Eq. (274), we get: $\left(1-P_{0}\right)\left[F \hat{J} \varphi_{0}\right] \equiv$ $\Delta_{0} \neq 0$, which demonstrates that (272) is not a solution to Eq. (274). Moreover, $\Delta_{0}$ splits in two natural pieces: $\Delta_{0}=\Delta_{0}^{\text {loc }}+\Delta_{0}^{\text {nloc }}$, where

$$
\begin{aligned}
& \Delta_{0}^{\text {loc }}=\left(1-P_{2}\right)\left[F \hat{L} \varphi_{2}\right], \\
& \Delta_{0}^{\text {nloc }}=\left(1-P_{0}\right)\left[-v_{T} F c_{i} \partial_{i} \varphi_{0}\right] .
\end{aligned}
$$

Here we have accounted for $P_{1}[F \hat{L} \varphi]=0$, and $\hat{L} \varphi_{1}=0$. The first piece of Eq. (275), $\Delta_{0}^{\text {loc }}$, can be termed local because it does not account for spatial gradients. Its origin is twofold. In the first place, recall that we are performing our analysis in a nonlocal-equilibrium state (the $13 \mathrm{M}$ approximation is not a zero point of the Boltzmann collision integral, hence $\hat{L} \varphi_{0} \neq 0$ ). In the second place, specializing to the linearized case under consideration, functions $\overline{c c}$ and $\boldsymbol{c}\left[c^{2}-(5 / 2)\right]$, in general, are not the eigenfunctions of the linearized collision integral, and hence $P_{2}\left[F \hat{L} \varphi_{0}\right] \neq F \hat{L} \varphi_{0}$, resulting in $\Delta_{0}^{\text {loc }} \neq 0 .{ }^{11}$

\footnotetext{
${ }^{11}$ Except for Maxwellian molecules (interaction potential $U \sim r^{-4}$ ) for which $\hat{L} \varphi_{0} \neq 0$ but $P_{2}\left[F \hat{L} \varphi_{\mathrm{G}}\right]=F \hat{L} \varphi_{0}$. Same goes for the relaxation time approximation of the collision integral $\left(\hat{L}=-\tau^{-1}\right)$.
} 
The nonlocal part may be written as

$$
\Delta_{0}^{\mathrm{nloc}}=-v_{T} F\left(\Pi_{1 \mid k r s} \partial_{k} \sigma_{r s}+\Pi_{2 \mid i k} \overline{\partial_{k} q_{i}}+\Pi_{3} \partial_{k} q_{k}\right),
$$

where $\Pi$ are velocity polynomials:

$$
\begin{aligned}
& \Pi_{1 \mid k r s}=c_{k}\left[c_{r} c_{s}-(1 / 3) \delta_{r s} c^{2}\right]-(2 / 5) \delta_{k s} c_{r} c^{2}, \\
& \Pi_{2 \mid i k}=(4 / 5)\left[c^{2}-(7 / 2)\right]\left[c_{i} c_{k}-(1 / 3) \delta_{i k} c^{2}\right], \\
& \Pi_{3}=(4 / 5)\left[c^{2}-(5 / 2)\right]\left[c^{2}-(3 / 2)\right]-c^{2} .
\end{aligned}
$$

We seek the dynamic correction of the form

$$
f=F\left[1+\varphi_{0}+\phi\right] .
$$

Substituting $\varphi=\varphi_{0}+\phi$, and $P=P_{0}$, into Eq. (274), we derive an equation for the correction $\phi$ :

$$
\left(1-P_{2}\right)\left[F \hat{L}\left(\varphi_{2}+\phi\right)\right]=\left(1-P_{0}\right)\left[v_{T} F c_{i} \partial_{i}\left(\varphi_{0}+\phi\right)\right] .
$$

Eq. (277) should be supplied with the additional condition, $P_{0}[F \phi]=0$.

Solution of the invariance equation. Let us apply the usual ordering to solve Eq. (277), introducing a small parameter $\epsilon$, multiplying the collision integral $\hat{L}$ with $\epsilon^{-1}$, and expanding $\phi=\sum_{n} \epsilon^{n} \phi^{(n)}$. Subject to the additional condition, the resulting sequence of linear integral equations is uniquely soluble. Let us consider the first two orders in $\epsilon$.

Because $\Delta_{0}^{\text {loc }} \neq 0$, the leading correction is of the order $\epsilon^{0}$, i.e. of the same order as the initial approximation $\varphi_{0}$. The function $\phi^{(0)}$ is due the following equation:

$$
\left(1-P_{2}\right)\left[F \hat{L}\left(\varphi_{2}+\phi^{(0)}\right)\right]=0,
$$

subject to the condition, $P_{0}\left[F \phi^{(0)}\right]=0$. Eq. (278) has the unique solution: $\varphi_{2}+\phi^{(0)}=\sigma_{i k} Y_{i k}^{(0)}+q_{i} Z_{i}^{(0)}$, where functions, $Y_{i k}^{(0)}$ and $Z_{i}^{(0)}$, are solutions to the integral equations:

$$
\hat{L} Y_{i k}^{(0)}=b Y_{i k}, \quad \hat{L} Z_{i}^{(0)}=a Z_{i}
$$

subject to the conditions, $P_{1}\left[F Y^{(0)}\right]=0$ and $P_{1}\left[F \mathbf{Z}^{(0)}\right]=0$. Factors $a$ and $b$ are:

$$
\begin{gathered}
a=\pi^{-3 / 2} \int \mathrm{e}^{-c^{2}} Z_{i}^{(0)} \hat{L} Z_{i}^{(0)} \mathrm{d} c, \\
b=\pi^{-3 / 2} \int \mathrm{e}^{-c^{2}} Y_{i k}^{(0)} \hat{L} Y_{i k}^{(0)} \mathrm{d} c .
\end{gathered}
$$

Now we are able to notice that Eq. (279) coincides with the CE equations [51] for the exact transport coefficients (viscosity and temperature conductivity). Emergency of these well known equations in the present context is important and rather unexpected: when the moment transport equations are closed with the locally corrected function $f^{\text {loc }}=F\left(1+\varphi_{0}+\phi^{(0)}\right)$, we come to a closed set of thirteen equations containing the exact $C E$ transport coefficients.

Let us analyze the next order $\left(\epsilon^{1}\right)$, where $\Delta_{0}^{\text {nloc }}$ comes into play. To simplify matters, we neglect the difference between the exact and the approximate $\mathrm{CE}$ transport coefficients. The correction $\phi^{(1)}$ is due to the equation,

$$
\left(1-P_{2}\right)\left[F \hat{L} \phi^{(1)}\right]+\Delta_{0}^{\text {nloc }}=0,
$$


the additional condition is: $P_{0}\left[F \phi^{(1)}\right]=0$. Problem (280) reduces to three integral equations of a familiar form

$$
\hat{L} \Psi_{1 \mid k r s}=\Pi_{1 \mid k r s}, \quad \hat{L} \Psi_{2 \mid i k}=\Pi_{2 \mid i k}, \quad \hat{L} \Psi_{3}=\Pi_{3},
$$

subject to conditions: $P_{1}\left[F \Psi_{1 \mid k r s}\right]=0, P_{1}\left[F \Psi_{2 \mid i k}\right]=0$, and $P_{1}\left[F \Psi_{3}\right]=0$. Integral equations $(281)$ are of the same structure as are the integral equations appearing in the CE method, and the methods to handle them are well developed [51]. In particular, a reasonable and simple approximation is to take $\Psi_{\alpha \mid \ldots}=-A_{\alpha} \Pi_{\alpha \mid \ldots}$. Then

$$
\phi^{(1)}=-v_{T}\left(A_{1} \Pi_{1 \mid k r s} \partial_{k} \sigma_{r s}+A_{2} \Pi_{2 \mid i k} \overline{\partial_{k} q_{i}}+A_{3} \Pi_{3} \partial_{k} q_{k}\right),
$$

where $A_{\alpha}$ are the approximate values of the kinetic coefficients, and which are expressed via matrix elements of the linearized collision integral:

$$
A_{\alpha}^{-1} \propto-\int \exp \left(-c^{2}\right) \Pi_{\alpha \mid \ldots} \hat{L} \Pi_{\alpha \mid \ldots} \mathrm{d} c>0 .
$$

The estimation can be extended to a computational scheme for any given molecular model (e.g., for the Lennard-Jones potential), in the manner of the transport coefficients computations in the $\mathrm{CE}$ method.

Corrected $13 \mathrm{M}$ equations. To summarize the results of the dynamic correction, we quote first the unclosed equations for the variables $M_{13}=M_{13}=\{n, \boldsymbol{u}, T, \boldsymbol{\sigma}, \boldsymbol{q}\}$ :

$$
\begin{aligned}
& \left(1 / v_{T}^{0}\right) \partial_{t} n+\partial_{i} u_{i}=0, \\
& \left(2 / v_{T}^{0}\right) \partial_{t} u_{i}+\partial_{i}(T+n)+\partial_{k} \sigma_{i k}=0, \\
& \left(1 / v_{T}^{0}\right) \partial_{t} T+(2 / 3) \partial_{i} u_{i}+(2 / 3) \partial_{i} q_{i}=0, \\
& \left(1 / v_{T}^{0}\right) \partial_{t} \sigma_{i k}+2 \overline{\partial_{i} u_{k}}-(2 / 3) \overline{\partial_{i} q_{k}}+\partial_{l} h_{i k l}=R_{i k}, \\
& \left(2 / v_{T}\right) \partial_{t} q_{i}-(5 / 2) \partial_{i} p-(5 / 2) \partial_{k} \sigma_{i k}+\partial_{k} g_{i k}=R_{i} .
\end{aligned}
$$

Terms spoiling the closure are: the higher moments of the distribution function,

$$
\begin{gathered}
h_{i k l}=2 \pi^{-3 / 2} \int \mathrm{e}^{-c^{2}} \varphi c_{i} c_{k} c_{l} \mathrm{~d} c, \\
g_{i k}=2 \pi^{-3 / 2} \int \mathrm{e}^{-c^{2}} \varphi c_{i} c_{k} c^{2} \mathrm{~d} c,
\end{gathered}
$$

and the "moments" of the collision integral,

$$
\begin{aligned}
R_{i k} & =\frac{2}{v_{T}} \pi^{-3 / 2} \int \mathrm{e}^{-c^{2}} c_{i} c_{k} \hat{L} \varphi \mathrm{d} c, \\
R_{i} & =\frac{2}{v_{T}} \pi^{-3 / 2} \int \mathrm{e}^{-c^{2}} c_{i} c^{2} \hat{L} \varphi \mathrm{d} c .
\end{aligned}
$$


The 13M Grad's distribution function (272) provides the zeroth-order closure approximation to both the higher-order moments and the "moments" of the collision integral:

$$
\begin{aligned}
& R_{i k}^{(0)}=-\mu_{0}^{-1} \sigma_{i k}, \quad R_{i}^{(0)}=-\lambda_{0}^{-1} q_{i}, \\
& \partial_{l} h_{i k l}^{(0)}=(2 / 3) \delta_{i k} \partial_{l} q_{l}+(4 / 5) \overline{\partial_{i} q_{k}}, \\
& \partial_{l} g_{l k}^{(0)}=(5 / 2) \partial_{k}(p+T)+(7 / 2) \partial_{l} \sigma_{l k},
\end{aligned}
$$

where $\mu_{0}$ and $\lambda_{0}$ are the first Sonine polynomial approximations to the viscosity and the temperature conductivity coefficients [51], respectively.

The local correction improves the closure of the "moments" of collision integral:

$$
R_{i k}=-\mu_{\mathrm{CE}}^{-1} \sigma_{i k}, \quad R_{i}=-\lambda_{\mathrm{CE}}^{-1} q_{i},
$$

where index CE corresponds to exact Chapman-Enskog values of the transport coefficients.

The nonlocal correction adds the following terms to the higher moments:

$$
\begin{gathered}
\partial_{l} g_{l k}=\partial_{l} g_{l k}^{(0)}-A_{3} \partial_{k} \partial_{l} q_{l}-A_{2} \partial_{l} \overline{\partial_{l} q_{k}}, \\
\partial_{l} h_{i k l}=\partial_{l} h_{i k l}^{(0)}-A_{1} \partial_{l} \partial_{l} \sigma_{i k},
\end{gathered}
$$

where $A_{i}$ are the kinetic coefficients derived above.

In order to illustrate what changes in Grad equations with the nonlocal correction, let us consider a model with two scalar variables, $T(x, t)$ and $q(x, t)$ (a simplified case of the one-dimensional corrected $13 \mathrm{M}$ system where one retains only the variables responsible for heat conduction):

$$
\partial_{t} T+\partial_{x} q=0, \quad \partial_{t} q+\partial_{x} T-a \partial_{x}^{2} q+q=0 .
$$

Parameter $a \geqslant 0$ controls "turning on" the nonlocal correction. Using $\{q(k, \omega), T(k, \omega)\} \exp (\omega t+\mathrm{i} k x)$, we come to a dispersion relation for the two roots $\omega_{1,2}(k)$. Without the correction $(a=0)$, there are two domains of $k$ : for $0 \leqslant k<k_{-}$, dispersion is diffusion-like $\left(\operatorname{Re} \omega_{1,2}(k) \leqslant 0, \operatorname{Im} \omega_{1,2}(k)=0\right)$, while as $k \geqslant k_{-}$, dispersion is wave-like $\left(\omega_{1}(k)=\omega_{2}^{*}(k), \operatorname{Im} \omega_{1}(k) \neq 0\right)$. For $a$ between 0 and 1 , the dispersion modifies in the following way: The wave-like domain becomes bounded, and exists for $k \in] k_{-}(a), k_{+}(a)$ [, while the diffusion-like domain consists of two pieces, $k<k_{-}(a)$ and $k>k_{+}(a)$.

The dispersion relation for $a=1 / 2$ is shown in Fig. 7. As $a$ increases to 1 , the boundaries of the wave-like domain, $k_{-}(a)$ and $k_{+}(a)$, move towards each other, and collapse at $a=1$. For $a>1$, the dispersion relation becomes purely diffusive $\left(\operatorname{Im} \omega_{1,2}=0\right)$ for all $k$.

Discussion: transport coefficients, destroying of the hyperbolicity, etc.

(i) Considering the $13 \mathrm{M}$ Grad ansatz as a suitable approximation to the closed dynamics of thirteen moments, we have found that the first correction leads to exact Chapman-Enskog transport coefficients. Further, the nonlocal part of this correction extends the Grad equations with terms containing spatial gradients of the heat flux and of the stress tensor, destroying the hyperbolic nature of the former. Corresponding kinetic coefficients are explicitly derived for the Boltzmann equation.

(ii) Extension of Grad equations with terms like in (291) was mentioned in many versions of the EIT [196]. These derivations were based on phenomenological and semi-phenomenological argument. In particular, the extension of the heat flux with appealing to nonlocality effects in dense fluids. Here 


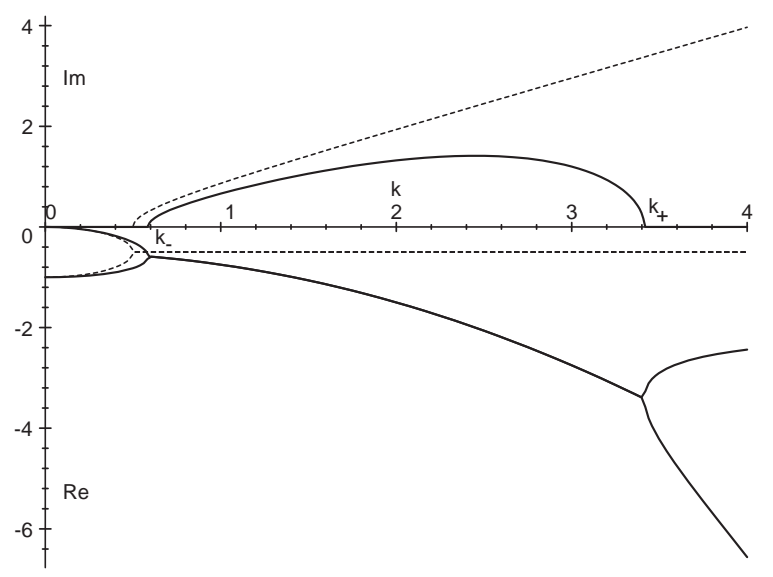

Fig. 7. Attenuation $\operatorname{Re} \omega_{1,2}(k)$ (lower pair of curves), frequency $\operatorname{Im} \omega_{1,2}(k)$ (upper pair of curves). Dashed lines—Grad case $(a=0)$, drawn lines-dynamic correction $(a=0.5)$.

we have derived the similar contribution from the simplest (i.e. dilute gas) kinetics, in fact, from the assumption about existence of the mesoscopic dynamics. The advantage of using the simplest kinetics is that corresponding kinetic coefficients (283) become a matter of a computation for any molecular model. This computational aspect will be discussed elsewhere, since it affects the dilute gas contribution to dense fluids fits. Here we would like to stress a formal support of relevancy of the above analysis: the nonlocal peace of dynamic correction is intermediated by the local correction, improving the $13 \mathrm{M}$ Grad estimation to the ordinary transport coefficients.

(iii) When the invariance principle is applied to derive hydrodynamics (closed equations for the variables $n, \boldsymbol{u}$ and $T$ ) then [6] the local Maxwellian $f_{\mathrm{lm}}$ is chosen as the input distribution function for the invariance equation. In the linear domain, $f_{\mathrm{lm}}=F\left[1+\varphi_{1}\right]$, and the projector is $P_{\operatorname{lm}}=P_{1}$, see Eqs. (272) and (273). When the latter expressions are substituted into the invariance equation (274), we obtain $\Delta_{\mathrm{lm}}=\Delta_{\mathrm{lm}}^{\text {nloc }}=-v_{T} F\left\{2 \partial_{i} u_{k} \overline{c_{i} c_{k}}+\partial_{i} T c_{i}\left[c^{2}-(5 / 2)\right]\right\}$, while $\Delta_{\mathrm{lm}}^{\mathrm{loc}} \equiv 0$ because the local Maxwellians are zero points of the Boltzmann collision integral. Consequently, the dynamic correction begins with the order $\epsilon$, and the analog of Eq. (280) reads:

$$
\hat{L} \phi_{\mathrm{lm}}^{(1)}=v_{T}\left\{2 \partial_{i} u_{k} \overline{c_{i} c_{k}}+\partial_{i} T c_{i}\left[c^{2}-(5 / 2)\right]\right\},
$$

subject to a condition, $P_{1}\left[F \phi_{1 \mathrm{~m}}^{(1)}\right]=0$. The latter is the familiar Chapman-Enskog equation, resulting in the Navier-Stokes correction to the Euler equations [51]. Thus, the nonlocal dynamic correction is related to the 13M Grad equations entirely in the same way as the Navier-Stokes are related to the Euler equations. As the final comment to this point, it was recently demonstrated with simple examples [24] that the invariance principle, as applied to derivation of hydrodynamics, is equivalent to the summation of the Chapman-Enskog expansion.

(iv) Let us discuss briefly the further corrections. The first local correction (the functions $Y_{1}$ and $Z_{1}$ in Eq. (279)) is not the limiting point of our iterational procedure. When the latter is continued, the subsequent local corrections are found from integral equations, $\hat{L} Y_{n+1}=b_{n+1} Y_{n}$, and $\hat{L} \mathbf{Z}_{n+1}=a_{n+1} \mathbf{Z}_{n}$. Thus, we are led to the following two eigenvalue problems: $\hat{L} \mathbf{Y}_{\infty}=b_{\infty} \mathrm{Y}_{\infty}$, and $\hat{L} \mathbf{Z}_{\infty}=a_{\infty} \mathbf{Z}_{\infty}$, 
where, in accord with general argument [6], $a_{\infty}$ and $b_{\infty}$ are the closest to zero eigenvalues among all the eigenvalue problems with the given tensorial structure [192].

(v) Approach of this example [9] can be extended to derive dynamic corrections to other (nonmoment) approximations of interest in the kinetic theory. The above analysis has demonstrated, in particular, the importance of the local correction, generically relevant to an approximation which is not a zero point of the collision integral. Very recently, this approach was successfully applied to improve the nonlinear Grad's 13-moment equations [197].

\section{Decomposition of motions, nonuniqueness of selection of fast motions, self-adjoint linearization, Onsager filter and quasi-chemical representation}

In Section 5 we used the second law of thermodynamics-existence of the entropy-in order to equip the problem of constructing slow invariant manifolds with a geometric structure. The requirement of the entropy growth (universally, for all the reduced models) restricts significantly the form of projectors (132).

In this section we introduce a different but equally important argument- the micro-reversibility ( $T$-invariance), and its macroscopic consequences, the reciprocity relations. As first discussed by Onsager in 1931 [150], the implication of the micro-reversibility is the self-adjointness of the linear approximation of system (76) in the equilibrium $x^{*}$ :

$$
\left\langle\left(D_{x} J\right)_{x^{*}} z \mid p\right\rangle_{x^{*}} \equiv\left\langle z \mid\left(D_{x} J\right)_{x^{*}} p\right\rangle_{x^{*}} .
$$

The main idea in the present section is to use the reciprocity relations (293) for the fast motions. In order to appreciate this idea, we should mention that the decomposition of motions into fast and slow is not unique. Requirement (293) for any equilibrium point of fast motions means the selection (filtration) of the fast motions. We term this Onsager filter. Equilibrium points of fast motions are all the points on manifolds of slow motions.

There exists a trivial way to symmetrization, linear operator $A$ is decomposed into symmetric and skew-symmetric parts, $A=\frac{1}{2}\left(A+A^{\dagger}\right)+\frac{1}{2}\left(A-A^{\dagger}\right)$. Here $A^{\dagger}$ is adjoint to $A$ with respect to a fixed scalar product (entropic scalar product in present context). However, replacement of an operator with its symmetric part can lead to catastrophic (from the physical standpoint) consequences such as, for example, loss of stability. In order to construct a sensible Onsager filter, we shall use the quasi-chemical representation.

The formalism of the quasi-chemical representation is one of the most developed means of modelling, it makes it possible to "assemble" complex processes out of elementary processes. There exist various presentations of the quasi-chemical formalism. Our presentation here is a generalization of the approach suggested first by Feinberg [187] (see also [186,188,59]).

Symbol $A_{i}$ ("quasi-substance") is put into correspondence to each variable $x_{i}$. The elementary reaction is defined accordingly to the stoichiometric equation,

$$
\sum_{i} \alpha_{i} A_{i} \rightleftharpoons \sum_{i} \beta_{i} A_{i}
$$

where $\alpha_{i}$ (loss stoichiometric coefficients) and $\beta_{i}$ (gain stoichiometric coefficients) are real numbers. Apart from the entropy, one specifies a monotonic function of one variable, $\Psi(a), \Psi^{\prime}(a)>0$. In particular, function $\Psi(a)=\exp (\lambda a), \lambda=$ const, is frequently encountered in applications. 
Given the elementary reaction (294), one defines the rates of the direct and of the inverse reactions:

$$
\begin{aligned}
& W^{+}=w^{*} \Psi\left(\sum_{i} \alpha_{i} \mu_{i}\right), \\
& W^{-}=w^{*} \Psi\left(\sum_{i} \beta_{i} \mu_{i}\right),
\end{aligned}
$$

where $\mu_{i}=\partial S / \partial x_{i}, x^{*}=$ const, $x^{*}>0$. The rate of the elementary reaction is then defined as, $W=W^{+}-W^{-}$.

The equilibrium of the elementary reaction (294) is given by the following equation:

$$
W^{+}=W^{-} \text {. }
$$

Thanks to the strict monotonicity of the function $\Psi$, equilibrium of the elementary reaction is reached when the arguments of the functions coincide in Eq. (295), that is, whenever

$$
\sum_{i}\left(\beta_{i}-\alpha_{i}\right) \mu_{i}=0
$$

Vector with the components $\gamma_{i}=\beta_{i}-\alpha_{i}$ is termed the stoichiometric vector of the reaction.

Let $x^{0}$ be a point of equilibrium of reaction (294). The linear approximation of the reaction rate has a particularly simple form:

$$
W\left(x^{0}+\delta\right)=-w^{*} \Psi^{\prime}\left(a\left(x^{0}\right)\right)\langle\gamma \mid \delta\rangle_{x^{0}}+\mathrm{o}(\delta),
$$

where $a\left(x^{0}\right)=\sum_{i} \alpha_{i} \mu_{i}\left(x^{0}\right)=\sum_{i} \beta_{i} \mu_{i}\left(x^{0}\right)$, and $\langle\mid\rangle_{x^{0}}$ is the entropic scalar product in the equilibrium. In other words,

$$
\left(D_{x} W\right)_{x^{0}}=-w^{*} \Psi^{\prime}\left(a\left(x^{0}\right)\right)\langle\gamma| .
$$

Let us write down the kinetic equation for one elementary reaction:

$$
\frac{\mathrm{d} x}{\mathrm{~d} t}=\gamma W(x) .
$$

Linearization of this equation in the equilibrium $x^{0}$ has the following form:

$$
\frac{\mathrm{d} \delta}{\mathrm{d} t}=-w^{*} \Psi^{\prime}\left(a\left(x^{0}\right)\right) \gamma\langle\gamma \mid \delta\rangle_{x^{0}} .
$$

That is, the matrix of the linear approximation has the form

$$
K=-k^{*}|\gamma\rangle\langle\gamma|
$$

where

$$
k^{*}=w^{*} \Psi^{\prime}\left(a\left(x^{0}\right)\right)>0,
$$

while the entropic scalar product of bra- and ket vectors is taken in the equilibrium point $x^{0}$.

If there are several elementary reactions, then the stoichiometric vectors $\gamma^{r}$ and the reaction rates $W_{r}(x)$ are specified for each reaction, while the kinetic equation is obtained by summing the right hand sides of Eq. (300) for individual reactions,

$$
\frac{\mathrm{d} x}{\mathrm{~d} t}=\sum_{r} \gamma^{r} W_{r}(x) \text {. }
$$


Let us assume that under the inversion of motions, the direct reaction transforms into the inverse reaction. Thus, the $T$-invariance of the equilibrium means that it is reached in the point of the detailed balance, where all the elementary reaction come to equilibrium simultaneously:

$$
W_{r}^{+}\left(x^{*}\right)=W_{r}^{-}\left(x^{*}\right) .
$$

This assumption is nontrivial if vectors $\gamma^{r}$ are linearly dependent (for example, if the number of reactions is greater than the number of species minus the number of conservation laws).

In the detailed balance case, the linearization of Eq. (303) about $x^{*}$ has the following form $\left(x=x^{*}+\delta\right)$ :

$$
\frac{\mathrm{d} \delta}{\mathrm{d} t}=-\sum_{r} k_{r}^{*} \gamma^{r}\left\langle\gamma^{r} \mid \delta\right\rangle_{x^{*}},
$$

where

$$
\begin{aligned}
k_{r}^{*} & =w_{r}^{*} \Psi_{r}^{\prime}\left(a_{r}^{*}\right)>0, \\
a_{r}^{*} & =\sum_{i} \alpha_{i}^{r} \mu_{i}\left(x^{*}\right)=\sum_{i} \beta_{i}^{r} \mu_{i}\left(x^{*}\right) .
\end{aligned}
$$

The following matrix of the linear approximation is obviously self-adjoint and stable:

$$
K=-\sum_{r} k_{r}^{*}\left|\gamma^{r}\right\rangle\left\langle\gamma^{r}\right| \text {. }
$$

Note that matrix $K$ is the sum of matrices of rank one.

Let us now extract the self-adjoint part of form (306) in the arbitrary point $x$. After linearizing the reaction rate about $x$, we obtain

$$
W(x+\delta)=w^{*}\left(\Psi^{\prime}(a(x))\langle\alpha \mid \delta\rangle_{x}-\Psi^{\prime}(b(x))\langle\beta \mid \delta\rangle_{x}\right)+\mathrm{o}(\delta),
$$

where

$$
\begin{aligned}
a(x) & =\sum_{i} \alpha_{i} \mu_{i}(x), \\
b(x) & =\sum_{i} \beta_{i} \mu_{i}(x) .
\end{aligned}
$$

Let us introduce notation,

$$
\begin{aligned}
& k^{\mathrm{SYM}}(x)=\frac{1}{2} w^{*}\left(\Psi^{\prime}(a(x))+\Psi^{\prime}(b(x))\right)>0, \\
& k^{\mathrm{A}}(x)=\frac{1}{2} w^{*}\left(\Psi^{\prime}(a(x))-\Psi^{\prime}(b(x))\right) .
\end{aligned}
$$

In terms of this notation, Eq. (307) may be rewritten as

$$
W(x+\delta)=-k^{\mathrm{SYM}}(x)\langle\gamma \mid \delta\rangle_{x}+k^{\mathrm{A}}(x)\langle\alpha+\beta \mid \delta\rangle_{x}+\mathrm{o}(\delta) .
$$

The second term vanishes in the equilibrium $\left(k^{\mathrm{A}}\left(x^{*}\right)=0\right.$, due to detailed balance).

Symmetric linearization (Onsager filter) consists in using only the first term in the linearized vector field (308) when analyzing the fast motion towards the (approximate) slow manifolds, instead 
of the full expression (307). Matrix $K(x)$ of the linear approximation becomes then the form similar to Eq. (306):

$$
K(x)=-\sum_{r} k_{r}^{\mathrm{SYM}}(x)\left|\gamma^{r}\right\rangle\left\langle\gamma^{r}\right|,
$$

where

$$
\begin{aligned}
& k_{r}^{\mathrm{SYM}}(x)=\frac{1}{2} w_{r}^{*}\left(\Psi_{r}^{\prime}(a(x))+\Psi_{r}^{\prime}(b(x))\right)>0, \\
& a_{r}(x)=\sum_{i} \alpha_{i}^{r} \mu_{i}(x), \\
& b_{r}(x)=\sum_{i} \beta_{i}^{r} \mu_{i}(x),
\end{aligned}
$$

while the entropic scalar product $\langle\mid\rangle_{x}$ is taken at the point $x$. For each index of the elementary reaction $r$, function $k_{r}^{\mathrm{SYM}}(x)$ is positive. Thus, stability of the symmetric matrix (309) is evident.

Symmetric linearization (309) is distinguished also by the fact that it preserves the rank of the elementary processes contributing to the complex mechanism: Same as in the equilibrium, matrix $K(x)$ is the sum of rank one operators corresponding to each individual process. This is not the case of the standard symmetrization.

Using the symmetric operator (309) in the above Newton method with incomplete linearization can be considered as a version of a heuristic strategy of "we act in such a way as if the manifold $F(W)$ were already slow invariant manifold". If this were the case, then, in particular, the fast motions were described by the self-adjoint linear approximation.

We describe the quasi-chemical formalism for finite-dimensional systems. Infinite-dimensional generalizations are almost obvious in many important cases, and are achieved by a mere replacement of summation by integration. The best example give us collisions in the Boltzmann equation: each velocity $v$ corresponds to a quasi-substance $A_{v}$, and a collision has a stoichiometric equation:

$$
A_{v}+A_{w} \rightleftharpoons A_{v^{\prime}}+A_{w^{\prime}} .
$$

In the example to this section we consider the Boltzmann collision integral from this standpoint in more details.

Example 6: Quasi-chemical representation and self-adjoint linearization of the Boltzmann collision operator

A decomposition of motions near thermodynamically nonequilibrium states results in a linear relaxation towards this state. A linear operator of this relaxation is explicitly constructed in the case of the Boltzmann equation.

An entropy-related specification of an equilibrium state is due to the two points of view. From the first, thermodynamic viewpoint, equilibria is a state in which the entropy is maximal. From the second, kinetic viewpoint, a quadratic form of entropy increases in a course of a linear regression towards this state. If an underlying microscopic dynamics is time-reversible, the kinetic viewpoint is realized due to known symmetric properties of a linearized kinetic operator. 
In most of near-equilibrium studies, a principle of a decomposition of motions into rapid and slow occupies a distinct place. In some special cases, decomposition of motions is taken into account explicitly, by introducing a small parameter into dynamic equations. More frequently, however, it comes into play implicitly, for example, through an assumption of a rapid decay of memory in projection operator formalism [154]. Even in presence of long-living dynamic effects (mode coupling), a decomposition of motions appears as a final instance to get a closed set of equations for slow variables.

However, for closed systems, there remains a question: whether and to what extend the two aforementioned entropy-related points of view are applicable to nonequilibrium states? Further, if an answer is positive, then how to make explicitly a corresponding specification?

This example is aimed at answering the questions just mentioned, and it is a straightforward continuation of results $[4,6]$. Namely, in $[4,6]$, it was demonstrated that the principle of a decomposition of motions alone constitutes a necessary and sufficient condition for the thermodynamic specification of a nonequilibrium state (this will be briefly reviewed in the next section). However, in a general situation, one deals with states $f$ other than $f_{0}$. A question is, whether these two ideas can be applied to $f \neq f_{0}$ (at least approximately), and if so, then how to make the presentation explicit.

A positive answer to this question was given partially in frames of the method of invariant manifolds [4-6]. Objects studied in [4-6] were manifolds in a space of distribution functions, and the goal was to construct iteratively a manifold that is tangent in all its points to a vector field of a dissipative system (an invariant manifold), beginning with some initial manifold with no such property. It was natural to employ methods of KAM-theory (Newton-type linear iterations to improve the initial manifold). However, an extra idea of a decomposition of motions into rapid and slow near the manifold was strongly necessary to adapt KAM-theory to dissipative systems. A geometrical formulation of this idea [4-6] results in a definition of a hyperplane of rapid motion, $\Gamma_{f}$, associated with the state $f$, and orthogonal to the gradient of the entropy in $f$. In a physical interpretation, $\Gamma_{f}$ contains all those states from a neighborhood of $f$, which come into $f$ in the course of rapid relaxation (as if $f$ were the final state of rapid processes occurring in its neighborhood). Usually, $\Gamma_{f}$ contains more states than can come into $f$ in a rapid relaxation because of conservation of some macroscopic quantities (e.g. density, momentum, and energy, as well as, possibly, higher moments of $f$ which practically do not vary in rapid processes). Extra states are eliminated by imposing additional restrictions, cutting out "thinner" linear manifolds, planes of rapid motions $P_{f}$, inside $\Gamma_{f}$. Extremal property of $f$ on $\Gamma_{f}$ is preserved on $P_{f}$ as well (cf. [4-6]).

Thus, decomposition of motions near a manifold results in the thermodynamical viewpoint: states $f$ belonging to the manifold are described as unique points of maximum of entropy on corresponding hyperplanes of rapid motions $\Gamma_{f}$. This formulation defines a slow dynamics on manifolds in agreement with the $H$-theorem for the Boltzmann equation, or with its analogs for other systems (see [4-6] for details). As it was shown in [4-6], decomposition of motions in a neighborhood of $f$ is a criteria (a necessary and sufficient condition) of an existence of the thermodynamic description of $f$.

Newton iteration gives a correction, $f+\delta f$, to states of a noninvariant manifold, while $\delta f$ is thought on $\Gamma_{f}$. Equation for $\delta f$ involves a linearization of the collision integral in state $f$. Here, if $f \neq f_{0}$, we come to a problem of how to perform a linearization of collision integral in concordance with the $H$-theorem (corrections to the manifold of local equilibrium states were studied in detail in [6]). 
Here we show that the aforementioned decomposition of motions results in the kinetic description of states on manifolds of slow motions, and that Onsager's principle can be applied in a natural way to linearize the Boltzmann collision integral.

Due to definition of $\Gamma_{f}$, the state $f$ is the unique point of minimum of the $H$-function on $\Gamma_{f}$. In the first nonvanishing approximation, we have the following expression for $H$ in the states on $\Gamma_{f}$ :

$$
H(f+\delta f) \approx H(f)+\frac{1}{2}\langle\delta f \mid \delta f\rangle_{f}
$$

Here $\langle\cdot \mid \cdot\rangle_{f}$ denotes a scalar product generated by the second derivative of $H$ in the state $f:\left\langle g_{1} \mid g_{2}\right\rangle_{f}=$ $\int f^{-1} g_{1} g_{2} \mathrm{~d} \boldsymbol{v}$.

Decomposition of motions means that quadratic form $\langle\delta f \mid \delta f\rangle_{f}$ decays monotonically in the course of the linear relaxation towards the state $f$. It is natural, therefore, to impose the requirement that this linear relaxation should obey Onsager's principle. Namely, the corresponding linear operator should be symmetric (formally self-adjoint) and nonpositively definite in scalar product $\langle\cdot \mid \cdot\rangle_{f}$, and its kernel should consist of linear combinations of conserved quantities $\left(1, \boldsymbol{v}\right.$, and $\left.v^{2}\right)$. In other words, decomposition of motions should give a picture of linear relaxation in a small neighborhood of $f$ similar to that in a small neighborhood of $f_{0}$. Following this idea, we will now decompose the linearized collision integral $L_{f}$ in two parts: $L_{f}^{\mathrm{SYM}}$ (satisfying Onsager's principle), and $L_{f}^{\mathrm{A}}$ (nonthermodynamic part).

In the state $f$, each direct encounter, $\left(\boldsymbol{v}, \boldsymbol{v}_{1}\right) \rightarrow\left(\boldsymbol{v}^{\prime}, \boldsymbol{v}_{1}^{\prime}\right)$, together with the reverse encounter, $\left(\boldsymbol{v}^{\prime}, \boldsymbol{v}_{1}^{\prime}\right) \rightarrow\left(\boldsymbol{v}, \boldsymbol{v}_{1}\right)$, contribute a rate, $G(f)-L(f)$, to the collision integral, where (see Section 2$)$ :

$$
\begin{gathered}
W(f)=W\left(\boldsymbol{v}^{\prime}, \boldsymbol{v}_{1}^{\prime} ; \boldsymbol{v}, \boldsymbol{v}_{1}\right) \exp \left\{\left.D_{f} H\right|_{f=f(\boldsymbol{v})}+\left.D_{f} H\right|_{f=f\left(\boldsymbol{v}_{1}\right)}\right\} \\
W^{\prime}(f)=W\left(\boldsymbol{v}^{\prime}, \boldsymbol{v}_{1}^{\prime} ; \boldsymbol{v}, \boldsymbol{v}_{1}\right) \exp \left\{\left.D_{f} H\right|_{f=f\left(\boldsymbol{v}^{\prime}\right)}+\left.D_{f} H\right|_{f=f\left(\boldsymbol{v}_{1}^{\prime}\right)}\right\} ;
\end{gathered}
$$

A deviation $\delta f$ from the state $f$ will change the rates of both the direct and the reverse processes. Resulting deviations of rates are:

$$
\begin{aligned}
& \delta W=W(f)\left\{\left.D_{f}^{2} H\right|_{f=f(\boldsymbol{v})} \cdot \delta f(\boldsymbol{v})+\left.D_{f}^{2} H\right|_{f=f\left(\boldsymbol{v}_{1}\right)} \cdot \delta f\left(\boldsymbol{v}_{1}\right)\right\} \\
& \delta W^{\prime}=W^{\prime}(f)\left\{\left.D_{f}^{2} H\right|_{f=f\left(\boldsymbol{v}^{\prime}\right)} \cdot \delta f\left(\boldsymbol{v}^{\prime}\right)+\left.D_{f}^{2} H\right|_{f=f\left(\boldsymbol{v}_{1}^{\prime}\right)} \cdot \delta f\left(\boldsymbol{v}_{1}^{\prime}\right)\right\}
\end{aligned}
$$

Symmetrization with respect to direct and reverse encounters will give a term proportional to a balanced rate, $W^{\mathrm{SYM}}(f)=\frac{1}{2}\left(W(f)+W^{\prime}(f)\right)$, in both of the expressions $\delta W$ and $\delta W^{\prime}$. Thus, we come to the decomposition of the linearized collision integral $L_{f}=L_{f}^{\mathrm{SYM}}+L_{f}^{\mathrm{A}}$, where

$$
\begin{gathered}
L_{f}^{\mathrm{SYM}} \delta f=\int w \frac{f^{\prime} f_{1}^{\prime}+f f_{1}}{2}\left\{\frac{\delta f^{\prime}}{f^{\prime}}+\frac{\delta f_{1}^{\prime}}{f_{1}^{\prime}}-\frac{\delta f_{1}}{f_{1}}-\frac{\delta f}{f}\right\} \mathrm{d} \boldsymbol{v}_{1}^{\prime} \mathrm{d} \boldsymbol{v}^{\prime} \mathrm{d} \boldsymbol{v}_{1} \\
L_{f}^{\mathrm{A}} \delta f=\int w \frac{f^{\prime} f_{1}^{\prime}-f f_{1}}{2}\left\{\frac{\delta f^{\prime}}{f^{\prime}}+\frac{\delta f_{1}^{\prime}}{f_{1}^{\prime}}+\frac{\delta f_{1}}{f_{1}}+\frac{\delta f}{f}\right\} \mathrm{d} \boldsymbol{v}_{1}^{\prime} \mathrm{d} \boldsymbol{v}^{\prime} \mathrm{d} \boldsymbol{v}_{1} ; \\
f=f(\boldsymbol{v}), f_{1}=f\left(\boldsymbol{v}_{1}\right), f^{\prime}=f\left(\boldsymbol{v}^{\prime}\right), f_{1}^{\prime}=f\left(\boldsymbol{v}_{1}^{\prime}\right), \delta f=\delta f(\boldsymbol{v}), \delta f_{1}=\delta f\left(\boldsymbol{v}_{1}\right), \delta f^{\prime}=\delta f\left(\boldsymbol{v}^{\prime}\right), \delta f_{1}^{\prime}=\delta f\left(\boldsymbol{v}_{1}^{\prime}\right) .
\end{gathered}
$$


Operator $L_{f}^{\mathrm{SYM}}(310)$ has the complete set of the aforementioned properties corresponding to the Onsager's principle, namely:

(i) $\left\langle g_{1}\left|L_{f}^{\mathrm{SYM}}\right| g_{2}\right\rangle_{f}=\left\langle g_{2}\left|L_{f}^{\mathrm{SYM}}\right| g_{1}\right\rangle_{f}$ (symmetry);

(ii) $\left\langle g\left|L_{f}^{\mathrm{SYM}}\right| g\right\rangle_{f} \leqslant 0$ (local entropy production inequality);

(iii) $f, \boldsymbol{v} f, v^{2} f \in \operatorname{ker} L_{f}^{\mathrm{SYM}}$ (conservation laws).

For an unspecified $f$, nonthermodynamic operator $L_{f}^{\mathrm{A}}(311)$ satisfies none of these properties. If $f=f_{0}$, then part (311) vanishes, while operator $L_{f_{0}}^{\mathrm{SYM}}$ becomes the usual linearized collision integral due to the balance $W\left(f_{0}\right)=W^{\prime}\left(f_{0}\right)$.

Nonnegative definite form $\langle\delta f \mid \delta f\rangle_{f}$ decays monotonically due to the equation of linear relaxation, $\partial_{t} \delta f=L_{f}^{\mathrm{SYM}} \delta f$, and the unique point of minimum, $\delta f=0$, of $\langle\delta f \mid \delta f\rangle_{f}$ corresponds to the equilibrium point of vector field $L_{f}^{\mathrm{SYM}} \delta f$.

Operator $L_{f}^{\text {SYM }}$ describes the state $f$ as the equilibrium state of a linear relaxation. Note that the method of extracting the symmetric part (310) is strongly based on the representation of direct and reverse processes, and it is not a simple procedure like, e.g., $\frac{1}{2}\left(L_{f}+L_{f}^{+}\right)$. The latter expression cannot be used as a basis for Onsager's principle since it would violate conditions (ii) and (iii).

Thus, if motions do decompose into a rapid motion towards the manifold and a slow motion along the manifold, then states on this manifold can be described from both the thermodynamical and kinetic points of view. Our consideration results in an explicit construction of operator $L_{f}^{\mathrm{SYM}}$ (310) responsible for the rapid relaxation towards the state $f$. It can be used, in particular, for obtaining corrections to such approximations as the Grad moment approximations and the TammMott-Smith approximation, in frames of the method [4-6]. The nonthermodynamic part (311) is always present in $L_{f}$, when $f \neq f_{0}$, but if trajectories of an equation $\partial_{t} \delta f=L_{f} \delta f$ are close to trajectories of an equation $\partial_{t} \delta f=L_{f}^{\mathrm{SYM}} \delta f$, then $L_{f}^{\mathrm{SYM}}$ gives a good approximation to $L_{f}$. A conclusion on a closeness of trajectories depends on particular features of $f$, and normally it can be made on a base of a small parameter. On the other hand, the explicit thermodynamic and kinetic presentation of states on a manifold of slow motions (the extraction of $L_{f}^{\mathrm{SYM}}$ performed above and construction of hyper-planes $\Gamma_{f}$ [4-6]) is based only the very idea of a decomposition of motions, and can be obtained with no consideration of a small parameter. Finally, though we have considered only the Boltzmann equation, the method of symmetrization can be applied to other dissipative systems with the same level of generality as the method [4-6].

\section{Relaxation methods}

Relaxation method is an alternative to the Newton iteration method described in Section 6: The initial approximation to the invariant manifold $F_{0}$ is moved with the film extension, Eq. (84),

$$
\frac{\mathrm{d} F_{t}(y)}{\mathrm{d} t}=\left(1-P_{t, y}\right) J\left(F_{t}(y)\right)=\Delta_{F(y)},
$$

till a fixed point is reached. Advantage of this method is a relative freedom in its implementation, because Eq. (84) needs not be solved exactly, one is interested only in finding fixed points. Therefore, "large stepping" in the direction of the defect, $\Delta_{F(y)}$ is possible, the termination point is defined by 
the condition that the vector field becomes orthogonal to $\Delta_{F(y)}$. For simplicity, let us consider the procedure of termination in the linear approximation of the vector field. Let $F_{0}(y)$ be the initial approximation to the invariant manifold, and we seek the first correction,

$$
F_{1}(y)=F_{0}(y)+\tau_{1}(y) \Delta_{F_{0}(y)},
$$

where function $\tau(y)$ has dimension of time, and is found from the condition that the linearized vector field attached to the points of the new manifold is orthogonal to the initial defect,

$$
\left\langle\Delta_{F_{0}(y)} \mid\left(1-P_{y}\right)\left[J\left(F_{0}(y)\right)+\tau_{1}(y)\left(D_{x} J\right)_{F_{0}(y)} \Delta_{F_{0}(y)}\right]\right\rangle_{F_{0}(y)}=0 .
$$

Explicitly,

$$
\tau_{1}(y)=-\frac{\left\langle\Delta_{F_{0}(y)} \mid \Delta_{F_{0}(y)}\right\rangle_{F_{0}(y)}}{\left\langle\Delta_{F_{0}(y)}\left|\left(D_{x} J\right)_{F_{0}(y)}\right| \Delta_{F_{0}(y)}\right\rangle_{F_{0}(y)}} .
$$

Further steps $\tau_{k}(y)$ are found in the same way. It is clear from the latter equations that the step of the relaxation method for the film extension is equivalent to the Galerkin approximation for solving the step of the Newton method with incomplete linearization. Actually, the relaxation method was first introduced in these terms in [12]. A partially similar idea of using the explicit Euler method to approximate the finite-dimensional invariant manifold on the basis of spectral decomposition was proposed earlier in Ref. [13].

An advantage of Eq. (313) is the explicit form of the size of the steps $\tau_{k}(y)$. This method was successfully applied to the Fokker-Plank equation [12].

\section{Example 7: Relaxation method for the Fokker-Planck equation}

Here we address the problem of closure for the FPE (31) in a general setting. First, we review the maximum entropy principle as a source of suitable quasi-equilibrium initial approximations for the closures. We also discuss a version of the maximum entropy principle, valid for a near-equilibrium dynamics, and which results in explicit formulae for arbitrary $U$ and $D$.

In this Example we consider the FPE of form (31):

$$
\partial_{t} W(\boldsymbol{x}, t)=\partial_{x} \cdot\left\{D \cdot\left[W \partial_{x} U+\partial_{x} W\right]\right\} .
$$

Here $W(\boldsymbol{x}, t)$ is the probability density over the configuration space $\boldsymbol{x}$, at the time $t$, while $U(\boldsymbol{x})$ and $D(\boldsymbol{x})$ are the potential and the positively semi-definite $(y \cdot D \cdot y \geqslant 0)$ diffusion matrix.

Quasi-equilibrium approximations for the Fokker-Planck equation. The quasi-equilibrium closures are almost never invariants of the true moment dynamics. For corrections to the quasiequilibrium closures, we apply the method of invariant manifold [6], which is carried out (subject to certain approximations explained below) to explicit recurrence formulae for one-moment near-equilibrium closures for arbitrary $U$ and $D$. These formulae give a method for computing the lowest eigenvalue of the problem, and which dominates the near-equilibrium FPE dynamics. Results are tested with model potential, including the FENE-like potentials [113-115].

Let us denote as $M$ the set of linearly independent moments $\left\{M_{0}, M_{1}, \ldots, M_{k}\right\}$, where $M_{i}[W]=$ $\int m_{i}(x) W(x) \mathrm{d} x$, and where $m_{0}=1$. We assume that there exists a function $W^{*}(M, x)$ which extremizes the entropy $S$ (32) under the constrains of fixed $M$. This quasi-equilibrium distribution function 
may be written

$$
W^{*}=W_{\mathrm{eq}} \exp \left[\sum_{i=0}^{k} \Lambda_{i} m_{i}(x)-1\right]
$$

where $\Lambda=\left\{\Lambda_{0}, \Lambda_{1}, \ldots, \Lambda_{k}\right\}$ are Lagrange multipliers. Closed equations for moments $M$ are derived in two steps. First, the quasi-equilibrium distribution (315) is substituted into the FPE (314) or (33) to give a formal expression: $\partial_{t} W^{*}=\left.\hat{M}_{W^{*}}(\delta S / \delta W)\right|_{W=W^{*}}$. Second, introducing a projector $\Pi^{*}$,

$$
\Pi^{*} \bullet=\sum_{i=0}^{k}\left(\partial W^{*} / \partial M_{i}\right) \int m(x) \bullet \mathrm{d} x,
$$

and applying $\Pi^{*}$ on both sides of the formal expression, we derive closed equations for $M$ in the quasi-equilibrium approximation. Further processing requires an explicit solution to the constrains, $\int W^{*}(\Lambda, x) m_{i}(x) \mathrm{d} x=M_{i}$, to get the dependence of Lagrange multipliers $\Lambda$ on the moments $M$. Though typically the functions $\Lambda(M)$ are not known explicitly, one general remark about the moment equations is readily available. Specifically, the moment equations in the quasi-equilibrium approximation have the form

$$
\dot{M}_{i}=\sum_{j=0}^{k} M_{i j}^{*}(M)\left(\partial S^{*}(M) / \partial M_{j}\right)
$$

where $S^{*}(M)=S\left[W^{*}(M)\right]$ is the macroscopic entropy, and where $M_{i j}^{*}$ is an $M$-dependent $(k+1) \times$ $(k+1)$ matrix:

$$
M_{i j}^{*}=\int W^{*}(M, x)\left[\partial_{x} m_{i}(x)\right] \cdot D(x) \cdot\left[\partial_{x} m_{j}(x)\right] \mathrm{d} x .
$$

The matrix $M_{i j}^{*}$ is symmetric, positive semi-definite, and its kernel is the vector $\delta_{0 i}$. Thus, the quasi-equilibrium closure reproduces the GENERIC structure on the macroscopic level, the vector field of macroscopic equations (316) is a metric transform of the gradient of the macroscopic entropy.

The following version of the quasi-equilibrium closures makes it possible to derive more explicit results in the general case [190-192,178]: In many cases, one can split the set of moments $M$ in two parts, $M_{\mathrm{I}}=\left\{M_{0}, M_{1}, \ldots, M_{l}\right\}$ and $M_{\mathrm{II}}=\left\{M_{l+1}, \ldots, M_{k}\right\}$, in such a way that the quasi-equilibrium distribution can be constructed explicitly for $M_{\mathrm{I}}$ as $W_{\mathrm{I}}^{*}\left(M_{\mathrm{I}}, x\right)$. The full quasi-equilibrium problem for $M=\left\{M_{\mathrm{I}}, M_{\mathrm{II}}\right\}$ in the "shifted" formulation reads: extremize the functional $S\left[W_{\mathrm{I}}^{*}+\Delta W\right]$ with respect to $\Delta W$, under the constrains $M_{\mathrm{I}}\left[W_{\mathrm{I}}^{*}+\Delta W\right]=M_{\mathrm{I}}$ and $M_{\mathrm{II}}\left[W_{\mathrm{I}}^{*}+\Delta W\right]=M_{\mathrm{II}}$. Let us denote as $\Delta M_{\mathrm{II}}=M_{\mathrm{II}}-M_{\mathrm{II}}\left(M_{\mathrm{I}}\right)$ deviations of the moments $M_{\mathrm{II}}$ from their values in the MEP state $W_{\mathrm{I}}^{*}$. For small deviations, the entropy is well approximated with its quadratic part

$$
\Delta S=-\int \Delta W\left[1+\ln \frac{W_{\mathrm{I}}^{*}}{W_{\mathrm{eq}}}\right] \mathrm{d} x-\frac{1}{2} \int \frac{\Delta W^{2}}{W_{\mathrm{I}}^{*}} \mathrm{~d} x .
$$

Taking into account the fact that $M_{\mathrm{I}}\left[W_{\mathrm{I}}^{*}\right]=M_{\mathrm{I}}$, we come to the following maximizaton problem:

$$
\Delta S[\Delta W] \rightarrow \max , \quad M_{\mathrm{I}}[\Delta W]=0, \quad M_{\mathrm{II}}[\Delta W]=\Delta M_{\mathrm{II}} .
$$

The solution to problem (317) is always explicitly found from a $(k+1) \times(k+1)$ system of linear algebraic equations for Lagrange multipliers. This method was applied to systems of Boltzmann equations for chemical reacting gases [190,191], and for an approximate solution to the Boltzmann 
equation: scattering rates "moments of collision integral" are treated as independent variables, and as an alternative to moments of the distribution function, to describe the rarefied gas near local equilibrium. Triangle version of the entropy maximum principle is used to derive the Grad-like description in terms of a finite number of scattering rates. The equations are compared to the Grad moment system in the heat nonconductive case. Estimations for hard spheres demonstrate, in particular, some $10 \%$ excess of the viscosity coefficient resulting from the scattering rate description, as compared to the Grad moment estimation [192].

In the remainder of this section we deal solely with one-moment near-equilibrium closures: $M_{\mathrm{I}}=$ $M_{0}$, (i.e. $W_{\mathrm{I}}^{*}=W_{\mathrm{eq}}$ ), and the set $M_{\mathrm{II}}$ contains a single moment $M=\int m W \mathrm{~d} x, m(x) \neq 1$. We shall specify notations for the near-equilibrium FPE, writing the distribution function as $W=W_{\text {eq }}(1+\Psi)$, where the function $\Psi$ satisfies an equation:

$$
\partial_{t} \Psi=W_{\mathrm{eq}}^{-1} \hat{J} \Psi
$$

where $\hat{J}=\partial_{x} \cdot\left[W_{\mathrm{eq}} D \cdot \partial_{x}\right]$. The triangle one-moment quasi-equilibrium function reads:

$$
W^{(0)}=W_{\text {eq }}\left[1+\Delta M m^{(0)}\right]
$$

where

$$
m^{(0)}=\left[\langle m m\rangle-\langle m\rangle^{2}\right]^{-1}[m-\langle m\rangle] .
$$

Here brackets $\langle\ldots\rangle=\int W_{\text {eq }} \ldots \mathrm{d} x$ denote equilibrium averaging. The superscript $(0)$ indicates that the triangle quasi-equilibrium function (319) will be considered as the initial approximation to the procedure which we address below. Projector for the approximation (319) has the form

$$
\Pi^{(0)} \bullet=W_{\text {eq }} \frac{m^{(0)}}{\left\langle m^{(0)} m^{(0)}\right\rangle} \int m^{(0)}(x) \mathrm{d} x .
$$

Substituting function (319) into the FPE (318), and applying projector (321) on both the sides of the resulting formal expression, we derive the equation for $M$ :

$$
\dot{M}=-\lambda_{0} \Delta M
$$

where $1 / \lambda_{0}$ is an effective time of relaxation of the moment $M$ to its equilibrium value, in the quasi-equilibrium approximation (319):

$$
\lambda_{0}=\left\langle m^{(0)} m^{(0)}\right\rangle^{-1}\left\langle\partial_{x} m^{(0)} \cdot D \cdot \partial_{x} m^{(0)}\right\rangle
$$

The invariance equation for the Fokker-Planck equation. Both the quasi-equilibrium and the triangle quasi-equilibrium closures are almost never invariants of the FPE dynamics. That is, the moments $M$ of solutions to the FPE (314) vary in time differently from the solutions to the closed moment equations like (316), and these variations are generally significant even for the near-equilibrium dynamics. Therefore, we ask for corrections to the quasi-equilibrium closures to finish with the invariant closures. This problem falls precisely into the framework of the method of invariant manifold [6], and we shall apply this method to the one-moment triangle quasi-equilibrium closing approximations. 
First, the invariant one-moment closure is given by an unknown distribution function $W^{(\infty)}=W_{\text {eq }}\left[1+\Delta M m^{(\infty)}(x)\right]$ which satisfies equation

$$
\left[1-\Pi^{(\infty)}\right] \hat{J} m^{(\infty)}=0 \text {. }
$$

Here $\Pi^{(\infty)}$ is a projector, associated with an unknown function $m^{(\infty)}$, and which is also yet unknown. Eq. (324) is a formal expression of the invariance principle for a one-moment near-equilibrium closure: considering $W^{(\infty)}$ as a manifold in the space of distribution functions, parameterized with the values of the moment $M$, we require that the microscopic vector field $\hat{J} m^{(\infty)}$ be equal to its projection, $\Pi^{(\infty)} \hat{J} m^{(\infty)}$, onto the tangent space of the manifold $W^{(\infty)}$.

Now we turn our attention to solving the invariance equation (324) iteratively, beginning with the triangle one-moment quasi-equilibrium approximation $W^{(0)}(319)$. We apply the following iteration process to Eq. (324):

$$
\left[1-\Pi^{(k)}\right] \hat{J} m^{(k+1)}=0,
$$

where $k=0,1, \ldots$, and where $m^{(k+1)}=m^{(k)}+\mu^{(k+1)}$, and the correction satisfies the condition $\left\langle\mu^{(k+1)} m^{(k)}\right\rangle=0$. Projector is updated after each iteration, and it has the form

$$
\Pi^{(k+1)} \bullet=W_{\text {eq }} \frac{m^{(k+1)}}{\left\langle m^{(k+1)} m^{(k+1)}\right\rangle} \int m^{(k+1)}(x) \mathrm{d} x .
$$

Applying $\Pi^{(k+1)}$ to the formal expression,

$$
W_{\mathrm{eq}} m^{(k+1)} \dot{M}=\Delta M\left[1-\Pi^{(k+1)}\right] m^{(k+1)},
$$

we derive the $(k+1)$ th update of the effective time (323):

$$
\lambda_{k+1}=\frac{\left\langle\partial_{x} m^{(k+1)} \cdot D \cdot \partial_{x} m^{(k+1)}\right\rangle}{\left\langle m^{(k+1)} m^{(k+1)}\right\rangle} .
$$

Specializing to the one-moment near-equilibrium closures, and following general argument [6], solutions to the invariance equation (324) are eigenfunctions of the operator $\hat{J}$, while the formal limit of the iteration process (325) is the eigenfunction which corresponds to the eigenvalue with the minimal nonzero absolute value.

Diagonal approximation. To obtain more explicit results, we shall now turn to an approximate solution to the problem (325) at each iteration. The correction $\mu^{(k+1)}$ satisfies the condition $\left\langle m^{(k)} \mu^{(k+1)}\right\rangle=0$, and can be decomposed as follows: $\mu^{(k+1)}=\alpha_{k} e^{(k)}+e_{\mathrm{ort}}^{(k)}$. Here $e^{(k)}$ is the variance of the $k$ th approximation: $e^{(k)}=W_{\text {eq }}^{-1}\left[1-\Pi^{(k)}\right] \hat{J} m^{(k)}=\lambda_{k} m^{(k)}+R^{(k)}$, where

$$
R^{(k)}=W_{\mathrm{eq}}^{-1} \hat{J} m^{(k)} .
$$

The function $e_{\text {ort }}^{(k)}$ is orthogonal to both $e^{(k)}$ and $m^{(k)}\left(\left\langle e^{(k)} e_{\text {ort }}^{(k)}\right\rangle=0\right.$, and $\left.\left\langle m^{(k)} e_{\text {ort }}^{(k)}\right\rangle=0\right)$.

Our diagonal approximation (DA) consists in disregarding the part $e_{\mathrm{ort}}^{(k)}$. In other words, we seek an improvement of the noninvariance of the $k$ th approximation along its variance $e^{(k)}$. Specifically, we consider the following ansatz at the $k$ th iteration:

$$
m^{(k+1)}=m^{(k)}+\alpha_{k} e^{(k)} .
$$


Table 2

Iterations $\lambda_{k}$ and the error $\delta_{k}$ for $U=x^{2} / 2$

\begin{tabular}{|c|c|c|c|c|c|c|c|c|}
\hline & & 0 & 1 & 4 & 8 & 12 & 16 & 20 \\
\hline \multirow[t]{3}{*}{ Ex. 1} & $\lambda$ & 1.99998 & 1.99993 & 1.99575 & 1.47795 & 1.00356 & 1.00001 & 1.00000 \\
\hline & $\delta$ & $0.16 \times 10^{-4}$ & $0.66 \times 10^{-4}$ & $0.42 \times 10^{-2}$ & 0.24 & $0.35 \times 10^{-2}$ & $0.13 \times 10^{-4}$ & $0.54 \times 10^{-7}$ \\
\hline & & 0 & 1 & 2 & 3 & 4 & 5 & 6 \\
\hline \multirow[t]{2}{*}{ Ex. 2} & $\lambda$ & 3.399 & 2.437 & 1.586 & 1.088 & 1.010 & 1.001 & 1.0002 \\
\hline & $\delta$ & 1.99 & 1.42 & 0.83 & 0.16 & $0.29 \times 10^{-1}$ & $0.27 \times 10^{-2}$ & $0.57 \times 10^{-3}$ \\
\hline
\end{tabular}

Substituting ansatz (329) into Eq. (325), and integrating the latter expression with the function $e^{(k)}$ to evaluate the coefficient $\alpha_{k}$ :

$$
\alpha_{k}=\frac{A_{k}-\lambda_{k}^{2}}{\lambda_{k}^{3}-2 \lambda_{k} A_{k}+B_{k}},
$$

where parameters $A_{k}$ and $B_{k}$ represent the following equilibrium averages:

$$
\begin{aligned}
& A_{k}=\left\langle m^{(k)} m^{(k)}\right\rangle^{-1}\left\langle R^{(k)} R^{(k)}\right\rangle \\
& B_{k}=\left\langle m^{(k)} m^{(k)}\right\rangle^{-1}\left\langle\partial_{x} R^{(k)} \cdot D \cdot \partial_{x} R^{(k)}\right\rangle .
\end{aligned}
$$

Finally, putting together Eqs. (327)-(331), we arrive at the following DA recurrence solution, and which is our main result:

$$
\begin{aligned}
& m^{(k+1)}=m^{(k)}+\alpha_{k}\left[\lambda_{k} m^{(k)}+R^{(k)}\right], \\
& \lambda_{k+1}=\frac{\lambda_{k}-\left(A_{k}-\lambda_{k}^{2}\right) \alpha_{k}}{1+\left(A_{k}-\lambda_{k}^{2}\right) \alpha_{k}^{2}} .
\end{aligned}
$$

Notice that the stationary points of the DA process (333) are the true solutions to the invariance equation (324). What may be lost within the DA is the convergency to the true limit of the procedure (325), i.e. to the minimal eigenvalue.

To test the convergency of the DA process (333) we have considered two potentials $U$ in the FPE (314) with a constant diffusion matrix $D$. The first test was with the square potential $U=x^{2} / 2$, in the three-dimensional configuration space, since for this potential the detail structure of the spectrum is well known. We have considered two examples of initial one-moment quasi-equilibrium closures with $m^{(0)}=x_{1}+100\left(x^{2}-3\right)$ (example 1), and $m^{(0)}=x_{1}+100 x^{6} x_{2}$ (example 2), in Eq. (320). The result of performance of the DA for $\lambda_{k}$ is presented in Table 2, together with the error $\delta_{k}$ which was estimated as the norm of the variance at each iteration: $\delta_{k}=\left\langle e^{(k)} e^{(k)}\right\rangle /\left\langle m^{(k)} m^{(k)}\right\rangle$. In both examples, we see a good monotonic convergency to the minimal eigenvalue $\lambda_{\infty}=1$, corresponding to the eigenfunction $x_{1}$. This convergency is even more striking in example 1, where the initial choice was very close to a different eigenfunction $x^{2}-3$, and which can be seen in the nonmonotonic behavior of the variance. Thus, we have an example to trust the DA approximation as converging to the proper object. 
Table 3

Iterations $\lambda_{k}$ for $U=-50 \ln \left(1-x^{2}\right)$

\begin{tabular}{|c|c|c|c|c|c|c|c|c|c|c|}
\hline & & 0 & 1 & 2 & 3 & 4 & 5 & 6 & 7 & 8 \\
\hline Ex. 3 & $\lambda$ & 213.17 & 212.186 & 211.914 & 211.861 & 211.849 & 211.845 & 211.843 & 211.842 & 211.841 \\
\hline Ex. 4 & $\lambda$ & 216.586 & 213.135 & 212.212 & 211.998 & 211.929 & 211.899 & 211.884 & 211.876 & 211.871 \\
\hline
\end{tabular}

For the second test, we have taken a one-dimensional potential $U=-50 \ln \left(1-x^{2}\right)$, the configuration space is the segment $|x| \leqslant 1$. Potentials of this type (so-called FENE potential) are used in applications of the FPE to models of polymer solutions [113-115]. Results are given in Table 3 for the two initial functions, $m^{(0)}=x^{2}+10 x^{4}-\left\langle x^{2}+10 x^{4}\right\rangle$ (example 3 ), and $m^{(0)}=x^{2}+10 x^{8}-\left\langle x^{2}+10 x^{8}\right\rangle$ (example 4). Both the examples demonstrate a stabilization of the $\lambda_{k}$ at the same value after some ten iterations.

In conclusion, we have developed the principle of invariance to obtain moment closures for the Fokker-Planck equation (314), and have derived explicit results for the one-moment near-equilibrium closures, particularly important to get information about the spectrum of the FP operator.

\section{Method of invariant grids}

Elsewhere above in this paper, we considered immersions $F(y)$, and methods for their construction, without addressing the question of how to implement $F$ in a constructive way. In most of the works (of us and of other people on similar problems), analytic forms were needed to represent manifolds (see, however, dual quasi-equilibrium integrators [198,199]). However, in order to construct manifolds of a relatively low dimension, grid-based representations of manifolds become a relevant option. The Method of invariant grids (MIG) was suggested recently in [10].

The principal idea of (MIG) is to find a mapping of finite-dimensional grids into the phase space of a dynamic system. That is we construct not just a point approximation of the invariant manifold $F^{*}(y)$, but an invariant grid. When refined, in the limit it is expected to converge, of course, to $F^{*}(y)$, but it is a separate, independently defined object.

Let's denote $L=R^{n}, G$ is a discrete subset of $R^{n}$. A natural choice would be a regular grid, but, this is not crucial from the point of view of the general formalism. For every point $y \in G$, a neighborhood of $y$ is defined: $V_{y} \subset G$, where $V_{y}$ is a finite set, and, in particular, $y \in V_{y}$. On regular grids, $V_{y}$ includes, as a rule, the nearest neighbors of $y$. It may also include the points, next to the nearest.

For our purposes, one should define a grid differential operator. For every function, defined on the grid, also all derivatives are defined:

$$
\left.\frac{\partial f}{\partial y_{i}}\right|_{y \in G}=\sum_{z \in V_{y}} q_{i}(z, y) f(z), \quad i=1, \ldots, n .
$$

where $q_{i}(z, y)$ are some coefficients. 
Here we do not specify the choice of the functions $q_{i}(z, y)$. We just mention in passing that, as a rule, Eq. (334) is established using some interpolation of $f$ in the neighborhood of $y$ in $R^{n}$ by some differentiable functions (for example, polynomial). This interpolation is based on the values of $f$ at the points of $V_{y}$. For regular grids, $q_{i}(z, y)$ are functions of the difference $z-y$. For some $y$ s which are close to the edges of the grid, functions are defined only on the part of $V_{y}$. In this case, the coefficients in (334) should be modified appropriately in order to provide an approximation using available values of $f$. Below we will assume this modification is always done. We also assume that the number of points in the neighborhood $V_{y}$ is always sufficient to make the approximation possible. This assumption restricts the choice of the grids $G$. Let's call admissible all such subsets $G$, on which one can define differentiation operator in every point.

Let $F$ be a given mapping of some admissible subset $G \subset R^{n}$ into $U$. For every $y \in V$ we define tangent vectors:

$$
T_{y}=\operatorname{Lin}\left\{g_{i}\right\}_{1}^{n},
$$

where vectors $g_{i}(i=1, \ldots, n)$ are partial derivatives (334) of the vector-function $F$ :

$$
g_{i}=\frac{\partial F}{\partial y_{i}}=\sum_{z \in V_{y}} q_{i}(z, y) F(z),
$$

or in the coordinate form

$$
\left(g_{i}\right)_{j}=\frac{\partial F_{j}}{\partial y_{i}}=\sum_{z \in V_{y}} q_{i}(z, y) F_{j}(z) .
$$

Here $\left(g_{i}\right)_{j}$ is the $j$ th coordinate of the vector $\left(g_{i}\right)$, and $F_{j}(z)$ is the $j$ th coordinate of the point $F(z)$. The grid $G$ is invariant, if for every node $y \in G$ the vector field $J(F(y))$ belongs to the tangent space $T_{y}$ (here $J$ is the right hand site of the kinetic equations (76)).

So, the definition of the invariant grid includes:

(1) Finite admissible subset $G \subset R^{n}$;

(2) A mapping $F$ of this admissible subset $G$ into $U$ (where $U$ is the phase space for kinetic Eqs. (76));

(3) The differentiation formulae (334) with given coefficients $q_{i}(z, y)$;

The grid invariance equation has a form of inclusion:

$$
J(F(y)) \in T_{y} \quad \text { for every } y \in G,
$$

or a form of equation:

$$
\left(1-P_{y}\right) J(F(y))=0 \text { for every } y \in G \text {, }
$$

where $P_{y}$ is the thermodynamic projector (132).

The grid differentiation formulae (334) are needed, in the first place, to establish the tangent space $T_{y}$, and the null space of the thermodynamic projector $P_{y}$ in each node. It is important to realise that locality of construction of thermodynamic projector enables this without a need for a global parametrization. 
Basically, in our approach, the grid specifics are in: (a) differentiation formulae, (b) grid construction strategy (the grid can be extended, contracted, refined, etc.) The invariance equations (78), equations of the film dynamics extension (84), the iteration Newton method (167), and the formulae of the relaxation approximation (313) do not change at all. For convenience, let us repeat all these formulae in the grid context.

Let $x=F(y)$ be position of a grid's node $y$ immersed into $U$. We have set of tangent vectors $g_{i}(x)$, defined in $x$ (336), (337). Thus, the tangent space $T_{y}$ is defined by (335). Also, one has entropy function $S(x)$, the linear functional $\left.D_{x} S\right|_{x}$, and the subspace $T_{0 y}=\left.T_{y} \cap \operatorname{ker} D_{x} S\right|_{x}$ in $T_{y}$. Let $T_{0 y} \neq T_{y}$. In this case we have a vector $\mathbf{e}_{y} \in T_{y}$, orthogonal to $T_{0 y},\left.D_{x} S\right|_{x}\left(\mathbf{e}_{y}\right)=1$. Then, the thermodynamic projector is defined as

$$
P_{y} \bullet=P_{0 y} \bullet+\left.\mathbf{e}_{y} D_{x} S\right|_{x} \bullet,
$$

where $P_{0 y}$ is the orthogonal projector on $T_{0 y}$ with respect to the entropic scalar product $\langle\mid\rangle_{x}$.

If $T_{0 y}=T_{y}$, then the thermodynamic projector is the orthogonal projector on $T_{y}$ with respect to the entropic scalar product $\langle\|\rangle_{x}$.

For the Newton method with incomplete linearization, the equations for calculating new node position $x^{\prime}=x+\delta x$ are:

$$
\begin{aligned}
& P_{y} \delta x=0, \\
& \left(1-P_{y}\right)(J(x)+D J(x) \delta x)=0 .
\end{aligned}
$$

Here $D J(x)$ is a matrix of derivatives of $J$, calculated in $x$. The self-adjoint linearization may be useful too (see Section 8).

Eq. (339) is a system of linear algebraic equations. In practice, it is convenient to choose some orthonormal (with respect to the entropic scalar product) basis $\mathbf{b}_{\mathbf{i}}$ in $\operatorname{ker} P_{y}$. Let $r=\operatorname{dim}\left(\operatorname{ker} P_{y}\right)$. Then $\delta x=\sum_{i=1}^{r} \delta_{i} \mathbf{b}_{\mathbf{i}}$, and the system looks like

$$
\sum_{k=1}^{r} \delta_{k}\left\langle\mathbf{b}_{\mathbf{i}} \mid D J(x) \mathbf{b}_{\mathbf{k}}\right\rangle_{x}=-\left\langle J(x) \mid \mathbf{b}_{\mathbf{i}}\right\rangle_{x}, \quad i=1 \ldots r .
$$

Here $\langle\mid\rangle_{x}$ is the entropic scalar product. This is the system of linear equations for adjusting the node position accordingly to the Newton method with incomplete linearization.

For the relaxation method, one needs to calculate the defect $\Delta_{x}=\left(1-P_{y}\right) J(x)$, and the relaxation step

$$
\tau(x)=-\frac{\left\langle\Delta_{x} \mid \Delta_{x}\right\rangle_{x}}{\left\langle\Delta_{x} \mid D J(x) \Delta_{x}\right\rangle_{x}} .
$$

Then, new node position $x^{\prime}$ is calculated as

$$
x^{\prime}=x+\tau(x) \Delta_{x} .
$$

This is the equation for adjusting the node position according to the relaxation method. 


\subsection{Grid construction strategy}

From all reasonable strategies of the invariant grid construction we will consider here the following two: growing lump and invariant flag.

\subsubsection{Growing lump}

In this strategy one chooses as initial the equilibrium point $y^{*}$. The first approximation is constructed as $F\left(y^{*}\right)=x^{*}$, and for some initial $V_{0}\left(V_{y^{*}} \subset V_{0}\right)$ one has $F(y)=x^{*}+A\left(y-y^{*}\right)$, where $A$ is an isometric embedding (in the standard Euclidean metrics) of $R^{n}$ in $E$.

For this initial grid one makes a fixed number of iterations of one of the methods chosen (Newton's method with incomplete linearization or the relaxation method), and, after that, puts $V_{1}=\bigcup_{y \in V_{0}} V_{y}$ and extends $F$ from $V_{0}$ onto $V_{1}$ using linear extrapolation and the process continues. One of the possible variants of this procedure is to extend the grid from $V_{i}$ to $V_{i+1}$ not after a fixed number of iterations, but when invariance defect $\Delta_{y}$ becomes smaller than a given $\epsilon$ (in a given norm, which is entropic, as a rule), for all nodes $y \in V_{i}$. The lump stops growing when it reaches the boundary and is within a given accuracy $\|\Delta\|<\epsilon$.

\subsubsection{Invariant flag}

For the invariant flag one uses sufficiently regular grids $G$, in which many points are situated on the coordinate lines, planes, etc. One considers the standard flag $R^{0} \subset R^{1} \subset R^{2} \subset \cdots \subset R^{n}$ (every next space is constructed by adding one more coordinate). It corresponds to a succession of grids $\{y\} \subset G^{1} \subset G^{2} \cdots \subset G^{n}$, where $\left\{y^{*}\right\}=R^{0}$, and $G^{i}$ is a grid in $R^{i}$.

First, $y^{*}$ is mapped in $x^{*}$ and further $F\left(y^{*}\right)=x^{*}$. Then an invariant grid is constructed on $V^{1} \subset G^{1}$ (up to the boundaries $U$ and within a given accuracy $\|\Delta\|<\epsilon$ ). After the neighborhoods in $G^{2}$ are added to the points $V^{1}$, and, using such extensions, the grid $V^{2} \subset G^{2}$ is constructed (up to the boundaries and within a given accuracy) and so on, until $V^{n} \subset G^{n}$ will be constructed.

We must underline here that, constructing the $k$ th grid $V^{k} \subset G^{k}$, the important role of the grids of smaller dimension $V^{0} \subset \cdots \subset V^{k-1} \subset V^{k}$ embedded in it, is preserved. The point $F\left(y^{*}\right)=x^{*}$ is preserved. For every $y \in V^{q}(q<k)$ the tangent vectors $g_{1}, \ldots, g_{q}$ are constructed, using the differentiation operators (334) on the whole $V^{k}$. Using the tangent space $T_{y}=\operatorname{Lin}\left\{g_{1}, \ldots, g_{q}\right\}$, the projector $P_{y}$ is constructed, the iterations are applied and so on. All this is done to obtain a succession of embedded invariant grids, given by the same map $F$.

\subsubsection{Boundaries check and the entropy}

We construct grid mapping of $F$ onto the finite set $V \in G$. The technique of checking if the grid still belongs to the phase space $U$ of kinetic system $U(F(V) \subset U)$ is quite straightforward: all the points $y \in V$ are checked to belong to $U$. If at the next iteration a point $F(y)$ leaves $U$, then it is returned inside by a homothety transform with the center in $x^{*}$. Since the entropy is a concave function, the homothety contraction with the center in $x^{*}$ increases the entropy monotonously. Another variant is cutting off the points leaving $U$.

By the way it was constructed, (132), the kernel of the entropic projector is annulled by the entropy differential. Thus, in the first order, steps in the Newton method with incomplete linearization (167) as well as in the relaxation methods (312), (313) do not change the entropy. But, if the steps are 


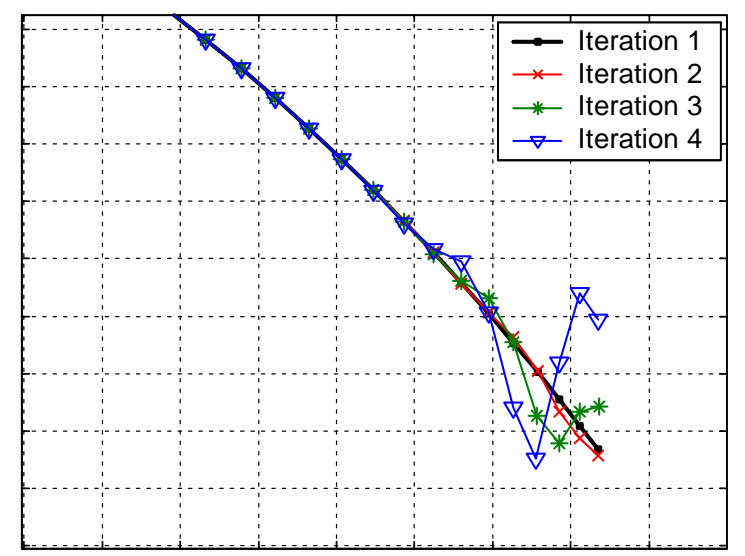

Fig. 8. Grid instability. For small grid steps approximations in the calculation of grid derivatives lead to the grid instability effect. On the figure several successive iterations of the algorithm without adaptation of the time step are shown that lead to undesirable "oscillations", which eventually destruct the grid starting from one of it's ends.

quite large, then the increasing of the entropy can become essential and the points are returned on their entropy level by the homothety contraction with the center in the equilibrium point.

\subsection{Instability of fine grids}

When one reduces the grid step (spacing between the nodes) in order to get a finer grid, then, starting from a definite step, it is possible to face the problem of the Courant instability [202-204]. Instead of converging, at the every iteration the grid becomes more entangled (see Fig. 8).

The way to get rid off this instability is well-known. This is decreasing the time step. Instead of the real time step, we have a shift in the Newtonian direction. Formally, we can assign for one complete step in the Newtonian direction a value $h=1$. Let us consider now the Newton method with an arbitrary $h$. For this, let us find $\delta x=\delta F(y)$ from (339), but we will change $\delta x$ proportionally to $h$ : the new value of $x_{n+1}=F_{n+1}(y)$ will be equal to

$$
F_{n+1}(y)=F_{n}(y)+h_{n} \delta F_{n}(y)
$$

where the lower index $n$ denotes the step number.

One way to choose the $h$ step value is to make it adaptive, controlling the average value of the invariance defect $\left\|\Delta_{y}\right\|$ at every step. Another way is the convergence control: then $\sum h_{n}$ plays a role of time.

Elimination of Courant instability for the relaxation method can be made quite analogously. Everywhere the step $h$ is maintained as big as it is possible without convergence problems.

\subsection{What space is the most appropriate for the grid construction?}

For the kinetics systems there are two distinguished representations of the phase space:

- The densities space (concentrations, energy or probability densities, etc.).

- The spaces of conjugate intensive quantities, potentials (temperature, chemical potentials, etc.). 
The density space is convenient for the construction of quasi-chemical representations. Here the balance relations are linear and the restrictions are in the form of linear inequalities (the densities themselves or some linear combinations of them must be positive).

The conjugate variables space is convenient in the sense that the equilibrium conditions, given the linear restrictions on the densities, are in the linear form (with respect to the conjugate variables). In these spaces the quasi-equilibrium manifolds exist in the form of linear subspaces and, vise versa, linear balance equations turns out to be equations of the conditional entropy maximum.

The duality we have just described is very well-known and studied in details in many works on thermodynamics and Legendre transformations [207,208]. This viewpoint of nonequilibrium thermodynamics unifies many well-established mesoscopic dynamical theories, as for example the Boltzmann kinetic theory and the Navier-Stokes-Fourier hydrodynamics [209]. In the previous section, the grids were constructed in the density space. But the procedure of constructing them in the space of the conjugate variables seems to be more consistent. The principal argument for this is the specific role of quasi-equilibrium, which exists as a linear manifold. Therefore, linear extrapolation gives a thermodynamically justified quasi-equilibrium approximation. Linear approximation of the slow invariant manifold in the neighborhood of the equilibrium in the conjugate variables space already gives the global quasi-equilibrium manifold, which corresponds to the motion separation (for slow and fast motions) in the neighborhood of the equilibrium point.

For the mass action law, transition to the conjugate variables is simply the logarithmic transformation of the coordinates.

\subsection{Carleman's formulae in the analytical invariant manifolds approximations. First benefit of analyticity: superresolution}

When constructing invariant grids, one must define the differential operators (334) for every grid node. For calculating the differential operators in some point $y$, an interpolation procedure in the neighborhood of $y$ is used. As a rule, it is an interpolation by a low-order polynomial, which is constructed using the function values in the nodes belonging to the neighbourhood of $y$ in $G$. This approximation (using values in the closest nodes) is natural for smooth functions. But, we are looking for the analytical invariant manifold (see discussion in the section: "film extension: analyticity instead of the boundary conditions"). Analytical functions have much more "rigid" structure than the smooth ones. One can change a smooth function in the neighborhood of any point in such a way, that outside this neighborhood the function will not change. In general, this is not possible for analytical functions: a kind of "long-range" effect takes place (as is well known).

The idea is to use this effect and to reconstruct some analytical function $f_{G}$ using function given on $G$. There is one important requirement: if these values on $G$ are values (given at the points of $G$ ) of some function $f$ which is analytical in the given neighborhood $U$, then if the $G$ is refined "correctly", one must have $f_{G} \rightarrow f$. The sequence of reconstructed function $f_{G}$ should converge to the "proper" function $f$.

What is the "correct refinement"? For smooth functions for the convergence $f_{G} \rightarrow f$ it is necessary and sufficient that, in the course of refinement, $G$ would approximate the whole $U$ with arbitrary accuracy. For analytical functions it is necessary only that, under the refinement, $G$ would 
approximate some uniqueness set ${ }^{12} A \subset U$. Suppose we have a sequence of grids $G$, each next is finer than previous, which approximates a set $A$. For smooth functions, using function values defined on the grids, one can reconstruct the function in $A$. For analytical functions, if the analyticity area $U$ is known, and $A$ is a uniqueness set in $U$, then one can reconstruct the function in $U$. The set $U$ can be essentially bigger than $A$; because of this such extension was named as superresolution effects [210]. There exist constructive formulae for construction of analytical functions $f_{G}$ for different areas $U$, uniqueness sets $A \subset U$ and for different ways of discrete approximation of $A$ by a sequence of fined grids $G$ [210]. Here we provide only one Carleman's formula which is the most appropriate for our purposes.

Let area $U=Q_{\sigma}^{n} \subset C^{n}$ be a product of strips $Q_{\sigma} \subset C, Q_{\sigma}=\{z \mid \operatorname{Im} z<\sigma\}$. We will construct functions holomorphic in $Q_{\sigma}^{n}$. This is effectively equivalent to the construction of real analytical functions $f$ in whole $R^{n}$ with a condition on the convergence radius $r(x)$ of the Taylor series for $f$ as a function of each coordinate: $r(x) \geqslant \sigma$ in every point $x \in R^{n}$.

The sequence of fined grids is constructed as follows: let for every $l=1, \ldots, n$ a finite sequence of distinct points $N_{l} \subset Q_{\sigma}$ be defined:

$$
N_{l}=\left\{x_{l j} \mid j=1,2,3 \ldots\right\}, \quad x_{l j} \neq x_{l i} \text { for } i \neq j .
$$

The uniqueness set $A$, which is approximated by a sequence of fined finite grids, has the form

$$
A=N_{1} \times N_{2} \times \cdots \times N_{n}=\left\{\left(x_{1 i_{1}}, x_{2 i_{2}}, \ldots, x_{n i_{n}}\right) \mid i_{1, \ldots, n}=1,2,3, \ldots\right\} .
$$

The grid $G_{m}$ is defined as the product of initial fragments $N_{l}$ of length $m$ :

$$
G_{m}=\left\{\left(x_{1 i_{1}}, x_{2 i_{2}} \ldots x_{n i_{n}}\right) \mid 1 \leqslant i_{1, \ldots, n} \leqslant m\right\} .
$$

Let's denote $\lambda=2 \sigma / \pi$ ( $\sigma$ is a half-width of the strip $Q_{\sigma}$ ). The key role in the construction of the Carleman's formula is played by the functional $\omega_{m}^{\lambda}(u, p, l)$ of three variables: $u \in U=Q_{\sigma}^{n}, p$ is an integer, $1 \leqslant p \leqslant m, l$ is an integer, $1 \leqslant p \leqslant n$. Further $u$ will be the coordinate value at the point where the extrapolation is calculated, $l$ will be the coordinate number, and $p$ will be an element of multi-index $\left\{i_{1}, \ldots, i_{n}\right\}$ for the point $\left(x_{1 i_{1}}, x_{2 i_{2}}, \ldots, x_{n i_{n}}\right) \in G$ :

$$
\omega_{m}^{\lambda}(u, p, l)=\frac{\left(e^{\lambda x_{l p}}+e^{\lambda \bar{x}_{l p}}\right)\left(e^{\lambda u}-e^{\lambda x_{l p}}\right)}{\lambda\left(e^{\lambda u}+e^{\lambda \bar{x}_{l p}}\right)\left(u-x_{l p}\right) e^{\lambda x_{l p}}} \prod_{j=1}^{m} \frac{\left(e^{\lambda x_{l p}}+e^{\lambda \bar{x}_{l j}}\right)\left(e^{\lambda u}-e^{\lambda x_{l j}}\right)}{\left(e^{\lambda x_{l p}}-e^{\lambda x_{l j}}\right)\left(e^{\lambda u}+e^{\lambda \bar{x}_{l j}}\right)}
$$

For real-valued $x_{p k}$ formula (347) becomes simpler:

$$
\omega_{m}^{\lambda}(u, p, l)=2 \frac{e^{\lambda u}-e^{\lambda x_{l p}}}{\lambda\left(e^{\lambda u}+e^{\lambda x_{l p}}\right)\left(u-x_{l p}\right)} \prod_{j=1}^{m} \frac{\left(e^{\lambda x_{l p}}+e^{\lambda x_{l j}}\right)\left(e^{\lambda u}-e^{\lambda x_{l j}}\right)}{\left(e^{\lambda x_{l p}}-e^{\lambda x_{l j}}\right)\left(e^{\lambda u}+e^{\lambda x_{l j}}\right)}
$$

The Carleman's formula for extrapolation from $G_{M}$ on $U=Q_{\sigma}^{n}(\sigma=\pi \lambda / 2)$ has the form $\left(z=\left(z_{1}, \ldots, z_{n}\right)\right)$ :

$$
f_{m}(z)=\sum_{k_{1}, \ldots, k_{n}=1}^{m} f\left(x_{k}\right) \prod_{j=1}^{n} \omega_{m}^{\lambda}\left(z_{j}, k_{j}, j\right),
$$

where $k=k_{1}, \ldots, k_{n}, x_{k}=\left(x_{1 k_{1}}, x_{2 k_{2}}, \ldots, x_{n k_{n}}\right)$.

\footnotetext{
${ }^{12}$ Let's remind to the reader that $A \subset U$ is called uniqueness set in $U$ if for analytical in $U$ functions $\psi$ and $\varphi$ from $\left.\left.\psi\right|_{A} \equiv \varphi\right|_{A}$ it follows $\psi=\varphi$.
} 
There exists a theorem [210]:

If $f \in H^{2}\left(Q_{\sigma}^{n}\right)$, then $f(z)=\lim _{m \rightarrow \infty} f_{m}(z)$, where $H^{2}\left(Q_{\sigma}^{n}\right)$ is the Hardy class of holomorphic in $Q_{\sigma}^{n}$ functions.

It is useful to present the asymptotics of (349) for big $\left|\operatorname{Re} z_{j}\right|$. For this we will consider the asymptotics of (349) for big $|\operatorname{Re} u|$ :

$$
\left|\omega_{m}^{\lambda}(u, p, l)\right|=\left|\frac{2}{\lambda u} \prod_{j=1}^{m} \frac{e^{\lambda x_{l p}}+e^{\lambda x_{l j}}}{e^{\lambda x_{l p}}-e^{\lambda x_{l j}}}\right|+\mathrm{o}\left(|\operatorname{Re} u|^{-1}\right) .
$$

From formula (349) one can see that for the finite $m$ and $\left|\operatorname{Re} z_{j}\right| \rightarrow \infty$ function $\left|f_{m}(z)\right|$ behaves like const $\cdot \prod_{j}\left|z_{j}\right|^{-1}$.

This property (zero asymptotics) must be taken into account when using formula (349). When constructing invariant manifolds $F(W)$, it is natural to use (349) not for the immersion $F(y)$, but for the deviation of $F(y)$ from some analytical ansatz $F_{0}(y)$ [211-214].

The analytical ansatz $F_{0}(y)$ can be obtained using Taylor series, just as in the Lyapunov auxiliary theorem [144] (also see above in the sections about the film extensions). Another variant is using Taylor series for the construction of Pade-approximations.

It is natural to use approximations (349) in dual variables as well, since there exists for them (as the examples demonstrate) a simple and very effective linear ansatz for the invariant manifold. This is the slow invariant subspace $E_{\text {slow }}$ of the operator of linearized system (76) in dual variables in the equilibrium point. This invariant subspace corresponds to the set of "slow" eigenvalues (with small $|\operatorname{Re} \lambda|, \operatorname{Re} \lambda<0$ ). In the initial space (of concentrations or densities) this invariant subspace is the quasi-equilibrium manifold. It consists of the maximal entropy points on the affine manifolds of the $x+E_{\text {fast }}$ form, where $E_{\text {fast }}$ is the "fast" invariant subspace of the operator of linearized system (76) in the initial variables in the equilibrium point. It corresponds to the "fast" eigenvalues (big $|\operatorname{Re} \lambda|$, $\operatorname{Re} \lambda<0)$.

In the problem of invariant grids constructing we can use the Carleman's formulae in two moments: first, for the definition grid differential operators (334), second, for the analytical continuation the manifold from the grid.

\section{Example 8: Two-step catalytic reaction}

Let us consider a two-step four-component reaction with one catalyst $A_{2}$ :

$$
A_{1}+A_{2} \leftrightarrow A_{3} \leftrightarrow A_{2}+A_{4}
$$

We assume the Lyapunov function of the form $S=-G=-\sum_{i=1}^{4} c_{i}\left[\ln \left(c_{i} / c_{i}^{\mathrm{eq}}\right)-1\right]$. The kinetic equation for the four-component vector of concentrations, $\mathbf{c}=\left(c_{1}, c_{2}, c_{3}, c_{4}\right)$, has the form

$$
\dot{\mathbf{c}}=\gamma_{1} W_{1}+\gamma_{2} W_{2} \text {. }
$$

Here $\gamma_{1,2}$ are stoichiometric vectors,

$$
\gamma_{1}=(-1,-1,1,0), \quad \gamma_{2}=(0,1,-1,1),
$$

while functions $W_{1,2}$ are reaction rates:

$$
W_{1}=k_{1}^{+} c_{1} c_{2}-k_{1}^{-} c_{3}, \quad W_{2}=k_{2}^{+} c_{3}-k_{2}^{-} c_{2} c_{4} .
$$




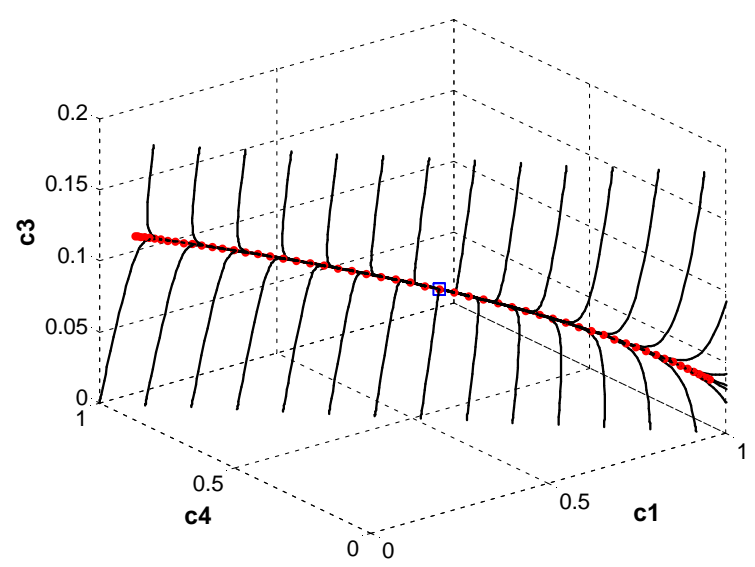

Fig. 9. One-dimensional invariant grid (circles) for two-dimensional chemical system. Projection into the $3 \mathrm{~d}$-space of $c_{1}$, $c_{4}, c_{3}$ concentrations. The trajectories of the system in the phase space are shown by lines. The equilibrium point is marked by square. The system quickly reaches the grid and further moves along it.

Here $k_{1,2}^{ \pm}$are reaction rate constants. The system under consideration has two conservation laws,

$$
c_{1}+c_{3}+c_{4}=B_{1}, \quad c_{2}+c_{3}=B_{2},
$$

or $\left\langle\mathbf{b}_{\mathbf{1}, \mathbf{2}}, \mathbf{c}\right\rangle=B_{1,2}$, where $\mathbf{b}_{\mathbf{1}}=(1,0,1,1)$ and $\mathbf{b}_{\mathbf{1}}=(0,1,1,0)$. The nonlinear system (351) is effectively two-dimensional, and we consider a one-dimensional reduced description. For our example, we chose the following set of parameters:

$$
\begin{aligned}
& k_{1}^{+}=0.3, \quad k_{1}^{-}=0.15, \quad k_{2}^{+}=0.8, \quad k_{2}^{-}=2.0 ; \\
& c_{1}^{\mathrm{eq}}=0.5, \quad c_{2}^{\mathrm{eq}}=0.1, \quad c_{3}^{\mathrm{eq}}=0.1, \quad c_{4}^{\mathrm{eq}}=0.4 ; \\
& B_{1}=1.0, \quad B_{2}=0.2 .
\end{aligned}
$$

In Fig. 9 one-dimensional invariant grid is shown in the $\left(c_{1}, c_{4}, c_{3}\right)$ coordinates. The grid was constructed by growing the grid, as described above. We used Newtonian iterations to adjust the nodes. The grid was grown up to the boundaries of the phase space.

The grid derivatives for calculating tangent vectors $g$ were taken as simple as $g\left(x_{i}\right)=\left(x_{i+1}-\right.$ $\left.x_{i-1}\right) /\left\|x_{i+1}-x_{i-1}\right\|$ for the internal nodes and $g\left(x_{1}\right)=\left(x_{1}-x_{2}\right) /\left\|x_{1}-x_{2}\right\|, g\left(x_{n}\right)=\left(x_{n}-x_{n-1}\right) /\left\|x_{n}-x_{n-1}\right\|$ for the grid's boundaries. Here $x_{i}$ denotes the vector of the $i$ th node position, $n$ is the number of nodes in the grid.

Close to the phase space boundaries we had to apply an adaptive algorithm for choosing the time step $h$ : if, after the next growing step and applying $N=20$ complete Newtonian steps, the grid did not converged, then we choose a new $h_{n+1}=h_{n} / 2$ and recalculate the grid. The final value for $h$ was $h \approx 0.001$.

The nodes positions are parametrized with entropic distance to the equilibrium point measured in the quadratic metrics given by $\mathbf{H}_{\mathbf{c}}=-\left\|\partial^{2} S(\mathbf{c}) / \partial c_{i} \partial c_{j}\right\|$ in the equilibrium $c^{\mathrm{eq}}$. It means that every node is on a sphere in this quadratic metrics with a given radius, which increases linearly. On this figure the step of the increase is chosen to be 0.05 . Thus, the first node is on the distance 0.05 from the equilibrium, the second is on the distance 0.10 and so on. Fig. 10 shows several basic values 


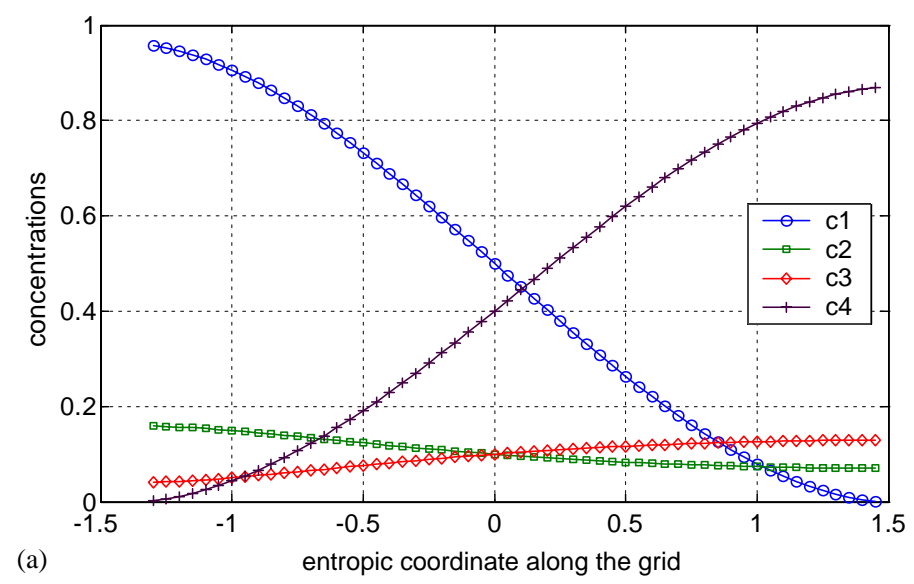

(a)

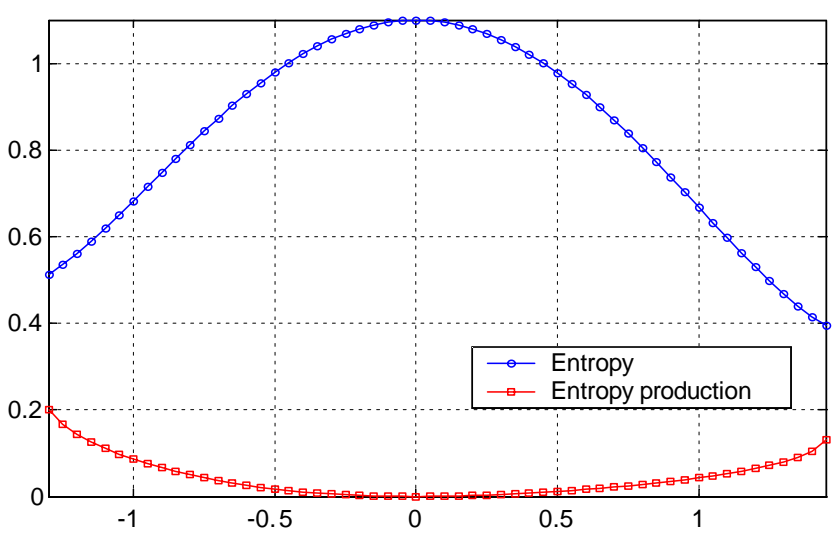

(b)

entropic coordinate along the grid

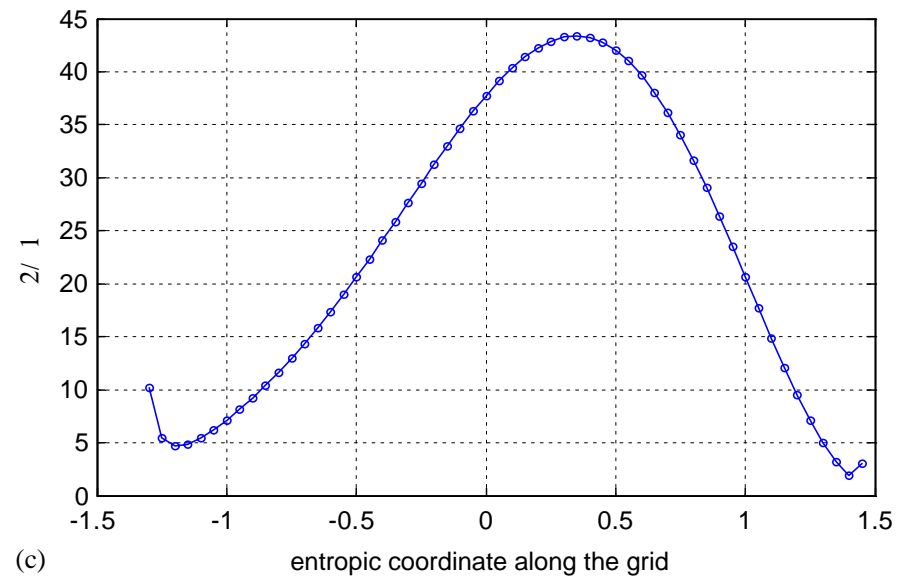

Fig. 10. One-dimensional invariant grid for two-dimensional chemical system. (a) Values of the concentrations along the grid. (b) Values of the entropy and the entropy production $(-\mathrm{d} G / \mathrm{d} t)$ along the grid. (c) Relation of the relaxation times "toward" and "along" the manifold. The nodes positions are parametrized with entropic distance measured in the quadratic metrics given by $\mathbf{H}_{\mathbf{c}}=-\left\|\partial^{2} S(\mathbf{c}) / \partial c_{i} \partial c_{j}\right\|$ in the equilibrium $c^{\text {eq }}$. Zero corresponds to the equilibrium. 
which facilitate understanding of the object (invariant grid) extracted. The sign on the $x$-axis of the graphs at Fig. 10 is meaningless, since the distance is always positive, but in this situation it denotes two possible directions from the equilibrium point.

Fig. 10a,b effectively represents the slow one-dimensional component of the dynamics of the system. Given any initial condition, the system quickly finds the corresponding point on the manifold and starting from this point the dynamics is given by a part of the graph on the Fig. 10a,b.

One of the useful values is shown on the Fig. 10c. It is the relation between the relaxation times "toward" and "along" the grid $\left(\lambda_{2} / \lambda_{1}\right.$, where $\lambda_{1}, \lambda_{2}$ are the smallest and the second smallest by absolute value nonzero eigenvalue of the system, symmetrically linearized at the point of the grid node). It shows that the system is very stiff close to the equilibrium point, and less stiff (by one order of magnitude) on the borders. This leads to the conclusion that the reduced model is more adequate in the neighborhood of the equilibrium where fast and slow motions are separated by two orders of magnitude. On the very end of the grid which corresponds to the positive absciss values, our one-dimensional consideration faces with definite problems (slow manifold is not well-defined).

\section{Example 9: Model hydrogen burning reaction}

In this section we consider a more interesting illustration, where the phase space is six-dimensional, and the system is four-dimensional. We construct an invariant flag which consists of one- and two-dimensional invariant manifolds.

We consider chemical system with six species called (provisionally) $\mathrm{H}_{2}$ (hydrogen), $\mathrm{O}_{2}$ (oxygen), $\mathrm{H}_{2} \mathrm{O}$ (water), $\mathrm{H}, \mathrm{O}, \mathrm{OH}$ (radicals). We assume the Lyapunov function of the form $S=-G=$ $-\sum_{i=1}^{6} c_{i}\left[\ln \left(c_{i} / c_{i}^{\mathrm{eq}}\right)-1\right]$. The subset of the hydrogen burning reaction and corresponding (direct) rate constants have been taken as:

1. $\mathrm{H}_{2} \leftrightarrow 2 \mathrm{H}, \quad k_{1}^{+}=2$,

2. $\mathrm{O}_{2} \leftrightarrow 2 \mathrm{O}, \quad k_{2}^{+}=1$,

3. $\mathrm{H}_{2} \mathrm{O} \leftrightarrow \mathrm{H}+\mathrm{OH}, \quad k_{3}^{+}=1$,

4. $\mathrm{H}_{2}+\mathrm{O} \leftrightarrow \mathrm{H}+\mathrm{OH}, \quad k_{4}^{+}=10^{3}$,

5. $\mathrm{O}_{2}+\mathrm{H} \leftrightarrow \mathrm{O}+\mathrm{OH}, \quad k_{5}^{+}=10^{3}$,

6. $\mathrm{H}_{2}+\mathrm{O} \leftrightarrow \mathrm{H}_{2} \mathrm{O}, \quad k_{6}^{+}=10^{2}$.

The conservation laws are:

$$
\begin{gathered}
2 c_{\mathrm{H}_{2}}+2 c_{\mathrm{H}_{2} \mathrm{O}}+c_{\mathrm{H}}+c_{\mathrm{OH}}=b_{\mathrm{H}}, \\
2 c_{\mathrm{O}_{2}}+c_{\mathrm{H}_{2} \mathrm{O}}+c_{\mathrm{O}}+c_{\mathrm{OH}}=b_{\mathrm{O}} .
\end{gathered}
$$

For parameter values we took $b_{\mathrm{H}}=2, b_{\mathrm{O}}=1$, and the equilibrium point:

$$
c_{\mathrm{H}_{2}}^{\mathrm{eq}}=0.27 \quad c_{\mathrm{O}_{2}}^{\mathrm{eq}}=0.135 \quad c_{\mathrm{H}_{2} \mathrm{O}}^{\mathrm{eq}}=0.7 \quad c_{\mathrm{H}}^{\mathrm{eq}}=0.05 \quad c_{\mathrm{O}}^{\mathrm{eq}}=0.02 \quad c_{\mathrm{OH}}^{\mathrm{eq}}=0.01 .
$$


Other rate constants $k_{i}^{-}, i=1 . .6$ were calculated from $\mathrm{c}^{\mathrm{eq}}$ value and $k_{i}^{+}$. For this system the stoichiometric vectors are:

$$
\begin{aligned}
& \gamma_{1}=(-1,0,0,2,0,0), \quad \gamma_{2}=(0,-1,0,0,2,0), \\
& \gamma_{3}=(0,0,-1,1,0,1), \quad \gamma_{4}=(-1,0,0,1,-1,1), \\
& \gamma_{5}=(0,-1,0,-1,1,1), \quad \gamma_{6}=(-1,0,1,0,-1,0) .
\end{aligned}
$$

We stress here once again that the system under consideration is fictional in that sense that the subset of equations corresponds to the simplified picture of this physical-chemical process and the constants do not correspond to any measured ones, but reflect only basic orders of magnitudes of the real-world system. In this sense we consider here a qualitative model system, which allows us to illustrate the invariant grids method without excessive complication. Nevertheless, modeling of real systems differs only in the number of species and equations. This leads, of course, to computationally harder problems, but not the crucial ones, and the efforts on the modeling of real-world systems are on the way.

Fig. 11a presents a one-dimensional invariant grid constructed for the system. Fig. 11b shows the picture of reduced dynamics along the manifold (for the explanation of the meaning of the $x$-coordinate, see the previous subsection). In Fig. 11c the three smallest by absolute value nonzero eigenvalues of the symmetrically linearized system $A^{\text {sym }}$ have been shown. One can see that the two smallest values almost "exchange" on one of the grid end. It means that one-dimensional "slow" manifold has definite problems in this region, it is just not well defined there. In practice, it means that one has to use at least two-dimensional grids there.

Fig. 12a gives a view onto the two-dimensional invariant grid, constructed for the system, using the "invariant flag" strategy. The grid was grown starting from the $1 \mathrm{D}$-grid constructed at the previous step. At the first iteration for every node of the initial grid, two nodes (and two edges) were added. The direction of the step was chosen as the direction of the eigenvector of the matrix $A^{\text {sym }}$ (at the point of the node), corresponding to the second "slowest" direction. The value of the step was chosen to be $\epsilon=0.05$ in terms of entropic distance. After several Newtonian iterations done until convergence, new nodes were added in the direction "orthogonal" to the 1D-grid. This time it is done by linear extrapolation of the grid on the same step $\epsilon=0.05$. When some new nodes have one or several negative coordinates (the grid reaches the boundaries) they were cut off. If a new node has only one edge, connecting it to the grid, it was excluded (since it does not allow calculating 2D-tangent space for this node). The process continues until the expansion is possible (after this, every new node has to be cut off).

The method for calculating tangent vectors for this regular rectangular 2D-grid was chosen to be quite simple. The grid consists of rows, which are co-oriented by construction to the initial 1D-grid, and columns that consist of the adjacent nodes in the neighboring rows. The direction of "columns" corresponds to the second slowest direction along the grid. Then, every row and column is considered as 1D-grid, and the corresponding tangent vectors are calculated as it was described before:

$$
g_{\text {row }}\left(x_{k, i}\right)=\left(x_{k, i+1}-x_{k, i-1}\right) /\left\|x_{k, i+1}-x_{k, i-1}\right\|
$$

for the internal nodes and

$$
g_{\text {row }}\left(x_{k, 1}\right)=\left(x_{k, 1}-x_{k, 2}\right) /\left\|x_{k, 1}-x_{k, 2}\right\|, \quad g_{\text {row }}\left(x_{k, n_{k}}\right)=\left(x_{k, n_{k}}-x_{k, n_{k}-1}\right) /\left\|x_{k, n_{k}}-x_{k, n_{k}-1}\right\|
$$



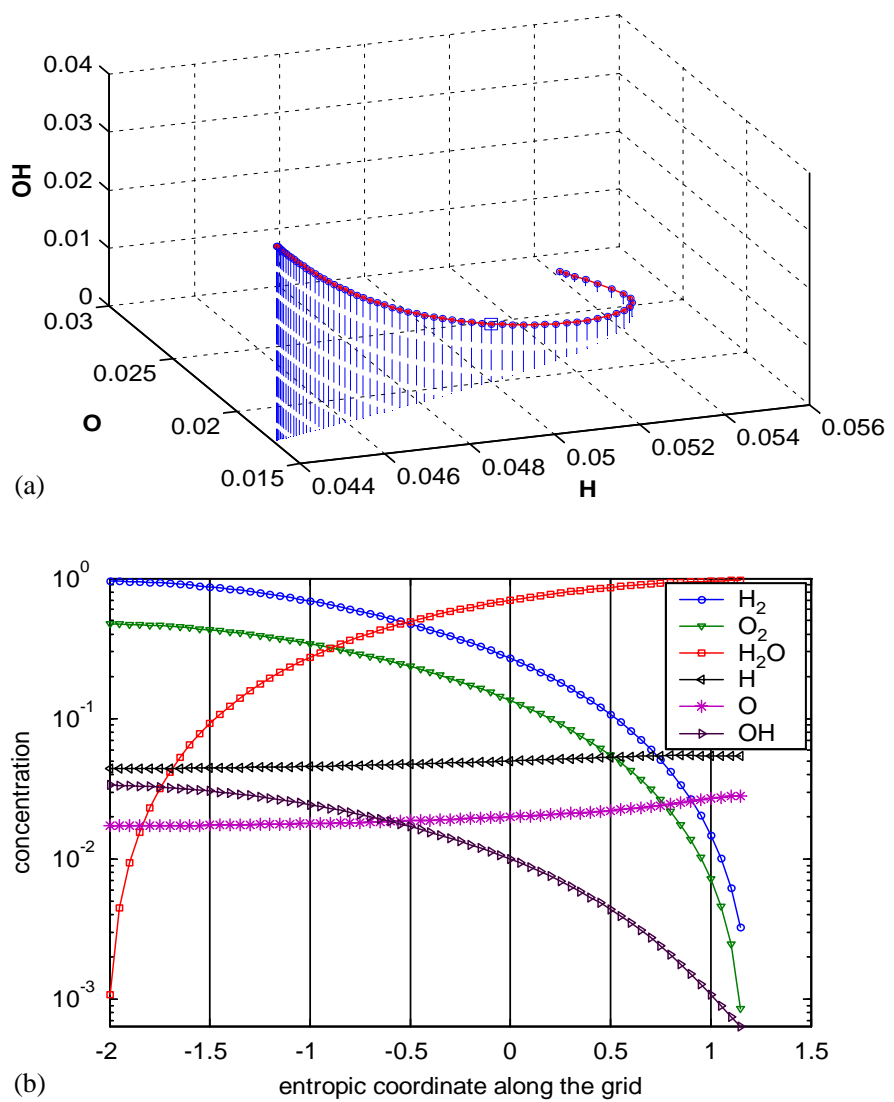

(b)

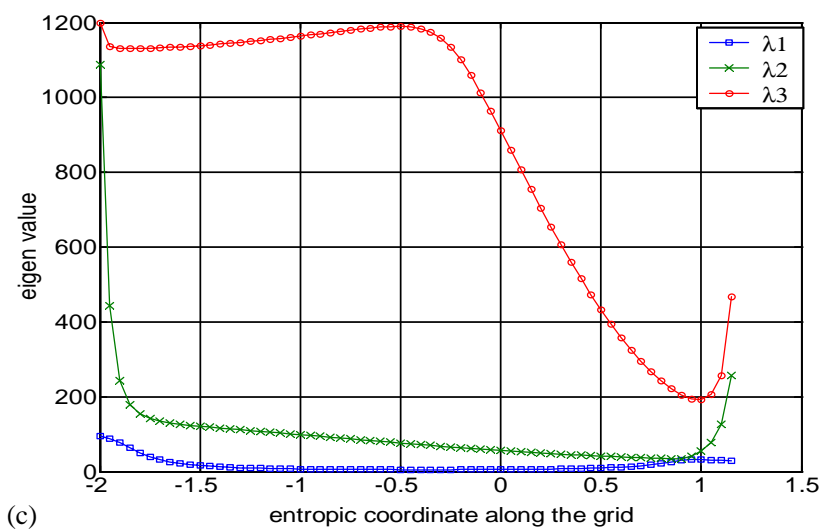

Fig. 11. One-dimensional invariant grid for model hydrogen burning system. (a) Projection into the $3 \mathrm{~d}$-space of $c_{\mathrm{H}}, c_{\mathrm{O}}$, $c_{\mathrm{OH}}$ concentrations. (b) Concentration values along the grid. (c) three smallest by absolute value nonzero eigenvalues of the symmetrically linearized system.

for the nodes which are close to the grid's edges. Here $x_{k, i}$ denotes the vector of the node in the $k$ th row, $i$ th column; $n_{k}$ is the number of nodes in the $k$ th row. Second tangent vector $g_{\text {col }}\left(x_{k, i}\right)$ is calculated completely analogously. In practice, it is convenient to orthogonalize $g_{\text {row }}\left(x_{k, i}\right)$ and $g_{\text {col }}\left(x_{k, i}\right)$. 
(a)
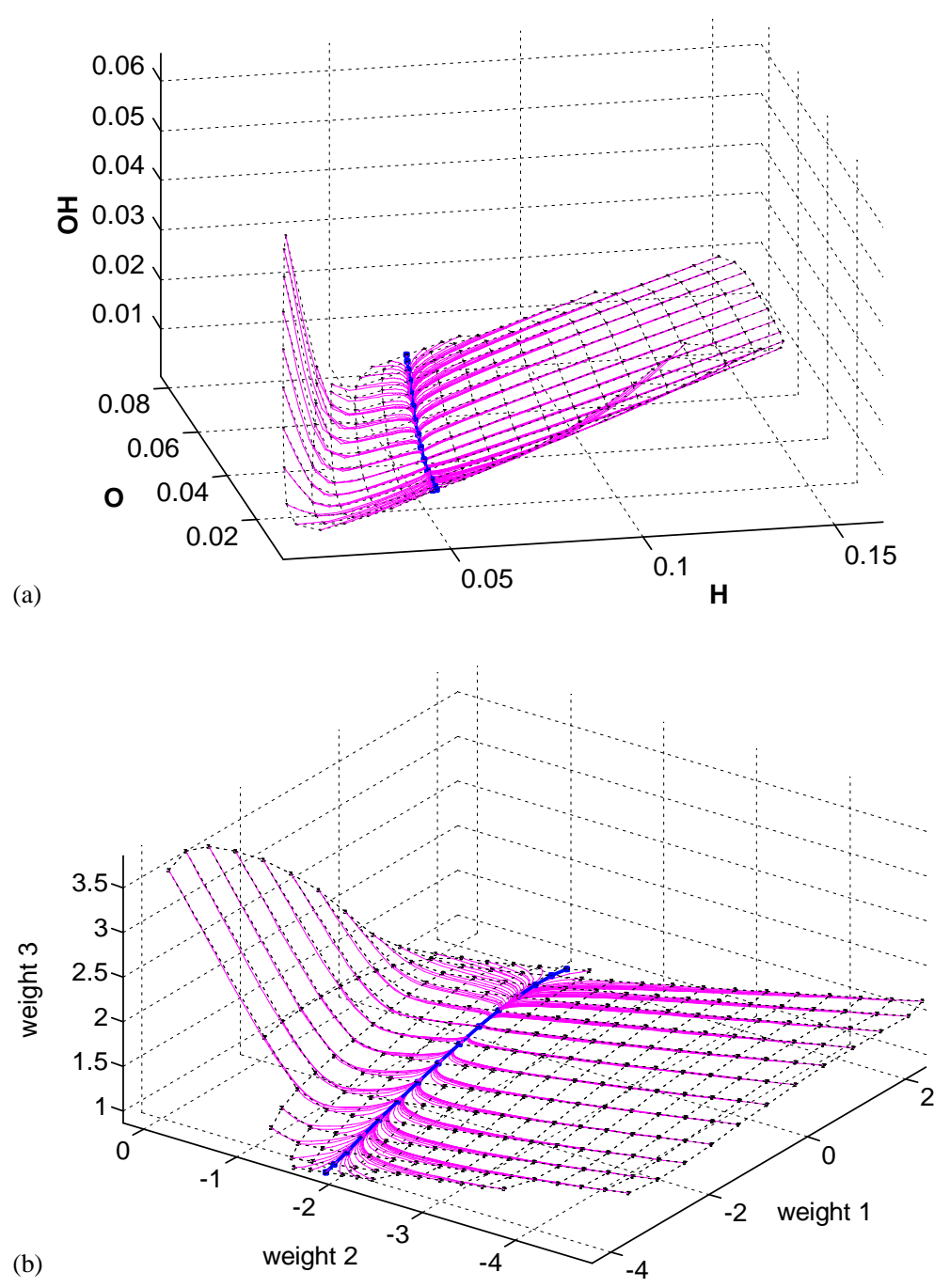

Fig. 12. Two-dimensional invariant grid for the model hydrogen burning system. (a) Projection into the $3 \mathrm{~d}$-space of $c_{\mathrm{H}}$, $c_{\mathrm{O}}, c_{\mathrm{OH}}$ concentrations. (b) Projection into the principal 3D-subspace. Trajectories of the system are shown coming out from the every grid node. Bold line denotes the one-dimensional invariant grid, starting from which the $2 \mathrm{D}$-grid was constructed.

Since the phase space is four-dimensional, it is impossible to visualize the grid in one of the coordinate 3D-views, as it was done in the previous subsection. To facilitate visualization one can utilize traditional methods of multi-dimensional data visualization. Here we make use of the principal components analysis (see, for example, [206]), which constructs a three-dimensional linear subspace with maximal dispersion of the othogonally projected data (grid nodes in our case). In other words, method of principal components constructs in multi-dimensional space such a three-dimensional box inside which the grid can be placed maximally tightly (in the mean square distance meaning). After projection of the grid nodes into this space, we get more or less adequate representation of the two-dimensional grid embedded into the six-dimensional concentrations space (Fig. 12b). The 
disadvantage of the approach is that the axes now do not have explicit meaning, being some linear combinations of the concentrations.

One attractive feature of two-dimensional grids is the possibility to use them as a screen, on which one can display different functions $f(\mathbf{c})$ defined in the concentrations space. This technology was exploited widely in the nonlinear data analysis by the elastic maps method [205]. The idea is to "unfold" the grid on a plane (to present it in the two-dimensional space, where the nodes form a regular lattice). In other words, we are going to work in the internal coordinates of the grid. In our case, the first internal coordinate (let's call it $s_{1}$ ) corresponds to the direction, co-oriented with the one-dimensional invariant grid, the second one (let's call it $s_{2}$ ) corresponds to the second slow direction. By how it was constructed, $s_{2}=0$ line corresponds to the one-dimensional invariant grid. Units of $s_{1}$ and $s_{2}$ are entropic distances in our case.

Every grid node has two internal coordinates $\left(s_{1}, s_{2}\right)$ and, simultaneously, corresponds to a vector in the concentration space. This allows us to map any function $f(\mathbf{c})$ from the multi-dimensional concentration space to the two-dimensional space of the grid. This mapping is defined in a finite number of points (grid nodes), and can be interpolated (linearly, in the simplest case) in between them. Using coloring and isolines one can visualize the values of the function in the neighborhood of the invariant manifold. This is meaningful, since, by the definition, the system spends most of the time in the vicinity of the invariant manifold, thus, one can visualize the behavior of the system. As a result of applying the technology, one obtains a set of color illustrations (a stack of information layers), put onto the grid as a map. This allows applying all the methods, working with stack of information layers, like geographical information systems (GIS) methods, which are very well developed.

In short words, the technique is a useful tool for exploration of dynamical systems. It allows to see simultaneously many different scenarios of the system behavior, together with different system's characteristics.

The simplest functions to visualize are the coordinates: $c_{i}(\mathbf{c})=c_{i}$. In Fig. 13 we displayed four colorings, corresponding to the four arbitrarily chosen concentrations functions $\left(\right.$ of $\mathrm{H}_{2}, \mathrm{O}, \mathrm{H}$ and $\mathrm{OH}$; Fig. $13 \mathrm{a}-\mathrm{d}$ ). The qualitative conclusions that can be made from the graphs are that, for example, the concentration of $\mathrm{H}_{2}$ practically does not change during the first fast motion (towards the 1D-grid) and then, gradually changes to the equilibrium value (the $\mathrm{H}_{2}$ coordinate is "slow"). The $\mathrm{O}$ coordinate is the opposite case, it is "fast" coordinate which changes quickly (on the first stage of motion) to the almost equilibrium value, and then it almost does not change. Basically, the slope angles of the coordinate isolines give some presentation of how "slow" a given concentration is. Fig. 13c shows interesting behavior of the $\mathrm{OH}$ concentration. Close to the $1 \mathrm{D}$ grid it behaves like "slow coordinate", but there is a region on the map where it has clear "fast" behavior (middle bottom of the graph).

The next two functions which one can want to visualize are the entropy $S$ and the entropy production $\sigma(\mathbf{c})=-\mathrm{d} G / \mathrm{d} t(\mathbf{c})=\sum_{i} \ln \left(c_{i} / c_{i}^{\mathrm{eq}}\right) \dot{c}_{i}$. They are shown in Fig. 14a,b.

Finally, we visualize the relation between the relaxation times of the fast motion towards the 2D-grid and along it. This is given in Fig. 14c. This picture allows to make a conclusion that two-dimensional consideration can be appropriate for the system (especially in the "high $\mathrm{H}_{2}$, high O" region), since the relaxation times "towards" and "along" the grid are definitely separated. One can compare this to Fig. 14d, where the relation between relaxation times towards and along the 1D-grid is shown. 

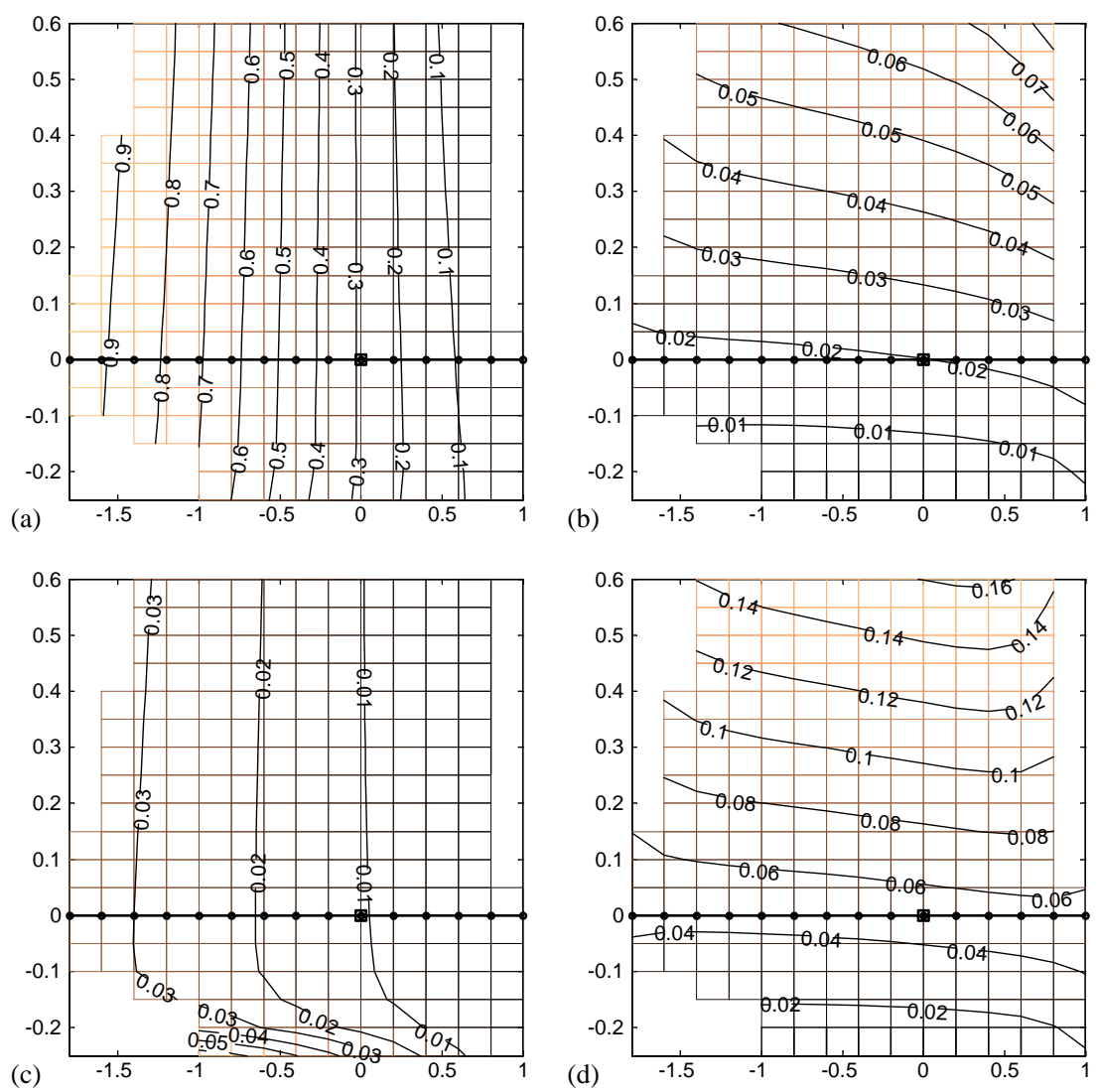

Fig. 13. Two-dimensional invariant grid as a screen for visualizing different functions defined in the concentrations space. The coordinate axes are entropic distances (see the text for the explanations) along the first and the second slowest directions on the grid. The corresponding 1D invariant grid is denoted by bold line, the equilibrium is denoted by square. (a) Concentration $\mathrm{H}_{2}$. (b) Concentration O. (c) Concentration OH. (d) Concentration H.

\section{Method of natural projector}

Ehrenfest suggested in 1911 a model of dynamics with a coarse-graining of the original conservative system in order to introduce irreversibility [215]. The idea of Ehrenfest is the following: One partitions the phase space of the Hamiltonian system into cells. The density distribution of the ensemble over the phase space evolves in time according to the Liouville equation within the time segments $n \tau<t<(n+1) \tau$, where $\tau$ is the fixed coarse-graining time step. Coarse-graining is executed at discrete times $n \tau$, densities are averaged over each cell. This alternation of the regular flow with the averaging describes the irreversible behavior of the system.

The formally most general construction extending the Ehrenfest idea is given below. Let us stay with notation of Section 3, and let a submanifold $F(W)$ be defined in the phase space $U$. Furthermore, we assume a map (a projection) is defined, $\Pi: U \rightarrow W$, with the properties:

$$
\Pi \circ F=1, \quad \Pi(F(y))=y .
$$



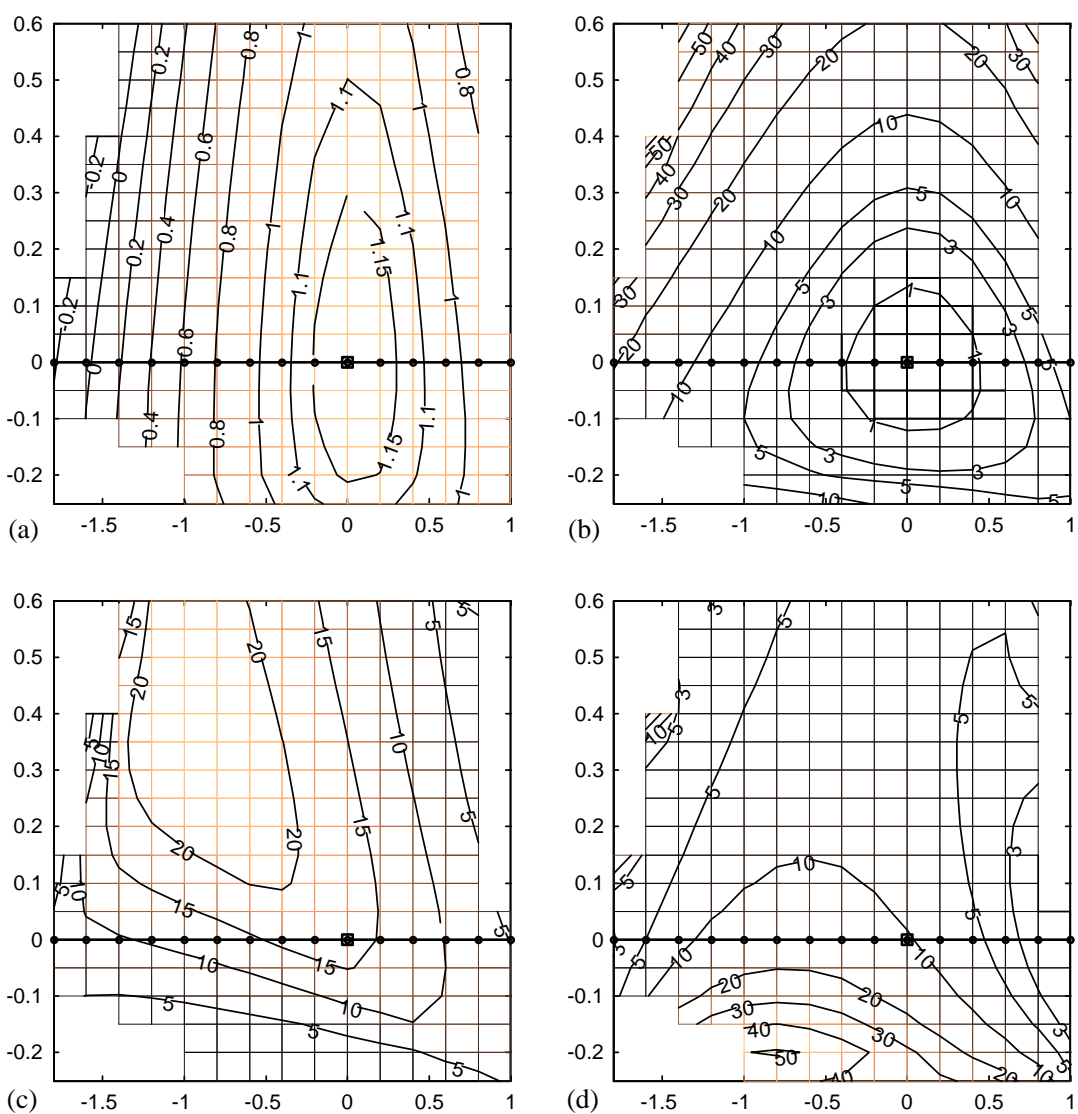

Fig. 14. Two-dimensional invariant grid as a screen for visualizing different functions defined in the concentrations space. The coordinate axes are entropic distances (see the text for the explanations) along the first and the second slowest directions on the grid. The corresponding 1D invariant grid is denoted by bold line, the equilibrium is denoted by square. (a) Entropy. (b) Entropy production. (c) $\lambda_{3} / \lambda_{2}$ relation. (d) $\lambda_{2} / \lambda_{1}$ relation.

In addition, one requires some mild properties of regularity, in particular, surjectivity of the differential, $D_{x} \Pi: E \rightarrow L$, in each point $x \in U$.

Let us fix the coarse-graining time $\tau>0$, and consider the following problem: Find a vector field $\Psi$ in $W$,

$$
\frac{\mathrm{d} y}{\mathrm{~d} t}=\Psi(y)
$$

such that, for every $y \in W$,

$$
\Pi\left(T_{\tau} F(y)\right)=\Theta_{\tau} y,
$$

where $T_{\tau}$ is the shift operator for system (76), and $\Theta_{\tau}$ is the (yet unknown!) shift operator for the system in question (362).

Eq. (363) means that one projects not the vector fields but segments of trajectories. Resulting vector field $\Psi(y)$ is called the natural projection of the vector field $J(x)$. 
Let us assume that there is a very stiff hierarchy of relaxation times in system (76): The motions of the system tend very rapidly to a slow manifold, and next proceed slowly along it. Then there is a smallness parameter, the ratio of these times. Let us take $F$ for the initial condition to the film equation (84). If the solution $F_{t}$ relaxes to the positively invariant manifold $F_{\infty}$, then, in the limit of a very stiff decomposition of motions, the natural projection of the vector field $J(x)$ tends to the usual infinitesimal projection of the restriction of $J$ on $F_{\infty}$, as $\tau \rightarrow \infty$ :

$$
\Psi_{\infty}(y)=\left.D_{x} \Pi\right|_{x=F_{\infty}(y)} J\left(F_{\infty}(y)\right) .
$$

For stiff dynamic systems, the limit (364) is qualitatively almost obvious: After some relaxation time $\tau_{0}$ (for $t>t_{0}$ ), the motion $T_{\tau}(x)$ is located in an $\epsilon$-neighborhood of $F_{\infty}(W)$. Thus, for $\tau \gg \tau_{0}$, the natural projection $\Psi$ (Eqs. (362) and (363)) is defined by the vector field attached to $F_{\infty}$ with any predefined accuracy. Rigorous proofs requires existence and uniqueness theorems, as well as homogeneous continuous dependence of solutions on initial conditions and right hand sides of equations.

The method of natural projector is applied not only to dissipative systems but also (and even mostly) to conservative systems. One of the methods to study the natural projector is based on series expansion ${ }^{13}$ in powers of $\tau$. Various other approximation schemes like Pade approximation are possible too.

The construction of natural projector was rediscovered in completely different context by Chorin et al. [217]. They constructed the optimal prediction methods for estimation the solution of nonlinear time-dependent problems when that solution is too complex to be fully resolved or when data are missing. The initial conditions for the unresolved components of the solution are drawn from a probability distribution, and their effect on a small set of variables that are actually computed is evaluated via statistical projection. The formalism resembles the projection methods of irreversible statistical mechanics, supplemented by the systematic use of conditional expectations and methods of solution for the orthogonal dynamics equation, needed to evaluate a nonMarkovian memory term. The result of the computations is close to the best possible estimate that can be obtained given the partial data.

Most of the methods of invariant manifold can be discussed as development of the ChapmanEnskog method. The idea is to construct the manifold of distribution functions, where the slow dynamics occurs. The change-over from solution of the Boltzmann equation to construction of an invariant manifold was a crucial idea of Enskog and Chapman. On the other hand, the method of natural projector gives development of ideas of the Hilbert method. This method was historically the first in the solution of the Boltzmann equation. The Hilbert method is not very popular now, nevertheless, for some purposes it may be more convenient than the Chapman-Enskog method, for example, for a studying of stationary solutions [218]. In the method of natural projector we are looking for a solutions of kinetic equations with quasi-equilibrium initial state (and in Hilbert method we start from the local equilibrium too). The main new element in the method of natural projector with respect to the Hilbert method is construction of the macroscopic equation (363). In the

\footnotetext{
${ }^{13}$ In the well known work of Lewis [216], this expansion was executed incorrectly (terms of different orders were matched on the left and on the right hand sides of Eq. (363)). This created an obstacle in a development of the method. See more detailed discussion in the section Example 10.
} 
next example the solution for the matching condition (363) will be found in a form of Taylor expansion.

Example 10: From reversible dynamics to Navier-Stokes and post-Navier-Stokes hydrodynamics by natural projector

The starting point of our construction are microscopic equations of motion. A traditional example of the microscopic description is the Liouville equation for classical particles. However, we need to stress that the distinction between "micro" and "macro" is always context dependent. For example, Vlasov's equation describes the dynamics of the one-particle distribution function. In one statement of the problem, this is a microscopic dynamics in comparison to the evolution of hydrodynamic moments of the distribution function. In a different setting, this equation itself is a result of reducing the description from the microscopic Liouville equation.

The problem of reducing the description includes a definition of the microscopic dynamics, and of the macroscopic variables of interest, for which equations of the reduced description must be found. The next step is the construction of the initial approximation. This is the well known quasi-equilibrium approximation, which is the solution to the variational problem, $S \rightarrow \max$, where $S$ in the entropy, under given constraints. This solution assumes that the microscopic distribution functions depend on time only through their dependence on the macroscopic variables. Direct substitution of the quasi-equilibrium distribution function into the microscopic equation of motion gives the initial approximation to the macroscopic dynamics. All further corrections can be obtained from a more precise approximation of the microscopic as well as of the macroscopic trajectories within a given time interval $\tau$ which is the parameter of our method.

The method described here has several clear advantages:

(i) It allows to derive complicated macroscopic equations, instead of writing them ad hoc. This fact is especially significant for the description of complex fluids. The method gives explicit expressions for relevant variables with one unknown parameter $(\tau)$. This parameter can be obtained from the experimental data.

(ii) Another advantage of the method is its simplicity. For example, in the case where the microscopic dynamics is given by the Boltzmann equation, the approach avoids evaluation of Boltzmann collision integral.

(iii) The most significant advantage of this formalization is that it is applicable to nonlinear systems. Usually, in the classical approaches to reduced description, the microscopic equation of motion is linear. In that case, one can formally write the evolution operator in the exponential form. Obviously, this does not work for nonlinear systems, such as, for example, systems with mean field interactions. The method which we are presenting here is based on mapping the expanded microscopic trajectory into the consistently expanded macroscopic trajectory. This does not require linearity. Moreover, the order-by-order recurrent construction can be, in principle, enhanced by restoring to other types of approximations, like Padé approximation, for example, but we do not consider these options here.

In the present section we discuss in detail applications of the method of natural projector $[16,17,21]$ to derivations of macroscopic equations in various cases, with and without mean field interaction 
potentials, for various choices of macroscopic variables, and demonstrate how computations are performed in the higher orders of the expansion. The structure of the Example is as follows: In the next subsection, for the sake of completeness, we describe briefly the formalization of Ehrenfest's approach [16,17]. We stress the role of the quasi-equilibrium approximation as the starting point for the constructions to follow. We derive explicit expressions for the correction to the quasi-equilibrium dynamics, and conclude this section with the entropy production formula and its discussion. In Section 3, we begin the discussion of applications. We use the present formalism in order to derive hydrodynamic equations. Zeroth approximation of the scheme is the Euler equations of the compressible nonviscous fluid. The first approximation leads to the system of Navier-Stokes equations. Moreover, the approach allows to obtain the next correction, so-called post-Navier-Stokes equations. The latter example is of particular interest. Indeed, it is well known that the post-NavierStokes equations as derived from the Boltzmann kinetic equation by the Chapman-Enskog method (Burnett and super-Burnett hydrodynamics) suffer from unphysical instability already in the linear approximation [53]. We demonstrate it by the explicit computation that the linearized higher-order hydrodynamic equations derived within our method are free from this drawback.

General construction. Let us consider a microscopic dynamics given by an equation,

$$
\dot{f}=J(f),
$$

where $f(x, t)$ is a distribution function over the phase space $x$ at time $t$, and where operator $J(f)$ may be linear or nonlinear. We consider linear macroscopic variables $M_{k}=\mu_{k}(f)$, where operator $\mu_{k}$ maps $f$ into $M_{k}$. The problem is to obtain closed macroscopic equations of motion, $\dot{M}_{k}=\phi_{k}(M)$. This is achieved in two steps: First, we construct an initial approximation to the macroscopic dynamics and, second, this approximation is further corrected on the basis of the coarse-gaining.

The initial approximation is the quasi-equilibrium approximation, and it is based on the entropy maximum principle under fixed constraints $[153,125]$ :

$$
S(f) \rightarrow \max , \quad \mu(f)=M,
$$

where $S$ is the entropy functional, which is assumed to be strictly concave, and $M$ is the set of the macroscopic variables $\{M\}$, and $\mu$ is the set of the corresponding operators. If the solution to the problem (366) exists, it is unique thanks to the concavity of the entropy functionals. Solution to Eq. (366) is called the quasi-equilibrium state, and it will be denoted as $f^{*}(M)$. The classical example is the local equilibrium of the ideal gas: $f$ is the one-body distribution function, $S$ is the Boltzmann entropy, $\mu$ are five linear operators, $\mu(f)=\int\left\{1, \boldsymbol{v}, v^{2}\right\} f \mathrm{~d} \boldsymbol{v}$, with $\boldsymbol{v}$ the particle's velocity; the corresponding $f^{*}(M)$ is called the local Maxwell distribution function.

If the microscopic dynamics is given by Eq. (365), then the quasi-equilibrium dynamics of the variables $M$ reads:

$$
\dot{M}_{k}=\mu_{k}\left(J\left(f^{*}(M)\right)\right)=\phi_{k}^{*} .
$$

The quasi-equilibrium approximation has important property, it conserves the type of the dynamics: If the entropy monotonically increases (or not decreases) due to Eq. (365), then the same is true for the quasi-equilibrium entropy, $S^{*}(M)=S\left(f^{*}(M)\right)$, due to the quasi-equilibrium dynamics (367). That is, if

$$
\dot{S}=\frac{\partial S(f)}{\partial f} \dot{f}=\frac{\partial S(f)}{\partial f} J(f) \geqslant 0,
$$


then

$$
\dot{S}^{*}=\sum_{k} \frac{\partial S^{*}}{\partial M_{k}} \dot{M}_{k}=\sum_{k} \frac{\partial S^{*}}{\partial M_{k}} \mu_{k}\left(J\left(f^{*}(M)\right)\right) \geqslant 0 .
$$

Summation in $k$ always implies summation or integration over the set of labels of the macroscopic variables.

Conservation of the type of dynamics by the quasi-equilibrium approximation is a simple yet a general and useful fact. If the entropy $S$ is an integral of motion of Eq. (365) then $S^{*}(M)$ is the integral of motion for the quasi-equilibrium equation (367). Consequently, if we start with a system which conserves the entropy (for example, with the Liouville equation) then we end up with the quasi-equilibrium system which conserves the quasi-equilibrium entropy. For instance, if $M$ is the one-body distribution function, and (365) is the (reversible) Liouville equation, then (367) is the Vlasov equation which is reversible, too. On the other hand, if the entropy was monotonically increasing on solutions to Eq. (365), then the quasi-equilibrium entropy also increases monotonically on solutions to the quasi-equilibrium dynamic equations (367). For instance, if Eq. (365) is the Boltzmann equation for the one-body distribution function, and $M$ is a finite set of moments (chosen in such a way that the solution to problem (366) exists), then (367) are closed moment equations for $M$ which increase the quasi-equilibrium entropy (this is the essence of a well known generalization of Grad's moment method).

\section{Enhancement of quasi-equilibrium approximations for entropy-conserving dynamics}

The goal of the present subsection is to describe the simplest analytic implementation, the microscopic motion with periodic coarse-graining. The notion of coarse-graining was introduced by Ehrenfest's in their seminal work [215]: The phase space is partitioned into cells, the coarse-grained variables are the amounts of the phase density inside the cells. Dynamics is described by the two processes, by the Liouville equation for $f$, and by periodic coarse-graining, replacement of $f(x)$ in each cell by its average value in this cell. The coarse-graining operation means forgetting the microscopic details, or of the history.

From the perspective of general quasi-equilibrium approximations, periodic coarse-graining amounts to the return of the true microscopic trajectory on the quasi-equilibrium manifold with the preservation of the macroscopic variables. The motion starts at the quasi-equilibrium state $f_{i}^{*}$. Then the true solution $f_{i}(t)$ of the microscopic equation (365) with the initial condition $f_{i}(0)=f_{i}^{*}$ is coarse-grained at a fixed time $t=\tau$, solution $f_{i}(\tau)$ is replaced by the quasi-equilibrium function $f_{i+1}^{*}=f^{*}\left(\mu\left(f_{i}(\tau)\right)\right)$. This process is sketched in Fig. 15.

From the features of the quasi-equilibrium approximation it follows that for the motion with periodic coarse-graining, the inequality is valid,

$$
S\left(f_{i}^{*}\right) \leqslant S\left(f_{i+1}^{*}\right)
$$

the equality occurs if and only if the quasi-equilibrium is the invariant manifold of the dynamic system (365). Whenever the quasi-equilibrium is not the solution to Eq. (365), the strict inequality in (369) demonstrates the entropy increase.

In other words, let us assume that the trajectory begins at the quasi-equilibrium manifold, then it takes off from this manifold according to the microscopic evolution equations. Then, after some time 


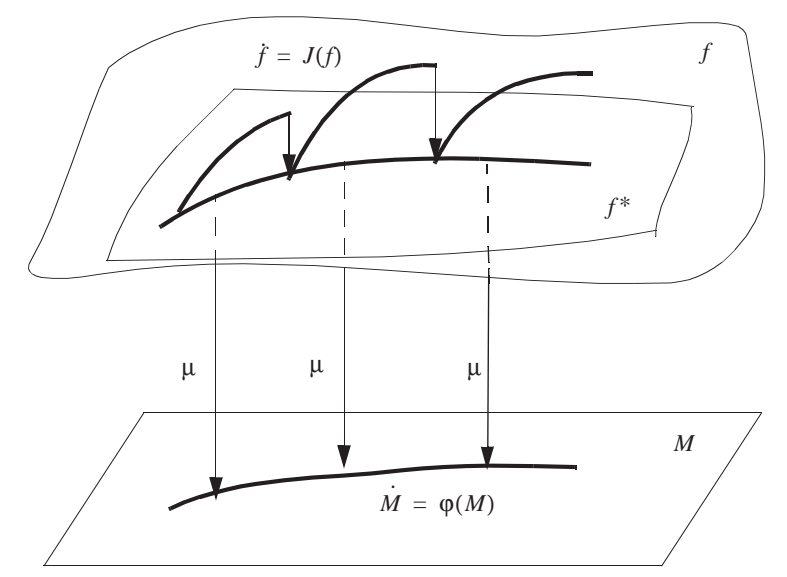

Fig. 15. Coarse-graining scheme. $f$ is the space of microscopic variables, $M$ is the space of the macroscopic variables, $f^{*}$ is the quasi-equilibrium manifold, $\mu$ is the mapping from the microscopic to the macroscopic space.

$\tau$, the trajectory is coarse-grained, that is the, state is brought back on the quasi-equilibrium manifold keeping the values of the macroscopic variables. The irreversibility is born in the latter process, and this construction clearly rules out quasi-equilibrium manifolds which are invariant with respect to the microscopic dynamics, as candidates for a coarse-graining. The coarse-graining indicates the way to derive equations for macroscopic variables from the condition that the macroscopic trajectory, $M(t)$, which governs the motion of the quasi-equilibrium states, $f^{*}(M(t))$, should match precisely the same points on the quasi-equilibrium manifold, $f^{*}(M(t+\tau))$, and this matching should be independent of both the initial time, $t$, and the initial condition $M(t)$. The problem is then how to derive the continuous time macroscopic dynamics which would be consistent with this picture. The simplest realization suggested in Refs. [16,17] is based on using an expansion of both the microscopic and the macroscopic trajectories. Here we present this construction to the third order accuracy, in a general form, whereas only the second-order accurate construction has been discussed in [16,17].

Let us write down the solution to the microscopic equation (365), and approximate this solution by the polynomial of third order in $\tau$. Introducing notation, $J^{*}=J\left(f^{*}(M(t))\right)$, we write,

$$
f(t+\tau)=f^{*}+\tau J^{*}+\frac{\tau^{2}}{2} \frac{\partial J^{*}}{\partial f} J^{*}+\frac{\tau^{3}}{3 !}\left(\frac{\partial J^{*}}{\partial f} \frac{\partial J^{*}}{\partial f} J^{*}+\frac{\partial^{2} J^{*}}{\partial f^{2}} J^{*} J^{*}\right)+\mathrm{o}\left(\tau^{3}\right) .
$$

Evaluation of the macroscopic variables on function (370) gives

$$
\begin{aligned}
M_{k}(t+\tau)= & M_{k}+\tau \phi_{k}^{*}+\frac{\tau^{2}}{2} \mu_{k}\left(\frac{\partial J^{*}}{\partial f} J^{*}\right) \\
& +\frac{\tau^{3}}{3 !}\left\{\mu_{k}\left(\frac{\partial J^{*}}{\partial f} \frac{\partial J^{*}}{\partial f} J^{*}\right)+\mu_{k}\left(\frac{\partial^{2} J^{*}}{\partial f^{2}} J^{*} J^{*}\right)\right\}+\mathrm{o}\left(\tau^{3}\right),
\end{aligned}
$$

where $\phi_{k}^{*}=\mu_{k}\left(J^{*}\right)$ is the quasi-equilibrium macroscopic vector field (the right hand side of Eq. (367)), and all the functions and derivatives are taken in the quasi-equilibrium state at time $t$. 
We shall now establish the macroscopic dynamic by matching the macroscopic and the microscopic dynamics. Specifically, the macroscopic dynamic equations (367) with the right hand side not yet defined, give the following third-order result:

$$
\begin{aligned}
M_{k}(t+\tau)= & M_{k}+\tau \phi_{k}+\frac{\tau^{2}}{2} \sum_{j} \frac{\partial \phi_{k}}{\partial M_{j}} \phi_{j} \\
& +\frac{\tau^{3}}{3 !} \sum_{i j}\left(\frac{\partial^{2} \phi_{k}}{\partial M_{i} M_{j}} \phi_{i} \phi_{j}+\frac{\partial \phi_{k}}{\partial M_{i}} \frac{\partial \phi_{i}}{\partial M_{j}} \phi_{j}\right)+\mathrm{o}\left(\tau^{3}\right) .
\end{aligned}
$$

Expanding functions $\phi_{k}$ into the series $\phi_{k}=R_{k}^{(0)}+\tau R_{k}^{(1)}+\tau^{2} R_{k}^{(2)}+\cdots,\left(R_{k}^{(0)}=\phi^{*}\right)$, and requiring that the microscopic and the macroscopic dynamics coincide to the order of $\tau^{3}$, we obtain the sequence of corrections for the right hand side of the equation for the macroscopic variables. Zeroth order is the quasi-equilibrium approximation to the macroscopic dynamics. The first-order correction gives

$$
R_{k}^{(1)}=\frac{1}{2}\left\{\mu_{k}\left(\frac{\partial J^{*}}{\partial f} J^{*}\right)-\sum_{j} \frac{\partial \phi_{k}^{*}}{\partial M_{j}} \phi_{j}^{*}\right\} .
$$

The next, second-order correction has the following explicit form:

$$
\begin{aligned}
R_{k}^{(2)}= & \frac{1}{3 !}\left\{\mu_{k}\left(\frac{\partial J^{*}}{\partial f} \frac{\partial J^{*}}{\partial f} J^{*}\right)+\mu_{k}\left(\frac{\partial^{2} J^{*}}{\partial f^{2}} J^{*} J^{*}\right)\right\}-\frac{1}{3 !} \sum_{i j}\left(\frac{\partial \phi_{k}^{*}}{\partial M_{i}} \frac{\partial \phi_{i}^{*}}{\partial M_{j}} \phi_{j}^{*}\right) \\
& -\frac{1}{3 !} \sum_{i j}\left(\frac{\partial^{2} \phi_{k}^{*}}{\partial M_{i} \partial M_{j}} \phi_{i}^{*} \phi_{j}^{*}\right)-\frac{1}{2} \sum_{j}\left(\frac{\partial \phi_{k}^{*}}{\partial M_{j}} R_{j}^{(1)}+\frac{\partial R_{j}^{(1)}}{\partial M_{j}} \phi_{j}^{*}\right),
\end{aligned}
$$

Further corrections are found by the same token. Eqs. (373)-(374) give explicit closed expressions for corrections to the quasi-equilibrium dynamics to the order of accuracy specified above. They are used below in various specific examples.

Entropy production. The most important consequence of the above construction is that the resulting continuous time macroscopic equations retain the dissipation property of the discrete time coarse-graining (369) on each order of approximation $n \geqslant 1$. Let us first consider the entropy production formula for the first-order approximation. In order to shorten notations, it is convenient to introduce the quasi-equilibrium projection operator,

$$
P^{*} g=\sum_{k} \frac{\partial f^{*}}{\partial M_{k}} \mu_{k}(g) .
$$

It has been demonstrated in [17] that the entropy production,

$$
\dot{S}_{(1)}^{*}=\sum_{k} \frac{\partial S^{*}}{\partial M_{k}}\left(R_{k}^{(0)}+\tau R_{k}^{(1)}\right),
$$

equals

$$
\dot{S}_{(1)}^{*}=-\left.\frac{\tau}{2}\left(1-P^{*}\right) J^{*} \frac{\partial^{2} S^{*}}{\partial f \partial f}\right|_{f^{*}}\left(1-P^{*}\right) J^{*} .
$$


Eq. (376) is nonnegative definite due to concavity of the entropy. Entropy production (376) is equal to zero only if the quasi-equilibrium approximation is the true solution to the microscopic dynamics, that is, if $\left(1-P^{*}\right) J^{*} \equiv 0$. While quasi-equilibrium approximations which solve the Liouville equation are uninteresting objects (except, of course, for the equilibrium itself), vanishing of the entropy production in this case is a simple test of consistency of the theory. Note that the entropy production (376) is proportional to $\tau$. Note also that projection operator does not appear in our consideration a priory, rather, it is the result of exploring the coarse-graining condition in the previous section.

Though Eq. (376) looks very natural, its existence is rather subtle. Indeed, Eq. (376) is a difference of the two terms, $\sum_{k} \mu_{k}\left(J^{*} \partial J^{*} / \partial f\right)$ (contribution of the second-order approximation to the microscopic trajectory), and $\sum_{i k} R_{i}^{(0)} \partial R_{k}^{(0)} / \partial M_{i}$ (contribution of the derivative of the quasi-equilibrium vector field). Each of these expressions separately gives a positive contribution to the entropy production, and Eq. (376) is the difference of the two positive definite expressions. In the higher order approximations, these subtractions are more involved, and explicit demonstration of the entropy production formulae becomes a formidable task. Yet, it is possible to demonstrate the increase-in-entropy without explicit computation, though at a price of smallness of $\tau$. Indeed, let us denote $\dot{S}_{(n)}^{*}$ the time derivative of the entropy on the $n$th order approximation. Then

$$
\int_{t}^{t+\tau} \dot{S}_{(n)}^{*}(s) \mathrm{d} s=S^{*}(t+\tau)-S^{*}(t)+\mathrm{O}\left(\tau^{n+1}\right),
$$

where $S^{*}(t+\tau)$ and $S^{*}(t)$ are true values of the entropy at the adjacent states of the $H$-curve. The difference $\delta S=S^{*}(t+\tau)-S^{*}(t)$ is strictly positive for any fixed $\tau$, and, by Eq. (376), $\delta S \sim \tau^{2}$ for small $\tau$. Therefore, if $\tau$ is small enough, the right hand side in the above expression is positive, and

$$
\tau \dot{S}_{(n)}^{*}\left(\theta_{(n)}\right)>0
$$

where $t \leqslant \theta_{(n)} \leqslant t+\tau$. Finally, since $\dot{S}_{(n)}^{*}(t)=\dot{S}_{(n)}^{*}(s)+\mathrm{O}\left(\tau^{n}\right)$ for any $s$ on the segment $[t, t+\tau]$, we can replace $\dot{S}_{(n)}^{*}\left(\theta_{(n)}\right)$ in the latter inequality by $\dot{S}_{(n)}^{*}(t)$. The sense of this consideration is as follows: Since the entropy production formula (376) is valid in the leading order of the construction, the entropy production will not collapse in the higher orders at least if the coarse-graining time is small enough. More refined estimations can be obtained only from the explicit analysis of the higher-order corrections.

Relation to the work of Lewis. Among various realizations of the coarse-graining procedures, the work of Lewis [216] appears to be most close to our approach. It is therefore pertinent to discuss the differences. Both methods are based on the coarse-graining condition,

$$
M_{k}(t+\tau)=\mu_{k}\left(T_{\tau} f^{*}(M(t))\right),
$$

where $T_{\tau}$ is the formal solution operator of the microscopic dynamics. Above, we applied a consistent expansion of both, the left hand side and the right hand side of the coarse-graining condition (377), in terms of the coarse-graining time $\tau$. In the work of Lewis [216], it was suggested, as a general way to exploring condition (377), to write the first-order equation for $M$ in the form of the differential pursuit,

$$
M_{k}(t)+\tau \frac{\mathrm{d} M_{k}(t)}{\mathrm{d} t} \approx \mu_{k}\left(T_{\tau} f^{*}(M(t))\right) .
$$


In other words, in the work of Lewis [216], the expansion to the first order was considered on the left (macroscopic) side of Eq. (377), whereas the right hand side containing the microscopic trajectory $T_{\tau} f^{*}(M(t))$ was not treated on the same footing. Clearly, expansion of the right hand side to first order in $\tau$ is the only equation which is common in both approaches, and this is the quasi-equilibrium dynamics. However, the difference occurs already in the next, second-order term (see Refs. [16,17] for details). Namely, the expansion to the second order of the right hand side of Lewis' equation [216] results in a dissipative equation (in the case of the Liouville equation, for example) which remains dissipative even if the quasi-equilibrium approximation is the exact solution to the microscopic dynamics, that is, when microscopic trajectories once started on the quasi-equilibrium manifold belong to it in all the later times, and thus no dissipation can be born by any coarse-graining.

On the other hand, our approach assumes a certain smoothness of trajectories so that application of the low-order expansion bears physical significance. For example, while using lower-order truncations it is not possible to derive the Boltzmann equation because in that case the relevant quasi-equilibrium manifold ( $N$-body distribution function is proportional to the product of one-body distributions, or uncorrelated states, see next section) is almost invariant during the long time (of the order of the mean free flight of particles), while the trajectory steeply leaves this manifold during the short-time pair collision. It is clear that in such a case lower-order expansions of the microscopic trajectory do not lead to useful results. It has been clearly stated by Lewis [216], that the exploration of the condition (377) depends on the physical situation, and how one makes approximations. In fact, derivation of the Boltzmann equation given by Lewis on the basis of the condition (377) does not follow the differential pursuit approximation: As is well known, the expansion in terms of particle's density of the solution to the BBGKY hierarchy is singular, and begins with the linear in time term. Assuming the quasi-equilibrium approximation for the $N$-body distribution function under fixed one-body distribution function, and that collisions are well localized in space and time, one gets on the right hand side of Eq. (377),

$$
f(t+\tau)=f(t)+n \tau J_{\mathrm{B}}(f(t))+\mathrm{o}(n),
$$

where $n$ is particle's density, $f$ is the one-particle distribution function, and $J_{\mathrm{B}}$ is the Boltzmann's collision integral. Next, using the mean-value theorem on the left hand side of Eq. (377), the Boltzmann equation is derived (see also a recent elegant renormalization-group argument for this derivation [36]).

We stress that our approach of matched expansion for exploring the coarse-graining condition (377) is, in fact, the exact (formal) statement that the unknown macroscopic dynamics which causes the shift of $M_{k}$ on the left hand side of Eq. (377) can be reconstructed order-by-order to any degree of accuracy, whereas the low-order truncations may be useful for certain physical situations. A thorough study of the cases beyond the lower-order truncations is of great importance which is left for future work.

Equations of hydrodynamics for simple fluid. The method discussed above enables one to establish in a simple way the form of equations of the macroscopic dynamics to various degrees of approximation. In this section, the microscopic dynamics is given by the Liouville equation, similar to the previous case. However, we take another set of macroscopic variables: density, average velocity, and average temperature of the fluid. Under this condition the solution to problem (366) is the local Maxwell distribution. For the hydrodynamic equations, the zeroth (quasi-equilibrium) approximation 
is given by Euler's equations of compressible nonviscous fluid. The next order approximation are the Navier-Stokes equations which have dissipative terms.

Higher-order approximations to the hydrodynamic equations, when they are derived from the Boltzmann kinetic equation (so-called Burnett approximation), are subject to various difficulties, in particular, they exhibit an instability of sound waves at sufficiently short wave length (see, e.g. [24] for a recent review). Here we demonstrate how model hydrodynamic equations, including postNavier-Stokes approximations, can be derived on the basis of coarse-graining idea, and investigate the linear stability of the obtained equations. We will find that the resulting equations are stable.

Two points need a clarification before we proceed further [17]. First, below we consider the simplest Liouville equation for the one-particle distribution, describing a free moving particle without interactions. The procedure of coarse-graining we use is an implementation of collisions leading to dissipation. If we had used the full interacting $N$-particle Liouville equation, the result would be different, in the first place, in the expression for the local equilibrium pressure. Whereas in the present case we have the ideal gas pressure, in the $N$-particle case the nonideal gas pressure would arise.

Second, and more essential is that, to the order of the Navier-Stokes equations, the result of our method is identical to the lowest-order Chapman-Enskog method as applied to the Boltzmann equation with a single relaxation time model collision integral (the Bhatnagar-Gross-Krook model [89]). However, this happens only at this particular order of approximation, because already the next, post-Navier-Stokes approximation, is different from the Burnett hydrodynamics as derived from the BGK model (the latter is linearly unstable).

Derivation of the Navier-Stokes equations. Let us assume that reversible microscopic dynamics is given by the one-particle Liouville equation,

$$
\frac{\partial f}{\partial t}=-v_{i} \frac{\partial f}{\partial r_{i}},
$$

where $f=f(\boldsymbol{r}, \boldsymbol{v}, t)$ is the one-particle distribution function, and index $i$ runs over spatial components $\{x, y, z\}$. Subject to appropriate boundary conditions which we assume, this equation conserves the Boltzmann entropy $S=-k_{\mathrm{B}} \int f \ln f \mathrm{~d} \boldsymbol{v} \mathrm{d} \boldsymbol{r}$.

We introduce the following hydrodynamic moments as the macroscopic variables: $M_{0}=\int f \mathrm{~d} \boldsymbol{v}$, $M_{i}=\int v_{i} f \mathrm{~d} \boldsymbol{v}, M_{4}=\int v^{2} f \mathrm{~d} \boldsymbol{v}$. These variables are related to the more conventional density, average velocity and temperature, $n, \boldsymbol{u}, T$ as follows:

$$
\begin{aligned}
& M_{0}=n, \quad M_{i}=n u_{i}, \quad M_{4}=\frac{3 n k_{\mathrm{B}} T}{m}+n u^{2}, \\
& n=M_{0}, \quad u_{i}=M_{0}^{-1} M_{i}, \quad T=\frac{m}{3 k_{\mathrm{B}} M_{0}}\left(M_{4}-M_{0}^{-1} M_{i} M_{i}\right) .
\end{aligned}
$$

The quasi-equilibrium distribution function (local Maxwellian) reads:

$$
f_{0}=n\left(\frac{m}{2 \pi k_{\mathrm{B}} T}\right)^{3 / 2} \exp \left(\frac{-m(v-u)^{2}}{2 k_{\mathrm{B}} T}\right) \text {. }
$$

Here and below, $n, \boldsymbol{u}$, and $T$ depend on $\boldsymbol{r}$ and $t$.

Based on the microscopic dynamics (379), the set of macroscopic variables (380), and the quasi-equilibrium (381), we can derive the equations of the macroscopic motion. 
A specific feature of the present example is that the quasi-equilibrium equation for the density (the continuity equation),

$$
\frac{\partial n}{\partial t}=-\frac{\partial n u_{i}}{\partial r_{i}}
$$

should be excluded out of the further corrections. This rule should be applied generally: If a part of the chosen macroscopic variables (momentum flux $n \boldsymbol{u}$ here) correspond to fluxes of other macroscopic variables, then the quasi-equilibrium equation for the latter is already exact, and has to be exempted of corrections.

The quasi-equilibrium approximation for the rest of the macroscopic variables is derived in the usual way. In order to derive the equation for the velocity, we substitute the local Maxwellian into the one-particle Liouville equation, and act with the operator $\mu_{k}=\int v_{k} \mathrm{~d} \boldsymbol{v}$ on both the sides of Eq. (379). We have

$$
\frac{\partial n u_{k}}{\partial t}=-\frac{\partial}{\partial r_{k}} \frac{n k_{\mathrm{B}} T}{m}-\frac{\partial n u_{k} u_{j}}{\partial r_{j}} .
$$

Similarly, we derive the equation for the energy density, and the complete system of equations of the quasi-equilibrium approximation reads (Euler equations):

$$
\begin{aligned}
& \frac{\partial n}{\partial t}=-\frac{\partial n u_{i}}{\partial r_{i}} \\
& \frac{\partial n u_{k}}{\partial t}=-\frac{\partial}{\partial r_{k}} \frac{n k_{\mathrm{B}} T}{m}-\frac{\partial n u_{k} u_{j}}{\partial r_{j}}, \\
& \frac{\partial \varepsilon}{\partial t}=-\frac{\partial}{\partial r_{i}}\left(\frac{5 k_{\mathrm{B}} T}{m} n u_{i}+u^{2} n u_{i}\right) .
\end{aligned}
$$

Now we are going to derive the next order approximation to the macroscopic dynamics (first order in the coarse-graining time $\tau$ ). For the velocity equation we have

$$
R_{n u_{k}}=\frac{1}{2}\left(\int v_{k} v_{i} v_{j} \frac{\partial^{2} f_{0}}{\partial r_{i} \partial r_{j}} \mathrm{~d} \boldsymbol{v}-\sum_{j} \frac{\partial \phi_{n u_{k}}}{\partial M_{j}} \phi_{j}\right),
$$

where $\phi_{j}$ are the corresponding right hand sides of the Euler equations (383). In order to take derivatives with respect to macroscopic moments $\left\{M_{0}, M_{i}, M_{4}\right\}$, we need to rewrite Eqs. (383) in terms of these variables instead of $\left\{n, u_{i}, T\right\}$. After some computation, we obtain

$$
R_{n u_{k}}=\frac{1}{2} \frac{\partial}{\partial r_{j}}\left(\frac{n k_{\mathrm{B}} T}{m}\left[\frac{\partial u_{k}}{\partial r_{j}}+\frac{\partial u_{j}}{\partial r_{k}}-\frac{2}{3} \frac{\partial u_{n}}{\partial r_{n}} \delta_{k j}\right]\right) .
$$

For the energy we obtain

$$
R_{\varepsilon}=\frac{1}{2}\left(\int v^{2} v_{i} v_{j} \frac{\partial^{2} f_{0}}{\partial r_{i} \partial r_{j}} \mathrm{~d} \boldsymbol{v}-\sum_{j} \frac{\partial \phi_{\varepsilon}}{\partial M_{j}} \phi_{j}\right)=\frac{5}{2} \frac{\partial}{\partial r_{i}}\left(\frac{n k_{\mathrm{B}}^{2} T}{m^{2}} \frac{\partial T}{\partial r_{i}}\right) .
$$


Thus, we get the system of the Navier-Stokes equation in the following form:

$$
\begin{aligned}
& \frac{\partial n}{\partial t}=-\frac{\partial n u_{i}}{\partial r_{i}}, \\
& \frac{\partial n u_{k}}{\partial t}=-\frac{\partial}{\partial r_{k}} \frac{n k_{\mathrm{B}} T}{m}-\frac{\partial n u_{k} u_{j}}{\partial r_{j}}+\frac{\tau}{2} \frac{\partial}{\partial r_{j}} \frac{n k_{\mathrm{B}} T}{m}\left(\frac{\partial u_{k}}{\partial r_{j}}+\frac{\partial u_{j}}{\partial r_{k}}-\frac{2}{3} \frac{\partial u_{n}}{\partial r_{n}} \delta_{k j}\right), \\
& \frac{\partial \varepsilon}{\partial t}=-\frac{\partial}{\partial r_{i}}\left(\frac{5 k_{\mathrm{B}} T}{m} n u_{i}+u^{2} n u_{i}\right)+\tau \frac{5}{2} \frac{\partial}{\partial r_{i}}\left(\frac{n k_{\mathrm{B}}^{2} T}{m^{2}} \frac{\partial T}{\partial r_{i}}\right) .
\end{aligned}
$$

We see that kinetic coefficients (viscosity and heat conductivity) are proportional to the coarsegraining time $\tau$. Note that they are identical with kinetic coefficients as derived from the BhatnagarGross-Krook model [89] in the first approximation of the Chapman-Enskog method [51] (also, in particular, no bulk viscosity).

Post-Navier-Stokes equations. Now we are going to obtain the second-order approximation to the hydrodynamic equations in the framework of the present approach. We will compare qualitatively the result with the Burnett approximation. The comparison concerns stability of the hydrodynamic modes near global equilibrium, which is violated for the Burnett approximation. Though the derivation is straightforward also in the general, nonlinear case, we shall consider only the linearized equations which is appropriate to our purpose here.

Linearizing the local Maxwell distribution function, we obtain

$$
\begin{aligned}
f & =n_{0}\left(\frac{m}{2 \pi k_{\mathrm{B}} T_{0}}\right)^{3 / 2}\left(\frac{n}{n_{0}}+\frac{m v_{n}}{k_{\mathrm{B}} T_{0}} u_{n}+\left(\frac{m v^{2}}{2 k_{\mathrm{B}} T_{0}}-\frac{3}{2}\right) \frac{T}{T_{0}}\right) \mathrm{e}^{-m v^{2} / 2 k_{\mathrm{B}} T_{0}} \\
& =\left\{M_{0}+2 M_{i} c_{i}+\left(\frac{2}{3} M_{4}-M_{0}\right)\left(c^{2}-\frac{3}{2}\right)\right\} \mathrm{e}^{-c^{2}}
\end{aligned}
$$

where we have introduced dimensionless variables: $c_{i}=v_{i} / v_{T}, v_{T}=\sqrt{2 k_{\mathrm{B}} T_{0} / m}$ is the thermal velocity, $M_{0}=\delta n / n_{0}, M_{i}=\delta u_{i} / v_{T}, M_{4}=(3 / 2)\left(\delta n / n_{0}+\delta T / T_{0}\right)$. Note that $\delta n$, and $\delta T$ determine deviations of these variables from their equilibrium values, $n_{0}$, and $T_{0}$.

The linearized Navier-Stokes equations read

$$
\begin{aligned}
& \frac{\partial M_{0}}{\partial t}=-\frac{\partial M_{i}}{\partial r_{i}} \\
& \frac{\partial M_{k}}{\partial t}=-\frac{1}{3} \frac{\partial M_{4}}{\partial r_{k}}+\frac{\tau}{4} \frac{\partial}{\partial r_{j}}\left(\frac{\partial M_{k}}{\partial r_{j}}+\frac{\partial M_{j}}{\partial r_{k}}-\frac{2}{3} \frac{\partial M_{n}}{\partial r_{n}} \delta_{k j}\right), \\
& \frac{\partial M_{4}}{\partial t}=-\frac{5}{2} \frac{\partial M_{i}}{\partial r_{i}}+\tau \frac{5}{2} \frac{\partial^{2} M_{4}}{\partial r_{i} \partial r_{i}} .
\end{aligned}
$$


Let us first compute the post-Navier-Stokes correction to the velocity equation. In accordance with Eq. (374), the first part of this term under linear approximation is

$$
\begin{aligned}
\frac{1}{3 !} \mu_{k}\left(\frac{\partial J^{*}}{\partial f} \frac{\partial J^{*}}{\partial f} J^{*}\right)-\frac{1}{3 !} \sum_{i j}\left(\frac{\partial \phi_{k}^{*}}{\partial M_{i}} \frac{\partial \phi_{i}^{*}}{\partial M_{j}} \phi_{j}^{*}\right) \\
=-\frac{1}{6} \int c_{k} \frac{\partial^{3}}{\partial r_{i} \partial r_{j} \partial r_{n}} c_{i} c_{j} c_{n}\left\{M_{0}+2 M_{i} c_{i}+\left(\frac{2}{3} M_{4}-M_{0}\right)\left(c^{2}-\frac{3}{2}\right)\right\} \mathrm{e}^{-c^{2} \mathrm{~d}^{3} c} \\
\quad+\frac{5}{108} \frac{\partial}{\partial r_{i}} \frac{\partial^{2} M_{4}}{\partial r_{s} \partial r_{s}}=\frac{1}{6} \frac{\partial}{\partial r_{k}}\left(\frac{3}{4} \frac{\partial^{2} M_{0}}{\partial r_{s} \partial r_{s}}-\frac{\partial^{2} M_{4}}{\partial r_{s} \partial r_{s}}\right)+\frac{5}{108} \frac{\partial}{\partial r_{k}} \frac{\partial^{2} M_{4}}{\partial r_{s} \partial r_{s}} \\
=\frac{1}{8} \frac{\partial}{\partial r_{k}} \frac{\partial^{2} M_{0}}{\partial r_{s} \partial r_{s}}-\frac{13}{108} \frac{\partial}{\partial r_{k}} \frac{\partial^{2} M_{4}}{\partial r_{s} \partial r_{s}} .
\end{aligned}
$$

The part of Eq. (374) proportional to the first-order correction is

$$
-\frac{1}{2} \sum_{j}\left(\frac{\partial \phi_{k}^{*}}{\partial M_{j}} R_{j}^{(1)}+\frac{\partial R_{k}^{(1)}}{\partial M_{j}} \phi_{j}^{*}\right)=\frac{5}{6} \frac{\partial}{\partial r_{k}} \frac{\partial^{2} M_{4}}{\partial r_{s} \partial r_{s}}+\frac{1}{9} \frac{\partial}{\partial r_{k}} \frac{\partial^{2} M_{4}}{\partial r_{s} \partial r_{s}}
$$

Combining together terms (389), and (390), we obtain

$$
R_{M_{k}}^{(2)}=\frac{1}{8} \frac{\partial}{\partial r_{k}} \frac{\partial^{2} M_{0}}{\partial r_{s} \partial r_{s}}+\frac{89}{108} \frac{\partial}{\partial r_{k}} \frac{\partial^{2} M_{4}}{\partial r_{s} \partial r_{s}}
$$

Similar calculation for the energy equation leads to the following result:

$$
\begin{gathered}
-\int c^{2} \frac{\partial^{3}}{\partial r_{i} \partial r_{j} \partial r_{k}} c_{i} c_{j} c_{k}\left\{M_{0}+2 M_{i} c_{i}+\left(\frac{2}{3} M_{4}-M_{0}\right)\left(c^{2}-\frac{3}{2}\right)\right\} \mathrm{e}^{-c^{2}} \mathrm{~d}^{3} c \\
+\frac{25}{72} \frac{\partial}{\partial r_{i}} \frac{\partial^{2} M_{i}}{\partial r_{s} \partial r_{s}}=-\frac{1}{6}\left(\frac{21}{4} \frac{\partial}{\partial r_{i}} \frac{\partial^{2} M_{i}}{\partial r_{s} \partial r_{s}}+\frac{25}{12} \frac{\partial}{\partial r_{i}} \frac{\partial^{2} M_{i}}{\partial r_{s} \partial r_{s}}\right)=-\frac{19}{36} \frac{\partial}{\partial r_{i}} \frac{\partial^{2} M_{i}}{\partial r_{s} \partial r_{s}} .
\end{gathered}
$$

The term proportional to the first-order corrections gives

$$
\frac{5}{6}\left(\frac{\partial^{2}}{\partial r_{s} \partial r_{s}} \frac{\partial M_{i}}{\partial r_{i}}\right)+\frac{25}{4}\left(\frac{\partial^{2}}{\partial r_{s} \partial r_{s}} \frac{\partial M_{i}}{\partial r_{i}}\right)
$$

Thus, we obtain

$$
R_{M_{4}}^{(2)}=\frac{59}{9}\left(\frac{\partial^{2}}{\partial r_{s} \partial r_{s}} \frac{\partial M_{i}}{\partial r_{i}}\right)
$$




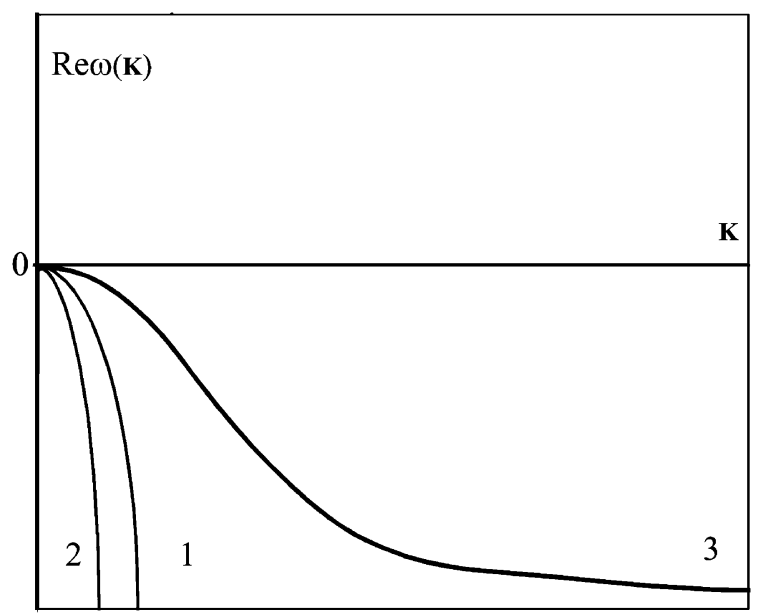

Fig. 16. Attenuation rates of various modes of the post-Navier-Stokes equations as functions of the wave vector. Attenuation rate of the twice degenerated shear mode is curve 1 . Attenuation rate of the two sound modes is curve 2 . Attenuation rate of the diffusion mode is curve 3 .

Finally, combining together all the terms, we obtain the following system of linearized hydrodynamic equations:

$$
\begin{aligned}
\frac{\partial M_{0}}{\partial t}= & -\frac{\partial M_{i}}{\partial r_{i}}, \\
\frac{\partial M_{k}}{\partial t}= & -\frac{1}{3} \frac{\partial M_{4}}{\partial r_{k}}+\frac{\tau}{4} \frac{\partial}{\partial r_{j}}\left(\frac{\partial M_{k}}{\partial r_{j}}+\frac{\partial M_{j}}{\partial r_{k}}-\frac{2}{3} \frac{\partial M_{n}}{\partial r_{n}} \delta_{k j}\right) \\
& +\tau^{2}\left\{\frac{1}{8} \frac{\partial}{\partial r_{k}} \frac{\partial^{2} M_{0}}{\partial r_{s} \partial r_{s}}+\frac{89}{108} \frac{\partial}{\partial r_{k}} \frac{\partial^{2} M_{4}}{\partial r_{s} \partial r_{s}}\right\}, \\
\frac{\partial M_{4}}{\partial t}= & -\frac{5}{2} \frac{\partial M_{i}}{\partial r_{i}}+\tau \frac{5}{2} \frac{\partial^{2} M_{4}}{\partial r_{i} \partial r_{i}}+\tau^{2} \frac{59}{9}\left(\frac{\partial^{2}}{\partial r_{s} \partial r_{s}} \frac{\partial M_{i}}{\partial r_{i}}\right) .
\end{aligned}
$$

Now we are in a position to investigate the dispersion relation of this system. Substituting $M_{i}=\tilde{M}_{i} \exp (\omega t+\mathrm{i}(\boldsymbol{k}, \boldsymbol{r}))(i=0, k, 4)$ into Eq. (392), we reduce the problem to finding the spectrum of the matrix:

$$
\left(\begin{array}{ccccc}
0 & -\mathrm{i} k_{x} & -\mathrm{i} k_{y} & -\mathrm{i} k_{z} & 0 \\
-\mathrm{i} k_{x} \frac{k^{2}}{8} & -\frac{1}{4} k^{2}-\frac{1}{12} k_{x}^{2} & -\frac{k_{x} k_{y}}{12} & -\frac{k_{x} k_{z}}{12} & -\mathrm{i} k_{x}\left(\frac{1}{3}+\frac{89 k^{2}}{108}\right) \\
-\mathrm{i} k_{y} \frac{k^{2}}{8} & -\frac{k_{x} k_{y}}{12} & -\frac{1}{4} k^{2}-\frac{1}{12} k_{y}^{2} & -\frac{k_{y} k_{z}}{12} & -\mathrm{i} k_{y}\left(\frac{1}{3}+\frac{89 k^{2}}{108}\right) \\
-\mathrm{i} k_{z} \frac{k^{2}}{8} & -\frac{k_{x} k_{z}}{12} & -\frac{k_{y} k_{z}}{12} & -\frac{1}{4} k^{2}-\frac{1}{12} k_{z}^{2} & -\mathrm{i} k_{z}\left(\frac{1}{3}+\frac{89 k^{2}}{108}\right) \\
0 & -\mathrm{i} k_{x}\left(\frac{5}{2}+\frac{59 k^{2}}{9}\right) & -\mathrm{i} k_{y}\left(\frac{5}{2}+\frac{59 k^{2}}{9}\right) & -\mathrm{i} k_{z}\left(\frac{5}{2}+\frac{59 k^{2}}{9}\right) & -\frac{5}{2} k^{2}
\end{array}\right) .
$$

This matrix has five eigenvalues. The real parts of these eigenvalues responsible for the decay rate of the corresponding modes are shown in Fig. 16 as functions of the wave vector $k$. We see that all 
real parts of all the eigenvalues are nonpositive for any wave vector. In other words, this means that the present system is linearly stable. For the Burnett hydrodynamics as derived from the Boltzmann or from the single relaxation time Bhatnagar-Gross-Krook model, it is well known that the decay rate of the acoustic becomes positive after some value of the wave vector $[53,24]$ which leads to the instability. While the method suggested here is clearly semi-phenomenological (coarse-graining time $\tau$ remains unspecified), the consistency of the expansion with the entropy requirements, and especially the latter result of the linearly stable post-Navier-Stokes correction strongly indicates that it might be more suited to establishing models of highly nonequilibrium hydrodynamics.

\section{Example 11: Natural projector for the McKean model}

In this section the fluctuation-dissipation formula recently derived by the method of natural projector [18] is illustrated by the explicit computation for McKean's kinetic model [219]. It is demonstrated that the result is identical, on the one hand, to the sum of the Chapman-Enskog expansion, and, on the other hand, to the exact solution of the invariance equation. The equality between all the three results holds up to the crossover from the hydrodynamic to the kinetic domain.

General scheme. Let us consider a microscopic dynamics (76) given by an equation for the distribution function $f(x, t)$ over a configuration space $x$ :

$$
\partial_{t} f=J(f),
$$

where operator $J(f)$ may be linear or nonlinear. Let $\boldsymbol{m}(f)$ be a set of linear functionals whose values, $\boldsymbol{M}=\boldsymbol{m}(f)$, represent the macroscopic variables, and also let $f(\boldsymbol{M}, x)$ be a set of distribution functions satisfying the consistency condition,

$$
\boldsymbol{m}(f(\boldsymbol{M}))=\boldsymbol{M} .
$$

The choice of the relevant distribution functions is the point of central importance which we discuss later on but for the time being we need only specification (394).

The starting point has been the following observation [16,17]: Given a finite time interval $\tau$, it is possible to reconstruct uniquely the macroscopic dynamics from a single condition. For the sake of completeness, we shall formulate this condition here. Let us denote as $\boldsymbol{M}(t)$ the initial condition at the time $t$ to the yet unknown equations of the macroscopic motion, and let us take $f(\boldsymbol{M}(t), x)$ for the initial condition of the microscopic equation (393) at the time $t$. Then the condition for the reconstruction of the macroscopic dynamics reads as follows: For every initial condition $\{\boldsymbol{M}(t), t\}$, solutions to the macroscopic dynamic equations at the time $t+\tau$ are equal to the values of the macroscopic variables on the solution to Eq. (393) with the initial condition $\{f(\boldsymbol{M}(t), x), t\}$ :

$$
\boldsymbol{M}(t+\tau)=\boldsymbol{m}\left(T_{\tau} f(\boldsymbol{M}(t))\right),
$$

where $T_{\tau}$ is the formal solution operator of the microscopic equation (393). The right hand side of Eq. (395) represents an operation on trajectories of the microscopic equation (393), introduced in a particular form by Ehrenfest's [215] (the coarse-graining): The solution at the time $t+\tau$ is replaced by the state on the manifold $f(\boldsymbol{M}, x)$. Notice that the coarse-graining time $\tau$ in Eq. (395) is finite, and we stress the importance of the required independence from the initial time $t$, and from the initial condition at $t$. 
The essence of the reconstruction of the macroscopic equations from the condition just formulated is in the following $[16,17]$ : Seeking the macroscopic equations in the form,

$$
\partial_{t} \boldsymbol{M}=\boldsymbol{R}(\boldsymbol{M}, \tau),
$$

we proceed with Taylor expansion of the unknown functions $\boldsymbol{R}$ in terms of powers $\tau^{n}$, where $n=$ $0,1, \ldots$, and require that each approximation, $\boldsymbol{R}^{(n)}$, of the order $n$, is such that resulting macroscopic solutions satisfy the condition (396) to the order $\tau^{n+1}$. This process of successive approximation is solvable. Thus, the unknown macroscopic equation (396) can be reconstructed to any given accuracy.

Coming back to the problem of choosing the distribution function $f(\boldsymbol{M}, x)$, we recall that many physically relevant cases of the microscopic dynamics (393) are characterized by existence of a concave functional $S(f)$ (the entropy functional; discussions of $S$ can be found in $[151,152,125]$ ). Traditionally, two cases are distinguished, the conservative $[\mathrm{d} S / \mathrm{d} t \equiv 0$ due to Eq. (393)], and the dissipative [ $\mathrm{d} S / \mathrm{d} t \geqslant 0$ due to Eq. (393), where equality sign corresponds to the stationary solution]. Approach (395) and (396) is applicable to both these situations. In both of these cases, among the possible sets of distribution functions $f(\boldsymbol{M}, x)$, the distinguished role is played by the well-known quasi-equilibrium approximations, $f^{*}(\boldsymbol{M}, x)$, which are maximizers of the functional $S(f)$ for fixed $\boldsymbol{M}$. We recall that, due to convexity of the functional $S$, if such a maximizer exists then it is unique. The special role of the quasi-equilibrium approximations is due to the well known fact that they preserve the type of dynamics: If $\mathrm{d} S / \mathrm{d} t \geqslant 0$ due to Eq. (393), then $\mathrm{d} S^{*} / \mathrm{d} t \geqslant 0$ due to the quasi-equilibrium dynamics, where $S^{*}(\boldsymbol{M})=S\left(f^{*}(\boldsymbol{M})\right)$ is the quasi-equilibrium entropy, and where the quasi-equilibrium dynamics coincides with the zeroth order in the above construction, $\boldsymbol{R}^{(0)}=$ $\boldsymbol{m}\left(J\left(f^{*}(\boldsymbol{M})\right)\right)$. We notice it in passing that, since the well known work of Jaynes [153], the usefulness of quasi-equilibrium approximations is well understood in various versions of projection operator formalism for the conservative case [95,154-156], as well as for the dissipative dynamics [125,46]. Relatively less studied remains the case of open or externally driven systems, where invariant quasi-equilibrium manifolds may become unstable [84]. The use of the quasi-equilibrium approximations for the above construction has been stressed in $[16,17,20]$. In particular, the strict increase in the quasi-equilibrium entropy has been demonstrated for the first and higher order approximations [17]. Examples have been provided [17], focusing on the conservative case, and demonstrating that several well known dissipative macroscopic equations, such as the Navier-Stokes equation and the diffusion equation for the one-body distribution function, are derived as the lowest order approximations of this construction.

The advantage of the approach $[16,17]$ is the locality of construction, because only Taylor series expansion of the microscopic solution is involved. This is also its natural limitation. From the physical standpoint, finite and fixed coarse-graining time $\tau$ remains a phenomenological device which makes it possible to infer the form of the macroscopic equations by a noncomplicated computation rather than to derive a full form thereof. For instance, the form of the Navier-Stokes equations can be derived from the simplest model of free motion of particles, in which case the coarse-graining is a substitution for collisions. Going away from the limitations imposed by the finite coarse graining time $[16,17]$ can be recognized as the major problem of a consistent formulation of the nonequilibrium statistical thermodynamics. Intuitively, this requires taking the limit $\tau \rightarrow \infty$, allowing for all the relevant correlations to be developed by the microscopic dynamics, rather than to be cut off at the finite $\tau$. Indeed, in the case of the dissipative dynamics, in particular, for the linearized Boltzmann equation, one typically expects an initial layer [87] which is completely cut off in the short-memory 
approximation, whereas those effects can be made small by taking $\tau$ large enough. A way of doing this in the general nonlinear setting for entropy-conserving systems still requires further work at the time of this writing.

Natural projector for linear systems. However, there is one important exception when the ' $\tau \rightarrow \infty$ problem' is readily solved [17,18]. This is the case where Eq. (393) is linear,

$$
\partial_{t} f=L f,
$$

and where the quasi-equilibrium is a linear function of $\boldsymbol{M}$. This is, in particular, the classical case of linear irreversible thermodynamics where one considers the linear macroscopic dynamics near the equilibrium, $f^{\mathrm{eq}}, L f^{\mathrm{eq}}=0$. We assume, for simplicity of presentation, that the macroscopic variables $\boldsymbol{M}$ vanish at equilibrium, and are normalized in such a way that $\boldsymbol{m}\left(f^{\mathrm{eq}} \boldsymbol{m}^{\dagger}\right)=\mathbf{1}$, where ${ }^{\dagger}$ denotes transposition, and $\mathbf{1}$ is an appropriate identity operator. In this case, the linear dynamics of the macroscopic variables $\boldsymbol{M}$ has the form,

$$
\partial_{t} \boldsymbol{M}=\boldsymbol{R} \boldsymbol{M},
$$

where the linear operator $\boldsymbol{R}$ is determined by the coarse-graining condition (395) in the limit $\tau \rightarrow \infty$ :

$$
\boldsymbol{R}=\lim _{\tau \rightarrow \infty} \frac{1}{\tau} \ln \left[\boldsymbol{m}\left(\mathrm{e}^{\tau L} f^{\mathrm{eq}} \boldsymbol{m}^{\dagger}\right)\right] .
$$

Formula (399) has been already briefly mentioned in [17], and its relation to the Green-Kubo formula has been demonstrated in [18]. In our case, the Green-Kubo formula reads:

$$
\boldsymbol{R}_{\mathrm{GK}}=\int_{0}^{\infty}\langle\dot{\boldsymbol{m}}(0) \dot{\boldsymbol{m}}(t)\rangle \mathrm{d} t,
$$

where angular brackets denote equilibrium averaging, and where $\dot{\boldsymbol{m}}=L^{\dagger} \boldsymbol{m}$. The difference between formulae (399) and (400) stems from the fact that condition (395) does not use an a priori hypothesis of the separation of the macroscopic and the microscopic time scales. For the classical $N$-particle dynamics, Eq. (399) is a complicated expression, involving a logarithm of noncommuting operators. It is therefore very desirable to gain its understanding in simple model situations.

Explicit example of the fluctuation-dissipation formula. In this section we want to give explicit example of formula (399). In order to make our point, we consider here dissipative rather than conservative dynamics in the framework of the well known toy kinetic model introduced by McKean [219] for the purpose of testing various ideas in kinetic theory. In the dissipative case with a clear separation of time scales, existence of formula (399) is underpinned by the entropy growth in both the rapid and the slow parts of the dynamics. This physical idea underlies generically the extraction of the slow (hydrodynamic) component of motion through the concept of normal solutions to kinetic equations, as pioneered by Hilbert [52], and has been discussed by many authors, e.g. [87,157,158]. Case studies for linear kinetic equation help clarifying the concept of this extraction [159,160,219].

Therefore, since for the dissipative case there exist well established approaches to the problem of reducing the description, and which are exact in the present setting, it is very instructive to see their relation to formula (399). Specifically, we compare the result with the exact sum of the Chapman-Enskog expansion [51], and with the exact solution in the framework of the method of invariant manifold [4-6]. We demonstrate that both the three approaches, different in their nature, give the same result as long as the hydrodynamic and the kinetic regimes are separated. 
The McKean model is the kinetic equation for the two-component vector function $f(r, t)=$ $\left(f_{+}(r, t), f_{-}(r, t)\right)^{\dagger}$ :

$$
\begin{aligned}
& \partial_{t} f_{+}=-\partial_{r} f_{+}+\epsilon^{-1}\left(\frac{f_{+}+f_{-}}{2}-f_{+}\right), \\
& \partial_{t} f_{-}=\partial_{r} f_{-}+\epsilon^{-1}\left(\frac{f_{+}+f_{-}}{2}-f_{-}\right) .
\end{aligned}
$$

Eq. (401) describes the one-dimensional kinetics of particles with velocities +1 and -1 as a combination of the free flight and a relaxation with the rate $\epsilon^{-1}$ to the local equilibrium. Using the notation, $(\boldsymbol{x}, \boldsymbol{y})$, for the standard scalar product of the two-dimensional vectors, we introduce the fields, $n(r, t)=(\boldsymbol{n}, \boldsymbol{f})$ [the local particle's density, where $\boldsymbol{n}=(1,1)]$, and $j(r, t)=(\boldsymbol{j}, \boldsymbol{f})$ [the local momentum density, where $\boldsymbol{j}=(1,-1)]$. Eq. (401) can be equivalently written in terms of the moments,

$$
\begin{aligned}
& \partial_{t} n=-\partial_{r} j, \\
& \partial_{t} j=-\partial_{r} n-\epsilon^{-1} j .
\end{aligned}
$$

The local equilibrium,

$$
\boldsymbol{f}^{*}(n)=\frac{n}{2} \boldsymbol{n},
$$

is the conditional maximum of the entropy,

$$
S=-\int\left(f_{+} \ln f_{+}+f_{-} \ln f_{-}\right) \mathrm{d} r
$$

under the constraint which fixes the density, $\left(\boldsymbol{n}, \boldsymbol{f}^{*}\right)=n$. The quasi-equilibrium manifold (403) is linear in our example, as well as is the kinetic equation.

The problem of reducing the description for model (401) amounts to finding the closed equation for the density field $n(r, t)$. When the relaxation parameter $\epsilon^{-1}$ is small enough (the relaxation dominance), then the first Chapman-Enskog approximation to the momentum variable, $j(r, t) \approx$ $-\epsilon \partial_{r} n(r, t)$, amounts to the standard diffusion approximation. Let us consider now how formula (399), and other methods, extend this result.

Because of the linearity of Eq. (401), and of the local equilibrium, it is natural to use the Fourier transform, $h_{k}=\int \exp (\mathrm{i} k r) h(r) \mathrm{d} r$. Eq. (401) is then written as

$$
\partial_{t} \boldsymbol{f}_{k}=\boldsymbol{L}_{k} \boldsymbol{f}_{k}
$$

where

$$
\boldsymbol{L}_{k}=\left(\begin{array}{cc}
-\mathrm{i} k-\frac{1}{2 \epsilon} & \frac{1}{2 \epsilon} \\
\frac{1}{2 \epsilon} & \mathrm{i} k-\frac{1}{2 \epsilon}
\end{array}\right) .
$$

Derivation of formula (399) in our example goes as follows: We seek the macroscopic dynamics of the form

$$
\partial_{t} n_{k}=R_{k} n_{k}
$$


where the function $R_{k}$ is yet unknown. In the left hand side of Eq. (395) we have

$$
n_{k}(t+\tau)=\mathrm{e}^{\tau R_{k}} n_{k}(t) .
$$

In the right hand side of Eq. (395) we have

$$
\left(\boldsymbol{n}, \mathrm{e}^{\tau \boldsymbol{L}_{k}} \boldsymbol{f}^{*}\left(n_{k}(t)\right)\right)=\frac{1}{2}\left(\boldsymbol{n}, \mathrm{e}^{\tau \boldsymbol{L}_{k}} \boldsymbol{n}\right) n_{k}(t) .
$$

After equating expressions (407) and (408), we require that the resulting equality holds in the limit $\tau \rightarrow \infty$ independently of the initial data $n_{k}(t)$. Thus, we arrive at formula (399):

$$
R_{k}=\lim _{\tau \rightarrow \infty} \frac{1}{\tau} \ln \left[\left(\boldsymbol{n}, \mathrm{e}^{\tau \boldsymbol{L}_{k}} \boldsymbol{n}\right)\right] .
$$

Eq. (409) defines the macroscopic dynamics (406) within the present approach. Explicit evaluation of expression (409) is straightforward in the present model. Indeed, operator $\boldsymbol{L}_{k}$ has two eigenvalues, $\Lambda_{k}^{ \pm}$, where

$$
\Lambda_{k}^{ \pm}=-\frac{1}{2 \epsilon} \pm \sqrt{\frac{1}{4 \epsilon^{2}}-k^{2}} .
$$

Let us denote as $\boldsymbol{e}_{k}^{ \pm}$two (arbitrary) eigenvectors of the matrix $\boldsymbol{L}_{k}$, corresponding to the eigenvalues $\Lambda_{k}^{ \pm}$. Vector $\boldsymbol{n}$ has a representation, $\boldsymbol{n}=\alpha_{k}^{+} \boldsymbol{e}_{k}^{+}+\alpha_{k}^{-} \boldsymbol{e}_{k}^{-}$, where $\alpha_{k}^{ \pm}$are complex-valued coefficients. With this, we obtain in Eq. (409),

$$
R_{k}=\lim _{\tau \rightarrow \infty} \frac{1}{\tau} \ln \left[\alpha_{k}^{+}\left(\boldsymbol{n}, \boldsymbol{e}_{k}^{+}\right) \mathrm{e}^{\tau \Lambda_{k}^{+}}+\alpha_{k}^{-}\left(\boldsymbol{n}, \boldsymbol{e}_{k}^{-}\right) \mathrm{e}^{\tau \Lambda_{k}^{-}}\right] .
$$

For $k \leqslant k_{\mathrm{c}}$, where $k_{\mathrm{c}}^{2}=4 \epsilon$, we have $\Lambda_{k}^{+}>\Lambda_{k}^{-}$. Therefore,

$$
R_{k}=\Lambda_{k}^{+} \text {for } k<k_{\mathrm{c}} \text {. }
$$

As was expected, formula (399) in our case results in the exact hydrodynamic branch of the spectrum of the kinetic equation (401). The standard diffusion approximation is recovered from Eq. (412) as the first nonvanishing approximation in terms of the $\left(k / k_{\mathrm{c}}\right)^{2}$.

At $k=k_{\mathrm{c}}$, the crossover from the extended hydrodynamic to the kinetic regime takes place, and $\operatorname{Re} \Lambda_{k}^{+}=\operatorname{Re} \Lambda_{k}^{-}$. However, we may still extend the function $R_{k}$ for $k \geqslant k_{\mathrm{c}}$ on the basis of formula (409):

$$
R_{k}=\operatorname{Re} \Lambda_{k}^{+} \quad \text { for } k \geqslant k_{\mathrm{c}} .
$$

Notice that the function $R_{k}$ as given by Eqs. (412) and (413) is continuous but nonanalytic at the crossover.

\section{Comparison with the Chapman-Enskog method and solution of invariance equation}

Let us now compare this result with the Chapman-Enskog method. Since the exact Chapman-Enskog solution for the systems like Eq. (403) has been recently discussed in detail elsewhere [23,24,162-165], we shall be brief here. Following the Chapman-Enskog method, we seek the momentum variable $j$ in terms of an expansion,

$$
j^{\mathrm{CE}}=\sum_{n=0}^{\infty} \epsilon^{n+1} j^{(n)} \text {. }
$$


The Chapman-Enskog coefficients, $j^{(n)}$, are found from the recurrence equations,

$$
j^{(n)}=-\sum_{m=0}^{n-1} \partial_{t}^{(m)} j^{(n-1-m)},
$$

where the Chapman-Enskog operators $\partial_{t}^{(m)}$ are defined by their action on the density $n$ :

$$
\partial_{t}^{(m)} n=-\partial_{r} j^{(m)} \text {. }
$$

The recurrence equations (414)-(416), become well defined as soon as the aforementioned zero-order approximation $j^{(0)}$ is specified,

$$
j^{(0)}=-\partial_{r} n \text {. }
$$

From Eqs. (415)-(417), it follows that the Chapman-Enskog coefficients $j^{(n)}$ have the following structure:

$$
j^{(n)}=b_{n} \partial_{r}^{2 n+1} n,
$$

where coefficients $b_{n}$ are found from the recurrence equation,

$$
b_{n}=\sum_{m=0}^{n-1} b_{n-1-m} b_{m}, \quad b_{0}=-1 .
$$

Notice that coefficients (419) are real-valued, by the sense of the Chapman-Enskog procedure. The Fourier image of the Chapman-Enskog solution for the momentum variable has the form,

$$
j_{k}^{\mathrm{CE}}=\mathrm{i} k B_{k}^{\mathrm{CE}} n_{k}
$$

where

$$
B_{k}^{\mathrm{CE}}=\sum_{n=0}^{\infty} b_{n}\left(-\epsilon k^{2}\right)^{n} .
$$

Equation for the function $B(421)$ is easily found upon multiplying Eq. (419) by $\left(-k^{2}\right)^{n}$, and summing in $n$ from zero to infinity:

$$
\epsilon k^{2} B_{k}^{2}+B_{k}+1=0 \text {. }
$$

Solution to the latter equation which respects condition (417), and which constitutes the exact Chapman-Enskog solution (421) is

$$
B_{k}^{\mathrm{CE}}= \begin{cases}k^{-2} \Lambda_{k}^{+}, & k<k_{\mathrm{c}}, \\ \text { none, } & k \geqslant k_{\mathrm{c}} .\end{cases}
$$

Thus, the exact Chapman-Enskog solution derives the macroscopic equation for the density as follows:

$$
\partial_{t} n_{k}=-\mathrm{i} k j_{k}^{\mathrm{CE}}=R_{k}^{\mathrm{CE}} n_{k},
$$

where

$$
R_{k}^{\mathrm{CE}}= \begin{cases}\Lambda_{k}^{+}, & k<k_{\mathrm{c}}, \\ \text { none, } & k \geqslant k_{\mathrm{c}} .\end{cases}
$$


The Chapman-Enskog solution does not extends beyond the crossover at $k_{\mathrm{c}}$. This happens because the full Chapman-Enskog solution appears as a continuation the diffusion approximation, whereas formula (409) is not based on such an extension a priori.

Finally, let us discuss briefly the comparison with the solution within the method of invariant manifold [4-6]. Specifically, the momentum variable $j_{k}^{\text {inv }}=\mathrm{i} k B_{k}^{\text {inv }} n_{k}$ is required to be invariant of both the microscopic and the macroscopic dynamics, that is, the time derivative of $j_{k}^{\text {inv }}$ due to the macroscopic subsystem,

$$
\frac{\partial j_{k}^{\text {inv }}}{\partial n_{k}} \partial_{t} n_{k}=\mathrm{i} k B_{k}^{\text {inv }}(-\mathrm{i} k)\left[\mathrm{i} k B_{k}^{\text {inv }}\right],
$$

should be equal to the derivative of $j_{k}^{\text {inv }}$ due to the microscopic subsystem,

$$
\partial_{t} j_{k}^{\text {inv }}=-\mathrm{i} k n_{k}-\epsilon^{-1} \mathrm{i} k B_{k}^{\text {inv }} n_{k},
$$

and that the equality between Eqs. (426) and (427) should hold independently of the specific value of the macroscopic variable $n_{k}$. This amounts to a condition for the unknown function $B_{k}^{\text {inv }}$, which is essentially the same as Eq. (422), and it is straightforward to show that the same selection procedure of the hydrodynamic root as above in the Chapman-Enskog case results in Eq. (425).

In conclusion, in this Example we have given the explicit illustration for formula (399). The example considered above demonstrates that formula (399) gives the exact macroscopic evolution equation, which is identical to the sum of the Chapman-Enskog expansion, as well as to the invariance principle. This identity holds up to the point where the hydrodynamics and the kinetics cease to be separated. Whereas the Chapman-Enskog solution does not extend beyond the crossover point, formula (399) demonstrates a nonanalytic extension. The example considered adds to the confidence of the correctness of the approach suggested in [16-19].

\section{Slow invariant manifold for a closed system has been found. What next?}

Suppose that the slow invariant manifold is found for a dissipative system. What have we constructed it for?

First of all, for solving the Cauchy problem, in order to separate motions. This means that the Cauchy problem is divided in the following two subproblems:

- Reconstruct the "fast" motion from the initial conditions to the slow invariant manifold (the initial layer problem).

- Solve the Cauchy problem for the "slow" motions on the manifold.

Thus, solving the Cauchy problem becomes easier (and in some complicated cases it just becomes possible).

Let us stress here that for any sufficiently reliable solution of the Cauchy problem one must solve not only the reduced Cauchy problem for the slow motion, but also the initial layer problem for fast motions.

While solving the latter problem it was found to be surprisingly effective to use piece-wise linear approximations with smoothing or even without it $[14,15]$. This method was used for the Boltzman equation, for chemical kinetics equations, and for the Pauli equation. 
There exists a different way to model the initial layer in kinetics problems: it is the route of model equations. For example, the Bhatnagar, Gross, Krook (BGK) equation [89] is the simplest model for the Boltzmann equation. It describes relaxation into a small neighborhood of the local Maxwell distribution. There are many types and hierarchies of the model equations $[89,87,90,10,129]$. The principal idea of any model equation is to replace the fast processes by a simple relaxation term. As a rule, it has a form $\mathrm{d} x / \mathrm{d} t=\cdots-\left(x-x_{\mathrm{sl}}(x)\right) / \tau$, where $x_{\mathrm{sl}}(x)$ is a point of the approximate slow manifold. Such form is used in the BGK-equation, or in the quasi-equilibrium models [90]. It also can take a gradient form, like in the gradient models [10,129]. These simplifications not only allows to study the fast motions separately but it also allows to zoom in the details of the interaction of fast and slow motions in the vicinity of the slow manifold.

What concerns solving the Cauchy problem for the "slow" motions, this is the basic problem of the hydrodynamics, of the gas dynamics (if the initial "big" systems describes kinetics of a gas or a fluid), etc. Here invariant manifold methods provide equations for a further study. However, even a preliminary consideration of the practical aspects of these studies shows a definite shortcoming. In practice, obtained equations are exploited not only for "closed" systems. The initial equations (76) describe a dissipative system that approaches the equilibrium. The equations of slow motion describe dissipative system too. Then these equations are supplied with various forces and flows, and after that they describe systems with more or less complex dynamics.

Because of this, there is a different answer to our question, what have we constructed the invariant manifold for?

First of all, in order to construct models of open system dynamics in the neighborhood of the slow manifold.

Various approaches to this modeling are described in the following subsections.

\subsection{Slow dynamics in open systems. Zero-order approximation and the thermodynamic} projector

Let the initial dissipative system (76) be "spoiled" by an additional term ("external vector field" $\left.J_{\text {ex }}(x, t)\right)$ :

$$
\frac{\mathrm{d} x}{\mathrm{~d} t}=J(x)+J_{\mathrm{ex}}(x, t), \quad x \subset U .
$$

For this new system the entropy does not increase everywhere. In the new system (428) different dynamic effects are possible, such as a nonuniqueness of stationary states, auto-oscillations, etc. The "inertial manifold" effect is well-known: solutions of (428) approach some relatively low-dimensional manifold on which all the nontrivial dynamics takes place [234,235,136]. This "inertial manifold" can have a finite dimension even for infinite-dimensional systems, for example, for the "reaction+ diffusion" systems [237].

In the theory of nonlinear control of partial differential equation systems a strategy based on approximate inertial manifolds [238] is proposed to facilitate the construction of finite-dimensional systems of ordinary differential equations (ODE), whose solutions can be arbitrarily close to the solutions of the infinite-dimensional system [240].

It is natural to expect that the inertial manifold of system (428) is located somewhere close to the slow manifold of the initial dissipative system (76). This hypothesis has the following basis. Suppose 
that the vector field $J_{\mathrm{ex}}(x, t)$ is sufficiently small. Let's introduce, for example, a small parameter $\varepsilon>0$, and consider $\varepsilon J_{\mathrm{ex}}(x, t)$ instead of $J_{\mathrm{ex}}(x, t)$. Let's assume that for system (76) a separation of motions into "slow" and "fast" takes place. In this case, there exists such interval of positive $\varepsilon$ that $\varepsilon J_{\mathrm{ex}}(x, t)$ is comparable to $J$ only in a small neighborhood of the given slow motion manifold of system (76). Outside this neighborhood, $\varepsilon J_{\mathrm{ex}}(x, t)$ is negligibly small in comparison with $J$ and only negligibly influences the motion (for this statement to be true, it is important that system (76) is dissipative and every solution comes in finite time to a small neighborhood of the given slow manifold).

Precisely this perspective on system (428) allows to exploit slow invariant manifolds constructed for the dissipative system (76) as the ansatz and the zero-order approximation in a construction of the inertial manifold of the open system (428). In the zero-order approximation, the right part of Eq. (428) is simply projected onto the tangent space of the slow manifold.

The choice of the projector is determined by the motion separation which was described above: fast motion is taken from the dissipative system (76). A projector which is suitable for all dissipative systems with given entropy function is unique. It is constructed in the following way (detailed consideration of this is given above in the sections "Entropic projector without a priori parametrization" and in Ref. [177]). Let a point $x \in U$ be defined and some vector space $T$, on which one needs to construct a projection ( $T$ is the tangent space to the slow manifold at the point $x$ ). We introduce the entropic scalar product $\langle\mid\rangle_{x}$ :

$$
\langle a \mid b\rangle_{x}=-\left(a, D_{x}^{2} S(b)\right) .
$$

Let us consider $T_{0}$ that is a subspace of $T$ and which is annulled by the differential $S$ at the point $x$.

$$
T_{0}=\left\{a \in T \mid D_{x} S(a)=0\right\} .
$$

Suppose ${ }^{14}$ that $T_{0} \neq T$. Let $e_{g} \in T, e_{g} \perp T_{0}$ with respect to the entropic scalar product $\langle\mid\rangle_{x}$, and $D_{x} S\left(e_{g}\right)=1$. These conditions define vector $e_{g}$ uniquely.

The projector onto $T$ is defined by the formula

$$
P(J)=P_{0}(J)+e_{g} D_{x} S(J)
$$

where $P_{0}$ is the orthogonal projector onto $T_{0}$ with respect to the entropic scalar product $\langle\mid\rangle_{x}$. For example, if $T$ a finite-dimensional space, then projector (431) is constructed in the following way. Let $e_{1}, \ldots, e_{n}$ be a basis in $T$, and for definiteness, $D_{x} S\left(e_{1}\right) \neq 0$.

1) Let us construct a system of vectors

$$
b_{i}=e_{i+1}-\lambda_{i} e_{1}, \quad(i=1, \ldots, n-1),
$$

where $\lambda_{i}=D_{x} S\left(e_{i+1}\right) / D_{x} S\left(e_{1}\right)$, and hence $D_{x} S\left(b_{i}\right)=0$. Thus, $\left\{b_{i}\right\}_{1}^{n-1}$ is a basis in $T_{0}$.

2) Let us orthogonalize $\left\{b_{i}\right\}_{1}^{n-1}$ with respect to the entropic scalar product $\langle\mid\rangle_{x}(76)$. We thus derived an orthonormal with respect to $\langle\mid\rangle_{x}$ basis $\left\{g_{i}\right\}_{1}^{n-1}$ in $T_{0}$.

3) We find $e_{g} \in T$ from the conditions:

$$
\left\langle e_{g} \mid g_{i}\right\rangle_{x}=0,(i=1, \ldots, n-1), \quad D_{x} S\left(e_{g}\right)=1
$$

\footnotetext{
${ }^{14}$ If $T_{0}=T$, then the thermodynamic projector is the orthogonal projector on $T$ with respect to the entropic scalar product $\langle\mid\rangle_{x}$.
} 
and, finally we get

$$
P(J)=\sum_{i=1}^{n-1} g_{i}\left\langle g_{i} \mid J\right\rangle_{x}+e_{g} D_{x} S(J) .
$$

If $D_{x} S(T)=0$, then the projector $P$ is simply the orthogonal projector with respect to the $\langle\mid\rangle_{x}$ scalar product. This is possible if $x$ is the global maximum of entropy point (equilibrium). Then

$$
P(J)=\sum_{i=1}^{n} g_{i}\left\langle g_{i} \mid J\right\rangle_{x},\left\langle g_{i} \mid g_{j}\right\rangle=\delta_{i j} .
$$

Remark. In applications, Eq. (76) often has additional linear balance constraints such as numbers of particles, momentum, energy, etc. Solving the closed dissipative system (76) we simply choose balance values and consider the dynamics of (76) on the corresponding affine balance subspace.

For driven system (428) the balances can be violated. Because of this, for the open system (428) the natural balance subspace includes the balance subspace of (76) with different balance values. For every set of balance values there is a corresponding equilibrium. Slow invariant manifold of the dissipative systems that is applied to the description of the driven systems (428) is usually the union of slow manifolds for all possible balance values. The equilibrium of the dissipative closed system corresponds to the entropy maximum given the balance values are fixed. In the phase space of the driven system (428) the entropy gradient in the equilibrium points of system (76) is not necessarily equal to zero.

In particular, for the Boltzmann entropy in the local finite-dimensional case one gets the thermodynamic projector in the following form:

$$
\begin{aligned}
& S=-\int f(v)(\ln (f(v))-1) \mathrm{d} v \\
& D_{f} S(J)=-\int J(v) \ln f(v) \mathrm{d} v, \\
& \langle\psi \mid \varphi\rangle_{f}=-\left(\psi, D_{f}^{2} S(\varphi)\right)=\int \frac{\psi(v) \varphi(v)}{f(v)} \mathrm{d} v, \\
& P(J)=\sum_{i=1}^{n-1} g_{i}(v) \int \frac{g_{i}(v) J(v)}{f(v)} \mathrm{d} v-e_{g}(v) \int J(v) \ln f(v) \mathrm{d} v,
\end{aligned}
$$

where $g_{i}(v)$ and $e_{g}(v)$ are constructed accordingly to the scheme described above,

$$
\begin{gathered}
\int \frac{g_{i}(v) g_{j}(v)}{f(v)} \mathrm{d} v=\delta_{i j}, \\
\int g_{i}(v) \ln f(v) \mathrm{d} v=0,
\end{gathered}
$$




$$
\begin{aligned}
& \int g_{i}(v) e_{g}(v) \mathrm{d} v=0, \\
& \int e_{g}(v) \ln f(v) \mathrm{d} v=1 .
\end{aligned}
$$

If for all $g \in T$ we have $\int g(v) \ln f(v) \mathrm{d} v=0$, then the projector $P$ is defined as the orthogonal projector with respect to the $\langle\mid\rangle_{f}$ scalar product.

\subsection{Slow dynamics in open systems. First-order approximation}

Thermodynamic projector (431) defines a "slow and fast motions" duality: if $T$ is the tangent space of the slow motion manifold then $T=\operatorname{im} P$, and $\operatorname{ker} P$ is the plane of fast motions. Let us denote by $P_{x}$ the projector at a point $x$ of a given slow manifold.

The vector field $J_{\text {ex }}(x, t)$ can be decomposed in two components:

$$
J_{\mathrm{ex}}(x, t)=P_{x} J_{\mathrm{ex}}(x, t)+\left(1-P_{x}\right) J_{\mathrm{ex}}(x, t) .
$$

Let us denote $J_{\text {ex } s}=P_{x} J_{\text {ex }}, J_{\text {ex } f}=\left(1-P_{x}\right) J_{\text {ex }}$. The slow component $J_{\text {ex } s}$ gives a correction to the motion along the slow manifold. This is a zero-order approximation. The "fast" component shifts the slow manifold in the fast motions plane. This shift changes $P_{x} J_{\mathrm{ex}}$ accordingly. Consideration of this effect gives a first-order approximation. In order to find it, let us rewrite the invariance equation taking $J_{\text {ex }}$ into account:

$$
\begin{aligned}
& \left(1-P_{x}\right)\left(J(x+\delta x)+\varepsilon J_{\text {ex }}(x, t)\right)=0 \\
& P_{x} \delta x=0 .
\end{aligned}
$$

The first iteration of the Newton method subject to incomplete linearization gives:

$$
\begin{aligned}
& \left(1-P_{x}\right)\left(D_{x} J(\delta x)+\varepsilon J_{\mathrm{ex}}(x, t)\right)=0 \\
& P_{x} \delta x=0 . \\
& \left(1-P_{x}\right) D_{x} J\left(1-P_{x}\right) J(\delta x)=-\varepsilon J_{\mathrm{ex}}(x, t) .
\end{aligned}
$$

Thus, we have derived a linear equation in the space $\operatorname{ker} P$. The operator $(1-P) D_{x} J(1-P)$ is defined in this space.

Utilization of the self-adjoint linearization instead of the traditional linearization $D_{x} J$ operator (see "Decomposition of motions, nonuniqueness of selection..." section) considerably simplifies solving and studying Eq. (444). It is necessary to take into account here that the projector $P$ is a sum of the orthogonal projector with respect to the $\langle\mid\rangle_{x}$ scalar product and a projector of rank one.

Assume that the first-order approximation equation (444) has been solved and the following function has been found:

$$
\delta_{1} x\left(x, \varepsilon J_{\mathrm{ex} f}\right)=-\left[\left(1-P_{x}\right) D_{x} J\left(1-P_{x}\right)\right]^{-1} \varepsilon J_{\mathrm{ex} f},
$$

where $D_{x} J$ is either the differential of $J$ or symmetrized differential of $J$ (309). 
Let $x$ be a point on the initial slow manifold. At the point $x+\delta x\left(x, \varepsilon J_{\mathrm{ex} f}\right)$ the right hand side of Eq. (428) in the first-order approximation is given by

$$
J(x)+\varepsilon J_{\mathrm{ex}}(x, t)+D_{x} J\left(\delta x\left(x, \varepsilon J_{\mathrm{ex} f}\right)\right) .
$$

Due to the first-order approximation (446), the motion of a point projection onto the manifold is given by the following equation:

$$
\frac{\mathrm{d} x}{\mathrm{~d} t}=P_{x}\left(J(x)+\varepsilon J_{\mathrm{ex}}(x, t)+D_{x} J\left(\delta x\left(x, \varepsilon J_{\mathrm{ex} f}(x, t)\right)\right)\right) .
$$

Note that, in Eq. (447), the vector field $J(x)$ enters only in the form of projection, $P_{x} J(x)$. For the invariant slow manifold it holds $P_{x} J(x)=J(x)$, but actually we always deal with approximately invariant manifolds, hence, it is necessary to use the projection $P_{x} J$ instead of $J$ in (447).

Remark. The notion "projection of a point onto the manifold" needs to be specified. For every point $x$ of the slow invariant manifold $M$ there are defined both the thermodynamic projector $P_{x}(431)$ and the fast motions plane $\operatorname{ker} P_{x}$. Let us define a projector $\Pi$ of some neighborhood of $M$ onto $M$ in the following way:

$$
\Pi(z)=x \quad \text { if } P_{x}(z-x)=0 .
$$

Qualitatively, it means that $z$, after all fast motions took place, comes into a small neighborhood of $x$. Operation (431) is defined uniquely in some small neighborhood of the manifold $M$.

A derivation of slow motions equations requires not only an assumption that $\varepsilon J_{\text {ex }}$ is small but it must be slow as well: $\mathrm{d} / \mathrm{d} t\left(\varepsilon J_{\text {ex }}\right)$ must be small too.

One can get the further approximations for slow motions of system (428), taking into account the time derivatives of $J_{\text {ex }}$. This is an alternative to the usage of the projection operators methods [154]. This is considered in a more detail in example 12 for a particularly interesting driven system of dilute polymeric solutions. A short scheme description is given in the next subsection.

11.3. Beyond the first-order approximation: higher-order dynamical corrections, stability loss and invariant manifold explosion

Let us pose formally the invariance problem for the driven system (428) in the neighborhood of the slow manifold $M$ of the initial (dissipative) system.

Let for a given neighborhood of $M$ an operator $\Pi$ (448) be defined. One needs to define the function $\delta x(x, \ldots)=\delta x\left(x, J_{\mathrm{ex}}, \dot{J}_{\mathrm{ex}}, \ddot{J}_{\mathrm{ex}}, \ldots\right), x \in M$, with the following properties:

$$
\begin{aligned}
& P_{x}(\delta x(x, \ldots))=0 \\
& J(x+\delta x(x, \ldots))+J_{\mathrm{ex}}(x+\delta x(x, \ldots), t)=\dot{x}_{\mathrm{sl}}+D_{x} \delta x(x, \ldots) \dot{x}_{\mathrm{sl}}+\sum_{n=0}^{\infty} D_{J_{\mathrm{ex}}^{(n)}} \delta x(x, \ldots) J_{\mathrm{ex}}^{(n+1)}
\end{aligned}
$$

where $\dot{x}_{\mathrm{sl}}=P_{x}\left(J(x+\delta x(x, \ldots))+J_{\mathrm{ex}}(x+\delta x(x, \ldots), t)\right), J_{\mathrm{ex}}^{(n)}=\mathrm{d}^{n} J_{\mathrm{ex}} / \mathrm{d} t^{n}, D_{J_{\mathrm{ex}}^{(n)}} \delta x(x, \ldots)$ is a partial differential of the function $\delta x\left(x, J_{\mathrm{ex}}, \dot{J}_{\mathrm{ex}}, \ddot{J}_{\mathrm{ex}}, \ldots, J_{\mathrm{ex}}^{(n)}, \ldots\right)$ with respect to the variable $J_{\mathrm{ex}}^{(n)}$. One can 
rewrite Eqs. (449) in the following form:

$$
\begin{aligned}
(1 & \left.-P_{x}-D_{x} \delta x(x, \ldots)\right)\left(J(x+\delta x(x, \ldots))+J_{\mathrm{ex}}(x+\delta x(x, \ldots), t)\right) \\
& =\sum_{n=0}^{\infty} D_{J_{\mathrm{ex}}^{(n)}} \delta x(x, \ldots) J_{\mathrm{ex}}^{(n+1)} .
\end{aligned}
$$

For solving Eq. (450) one can use an iteration method and take into account smallness consideration. The series in the right hand side of Eq. (450) can be rewritten as

$$
\text { RHS }=\sum_{n=0}^{k-1} \varepsilon^{n+1} D_{J_{\mathrm{ex}}^{(n)}} \delta x(x, \ldots) J_{\mathrm{ex}}^{(n+1)}
$$

at the $k$ th iteration, considering expansion terms only to order less than $k$. The first iteration equation was solved in the previous subsection. On second iteration one gets the following equation:

$$
\begin{aligned}
(1 & \left.-P_{x}-D_{x} \delta_{1} x\left(x, J_{\mathrm{ex}}\right)\right)\left(J\left(x+\delta_{1} x\left(x, J_{\mathrm{ex}}\right)\right)\right. \\
& \left.+\left.D_{z} J(z)\right|_{z=x+\delta_{1} x\left(x, J_{\mathrm{ex}}\right)} \cdot\left(\delta_{2} x-\delta_{1} x\left(x, J_{\mathrm{ex}}\right)\right)+J_{\mathrm{ex}}\right) \\
& =D_{J_{\mathrm{ex}}} \delta_{1} x\left(x, J_{\mathrm{ex}}\right) \dot{J_{\mathrm{ex}}} .
\end{aligned}
$$

This is a linear equation with respect to $\delta_{2} x$. The solution $\delta_{2} x\left(x, J_{\mathrm{ex}}, \dot{J}_{\mathrm{ex}}\right)$ depends linearly on $\dot{J}_{\text {ex }}$, but nonlinearly on $J_{\text {ex }}$. Let us remind that the first iteration equation solution depends linearly on $J_{\text {ex }}$.

In all these iteration equations the field $J_{\mathrm{ex}}$ and it's derivatives are included in the formulas as if they were functions of time $t$ only. Indeed, for any solution $x(t)$ of Eqs. (428) $J_{\text {ex }}(x, t)$ can be substituted for $J_{\mathrm{ex}}(x(t), t)$. The function $x(t)$ will be a solution of system (428) in which $J_{\mathrm{ex}}(x, t)$ is substituted for $J_{\mathrm{ex}}(t)$ in this way.

However, in order to obtain the macroscopic equations (447) one must return to $J_{\mathrm{ex}}(x, t)$. For the first iteration such return is quite simple as one can see from (446). There $J_{\text {ex }}(x, t)$ is calculated in points of the initial slow manifold. For general case, suppose that $\delta x=\delta x\left(x, J_{\mathrm{ex}}, \dot{J}_{\mathrm{ex}}, \ldots, J_{\mathrm{ex}}^{(k)}\right)$ has been found. The motion equations for $x$ (447) have the following form:

$$
\frac{\mathrm{d} x}{\mathrm{~d} t}=P_{x}\left(J(x+\delta x)+J_{\mathrm{ex}}(x+\delta x, t)\right) .
$$

In these equations the shift $\delta x$ must be a function of $x$ and $t$ (or a function of $x, t, \alpha$, where $\alpha$ are external fields, see example 12, but from the point of view of this consideration dependence on the external fields is not essential). One calculates the shift $\delta x(x, t)$ using the following equation:

$$
J_{\mathrm{ex}}=J_{\mathrm{ex}}\left(x+\delta x\left(x, J_{\mathrm{ex}}, \dot{J}_{\mathrm{ex}}, \ldots, J_{\mathrm{ex}}^{(k)}\right), t\right) .
$$

It can be solved, for example, by the iterative method, taking $J_{\mathrm{ex} 0}=J_{\mathrm{ex}}(x, t)$ :

$$
J_{\mathrm{ex}(n+1)}=J_{\mathrm{ex}}\left(x+\delta x\left(x, J_{\mathrm{ex}(n)}, \dot{J}_{\mathrm{ex}(n)}, \ldots, J_{\mathrm{ex}(n)}^{(k)}\right), t\right) .
$$

We hope that using $J_{\text {ex }}$ in Eqs. (454) and (455) both as a variable and as a symbol of unknown function $J_{\text {ex }}(x, t)$ will not lead to a confusion.

In all the constructions introduced above it was assumed that $\delta x$ is sufficiently small and the driven system (428) will not deviate too far from the slow invariant manifold of the initial system. However, 
a stability loss is possible: solutions of Eq. (428) can deviate arbitrarily far given some perturbations level. The invariant manifold can loose it's stability. Qualitatively, this effect of invariant manifold explosion can be represented as follows.

Suppose that $J_{\mathrm{ex}}$ includes the parameter $\varepsilon$ : one has $\varepsilon J_{\mathrm{ex}}$ in Eq. (428). When $\varepsilon$ is small, system motions are located in a small neighborhood of the initial manifold. This neighborhood grows monotonically and continuously with increase of $\varepsilon$, but after some $\varepsilon_{0}$ a sudden change happens ("explosion") and the neighborhood, in which the motion takes place, becomes significantly wider at $\varepsilon>\varepsilon_{0}$ than at $\varepsilon<\varepsilon_{0}$. The stability loss is not necessarily connected with the invariance loss. In example 13 it is shown how the invariant manifold (which is at the same time the quasi-equilibrium manifold in the example) can loose it's stability. This "explosion" of the invariant manifold leads to essential physical consequences (see example 13).

\subsection{Lyapunov norms, finite-dimensional asymptotic and volume contraction}

In a general case, it is impossible to prove the existence of a global Lyapunov function on the basis of local data. We can only verify or falsify the hypothesis about a given function, is it a global Lyapunov function, or is it not. On the other hand, there exists a more strict stability property which can be verified or falsified (in principle) on the base of local data analysis. This is a Lyapunov norm existence.

A norm $\|\bullet\|$ is the Lyapunov norm for system (428), if for any two solutions $x^{(1)}(t), x^{(2)}(t), t \geqslant 0$, the function $\left\|x^{(1)}(t)-x^{(2)}(t)\right\|$ is nonincreasing in time.

Linear operator $A$ is dissipative with respect to a norm $\|\bullet\|$, if $\exp (A t)(t \geqslant 0)$ is a semigroup of contractions: $\|\exp (A t) x\| \leqslant\|x\|$ for any $x$ and $t \geqslant 0$. The family of linear operators $\left\{A_{\alpha}\right\}_{\alpha \in K}$ is simultaneously dissipative, if all operators $A_{\alpha}$ are dissipative with respect to some norm $\|\bullet\|$ (it should be stressed that there exists one norm for all $A_{\alpha}, \alpha \in K$ ). The mathematical theory of simultaneously dissipative operators for finite-dimensional spaces was developed in Refs. [220-224].

Let system (428) be defined in a convex set $U \subset E$, and $A_{x}$ be Jacobi operator at the point $x$ : $A_{x}=D_{x}\left(J(x)+J_{\text {ex }}(x)\right)$. This system has a Lyapunov norm, if the family of operators $\left\{A_{x}\right\}_{x \in U}$ is simultaneously dissipative. If one can choose such $\varepsilon>0$ that for all $A_{x}, t>0$, any vector $z$, and this Lyapunov norm $\left\|\exp \left(A_{x} t\right) z\right\| \leqslant \exp (-\varepsilon t)\|z\|$, then for any two solutions $x^{(1)}(t), x^{(2)}(t), t \geqslant 0$ of Eqs. (428) $\left\|x^{(1)}(t)-x^{(2)}(t)\right\| \leqslant \exp (-\varepsilon t)\left\|x^{(1)}(0)-x^{(2)}(0)\right\|$.

The simplest class of nonlinear kinetic (open) systems with Lyapunov norms was described in the paper [225]. These are reaction systems without interactions of various substances. The stoichiometric equation of each elementary reaction has a form

$$
\alpha_{r i} A_{i} \rightarrow \sum_{j} \beta_{r j} A_{j}
$$

where $r$ enumerates reactions, $\alpha_{r i}, \beta_{r j}$ are nonnegative stoichiometric coefficients (usually they are integer), $A_{i}$ are symbols of substances.

In the right hand side of Eq. (456) there is one initial reagent, though $\alpha_{r i}>1$ is possible (there may be several copies of $A_{i}$, for example $\left.3 A \rightarrow 2 B+C\right)$.

Kinetic equations for reaction system (456) have a Lyapunov norm [225]. This is $l^{1}$ norm with weights: $\|x\|=\sum_{i} w_{i}\left|x_{i}\right|, w_{i}>0$. There exists no quadratic Lyapunov norm for reaction systems without interaction of various substances. 
Existence of the Lyapunov norm is a very strong restriction on nonlinear systems, and such systems are not wide spread in applications. But if we go from distance contraction to contraction of $k$-dimensional volumes $(k=2,3, \ldots$,$) [231], the situation changes. There exist many kinetic systems$ with a monotonous contraction of $k$-dimensional volumes for sufficiently big $k$ (see, for example, [234-237]). Let $x(t), t \geqslant 0$ be a solution of Eq. (428). Let us write a first approximation equation for small deviations from $x(t)$ :

$$
\frac{\mathrm{d} \Delta x}{\mathrm{~d} t}=A_{x(t)} \Delta x .
$$

This is linear system with coefficients depending on $t$. Let us study how system (457) changes $k$-dimensional volumes. A $k$-dimensional parallelepiped with edges $x^{(1)}, x^{(2)}, \ldots, x^{(k)}$ is an element of the $k$ th exterior power:

$$
x^{(1)} \wedge x^{(2)} \wedge \cdots \wedge x^{(k)} \in \underbrace{E \wedge E \wedge \cdots \wedge E}_{k}
$$

(this is an antisymmetric tensor). A norm in the $k$ th exterior power of the space $E$ is a measure of $k$-dimensional volumes (one of the possible measures). Dynamics of parallelepipeds induced by system (457) can be described by equations

$$
\begin{aligned}
& \frac{\mathrm{d}}{\mathrm{d} t}\left(\Delta x^{(1)} \wedge \Delta x^{(2)} \wedge \cdots \wedge \Delta x^{(k)}\right) \\
& =\left(A_{x(t)} \Delta x^{(1)}\right) \wedge \Delta x^{(2)} \wedge \cdots \wedge \Delta x^{(k)}+\Delta x^{(1)} \wedge\left(A_{x(t)} \Delta x^{(2)}\right) \wedge \cdots \wedge \Delta x^{(k)}+\cdots \\
& \quad+\Delta x^{(1)} \wedge \Delta x^{(2)} \wedge \cdots \wedge\left(A_{x(t)} \Delta x^{(k)}\right)=A_{x(t)}^{D \wedge k}\left(\Delta x^{(1)} \wedge \Delta x^{(2)} \wedge \cdots \wedge \Delta x^{(k)}\right) .
\end{aligned}
$$

Here $A_{x(t)}^{D \wedge k}$ are operators of induced action of $A_{x(t)}$ on the $k$ th exterior power of $E$. Again, a decreasing of $\left\|\Delta x^{(1)} \wedge \Delta x^{(2)} \wedge \cdots \wedge \Delta x^{(k)}\right\|$ in time is equivalent to dissipativity of all operators $A_{x(t)}^{D \wedge k}, t \geqslant 0$ in the norm $\|\bullet\|$. Existence of such norm for all $A_{x}^{D \wedge k}(x \in U)$ is equivalent to decreasing of volumes of all parallelepipeds due to first approximation system (457) for any solution $x(t)$ of Eqs. (428). If one can choose such $\varepsilon>0$ that for all $A_{x}(x \in U)$, any vector $z \in E \wedge E \wedge \cdots \wedge E$, and this norm $\left\|\exp \left(A_{x}^{D \wedge k} t\right) z\right\| \leqslant \exp (-\varepsilon t)\|z\|$, then the volumes of parallelepipeds decrease exponentially as $\exp (-\varepsilon t)$.

For such systems we can estimate the Hausdorff dimension of the attractor [226] (under some additional technical conditions about solutions boundedness): it cannot exceed $k$. It is necessary to stress here that this estimation of the Hausdorff dimension does not solve the problem of construction of the invariant manifold containing this attractor, and one needs special technique and additional restriction on the system to obtain this manifold (see [235,242,238,243]).

The simplest way for construction slow invariant manifold is possible for systems with a dominance of linear part in highest dimensions. Let an infinite-dimensional system have a form: $\dot{u}+A u=$ $R(u)$, where $A$ is self-adjoint, and has discrete spectrum $\lambda_{i} \rightarrow \infty$ with sufficiently big gaps between $\lambda_{i}$, and $R(u)$ is continuous. One can build the slow manifold as the graph over a root space of $A$. Let the basis consists of eigenvectors of $A$. In this basis $\dot{u}_{i}=-\lambda_{i} u_{i}+R_{i}(u)$, and it seems very plausible that for some $k$ and sufficiently big $i$ functions $u_{i}(t)$ exponentially fast tend to $u_{i}\left(u_{1}(t), \ldots u_{k}(t)\right)$, if $R_{i}(u)$ are bounded and continuous in a suitable sense.

Different variants of rigorous theorems about systems with such a dominance of linear part in highest dimensions linear may be found in literature (see, for example, the textbook [76]). Even if 
all the sufficient conditions hold, it remains the problem of efficient computing of these manifold, and different ways for calculation are proposed: from Euler method for manifold correction [13] to different algorithms of discretisations [3,81,239].

The simplest conditions of simultaneous dissipativity for the family of operators $\left\{A_{x}\right\}$ can be created in a following way: let us take a norm $\|\bullet\|$. If all operators $A_{x}$ are dissipative with respect to this norm, then the family $A_{x}$ is (evidently) simultaneously dissipative in this norm. So, we can verify or falsify a hypothesis about simultaneous dissipativity for a given norm. Simplest examples give us quadratic and $l^{1}$ norms.

For quadratic norm associated with a scalar product $\langle\mid\rangle$ dissipativity of operator $A$ is equivalent to nonpositivity of all points of spectrum $A+A^{+}$, where $A^{+}$is the adjoint to $A$ operator with respect to scalar product $\langle\|\rangle$.

For $l^{1}$ norm with weights $\|x\|=\sum_{i} w_{i}\left|x_{i}\right|, w_{i}>0$. The condition of operator $A$ dissipativity for this norm is the weighted diagonal predominance for columns of the $A$ matrix $A=\left(a_{i j}\right)$ :

$$
a_{i i}<0, \quad w_{i}\left|a_{i i}\right| \geqslant \sum_{j, j \neq i} w_{j}\left|a_{j i}\right| .
$$

For exponential contraction it is necessary and sufficient that some gap exists in the dissipativity inequalities:

for quadratic norm $\sigma\left(A+A^{+}\right)<\varepsilon<0$, where $\sigma\left(A+A^{+}\right)$is the spectrum of $A+A^{+}$;

for $l^{1}$ norm with weights $a_{i i}<0, w_{i}\left|a_{i i}\right| \geqslant \sum_{j, j \neq i} w_{j}\left|a_{j i}\right|+\varepsilon, \varepsilon>0$.

The sufficient conditions of simultaneous dissipativity can have another form (not only the form of dissipativity checking with respect to a given norm) [221-224], but the problem of necessary and sufficient conditions in general case is open.

The dissipativity conditions for operators $A_{x}^{D \wedge k}$ of induced action of $A_{x}$ on the $k$ th exterior power of $E$ have the similar form, for example, if we know the spectrum of $A+A^{+}$, then it is easy to find the spectrum of $A_{x(t)}^{D \wedge k}+\left(A_{x(t)}^{D \wedge k}\right)^{+}$: each eigenvalue of this operator is a sum of $k$ distinct eigenvalues of $A+A^{+}$; the $A_{x(t)}^{D \wedge k}+\left(A_{x(t)}^{D \wedge k}\right)^{+}$spectrum is a closure of set of sums of $k$ distinct points $A+A^{+}$ spectrum.

A basis the $k$ th exterior power of $E$ can be constructed from the basis $\left\{e_{i}\right\}$ of $E$ : it is

$$
\left\{e_{i_{1} i_{2} \ldots i_{k}}\right\}=\left\{e_{i_{1}} \wedge e_{i_{2}} \wedge \cdots \wedge e_{i_{k}}\right\}, \quad i_{1}<i_{2}<\cdots<i_{k} .
$$

For $l^{1}$ norm with weights in the $k$ th exterior power of $E$ the set of weights is $\left\{w_{i_{1} i_{2} \ldots i_{k}}>0\right.$, $\left.i_{1}<i_{2}<\cdots<i_{k}\right\}$. The norm of a vector $z$ is

$$
\|z\|=\sum_{i_{1}<i_{2}<\cdots<i_{k}} w_{i_{1} i_{2} \cdots i_{k}}\left|z_{i_{1} i_{2} \ldots i_{k}}\right| \text {. }
$$

The dissipativity conditions for operators $A^{D \wedge k}$ of induced action of $A$ in $l^{1}$ norm with weights have the form

$$
\begin{aligned}
& a_{i_{1} i_{1}}+a_{i_{2} i_{2}}+\cdots+a_{i_{k} i_{k}}<0, \\
& w_{i_{1} i_{2} \ldots i_{k}}\left|a_{i_{1} i_{1}}+a_{i_{2} i_{2}}+\cdots+a_{i_{k} i_{k}}\right| \geqslant \sum_{l=1}^{k} \sum_{j, j \neq i_{1}, i_{2}, \ldots, i_{k}} w_{i_{1} i_{2} \ldots i_{k}}^{l, j}\left|a_{j i_{l}}\right|
\end{aligned}
$$


for any

$$
i_{1}<i_{2}<\cdots<i_{k}
$$

where $w_{i_{1} i_{2} \ldots i_{k}}^{l, j}=w_{I}$, multiindex $I$ consists of indices $i_{p}(p \neq l)$, and $j$.

For infinite-dimensional systems the problem of volume contraction and Lyapunov norms for exterior powers of $E$ consists of three parts: geometrical part concerning the choice of norm for simultaneous dissipativity of operator families, topological part concerning topological nonequivalence of constructed norms, and estimation of the bounded set containing compact attractor.

The difficult problem may concern the appropriate a priori estimations of the bounded convex positively invariant set $V \subset U$ where the compact attractor is situated. It may be crucial to solve the problem of simultaneous dissipativity for the most narrow family of operators $\left\{A_{x}, x \in V\right\}$ (and their induced action on the $k$ th exterior power of $E$ ).

The estimation of attractor dimension based on Lyapunov norms in the exterior powers is rather rough. This is a local estimation. More exact estimations are based on global Lyapunov exponents (Lyapunov or Kaplan-Yorke dimension [228,229]). There are many different measures of dimension [227,230], and many efforts are applied to create good estimations for different dimensions [241]. Estimations of attractor dimension was given for different systems: from the Navier-Stokes hydrodynamic [233] to climate dynamics [232]. The introduction and review of many results is given in the book [235]. But local estimations remain the main tools for estimation of attractors dimension, because global estimations for complex systems are much more complicated and often unattainable because of computation complexity.

\section{Example 12: The universal limit in dynamics of dilute polymeric solutions}

The method of invariant manifold is developed for a derivation of reduced description in kinetic equations of dilute polymeric solutions. It is demonstrated that this reduced description becomes universal in the limit of small Deborah and Weissenberg numbers, and it is represented by the (revised) Oldroyd 8 constants constitutive equation for the polymeric stress tensor. Coefficients of this constitutive equation are expressed in terms of the microscopic parameters. A systematic procedure of corrections to the revised Oldroyd 8 constants equations is developed. Results are tested with simple flows.

Kinetic equations arising in the theory of polymer dynamics constitute a wide class of microscopic models of complex fluids. Same as in any branch of kinetic theory, the problem of reduced description becomes actual as soon as the kinetic equation is established. However, in spite of an enormous amount of work in the field of polymer dynamics [113-115,249,259], this problem remains less studied as compared to other classical kinetic equations.

It is the purpose of this section to suggest a systematic approach to the problem of reduced description for kinetic models of polymeric fluids. First, we would like to specify our motivation by comparing the problem of the reduced description for that case with a similar problem in the familiar case of the rarefied gas obeying the classical Boltzmann kinetic equation [87,51].

The problem of reduced description begins with establishing a set of slow variables. For the Boltzmann equation, this set is represented by five hydrodynamic fields (density, momentum and energy) which are low-order moments of the distribution function, and which are conserved quantities of the dissipation process due to particle's collisions. The reduced description is a closed system of 
equations for these fields. One starts with the manifold of local equilibrium distribution functions (local Maxwellians), and finds a correction by the Chapman-Enskog method [51]. The resulting reduced description (the Navier-Stokes hydrodynamic equations) is universal in the sense that the form of equations does not depend on details of particle's interaction whereas the latter shows up explicitly only in the transport coefficients (viscosity, temperature conductivity, etc.).

Coming back to the complex fluids, we shall consider the simplest case of dilute polymer solutions represented by dumbbell models studied below. Two obstacles preclude an application of the traditional techniques. First, the question which variables should be regarded as slow is at least less evident because the dissipative dynamics in the dumbbell models has no nontrivial conservation laws compared to the Boltzmann case. Consequently, a priori, there are no distinguished manifolds of distribution functions like the local equilibria which can be regarded as a starting point. Second, while the Boltzmann kinetic equation provides a self-contained description, the dumbbell kinetic equations are coupled to the hydrodynamic equations. This coupling manifests itself as an external flux in the kinetic equation.

The well-known distinguished macroscopic variable associated with the dumbbell kinetic equations is the polymeric stress tensor [113,259]. This variable is not the conserved quantity but nevertheless it should be treated as a relevant slow variable because it actually contributes to the macroscopic (hydrodynamic) equations. Equations for the stress tensor are known as the constitutive equations, and the problem of reduced description for the dumbbell models consists in deriving such equations from the kinetic equation.

Our approach is based on the method of invariant manifold [6], modified for systems coupled with external fields. This method suggests constructing invariant sets (or manifolds) of distribution functions that represent the asymptotic states of slow evolution of the kinetic system. In the case of dumbbell models, the reduced description is produced by equations which constitute stress-strain relations, and two physical requirements are met by our approach: The first is the principle of frame-indifference with respect to any time-dependent reference frame. This principle requires that the resulting equations for the stresses contain only frame-indifferent quantities. For example, the frame-dependent vorticity tensor should not show up in these equations unless being presented in frame-indifferent combinations with another tensors. The second principle is the thermodynamic stability: In the absence of the flow, the constitutive model should be purely dissipative, in other words, it should describe the relaxation of stresses to their equilibrium values.

The physical picture addressed below takes into account two assumptions: (i) In the absence of the flow, deviations from the equilibrium are small. Then the invariant manifold is represented by eigenvectors corresponding to the slowest relaxation modes. (ii) When the external flow is taken into account, it is assumed to cause a small deformation of the invariant manifolds of the purely dissipative dynamics. Two characteristic parameters are necessary to describe this deformation. The first is the characteristic time variation of the external field. The second is the characteristic intensity of the external field. For dumbbell models, the first parameter is associated with the conventional Deborah number while the second one is usually called the Weissenberg number. An iteration approach which involves these parameters is developed.

Two main results of the analysis are as follows: First, the lowest-order constitutive equations with respect to the characteristic parameters mentioned above has the form of the revised phenomenological Oldroyd 8 constants model. This result is interpreted as the macroscopic limit of the microscopic dumbbell dynamics whenever the rate of the strain is low, and the Deborah number is small. This 
limit is valid generically, in the absence or in the presence of the hydrodynamic interaction, and for the arbitrary nonlinear elastic force. The phenomenological constants of the Oldroyd model are expressed in a closed form in terms of the microscopic parameters of the model. The universality of this limit is similar to that of the Navier-Stokes equations which are the macroscopic limit of the Boltzmann equation at small Knudsen numbers for arbitrary hard-core molecular interactions. The test calculation for the nonlinear FENE force demonstrates a good quantitative agreement of the constitutive equations with solutions to the microscopic kinetic equation within the domain of their validity.

The second result is a regular procedure of finding corrections to the zero-order model. These corrections extend the model into the domain of higher rates of the strain, and to flows which alternate faster in time. Same as in the zero-order approximation, the higher-order corrections are linear in the stresses, while their dependence on the gradients of the flow velocity and its time derivatives becomes highly nonlinear.

The section is organized as follows: For the sake of completeness, we present the nonlinear dumbbell kinetic models in the next subsection, "The problem of reduced description in polymer dynamics". In the section, "The method of invariant manifold for weakly driven systems", we describe in details our approach to the derivation of macroscopic equations for an abstract kinetic equation coupled to external fields. This derivation is applied to the dumbbell models in the section, "Constitutive equations". The zero-order constitutive equation is derived and discussed in detail in this section, as well as the structure of the first correction. Tests of the zero-order constitutive equation for simple flow problems are given in the section, "Tests on the FENE dumbbell model".

\section{The problem of reduced description in polymer dynamics}

Elastic dumbbell models. The elastic dumbbell model is the simplest microscopic model of polymer solutions [113]. The dumbbell model reflects the two features of real-world macromolecules to be orientable and stretchable by a flowing solvent. The polymeric solution is represented by a set of identical elastic dumbbells placed in an isothermal incompressible liquid. In this example we adopt notations used in kinetic theory of polymer dynamics [113]. Let $\boldsymbol{Q}$ be the connector vector between the beads of a dumbbell, and $\Psi(\boldsymbol{x}, \boldsymbol{Q}, t)$ be the configuration distribution function which depends on the location in the space $\boldsymbol{x}$ at time $t$. We assume that dumbbells are distributed uniformly, and consider the normalization, $\int \Psi(\boldsymbol{x}, \boldsymbol{Q}, t) \mathrm{d} \boldsymbol{Q}=1$. The Brownian motion of beads in the physical space causes a diffusion in the phase space described by the Fokker-Planck equation (FPE) [113]:

$$
\frac{D \Psi}{D t}=-\frac{\partial}{\partial \boldsymbol{Q}} \cdot \boldsymbol{k} \cdot \boldsymbol{Q} \Psi+\frac{2 k_{\mathrm{B}} T}{\xi} \frac{\partial}{\partial \boldsymbol{Q}} \cdot \boldsymbol{D} \cdot\left(\frac{\partial}{\partial \boldsymbol{Q}} \Psi+\frac{\boldsymbol{F}}{k_{\mathrm{B}} T} \Psi\right) \cdot
$$

Here $D / D t=\partial / \partial t+\boldsymbol{v} \cdot \nabla$ is the material derivative, $\nabla$ is the spatial gradient, $\boldsymbol{k}(\boldsymbol{x}, t)=(\nabla \boldsymbol{v})^{\dagger}$ is the gradient of the velocity of the solvent $\boldsymbol{v}$, $\dagger$ denotes transposition of tensors, $\boldsymbol{D}$ is the dimensionless diffusion matrix, $k_{\mathrm{B}}$ is the Boltzmann constant, $T$ is the temperature, $\xi$ is the dimensional coefficient characterizing a friction exerted by beads moving through solvent media (friction coefficient $[113,114])$, and $\boldsymbol{F}=\partial \phi / \partial \boldsymbol{Q}$ is the elastic spring force defined by the potential $\phi$. We consider forces of the form $\boldsymbol{F}=H f\left(Q^{2}\right) \boldsymbol{Q}$, where $f\left(Q^{2}\right)$ is a dimensionless function of the variable $Q^{2}=\boldsymbol{Q} \cdot \boldsymbol{Q}$, and $H$ is the dimensional constant. Incompressibility of solvent implies $\sum_{i} k_{i i}=0$. 
Let us introduce a time dimensional constant

$$
\lambda_{\mathrm{r}}=\frac{\xi}{4 H},
$$

which coincides with a characteristic relaxation time of dumbbell configuration in the case when the force $\boldsymbol{F}$ is linear: $f\left(Q^{2}\right)=1$. It proves convenient to rewrite the FPE (460) in the dimensionless form

$$
\frac{D \Psi}{D \widehat{t}}=-\frac{\partial}{\partial \widehat{\mathbf{Q}}} \cdot \widehat{\mathbf{k}} \cdot \widehat{\mathbf{Q}} \Psi+\frac{\partial}{\partial \widehat{\mathbf{Q}}} \cdot \boldsymbol{D} \cdot\left(\frac{\partial}{\partial \widehat{\mathbf{Q}}} \Psi+\widehat{\mathbf{F}} \Psi\right)
$$

Various dimensionless quantities used are: $\widehat{\mathbf{Q}}=\left(H / k_{\mathrm{B}} T\right)^{1 / 2} \boldsymbol{Q}, D / D \widehat{t}=\partial / \partial \widehat{t}+\boldsymbol{v} \cdot \bar{\nabla}, \widehat{t}=t / \lambda_{\mathrm{r}}$ is the dimensionless time, $\bar{\nabla}=\lambda_{\mathrm{r}} \nabla$ is the reduced space gradient, and $\widehat{\mathbf{k}}=\boldsymbol{k} \lambda_{\mathrm{r}}=(\bar{\nabla} \boldsymbol{v})^{\dagger}$ is the dimensionless tensor of the gradients of the velocity. In the sequel, only dimensionless quantities $\widehat{\mathbf{Q}}$ and $\widehat{\mathbf{F}}$ are used, and we keep notations $\boldsymbol{Q}$ and $\boldsymbol{F}$ for them for the sake of simplicity.

The quantity of interest is the stress tensor introduced by Kramers [113]:

$$
\tau=-v_{\mathrm{s}} \dot{\gamma}+n k_{\mathrm{B}} T(\mathbf{1}-\langle\boldsymbol{F} \boldsymbol{Q}\rangle),
$$

where $v_{\mathrm{s}}$ is the viscosity of the solvent, $\dot{\gamma}=\boldsymbol{k}+\boldsymbol{k}^{\dagger}$ is the rate-of-strain tensor, $n$ is the concentration of polymer molecules, and the angle brackets stand for the averaging with the distribution function $\Psi:\langle\bullet\rangle \equiv \int \bullet \Psi(\boldsymbol{Q}) \mathrm{d} \boldsymbol{Q}$. The tensor

$$
\tau_{\mathrm{p}}=n k_{\mathrm{B}} T(\mathbf{1}-\langle\boldsymbol{F} \boldsymbol{Q}\rangle)
$$

gives a contribution to stresses caused by the presence of polymer molecules.

The stress tensor is required in order to write down a closed system of hydrodynamic equations:

$$
\frac{D v}{D t}=-\rho^{-1} \nabla p-\nabla \cdot \tau[\Psi] .
$$

Here $p$ is the pressure, and $\rho=\rho_{\mathrm{s}}+\rho_{\mathrm{p}}$ is the mass density of the solution where $\rho_{\mathrm{s}}$ is the solvent, and $\rho_{\mathrm{p}}$ is the polymeric contributions.

Several models of the elastic force are known in the literature. The Hookean law is relevant to small perturbations of the equilibrium configuration of the macromolecule:

$$
\boldsymbol{F}=\boldsymbol{Q} .
$$

In that case, the differential equation for $\tau$ is easily derived from the kinetic equation, and is the well known Oldroyd-B constitutive model [113].

The second model, the FENE force law [250], was derived as an approximation to the inverse Langevin force law [113] for a more realistic description of the elongation of a polymeric molecule in a solvent:

$$
\boldsymbol{F}=\frac{\boldsymbol{Q}}{1-\boldsymbol{Q}^{2} / \boldsymbol{Q}_{0}^{2}}
$$

This force law takes into account the nonlinear stiffness and the finite extendibility of dumbbells, where $\boldsymbol{Q}_{0}$ is the maximal extendibility.

The features of the diffusion matrix are important for both the microscopic and the macroscopic behavior. The isotropic diffusion is represented by the simplest diffusion matrix

$$
D_{\mathrm{I}}=\frac{1}{2} \mathbf{1} \text {. }
$$


Here 1 is the unit matrix. When the hydrodynamic interaction between the beads is taken into account, this results in an anisotropic contribution to the diffusion matrix (467). The original form of this contribution is the Oseen-Burgers tensor $\boldsymbol{D}_{\mathrm{H}}[251,252]$ :

$$
\boldsymbol{D}=\boldsymbol{D}_{\mathrm{I}}-\kappa \boldsymbol{D}_{\mathrm{H}}, \quad \boldsymbol{D}_{\mathrm{H}}=\frac{1}{Q}\left(\mathbf{1}+\frac{\boldsymbol{Q Q}}{Q^{2}}\right),
$$

where

$$
\kappa=\left(\frac{H}{k_{\mathrm{B}} T}\right)^{1 / 2} \frac{\xi}{16 \pi v_{\mathrm{s}}} .
$$

Several modifications of the Oseen-Burgers tensor can be found in the literature (the Rotne-PragerYamakawa tensor $[253,254])$, but here we consider only the classical version.

Properties of the Fokker-Planck operator. Let us review some of the properties of the FokkerPlanck operator $J$ in the right hand side of Eq. (461) relevant to what will follow. This operator can be written as $J=J_{\mathrm{d}}+J_{\mathrm{h}}$, and it represents two processes.

The first term, $J_{\mathrm{d}}$, is the dissipative part,

$$
J_{\mathrm{d}}=\frac{\partial}{\partial \boldsymbol{Q}} \cdot \boldsymbol{D} \cdot\left(\frac{\partial}{\partial \boldsymbol{Q}}+\boldsymbol{F}\right) .
$$

This part is responsible for the diffusion and friction which affect internal configurations of dumbbells, and it drives the system to the unique equilibrium state,

$$
\Psi_{\mathrm{eq}}=c^{-1} \exp \left(-\phi\left(Q^{2}\right)\right),
$$

where $c=\int \exp (-\phi) \mathrm{d} \boldsymbol{Q}$ is the normalization constant.

The second part, $J_{\mathrm{h}}$, describes the hydrodynamic drag of the beads in the flowing solvent:

$$
J_{\mathrm{h}}=-\frac{\partial}{\partial \boldsymbol{Q}} \cdot \widehat{\mathbf{k}} \cdot \boldsymbol{Q} .
$$

The dissipative nature of the operator $J_{\mathrm{d}}$ is reflected by its spectrum. We assume that this spectrum consists of real-valued nonpositive eigenvalues, and that the zero eigenvalue is not degenerated. In the sequel, the following scalar product will be useful:

$$
\langle g, h\rangle_{\mathrm{s}}=\int \Psi_{\mathrm{eq}}^{-1} g h \mathrm{~d} \boldsymbol{Q} .
$$

The operator $J_{\mathrm{d}}$ is symmetric and nonpositive definite in this scalar product:

$$
\left\langle J_{\mathrm{d}} g, h\right\rangle_{\mathrm{s}}=\left\langle g, J_{\mathrm{d}} h\right\rangle_{\mathrm{s}} \quad \text { and } \quad\left\langle J_{\mathrm{d}} g, g\right\rangle_{\mathrm{s}} \leqslant 0 .
$$

Since

$$
\left\langle J_{\mathrm{d}} g, g\right\rangle_{\mathrm{s}}=-\int \Psi_{\mathrm{eq}}^{-1}(\partial g / \partial \boldsymbol{Q}) \cdot \Psi_{\mathrm{eq}} \boldsymbol{D} \cdot(\partial g / \partial \boldsymbol{Q}) \mathrm{d} \boldsymbol{Q},
$$

the above inequality is valid if the diffusion matrix $\boldsymbol{D}$ is positive semidefinite. This happens if $\boldsymbol{D}=\boldsymbol{D}_{\mathrm{I}}$ (467) but is not generally valid in the presence of the hydrodynamic interaction (468). Let us split the operator $J_{\mathrm{d}}$ according to the splitting of the diffusion matrix $\boldsymbol{D}$ :

$$
J_{\mathrm{d}}=J_{\mathrm{d}}^{\mathrm{I}}-\kappa J_{\mathrm{d}}^{\mathrm{H}} \quad \text { where } J_{\mathrm{d}}^{\mathrm{I}, \mathrm{H}}=\partial / \partial \boldsymbol{Q} \cdot \boldsymbol{D}_{\mathrm{I}, \mathrm{H}} \cdot(\partial / \partial \boldsymbol{Q}+\boldsymbol{F}) .
$$


Both the operators $J_{\mathrm{d}}^{\mathrm{I}}$ and $J_{\mathrm{d}}^{\mathrm{H}}$ have nondegenerated eigenvalue 0 which corresponds to their common eigenfunction $\Psi_{\text {eq }}: J_{\mathrm{d}}^{\mathrm{I}, \mathrm{H}} \Psi_{\text {eq }}=0$, while the rest of the spectrum of both operators belongs to the nonpositive real semi-axis. Then the spectrum of the operator $J_{\mathrm{d}}=J_{\mathrm{d}}^{\mathrm{I}}-\kappa J_{\mathrm{d}}^{\mathrm{H}}$ remains nonpositive for sufficiently small values of the parameter $\kappa$. The spectral properties of both operators $J_{\mathrm{d}}^{\mathrm{I}, \mathrm{H}}$ depend only on the choice of the spring force $\boldsymbol{F}$. Thus, in the sequel we assume that the hydrodynamic interaction parameter $\kappa$ is sufficiently small so that the thermodynamic stability property (471) holds.

We note that the scalar product $\langle\bullet, \bullet\rangle_{\mathrm{s}}$ coincides with the second differential $\left.D^{2} S\right|_{\Psi_{\text {eq }}}$ of an entropy functional $S[\Psi]:\langle\bullet, \bullet\rangle_{\mathrm{s}}=-\left.D^{2} S\right|_{\Psi_{\mathrm{eq}}}[\bullet, \bullet]$, where the entropy has the form

$$
S[\Psi]=-\int \Psi \ln \left(\frac{\Psi}{\Psi_{\text {eq }}}\right) \mathrm{d} \boldsymbol{Q}=-\left\langle\ln \left(\frac{\Psi}{\Psi_{\text {eq }}}\right)\right\rangle .
$$

The entropy $S$ grows in the course of dissipation:

$$
D S\left[J_{\mathrm{d}} \Psi\right] \geqslant 0 .
$$

This inequality similar to inequality (471) is satisfied for sufficiently small $\kappa$. Symmetry and nonpositiveness of operator $J_{\mathrm{d}}$ in the scalar product defined by the second differential of the entropy is a common property of linear dissipative systems.

Statement of the problem. Given the kinetic equation (460), we aim at deriving differential equations for the stress tensor $\tau$ (462). The latter includes the moments $\langle\boldsymbol{F} \boldsymbol{Q}\rangle=\int \boldsymbol{F} \boldsymbol{Q} \Psi \mathrm{d} \boldsymbol{Q}$.

In general, when the diffusion matrix is nonisotropic and/or the spring force is nonlinear, closed equations for these moments are not available, and approximations are required. With this, any derivation should be consistent with the three requirements:

(i) Dissipativity or thermodynamic stability: the macroscopic dynamics should be dissipative in the absence of the flow.

(ii) Slowness: the macroscopic equations should represent slow degrees of freedom of the kinetic equation.

(iii) Material frame indifference: the form of equations for the stresses should be invariant with respect to the Eucluidian, time dependent transformations of the reference frame $[113,255]$.

While these three requirements should be met by any approximate derivation, the validity of our approach will be restricted by two additional assumptions:

(a) Let us denote $\theta_{1}$ the inertial time of the flow, which we define via characteristic value of the gradient of the flow velocity: $\theta_{1}=|\nabla \boldsymbol{v}|^{-1}$, and $\theta_{2}$ the characteristic time of the variation of the flow velocity. We assume that the characteristic relaxation time of the molecular configuration $\theta_{\mathrm{r}}$ is small as compared to both the characteristic times $\theta_{1}$ and $\theta_{2}$ :

$$
\theta_{\mathrm{r}} \ll \theta_{1} \quad \text { and } \quad \theta_{\mathrm{r}} \ll \theta_{2} .
$$

(b) In the absence of the flow, the initial deviation of the distribution function from the equilibrium is small so that the linear approximation is valid.

While assumption (b) is merely of a technical nature, and it is intended to simplify the treatment of the dissipative part of the Fokker-Planck operator (469) for elastic forces of a complicated form, assumption (a) is crucial for taking into account the flow in an adequate way. We have assumed 
that the two parameters characterizing the composed system 'relaxing polymer configuration+flowing solvent' should be small: These two parameters are:

$$
\varepsilon_{1}=\theta_{\mathrm{r}} / \theta_{1}, \quad \varepsilon_{2}=\theta_{\mathrm{r}} / \theta_{2} .
$$

The characteristic relaxation time of the polymeric configuration is defined via the coefficient $\lambda_{\mathrm{r}}$ : $\theta_{\mathrm{r}}=c \lambda_{\mathrm{r}}$, where $c$ is some positive dimensionless constant which is estimated by the absolute value of the lowest nonzero eigenvalue of the operator $J_{\mathrm{d}}$. The first parameter $\varepsilon_{1}$ is usually termed the Weissenberg number while the second one $\varepsilon_{2}$ is the Deborah number (cf. Ref. [256, Section 7-2]).

\section{The method of invariant manifold for weakly driven systems}

The Newton iteration scheme. In this section we introduce an extension of the method of invariant manifold [6] onto systems coupled with external fields. We consider a class of dynamic systems of the form

$$
\frac{\mathrm{d} \Psi}{\mathrm{d} t}=J_{\mathrm{d}} \Psi+J_{\mathrm{ex}}(\alpha) \Psi
$$

where $J_{\mathrm{d}}$ is a linear operator representing the dissipative part of the dynamic vector field, while $J_{\text {ex }}(\alpha)$ is a linear operator which represents an external flux and depends on a set of external fields $\alpha=\left\{\alpha_{1}, \ldots, \alpha_{k}\right\}$. Parameters $\alpha$ are either known functions of the time, $\alpha=\alpha(t)$, or they obey a set of equations,

$$
\frac{\mathrm{d} \alpha}{\mathrm{d} t}=\Phi(\Psi, \alpha)
$$

Without any restriction, parameters $\alpha$ are adjusted in such a way that $J_{\mathrm{ex}}(\alpha=0) \equiv 0$. Kinetic equation (461) has form (475), and general results of this section will be applied to the dumbbell models below in a straightforward way.

We assume that the vector field $J_{\mathrm{d}} \Psi$ has the same dissipative properties as the Fokker-Planck operator (469). Namely there exists the globally convex entropy function $S$ which obeys: $D S\left[J_{\mathrm{d}} \Psi\right] \geqslant 0$, and the operator $J_{\mathrm{d}}$ is symmetric and nonpositive in the scalar product $\langle\bullet, \bullet\rangle_{\mathrm{s}}$ defined by the second differential of the entropy: $\langle g, h\rangle_{\mathrm{s}}=-D^{2} S[g, h]$. Thus, the vector field $J_{\mathrm{d}} \Psi$ drives the system irreversibly to the unique equilibrium state $\Psi_{\text {eq }}$.

We consider a set of $n$ real-valued functionals, $M_{i}^{*}[\Psi]$ (macroscopic variables), in the phase space $\mathscr{F}$ of system (475). A macroscopic description is obtained once we have derived a closed set of equations for the variables $M_{i}^{*}$.

Our approach is based on constructing a relevant invariant manifold in phase space $\mathscr{F}$. This manifold is thought as a finite-parametric set of solutions $\Psi(M)$ to Eqs. (475) which depends on time implicitly via the $n$ variables $M_{i}[\Psi]$. The latter may differ from the macroscopic variables $M_{i}^{*}$. For systems with external fluxes (475), we assume that the invariant manifold depends also on the parameters $\alpha$, and on their time derivatives taken to arbitrary order: $\Psi(M, \mathscr{A})$, where $\mathscr{A}=\left\{\alpha, \alpha^{(1)}, \ldots\right\}$ is the set of time derivatives $\alpha^{(k)}=\mathrm{d}^{k} \alpha / \mathrm{d} t^{k}$. It is convenient to consider time derivatives of $\alpha$ as independent parameters. This assumption is important because then we do not need an explicit form of the Eqs. (476) in the course of construction of the invariant manifold. 
By a definition, the dynamic invariance postulates the equality of the "macroscopic" and the "microscopic" time derivatives:

$$
J \Psi(M, \mathscr{A})=\sum_{i=1}^{n} \frac{\partial \Psi(M, \mathscr{A})}{\partial M_{i}} \frac{\mathrm{d} M_{i}}{\mathrm{~d} t}+\sum_{n=0}^{\infty} \sum_{j=1}^{k} \frac{\partial \Psi(M, \mathscr{A})}{\partial \alpha_{j}^{(n)}} \alpha_{j}^{(n+1)},
$$

where $J=J_{\mathrm{d}}+J_{\mathrm{ex}}(\alpha)$. The time derivatives of the macroscopic variables, $\mathrm{d} M_{i} / \mathrm{d} t$, are calculated as follows:

$$
\frac{\mathrm{d} M_{i}}{\mathrm{~d} t}=D M_{i}[J \Psi(M, \mathscr{A})],
$$

where $D M_{i}$ stands for differentials of the functionals $M_{i}$.

Let us introduce the projector operator associated with the parameterization of the manifold $\Psi(M, \mathscr{A})$ by the values of the functionals $M_{i}[\Psi]$ :

$$
P_{M}=\sum_{i=1}^{n} \frac{\partial \Psi(M, \mathscr{A})}{\partial M_{i}} D M_{i}[\bullet] .
$$

It projects vector fields from the phase space $\mathscr{F}$ onto tangent bundle $T \Psi(M, \mathscr{A})$ of the manifold $\Psi(M, \mathscr{A})$. Then Eq. (477) is rewritten as the invariance equation:

$$
\left(1-P_{M}\right) J \Psi(M, \mathscr{A})=\sum_{n=0}^{\infty} \sum_{j=1}^{k} \frac{\partial \Psi}{\partial \alpha_{j}^{(n)}} \alpha_{j}^{(n+1)},
$$

which has the invariant manifolds as its solutions.

Furthermore, we assume the following: (i) The external flux $J_{\text {ex }}(\alpha) \Psi$ is small in comparison to the dissipative part $J_{\mathrm{d}} \Psi$, i.e. with respect to some norm we require: $\left|J_{\mathrm{ex}}(\alpha) \Psi\right| \ll\left|J_{\mathrm{d}} \Psi\right|$. This allows us to introduce a small parameter $\varepsilon_{1}$, and to replace the operator $J_{\text {ex }}$ with $\varepsilon_{1} J_{\text {ex }}$ in Eq. (475). Parameter $\varepsilon_{1}$ is proportional to the characteristic value of the external variables $\alpha$. (ii) The characteristic time $\theta_{\alpha}$ of the variation of the external fields $\alpha$ is large in comparison to the characteristic relaxation time $\theta_{\mathrm{r}}$, and the second small parameter is $\varepsilon_{2}=\theta_{\mathrm{r}} / \theta_{\alpha} \ll 1$. The parameter $\varepsilon_{2}$ does not enter the vector field $J$ explicitly but it shows up in the invariance equation. Indeed, with a substitution, $\alpha^{(i)} \rightarrow \varepsilon_{2}^{i} \alpha^{(i)}$, the invariance equation (477) is rewritten in a form which incorporates both the parameters $\varepsilon_{1}$ and $\varepsilon_{2}$ :

$$
\left(1-P_{M}\right)\left\{J_{\mathrm{d}}+\varepsilon_{1} J_{\mathrm{ex}}\right\} \Psi=\varepsilon_{2} \sum_{i} \sum_{j=1}^{k} \frac{\partial \Psi}{\partial \alpha_{j}^{(i)}} \alpha_{j}^{(i+1)} .
$$

We develop a modified Newton scheme for solution of this equation. Let us assume that we have some initial approximation to desired manifold $\Psi_{(0)}$. We seek the correction of the form $\Psi_{(1)}=\Psi_{(0)}+\Psi_{1}$. Substituting this expression into Eq. (481), we derive

$$
\begin{aligned}
& \left(1-P_{M}^{(0)}\right)\left\{J_{\mathrm{d}}+\varepsilon_{1} J_{\mathrm{ex}}\right\} \Psi_{1}-\varepsilon_{2} \sum_{i} \sum_{j=1}^{k} \frac{\partial \Psi_{1}}{\partial \alpha_{j}^{(i)}} \alpha_{j}^{(i+1)} \\
& =-\left(1-P_{M}^{(0)}\right) J \Psi_{(0)}+\varepsilon_{2} \sum_{i} \sum_{j=1}^{k} \frac{\partial \Psi_{(0)}}{\partial \alpha_{j}^{(i)}} \alpha_{j}^{(i+1)} .
\end{aligned}
$$


Here $P_{M}^{(0)}$ is a projector onto tangent bundle of the manifold $\Psi_{(0)}$. Further, we neglect two terms in the left hand side of this equation, which are multiplied by parameters $\varepsilon_{1}$ and $\varepsilon_{2}$, regarding them small in comparison to the first term. In the result we arrive at the equation,

$$
\left(1-P_{M}^{(0)}\right) J_{\mathrm{d}} \Psi_{1}=-\left(1-P_{M}^{(0)}\right) J \Psi_{(0)}+\varepsilon_{2} \sum_{i} \sum_{j=1}^{k} \frac{\partial \Psi_{(0)}}{\partial \alpha_{j}^{(i)}} \alpha_{j}^{(i+1)} .
$$

For $(n+1)$ th iteration we obtain

$$
\left(1-P_{M}^{(n)}\right) J_{\mathrm{d}} \Psi_{n+1}=-\left(1-P_{M}^{(0)}\right) J \Psi_{(n)}+\varepsilon_{2} \sum_{i} \sum_{j=1}^{k} \frac{\partial \Psi_{(n)}}{\partial \alpha_{j}^{(i)}} \alpha_{j}^{(i+1)},
$$

where $\Psi_{(n)}=\sum_{i=0}^{n} \Psi_{i}$ is the approximation of $n$th order and $P_{M}^{(n)}$ is the projector onto its tangent bundle.

It should be noted that deriving Eqs. (483) and (484) we have not varied the projector $P_{M}$ with respect to yet unknown term $\Psi_{n+1}$, i.e. we have kept $P_{M}=P_{M}^{(n)}$ and have neglected the contribution from the term $\Psi_{n+1}$. The motivation for this action comes from the original paper [6], where it was shown that such modification generates iteration schemes properly converging to slow invariant manifold.

In order to gain the solvability of Eq. (484) an additional condition is required:

$$
P_{M}^{(n)} \Psi_{n+1}=0
$$

This condition is sufficient to provide the existence of the solution to linear system (484), while the additional restriction onto the choice of the projector is required in order to guarantee the uniqueness of the solution. This condition is

$$
\operatorname{ker}\left[\left(1-P_{M}^{(n)}\right) J_{\mathrm{d}}\right] \cap \operatorname{ker} P_{M}^{(n)}=\mathbf{0} .
$$

Here ker denotes a null space of the corresponding operator. How this condition can be met is discussed in the next subsection.

It is natural to begin the iteration procedure (484) starting from the invariant manifold of the nondriven system. In other words, we choose the initial approximation $\Psi_{(0)}$ as the solution of the invariance equation (481) corresponding to $\varepsilon_{1}=0$ and $\varepsilon_{2}=0$ :

$$
\left(1-P_{M}^{(0)}\right) J_{\mathrm{d}} \Psi_{(0)}=0 \text {. }
$$

We shall return to the question how to construct solutions to this equation in the subsection "Linear zero-order equations".

The above recurrent equations (484), (485) present the simplified Newton method for the solution of invariance equation (481), which involves the small parameters. A similar procedure for the Grad equations of the Boltzmann kinetic theory was used recently in the Ref. [9]. When these parameters are not small, one should proceed directly with Eqs. (482).

Above, we have focused our attention on how to organize the iterations to construct invariant manifolds of weakly driven systems. The only question we have not yet answered is how to choose projectors in iterative equations in a consistent way. In the next subsection we discuss the problem of derivation of the reduced dynamics and its relation to the problem of the choice of projector. 
Projector and reduced dynamics. Below we suggest the projector which is equally applicable for constructing invariant manifolds by the iteration method (484), (485) and for generating macroscopic equations based on given manifold.

Let us discuss the problem of constructing closed equations for macroparameters. Having some approximation to the invariant manifold, we nevertheless deal with a noninvariant manifold and we face the problem how to construct the dynamics on it. If the $n$-dimensional manifold $\widetilde{\Psi}$ is found the macroscopic dynamics is induced by any projector $P$ onto the tangent bundle of $\widetilde{\Psi}$ as follows [6]:

$$
\frac{\mathrm{d} M_{i}^{*}}{\mathrm{~d} t}=\left.D M_{i}^{*}\right|_{\tilde{\Psi}}[P J \widetilde{\Psi}] \text {. }
$$

To specify the projector we involve the two above mentioned principles: dissipativity and slowness. The dissipativity is required to have the unique and stable equilibrium solution for macroscopic equations, when the external fields are absent $(\alpha=0)$. The slowness condition requires the induced vector field $P J \Psi$ to match the slow modes of the original vector field $J \Psi$.

Let us consider the parameterization of the manifold $\widetilde{\Psi}(M)$ by the parameters $M_{i}[\Psi]$. This parameterization generates associated projector $P=P_{M}$ by Eq. (479). This leads us to look for the admissible parameterization of this manifold, where by admissibility we understand the concordance with the dissipativity and the slowness requirements. We solve the problem of the admissible parameterization in the following way. Let us define the functionals $M_{i} i=1, \ldots, n$ by the set of the lowest eigenvectors $\varphi_{i}$ of the operator $J_{\mathrm{d}}$ :

$$
M_{i}[\widetilde{\Psi}]=\left\langle\varphi_{i}, \widetilde{\Psi}\right\rangle_{\mathrm{s}}
$$

where $J_{\mathrm{d}} \varphi_{i}=\lambda_{i} \varphi_{i}$. The lowest eigenvectors $\varphi_{1}, \ldots, \varphi_{n}$ are taken as a join of basises in the eigenspaces of the eigenvalues with smallest absolute values: $0<\left|\lambda_{1}\right| \leqslant\left|\lambda_{2}\right| \leqslant \cdots \leqslant\left|\lambda_{n}\right|$. For simplicity we shall work with the orthonormal set of eigenvectors: $\left\langle\varphi_{i}, \varphi_{j}\right\rangle_{\mathrm{s}}=\delta_{i j}$ with $\delta_{i j}$ the Kronecker symbol. Since the function $\Psi_{\text {eq }}$ is the eigenvector of the zero eigenvalue we have: $M_{i}\left[\Psi_{\text {eq }}\right]=\left\langle\varphi_{i}, \Psi_{\text {eq }}\right\rangle_{\mathrm{s}}=0$.

Then the associated projector $P_{M}$, written as

$$
P_{M}=\sum_{i=1}^{n} \frac{\partial \widetilde{\Psi}}{\partial M_{i}}\left\langle\varphi_{i}, \bullet\right\rangle_{\mathrm{s}},
$$

will generate the equations in terms of the parameters $M_{i}$ as follows:

$$
\mathrm{d} M_{i} / \mathrm{d} t=\left\langle\varphi_{i} P_{M} J \widetilde{\Psi}\right\rangle_{\mathrm{s}}=\left\langle\varphi_{i} J \widetilde{\Psi}\right\rangle_{\mathrm{s}}
$$

Their explicit form is

$$
\frac{\mathrm{d} M_{i}}{\mathrm{~d} t}=\lambda_{i} M_{i}+\left\langle J_{\mathrm{ex}}^{+}(\alpha) g_{i}, \widetilde{\Psi}(M)\right\rangle_{\mathrm{s}},
$$

where the $J_{\mathrm{ex}}^{+}$is the adjoint to operator $J_{\mathrm{ex}}$ with respect to the scalar product $\langle\bullet, \bullet\rangle_{\mathrm{s}}$.

Apparently, in the absence of forcing $(\alpha \equiv 0)$ the macroscopic equations $\mathrm{d} M_{i} / \mathrm{d} t=\lambda_{i} M_{i}$ are thermodynamically stable. They represent the dynamics of slowest eigenmodes of equations $\mathrm{d} \Psi / \mathrm{d} t=J_{\mathrm{d}} \Psi$. Thus, projector (489) complies with the above stated requirements of dissipativity and slowness in the absence external flux.

To rewrite the macroscopic equations (490) in terms of the required set of macroparameters, $M_{i}^{*}[\Psi]=\left\langle m_{i}^{*}, \Psi\right\rangle_{\mathrm{s}}$, we use formula (488) which is equivalent to the change of variables 
$\{M\} \rightarrow\left\{M^{*}(M)\right\}, M_{i}^{*}=\left\langle m_{i}^{*}, \widetilde{\Psi}(M)\right\rangle_{\mathrm{s}}$ in Eqs. (490). Indeed, this is seen from the relation:

$$
\left.D M_{i}^{*}\right|_{\tilde{\Psi}}\left[P_{M} J \widetilde{\Psi}\right]=\left.\sum_{j} \frac{\partial M_{i}^{*}}{\partial M_{j}} D M_{j}\right|_{\tilde{\Psi}}[J \widetilde{\Psi}] .
$$

We have constructed the dynamics with the help of the projector $P_{M}$ associated with the lowest eigenvectors of the operator $J_{\mathrm{d}}$. It is directly verified that such projector (489) fulfills condition (485) for arbitrary manifold $\Psi_{(n)}=\widetilde{\Psi}$. For this reason it is natural to use the projector (489) for both procedures, constructing the invariant manifold, and deriving the macroscopic equations.

We have to note that the above described approach to defining the dynamics via the projector is different from the concept of "thermodynamic parameterization" proposed in Refs. [6,5]. The latter was applicable for arbitrary dissipative systems including nonlinear ones, whereas the present derivations are applied solely for linear systems.

Linear zero-order equations. In this section we focus our attention on the solution of the zero-order invariance equation (487). We seek the linear invariant manifold of the form

$$
\Psi_{(0)}(a)=\Psi_{\mathrm{eq}}+\sum_{i=1}^{n} a_{i} m_{i}
$$

where $a_{i}$ are coordinates on this manifold. This manifold can be considered as an expansion of the relevant slow manifold near the equilibrium state. This limits the domain of validity of manifolds (491) because they are not generally positively definite. This remark indicates that nonlinear invariant manifolds should be considered for large deviations from the equilibrium but this goes beyond the scope of this Example.

The linear $n$-dimensional manifold representing the slow motion for the linear dissipative system (475) is associated with $n$ slowest eigenmodes. This manifold should be built up as the linear hull of the eigenvectors $\varphi_{i}$ of the operator $J_{\mathrm{d}}$, corresponding to the lower part of its spectrum. Thus we choose $m_{i}=\varphi_{i}$.

Dynamic equations for the macroscopic variables $M^{*}$ are derived in two steps. First, following the subsection, "Projector and reduced dynamics", we parameterize the linear manifold $\Psi_{(0)}$ with the values of the moments $M_{i}[\Psi]=\left\langle\varphi_{i}, \Psi\right\rangle_{\mathrm{s}}$. We obtain that the parameterization of manifold (491) is given by $a_{i}=M_{i}$, or:

$$
\Psi_{(0)}(M)=\Psi_{\mathrm{eq}}+\sum_{i=1}^{n} M_{i} \varphi_{i}
$$

Then the reduced dynamics in terms of variables $M_{i}$ reads

$$
\frac{\mathrm{d} M_{i}}{\mathrm{~d} t}=\lambda_{i} M_{i}+\sum_{j}\left\langle J_{\mathrm{ex}}^{+} \varphi_{i}, \varphi_{j}\right\rangle_{\mathrm{s}} M_{j}+\left\langle J_{\mathrm{ex}}^{+} \varphi_{i}, \Psi_{\mathrm{eq}}\right\rangle_{\mathrm{s}},
$$

where $\lambda_{i}=\left\langle\varphi_{i}, J_{\mathrm{d}} \varphi_{i}\right\rangle_{\mathrm{s}}$ are eigenvalues which correspond to eigenfunctions $\varphi_{i}$. 
Second, we switch from the variables $M_{i}$ to the variables $M_{i}^{*}(M)=\left\langle m_{i}^{*}, \Psi_{(0)}(M)\right\rangle_{\mathrm{s}}$ in Eq. (492). Resulting equations for the variables $M^{*}$ are also linear:

$$
\begin{aligned}
\frac{\mathrm{d} M_{i}^{*}}{\mathrm{~d} t}= & \sum_{j k l}\left(B^{-1}\right)_{i j} \Lambda_{j k} B_{k l} \Delta M_{l}^{*}+\sum_{j k}\left(B^{-1}\right)_{i j}\left\langle J_{\mathrm{ex}}^{+} \varphi_{j}, \varphi_{k}\right\rangle_{\mathrm{s}} \Delta M_{k}^{*} \\
& +\sum_{j}\left(B^{-1}\right)_{i j}\left\langle J_{\mathrm{ex}}^{+} \varphi_{j}, \Psi_{\mathrm{eq}}\right\rangle_{\mathrm{s}} .
\end{aligned}
$$

Here $\Delta M_{i}^{*}=M_{i}^{*}-M_{\mathrm{eq} \mid i}^{*}$ is the deviation of the variable $M_{i}^{*}$ from its equilibrium value $M_{\mathrm{eq} \mid i}^{*}$, and $B_{i j}=\left\langle m_{i}^{*}, \varphi_{j}\right\rangle$ and $\Lambda_{i j}=\lambda_{i} \delta_{i j}$.

Auxiliary formulas: 1. Approximations to eigenfunctions of the Fokker-Planck operator

In this subsection we discuss the question how to find the lowest eigenvectors $\Psi_{\text {eq }} m_{0}\left(Q^{2}\right)$ and $\Psi_{\mathrm{eq}}\left(Q^{2}\right) \mathbf{Q Q}$ of the operator $J_{\mathrm{d}}(469)$ in the classes of functions having a form: $w_{0}(Q)$ and $w_{1}(Q) \mathbf{Q}$. The results presented in this subsection were used in the subsections: "Constitutive equations" and "Tests on the FENE dumbbell model". It is directly verified that

$$
\begin{aligned}
& J_{\mathrm{d}} w_{0}=G_{0}^{\mathrm{h}} w_{0}, \\
& J_{\mathrm{d}} w_{1} \mathbf{Q} \mathbf{Q}=\left(G_{1}^{\mathrm{h}} w_{1}\right) \mathbf{Q} \mathbf{Q},
\end{aligned}
$$

where the operators $G_{0}^{\mathrm{h}}$ and $G_{1}^{\mathrm{h}}$ are given by

$$
G_{0}^{\mathrm{h}}=G_{0}-\kappa H_{0}, \quad G_{1}^{\mathrm{h}}=G_{1}-\kappa H_{1} .
$$

The operators $G_{0,1}$ and $H_{0,1}$ act in the space of isotropic functions (i.e. dependent only on $\left.Q=(\boldsymbol{Q} \cdot \boldsymbol{Q})^{1 / 2}\right)$ as follows:

$$
\begin{aligned}
G_{0} & =\frac{1}{2}\left(\frac{\partial^{2}}{\partial Q^{2}}-f Q \frac{\partial}{\partial Q}+\frac{2}{Q} \frac{\partial}{\partial Q}\right), \\
G_{1} & =\frac{1}{2}\left(\frac{\partial^{2}}{\partial Q^{2}}-f Q \frac{\partial}{\partial Q}+\frac{6}{Q} \frac{\partial}{\partial Q}-2 f\right), \\
H_{0} & =\frac{2}{Q}\left(\frac{\partial^{2}}{\partial Q^{2}}-f Q \frac{\partial}{\partial Q}+\frac{2}{Q} \frac{\partial}{\partial Q}\right), \\
H_{1} & =\frac{2}{Q}\left(\frac{\partial^{2}}{\partial Q^{2}}-f Q \frac{\partial}{\partial Q}+\frac{5}{Q} \frac{\partial}{\partial Q}-2 f+\frac{1}{Q^{2}}\right) .
\end{aligned}
$$

The following two properties of the operators $G_{0,1}^{\mathrm{h}}$ are important for our analysis: Let us define two scalar products $\langle\bullet, \bullet\rangle_{0}$ and $\langle\bullet, \bullet\rangle_{1}$ :

$$
\begin{aligned}
& \langle y, x\rangle_{0}=\langle x y\rangle_{\mathrm{e}}, \\
& \langle y, x\rangle_{1}=\left\langle x y Q^{4}\right\rangle_{\mathrm{e}} .
\end{aligned}
$$


Here $\langle\bullet\rangle_{\mathrm{e}}$ is the equilibrium average as defined in (511). Then we state that for sufficiently small $\kappa$ the operators $G_{0}^{\mathrm{h}}$ and $G_{1}^{\mathrm{h}}$ are symmetric and nonpositive in the scalar products $\langle\bullet, \bullet\rangle_{0}$ and $\langle\bullet, \bullet\rangle_{1}$, respectively. Thus for obtaining the desired eigenvectors of the operator $J_{\mathrm{d}}$ we need to find the eigenfunctions $m_{0}$ and $m_{1}$ related to the lowest nonzero eigenvalues of the operators $G_{0,1}^{\mathrm{h}}$.

Since we regard the parameter $\kappa$ small it is convenient, first, to find lowest eigenfunctions $g_{0,1}$ of the operators $G_{0,1}$ and, then, to use standard perturbation technique in order to obtain $m_{0,1}$. For the perturbation of the first order one finds [262]:

$$
\begin{aligned}
& m_{0}=g_{0}+\kappa h_{0}, \quad h_{0}=-g_{0} \frac{\left\langle g_{0} H_{0} G_{0} g_{0}\right\rangle_{0}}{\left\langle g_{0}, g_{0}\right\rangle_{0}}-G_{0} H_{0} g_{0} ; \\
& m_{1}=g_{1}+\kappa h_{1}, \quad h_{1}=-g_{1} \frac{\left\langle g_{1} H_{1} G_{1} g_{1}\right\rangle_{1}}{\left\langle g_{1}, g_{1}\right\rangle_{1}}-G_{1} H_{1} g_{1} .
\end{aligned}
$$

For the rest of this subsection we describe one recurrent procedure for obtaining the functions $m_{0}$ and $m_{1}$ in a constructive way. Let us solve this problem by minimizing the functionals $\boldsymbol{\Lambda}_{0,1}$ :

$$
\Lambda_{0,1}\left[m_{0,1}\right]=-\frac{\left\langle m_{0,1}, G_{0,1}^{\mathrm{h}} m_{0,1}\right\rangle_{0,1}}{\left\langle m_{0,1}, m_{0,1}\right\rangle_{0,1}} \rightarrow \min ,
$$

by means of the gradient descent method.

Let us denote $e_{0,1}$ the eigenfunctions of the zero eigenvalues of the operators $G_{0,1}^{\mathrm{h}}$. Their explicit values are $e_{0}=1$ and $e_{1}=0$. Let the initial approximations $m_{0,1}^{(0)}$ to the lowest eigenfunctions $m_{0,1}$ be chosen so that $\left\langle m_{0,1}^{(0)}, e_{0,1}\right\rangle_{0,1}=0$. We define the variation derivative $\delta \Lambda_{0,1} / \delta m_{0,1}$ and look for the correction in the form

$$
m_{0,1}^{(1)}=m_{0,1}^{(0)}+\delta m_{0,1}^{(0)}, \quad \delta m_{0,1}^{(0)}=\alpha \frac{\delta \Lambda_{0,1}}{\delta m_{0,1}},
$$

where scalar parameter $\alpha<0$ is found from the condition:

$$
\frac{\partial \Lambda_{0,1}\left[m_{0,1}^{(1)}(\alpha)\right]}{\partial \alpha}=0 .
$$

In the explicit form the result reads:

$$
\delta m_{0,1}^{(0)}=\alpha_{0,1}^{(0)} \Delta_{0,1}^{(0)}
$$

where

$$
\begin{aligned}
& \Delta_{0,1}^{(0)}=\frac{2}{\left\langle m_{0,1}^{(0)}, m_{0,1}^{(0)}\right\rangle_{0,1}}\left(m_{0,1}^{(0)} \lambda_{0,1}^{(0)}-G_{0,1}^{\mathrm{h}} m_{0,1}^{(0)}\right), \\
& \lambda_{0,1}^{(0)}=\frac{\left\langle m_{0,1}^{(0)}, G_{0,1}^{\mathrm{h}} m_{0,1}^{(0)}\right\rangle_{0,1}}{\left\langle m_{0,1}^{(0)}, m_{0,1}^{(0)}\right\rangle_{0,1}},
\end{aligned}
$$




$$
\begin{aligned}
& \alpha_{0,1}^{(0)}=q_{0,1}-\sqrt{q_{0,1}^{2}+\frac{\left\langle m_{0,1}^{(0)}, m_{0,1}^{(0)}\right\rangle_{0,1}}{\left\langle\Delta_{0,1}^{(0)}, \Delta_{0,1}^{(0)}\right\rangle_{0,1}}}, \\
& q_{0,1}=\frac{1}{\left\langle\Delta_{0,1}^{(0)}, \Delta_{0,1}^{(0)}\right\rangle_{0,1}}\left(\frac{\left\langle m_{0,1}^{(0)}, G_{0,1}^{\mathrm{h}} m_{0,1}^{(0)}\right\rangle_{0,1}}{\left\langle m_{0,1}^{(0)}, m_{0,1}^{(0)}\right\rangle_{0,1}}-\frac{\left\langle\Delta_{0,1}^{(0)}, G_{0,1}^{\mathrm{h}} \Delta_{0,1}^{(0)}\right\rangle_{0,1}}{\left\langle\Delta_{0,1}^{(0)}, \Delta_{0,1}^{(0)}\right\rangle_{0,1}}\right) .
\end{aligned}
$$

Having the new correction $m_{0,1}^{(1)}$ we can repeat the procedure and eventually generate the recurrence scheme. Since by the construction all iterative approximations $m_{0,1}^{(n)}$ remain orthogonal to zero eigenfunctions $e_{0,1}:\left\langle m_{0,1}^{(n)}, e_{0,1}\right\rangle_{0,1}=0$ we avoid the convergence of this recurrence procedure to the eigenfunctions $e_{0,1}$.

The quantities $\delta_{0,1}^{(n)}$ :

$$
\delta_{0,1}^{(n)}=\frac{\left\langle\Delta_{0,1}^{(n)}, \Delta_{0,1}^{(n)}\right\rangle_{0,1}}{\left\langle m_{0,1}^{(n)}, m_{0,1}^{(n)}\right\rangle_{0,1}}
$$

can serve as relative error parameters for controlling the convergence of the iteration procedure (501).

Auxiliary formulas: 2. Integral relations

Let $\Omega$ be a sphere in $\mathbf{R}^{3}$ with the center at the origin of the coordinate system or be the entire space $\mathbf{R}^{3}$. For any function $s\left(x^{2}\right)$, where $x^{2}=\boldsymbol{x} \cdot \boldsymbol{x}, \boldsymbol{x} \in \mathbf{R}^{3}$, and any square $3 \times 3$ matrices $\mathbf{A}, \boldsymbol{B}, \boldsymbol{C}$ independent of $\boldsymbol{x}$ the following integral relations are valid:

$$
\begin{aligned}
& \int_{\Omega} s\left(x^{2}\right) \mathbf{x} \mathbf{x}(\mathbf{x} \mathbf{x}: \mathbf{A}) \mathrm{d} \boldsymbol{x}=\frac{2}{15} \stackrel{\circ}{\mathbf{A}} \int_{\Omega} s x^{4} \mathrm{~d} \boldsymbol{x} ; \\
& \int_{\Omega} s\left(x^{2}\right) \mathbf{x} \mathbf{x}(\stackrel{\circ}{\mathbf{x}}: \mathbf{A})(\stackrel{\circ}{\mathbf{x}}: \boldsymbol{B}) \mathrm{d} \boldsymbol{x}=\frac{4}{105}(\mathbf{A} \cdot \boldsymbol{B} \stackrel{\circ}{+} \boldsymbol{B} \cdot \mathbf{A}) \int_{\Omega} s x^{6} \mathrm{~d} \boldsymbol{x} ; \\
& \int_{\Omega} s\left(x^{2}\right) \stackrel{\circ}{\mathbf{x}}(\mathbf{x} \mathbf{x}: \mathbf{A})(\mathbf{x} \mathbf{x}: \boldsymbol{B})(\mathbf{x} \mathbf{0}: \boldsymbol{C}) \mathrm{d} \boldsymbol{x} \\
& =\frac{4}{315}\{\stackrel{\circ}{\mathbf{A}}(\boldsymbol{B}: \boldsymbol{C})+\stackrel{\circ}{\mathbf{B}}(\mathbf{A}: \boldsymbol{C})+\stackrel{\circ}{\mathbf{C}}(\mathbf{A}: \boldsymbol{B})\} \int_{\Omega} s x^{8} \mathrm{~d} \boldsymbol{x} .
\end{aligned}
$$

Microscopic derivation of constitutive equations

Iteration scheme. In this section we apply the above developed formalism to the elastic dumbbell model (461). External field variables $\alpha$ are the components of the tensor $\widehat{\mathbf{k}}$.

Since we aim at constructing a closed description for the stress tensor $\tau$ (462) with the six independent components, the relevant manifold in our problem should be six-dimensional. Moreover, we allow a dependence of the manifold on the material derivatives of the tensor $\widehat{\mathbf{k}}: \widehat{\mathbf{k}}^{(i)}=D^{i} \boldsymbol{k} / D t^{i}$. Let $\Psi^{*}(M, \mathscr{K}) \mathscr{K}=\left\{\widehat{\mathbf{k}}, \widehat{\mathbf{k}}^{(1)}, \ldots\right\}$ be the desired manifold parameterized by the six variables $M_{i}$ $i=1, \ldots, 6$ and the independent components (maximum eight for each $\widehat{\mathbf{k}}^{(l)}$ ) of the tensors $\widehat{\mathbf{k}}^{(l)}$. Small 
parameters $\varepsilon_{1}$ and $\varepsilon_{2}$, introduced in the section: "The problem of reduced description in polymer dynamics", are established by Eq. (474). Then we define the invariance equation:

$$
\left(1-P_{M}\right)\left(J_{\mathrm{d}}+\varepsilon_{1} J_{\mathrm{h}}\right) \Psi=\varepsilon_{2} \sum_{i=0}^{\infty} \sum_{l m} \frac{\partial \Psi}{\partial \widehat{h}_{l m}^{(i)}} \widehat{h}_{l m}^{(i+1)},
$$

where $P_{M}=\left(\partial \Psi / \partial M_{i}\right) D M_{i}[\bullet]$ is the projector associated with chosen parameterization and summation indexes $l, m$ run only eight independent components of tensor $\widehat{\mathbf{k}}$.

Following the further procedure we straightforwardly obtain the recurrent equations:

$$
\begin{aligned}
& \left(1-P_{M}^{(n)}\right) J_{\mathrm{d}} \Psi_{n+1}=-\left(1-P_{M}^{(n)}\right)\left[J_{\mathrm{d}}+\varepsilon_{1} J_{\mathrm{h}}\right] \Psi_{(n)}+\varepsilon_{2} \sum_{i} \sum_{l m} \frac{\partial \Psi_{(n)}}{\partial \widehat{k}_{l m}^{(i)}} \widehat{k}_{l m}^{(i+1)}, \\
& P_{M}^{(n)} \Psi_{n+1}=0,
\end{aligned}
$$

where $\Psi_{n+1}$ is the correction to the manifold $\Psi_{(n)}=\sum_{i=0}^{n} \Psi_{i}$.

The zero-order manifold is found as the relevant solution to equation:

$$
\left(1-P_{M}^{(0)}\right) J_{\mathrm{d}} \Psi_{(0)}=0 \text {. }
$$

We construct zero-order manifold $\Psi_{(0)}$ in the subsection, "Zero-order constitutive equation".

The dynamics in general form. Let us assume that some approximation to invariant manifold $\widetilde{\Psi}(a, \mathscr{K})$ is found (here $a=\left\{a_{1}, \ldots, a_{6}\right\}$ are some coordinates on this manifold). The next step is constructing the macroscopic dynamic equations.

In order to comply with dissipativity and slowness by means of the recipe from the previous section we need to find six lowest eigenvectors of the operator $J_{\mathrm{d}}$. We shall always assume in a sequel that the hydrodynamic interaction parameter $\kappa$ is small enough that the dissipativity of $J_{\mathrm{d}}(471)$ is not violated.

Let us consider two classes of functions: $\mathscr{C}_{1}=\left\{w_{0}\left(Q^{2}\right)\right\}$ and $\mathscr{C}_{2}=\left\{w_{1}\left(Q^{2}\right) \mathbf{Q Q}\right\}$, where $w_{0,1}$ are functions of $Q^{2}$ and the notation $\circ$ indicates traceless parts of tensor or matrix, e.g. for the dyad $Q Q$ :

$$
(\mathbf{Q} \mathbf{Q})_{i j}=Q_{i} Q_{j}-\frac{1}{3} \delta_{i j} Q^{2}
$$

Since the sets $\mathscr{C}_{1}$ and $\mathscr{C}_{2}$ are invariant with respect to operator $J_{\mathrm{d}}$, i.e. $J_{\mathrm{d}} \mathscr{C}_{1} \subset \mathscr{C}_{1}$ and $J_{\mathrm{d}} \mathscr{C}_{2} \subset \mathscr{C}_{2}$, and densities $\boldsymbol{F} \boldsymbol{Q}=f \mathbf{Q} \mathbf{Q}+(1 / 3) \mathbf{1} f Q^{2}$ of the moments comprising the stress tensor $\tau_{p}(463)$ belong to the space $\mathscr{C}_{1}+\mathscr{C}_{2}$, we shall seek the desired eigenvectors in the classes $\mathscr{C}_{1}$ and $\mathscr{C}_{2}$. Namely, we intend to find one lowest isotropic eigenvector $\Psi_{\text {eq }} m_{0}\left(Q^{2}\right)$ of eigenvalue $-\lambda_{0}\left(\lambda_{0}>0\right)$ and five nonisotropic eigenvectors $m_{i j}=\Psi_{\text {eq }} m_{1}\left(Q^{2}\right)(\mathbf{Q Q})_{i j}$ of another eigenvalue $-\lambda_{1}\left(\lambda_{1}>0\right)$. The method of derivation and analytic evaluation of these eigenvalues are discussed in the subsection "Auxiliary formulas, 1". For a while we assume that these eigenvectors are known.

In the next step we parameterize given manifold $\widetilde{\Psi}$ by the values of the functionals:

$$
\begin{aligned}
& M_{0}=\left\langle\Psi_{\mathrm{eq}} m_{0}, \widetilde{\Psi}\right\rangle_{\mathrm{s}}=\int m_{0} \widetilde{\Psi} \mathrm{d} \boldsymbol{Q}, \\
& \stackrel{\circ}{\mathbf{M}}=\left\langle\Psi_{\mathrm{eq}} m_{1} \stackrel{\circ}{\mathbf{Q}}, \widetilde{\Psi}\right\rangle_{\mathrm{s}}=\int m_{1} \mathbf{Q} \mathbf{Q} \widetilde{\Psi} \mathrm{d} \boldsymbol{Q} .
\end{aligned}
$$


Once a desired parameterization $\widetilde{\Psi}\left(M_{0}, \stackrel{\circ}{\mathbf{M}}, \mathscr{K}\right)$ is obtained, the dynamic equations are found as

$$
\begin{aligned}
& \frac{D M_{0}}{D \widehat{t}}+\lambda_{0} M_{0}=\left\langle(\hat{\dot{\gamma}}: \mathbf{Q} \mathbf{Q}) m_{0}^{\prime}\right\rangle, \\
& \stackrel{\circ}{\mathbf{M}_{[1]}}+\lambda_{1} \stackrel{\circ}{\mathbf{M}}=-\frac{1}{3} \mathbf{1} \hat{\dot{\gamma}}: \stackrel{\circ}{\mathbf{M}}-\frac{1}{3} \hat{\dot{\gamma}}\left\langle m_{1} Q^{2}\right\rangle+\left\langle\mathbf{Q} \mathbf{Q}(\hat{\dot{\gamma}}: \mathbf{Q} \mathbf{Q}) m_{1}^{\prime}\right\rangle,
\end{aligned}
$$

where all averages are calculated with the df $\widetilde{\Psi}$, i.e. $\langle\bullet\rangle=\int \bullet \widetilde{\Psi} \mathrm{d} \boldsymbol{Q}, m_{0,1}^{\prime}=\mathrm{d} m_{0,1}\left(Q^{2}\right) / \mathrm{d}\left(Q^{2}\right)$ and subscript [1] represents the upper convective derivative of tensor:

$$
\Lambda_{[1]}=\frac{D \boldsymbol{\Lambda}}{D \widehat{t}}-\left\{\widehat{\mathbf{k}} \cdot \boldsymbol{\Lambda}+\boldsymbol{\Lambda} \cdot \widehat{\mathbf{k}}^{\dagger}\right\}
$$

The parameters $\lambda_{0,1}$, which are absolute values of eigenvalues of operator $J_{\mathrm{d}}$, are calculated by formulas (for definition of operators $G_{1}$ and $G_{2}$ see subsection "Auxiliary formulas, 1"):

$$
\begin{aligned}
& \lambda_{0}=-\frac{\left\langle m_{0} G_{0} m_{0}\right\rangle_{\mathrm{e}}}{\left\langle m_{0} m_{0}\right\rangle_{\mathrm{e}}}>0, \\
& \lambda_{1}=-\frac{\left\langle Q^{4} m_{1} G_{1} m_{1}\right\rangle_{\mathrm{e}}}{\left\langle m_{1} m_{1} Q^{4}\right\rangle_{\mathrm{e}}}>0,
\end{aligned}
$$

where we have introduced the notation of the equilibrium average:

$$
\langle y\rangle_{\mathrm{e}}=\int \Psi_{\text {eq }} y \mathrm{~d} \boldsymbol{Q} .
$$

Equations on components of the polymeric stress tensor $\tau_{\mathrm{p}}(463)$ are constructed as a change of variables $\left\{M_{0}, \stackrel{\circ}{\mathbf{M}}\right\} \rightarrow \tau_{\mathrm{p}}$. The use of the projector $\widetilde{P}$ makes this operation straightforward:

$$
\frac{D \tau_{\mathrm{p}}}{D \widehat{t}}=-n k_{\mathrm{B}} T \int \boldsymbol{F} \boldsymbol{Q} \widetilde{P} J \widetilde{\Psi}\left(M_{0}\left(\tau_{\mathrm{p}}, \mathscr{K}\right), \stackrel{\circ}{\mathbf{M}}\left(\tau_{\mathrm{p}}, \mathscr{K}\right), \mathscr{K}\right) \mathrm{d} \boldsymbol{Q}
$$

Here, the projector $\widetilde{P}$ is associated with the parameterization by the variables $M_{0}$ and $\stackrel{\circ}{\mathbf{M}}$ :

$$
\widetilde{P}=\frac{\partial \widetilde{\Psi}}{\partial M_{0}}\left\langle\Psi_{\mathrm{eq}} m_{0}, \bullet\right\rangle_{\mathrm{s}}+\sum_{k l} \frac{\partial \widetilde{\Psi}}{\partial \dot{\mathbf{M}}_{k l}}\left\langle\Psi_{\mathrm{eq}} m_{1}(\mathbf{Q} \mathbf{Q})_{k l}, \bullet\right\rangle_{\mathrm{s}} .
$$

We note that sometimes it is easier to make transition to the variables $\tau_{\mathrm{p}}$ after solving Eqs. (508) rather than to construct explicitly and solve equations in terms of $\tau_{\mathrm{p}}$. It allows to avoid inverting the functions $\tau_{\mathrm{p}}\left(M_{0}, \dot{\mathbf{M}}\right)$ and to deal with simpler equations.

Zero-order constitutive equation. In this subsection we derive the closure based on the zero-order manifold $\Psi_{(0)}$ found as appropriate solution to Eq. (506). Following the approach described in subsection, "Linear zero-order equations", we construct such a solution as the linear expansion near the equilibrium state $\Psi_{\text {eq }}(491)$. After parameterization by the values of the variables $M_{0}$ and $\stackrel{\circ}{\mathbf{M}}$ 
associated with the eigenvectors $\Psi_{\text {eq }} m_{0}$ and $\Psi_{\text {eq }} m_{1} \mathbf{Q Q}$ we find:

$$
\Psi_{(0)}=\Psi_{\text {eq }}\left(1+M_{0} \frac{m_{0}}{\left\langle m_{0} m_{0}\right\rangle_{\mathrm{e}}}+\frac{15}{2} \stackrel{\circ}{\mathbf{M}}: \mathbf{Q} \mathbf{Q} \frac{m_{1}}{\left\langle m_{1} m_{1} Q^{4}\right\rangle_{\mathrm{e}}}\right) .
$$

Then with the help of projector (513):

$$
P_{M}^{(0)}=\Psi_{\text {eq }}\left\{\frac{m_{0}}{\left\langle m_{0} m_{0}\right\rangle_{\mathrm{e}}}\left\langle m_{0}, \bullet\right\rangle_{\mathrm{e}}+\frac{15}{2} \frac{m_{1}}{\left\langle m_{1} m_{1} Q^{4}\right\rangle_{\mathrm{e}}} \mathbf{Q} \mathbf{Q}:\left\langle m_{1} \mathbf{Q} \mathbf{Q}, \bullet\right\rangle_{\mathrm{e}}\right\}
$$

by formula (512) we obtain

$$
\begin{aligned}
& \frac{D \operatorname{tr} \tau_{\mathrm{p}}}{D \widehat{t}}+\lambda_{0} \operatorname{tr} \tau_{\mathrm{p}}=a_{0}\left(\stackrel{\circ}{\tau}_{\mathrm{p}}: \hat{\dot{\gamma}}\right), \\
& {\stackrel{\circ}{\tau_{p[1]}}}+\lambda_{0} \stackrel{\circ}{\mathrm{\tau}}_{\mathrm{p}}=b_{0}\left[\stackrel{\circ}{\tau}_{\mathrm{p}} \cdot \hat{\gamma}+\hat{\dot{\gamma}} \cdot \stackrel{\circ}{\tau}_{\mathrm{p}}\right]-\frac{1}{3} \mathbf{1}\left(\stackrel{\circ}{\tau}_{\mathrm{p}}: \hat{\dot{\gamma}}\right)+\left(b_{1} \operatorname{tr} \tau_{\mathrm{p}}-b_{2} n k_{\mathrm{B}} T\right) \hat{\dot{\gamma}},
\end{aligned}
$$

where the constants $b_{i}, a_{0}$ are

$$
\begin{aligned}
a_{0} & =\frac{\left\langle f m_{0} Q^{2}\right\rangle_{\mathrm{e}}\left\langle m_{0} m_{1} Q^{4} m_{1}^{\prime}\right\rangle_{\mathrm{e}}}{\left\langle f m_{0} Q^{4}\right\rangle_{\mathrm{e}}\left\langle m_{0}^{2}\right\rangle_{\mathrm{e}}}, \\
b_{0} & =\frac{2}{7} \frac{\left\langle m_{1} m_{2}^{\prime} Q^{6}\right\rangle_{\mathrm{e}}}{\left\langle m_{1}^{2} Q^{4}\right\rangle_{\mathrm{e}}}, \\
b_{1} & =\frac{1}{15} \frac{\left\langle f m_{1} Q^{4}\right\rangle_{\mathrm{e}}}{\left\langle f m_{0} Q^{2}\right\rangle_{\mathrm{e}}}\left\{2 \frac{\left\langle m_{0} m_{2}^{\prime} Q^{4}\right\rangle_{\mathrm{e}}}{\left\langle m_{1}^{2} Q^{4}\right\rangle_{\mathrm{e}}}+5 \frac{\left\langle m_{0} m_{1} Q^{2}\right\rangle_{\mathrm{e}}}{\left\langle m_{1} m_{1} Q^{4}\right\rangle_{\mathrm{e}}}\right\}, \\
b_{2} & =\frac{1}{15} \frac{\left\langle f m_{1} Q^{4}\right\rangle_{\mathrm{e}}}{\left\langle m_{1} m_{1} Q^{4}\right\rangle_{\mathrm{e}}}\left\{2\left\langle m_{2}^{\prime} Q^{4}\right\rangle_{\mathrm{e}}+5\left\langle m_{1} Q^{2}\right\rangle_{\mathrm{e}}\right\} .
\end{aligned}
$$

We remind that $m_{0,1}^{\prime}=\partial m_{0,1} / \partial\left(Q^{2}\right)$. These formulas were obtained using the auxiliary results from subsection "Auxiliary formulas, 2 ".

Revised Oldroyd 8 constant constitutive equation for the stress. It is remarkable that being rewritten in terms of the full stresses $\tau=-v_{\mathrm{s}} \dot{\gamma}+\tau_{\mathrm{p}}$ the dynamic system (516) takes a form:

$$
\begin{aligned}
\tau+ & c_{1} \tau_{[1]}+c_{3}\{\dot{\gamma} \cdot \tau+\tau \cdot \dot{\gamma}\}+c_{5}(\operatorname{tr} \tau) \dot{\gamma}+\mathbf{1}\left(c_{6} \tau: \dot{\gamma}+c_{8} \operatorname{tr} \tau\right) \\
& =-v\left\{\dot{\gamma}+c_{2} \dot{\gamma}_{[1]}+c_{4} \dot{\gamma} \cdot \dot{\gamma}+c_{7}(\dot{\gamma}: \dot{\gamma}) \mathbf{1}\right\},
\end{aligned}
$$

where the parameters $v, c_{i}$ are given by the following relationships:

$$
\begin{aligned}
& v=\lambda_{\mathrm{r}} v_{\mathrm{s}} \mu, \quad \mu=1+n k_{\mathrm{B}} T \lambda_{1} b_{2} / v_{\mathrm{s}}, \\
& c_{1}=\lambda_{\mathrm{r}} / \lambda_{1}, \quad c_{2}=\lambda_{\mathrm{r}} /\left(\mu \lambda_{1}\right), \\
& c_{3}=-b_{0} \lambda_{\mathrm{r}} / \lambda_{0}, \quad c_{4}=-2 b_{0} \lambda_{\mathrm{r}} /\left(\mu \lambda_{1}\right), \\
& c_{5}=\frac{\lambda_{\mathrm{r}}}{3 \lambda_{1}}\left(2 b_{0}-3 b_{1}-1\right), \quad c_{6}=\frac{\lambda_{\mathrm{r}}}{\lambda_{1}}\left(2 b_{0}+1-a_{0}\right), \\
& c_{7}=\frac{\lambda_{\mathrm{r}}}{\lambda_{1} \mu}\left(2 b_{0}+1-a_{0}\right), \quad c_{8}=\frac{1}{3}\left(\lambda_{0} / \lambda_{1}-1\right) .
\end{aligned}
$$


In the last two formulas we returned to the original dimensional quantities: time $t$ and gradient of velocity tensor $\boldsymbol{k}=\nabla \boldsymbol{v}$, and at the same time we kept the old notations for the dimensional convective derivative $\boldsymbol{\Lambda}_{[1]}=D \boldsymbol{\Lambda} / D t-\boldsymbol{k} \cdot \boldsymbol{\Lambda}-\boldsymbol{\Lambda} \cdot \boldsymbol{k}^{\dagger}$.

Note that all parameters (519) are related to the entropic spring law $f$ due to Eq. (517). Thus, the constitutive relation for the stress $\tau$ (518) is fully derived from the microscopic kinetic model.

If the constant $c_{8}$ were equal to zero, then the form of Eq. (518) would be recognized as the Oldroyd 8 constant model [257], proposed by Oldroyd about 40 years ago on a phenomenological basis. Nonzero $c_{8}$ indicates a presence of difference between $\lambda_{\mathrm{r}} / \lambda_{0}$ and $\lambda_{\mathrm{r}} / \lambda_{1}$ which are relaxation times of trace $\operatorname{tr} \tau$ and traceless components $\stackrel{\circ}{\tau}$ of the stress tensor $\tau$.

Higher-order constitutive equations. In this subsection we discuss the properties of corrections to the zero-order model (518). Let $P_{M}^{(0)}(515)$ be the projector onto the zero-order manifold $\Psi_{(0)}(514)$. The invariance equation (504) for the first-order correction $\Psi_{(1)}=\Psi_{(0)}+\Psi_{1}$ takes a form

$$
\begin{aligned}
& L \Psi_{1}=-\left(1-P_{M}^{(0)}\right)\left(J_{\mathrm{d}}+J_{\mathrm{h}}\right) \Psi_{(0)}, \\
& P_{M}^{(0)} \Psi_{1}=0
\end{aligned}
$$

where $L=\left(1-P_{M}^{(0)}\right) J_{\mathrm{d}}\left(1-P_{M}^{(0)}\right)$ is the symmetric operator. If the manifold $\Psi_{(0)}$ is parameterized by the functionals $M_{0}=\int g_{0} \Psi_{(0)} \mathrm{d} \boldsymbol{Q}$ and $\stackrel{\grave{M}}{=}=\int m_{1} \mathbf{Q} \mathbf{Q} \Psi_{(0)} \mathrm{d} \boldsymbol{Q}$, where $\Psi_{\text {eq }} m_{0}$ and $\Psi_{\text {eq }} \mathbf{Q} \mathbf{Q} m_{1}$ are lowest eigenvectors of $J_{\mathrm{d}}$, then the general form of the solution is given by

$$
\begin{aligned}
\Psi_{1}= & \Psi_{\mathrm{eq}}\left\{z_{0} M_{0}(\dot{\gamma}: \mathbf{Q} \mathbf{Q})+z_{1}(\stackrel{\circ}{\mathbf{M}}: \mathbf{Q} \mathbf{Q})(\dot{\gamma}: \mathbf{Q} \mathbf{Q})\right. \\
& \left.+z_{2}\{\dot{\gamma} \cdot \stackrel{\circ}{\mathbf{M}}+\stackrel{\circ}{\mathbf{M}} \cdot \dot{\gamma}\}: \mathbf{Q} \mathbf{Q}+z_{3} \dot{\gamma}: \stackrel{\circ}{\mathbf{M}}+\frac{1}{2} \dot{\gamma}: \mathbf{Q} \mathbf{Q}\right\} .
\end{aligned}
$$

The terms $z_{0}-z_{3}$ are the functions of $Q^{2}$ found as the solutions to some linear differential equations.

We observe two features of the new manifold: first, it remains linear in variables $M_{0}$ and $\stackrel{\circ}{\mathbf{M}}$ and second it contains the dependence on the rate of strain tensor $\dot{\gamma}$. As the consequence, the transition to variables $\tau$ is given by the linear relations:

$$
\begin{aligned}
& -\frac{\stackrel{\circ}{\mathrm{p}}_{\mathrm{p}}}{n k_{\mathrm{B}} T}=r_{0} \stackrel{\circ}{\mathbf{M}}+r_{1} M_{0} \dot{\gamma}+r_{2}\{\dot{\gamma} \cdot \stackrel{\circ}{\mathbf{M}}+\stackrel{\circ}{\mathbf{M}} \cdot \dot{\gamma}\}+r_{3} \dot{\gamma} \cdot \dot{\gamma}, \\
& -\frac{\operatorname{tr} \tau_{\mathrm{p}}}{n k_{\mathrm{B}} T}=p_{0} M_{0}+p_{1} \dot{\gamma}: \stackrel{\circ}{\mathbf{M}},
\end{aligned}
$$

where $r_{i}$ and $p_{i}$ are some constants. Finally the equations in terms of $\tau$ should be also linear. Analysis shows that the first-order correction to the modified Oldroyd 8 constants model (518) will be transformed into the equations of the following general structure:

$$
\tau+c_{1} \tau_{[1]}+\left\{\boldsymbol{\Gamma}_{1} \cdot \tau \cdot \boldsymbol{\Gamma}_{2}+\boldsymbol{\Gamma}_{2}^{\dagger} \cdot \tau \cdot \boldsymbol{\Gamma}_{1}^{\dagger}\right\}+\boldsymbol{\Gamma}_{3}(\operatorname{tr} \tau)+\boldsymbol{\Gamma}_{4}\left(\boldsymbol{\Gamma}_{5}: \tau\right)=-v_{0} \boldsymbol{\Gamma}_{6},
$$

where $\boldsymbol{\Gamma}_{1}-\boldsymbol{\Gamma}_{6}$ are tensors dependent on the rate-of-strain tensor $\dot{\gamma}$ and its first convective derivative $\dot{\gamma}_{[1]}$, constant $c_{1}$ is the same as in Eq. (519) and $v_{0}$ is a positive constant.

Because the explicit form of the tensors $\boldsymbol{\Gamma}_{i}$ is quite extensive we do not present them in this section. Instead we give several general remarks about the structure of the first- and higher-order 
corrections:

(1) Since manifold (521) does not depend on the vorticity tensor $\omega=\boldsymbol{k}-\boldsymbol{k}^{\dagger}$ the latter enters Eqs. (523) only via convective derivatives of $\tau$ and $\dot{\gamma}$. This is sufficient to acquire the frame indifference feature, since all the tensorial quantities in dynamic equations are indifferent in any time dependent reference frame [256].

(2) When $\boldsymbol{k}=0$ the first order equations (523) as well as equations for any order reduce to linear relaxation dynamics of slow modes:

$$
\begin{aligned}
& \frac{D \tau}{D t}+\frac{\lambda_{1}}{\lambda_{\mathrm{r}}} \stackrel{\circ}{\tau}=0, \\
& \frac{D \operatorname{tr} \tau}{D t}+\frac{\lambda_{0}}{\lambda_{\mathrm{r}}} \operatorname{tr} \tau=0,
\end{aligned}
$$

which is obviously concordant with the dissipativity and the slowness requirements.

(3) In all higher-order corrections one will be always left with linear manifolds if the projector associated with functionals $M_{0}[\Psi]$ and $\stackrel{\circ}{\mathbf{M}}[\Psi]$ is used in every step. It follows that the resulting constitutive equations will always take a linear form (523), where all tensors $\gamma_{i}$ depend on higher order convective derivatives of $\dot{\gamma}$ (the highest possible order is limited by the order of the correction). Similarly to the first and zero orders the frame indifference is guaranteed if the manifold does not depend on the vorticity tensor unless the latter is incorporated in any frame invariant time derivatives. It is reasonable to eliminate the dependence on vorticity (if any) at the stage of constructing the solution to iteration equations (504).

(4) When the force $\boldsymbol{F}$ is linear $\boldsymbol{F}=\boldsymbol{Q}$ our approach is proven to be also correct since it leads the Oldroyd-B model (Eq. (518) with $c_{i}=0$ for $i=3, \ldots, 8$ ). This follows from the fact that the spectrum of the corresponding operator $J_{\mathrm{d}}$ is more degenerated, in particular $\lambda_{0}=\lambda_{1}=1$ and the corresponding lowest eigenvectors comprise a simple dyad $\Psi_{\text {eq }} Q \boldsymbol{Q}$.

\section{Tests on the FENE dumbbell model}

In this section we specify the choice of the force law as the FENE springs (466) and present results of test calculations for the revised Oldroyd 8 constants (516) equations on the examples of two simple viscometric flows.

We introduce the extensibility parameter of FENE dumbbell model $b$ :

$$
b=\widehat{\mathbf{Q}}_{0}^{2}=\frac{H \boldsymbol{Q}_{0}^{2}}{k_{\mathrm{B}} T} .
$$

It was estimated [113] that $b$ is proportional to the length of polymeric molecule and has a meaningful variation interval 50-1000. The limit $b \rightarrow \infty$ corresponds to the Hookean case and therefore to the Oldroyd-B constitutive relation.

In our test calculations we will compare our results with the Brownian dynamic (BD) simulation data made on FENE dumbbell equations [258], and also with one popular approximation to the FENE model known as FENE-P (FENE-Peterelin) model [259,113,260]. The latter is obtained by self-consistent approximation to FENE force:

$$
\boldsymbol{F}=\frac{1}{1-\left\langle\boldsymbol{Q}^{2}\right\rangle / b} \boldsymbol{Q} .
$$


Table 4

Values of constants to the revised Oldroyd 8 constants model computed on the base of the FENE dumbbells model

\begin{tabular}{lllllll}
\hline$b$ & $\lambda_{0}$ & $\lambda_{1}$ & $b_{0}$ & $b_{1}$ & $b_{2}$ & $a_{0}$ \\
\hline 20 & 1.498 & 1.329 & -0.0742 & 0.221 & 1.019 & 0.927 \\
50 & 1.198 & 1.135 & -0.0326 & 0.279 & 1.024 & 0.982 \\
100 & 1.099 & 1.068 & -0.0179 & 0.303 & 1.015 & 0.990 \\
200 & 1.050 & 1.035 & 0.000053 & 0.328 & 1.0097 & 1.014 \\
$\infty$ & 1 & 1 & 0 & $1 / 3$ & 1 \\
\hline
\end{tabular}

Table 5

Corrections due to hydrodynamic interaction to the constants of the revised Oldroyd 8 constants model based on FENE force

\begin{tabular}{rllcccc}
\hline$b$ & $\delta \lambda_{0}$ & $\delta \lambda_{1}$ & $\delta b_{0}$ & $\delta b_{1}$ & $\delta b_{2}$ & $\delta a_{0}$ \\
\hline 20 & -0.076 & -0.101 & 0.257 & -0.080 & -0.0487 & -0.0664 \\
50 & -0.0618 & -0.109 & -0.365 & 0.0885 & -0.0205 & -0.0691 \\
100 & -0.0574 & -0.111 & -1.020 & 0.109 & -0.020 & -0.0603 \\
\hline
\end{tabular}

This force law like Hookean case allows for the exact moment closure leading to nonlinear constitutive equations $[113,260]$. Specifically we will use the modified variant of FENE-P model, which matches the dynamics of original FENE in near equilibrium region better than the classical variant. This modification is achieved by a slight modification of Kramers definition of the stress tensor:

$$
\tau_{\mathrm{p}}=n k_{\mathrm{B}} T(1-\theta b) \mathbf{1}-\langle\boldsymbol{F} \boldsymbol{Q}\rangle .
$$

The case $\theta=0$ gives the classical definition of FENE-P, while more thorough estimation $[249,260]$ is $\theta=(b(b+2))^{-1}$.

Constants. The specific feature of the FENE model is that the length of dumbbells $\boldsymbol{Q}$ can vary only in a bounded domain of $\mathbf{R}^{3}$, namely inside a sphere $S_{b}=\left\{Q^{2} \leqslant b\right\}$. The sphere $S_{b}$ defines the domain of integration for averages $\langle\bullet\rangle_{\mathrm{e}}=\int_{S_{b}} \Psi_{\mathrm{eq}} \bullet \mathrm{d} \boldsymbol{Q}$, where the equilibrium distribution reads $\Psi_{\text {eq }}=c^{-1}\left(1-Q^{2} / b\right)^{b / 2}, c=\int_{S_{b}}\left(1-Q^{2} / b\right)^{b / 2} \mathrm{~d} \boldsymbol{Q}$.

In order to find constants for the zero-order model (516) we do the following: First we analytically compute the lowest eigenfunctions of operator $J_{\mathrm{d}}: g_{1}\left(Q^{2}\right) \mathbf{Q} \mathbf{Q}$ and $g_{0}\left(Q^{2}\right)$ without account of the hydrodynamic interaction $(\kappa=0)$. The functions $g_{0}$ and $g_{1}$ are computed by a procedure presented in subsection "Auxiliary formulas, 1 " with the help of the symbolic manipulation software Maple $V .3$ [261]. Then we calculate the perturbations terms $h_{0,1}$ by formulas (499) introducing the account of hydrodynamic interaction. Table 4 presents the constants $\lambda_{0,1}, a_{i}, b_{i}(510),(517)$ of the zero-order model (516) without inclusion of hydrodynamic interaction $\kappa=0$ for several values of extensibility parameter $b$. The relative error $\delta_{0,1}$ (see subsection "Auxiliary formulas, 1") of approximation for these calculations did not exceed the value 0.02. Table 5 shows the linear correction terms for constants from Table 4 which account a hydrodynamic interaction effect: $\lambda_{0,1}^{\mathrm{h}}=\lambda_{0,1}\left(1+\kappa\left(\delta \lambda_{0,1}\right)\right)$, $a_{i}^{\mathrm{h}}=a_{i}\left(1+\kappa\left(\delta a_{i}\right)\right), b_{i}^{\mathrm{h}}=b_{i}\left(1+\kappa\left(\delta b_{i}\right)\right)$. The latter are calculated by substituting the perturbed functions 
$m_{0,1}=g_{0,1}+\kappa h_{0,1}$ into (510) and (517), and expanding them up to first-order in $\kappa$. One can observe, since $\kappa>0$, the effect of hydrodynamic interaction results in the reduction of the relaxation times.

Dynamic problems. The rest of this section concerns the computations for two particular flows. The shear flow is defined by

$$
\boldsymbol{k}(t)=\dot{\gamma}(t)\left[\begin{array}{lll}
0 & 1 & 0 \\
0 & 0 & 0 \\
0 & 0 & 0
\end{array}\right],
$$

where $\dot{\gamma}(t)$ is the shear rate, and the elongation flow corresponds to the choice:

$$
\boldsymbol{k}(t)=\dot{\varepsilon}(t)\left[\begin{array}{ccc}
1 & 0 & 0 \\
0 & -1 / 2 & 0 \\
0 & 0 & -1 / 2
\end{array}\right]
$$

where $\dot{\varepsilon}(t)$ is the elongation rate.

In test computations we will look at the so-called viscometric material functions defined through the components of the polymeric part of the stress tensor $\tau_{\mathrm{p}}$. Namely, for shear flow they are the shear viscosity $v$, the first and the second normal stress coefficients $\psi_{1}, \psi_{2}$, and for elongation flow the only function is the elongation viscosity $\bar{v}$. In dimensionless form they are written as

$$
\begin{aligned}
& \widehat{v}=\frac{v-v_{\mathrm{s}}}{n k_{\mathrm{B}} T \lambda_{\mathrm{r}}}=-\frac{\tau_{\mathrm{p}, 12}}{\bar{\gamma} n k_{\mathrm{B}} T}, \\
& \widehat{\psi}_{1}=\frac{\psi_{1}}{n k_{\mathrm{B}} T \lambda_{\mathrm{r}}^{2}}=\frac{\tau_{\mathrm{p}, 22}-\tau_{\mathrm{p}, 11}}{\bar{\gamma}^{2} n k_{\mathrm{B}} T}, \\
& \widehat{\psi}_{2}=\frac{\psi_{2}}{n k_{\mathrm{B}} T \lambda_{\mathrm{r}}^{2}}=\frac{\tau_{p, 33}-\tau_{p, 22}}{\bar{\gamma}^{2} n k_{\mathrm{B}} T}, \\
& \vartheta=\frac{\bar{v}-3 v_{\mathrm{s}}}{n k_{\mathrm{B}} T \lambda_{\mathrm{r}}}=\frac{\tau_{\mathrm{p}, 22}-\tau_{\mathrm{p}, 11}}{\bar{\varepsilon} n k_{\mathrm{B}} T},
\end{aligned}
$$

where $\bar{\gamma}=\dot{\gamma} \lambda_{\mathrm{r}}$ and $\bar{\varepsilon}=\dot{\varepsilon} \lambda_{\mathrm{r}}$ are dimensionless shear and elongation rates. Characteristic values of latter parameters $\bar{\gamma}$ and $\bar{\varepsilon}$ allow to estimate the parameter $\varepsilon_{1}$ (474). For all flows considered below the second flow parameter (Deborah number) $\varepsilon_{2}$ is equal to zero.

Let us consider the steady state values of viscometric functions in steady shear and elongation flows: $\dot{\gamma}=$ const, $\dot{\varepsilon}=$ const. For the shear flow the steady values of these functions are found from Eqs. (516) as follows:

$$
\widehat{v}=b_{2} /\left(\lambda_{1}-c \bar{\gamma}^{2}\right), \quad \widehat{\psi}_{1}=2 \widehat{v} / \lambda_{1}, \quad \widehat{\psi}_{2}=2 b_{0} \widehat{v} / \lambda_{1}
$$

where $c=2 / 3\left(2 b_{0}^{2}+2 b_{0}-1\right) / \lambda_{1}+2 b_{1} a_{0} / \lambda_{0}$. Estimations for the constants (see Table 1$)$ shows that $c \leqslant 0$ for all values of $b$ (case $c=0$ corresponds to $b=\infty$ ), thus all three functions are monotonically decreasing in absolute value with increase of quantity $\bar{\gamma}$, besides the case when $b=\infty$. Although 

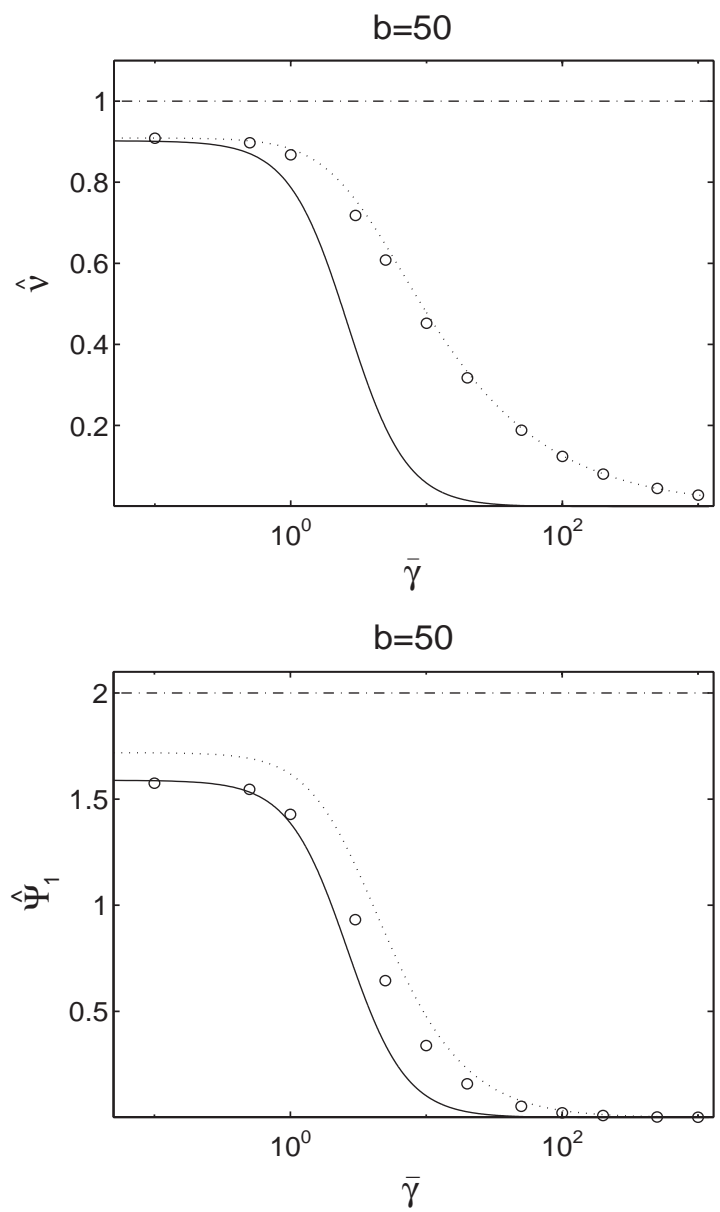

Fig. 17. Dimensionless shear viscosity $\widehat{v}$ and first normal stress coefficient $\widehat{\psi}_{1}$ vs. shear rate: (——) revised Oldroyd 8 constants model; $(\cdots \cdots)$ ) FENE-P model; (०००) BD simulations on the FENE model; $(-\cdot-\cdot-)$ Hookean dumbbell model.

they qualitatively correctly predict the shear thinning for large shear rates due to power law, but the exponent -2 of power dependence in the limit of large $\bar{\gamma}$ from the values -0.66 for parameter $\widehat{v}$ and -1.33 for $\widehat{\psi}_{1}$ observed in Brownian dynamic simulations [258]. It is explained by the fact that slopes of shear thining lie out of the applicability domain of our model. A comparison with BD simulations and modified FENE-P model is depicted in Fig. 17.

The predictions for the second normal stress coefficient indicate one more difference between revised Oldroyd 8 constant equation and FENE-P model. FENE-P model shows identically zero values for $\widehat{\psi}_{2}$ in any shear flow, either steady or time dependent, while the model (516), as well as BD simulations (see Fig. 9 in Ref. [258]) predict small, but nonvanishing values for this quantity. Namely, due to model (516) in shear flows the following relation $\widehat{\psi}_{2}=b_{0} \widehat{\psi}_{1}$ is always valid, with proportionality coefficient $b_{0}$ small and mostly negative, what leads to small and mostly negative values of $\widehat{\psi}_{2}$. 


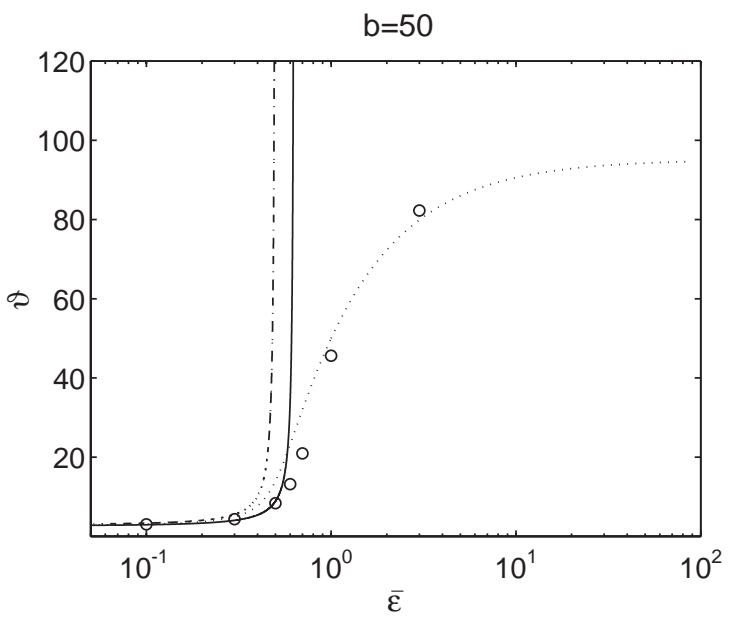

Fig. 18. Dimensionless elongation viscosity vs. elongation rate: $(--)$ revised Oldroyd 8 constants model, $(\cdots \cdots)$ FENE-P model, (०००) BD simulations on the FENE model; $(-\cdot-\cdot-)$ Hookean dumbbell model.

Table 6

Singular values of elongation rate

\begin{tabular}{lllllll}
\hline$b$ & 20 & 50 & 100 & 120 & 200 & $\infty$ \\
$\bar{\varepsilon}^{*}$ & 0.864 & 0.632 & 0.566 & 0.555 & 0.520 & 0.5 \\
\hline
\end{tabular}

In the elongation flow the steady state value to $\vartheta$ is found as

$$
\vartheta=\frac{3 b_{2}}{\lambda_{1}-\frac{5}{6}\left(2 b_{0}+1\right) \bar{\varepsilon}-7 b_{1} a_{0} \bar{\varepsilon}^{2} / \lambda_{0}} .
$$

The denominator has one root on positive semi-axis

$$
\bar{\varepsilon}_{*}=-\frac{5 \lambda_{0}\left(2 b_{0}+1\right)}{84 b_{1} a_{0}}+\left(\left(\frac{5 \lambda_{0}\left(2 b_{0}+1\right)}{84 b_{1} a_{0}}\right)^{2}+\frac{\lambda_{1} \lambda_{0}}{7 b_{1} a_{0}}\right)^{1 / 2},
$$

which defines a singularity point for the dependence $\vartheta(\bar{\varepsilon})$. The BD simulation experiments [258] on the FENE dumbbell models shows that there is no divergence of elongation viscosity for all values of elongation rate (see Fig. 18). For Hookean springs $\bar{\varepsilon}_{*}=1 / 2$ while in our model (516) the singularity point shifts to higher values with respect to decreasing values of $b$ as it is demonstrated in Table 6.

Fig. 19 gives an example of dynamic behavior for elongation viscosity in the instant start-up of the elongational flow. Namely it shows the evolution of initially vanishing polymeric stresses after instant jump of elongation rate at the time moment $t=0$ from the value $\bar{\varepsilon}=0$ to the value $\bar{\varepsilon}=0.3$.

It is possible to conclude that the revised Oldroyd 8 constants model (516) with estimations given by (517) for small and moderate rates of strain up to $\varepsilon_{1}=\lambda_{\mathrm{r}}|\dot{\gamma}| /\left(2 \lambda_{1}\right) \sim 0.5$ yields a good approximation to original FENE dynamics. The quality of the approximation in this interval is the same or better than the one of the nonlinear FENE-P model. 

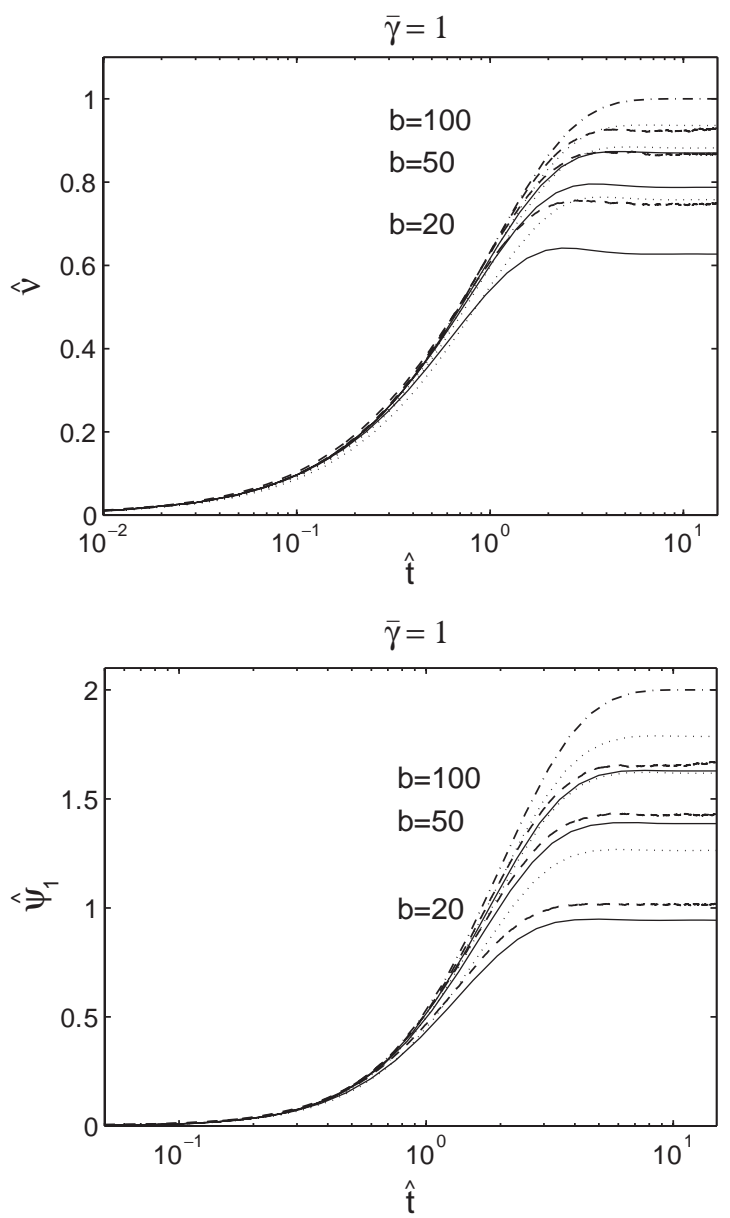

Fig. 19. Time evolution of elongation viscosity after inception of the elongation flow with elongation rate $\bar{\varepsilon}=0.3$ : $(--)$ revised Oldroyd 8 constants model, $(\cdots \cdots)$ FENE-P model, $(---)$ BD simulations on FENE model; $(-\cdot-\cdot-)$ Hookean dumbbell model.

\section{The main results of this Example are as follows:}

(i) We have developed a systematic method of constructing constitutive equations from the kinetic dumbbell models for the polymeric solutions. The method is free from a priori assumptions about the form of the spring force and is consistent with basic physical requirements: frame invariance and dissipativity of internal motions of fluid. The method extends so-called the method of invariant manifold onto equations coupled with external fields. Two characteristic parameters of fluid flow were distinguished in order to account for the effect of the presence of external fields. The iterative Newton scheme for obtaining a slow invariant manifold of the system driven by the flow with relatively low values of both characteristic parameters was developed. 
(ii) We demonstrated that the revised phenomenological Oldroyd 8 constants constitutive equations represent the slow dynamics of microscopic elastic dumbbell model with any nonlinear spring force in the limit when the rate of strain and frequency of time variation of the flow are sufficiently small and microscopic states at initial time of evolution are taken not far from the equilibrium.

(iii) The corrections to the zero-order manifold lead generally to linear in stresses equations but with highly nonlinear dependence on the rate of strain tensor and its convective derivatives.

(iv) The zero-order constitutive equation is compared to the direct Brownian dynamics simulation for FENE dumbbell model as well as to predictions of FENE-P model. This comparison shows that the zero-order constitutive equation gives the correct predictions in the domain of its validity, but does not exclude qualitative discrepancy occurring out of this domain, particularly in elongation flows.

This discrepancy calls for a further development, in particular, the use of nonlinear manifolds for derivation of zero-order model. The reason is in the necessity to provide concordance with the requirement of the positivity of distribution function. It may lead to nonlinear constitutive equation on any order of correction. These issues are currently under consideration and results will be reported separately.

Example 13: Explosion of invariant manifold, limits of macroscopic description for polymer molecules, molecular individualism, and multimodal distributions

Derivation of macroscopic equations from the simplest dumbbell models is revisited [84]. It is demonstrated that the onset of the macroscopic description is sensitive to the flows. For the FENE-P model, small deviations from the Gaussian solution undergo a slow relaxation before the macroscopic description sets on. Some consequences of these observations are discussed. A new class of closures is discussed, the kinetic multipeak polyhedra. Distributions of this type are expected in kinetic models with multidimensional instability as universally, as the Gaussian distribution appears for stable systems. The number of possible relatively stable states of a nonequilibrium system grows as $2^{m}$, and the number of macroscopic parameters is in order $m n$, where $n$ is the dimension of configuration space, and $m$ is the number of independent unstable directions in this space. The elaborated class of closures and equations pretends to describe the effects of "molecular individualism".

\section{Dumbbell models and the problem of the classical Gaussian solution stability}

We shall consider the simplest case of dilute polymer solutions represented by dumbbell models. The dumbbell model reflects the two features of real-world macromolecules to be orientable and stretchable by a flowing solvent [113].

Let us consider the simplest one-dimensional kinetic equation for the configuration distribution function $\Psi(q, t)$, where $q$ is the reduced vector connecting the beads of the dumbbell. This equation is slightly different from the usual Fokker-Planck equation. It is nonlinear, because of the dependence of potential energy $U$ on the moment $M_{2}[\Psi]=\int q^{2} \Psi(q) \mathrm{d} q$. This dependence allows us to get the exact quasi-equilibrium equations on $M_{2}$, but this equations are not solving the problem: this 
quasi-equilibrium manifold may become unstable when the flow is present [84]. Here is this model:

$$
\partial_{t} \Psi=-\partial_{q}\{\alpha(t) q \Psi\}+\frac{1}{2} \partial_{q}^{2} \Psi
$$

Here

$$
\alpha(t)=\kappa(t)-\frac{1}{2} f\left(M_{2}(t)\right),
$$

$\kappa(t)$ is the given time-independent velocity gradient, $t$ is the reduced time, and the function $-f q$ is the reduced spring force. Function $f$ may depend on the second moment of the distribution function $M_{2}=\int q^{2} \Psi(q, t) \mathrm{d} q$. In particular, the case $f \equiv 1$ corresponds to the linear Hookean spring, while $f=\left[1-M_{2}(t) / b\right]^{-1}$ corresponds to the self-consistent finite extension nonlinear elastic spring (the FENE-P model, first introduced in [260]). The second moment $M_{2}$ occurs in the FENE-P force $f$ as the result of the pre-averaging approximation to the original FENE model (with nonlinear spring force $\left.f=\left[1-q^{2} / b\right]^{-1}\right)$. The parameter $b$ changes the characteristics of the force law from Hookean at small extensions to a confining force for $q^{2} \rightarrow b$. Parameter $b$ is roughly equal to the number of monomer units represented by the dumbbell and should therefore be a large number. In the limit $b \rightarrow \infty$, the Hookean spring is recovered. Recently, it has been demonstrated that FENE-P model appears as first approximation within a systematic self-confident expansion of nonlinear forces [16].

Eq. (535) describes an ensemble of noninteracting dumbbells subject to a pseudo-elongational flow with fixed kinematics. As is well known, the Gaussian distribution function,

$$
\Psi^{G}\left(M_{2}\right)=\frac{1}{\sqrt{2 \pi M_{2}}} \exp \left[-\frac{q^{2}}{2 M_{2}}\right],
$$

solves Eq. (535) provided the second moment $M_{2}$ satisfies

$$
\frac{\mathrm{d} M_{2}}{\mathrm{~d} t}=1+2 \alpha(t) M_{2} .
$$

Solution (537) and (538) is the valid macroscopic description if all other solutions of Eq. (535) are rapidly attracted to the family of Gaussian distributions (537). In other words [6], the special solution (537) and (538) is the macroscopic description if Eq. (537) is the stable invariant manifold of the kinetic equation (535). If not, then the Gaussian solution is just a member of the family of solutions, and Eq. (538) has no meaning of the macroscopic equation. Thus, the complete answer to the question of validity of Eq. (538) as the macroscopic equation requires a study of dynamics in the neighborhood of manifold (537). Because of the simplicity of model (535), this is possible to a satisfactory level even for $M_{2}$-dependent spring forces.

\section{Dynamics of the moments and explosion of the Gaussian manifold}

In the paper [84] it was shown, that there is a possibility of "explosion" of the Gaussian manifold: with the small initial deviation from it, the solutions of Eq. (535) are very fast going far from, and then slowly come back to the stationary point which is located on the Gaussian manifold. The distribution function $\Psi$ is stretched fast, but loses the Gaussian form, and after that the Gaussian form recovers slowly with the new value of $M_{2}$. Let us describe briefly the results of [84]. 
Let $M_{2 n}=\int q^{2 n} \Psi \mathrm{d} q$ denote the even moments (odd moments vanish by symmetry). We consider deviations $\mu_{2 n}=M_{2 n}-M_{2 n}^{\mathrm{G}}$, where $M_{2 n}^{\mathrm{G}}=\int q^{2 n} \Psi^{\mathrm{G}} \mathrm{d} q$ are moments of the Gaussian distribution function (537). Let $\Psi\left(q, t_{0}\right)$ be the initial condition to Eq. (535) at time $t=t_{0}$. Introducing functions,

$$
p_{2 n}\left(t, t_{0}\right)=\exp \left[4 n \int_{t_{0}}^{t} \alpha\left(t^{\prime}\right) \mathrm{d} t^{\prime}\right]
$$

where $t \geqslant t_{0}$, and $2 n \geqslant 4$, the exact time evolution of the deviations $\mu_{2 n}$ for $2 n \geqslant 4$ reads

$$
\mu_{4}(t)=p_{4}\left(t, t_{0}\right) \mu_{4}\left(t_{0}\right),
$$

and

$$
\mu_{2 n}(t)=\left[\mu_{2 n}\left(t_{0}\right)+2 n(4 n-1) \int_{t_{0}}^{t} \mu_{2 n-2}\left(t^{\prime}\right) p_{2 n}^{-1}\left(t^{\prime}, t_{0}\right) \mathrm{d} t^{\prime}\right] p_{2 n}\left(t, t_{0}\right),
$$

for $2 n \geqslant 6$. Eqs. (539)-(541) describe evolution near the Gaussian solution for arbitrary initial condition $\Psi\left(q, t_{0}\right)$. Notice that explicit evaluation of the integral in Eq. (539) requires solution to the moment equation (538) which is not available in the analytical form for the FENE-P model.

It is straightforward to conclude that any solution with a nonGaussian initial condition converges to the Gaussian solution asymptotically as $t \rightarrow \infty$ if

$$
\lim _{t \rightarrow \infty} \int_{t_{0}}^{t} \alpha\left(t^{\prime}\right) \mathrm{d} t^{\prime}<0
$$

However, even if this asymptotic condition is met, deviations from the Gaussian solution may survive for considerable finite times. For example, if for some finite time $T$, the integral in Eq. (539) is estimated as $\int_{t_{0}}^{t} \alpha\left(t^{\prime}\right) \mathrm{d} t^{\prime}>\alpha\left(t-t_{0}\right), \alpha>0, t \leqslant T$, then the Gaussian solution becomes exponentially unstable during this time interval. If this is the case, the moment equation (538) cannot be regarded as the macroscopic equation. Let us consider specific examples.

For the Hookean spring $(f \equiv 1)$ under a constant elongation $(\kappa=$ const $)$, the Gaussian solution is exponentially stable for $\kappa<0.5$, and it becomes exponentially unstable for $\kappa>0.5$. The exponential instability in this case is accompanied by the well known breakdown of the solution to Eq. (538) due to infinite stretching of the dumbbell. The situation is much more interesting for the FENE-P model because this nonlinear spring force does not allow the infinite stretching of the dumbbell $[274,275]$.

Eqs. (538) and (540) were integrated by the 5th order Runge-Kutta method with adaptive time step. The FENE-P parameter $b$ was set equal to 50 . The initial condition was $\Psi(q, 0)=C\left(1-q^{2} / b\right)^{b / 2}$, where $C$ is the normalization (the equilibrium of the FENE model, notoriously close to the FENE-P equilibrium [258]). For this initial condition, in particular, $\mu_{4}(0)=-6 b^{2} /\left[(b+3)^{2}(b+5)\right]$ which is about $4 \%$ of the value of $M_{4}$ in the Gaussian equilibrium for $b=50$. In Fig. 20 we demonstrate deviation $\mu_{4}(t)$ as a function of time for several values of the flow. Function $M_{2}(t)$ is also given for comparison. For small enough $\kappa$ we find an adiabatic regime, that is $\mu_{4}$ relaxes exponentially to zero. For stronger flows, we observe an initial fast runaway from the invariant manifold with $\left|\mu_{4}\right|$ growing over three orders of magnitude compared to its initial value. After the maximum deviation has been reached, $\mu_{4}$ relaxes to zero. This relaxation is exponential as soon as the solution to Eq. (538) approaches the steady state. However, the time constant for this exponential relaxation $\left|\alpha_{\infty}\right|$ is very small. Specifically, for large $\kappa$,

$$
\alpha_{\infty}=\lim _{t \rightarrow \infty} \alpha(t)=-\frac{1}{2 b}+\mathrm{O}\left(\kappa^{-1}\right) .
$$




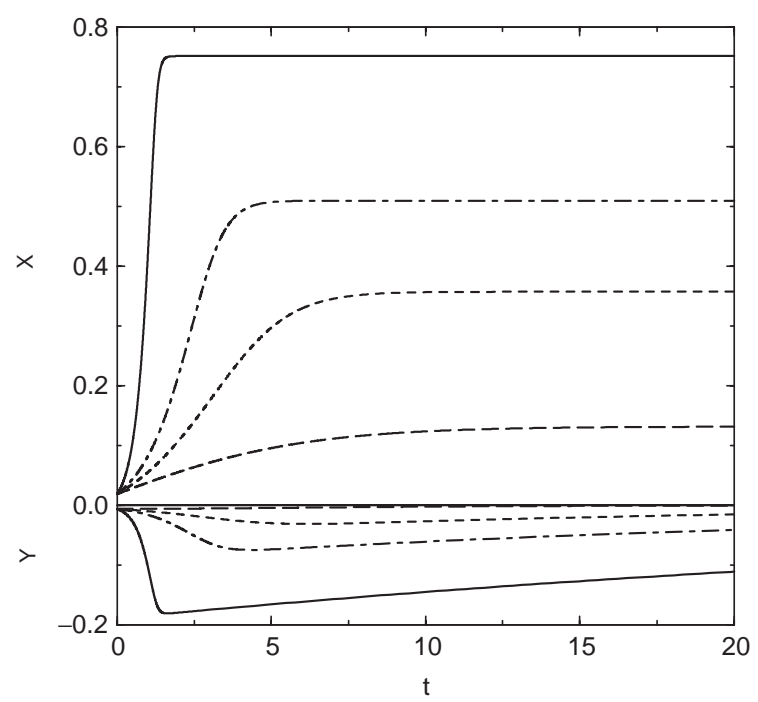

Fig. 20. Deviations of reduced moments from the Gaussian solution as a function of reduced time $t$ in pseudo-elongation flow for the FENE-P model. Upper part: Reduced second moment $X=M_{2} / b$. Lower part: Reduced deviation of fourth moment from Gaussian solution $Y=-\mu_{4}^{1 / 2} / b$. Solid: $\kappa=2$, dash-dot: $\kappa=1$, dash: $\kappa=0.75$, long dash: $\kappa=0.5$. (The figure from the paper [84], computed by P. Ilg.)

Thus, the steady state solution is unique and Gaussian but the stronger is the flow, the larger is the initial runaway from the Gaussian solution, while the return to it thereafter becomes flow-independent. Our observation demonstrates that, though the stability condition (542) is met, significant deviations from the Gaussian solution persist over the times when the solution of Eq. (538) is already reasonably close to the stationary state. If we accept the usually quoted physically reasonable minimal value of parameter $b$ of the order 20 then the minimal relaxation time is of order 40 in the reduced time units of Fig. 20. We should also stress that the two limits, $\kappa \rightarrow \infty$ and $b \rightarrow \infty$, are not commutative, thus it is not surprising that estimation (543) does not reduce to the above mentioned Hookean result as $b \rightarrow \infty$. Finally, peculiarities of convergence to the Gaussian solution are even furthered if we consider more complicated (in particular, oscillating) flows $\kappa(t)$. Further numerical experiments are presented in [85]. The statistics of FENE-P solutions with random strains was studied recently by J.-L. Thiffeault [263].

Two-peak approximation for polymer stretching in flow and explosion of the Gaussian manifold

In accordance with [264] the ansatz for $\Psi$ can be suggested in the following form:

$$
\Psi^{A n}(\{\sigma, \varsigma\}, q)=\frac{1}{2 \sigma \sqrt{2 \pi}}\left(\mathrm{e}^{-(q+\varsigma)^{2} / 2 \sigma^{2}}+\mathrm{e}^{-(q-\varsigma)^{2} / 2 \sigma^{2}}\right) .
$$

Natural inner coordinates on this manifold are $\sigma$ and $\varsigma$. Note, that now $\sigma^{2} \neq M_{2}$. The value $\sigma^{2}$ is a dispersion of one of the Gaussian summands in (544),

$$
M_{2}\left(\Psi^{A n}(\{\sigma, \varsigma\}, q)\right)=\sigma^{2}+\varsigma^{2} .
$$


To build the thermodynamic projector on manifold (544), the thermodynamic Lyapunov function is necessary. It is necessary to emphasize, that Eqs. (535) are nonlinear. For such equations, the arbitrarity in the choice of the thermodynamic Lyapunov function is much smaller than for the linear Fokker-Planck equation. Nevertheless, such a function exists. It is the free energy

$$
F=U\left(M_{2}[\Psi]\right)-T S[\Psi]
$$

where

$$
S[\Psi]=-\int \Psi(\ln \Psi-1) \mathrm{d} q
$$

$U\left(M_{2}[\Psi]\right)$ is the potential energy in the mean field approximation, $T$ is the temperature (further we assume that $T=1)$.

Note, that Kullback-form entropy [118] $S_{k}=-\int \Psi \ln \left(\Psi / \Psi^{*}\right)$ also has the form $S_{k}=-F / T$ :

$$
\begin{aligned}
& \Psi^{*}=\exp (-U), \\
& S_{k}[\Psi]=-\langle U\rangle-\int \Psi \ln \Psi \mathrm{d} q .
\end{aligned}
$$

If $U\left(M_{2}[\Psi]\right)$ in the mean field approximation is the convex function of $M_{2}$, then the free energy (545) is the convex functional too.

For the FENE-P model $U=-\ln \left[1-M_{2} / b\right]$.

In accordance to the thermodynamics the vector $I$ of flow of $\Psi$ must be proportional to the gradient of the corresponding chemical potential $\mu$ :

$$
I=-B(\Psi) \nabla_{q} \mu
$$

where $\mu=\delta F / \delta \Psi, B \geqslant 0$. From Eq. (545) it follows, that

$$
\begin{aligned}
& \mu=\frac{\mathrm{d} U\left(M_{2}\right)}{\mathrm{d} M_{2}} \cdot q^{2}+\ln \Psi \\
& I=-B(\Psi)\left[2 \frac{\mathrm{d} U}{\mathrm{~d} M_{2}} \cdot q+\Psi^{-1} \nabla_{q} \Psi\right] .
\end{aligned}
$$

If we suppose here $B=D / 2 \Psi$, then we get

$$
\begin{aligned}
& I=-D\left[\frac{\mathrm{d} U}{\mathrm{~d} M_{2}} \cdot q \Psi+\frac{1}{2} \nabla_{q} \Psi\right] \\
& \frac{\partial \Psi}{\partial t}=\operatorname{div}_{q} I=D \frac{\mathrm{d} U\left(M_{2}\right)}{\mathrm{d} M_{2}} \partial_{q}(q \Psi)+\frac{D}{2} \partial^{2} q \Psi
\end{aligned}
$$

When $D=1$ this equations coincide with (535) in the absence of the flow: due to Eq. (548) $\mathrm{d} F / \mathrm{d} t \leqslant 0$. 
Let us construct the thermodynamic projector with the help of the thermodynamic Lyapunov function $F$ (545). Corresponding entropic scalar product at the point $\Psi$ has the form

$$
\langle f \mid g\rangle_{\Psi}=\left.\frac{\mathrm{d}^{2} U}{\mathrm{~d} M_{2}^{2}}\right|_{M_{2}=M_{2}[\Psi]} \cdot \int q^{2} f(q) \mathrm{d} q \cdot \int q^{2} g(q) \mathrm{d} q+\int \frac{f(q) g(q)}{\Psi(q)} \mathrm{d} q .
$$

During the investigation of ansatz (544) the scalar product (549), constructed for the corresponding point of the Gaussian manifold with $M_{2}=\sigma^{2}$, will be used. It will let us to investigate the neighborhood of the Gaussian manifold (and to get all the results in the analytical form):

$$
\langle f \mid g\rangle_{\sigma^{2}}=\left.\frac{\mathrm{d}^{2} U}{\mathrm{~d} M_{2}^{2}}\right|_{M_{2}=\sigma^{2}} \cdot \int q^{2} f(q) \mathrm{d} q \cdot \int q^{2} g(q) \mathrm{d} q+\sigma \sqrt{2 \pi} \int \mathrm{e}^{q^{2} / 2 \sigma^{2}} f(q) g(q) \mathrm{d} q .
$$

Also we will need to know the functional DF at the point of Gaussian manifold:

$$
\mathrm{DF}_{\sigma^{2}}(f)=\left(\left.\frac{\mathrm{d} U\left(M_{2}\right)}{\mathrm{d} M_{2}}\right|_{M_{2}=\sigma^{2}}-\frac{1}{2 \sigma^{2}}\right) \int q^{2} f(q) \mathrm{d} q,
$$

(with the condition $\int f(q) \mathrm{d} q=0$ ). The point

$$
\left.\frac{\mathrm{d} U\left(M_{2}\right)}{\mathrm{d} M_{2}}\right|_{M_{2}=\sigma^{2}}=\frac{1}{2 \sigma^{2}},
$$

corresponds to the equilibrium.

The tangent space to manifold (544) is spanned by the vectors

$$
\begin{aligned}
& f_{\sigma}=\frac{\partial \Psi^{A n}}{\partial\left(\sigma^{2}\right)} ; \quad f_{\varsigma}=\frac{\partial \Psi^{A n}}{\partial\left(\varsigma^{2}\right)} ; \\
& f_{\sigma}=\frac{1}{4 \sigma^{3} \sqrt{2 \pi}}\left[\mathrm{e}^{-(q+\varsigma)^{2} / 2 \sigma^{2}} \frac{(q+\varsigma)^{2}-\sigma^{2}}{\sigma^{2}}+\mathrm{e}^{-(q-\varsigma)^{2} / 2 \sigma^{2}} \frac{(q-\varsigma)^{2}-\sigma^{2}}{\sigma^{2}}\right] ; \\
& f_{\varsigma}=\frac{1}{4 \sigma^{2} \varsigma \sqrt{2 \pi}}\left[-\mathrm{e}^{-(q+\varsigma)^{2} / 2 \sigma^{2}} \frac{q+\varsigma}{\sigma}+\mathrm{e}^{-(q-\varsigma)^{2} / 2 \sigma^{2}} \frac{(q-\varsigma)}{\sigma}\right] .
\end{aligned}
$$

The Gaussian entropy (free energy) production in the directions $f_{\sigma}$ and $f_{\varsigma}(551)$ has a very simple form

$$
\mathrm{DF}_{\sigma^{2}}\left(f_{\varsigma}\right)=\mathrm{DF}_{\sigma^{2}}\left(f_{\sigma}\right)=\left.\frac{\mathrm{d} U\left(M_{2}\right)}{\mathrm{d} M_{2}}\right|_{M_{2}=\sigma^{2}}-\frac{1}{2 \sigma^{2}} .
$$

The linear subspace $\operatorname{ker}^{\mathrm{DF}} \sigma_{\sigma^{2}}$ in $\operatorname{lin}\left\{f_{\sigma}, f_{\varsigma}\right\}$ is spanned by the vector $f_{\varsigma}-f_{\sigma}$.

Let us have the given vector field $\mathrm{d} \Psi / \mathrm{d} t=J(\Psi)$ at the point $\Psi(\{\sigma, \varsigma\})$. We need to build the projection of $J$ onto the tangent space $T_{\sigma, \varsigma}$ at the point $\Psi(\{\sigma, \varsigma\})$ :

$$
P_{\sigma, \varsigma}^{\text {th }}(J)=\varphi_{\sigma} f_{\sigma}+\varphi_{\varsigma} f_{\varsigma} .
$$

This equation means, that the equations for $\sigma^{2}$ and $\varsigma^{2}$ will have the form

$$
\frac{\mathrm{d} \sigma^{2}}{\mathrm{~d} t}=\varphi_{\sigma} ; \quad \frac{\mathrm{d} \varsigma^{2}}{\mathrm{~d} t}=\varphi_{\varsigma} .
$$


Projection $\left(\varphi_{\sigma}, \varphi_{\varsigma}\right)$ can be found from the following two equations:

$$
\begin{aligned}
& \varphi_{\sigma}+\varphi_{\varsigma}=\int q^{2} J(\Psi)(q) \mathrm{d} q ; \\
& \left\langle\varphi_{\sigma} f_{\sigma}+\varphi_{\varsigma} f_{\varsigma} \mid f_{\sigma}-f_{\varsigma}\right\rangle_{\sigma^{2}}=\left\langle J(\Psi) \mid f_{\sigma}-f_{\varsigma}\right\rangle_{\sigma^{2}},
\end{aligned}
$$

where $\langle f \mid g\rangle_{\sigma^{2}}=\left\langle J(\Psi) \mid f_{\sigma}-f_{\varsigma}\right\rangle_{\sigma^{2}}$, (549). First equation of (556) means, that the time derivative $\mathrm{d} M_{2} / \mathrm{d} t$ is the same for the initial and the reduced equations. Due to the formula for the dissipation of the free energy (551), this equality is equivalent to the persistence of the dissipation in the neighborhood of the Gaussian manifold. Indeed, in according to $(551) \mathrm{d} F / \mathrm{d} t=A\left(\sigma^{2}\right) \int q^{2} J(\Psi)(q) \mathrm{d} q=$ $A\left(\sigma^{2}\right) \mathrm{d} M_{2} / \mathrm{d} t$, where $A\left(\sigma^{2}\right)$ does not depend of $J$. On the other hand, time derivative of $M_{2}$ due to projected equation (555) is $\varphi_{\sigma}+\varphi_{\varsigma}$, because $M_{2}=\sigma^{2}+\varsigma^{2}$.

The second equation in (556) means, that $J$ is projected orthogonally on ker DS $\cap T_{\sigma, \varsigma}$. Let us use the orthogonality with respect to the entropic scalar product (550). The solution of Eqs. (556) has the form

$$
\begin{aligned}
\frac{\mathrm{d} \sigma^{2}}{\mathrm{~d} t} & =\varphi_{\sigma}=\frac{\left\langle J \mid f_{\sigma}-f_{\varsigma}\right\rangle_{\sigma^{2}}+M_{2}(J)\left(\left\langle f_{\varsigma} \mid f_{\varsigma}\right\rangle_{\sigma^{2}}-\left\langle f_{\sigma} \mid f_{\varsigma}\right\rangle_{\sigma^{2}}\right)}{\left\langle f_{\sigma}-f_{\varsigma} \mid f_{\sigma}-f_{\varsigma}\right\rangle_{\sigma^{2}}}, \\
\frac{\mathrm{d} \varsigma^{2}}{\mathrm{~d} t} & =\varphi_{\varsigma}=\frac{-\left\langle J \mid f_{\sigma}-f_{\varsigma}\right\rangle_{\sigma^{2}}+M_{2}(J)\left(\left\langle f_{\sigma} \mid f_{\sigma}\right\rangle_{\sigma^{2}}-\left\langle f_{\sigma} \mid f_{\varsigma}\right\rangle_{\sigma^{2}}\right)}{\left\langle f_{\sigma}-f_{\varsigma} \mid f_{\sigma}-f_{\varsigma}\right\rangle_{\sigma^{2}}},
\end{aligned}
$$

where $J=J(\Psi), M_{2}(J)=\int q^{2} J(\Psi) \mathrm{d} q$.

It is easy to check, that formulas (557) are indeed defining the projector: if $f_{\sigma}$ (or $f_{\varsigma}$ ) is substituted there instead of the function $J$, then we will get $\varphi_{\sigma}=1, \varphi_{\varsigma}=0$ (or $\varphi_{\sigma}=0, \varphi_{\varsigma}=1$, respectively). Let us substitute the right part of the initial kinetic equations (535), calculated at the point $\Psi(q)=$ $\Psi(\{\sigma, \varsigma\}, q)$ (see Eq. (544)) in Eq. (557) instead of $J$. We will get the closed system of equations on $\sigma^{2}, \varsigma^{2}$ in the neighborhood of the Gaussian manifold.

This system describes the dynamics of the distribution function $\Psi$. The distribution function is represented as the half-sum of two Gaussian distributions with the averages of distribution $\pm \varsigma$ and mean-square deviations $\sigma$. All integrals in the right hand part of (557) are possible to calculate analytically.

Basis $\left(f_{\sigma}, f_{\varsigma}\right)$ is convenient to use everywhere, except the points in the Gaussian manifold, $\varsigma=0$, because if $\varsigma$, then

$$
f_{\sigma}-f_{\varsigma}=\mathrm{O}\left(\frac{\varsigma^{2}}{\sigma^{2}}\right) \rightarrow 0 .
$$

Let us analyze the stability of the Gaussian manifold to the "dissociation" of the Gaussian peak in two peaks (544). To do this, it is necessary to find first nonzero term in the Taylor expansion in $\varsigma^{2}$ of the right hand side of the second equation in system (557). The denominator has the order of $\varsigma^{4}$, the numerator has, as it is easy to see, the order not less, than $\varsigma^{6}$ (because the Gaussian manifold is invariant with respect to the initial system).

With the accuracy up to $\varsigma^{4}$ :

$$
\frac{1}{\sigma^{2}} \frac{\mathrm{d} \varsigma^{2}}{\mathrm{~d} t}=2 \alpha \frac{\varsigma^{2}}{\sigma^{2}}+\mathrm{o}\left(\frac{\varsigma^{4}}{\sigma^{4}}\right),
$$




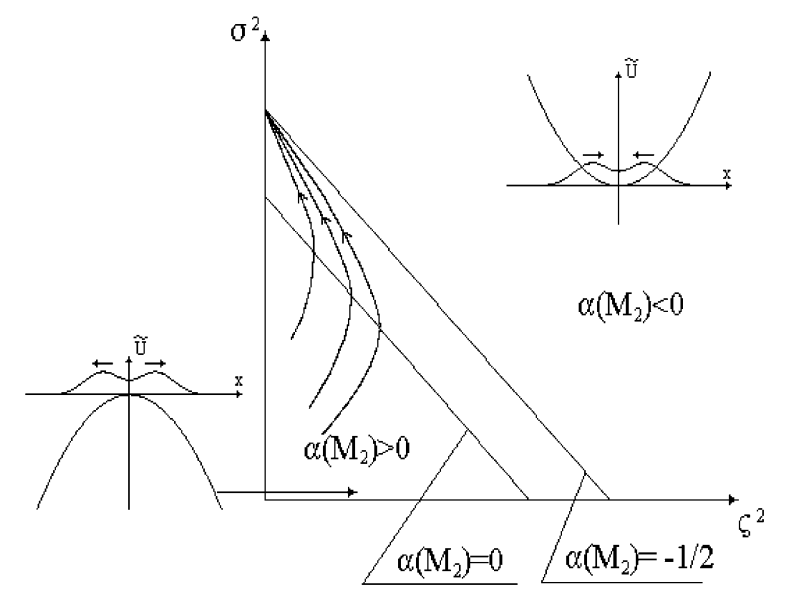

Fig. 21. Phase trajectories for the two-peak approximation, FENE-P model. The vertical axis $(\varsigma=0)$ corresponds to the Gaussian manifold. The triangle with $\alpha\left(M_{2}\right)>0$ is the domain of exponential instability.
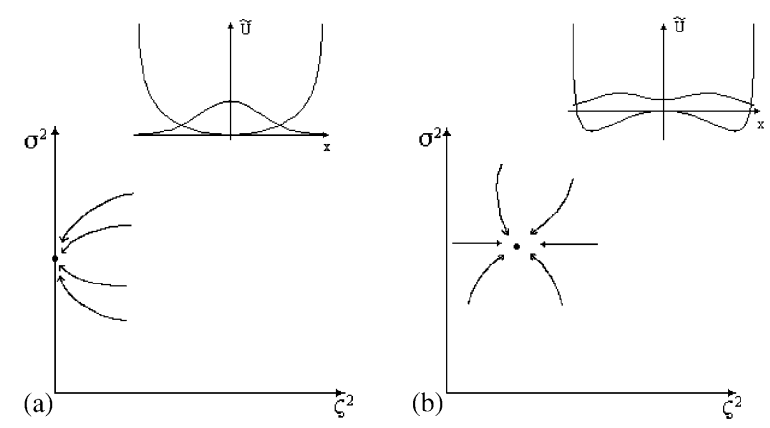

Fig. 22. Phase trajectories for the two-peak approximation, FENE model: (a) A stable equilibrium on the vertical axis, one stable peak; (b) A stable equilibrium with $\varsigma>0$, stable two-peak configuration.

where

$$
\alpha=\kappa-\left.\frac{\mathrm{d} U\left(M_{2}\right)}{\mathrm{d} M_{2}}\right|_{M_{2}=\sigma^{2}} .
$$

So, if $\alpha>0$, then $\varsigma^{2}$ grows exponentially $\left(\varsigma \sim \mathrm{e}^{\alpha t}\right)$ and the Gaussian manifold is unstable; if $\alpha<0$, then $\varsigma^{2}$ decreases exponentially and the Gaussian manifold is stable.

Near the vertical axis $\mathrm{d} \sigma^{2} / \mathrm{d} t=1+2 \alpha \sigma^{2} .{ }^{15}$ The form of the phase trajectories is shown qualitative on Fig. 21. Note that this result completely agrees with Eq. (540).

For the real equation FPE (for example, with the FENE potential) the motion in presence of the flow can be represented as the motion in the effective potential well $\tilde{U}(q)=U(q)-\frac{1}{2} \kappa q^{2}$. Different variants of the phase portrait for the FENE potential are present on Fig. 22. Instability and dissociation of the unimodal distribution functions ("peaks") for the FPE is the general effect when the flow is present.

\footnotetext{
${ }^{15}$ Pavel Gorban calculated the projector (557) analytically without Taylor expansion and with the same, but exact result: $\mathrm{d} \varsigma^{2} / \mathrm{d} t=2 \alpha \varsigma^{2} \mathrm{~d} \sigma^{2} / \mathrm{d} t=1+2 \alpha \sigma^{2}$.
} 
The instability occurs when the matrix $\partial^{2} \tilde{U} / \partial q_{i} \partial q_{j}$ starts to have negative eigenvalues ( $\tilde{U}$ is the effective potential energy, $\left.\tilde{U}(q)=U(q)-\frac{1}{2} \sum_{i, j} \kappa_{i, j} q_{i} q_{j}\right)$.

\section{Polymodal polyhedron and molecular individualism}

The discovery of the molecular individualism for dilute polymers in the flow [265] was the challenge to theory from the very beginning. "Our data should serve as a guide in developing improved microscopic theories for polymer dynamics"... was the concluding sentence of the paper [265]. P. de Gennes invented the term "molecular individualism" [266]. He stressed that in this case the usual averaging procedures are not applicable. At the highest strain rates distinct conformation shapes with different dynamics were observed [265]. Further works for shear flow demonstrated not only shape differences, but different large temporal fluctuations [267].

Equation for the molecules in a flow are known. These are the Fokker-Planck equations with external force. The theory of the molecular individualism is hidden inside these equations. Following the logic of model reduction we should solve two problems: to construct the slow manifold, and to project the equation on this manifold. The second problem is solved: the thermodynamic projector is necessary for this projection.

How to solve the first problem? We can find a hint in previous subsections. The Gaussian distributions form the invariant manifold for the FENE-P model of polymer dynamics, but, as it was discovered in [84], this manifold can become unstable in the presence of a flow. We propose to model this instability as dissociation of the Gaussian peak into two peaks. This dissociation describes appearance of an unstable direction in the configuration space.

In the classical FENE-P model of polymer dynamics a polymer molecule is represented by one coordinate: the stretching of molecule (the connector vector between the beads). There exists a simple mean field generalized models for multidimensional configuration spaces of molecules. In these models dynamics of distribution functions is described by the Fokker-Planck equation in a quadratic potential well. The matrix of coefficients of this quadratic potential depends on the matrix of the second order moments of the distribution function. The Gaussian distributions form the invariant manifold for these models, and the first dissociation of the Gaussian peak after appearance of the unstable direction in the configuration space has the same nature and description, as for the one-dimensional models of molecules considered below.

At the highest strain there can appear new unstable directions, and corresponding dissociations of Gaussian peaks form a cascade of dissociation. For $m$ unstable directions we get the Gaussian parallelepiped: The distribution function is represented as a sum of $2^{m}$ Gaussian peaks located in the vertixes of parallelepiped:

$$
\Psi(q)=\frac{1}{2^{m}(2 \pi)^{n / 2} \sqrt{\operatorname{det} \Sigma}} \sum_{\varepsilon_{i}= \pm 1,(i=1, \ldots, m)} \exp \left(-\frac{1}{2}\left(\Sigma^{-1}\left(q+\sum_{i=1}^{m} \varepsilon_{i} \varsigma_{i}\right), q+\sum_{i=1}^{m} \varepsilon_{i} \varsigma_{i}\right)\right),
$$

where $n$ is dimension of configuration space, $2 \varsigma_{i}$ is the vector of the $i$ th edge of the parallelepiped, $\Sigma$ is the one peak covariance matrix (in this model $\Sigma$ is the same for all peaks). The macroscopic variables for this model are:

1. The covariance matrix $\Sigma$ for one peak;

2. The set of vectors $\varsigma_{i}$ (or the parallelepiped edges). 
The stationary polymodal distribution for the Fokker-Planck equation corresponds to the persistence of several local minima of the function $\tilde{U}(q)$. The multidimensional case is different from one-dimensional because it has the huge amount of possible configurations. An attempt to describe this picture quantitative meet the following obstacle: we do not know the potential $U$, on the other hand, the effect of molecular individualism [265-267] seems to be universal in its essence, without dependence of the qualitative picture on details of interactions. We should find a mechanism that is as general, as the effect. The simplest dumbbell model which we have discussed in previous subsection does not explain the effect, but it gives us a hint: the flow can violate the stability of unimodal distribution. If we assume that the whole picture is hidden insight a multidimensional Fokker-Planck equation for a large molecule in a flow, then we can use this hint in such a way: when the flow strain grows there appears a sequence of bifurcations, and for each of them a new unstable direction arises. For qualitative description of such a picture we can apply a language of normal forms [268], but with some modification.

The bifurcation in dimension one with appearance of two point of minima from one point has the simplest polynomial representation: $U(q, \alpha)=q^{4}+\alpha q^{2}$. If $\alpha \geqslant 0$, then this potential has one minimum, if $\alpha<0$, then there are two points of minima. The normal form of degenerated singularity is $U(q)=q^{4}$. Such polynomial forms as $q^{4}+\alpha q^{2}$ are very simple, but they have inconvenient asymptotic at $q \rightarrow \infty$. For our goals it is more appropriate to use logarithms of convex combinations of Gaussian distributions instead of polynomials. It is the same class of jets near the bifurcation, but with given quadratic asymptotic $q \rightarrow \infty$. If one needs another class of asymptotic, it is possible just to change the choice of the basic peak. All normal forms of the critical form of functions, and families of versal deformations are well investigated and known [268].

Let us represent the deformation of the probability distribution under the strain in multidimensional case as a cascade of peak dissociation. The number of peaks will duplicate on the each step. The possible cascade of peaks dissociation is presented qualitatively on Fig. 23. The important property of this qualitative picture is the linear complexity of dynamical description with exponential complexity of geometrical picture. Let $m$ be the number of bifurcation steps in the cascade. Then

- For description of parallelepiped it is sufficient to describe $m$ edges;

- There are $2^{m-1}$ geometrically different conformations associated with $2^{m}$ vertex of parallelepiped (central symmetry halved this number).

Another important property is the threshold nature of each dissociation: It appears in points of stability loss for new directions, in these points the dimension of unstable direction increases.

Positions of peaks correspond to parallelepiped vertices. Different vertices in configuration space present different geometric forms. So, it seems plausible ${ }^{16}$ that observed different forms ("dumbbells", "half-dumbbells", "kinked", "folded" and other, not classified forms) correspond to

\footnotetext{
${ }^{16}$ We cannot prove it now, and it is necessary to determine the status of proposed qualitative picture: it is much more general than a specific model, it is the mechanism which acts in a wide class of models. The cascade of instabilities can appear and, no doubt, it appears for the Fokker-Planck equation for a large molecule in a flow. But it is not proven yet that the effects observed in well-known experiments have exactly this mechanism. This proof requires quantitative verification of a specific model. And now we talk not about a proven, but about the plausible mechanism which typically appears for systems with instabilities.
} 


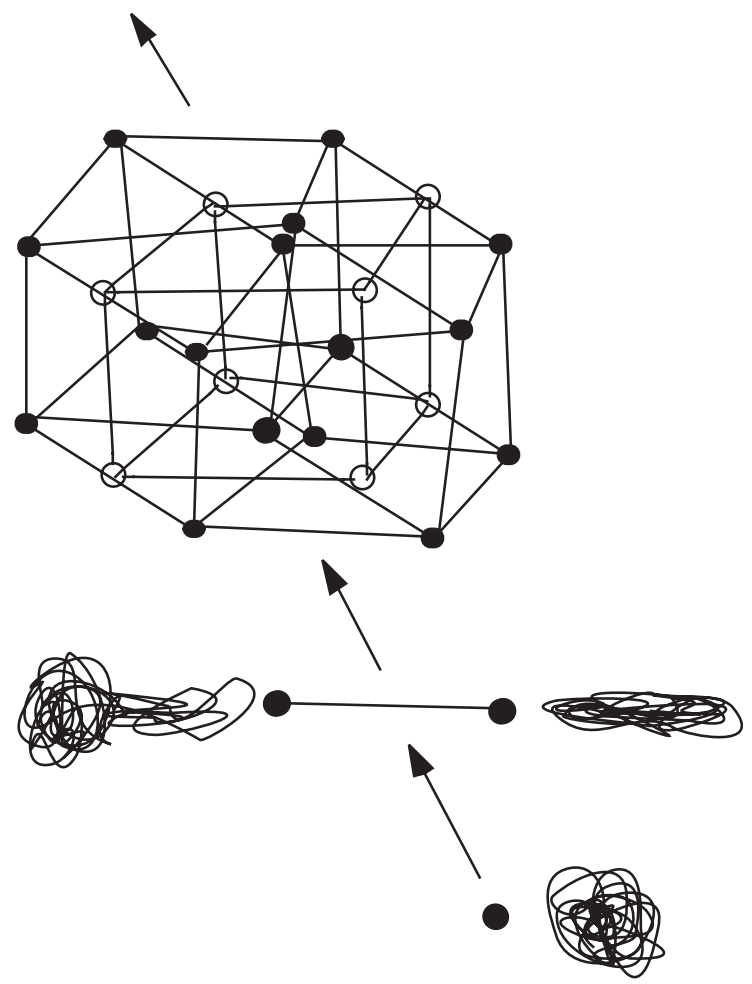

Fig. 23. Cartoon representing the steps of molecular individualism. Black dots are vertices of Gaussian parallelepiped. Zero, one, and four-dimensional polyhedrons are drawn. The three-dimensional polyhedron used to draw the four-dimensional object is also presented. Each new dimension of the polyhedron adds as soon as the corresponding bifurcation occurs. Quasi-stable polymeric conformations are associated with each vertex. First bifurcation pertinent to the instability of a dumbbell model in elongational flow is described in the text.

these vertices of parallelepiped. Each vertex is a metastable state of a molecule and has its own basin of attraction. A molecule goes to the vertex which depends strongly on details of initial conditions.

The simplest multidimensional dynamic model is the Fokker-Planck equation with quadratic mean field potential. This is direct generalization of the FENE-P model: the quadratic potential $U(q)$ depends on the tensor of second moments $\boldsymbol{M}_{2}=\left\langle q_{i} q_{j}\right\rangle$ (here the angle brackets denote the averaging). This dependence should provide the finite extensibility. This may be, for example, a simple matrix generalization of the FENE-P energy:

$$
U(q)=\sum_{i j} K_{i j} q_{i} q_{j}, \quad \mathbf{K}=\mathbf{K}^{0}+\phi\left(\boldsymbol{M}_{2} / b\right), \quad\langle U(q)\rangle=\operatorname{tr}\left(\mathbf{K} \boldsymbol{M}_{2} / b\right)
$$

where $b$ is a constant (the limit of extensibility), $\mathbf{K}^{0}$ is a constant matrix, $\boldsymbol{M}_{2}$ is the matrix of second moments, and $\phi$ is a positive analytical monotone increasing function of one variable on the interval $(0,1), \phi(x) \rightarrow \infty$ for $x \rightarrow 1$ (for example, $\phi(x)=-\ln (1-x) / x$, or $\phi(x)=(1-x)^{-1}$ ).

For quadratic multidimensional mean field models persists the qualitative picture of Fig. 21: there is nonstationary molecular individualism for stationary "molecular collectivism". The stationary 
distribution is the Gaussian distribution, and on the way to this stationary point there exists an unstable region, where the distribution dissociates onto $2^{m}$ peaks ( $m$ is the number of unstable degrees of freedom).

Dispersion of individual peak in unstable region increases too. This effect can deform the observed situation: If some of the peaks have significant intersection, then these peaks join into new extended classes of observed molecules. The stochastic walk of molecules between connected peaks can be observed as "large nonperiodical fluctuations". This walk can be unexpected fast, because it can be effectively a motion in a low-dimensional space, for example, in one-dimensional space (in a neighborhood of a part of one-dimensional skeleton of the polyhedron).

We discussed the important example of ansatz: the multipeak models. Two examples of these type of models demonstrated high efficiency during decades: the Tamm-Mott-Smith bimodal ansatz for shock waves, and the Langer-Bar-on-Miller [269-271] approximation for spinodal decomposition.

The multimodal polyhedron appears every time as an appropriate approximation for distribution functions for systems with instabilities. We create such an approximation for the Fokker-Planck equation for polymer molecules in a flow. Distributions of this type are expected to appear in each kinetic model with multidimensional instability as universally, as Gaussian distribution appears for stable systems. This statement needs a clarification: everybody knows that the Gaussian distribution is stable with respect to convolutions, and the appearance of this distribution is supported by central limit theorem. Gaussian polyhedra form a stable class: convolution of two Gaussian polyhedra is a Gaussian polyhedron, convolution of a Gaussian polyhedron with a Gaussian distribution is a Gaussian polyhedron with the same number of vertices. On the other hand, a Gaussian distribution in a potential well appears as an exponent of a quadratic form which represents the simplest stable potential (a normal form of a nondegenerated critical point). Families of Gaussian parallelepipeds appear as versal deformations with given asymptotic for systems with cascade of simplest bifurcations.

The usual point of view is: The shape of the polymers in a flow is either a coiled ball, or a stretched ellipsoid, and the Fokker-Planck equation describes the stretching from the ball to the ellipsoid. It is not the whole truth, even for the FENE-P equation, as it was shown in Refs. [84,264]. The Fokker-Planck equation describes the shape of a probability cloud in the space of conformations. In the flow with increasing strain this shape changes from the ball to the ellipsoid, but, after some thresholds, this ellipsoid transforms into a multimodal distribution which can be modeled as the peak parallelepiped. The peaks describe the finite number of possible molecule conformations. The number of this distinct conformations grows for a parallelepiped as $2^{m}$ with the number $m$ of independent unstable direction. Each vertex has its own basin of attraction. A molecule goes to the vertex which depends strongly on details of initial conditions.

These models pretend to be the kinetic basis for the theory of molecular individualism. The detailed computations will be presented in following works, but some of the qualitative features of the models are in agreement with some of qualitative features of the picture observed in experiment [265-267]: effect has the threshold character, different observed conformations depend significantly on the initial conformation and orientation.

Some general questions remain open:

- Of course, appearance of $2^{m}$ peaks in the Gaussian parallelepiped is possible, but some of these peaks can join in following dynamics, hence the first question is: what is the typical number of significantly different peaks for a $m$-dimensional instability? 
- How can we decide what scenario is more realistic from the experimental point of view: the proposed universal kinetic mechanism, or the scenario with long living metastable states (for example, the relaxation of knoted molecules in the flow can give an other picture than the relaxation of unknoted molecules)?

- The analysis of random walk of molecules from peak to peak should be done, and results of this analysis should be compared with observed large fluctuations.

The systematic discussion of the difference between the Gaussian elipsoid (and its generalizations) and the Gaussian multipeak polyhedron (and its generalizations) seems to be necessary. This polyhedron appears generically as the effective ansatz for kinetic systems with instabilities.

\section{Accuracy estimation and postprocessing in invariant manifolds construction}

Assume that for the dynamical system (76) the approximate invariant manifold has been constructed and the slow motion equations have been derived:

$$
\frac{\mathrm{d} x_{\mathrm{sl}}}{\mathrm{d} t}=P_{x_{\mathrm{sl}}}\left(J\left(x_{\mathrm{sl}}\right)\right), \quad x_{\mathrm{sl}} \in M,
$$

where $P_{x_{\mathrm{sl}}}$ is the corresponding projector onto the tangent space $T_{x_{\mathrm{sl}}}$ of $M$. Suppose that we have solved system (560) and have obtained $x_{\mathrm{sl}}(t)$. Let's consider the following two questions:

- How well this solution approximates the true solution $x(t)$ given the same initial conditions?

- Is it possible to use the solution $x_{\mathrm{sl}}(t)$ for it's refinement?

These two questions are interconnected. The first question states the problem of the accuracy estimation. The second one states the problem of postprocessing [244-246,277].

The simplest ("naive") estimation is given by the "invariance defect":

$$
\Delta_{x_{\mathrm{sl}}}=\left(1-P_{x_{\mathrm{sl}}}\right) J\left(x_{\mathrm{sl}}\right),
$$

which can be compared with $J\left(x_{\mathrm{s} 1}\right)$. For example, this estimation is given by $\epsilon=\left\|\Delta_{x_{\mathrm{sl}}}\right\| /\left\|J\left(x_{\mathrm{s} 1}\right)\right\|$ using some appropriate norm.

Probably, the most comprehensive answer to this question can be given by solving the following equation:

$$
\frac{\mathrm{d}(\delta x)}{\mathrm{d} t}=\Delta_{x_{\mathrm{sl}}(t)}+\left.D_{x} J(x)\right|_{x_{\mathrm{sl}}(t)} \delta x .
$$

This linear equation describes the dynamics of the deviation $\delta x(t)=x(t)-x_{\mathrm{sl}}(t)$ using the linear approximation. The solution with zero initial condition $\delta x(0)=0$ allows to estimate the robustness of $x_{\mathrm{sl}}$, as well as the error value. Substituting $x_{\mathrm{sl}}(t)$ for $x_{\mathrm{sl}}(t)+\delta x(t)$ gives the required solution refinement. This dynamical postprocessing [246] allows to refine the solution substantially and to estimate its accuracy and robustness. However, the price for this is solving Eq. (562) with variable coefficients. Thus, this dynamical postprocessing can be addressed by a whole hierarchy of simplifications, both dynamical and static. Let us mention some of them, starting from the dynamical ones. 
(1) Freezing the coefficients. In Eq. (562) the linear operator $\left.D_{x} J(x)\right|_{x_{\mathrm{sl}}(t)}$ is replaced by it's value in some distinguished point $x^{*}$ (for example, in the equilibrium) or it is frozen somehow else. As a result, one gets the equation with constant coefficients and the explicit integration formula:

$$
\delta x(t)=\int_{0}^{t} \exp \left(D^{*}(t-\tau)\right) \Delta_{x_{\mathrm{sl}}(\tau)} \mathrm{d} \tau,
$$

where $D^{*}$ is the "frozen" operator and $\delta x(0)=0$.

Another important way of freezing is substituting (562) for some model equation, i.e. substituting $D_{x} J(x)$ for $-1 / \tau^{*}$, where $\tau^{*}$ is the relaxation time. In this case the formula for $\delta x(t)$ has very simple form:

$$
\delta x(t)=\int_{0}^{t} \mathrm{e}^{(\tau-t) / \tau^{*}} \Delta_{x_{\mathrm{sl}}(\tau)} \mathrm{d} \tau .
$$

(2) One-dimensional Galerkin-type approximation. Another "scalar" approximation is given by projecting $(562)$ on $\Delta(t)=\Delta_{x_{\mathrm{sl}}(t)}$. Using the ansatz

$$
\delta x(t)=\delta(t) \cdot \Delta(t),
$$

substituting it into Eq. (562) after orthogonal projection on $\Delta(t)$ we obtain

$$
\frac{\mathrm{d} \delta(t)}{\mathrm{d} t}=1+\delta \frac{\langle\Delta \mid D \Delta\rangle-\langle\Delta \mid \dot{\Delta}\rangle}{\langle\Delta \mid \Delta\rangle},
$$

where $\langle\mid\rangle$ is an appropriate scalar product, which can depend on the point $x_{\mathrm{sl}}$ (for example, the entropic scalar product), $D=\left.D_{x} J(x)\right|_{x_{\mathrm{sl}}(t)}$ or the self-adjoint linearizarion of this operator, or some approximation of it, $\dot{\Delta}=\mathrm{d} \Delta(t) / \mathrm{d} t$.

A "hybrid" between Eqs. (566) and (562) has the simplest form (but it is more difficult for computation than Eq. (566)):

$$
\frac{\mathrm{d}(\delta x)}{\mathrm{d} t}=\Delta(t)+\frac{\langle\Delta \mid D \Delta\rangle}{\langle\Delta \mid \Delta\rangle} \delta x .
$$

Here one uses the normalized matrix element $\langle\Delta \mid D \Delta\rangle /\langle\Delta \mid \Delta\rangle$ instead of the linear operator $D=\left.D_{x} J(x)\right|_{x_{\mathrm{sl}}(t)}$.

Both Eqs. (566) and (567) can be solved explicitly:

$$
\begin{aligned}
& \delta(t)=\int_{0}^{t} \mathrm{~d} \tau \exp \left(\int_{\tau}^{t} k(\theta) \mathrm{d} \theta\right), \\
& \delta x(t)=\int_{0}^{t} \Delta(\tau) \mathrm{d} \tau \exp \left(\int_{\tau}^{t} k_{1}(\theta) \mathrm{d} \theta\right),
\end{aligned}
$$

where $k(t)=\langle\Delta \mid D \Delta\rangle-\langle\Delta \mid \dot{\Delta}\rangle /\langle\Delta \mid \Delta\rangle, k_{1}(t)=\langle\Delta \mid D \Delta\rangle /\langle\Delta \mid \Delta\rangle$.

The projection of $\Delta_{x_{\mathrm{sl}}}(t)$ on the slow motion is equal to zero, hence, for the post-processing analysis of the slow motion, the one-dimensional model (566) should be supplemented by one more 
iteration in order to find the first nonvanishing term in $\delta x_{\mathrm{sl}}(t)$ :

$$
\begin{aligned}
& \frac{\mathrm{d}\left(\delta x_{\mathrm{s} 1}(t)\right)}{\mathrm{d} t}=\delta(t) P_{x_{\mathrm{s} 1}(t)}\left(\left.D_{x} J(x)\right|_{x_{\mathrm{s} 1}(\tau)}\right)(\Delta(t)) ; \\
& \delta x_{\mathrm{sl}}(t)=\int_{0}^{t} \delta(\tau) P_{x_{\mathrm{sl}}(\tau)}\left(\left.D_{x} J(x)\right|_{x_{\mathrm{sl}}(\tau)}\right)(\Delta(\tau)) \mathrm{d} \tau .
\end{aligned}
$$

where $\delta(t)$ is the solution of (566).

(3) For a static post-processing one uses stationary points of dynamical equations (562) or their simplified versions (563), (566). Instead of (562) one gets:

$$
\left.D_{x} J(x)\right|_{x_{\mathrm{sl}}(t)} \delta x=-\Delta_{x_{\mathrm{sl}}(t)}
$$

with one additional condition $P_{x_{\mathrm{sl}}} \delta x=0$. This is exactly the iteration equation of the Newton's method in solving the invariance equation. A clarification is in order here. Static post-processing (571) as well as other post-processing formulas should not be confused with the Newton method and other for correcting the approximately invariant manifold. Here, only a single trajectory $x_{\mathrm{sl}}(t)$ on the manifold is corrected, not the whole manifold.

The corresponding stationary problems for the model equations and for the projections of (562) on $\Delta$ are evident. We only mention that in the projection on $\Delta$ one gets a step of the relaxation method for the invariant manifold construction.

In Example 14 it will be demonstrated how one can use function $\Delta\left(x_{\mathrm{sl}}(t)\right)$ in the accuracy estimation of macroscopic equations on example of polymer solution dynamics.

Example 14: Defect of invariance estimation and switching from the microscopic simulations to macroscopic equations

A method which recognizes the onset and breakdown of the macroscopic description in microscopic simulations was developed in [16,276,201]. The method is based on the invariance of the macroscopic dynamics relative to the microscopic dynamics, and it is demonstrated for a model of dilute polymeric solutions where it decides switching between Direct Brownian Dynamics simulations and integration of constitutive equations.

Invariance principle and micro-macro computations. Derivation of reduced (macroscopic) dynamics from the microscopic dynamics is the dominant theme of nonequilibrium statistical mechanics. At the present time, this very old theme demonstrates new facets in view of a massive use of simulation techniques on various levels of description. A two-side benefit of this use is expected: on the one hand, simulations provide data on molecular systems which can be used to test various theoretical constructions about the transition from micro to macro description. On the other hand, while the microscopic simulations in many cases are based on limit theorems [such as, for example, the central limit theorem underlying the Direct Brownian Dynamics simulations (BD)] they are extremely time-consuming in any real situation, and a timely recognition of the onset of a macroscopic description may considerably reduce computational efforts.

In this section, we aim at developing a "device" which is able to recognize the onset and the breakdown of a macroscopic description in the course of microscopic computations.

Let us first present the main ideas of the construction in an abstract setting. We assume that the microscopic description is set up in terms of microscopic variables $\xi$. In the examples considered 
below, microscopic variables are distribution functions over the configuration space of polymers. The microscopic dynamics of variables $\xi$ is given by the microscopic time derivative $\mathrm{d} \xi / \mathrm{d} t=\dot{\xi}(\xi)$. We also assume that the set of macroscopic variables $\boldsymbol{M}$ is chosen. Typically, the macroscopic variables are some lower-order moments if the microscopic variables are distribution functions. The reduced (macroscopic) description assumes (a) The dependence $\xi(\boldsymbol{M})$, and (b) The macroscopic dynamics $\mathrm{d} \boldsymbol{M} / \mathrm{d} t=\dot{\boldsymbol{M}}(\boldsymbol{M})$. We do not discuss here in any detail the way one gets the dependence $\xi(\boldsymbol{M})$, however, we should remark that, typically, it is based on some (explicit or implicit) idea about decomposition of motions into slow and fast, with $M$ as slow variables. With this, such tools as maximum entropy principle, quasi-stationarity, cumulant expansion etc. become available for constructing the dependence $\xi(\boldsymbol{M})$.

Let us compare the microscopic time derivative of the function $\xi(\boldsymbol{M})$ with its macroscopic time derivative due to the macroscopic dynamics:

$$
\Delta(\boldsymbol{M})=\frac{\partial \xi(\boldsymbol{M})}{\partial \boldsymbol{M}} \cdot \dot{\boldsymbol{M}}(\boldsymbol{M})-\dot{\xi}(\xi(\boldsymbol{M}))
$$

If the defect of invariance $\Delta(\boldsymbol{M})(572)$ is equal to zero on the set of admissible values of the macroscopic variables $M$, it is said that the reduced description $\xi(\boldsymbol{M})$ is invariant. Then the function $\xi(\boldsymbol{M})$ represents the invariant manifold in the space of microscopic variables. The invariant manifold is relevant if it is stable. Exact invariant manifolds are known in a very few cases (for example, the exact hydrodynamic description in the kinetic Lorentz gas model [159], in Grad's systems [23,24], and one more example will be mentioned below). Corrections to the approximate reduced description through minimization of the defect of invariance is a part of the so-called method of invariant manifolds [6]. We here consider a different application of the invariance principle for the purpose mentioned above.

The time dependence of the macroscopic variables can be obtained in two different ways: First, if the solution of the microscopic dynamics at time $t$ with initial data at $t_{0}$ is $\xi_{t, t_{0}}$, then evaluation of the macroscopic variables on this solution gives $\boldsymbol{M}_{t, t_{0}}^{\text {micro }}$. On the other hand, solving dynamic equations of the reduced description with initial data at $t_{0}$ gives $\boldsymbol{M}_{t, t_{0}}^{\text {macro }}$. Let $\|\Delta\|$ be a value of defect of invariance with respect to some norm, and $\epsilon>0$ is a fixed tolerance level. Then, if at the time $t$ the following inequality is valid,

$$
\left\|\Delta\left(\boldsymbol{M}_{t, t_{0}}^{\text {micro }}\right)\right\|<\epsilon,
$$

this indicates that the accuracy provided by the reduced description is not worse than the true microscopic dynamics (the macroscopic description sets on). On the other hand, if

$$
\left\|\Delta\left(\boldsymbol{M}_{t, t_{0}}^{\text {macro }}\right)\right\|>\epsilon,
$$

then the accuracy of the reduced description is insufficient (the reduced description breaks down), and we must use the microscopic dynamics.

Thus, evaluating the defect of invariance (572) on the current solution to macroscopic equations, and checking the inequality (574), we are able to answer the question whether we can trust the solution without looking at the microscopic solution. If the tolerance level is not exceeded then we can safely integrate the macroscopic equation. We now proceed to a specific example of this approach. We consider a well-known class of microscopic models of dilute polymeric solutions. 


\section{Application to dynamics of dilute polymer solution}

A well-known problem of the nonNewtonian fluids is the problem of establishing constitutive equations on the basis of microscopic kinetic equations. We here consider a model introduced by Lielens et al. [272]:

$$
\dot{f}(q, t)=-\partial_{q}\left\{\kappa(t) q f-\frac{1}{2} f \partial_{q} U\left(q^{2}\right)\right\}+\frac{1}{2} \partial_{q}^{2} f .
$$

With the potential $U(x)=-(b / 2) \ln (1-x / b)$ Eq. (575) becomes the one-dimensional version of the FENE dumbbell model which is used to describe the elongational behavior of dilute polymer solutions.

The reduced description seeks a closed time evolution equation for the stress $\tau=\left\langle q \partial_{q} U\left(q^{2}\right)\right\rangle-1$. Due to its nonpolynomial character, the stress $\tau$ for the FENE potential depends on all moments of $f$. We have shown in [273] how such potentials can be approximated systematically by a set of polynomial potentials $U_{n}(x)=\sum_{j=1}^{n} 1 / 2 j c_{j} x^{j}$ of degree $n$ with coefficients $c_{j}$ depending on the even moments $M_{j}=\left\langle q^{2 j}\right\rangle$ of $f$ up to order $n$, with $n=1,2, \ldots$, formally converging to the original potential as $n$ tends to infinity. In this approximation, the stress $\tau$ becomes a function of the first $n$ even moments of $f, \tau(\boldsymbol{M})=\sum_{j=1}^{n} c_{j} M_{j}-1$, where the set of macroscopic variables is denoted by $\boldsymbol{M}=\left\{M_{1}, \ldots, M_{n}\right\}$.

The first two potentials approximating the FENE potential are:

$$
\begin{aligned}
& U_{1}\left(q^{2}\right)=U^{\prime}\left(M_{1}\right) q^{2}, \\
& U_{2}\left(q^{2}\right)=\frac{1}{2}\left(q^{4}-2 M_{1} q^{2}\right) U^{\prime \prime}\left(M_{1}\right)+\frac{1}{2}\left(M_{2}-M_{1}^{2}\right) q^{2} U^{\prime \prime \prime}\left(M_{1}\right),
\end{aligned}
$$

where $U^{\prime}, U^{\prime \prime}$ and $U^{\prime \prime \prime}$ denote the first, second and third derivative of the potential $U$, respectively. The potential $U_{1}$ corresponds to the well-known FENE-P model. The kinetic equation (575) with the potential $U_{2}$ (577) will be termed the FENE-P +1 model below. Direct Brownian Dynamics simulation (BD) of the kinetic equation (575) with the potential $U_{2}$ for the flow situations studied in [272] demonstrates that it is a reasonable approximation to the true FENE dynamics whereas the corresponding moment chain is of a simpler structure. In [16] this was shown for a periodic flow, while Fig. 24 shows results for the flow

$$
\kappa(t)= \begin{cases}100 t(1-t) \mathrm{e}^{-4 t}, & 0 \leqslant t \leqslant 1, \\ 0, & \text { else } .\end{cases}
$$

The quality of the approximation indeed increases with the order of the polynomial.

For any potential $U_{n}$, the invariance equation can be studied directly in terms of the full set of the moments, which is equivalent to studying the distribution functions. The kinetic equation (575) can be rewritten equivalently in terms of moment equations,

$$
\begin{aligned}
& \dot{M}_{k}=F_{k}\left(M_{1}, \ldots, M_{k+n-1}\right), \\
& F_{k}=2 k \kappa(t) M_{k}+k(2 k-1) M_{k-1}-k \sum_{j=1}^{n} c_{j} M_{k+j-1} .
\end{aligned}
$$




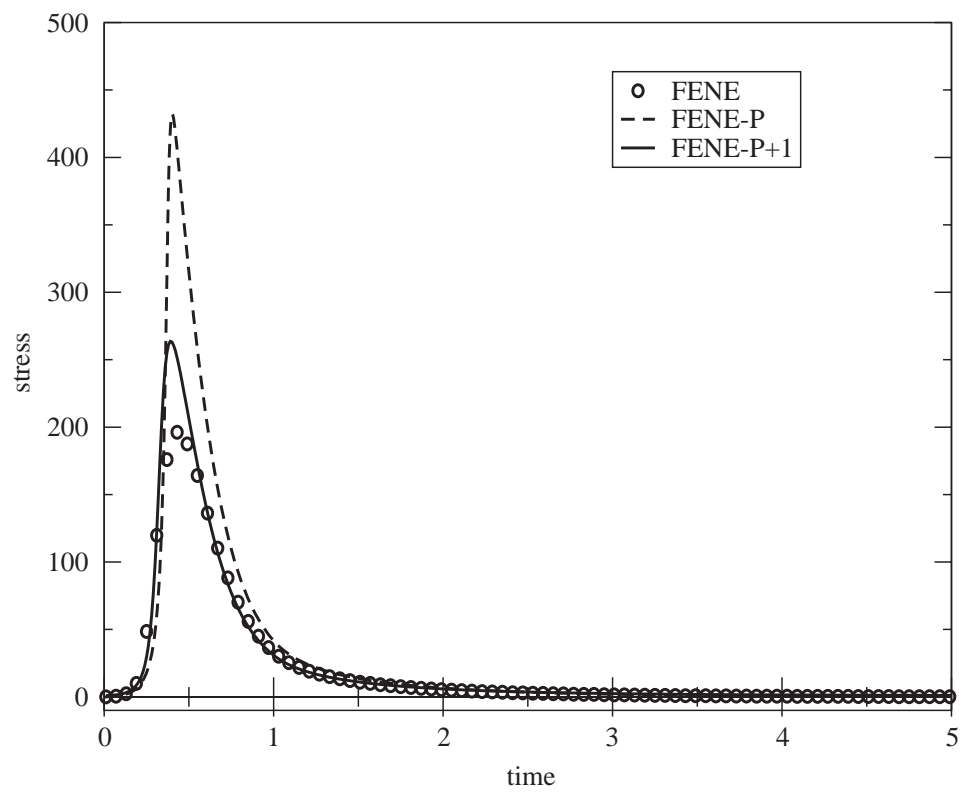

Fig. 24. Stress $\tau$ versus time from direct Brownian dynamics simulation: symbols-FENE, dashed line-FENE-P, solid line-FENE-P +1 .

We seek functions $M_{k}^{\text {macro }}(\boldsymbol{M}), k=n+1, \ldots$ which are form-invariant under the dynamics:

$$
\sum_{j=1}^{n} \frac{\partial M_{k}^{\text {macro }}(\boldsymbol{M})}{\partial M_{j}} F_{j}(\boldsymbol{M})=F_{k}\left(M_{1}, \ldots, M_{n}, M_{n+1}(\boldsymbol{M}), \ldots, M_{n+k}(\boldsymbol{M})\right) .
$$

This set of invariance equations states the following: The time derivative of the form $M_{k}^{\text {macro }}(\boldsymbol{M})$ when computed due to the closed equation for $\boldsymbol{M}$ (the first contribution on the left hand side of Eq. (580), or the 'macroscopic' time derivative) equals the time derivative of $M_{k}$ as computed by true moment equation with the same form $M_{k}(\boldsymbol{M})$ (the second contribution, or the 'microscopic' time derivative), and this equality should hold whatsoever values of the moments $\boldsymbol{M}$ are.

Eqs. (580) in case $n=1$ (FENE-P) are solvable exactly with the result

$$
M_{k}^{\text {macro }}=a_{k} M_{1}^{k} \quad \text { with } a_{k}=(2 k-1) a_{k-1}, a_{0}=1 .
$$

This dependence corresponds to the Gaussian solution in terms of the distribution functions. As expected, the invariance principle give just the same result as the usual method of solving the FENE-P model.

Let us briefly discuss the potential $U_{2}$, considering a simple closure approximation

$$
M_{k}^{\text {macro }}\left(M_{1}, M_{2}\right)=a_{k} M_{1}^{k}+b_{k} M_{2} M_{1}^{k-2},
$$

where $a_{k}=1-k(k-1) / 2$ and $b_{k}=k(k-1) / 2$. The function $M_{3}^{\text {macro }}$ closes the moment equations for

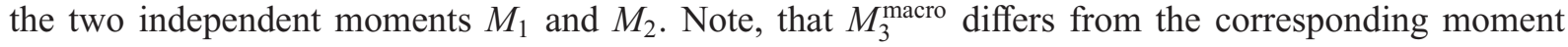
$M_{3}$ of the actual distribution function by the neglect of the 6th cumulant. The defect of invariance 


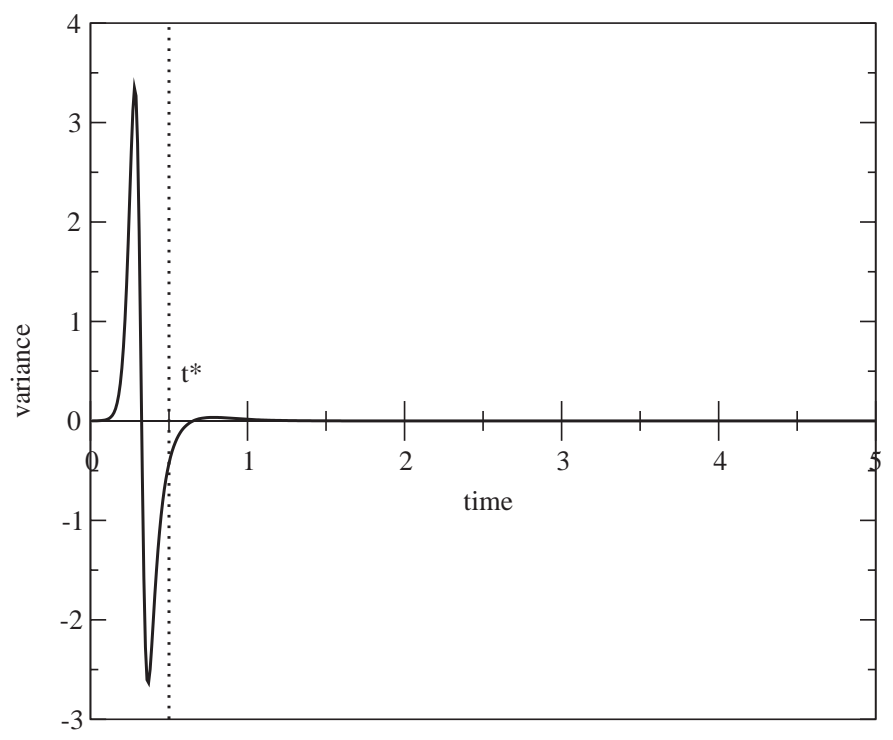

Fig. 25. Defect of invariance $\Delta_{3} / b^{3}$, Eq. (582), versus time extracted from BD simulation (the FENE-P +1 model) for the flow situation of Eq. (578).

of this approximation is a set of functions $\Delta_{k}$ where

$$
\Delta_{3}\left(M_{1}, M_{2}\right)=\frac{\partial M_{3}^{\text {macro }}}{\partial M_{1}} F_{1}+\frac{\partial M_{3}^{\text {macro }}}{\partial M_{2}} F_{2}-F_{3},
$$

and analogously for $k \geqslant 3$. In the sequel, we make all conclusions based on the defect of invariance $\Delta_{3}(582)$.

It is instructive to plot the defect of invariance $\Delta_{3}$ versus time, assuming the functions $M_{1}$ and $M_{2}$ are extracted from the BD simulation (see Fig. 25). We observe that the defect of invariance is a nonmonotonic function of the time, and that there are three pronounced domains: From $t_{0}=0-t_{1}$ the defect of invariance is almost zero which means that the ansatz is reasonable. In the intermediate domain, the defect of invariance jumps to high values (so the quality of approximation is poor). However, after some time $t=t^{*}$, the defect of invariance again becomes negligible, and remains so for later times. Such behavior is typical of so-called "kinetic layer".

Instead of attempting to improve the closure, the invariance principle can be used directly to switch from the BD simulation to the solution of the macroscopic equation without loosing the accuracy to a given tolerance. Indeed, the defect of invariance is a function of $M_{1}$ and $M_{2}$, and it can be easily evaluated both on the data from the solution to the macroscopic equation, and the BD data. If the defect of invariance exceeds some given tolerance on the macroscopic solution this signals to switch to the $\mathrm{BD}$ integration. On the other hand, if the defect of invariance becomes less than the tolerance level on the $\mathrm{BD}$ data signals that the $\mathrm{BD}$ simulation is not necessary anymore, and one can continue with the integration of the macroscopic equations. This reduces the necessity of using BD simulations only to get through the kinetic layers. A realization of this hybrid approach is demonstrated in Fig. 26: for the same flow we have used the BD dynamics only for the first period of the flow while integrated the macroscopic equations in all the later times. The quality of the result is comparable to 


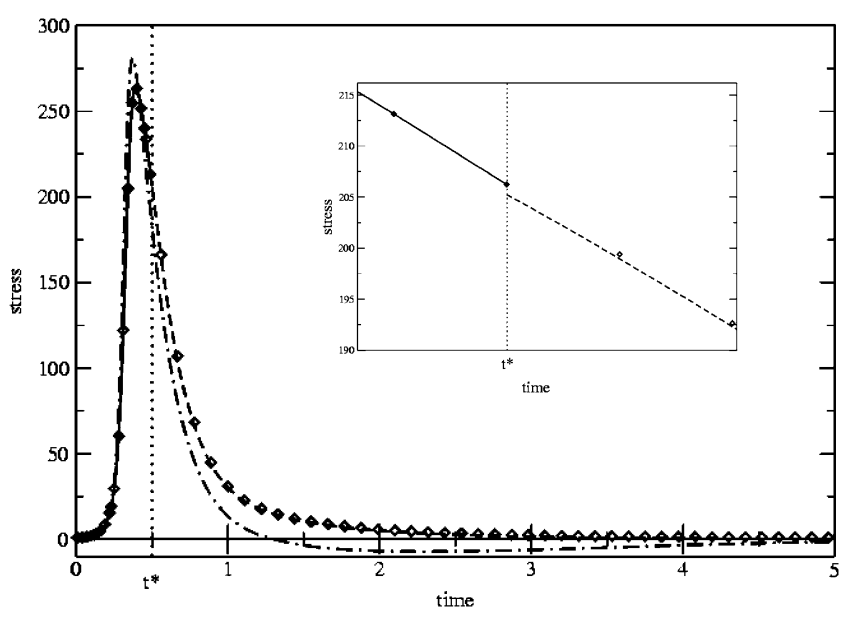

Fig. 26. Switching from the BD simulations to macroscopic equations after the defect of invariance has reached the given tolerance level (the FENE-P+1 model): symbols - the BD simulation, solid line- the BD simulation from time $t=0$ up to time $t^{*}$, dashed line - integration of the macroscopic dynamics with initial data from BD simulation at time $t=t^{*}$. For comparison, the dot-dashed line gives the result for the integration of the macroscopic dynamics with equilibrium conditions from $t=0$. Inset: Transient dynamics at the switching from BD to macroscopic dynamics on a finer time scale.

the BD simulation whereas the total integration time is much shorter. The transient dynamics at the point of switching from the BD scheme to the integration of the macroscopic equations (shown in the inset in Fig. 26) deserves a special comment: The initial conditions at $t^{*}$ are taken from the BD data. Therefore, we cannot expect that at the time $t^{*}$ the solution is already on the invariant manifold, rather, at best, close to it. Transient dynamics therefore signals the stability of the invariant manifold we expect: Even though the macroscopic solution starts not on this manifold, it nevertheless attracts to it. The transient dynamics becomes progressively less pronounced if the switching is done at later times. The stability of the invariant manifold in case of the FENE-P model is studied in detail in [84].

The present approach of combined microscopic and macroscopic simulations can be realized on the level of moment closures (which then needs reconstruction of the distribution function from the moments at the switching from macroscopic integration to BD procedures), or for parametric sets of distribution functions if they are available [272]. It can be used for a rigorous construction of domain decomposition methods in various kinetic problems.

\section{Conclusion}

To construct slow invariant manifolds is useful. Effective model reduction becomes impossible without them for complex kinetic systems.

Why to reduce description in the times of supercomputers?

First, in order to gain understanding. In the process of reducing the description one is often able to extract the essential, and the mechanisms of the processes under study become more transparent.

Second, if one is given the detailed description of the system, then one should be able also to solve the initial-value problem for this system. But what should one do in the case where the system 
is representing just a small part of the huge number of interacting systems? For example, a complex chemical reaction system may represent only a point in a three-dimensional flow.

Third, without reducing the kinetic model, it is impossible to construct this model. This statement seems paradoxal only at the first glance: How come, the model is first simplified, and is constructed only after the simplification is done? However, in practice, the typical for a mathematician statement of the problem, (Let the system of differential equations be given, then ...) is rather rarely applicable for detailed kinetics. Quite on the contrary, the thermodynamic data (energies, enthalpies, entropies, chemical potentials etc) for sufficiently rarefied systems are quite reliable. Final identification of the model is always done on the basis of comparison with the experiment and with a help of fitting. For this purpose, it is extremely important to reduce the dimension of the system, and to reduce the number of tunable parameters.

And, finally, for every supercomputer there exist too complicated problems. Model reduction makes these problems less complicated and sometimes gives us the possibility to solve them.

It is useful to apply thermodynamics and the quasi-equilibrium concept while seeking slow invariant manifolds

Though the open systems are important for many applications, however, it is useful to begin their study and model reduction with the analysis of closed (sub)systems. Then the thermodynamics equips these systems with Lyapunov functions (entropy, free energy, free enthalpy, depending on the context). These Lyapunov functions are usually known much better than the right hand sides of kinetic equations (in particular, this is the case in reaction kinetics). Using this Lyapunov function, one constructs the initial approximation to the slow manifold, that is, the quasi-equilibrium manifold, and also one constructs the thermodynamic projector.

The thermodynamic projector is the unique operator which transforms the arbitrary vector field equipped with the given Lyapunov function into a vector field with the same Lyapunov function (and also this happens on any manifold which is not tangent to the level of the Lyapunov function).

The quasi-chemical approximation is an extremely rich toolbox for assembling equations. It enables to construct and study wide classes of evolution equations equipped with prescribed Lyapunov functions, with Onsager reciprocity relations and like.

Slow invariant manifolds of thermodynamically closed systems are useful for constructing slow invariant manifolds of corresponding open systems. The necessary technic is developed.

Postprocessing of the invariant manifold construction is important both for estimation of the accuracy and for the accuracy improvement.

The main result of this work can be formulated as follows: It is possible indeed to construct invariant manifolds. The problem of constructing invariant manifolds can be formulated as the invariance equation, subject to additional conditions of slowness (stability). The Newton method with incomplete linearization, relaxation methods, the method of natural projector, and the method of invariant grids enables educated approximations to the slow invariant manifolds.

Studies on invariant manifolds were initiated by Lyapunov [144] and Poincare [145] (see [146]). Essential stages of the development of these ideas in the XX century are reflected in the books $[146,278,136,235]$. It becomes more and more evident at the present time that the constructive methods of invariant manifold are useful on a host of subjects, from applied hydrodynamics [279] to physical and chemical kinetics. 


\section{Acknowledgements}

First of all, we are grateful to our coauthors: M.S. S. Ansumali (Zürich), Prof. V.I. Bykov (Krasnoyarsk), Prof. M. Deville (Lausanne), Dr. G. Dukek (Ulm), Dr. P. Ilg (Zürich-Berlin), Prof. T.F. Nonnenmacher (Ulm), Prof. H.C. Öttinger (Zürich), M.S. P.A. Gorban (Krasnoyarsk-OmskZürich), M.S. A. Ricksen (Zürich), Prof. S. Succi (Roma), Dr. L.L. Tatarinova (Krasnoyarsk-Zürich), Prof. G.S. Yablonskii (Novosibirsk-Saint-Louis), Dr. V.B. Zmievskii (Krasnoyarsk-LausanneMontreal) for years of collaboration, stimulating discussion and support. We thank Prof. M. Grmela (Montreal) for detailed and encouraging discussion of the geometrical foundations of nonequilibrium thermodynamics. Prof. M. Shubin (Moscow-Boston) explained us some important chapters of the pseudodifferential operators theory. Finally, it is our pleasure to thank Prof. Misha Gromov (IHES, Bures-sur-Yvette) for encouragement and the spirit of Geometry.

\section{References}

[1] N.G. Van Kampen, Elimination of fast variables, Phys. Rep. 124 (1985) 69-160.

[2] N.N. Bogolyubov, Dynamic Theory problems in Statistical Physics, Gostekhizdat, Moscow, Leningrad, 1946.

[3] A.J. Roberts, Low-dimensional modelling of dynamical systems applied to some dissipative fluid mechanics, in: R. Ball, N. Akhmediev (Eds.), Nonlinear Dynamics from Lasers to Butterflies, Lecture Notes in Complex Systems, Vol. 1, World Scientific, Singapore, 2003, pp. 257-313.

[4] A.N. Gorban, I.V. Karlin, The constructing of invariant manifolds for the Boltzmann equation, Adv. Model. Anal. C 33 (3) (1992) 39-54.

[5] A.N. Gorban, I.V. Karlin, Thermodynamic parameterization, Physica A 190 (1992) 393-404.

[6] A.N. Gorban, I.V. Karlin, Method of invariant manifolds and regularization of acoustic spectra, Transp. Theory Stat. Phys. 23 (1994) 559-632.

[7] I.V. Karlin, G. Dukek, T.F. Nonnenmacher, Invariance principle for extension of hydrodynamics: nonlinear viscosity, Phys. Rev. E 55 (2) (1997) 1573-1576.

[8] V.B. Zmievskii, I.V. Karlin, M. Deville, The universal limit in dynamics of dilute polymeric solutions, Physica A 275 (1-2) (2000) 152-177.

[9] I.V. Karlin, A.N. Gorban, G. Dukek, T.F. Nonnenmacher, Dynamic correction to moment approximations, Phys. Rev. E 57 (1998) 1668-1672.

[10] A.N. Gorban, I.V. Karlin, Method of invariant manifold for chemical kinetics, Chem. Eng. Sci. 58 (21) (2003) 4751-4768, Preprint online: http://arxiv.org/abs/cond-mat/0207231.

[11] A.N. Gorban, I.V. Karlin, V.B. Zmievskii, S.V. Dymova, Reduced description in reaction kinetics, Physica A 275 (3-4) (2000) 361-379.

[12] I.V. Karlin, V.B. Zmievskii, Invariant closure for the Fokker-Planck equation, 1998. Preprint online: http://arxiv.org/abs/adap-org/9801004.

[13] C. Foias, M.S. Jolly, I.G. Kevrekidis, G.R. Sell, E.S. Titi, On the computation of inertial manifolds, Phys. Lett. A $131(7-8)(1988)$ 433-436.

[14] A.N. Gorban, I.V. Karlin, V.B. Zmievskii, T.F. Nonnenmacher, Relaxational trajectories: global approximations, Physica A 231 (1996) 648-672.

[15] A.N. Gorban, I.V. Karlin, V.B. Zmievskii, Two-step approximation of space-independent relaxation, Transp. Theory Stat. Phys. 28 (3) (1999) 271-296.

[16] A.N. Gorban, I.V. Karlin, P. Ilg, H.C. Öttinger, Corrections and enhancements of quasi-equilibrium states, J. Non-Newtonian Fluid Mech. 96 (2001) 203-219.

[17] A.N. Gorban, I.V. Karlin, H.C. Öttinger, L.L. Tatarinova, Ehrenfests argument extended to a formalism of nonequilibrium thermodynamics, Phys. Rev. E 63 (2001) 066124.

[18] A.N. Gorban, I.V. Karlin, Reconstruction lemma and fluctuation-dissipation theorem, Rev. Mex. Fis. 48 (Suppl. 1) (2002) 238-242. 
[19] A.N. Gorban, I.V. Karlin, Macroscopic dynamics through coarse-graining: a solvable example, Phys. Rev. E 56 (2002) 026116.

[20] A.N. Gorban, I.V. Karlin, Geometry of irreversibility, in: F. Uribe (Ed.), Recent Developments in Mathematical and Experimental Physics, Vol. C, Hydrodynamics and Dynamical Systems, Kluwer, Dordrecht, 2002, pp. 19-43.

[21] I.V. Karlin, L.L. Tatarinova, A.N. Gorban, H.C. Öttinger, Irreversibility in the short memory approximation, Physica A 327 (3-4) (2003) 399-424, Preprint online: http://arXiv.org/abs/cond-mat/0305419 v1 18 May 2003.

[22] I.V. Karlin, A. Ricksen, S. Succi, Dissipative quantum dynamics from Wigner distributions, in: Quantum Limits to the Second Law: First International Conference on Quantum Limits to the Second Law, San Diego, California (USA), 29-31 July 2002; AIP Conf. Proc. 643 (2002) 19-24.

[23] A.N. Gorban, I.V. Karlin, Short-wave limit of hydrodynamics: a soluble example, Phys. Rev. Lett. 77 (1996) 282-285.

[24] I.V. Karlin, A.N. Gorban, Hydrodynamics from Grad's equations: what can we learn from exact solutions? Ann. Phys. (Leipzig) 11 (2002) 783-833, Preprint online: http://arXiv.org/abs/cond-mat/0209560.

[25] A.N. Gorban, I.V. Karlin, Structure and approximations of the Chapman-Enskog expansion, Sov. Phys. JETP 73 (1991) 637-641.

[26] A.N. Gorban, I.V. Karlin, Structure and approximations of the Chapman-Enskog expansion for linearized Grad equations, Transp. Theory Stat. Phys. 21 (1992) 101-117.

[27] I.V. Karlin, Simplest nonlinear regularization, Transp. Theory Stat. Phys. 21 (1992) 291-293.

[28] A.N. Kolmogorov, On conservation of conditionally periodic motions under small perturbations of the Hamiltonian, Dokl. Akad. Nauk SSSR 98 (1954) 527-530.

[29] V.I. Arnold, Proof of a theorem of A N Kolmogorov on the invariance of quasi-periodic motions under small perturbations of the Hamiltonian, Russ. Math. Surv. 18 (1963) 9-36 (English translation).

[30] J. Moser, Convergent series expansions for quasi-periodic motions, Math. Ann. 169 (1967) 136-176.

[31] D.A. Jones, A.M. Stuart, E.S. Titi, Persistence of invariant sets for dissipative evolution equations, J. Math. Anal. Appl. 219 (2) (1998) 479-502.

[32] W.-J. Beyn, W. Kless, Numerical Taylor expansions of invariant manifolds in large dynamical systems, Numer. Math. 80 (1998) 1-38.

[33] N. Kazantzis, Singular PDEs and the problem of finding invariant manifolds for nonlinear dynamical systems, Phys. Lett. A 272 (4) (2000) 257-263.

[34] D.V. Shirkov, V.F. Kovalev, Bogoliubov renormalization group and symmetry of solution in mathematical physics, Phys. Rep. 352 (2001) 219-249, Preprint online: http://arxiv.org/abs/hep-th/0001210.

[35] J. Zinn-Justin, Quantum Field Theory and Critical Phenomena, Clarendon Press, Oxford, 1989.

[36] O. Pashko, Y. Oono, The Boltzmann equation is a renormalization group equation, Int. J. Mod. Phys. B 14 (2000) 555-561.

[37] T. Kunihiro, A geometrical formulation of the renormalization group method for global analysis, Prog. Theor. Phys. 94 (1995) 503-514 Erratum: ibid. 95 (1996) 835. Preprint online: http://arxiv.org/abs/hep-th/9505166.

[38] S.-I. Ei, K. Fujii, T. Kunihiro, Renormalization-group method for reduction of evolution equations; invariant manifolds and envelopes, Ann. Phys. 280 (2000) 236-298, Preprint online: http://arxiv.org/abs/hep-th/9905088.

[39] Y. Hatta, T. Kunihiro, Renormalization group method applied to kinetic equations: roles of initial values and time, Ann. Phys. 298 (2002) 24-57, Preprint online: http://arxiv.org/abs/hep-th/0108159.

[40] A. Degenhard, J. Rodrigues-Laguna, Towards the evaluation of the relevant degrees of freedom in nonlinear partial differential equations, J. Stat. Phys. 106 (516) (2002) 1093-1119.

[41] D. Forster, D.R. Nelson, M.J. Stephen, Long-time tails and the large-eddy behavior of a randomly stirred fluid, Phys. Rev. Lett. 36 (1976) 867-870.

[42] D. Forster, D.R. Nelson, M.J. Stephen, Large-distance and long-time properties of a randomly stirred fluid, Phys. Rev. A 16 (1977) 732-749.

[43] L.Ts. Adzhemyan, N.V. Antonov, M.V. Kompaniets, A.N. Vasil'ev, Renormalization-group approach to the stochastic Navier Stokes equation: two-loop approximation, Int. J. Mod. Phys. B 17 (10) (2003) 2137-2170.

[44] H. Chen, S. Succi, S. Orszag, Analysis of subgrid scale turbulence using Boltzmann Bhatnagar-Gross-Krook kinetic equation, Phys. Rev. E 59 (1999) R2527-R2530.

[45] H. Chen, S. Kandasamy, S. Orszag, R. Shock, S. Succi, V. Yakhot, Extended Boltzmann kinetic equation for turbulent flows, Science 301 (2003) 633-636. 
[46] P. Degond, M. Lemou, Turbulence models for incompressible fluids derived from kinetic theory, J. Math. Fluid Mech. 4 (3) (2002) 257-284.

[47] S. Ansumali, I.V. Karlin, S. Succi, Kinetic theory of turbulence modeling: smallness parameter, scaling and microscopic derivation of Smagorinsky model, Physica A (2004) to appear. Preprint online: http://arxiv.org/abs/cond-mat/0310618.

[48] J. Smagorinsky, General circulation experiments with the primitive equations: I. The basic equations, Mon. Weather Rev. 91 (1963) 99-164.

[49] J. Bricmont, K. Gawedzki, A. Kupiainen, KAM theorem and quantum field theory, Commun. Math. Phys. 201 (1999) 699-727, E-print mp_arc 98-526, online: http://mpej.unige.ch/mp_arc/c/98/98-517.ps.gz.

[50] A.N. Gorban, I.V. Karlin, Methods of nonlinear kinetics, in: Encyclopedia of Life Support Systems, EOLSS Publishers, Oxford, 2004, http://www.eolss.net. Preprint online: http://arXiv.org/abs/cond-mat/0306062.

[51] S. Chapman, T. Cowling, Mathematical Theory of Non-uniform Gases, 3rd Edition, Cambridge University Press, Cambridge, 1970.

[52] D. Hilbert, Begründung der kinetischen Gastheorie, Math. Ann. 72 (1912) 562-577.

[53] A.V. Bobylev, The Chapman-Enskog and Grad methods for solving the Boltzmann equation, Sov. Phys. Dokl. 27 (1) (1982) 29-31.

[54] L.S. Garca-Coln, M.S. Green, F. Chaos, The Chapman-Enskog solution of the generalized Boltzmann equation, Physica 32 (2) (1966) 450-478.

[55] J.R. Bowen, A. Acrivos, A.K. Oppenheim, Singular perturbation refinement to quasi-steady state approximation in chemical kinetics, Chem. Eng. Sci. 18 (1963) 177-188.

[56] L.A. Segel, M. Slemrod, The quasi-steady-state assumption: a case study in perturbation, SIAM Rev. 31 (1989) 446-477.

[57] S.J. Fraser, The steady state and equilibrium approximations: a geometrical picture, J. Chem. Phys. 88 (8) (1988) 4732-4738.

[58] M.R. Roussel, S.J. Fraser, Geometry of the steady-state approximation: perturbation and accelerated convergence methods, J. Chem. Phys. 93 (1990) 1072-1081.

[59] G.S. Yablonskii, V.I. Bykov, A.N. Gorban, V.I. Elokhin, Kinetic models of catalytic reactions, in: R.G. Compton (Ed.), Comprehensive Chemical Kinetics, Vol. 32, Elsevier, Amsterdam, 1991.

[60] A.B. Vasil'eva, V.F. Butuzov, L.V. Kalachev, The Boundary Function Method for Singular Perturbation Problems, SIAM, Philadelphia, PA, 1995.

[61] V.V. Strygin, V.A. Sobolev, Spliting of Motion by Means of Integral Manifolds, Nauka, Moscow, 1988.

[62] H.G. Roos, M. Stynes, L. Tobiska, Numerical Methods for Singularly Perturbed Differential Equations: Convection-Diffusion and Flow Problems, Springer, Berlin, 1996.

[63] E.F. Mishchenko, Y.S. Kolesov, A.U. Kolesov, N.Kh. Rozov, Asymptotic Methods in Singularly Perturbed Systems, Consultants Bureau, New York, 1994.

[64] I.V. Novozhilov, Fractional Analysis: Methods of Motion Decomposition, Birkhäuser, Boston, 1997.

[65] A. Milik, Singular perturbation on the Web, 1997. ik/singdir.html\#geo.sing.

[66] C.W. Gear, Numerical Initial Value Problems in Ordinary Differential Equations, Prentice-Hall, Englewood Cliffs, NJ, 1971.

[67] H. Rabitz, M. Kramer, D. Dacol, Sensitivity analysis in chemical kinetics, Ann. Rev. Phys. Chem. 34 (1983) 419-461.

[68] S.H. Lam, D.A. Goussis, The CSP method for simplifying kinetics, Int. J. Chem. Kinet. 26 (1994) 461-486.

[69] U. Maas, S.B. Pope, Simplifying chemical kinetics: intrinsic low-dimensional manifolds in composition space, Combust. Flame 88 (1992) 239-264.

[70] H.G. Kaper, T.J. Kaper, Asymptotic analysis of two reduction methods for systems of chemical reactions, Physica D 165 (2002) 66-93.

[71] A. Zagaris, H.G. Kaper, T.J. Kaper, Analysis of the computational singular perturbation reduction method for chemical kinetics, J. Nonlinear Sci. 14(1) (2004) 59-91. Preprint on-line: http://arxiv.org/abs/math.DS/0305355.

[72] A. Debussche, R. Temam, Inertial manifolds and slow manifolds, Appl. Math. Lett. 4 (4) (1991) 73-76.

[73] C. Foias, G. Prodi, Sur le comportement global des solutions non stationnaires des equations de Navier-Stokes en dimension deux, Rend. Sem. Mat. Univ. Padova. 39 (1967) 1-34.

[74] O.A. Ladyzhenskaya, A dynamical system generated by Navier-Stokes equations, J. Sov. Math. 3 (1975) 458-479. 
[75] I.D. Chueshov, Theory of functionals that uniquely determine the asymptotic dynamics of infinite-dimensional dissipative systems, Russian Math. Surv. 53 (4) (1998) 731-776.

[76] I.D. Chueshov, Introduction to the theory of infinite-dimensional dissipative systems, The Electronic Library of Mathematics, 2002, http://rattler.cameron.edu/EMIS/monographs/Chueshov/ (Translated from Russian edition, ACTA Scientific Publishing House, Kharkov, Ukraine, 1999).

[77] M. Dellnitz, O. Junge, Set oriented numerical methods for dynamical systems, in: B. Fiedler, G. Iooss, N. Kopell (Eds.), Handbook of Dynamical Systems II: Towards Applications, World Scientific, Singapore, 2002, pp. 221-264. http://math-www.upb.de/ agdellnitz/papers/handbook.pdf.

[78] M. Dellnitz, A. Hohmann, The computation of unstable manifolds using subdivision and continuation, in: H.W. Broer, et al. (Eds.), Progress in Nonlinear Differential Equations and Their Applications, Vol. 19, Birkhäuser, Basel/Switzerland, 1996, pp. 449-459.

[79] H.W. Broer, H.M. Osinga, G. Vegter, Algorithms for computing normally hyperbolic invariant manifolds, Z. Angew. Math. Phys. 48 (1997) 480-524.

[80] B.M. Garay, Estimates in discretizing normally hyperbolic compact invariant manifolds of ordinary differential equations, Comput. Math. Appl. 42 (2001) 1103-1122.

[81] A.N. Gorban, I.V. Karlin, A.Yu. Zinovyev, Invariant grids for reaction kinetics, Physica A 333 (2004) $106-154$. Preprint online: http:/www.ihes.fr/PREPRINTS/P03/Resu/resu-P03-42.html.

[82] C. Theodoropoulos, Y.H. Qian, I.G. Kevrekidis, Coarse stability and bifurcation analysis using time-steppers: a reaction-diffusion example, Proc. Natl. Acad. Sci. 97 (2000) 9840-9843.

[83] I.G. Kevrekidis, C.W. Gear, J.M. Hyman, P.G. Kevrekidis, O. Runborg, C. Theodoropoulos, Equation-free, coarse-grained multiscale computation: enabling microscopic simulators to perform system-level analysis, Comm. Math. Sci. 1 (4) (2003) 715-762.

[84] P. Ilg, I.V. Karlin, Validity of macroscopic description in dilute polymeric solutions, Phys. Rev. E 62 (2000) $1441-1443$.

[85] P. Ilg, E. De Angelis, I.V. Karlin, C.M. Casciola, S. Succi, Polymer dynamics in wall turbulent flow, Europhys. Lett. 58 (2002) 616-622.

[86] L. Boltzmann, Lectures on Gas Theory, University of California Press, Berkely, CA, 1964.

[87] C. Cercignani, The Boltzmann Equation and its Applications, Springer, New York, 1988.

[88] C. Cercignani, R. Illner, M. Pulvirent, The Mathematical Theory of Dilute Gases, Springer, New York, 1994.

[89] P.L. Bhatnagar, E.P. Gross, M. Krook, A model for collision processes in gases. I. Small amplitude processes in charged and neutral one-component systems, Phys. Rev. 94 (3) (1954) 511-525.

[90] A.N. Gorban, I.V. Karlin, General approach to constructing models of the Boltzmann equation, Physica A 206 (1994) 401-420.

[91] J. Lebowitz, H. Frisch, E. Helfand, Non-equilibrium distribution functions in a fluid, Phys. Fluids 3 (1960) 325.

[92] R.J. DiPerna, P.L. Lions, On the Cauchy problem for Boltzmann equation: global existence and weak stability, Ann. Math. 130 (1989) 321-366.

[93] D. Enskog, Kinetische theorie der Vorange in massig verdunnten Gasen. I Allgemeiner Teil, Almqvist and Wiksell, Uppsala, 1917.

[94] J.E. Broadwell, Study of shear flow by the discrete velocity method, J. Fluid Mech. 19 (1964) $401-414$.

[95] B. Robertson, Equations of motion in nonequilibrium statistical mechanics, Phys. Rev. 144 (1966) 151-161.

[96] G.A. Bird, Molecular Gas Dynamics and the Direct Simulation of Gas Flows, Clarendon Press, Oxford, 1994.

[97] E.S. Oran, C.K. Oh, B.Z. Cybyk, Direct simulation Monte Carlo: recent advances and applications, Annu. Rev. Fluid Mech. 30 (1998) 403-441.

[98] R. Gatignol, Theorie cinetique des gaz a repartition discrete de vitesses, in: Lecture Notes in Physics, Vol. 36, Springer, Berlin, 1975.

[99] U. Frisch, B. Hasslacher, Y. Pomeau, Lattice-gas automata for the Navier-Stokes equation, Phys. Rev. Lett. 56 (1986) 1505-1509.

[100] Gr. Mcnamara, G. Zanetti, Use of the Boltzmann-equation to simulate lattice-gas automata, Phys. Rev. Lett. 61 (1988) 2332-2335.

[101] F. Higuera, S. Succi, R. Benzi, Lattice gas-dynamics with enhanced collisions, Europhys. Lett. 9 (1989) $345-349$. 
[102] R. Benzi, S. Succi, M. Vergassola, The lattice Boltzmann-equation-theory and applications, Phys. Rep. 222 (3) (1992) 145-197.

[103] S. Chen, G.D. Doolen, Lattice Boltzmann method for fluid flows, Annu. Rev. Fluid Mech. 30 (1998) 329-364.

[104] S. Succi, The Lattice Boltzmann Equation for Fluid Dynamics and Beyond, Clarendon Press, Oxford, 2001.

[105] S. Succi, I.V. Karlin, H. Chen, Role of the $H$ theorem in lattice Boltzmann hydrodynamic simulations, Rev. Mod. Phys. 74 (2002) 1203-1220.

[106] I.V. Karlin, A.N. Gorban, S. Succi, V. Boffi, Maximum entropy principle for lattice kinetic equations, Phys. Rev. Lett. 81 (1998) 6-9.

[107] I.V. Karlin, A. Ferrante, H.C. Öttinger, Perfect entropy functions of the Lattice Boltzmann method, Europhys. Lett. 47 (1999) 182-188.

[108] S. Ansumali, I.V. Karlin, Stabilization of the lattice Boltzmann method by the $H$ theorem: a numerical test, Phys. Rev. E 62 (6) (2000) 7999-8003.

[109] S. Ansumali, I.V. Karlin, Entropy function approach to the lattice Boltzmann method, J. Stat. Phys. 107 (1/2) (2002) 291-308.

[110] S. Ansumali, I.V. Karlin, H.C. Öttinger, Minimal entropic kinetic models for hydrodynamics, Europhys. Lett. 63 (2003) 798-804.

[111] N.G. Van Kampen, Stochastic Processes in Physics and Chemistry, North-Holland, Amsterdam, 1981.

[112] H. Risken, The Fokker-Planck equation, Springer, Berlin, 1984.

[113] R.B. Bird, C.F. Curtiss, R.C. Armstrong, O. Hassager, Dynamics of Polymer Liquids, 2nd Edition, Wiley, New York, 1987.

[114] M. Doi, S.F. Edwards, The Theory of Polymer Dynamics, Clarendon Press, Oxford, 1986.

[115] H.C. Öttinger, Stochastic Processes in Polymeric Fluids, Springer, Berlin, 1996.

[116] M. Grmela, H.C. Öttinger, Dynamics and thermodynamics of complex fluids. I. Development of a general formalism, Phys. Rev. E 56 (1997) 6620-6632.

[117] H.C. Öttinger, M. Grmela, Dynamics and thermodynamics of complex fluids. II. Illustrations of a general formalism, Phys. Rev. E 56 (1997) 6633-6655.

[118] S. Kullback, Information Theory and Statistics, Wiley, New York, 1959.

[119] A.R. Plastino, H.G. Miller, A. Plastino, Minimum Kullback entropy approach to the Fokker-Planck equation, Phys. Rev. E 56 (1997) 3927-3934.

[120] A.N. Gorban, I.V. Karlin, Family of additive entropy functions out of thermodynamic limit, Phys. Rev. E 67 (2003) 016104. Preprint online: http://arxiv.org/abs/cond-mat/0205511.

[121] A.N. Gorban, I.V. Karlin, H.C. Öttinger, The additive generalization of the Boltzmann entropy, Phys. Rev. E 67 (2003) 067104. Preprint online: http://arxiv.org/abs/cond-mat/0209319.

[122] P. Gorban, Monotonically equivalent entropies and solution of additivity equation, Physica A (2003) 380-390. Preprint online: http://arxiv.org/pdf/cond-mat/0304131.

[123] C. Tsallis, Possible generalization of Boltzmann-Gibbs statistics, J. Stat. Phys. 52 (1988) 479-487.

[124] S. Abe, Y. Okamoto (Eds.), Nonextensive Statistical Mechanics and its Applications, Springer, Heidelberg, 2001.

[125] A.N. Gorban, Equilibrium encircling. Equations of chemical kinetics and their thermodynamic analysis, Nauka, Novosibirsk, 1984.

[126] G. Dukek, I.V. Karlin, T.F. Nonnenmacher, Dissipative brackets as a tool for kinetic modeling, Physica A 239 (4) (1997) 493-508.

[127] N.N. Orlov, L.I. Rozonoer, The macrodynamics of open systems and the variational principle of the local potential, J. Franklin Inst. 318 (1984) 283-314, 315-347.

[128] A.I. Volpert, S.I. Hudjaev, Analysis in Classes of Discontinuous Functions and the Equations of Mathematical Physics, Nijhoff, Dordrecht, 1985.

[129] S. Ansumali, I.V. Karlin, Single relaxation time model for entropic lattice Boltzmann methods, Phys. Rev. E 65 (2002) 056312.

[130] V.I. Bykov, G.S. Yablonskii, T.A. Akramov, The rate of the free energy decrease in the course of the complex chemical reaction, Dokl. Akad. Nauk USSR 234 (3) (1977) 621-634.

[131] H. Struchtrup, W. Weiss, Maximum of the local entropy production becomes minimal in stationary processes, Phys. Rev. Lett. 80 (1998) 5048-5051. 
[132] M. Grmela, I.V. Karlin, V.B. Zmievski, Boundary layer minimum entropy principles: a case study, Phys. Rev. E 66 (2002) 011201.

[133] V.I. Dimitrov, Simple Kinetics, Nauka, Novosibirsk, 1982.

[134] I. Prigogine, Thermodynamics of Irreversible Processes, Interscience, New York, 1961.

[135] E.M. Lifshitz, L.P. Pitaevskii, Physical kinetics, in: L.D. Landau, E.M. Lifshitz (Eds.), Course of Theoretical Physics, Vol. 10, Pergamon Press, Oxford, 1968.

[136] P. Constantin, C. Foias, B. Nicolaenko, R. Temam, Integral manifolds and inertial manifolds for dissipative partial differential equations, in: Applied Mathematical Science, Vol. 70, Springer, New York, 1988.

[137] J.C. Robinson, A concise proof of the "geometric" construction of inertial manifolds, Phys. Lett. A 200 (1995) 415-417.

[138] L.B. Ryashko, E.E. Shnol, On exponentially attracting invariant manifolds of ODEs, Nonlinearity 16 (2003) 147-160.

[139] W. Walter, An elementary proof of the Cauchy-Kovalevsky theorem, Amer. Math. Monthly 92 (1985) $115-126$.

[140] L.C. Evans, Partial Differential Equations, AMS, Providence, RI, USA, 1998.

[141] Ju.A. Dubinskii, Analytic Pseudo-differential Operators and Their Applications, in: Book Series: Mathematics and its Applications, Soviet Series, Vol. 68, Kluwer Academic Publishers, Dordrecht, 1991.

[142] C.D. Levermore, M. Oliver, Analyticity of solutions for a generalized Euler equation, J. Differential Equations 133 (1997) 321-339.

[143] M. Oliver, E.S. Titi, On the domain of analyticity for solutions of second order analytic nonlinear differential equations, J. Differential Equations 174 (2001) 55-74.

[144] A.M. Lyapunov, The General Problem of the Stability of Motion, Taylor \& Francis, London, 1992.

[145] H. Poincaré, Les méthodes nouvelles de la mécanique céleste, Vols. 1-3. Gauthier-Villars, Paris, 1892/1893/1899.

[146] V.I. Arnold, Geometrical Methods in the Theory of Differential Equations, Springer, New York, Berlin, 1983.

[147] N. Kazantzis, C. Kravaris, Nonlinear observer design using Lyapunovs auxiliary theorem, Systems Control Lett. 34 (1998) 241-247.

[148] A.J. Krener, M. Xiao, Nonlinear observer design in the Siegel domain, SIAM J. Control Optim. 41 (3) (2002) 932-953.

[149] N. Kazantzis, Th. Good, Invariant manifolds and the calculation of the long-term asymptotic response of nonlinear processes using singular PDEs, Comput. Chem. Eng. 26 (2002) 999-1012.

[150] L. Onsager, Reciprocal relations in irreversible processes. I, Phys. Rev. 37 (1931) 405-426 II. Phys. Rev. 38 (1931) 2265-2279.

[151] A. Wehrl, General properties of entropy, Rev. Mod. Phys. 50 (2) (1978) 221-260.

[152] F. Schlögl, Stochastic measures in nonequilibrium thermodynamics, Phys. Rep. 62 (4) (1980) $267-380$.

[153] E.T. Jaynes, Information theory and statistical mechanics, in: K.W. Ford (Ed.), Statistical Physics. Brandeis Lectures, Vol. 3, Benjamin, New York, 1963, pp. 160-185.

[154] H. Grabert, Projection Operator Techniques in Nonequilibrium Statistical Mechanics, Springer, Berlin, 1982.

[155] D. Zubarev, V. Morozov, G. Röpke, Statistical mechanics of nonequilibrium processes, Vol. 1, Basic Concepts, Kinetic Theory, Akademie Verlag, Berlin, 1996, Vol. 2, Relaxation and Hydrodynamic Processes, Akademie Verlag, Berlin, 1997.

[156] M.W. Evans, P. Grigolini, G. Pastori Parravicini (Eds.), Memory function approaches to stochastic problems in condensed matter, Advances in Chemical Physics, Vol. 62, Wiley, New York, 1985.

[157] G.E. Uhlenbeck, in: E.G.D. Cohen (Ed.), Fundamental Problems in Statistical Mechanics, Vol. II, North-Holland, Amsterdam, 1968.

[158] H. Grad, On the kinetic theory of rarefied gases, Comm. Pure Appl. Math. 2 (4) (1949) 331-407.

[159] E.H. Hauge, Exact and Chapman-Enskog solutions of the Boltzmann equation for the Lorentz model, Phys. Fluids 13 (1970) 1201-1208.

[160] U.M. Titulaer, A systematic solution procedure for the Fokker-Planck equation of a Brownian particle in the high-friction case, Physica A 91 (3-4) (1978) 321-344.

[161] M.E. Widder, U.M. Titulaer, Two kinetic models for the growth of small droplets from gas mixtures, Physica A 167 (3) (1990) 663-675.

[162] I.V. Karlin, G. Dukek, T.F. Nonnenmacher, Gradient expansions in kinetic theory of phonons, Phys. Rev. B 55 (1997) 6324-6329. 
[163] I.V. Karlin, Exact summation of the Chapman-Enskog expansion from moment equations, J. Phys. A: Math. Gen. 33 (2000) 8037-8046.

[164] M. Slemrod, Constitutive relations for monatomic gases based on a generalized rational approximation to the sum of the Chapman-Enskog expansion, Arch. Rat. Mech. Anal. 150 (1) (1999) 1-22.

[165] M. Slemrod, Renormalization of the Chapman-Enskog expansion: isothermal fluid flow and Rosenau saturation, J. Stat. Phys. 91 (1-2) (1998) 285-305.

[166] G.W. Gibbs, Elementary Principles of Statistical Mechanics, Dover, New York, 1960.

[167] A.M. Kogan, L.I. Rozonoer, On the macroscopic description of kinetic processes, Dokl. AN SSSR 158 (3) (1964) $566-569$.

[168] A.M. Kogan, Derivation of Grad-type equations and study of their properties by the method of entropy maximization, Prikl. Math. Mech. 29 (1) (1965) 122-133.

[169] L.I. Rozonoer, Thermodynamics of nonequilibrium processes far from equilibrium, Thermodynamics and Kinetics of Biological Processes, Nauka, Moscow, 1980, pp. 169-186.

[170] J. Karkheck, G. Stell, Maximization of entropy, kinetic equations, and irreversible thermodynamics, Phys. Rev. A 25 (6) (1984) 3302-3327.

[171] R.E. Nettleton, E.S. Freidkin, Nonlinear reciprocity and the maximum entropy formalism, Physica A 158 (2) (1989) $672-690$.

[172] J.T. Alvarez-Romero, L.S. Garca-Coln, The foundations of informational statistical thermodynamics revisited, Physica A 232 (1-2) (1996) 207-228.

[173] B.C. Eu, Kinetic Theory and Irreversible Thermodynamics, Wiley, New York, 1992.

[174] N.N. Bugaenko, A.N. Gorban, I.V. Karlin, Universal expansion of the triplet distribution function, Teor. Mat. Fis. 88 (3) (1991) 430-441 (Transl.: Theoret. Math. Phys. (1992) 977-985).

[175] C.D. Levermore, Moment closure hierarchies for kinetic theories, J. Stat. Phys. 83 (1996) 1021-1065.

[176] R. Balian, Y. Alhassid, H. Reinhardt, Dissipation in many-body systems: a geometric approach based on information theory, Phys. Rep. 131 (1) (1986) 1-146.

[177] A.N. Gorban, I.V. Karlin, Uniqueness of thermodynamic projector and kinetic basis of molecular individualism, Physica A 336(3-4) (2004) 391-432. Preprint online: http://arxiv.org/abs/cond-mat/0309638.

[178] A.N. Gorban, I.V. Karlin, Quasi-equilibrium approximation and non-standard expansions in the theory of the Boltzmann kinetic equation, in: R.G. Khlebopros (Ed.), Mathematical Modelling in Biology and Chemistry. New Approaches, Nauka, Novosibirsk, 1991, pp. 69-117, [in Russian].

[179] A.N. Gorban, I.V Karlin, Quasi-equilibrium closure hierarchies for the Boltzmann equation [Translation of the first part of the paper [178]], Preprint, 2003, Preprint online: http://arXiv.org/abs/cond-mat/0305599.

[180] D. Jou, J. Casas-Vázquez, G. Lebon, Extended Irreversible Thermodynamics, Springer, Berlin, 1993.

[181] A. Gorban, I. Karlin, New Methods for Solving the Boltzmann Equations, AMSE Press, Tassin, France, 1994.

[182] J.O. Hirschfelder, C.F. Curtiss, R.B. Bird, Molecular Theory of Gases and Liquids, Wiley, NY, 1954.

[183] J. Dorfman, H. van Beijeren, in: B. Berne (Ed.), Statistical Mechanics B, Plenum, NY, 1977.

[184] P. Résibois, M. De Leener, Classical Kinetic Theory of Fluids, Wiley, NY, 1977.

[185] G. Ford, J. Foch, in: G. Uhlenbeck, J. de Boer (Eds.), Studies in Statistical Mechanics, Vol. 5, North-Holland, Amsterdam, 1970.

[186] P. Van Rysselberge, Reaction rates and affinities, J. Chem. Phys. 29 (3) (1958) 640-642.

[187] M. Feinberg, Chemical kinetics of a certain class, Arch. Rat. Mech. Anal. 46 (1) (1972) 1-41.

[188] V.I. Bykov, A.N. Gorban, G.S. Yablonskii, Description of nonisothermal reactions in terms of Marcelin-de Donder kinetics and its generalizations, React. Kinet. Catal. Lett. 20 (3-4) (1982) 261-265.

[189] T. De Donder, P. Van Rysselberghe, Thermodynamic Theory of Affinity. A Book of Principles, University Press, Stanford, 1936.

[190] I.V. Karlin, On the relaxation of the chemical reaction rate, in: K.I. Zamaraev, G.S. Yablonskii (Eds.), Mathematical Problems of Chemical Kinetics, Nauka, Novosibirsk, 1989, pp. 7-42, [in Russian].

[191] I.V. Karlin, The problem of reduced description in kinetic theory of chemically reacting gas, Model. Measure. Control C 34 (4) (1993) 1-34.

[192] A.N. Gorban, I.V. Karlin, Scattering rates versus moments: alternative Grad equations, Phys. Rev. E 54 (1996) R3109.

[193] F. Treves, Introduction to Pseudodifferential and Fourier Integral Operators, Plenum, NY, 1982. 
[194] M.A. Shubin, Pseudodifferential Operators and Spectral Theory, Nauka, Moscow, 1978.

[195] T. Dedeurwaerdere, J. Casas-Vzquez, D. Jou, G. Lebon, Foundations and applications of a mesoscopic thermodynamic theory of fast phenomena, Phys. Rev. E 53 (1) (1996) 498-506.

[196] R.F. Rodríguez, L.S. García-Colín, M. Lpez de Haro, D. Jou, C. Prez-Garca, The underlying thermodynamic aspects of generalized hydrodynamics, Phys. Lett. A 107 (1) (1985) 17-20.

[197] H. Struchtrup, M. Torrilhon, Regularization of Grad's 13 moment equations: derivation and linear analysis, Phys. Fluids 15 (2003) 2668-2680.

[198] P. Ilg, I.V. Karlin, H.C. Öttinger, Canonical distribution functions in polymer dynamics: I. Dilute solutions of flexible polymers, Physica A 315 (2002) 367-385.

[199] P. Ilg, I.V. Karlin, M. Kröger, H.C. Öttinger, Canonical distribution functions in polymer dynamics: II liquid-crystalline polymers, Physica A 319 (2003) 134-150.

[200] P. Ilg, M. Kröger, Magnetization dynamics, rheology, and an effective description of ferromagnetic units in dilute suspension, Phys. Rev. E 66 (2002) 021501 Erratum, Phys. Rev. E 67 (2003) 049901(E).

[201] P. Ilg, I.V. Karlin, Combined micro-macro integration scheme from an invariance principle: application to ferrofluid dynamics, J. Non-Newtonian Fluid Mech. (2004) to appear. Preprint online: http://arxiv.org/abs/cond-mat/0401383.

[202] R. Courant, K.O. Friedrichs, H. Lewy, On the partial difference equations of mathematical physics, Int. Br. Med. (1967) 215-234.

[203] W.F. Ames, Numerical Methods for Partial Differential Equations, 2nd Edition, Academic Press, New York, 1977.

[204] R.D. Richtmyer, K.W. Morton, Difference Methods for Initial Value Problems, 2nd Edition, Wiley-Interscience, New York, 1967.

[205] A.N. Gorban, A.Yu. Zinovyev, Visualization of data by method of elastic maps and its applications in genomics, economics and sociology, Institut des Hautes Etudes Scientifiques, Preprint. IHES M/01/36. (2001). Online: http://www.ihes.fr/PREPRINTS/M01/Resu/resu-M01-36.html.

[206] I.T. Jolliffe, Principal Component Analysis, Springer, Berlin, 1986.

[207] H.B. Callen, Thermodynamics and an Introduction to Thermostatistics, Wiley, New York, 1985.

[208] Use of Legendre transforms in chemical thermodynamics, IUPAC Technical Report, Prepared for publication by R.A. Alberty (Pure Appl. Chem. 73(8) (2001) 1349-1380. Online: http://www.iupac.org/publications/pac/2001/pdf/ 7308x1349.pdf).

[209] M. Grmela, Reciprocity relations in thermodynamics, Physica A 309 (3-4) (2002) 304-328.

[210] L. Aizenberg, Carleman's formulas in complex analysis: theory and applications, in: Mathematics and its Applications, Vol. 244, Kluwer, Dordrecht, 1993.

[211] A.N. Gorban, A.A. Rossiev, D.C. Wunsch II, Neural network modeling of data with gaps: method of principal curves, Carleman's formula, and other, The talk was given at the USA-NIS Neurocomputing opportunities workshop, Washington DC, July 1999 (Associated with IJCNN'99). Preprint online: http://arXiv.org/abs/cond-mat/0305508.

[212] A.N. Gorban, A.A. Rossiev, Neural network iterative method of principal curves for data with gaps, J. Comput. System Sci. Int. 38 (5) (1999) 825-831.

[213] V.A. Dergachev, A.N. Gorban, A.A. Rossiev, L.M. Karimova, E.B. Kuandykov, N.G. Makarenko, P. Steier, The filling of gaps in geophysical time series by artificial neural networks, Radiocarbon 43 (2A) (2001) 365-371.

[214] A. Gorban, A. Rossiev, N. Makarenko, Y. Kuandykov, V. Dergachev, Recovering data gaps through neural network methods, Int. J. Geomagn. Aeronomy 3 (2) (2002) 191-197.

[215] P. Ehrenfest, Collected Scientific Papers, North-Holland, Amsterdam, 1959, pp. 213-300.

[216] R.M. Lewis, A unifying principle in statistical mechanics, J. Math. Phys. 8 (1967) 1448-1460.

[217] A.J. Chorin, O.H. Hald, R. Kupferman, Optimal prediction with memory, Physica D 166 (2002) $239-257$.

[218] Y. Sone, Kinetic Theory and Fluid Dynamics, Birkhäuser, Boston, 2002.

[219] H.P. McKean Jr., J. Math. Phys. 8 (1967) 547.

[220] A.N. Gorban, V.I. Bykov, G.S. Yablonskii, Essays on Chemical Relaxation, Nauka, Novosibirsk, 1986.

[221] V.I. Verbitskii, A.N. Gorban, G.Sh. Utjubaev, Yu.I. Shokin, Moore effect in interval spaces, Dokl. AN SSSR. 304 (1) (1989) 17-21.

[222] V.I. Bykov, V.I. Verbitskii, A.N. Gorban, On one estimation of solution of Cauchy problem with uncertainty in initial data and right part, Izv. vuzov, Ser. Mat. N 12 (1991) 5-8. 
[223] V.I. Verbitskii, A.N. Gorban, Simultaneously dissipative operators and their applications, Sib. Mat. J. 33 (1) (1992) $26-31$.

[224] A.N. Gorban, Yu.I. Shokin, V.I. Verbitskii, Simultaneously dissipative operators and the infinitesimal Moore effect in interval spaces, Preprint, 1997, Preprint online: http://arXiv.org/abs/physics/9702021.

[225] A.N. Gorban, V.I. Bykov, G.S. Yablonskii, Thermodynamic function analogue for reactions proceeding without interaction of various substances, Chem. Eng. Sci. 41 (11) (1986) 2739-2745.

[226] P. Grassberger, On the Hausdorff dimension of fractal attractors, J. Stat. Phys. 26 (1981) 173-179.

[227] P. Grassberger, I. Procaccia, Measuring the strangeness of strange attractors, Physica D 9 (1983) 189-208.

[228] P. Frederickson, J.L. Kaplan, E.D. Yorke, J.A. Yorke, The Lyapunov dimension of strange attractors, J. Differential Equations 49 (1983) 185-207.

[229] F. Ledrappier, L.-S. Young, The metric entropy of diffeomorphisms: I. Characterization of measures satisfying Pesin's formula; II. Relations between entropy, exponents and dimensions, Ann. Math. 122 (1985) 509-539, $540-574$.

[230] H.G.E. Hentschel, I. Procaccia, The infinite number of generalized dimensions of fractals and strange attractors, Physica D: Nonlinear Phenomena 8 (3) (1983) 435-444.

[231] Yu.S. Ilyashenko, On dimension of attractors of $k$-contracting systems in an infinite dimensional space, Vest. Mosk. Univ. Ser. 1 Mat. Mekh. 3 (1983) 52-58.

[232] C. Nicolis, G. Nicolis, Is there a climate attractor? Nature 311 (1984) 529-532.

[233] C. Foias, O.P. Manley, R. Temam, An estimate of the Hausdorff dimension of the attractor for homogeneous decaying turbulence, Phys. Lett. A 122 (3-4) (1987) 140-144.

[234] C. Foias, G.R. Sell, R. Temam, Inertial manifolds for dissipative nonlinear evolution equations, J. Differential Equations 73 (1988) 309-353.

[235] R. Temam, Infinite-dimensional dynamical systems in mechanics and physics, Applied Mathematical Science, Vol. 68, Springer, New York, 1988. (Second Edition, 1997).

[236] C. Foias, G.R. Sell, E.S. Titi, Exponential tracking and approximation of inertial manifolds for dissipative nonlinear equations, J. Dyn. Differ. Equations 1 (1989) 199-244.

[237] M.I. Vishik, Asymptotic Behaviour of Solutions of Evolutionary Equations, Cambridge University Press, Cambridge, 1993.

[238] D.A. Jones, E.S. Titi, $C^{1}$ Approximations of inertial manifolds for dissipative nonlinear equations, J. Differential Equations 127 (1) (1996) 54-86.

[239] J.C. Robinson, Computing inertial manifolds, Discrete Contin. Dyn. Systems 8 (4) (2002) 815-833.

[240] P.D. Christofides, Nonlinear and Robust Control of Partial Differential Equation Systems: Methods and Applications to Transport-Reaction Processes, Birkhäuser, Boston, 2001.

[241] V.V. Chepyzhov, A.A. Ilyin, A note on the fractal dimension of attractors of dissipative dynamical systems, Nonlinear Anal. 44 (2001) 811-819.

[242] M. Marion, R. Temam, Nonlinear Galerkin methods, SIAM J. Numer. Anal. 26 (1989) 1139-1157.

[243] C. Jones, T. Kaper, N. Kopell, Tracking invariant manifolds up to exponentially small errors, SIAM J. Math. Anal. 27 (1996) 558-577.

[244] He. Yinnian, R.M.M. Mattheij, Stability and convergence for the reform postprocessing Galerkin method, Nonlinear Anal. Real World Appl. 4 (2000) 517-533.

[245] B. Garsia-Archilla, J. Novo, E.S. Titi, Postprocessing the Galerkin method: a novel approach to approximate inertial manifolds, SIAM J. Numer. Anal. 35 (1998) 941-972.

[246] L.G. Margolin, E.S. Titi, S. Wynne, The postprocessing Galerkin and nonlinear Galerkin methods-a truncation analysis point of view, SIAM J. Numer. Anal. (to appear) Online: http://math.uci.edu/\%7Eetiti/Publications/Wynne/ DPP/DPP_final_revised.pdf.

[247] N. Fenichel, Geometric singular perturbation theory for ordinary differential equations, J. Differential Equations 31 (1979) 59-93.

[248] C.K.R.T. Jones, Geometric singular perturbation theory, in: L. Arnold (Ed.), Dynamical Systems, Montencatini Terme, Lecture Notes in Mathematics, Vol. 1609, Springer, Berlin, 1994, pp. 44-118.

[249] R.B. Bird, J.M. Wiest, Constitutive equations for polymeric liquids, Annu. Rev. Fluid Mech. 27 (1995) 169.

[250] H.R. Warner, Kinetic theory and rheology of dilute suspensions of finitely extendible dumbbells, Ind. Eng. Chem. Fund. 11 (1972) 379. 
[251] C.W. Oseen, Ark. f. Mat. Astr. og Fys. 6 (29) (1910) 1.

[252] J.M. Burgers, Verhandelingen Koninkl. Ned. Akad. Wetenschap. 16 (Section 1, Chapter 3 ) (1938) 113.

[253] J. Rotne, S. Prager, Variational treatment of hydrodynamic interaction, J. Chem. Phys. 50 (1969) 4831.

[254] H. Yamakawa, Transport properties of polymer chain in dilute solution: hydrodynamic interaction, J. Chem. Phys. 53 (1970) 436.

[255] W. Noll, A mathematical theory of the mechanical behavior of continuous media, Arch. Rat. Mech. Anal. 2 (1958) 197.

[256] G. Astarita, G. Marrucci, Principles of Non-Newtonian Fluid Mechanics, McGraw-Hill, London, 1974.

[257] J.G. Oldroyd, Non-Newtonian effects in steady motion of some idealized elastico-viscous liquids, Proc. R. Soc. A 245 (1958) 278.

[258] M. Herrchen, H.C. Öttinger, A detailed comparison of various FENE dumbbell models, J. Non-Newtonian Fluid Mech. 68 (1997) 17.

[259] M. Kröger, Simple models for complex nonequilibrium fluids, Phys. Rep. 390 (6) (2004) 453-551.

[260] R.B. Bird, R.B. Dotson, N.J. Jonson, Polymer solution rheology based on a finitely extensible bead-spring chain model, J. Non-Newtonian Fluid Mech. 7 (1980) 213-235 Corrigendum 8 (1981) 193.

[261] B.W. Char, et al., Maple V Language Reference Manual, Springer, New York, 1991.

[262] T. Kato, Perturbation Theory for Linear Operators, Springer, Berlin, 1976.

[263] J.-L. Thiffeault, Finite extension of polymers in turbulent flow, Phys. Lett. A 308 (5-6) (2003) 445-450.

[264] A.N. Gorban, P.A. Gorban, I.V. Karlin, Legendre integrators, post-processing and quasiequilibrium, J. Non-Newtonian Fluid Mech. (2004). Preprint on-line: http://arxiv.org/pdf/cond-mat/0308488, to appear.

[265] Th.T. Perkins, D.E. Smith, S. Chu, Single polymer dynamics in an elongational flow, Science 276 (5321) (1997) 2016-2021.

[266] P.G. De Gennes, Molecular individualism, Science 276 (5321) (1997) 1999-2000.

[267] D.E. Smith, H.P. Babcock, S. Chu, Single-polymer dynamics in steady shear flow, Science 283 (1999) $1724-1727$.

[268] V.I. Arnold, A.N. Varchenko, S.M. Gussein-Zade, Singularities of Differentiable Maps, 2 Vols, Brickhäuser, Boston, 1985-1988.

[269] J.S. Langer, M. Bar-on, H.D. Miller, New computational method in the theory of spinodal decomposition, Phys. Rev. A 11 (4) (1975) 1417-1429.

[270] M. Grant, M. San Miguel, J. Vinals, J.D. Gunton, Theory for the early stages of phase separation: the long-range-force limit, Phys. Rev. B 31 (5) (1985) 3027-3039.

[271] V. Kumaran, G.H. Fredrickson, Early stage spinodal decomposition in viscoelastic fluids, J. Chem. Phys. 105 (18) (1996) 8304-8313.

[272] G. Lielens, P. Halin, I. Jaumin, R. Keunings, V. Legat, New closure approximations for the kinetic theory of finitely extensible dumbbells, J. Non-Newtonian Fluid Mech. 76 (1998) 249-279.

[273] P. Ilg, I.V. Karlin, H.C. Öttinger, Generating moment equations in the Doi model of liquid-crystalline polymers, Phys. Rev. E 60 (1999) 5783-5787.

[274] N. Phan-Thien, C.G. Goh, J.D. Atkinson, The motion of a dumbbell molecule in a torsional flow is unstable at high Weissenberg number, J. Non-Newtonian Fluid Mech. 18 (1) (1985) 1-17.

[275] C.G. Goh, N. Phan-Thien, J.D. Atkinson, On the stability of a dumbbell molecule in a continuous squeezing flow, J. Non-Newtonian Fluid Mech. 18 (1) (1985) 19-23.

[276] I.V. Karlin, P. Ilg, H.C. Öttinger, Invariance principle to decide between micro and macro computation, in: F. Uribe (Ed.), Recent Developments in Mathematical and Experimental Physics, Vol. C: Hydrodynamics and Dynamical Systems, Kluwer, Dordrecht, 2002, pp. 45-52.

[277] J. Novo, E.S. Titi, S. Wynne, Efficient methods using high accuracy approximate inertial manifolds, Numer. Math. 87 (2001) 523-554.

[278] M.W. Hirsch, C. Pugh, M. Shub, Invariant manifolds, in: Lecture Notes in Mathematics, Vol. 583, Springer, Berlin, 1977.

[279] Cr. Jones, S. Winkler, Do invariant manifolds hold water? in: B. Fiedler, G. Iooss, N. Koppell (Eds.), Handbook of Dynamical Systems III: Towards Applications, World Scientific, Singapore, to appear. Online: http://www.cfm.brown.edu/people/sean/JonesWinkler.ps.zip. 